
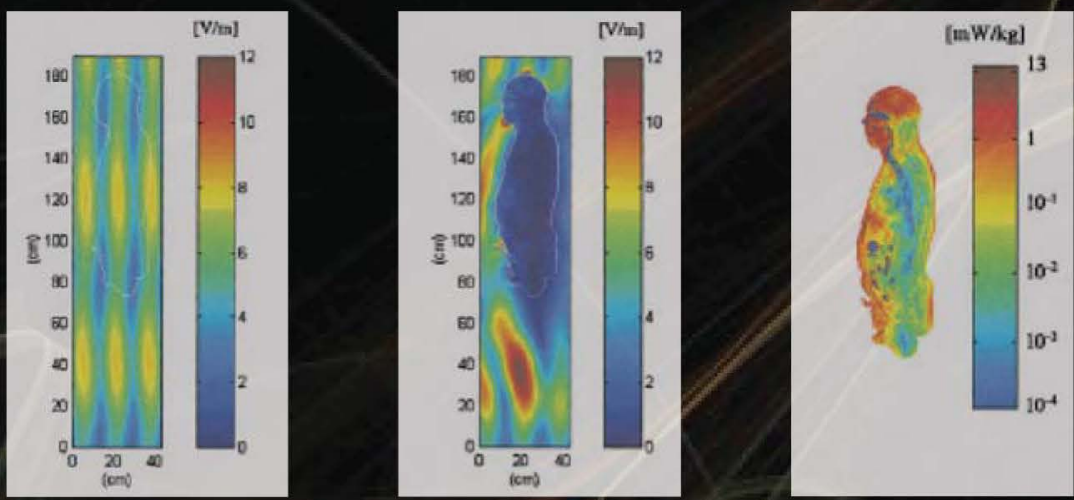

\title{
ELECTROMAGNETIC FIELDS IN BIOLOGICAL SYSTEMS
}
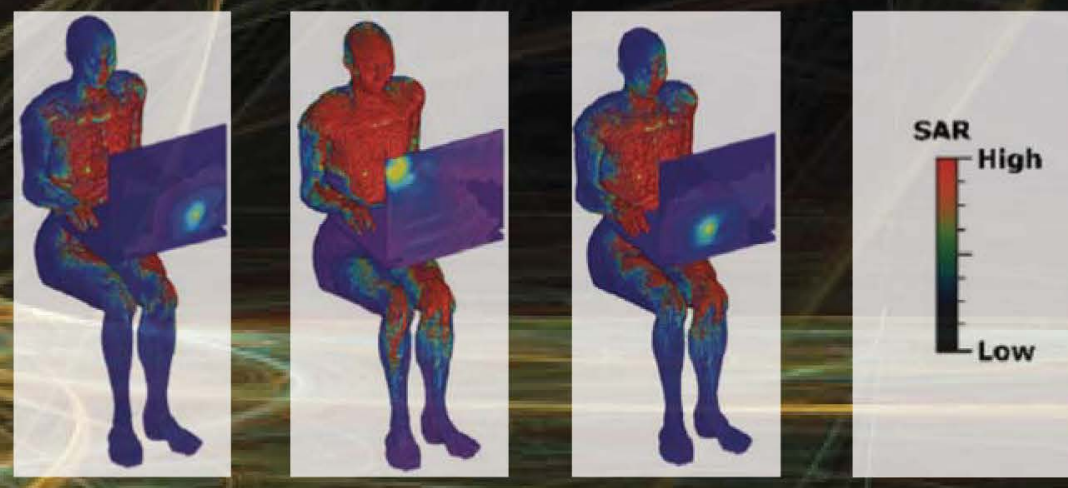

\section{EDITED BY JAMES C. LIN}




\section{ELECTROMAGNETIC FIELDS IN BIOLOGICAL SYSTEMS}

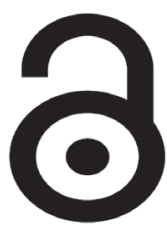




\title{
Biological Effects of Electromagnetics Series
}

\author{
Series Editors \\ Frank Barnes \\ University of Colorado \\ Boulder, Colorado, U.S.A. \\ Ben Greenebaum \\ University of Wisconsin-Parkside \\ Somers, Wisconsin, U.S.A.
}

Electromagnetic Fields in Biological Systems, edited by James C. Lin

The Physiology of Bioelectricity in Development, Tissue Regeneration, and Cancer, edited by Christine E. Pullar

Advanced Electroporation Techniques in Biology and Medicine, edited by Andrei G. Pakhomov, Damijan Miklavčič, and Marko S. Markov 


\section{ELECTROMAGNETIC FIELDS IN BIOLOGICAL SYSTEMS}

\section{EDITED BY \\ JAMES C. LIN}

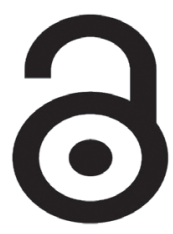


CRC Press

Taylor \& Francis Group

6000 Broken Sound Parkway NW, Suite 300

Boca Raton, FL 33487-2742

(C) 2012 by Taylor \& Francis Group, LLC

CRC Press is an imprint of Taylor \& Francis Group, an Informa business

No claim to original U.S. Government works

Printed in the United States of America on acid-free paper

Version Date: 20110707

International Standard Book Number: 978-1-4398-5999-5 (Hardback)

This book contains information obtained from authentic and highly regarded sources. Reasonable efforts have been made to publish reliable data and information, but the author and publisher cannot assume responsibility for the validity of all materials or the consequences of their use. The authors and publishers have attempted to trace the copyright holders of all material reproduced in this publication and apologize to copyright holders if permission to publish in this form has not been obtained. If any copyright material has not been acknowledged please write and let us know so we may rectify in any future reprint.

The Open Access version of this book, available at www.taylorfrancis.com, has been made available under a Creative Commons Attribution-Non Commercial-No Derivatives 4.0 license.

For permission to photocopy or use material electronically from this work, please access www.copyright. com (http://www.copyright.com/) or contact the Copyright Clearance Center, Inc. (CCC), 222 Rosewood Drive, Danvers, MA 01923, 978-750-8400. CCC is a not-for-profit organization that provides licenses and registration for a variety of users. For organizations that have been granted a photocopy license by the CCC, a separate system of payment has been arranged.

Trademark Notice: Product or corporate names may be trademarks or registered trademarks, and are used only for identification and explanation without intent to infringe.

\section{Library of Congress Cataloging-in-Publication Data}

Electromagnetic fields in biological systems / editor, James C. Lin. p. ; cm. -- (Biological effects of electromagnetics series)

Includes bibliographical references and index.

ISBN 978-1-4398-5999-5 (hardcover : alk. paper)

1. Electromagnetism--Physiological effect. I. Lin, James C. II. Series: Biological effects of electromagnetics series.

[DNLM: 1. Electromagnetic Fields--adverse effects. 2. Radiation, Nonionizing. QT 162.U4]

QP82.2.E43.E453 2011

612 '.01442--dc23

2011020823

Visit the Taylor \& Francis Web site at
http://www.taylorandfrancis.com

and the CRC Press Web site at

http://www.crcpress.com 


\section{Contents}

Preface

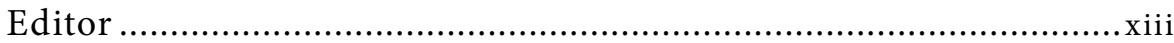

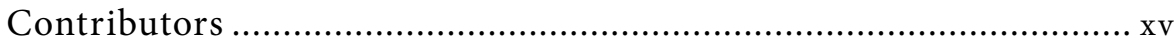

1 Coupling of Electromagnetic Fields into Biological Systems........1 James C. Lin

2 Pulsed Electric Fields in Biological Cells and Membranes .71 R. P. Joshi and K. H. Schoenbach

3 Static, Low-Frequency, and Pulsed Magnetic Fields in Biological Systems.

Shoogo Ueno and Hideyuki Okano

4 Interaction of Extremely Low-Frequency Electromagnetic Fields with Biological Systems.

Tsukasa Shigemitsu and Kenichi Yamazaki

5 Mobile Communication Fields in Biological Systems 261 Konstantina S. Nikita and Asimina Kiourti

6 Medical Devices and Systems Exposure and Dosimetry. N. Leitgeb

7 Terahertz Radiation: Sources, Applications, and Biological Effects

Gerald J. Wilmink and Jessica E. Grundt

Index 



\section{Preface}

The topic of interactions of electromagnetic fields with biological systems is not only drawing attention from research scientists but also capturing interest from members of the general public. In the United States, the National Center for Complementary and Alternative Medicine, which is part of the National Institutes of Health, conducts and supports research that is generally not considered part of conventional or western medicine. The scope of research conducted by the Center is very broad and includes a group of diverse medical and health-care practices and treatments. In particular, the institution has provided research support for several projects investigating the therapeutic effectiveness of electromagnetic energy in the millimeter wavelength (mmW) region.

The use of nonionizing electromagnetic energy (in the mode of fields, waves, or radiation) for medical purposes dates back to the late 1800s. Shortly after Heinrich Hertz's confirmation of James C. Maxwell's prediction of the existence of electromagnetic waves, Arsene d'Arsonval applied the newly discovered high-frequency $(10 \mathrm{kHz})$ current on himself and found that it produced warming of muscles without any accompanying sensation of muscular contraction. Indeed, the application of electromagnetic fields in biology and medicine has a long and illustrious history of research that continues to this date. Perhaps the most prominent application today is its use in medicine for magnetic resonance imaging and spectroscopy.

Moreover, interest in wireless communication and widespread use of wireless communication devices such as cellular mobile telephones have grown significantly all over the world during the past two decades. As a consequence, for the first time in human history a source of electromagnetic radiation has come to be located right next to the head or the body. This trend will likely continue and it promises to expand at a greater rate in the near future. Almost all human beings will soon become immersed a vast milieu of electromagnetic fields, waves, and radiation generated by human activity. Aside from cellular mobile telephones, such fields may come from, among others, the hubs (base stations) that enable wireless connections of all mobile and location-fixed devices, smart grids associated with the distribution and delivery of electricity, and global positioning systems (GPSs) employed for navigation to enable and facilitate mobility with safety and reliability.

Without question, human-made electromagnetic radiation will increase both in strength and in the spread of the frequency spectrum. The fact that electromagnetic fields and radiation can interact with biological systems is beyond debate. For instance, 
nuclear magnetic resonance takes place in biological medium under the influence of a radiofrequency $(\mathrm{RF})$ field to allow imaging and spectroscopy of tissues inside the human body. Clearly, a biological effect is a prerequisite to any potential medical application of electromagnetic fields. However, an unintended or deleterious biological effect of electromagnetic fields and radiation may indicate grounds for health and safety precautions in the communication, industrial, scientific, and medical use of electromagnetic fields and radiation. Regardless, to help advance knowledge of the biological effects of such fields and to exploit their potential medical applications, it is essential to describe the characteristics of not only the applied electromagnetic fields and radiation but also the resulting electromagnetic fields and radiation inside the biological system.

A quantitative relationship between applied and induced electromagnetic fields and radiation would relate them to specific responses of the biological system. It should also facilitate an understanding of the biological responses. The induced field is the primary source of energy driving the interaction of electromagnetic energy with the biological system. Although it may contribute to the formulation of mechanisms of interaction, it is independent of any mechanism of interaction. Moreover, knowledge of applied and induced fields would aid in analyzing relationships among various observed biological effects in different experimental models and subjects. It could also serve as an index for comparison and extrapolation of experimental results from cell to cell, tissue to tissue, and tissue to animal, and from animal to animal, animal to human, and human to human exposures.

Experiments designed to study the interaction of electromagnetic fields and radiation with biological systems and the possible effects of such fields on the systems can be divided into three categories: (1) in vitro biological experiments, (2) in vivo animal experiments, and (3) laboratory or epidemiological studies on humans. In vitro biological experiments typically involve biological entities constituted by cells contained within flasks or petri dishes and exposed to well-defined electromagnetic fields and radiation. These experiments are most suited to study the possible effects of exposure on specific biological targets or to study postulates and verify proposed interaction mechanisms aimed toward explaining observed biological responses. Epidemiological studies can offer the most direct evidence on the health effects of human exposure to electromagnetic fields and radiation. Apart from the difficulties faced in exposure assessment, that is, the quantification of levels of applied and induced electromagnetic fields and radiation, which is rather difficult if not impossible to obtain with a high degree of accuracy, the major limitation of epidemiological studies is the prolonged period of time typically required for observation in most cases and the related confounding factors.

Moreover, the implications of any effect obtained at the cellular level from in vitro investigations are not always obvious in terms of health effects on the whole organism. Thus, it is often necessary to conduct in vivo experiments, where whole animals, such as mice and rats, are directly exposed to electromagnetic fields and radiation and the potential for induction of specific health effects is studied. In vivo experiments are not only important in assessing possible health effects of electromagnetic fields and the thresholds for their induction but also useful in allowing extrapolation of animal observations to human subjects, provided knowledge is available to specify the relationship between applied and induced electromagnetic fields and radiation. 
The objective of this book is to provide a comprehensive discussion of the interactions of electromagnetic fields and radiation with biological systems, spanning a variety of topics from static fields to terahertz waves in seven chapters. Each chapter includes materials written by scientists who have made major contributions to the relevant subjects. Particular emphasis is placed on the coupling of electromagnetic fields and radiation into biological systems. Each chapter focuses on induced fields and absorbed energy from applied or exposure fields, and a review of the literature is included to explain the motivation for writing the chapter. The relevant literature is summarized so that the reader can understand why the topic is of interest or importance and this summary discusses current progress on the subject. The aim is to achieve a quantitative understanding of the relationships between the applied and induced electromagnetic fields and radiation that cause biological effects and enable medical applications.

A wide range of analytical techniques, computational algorithms, and/or experimental methods may be employed to determine the coupling of electromagnetic fields and radiation into biological systems. Hybrid approaches involving both analytical and numerical methods have been used. Although this book spotlights advanced experimental and computational techniques and current results on isolated cells and realistic anatomical models, it begins with a brief introduction of Maxwell's equations, which is the fundamental mathematical statement of the physical laws that govern all electromagnetic phenomena. It is significant to note that although most theories of classical physics were fundamentally modified as a result of the introduction of Albert Einstein's special theory of relativity, Maxwell's equations have remained consistent and intact over the years in describing the known physical phenomena over the entire experimentally observed nonionizing electromagnetic spectrum.

Knowledge of internal electric and magnetic fields, induced current densities, and specific absorption rates (SAR) inside the biological medium is fundamental in studying biological responses to, health effects of, and medical applications of electromagnetic fields and radiation. The discussions in Chapter 1 provide a basic understanding of essential interactions and field coupling phenomena to facilitate better appreciation and understanding of their use and importance. Electromagnetic energy at both high and low frequencies can be transmitted into a biological medium. Using canonical geometries, phantom models, and anatomically based representations of the human body, results obtained from closed-form analytical solutions and computer methods to predict the internal fields and their distributions are discussed and salient features are summarized. Specifically, they include induced static electric and magnetic fields, transmission of low-frequency and quasistatic electric and magnetic fields, transmission and reflection of RF fields at planar interfaces, RF coupling to bodies with curvature and in the near field, dosimetry and energy absorption from handheld cell phones in anatomical human models, SAR in childlike head models, fields from body-worn devices, and whole-body exposure from cell phone base stations. The coupling of short (narrowwidth) and ultra-wideband (UWB) pulses into the human body is analyzed for planar, spherical, and full-scale anatomical models. Recent advances in mmW technologies and beyond have motivated a wide range of telecommunication, industrial, medical, and scientific applications including security screening in the form of whole-body image 
scanning. The chapter concludes with a summary of available information on transmitted, reflected, and distributed mmW energy in skin tissues.

Short electric pulses can extend deeply into the cell interior (see Chapter 2) and have been briefly discussed in Chapter 1. The classical understanding of a majority of the electric field induced biological effect is the induction of a potential difference across the cell membrane by the external electric field. For example, the electric field strength required to achieve electroporation depends on the duration of the applied pulse, because this process involves the gradual charging of the cell membrane followed by rearrangement of the lipid molecules. The typical pulses range from tens of milliseconds (ms) with amplitudes in the $10 \mathrm{kV} / \mathrm{m}$ ranges to pulses of a few microseconds (ms) or less at field strengths of several $100 \mathrm{kV} / \mathrm{m}$. More recently, the electrical pulses in the nanosecond (ns) duration range and pulse amplitudes as high as $30,000 \mathrm{kV} / \mathrm{m}$ have been investigated for triggering purely electrically driven responses without any thermal heating. Such fast processes as electron transfers between molecules, electrophoretic separation and selforganization, or field induced changes in reaction kinetics are being explored. Chapter 2 presents many potential applications based on intracellular effects produced by the use of nanosecond, pulsed electric fields (nsPEF) of high-intensity. It is anticipated that nsPEF may provide versatile non-thermal tools capable of producing cellular electroporation, intra-cellular calcium release, shrinkage of tumors and cellular apoptosis, temporary blockage of action potential propagation in nerves, activation of platelets, and release of growth factors for accelerated wound healing.

Exposure to naturally occurring and human-made static, low-frequency, and pulsed magnetic fields in biological systems forms the topic of Chapter 3. It is of interest because changes in the strength and distribution of such fields have been found to be biologically effective; many questions have been raised as to whether exposure to these fields may be linked to adverse health effects, and a wide range of biomedical applications have been developed to take advantage of the interaction of magnetic fields with biological systems. In addition to discussing the coupling of static, low-frequency, and pulsed magnetic fields into biological systems, the chapter summarizes more recent information on biological effects and medical applications of such fields and discusses mechanisms by which biological systems sense and respond to magnetic fields.

During the past few decades, the interaction of extremely low frequency (ELF) electromagnetic fields with biological systems has become a major source of health concern, especially for the 50 and $60 \mathrm{~Hz}$ frequencies used by electric power distribution systems. An essential aspect of health investigations has been the evaluation of induced electric fields and current densities inside human subjects and phantom models of human and animal bodies. Chapter 4 is devoted to dosimetry or coupling of ELF fields into biological systems. It provides a description of the historical developments and recent trends in numerical dosimetry. It also includes a comprehensive review of research efforts from Japan on induced electric fields and current densities inside phantom models resulting from ELF exposures.

Cellular communication has evolved into one of the most successfully commercialized technologies. Today, the immense popularity of cell phones is beyond debate. Worldwide, there are more people who own and use cell or mobile phones than any other electronic device, regardless of whether the country is rich or poor, developed 
or developing. Some predict that the entire world population will soon be exposed to $\mathrm{RF}$ electromagnetic fields or radiation from sources located near the human body that enable wireless communication. The coupling or dosimetry of RF radiation into the body in presented in Chapter 5. It discusses numerical tools and experimental methods that may be used to model and assess the interaction of mobile communication devices with the human body. Given the proximity of the cell phone handset to the human head in common use, the interaction between the cell phone and the user's head has been extensively studied. Moreover, there is increasing scientific interest in assessing human exposure to base station antennas, local area networks, and newly emerging mobile communication technologies. These scenarios suggest continuous and simultaneous exposure to multiple sources of RF and microwave radiation over an extended period of time or even the entire lifetime of the world population.

Electromagnetic fields and waves are widely used in medicine, and they are playing progressively more important roles in health care each day. The medical applications are based on either the direct interaction of the field with biological tissues to illicit functional responses or the conversion of field energy to heat through the delivery of electromagnetic energy into the treatment region. They enable the acquisition of diagnostic information such as distribution and binding status of nuclei in magnetic resonance imaging or spectroscopy. The applications encompass a wide range of electromagnetic frequencies, ranging from static to ELF and RF fields. In addition, they may involve rapidly changing field transients and spatial gradients, depending on the specific application. The exposure and dosimetry associated with these medical devices and systems are explained in Chapter 6 . This chapter also includes discussions on the unintentional exposure of patients or staff or both through the coupling of stray electromagnetic fields from medical devices.

An emerging area of significant research activity over the past decade has been the terahertz region of the electromagnetic spectrum because of the development of highpower terahertz sources. While there is a paucity of biological studies at terahertz frequencies, information on exposure and dosimetry is even scarcer. However, terahertz sources are aggressively being explored for practical medical, military, and security applications. Current examples include early cancer detection and diagnosis; identification of concealed explosives, drugs, and weapons; and terahertz imaging and sensing techniques for security screening. Chapter 7 reviews available data on the biological effects of terahertz radiation and discusses the current understanding of the physical events that transpire when terahertz radiation interacts with biological tissues, cells, and organelles.

The editor thanks the authors for their intellectual contributions; Sarah Coffey for valuable assistance in preparing the manuscripts for this volume; Michael Slaughter, executive editor; and Jessica Vakili, production coordinator, editorial project development of CRC Press/Taylor \& Francis for their interest, and Frank Barnes and Ben Greenebaum, serial editors for their support in publishing this project. 



\section{Editor}

Dr. James C. Lin is a professor of electrical engineering, bioengineering, physiology, and biophysics at the University of Illinois in Chicago, where he has served as head of the Bioengineering Department, director of the Robotics and Automation Laboratory, and director of special projects in the College of Engineering.

$\mathrm{He}$ is a fellow of the American Association for the Advancement of Science, the American Institute for Medical and Biological Engineering, and the Institute of Electrical and Electronics Engineers (IEEE). He held an NSC research chair from 1993 to 1997 and is an IEEE-Engineering in Medicine and Biology Society (EMBS) distinguished lecturer. He is a recipient of the d'Arsonval Medal from the Bioelectromagnetics Society, IEEE EMC Transactions Prize Paper Award, IEEE COMAR Recognition Award, CAPAMA Outstanding Leadership and Service Awards, and UIC Best Engineering Advisor Award. He received his BS, MS, and PhD from the University of Washington, Seattle.

Dr. Lin has served in leadership positions of several scientific and professional organizations including president of the Bioelectromagnetics Society, chairman of the International Scientific Radio Union (URSI) Commission on Electromagnetics in Biology and Medicine, chairman of the IEEE Committee on Man and Radiation, vice president of the U.S. National Council on Radiation Protection and Measurements (NCRP) and chairman of its Committee on Biological Effects and Exposure Criteria for Radiofrequency Fields, and chairman of the International Commission on Non-Ionizing Radiation Protection (ICNIRP) Scientific Committee. He has served on numerous advisory committees and panels for the Office of the U.S. President, National Academy of Sciences, National Research Council, National Science Foundation, National Institutes of Health, Marconi Foundation (Italy), and the World Health Organization.

Dr. Lin has authored or edited nine books and authored more than 170 journal papers and book chapters. In addition, his columns with more than 100 articles on the health and safety of wireless mobile communication radiation appear in four professional magazines. He is the editor-in-chief of the Bioelectromagnetics Journal and has served as editor and member on the editorial boards of several journals. He is a member of the Sigma Xi, Phi Tau Phi, Tau Beta Pi, and Golden Key honorary societies, and is listed in American Men and Women of Science, Who's Who in America, Who's Who in Engineering, Who's Who in the World, and Men of Achievement. 



\section{Contributors}

Jessica E. Grundt

711th Human Performance Wing

Radio Frequency Radiation Branch

Air Force Research Laboratory

Brooks City-Base, Texas

\section{R. P. Joshi}

Department of Electrical and Computer Engineering and

Frank Reidy Research Center for Bioelectrics

Old Dominion University

Norfolk, Virginia

\section{Asimina Kiourti}

School of Electrical and Computer Engineering

National Technical University of Athens Athens, Greece

\section{N. Leitgeb}

Institute of Health Care Engineering European Notified Body of Medical Devices Graz University of Technology

Graz, Austria

James C. Lin

Department of Electrical and Computer Engineering and Department of Bioengineering University of Illinois

Chicago, Illinois
Konstantina S. Nikita

School of Electrical and Computer Engineering

National Technical University of Athens

Athens, Greece

\section{Hideyuki Okano}

Research Center for Frontier Medical Engineering

Chiba University

Chiba, Japan

K. H. Schoenbach

Department of Electrical and Computer Engineering and

Frank Reidy Research Center for

Bioelectrics

Old Dominion University

Norfolk, Virginia

Tsukasa Shigemitsu

Japan EMF Information Center

Tokyo, Japan

\section{Shoogo Ueno}

Department of Applied Quantum

Physics

Graduate School of Engineering

Kyushu University

Fukuoka, Japan 


\section{Gerald J. Wilmink}

711th Human Performance Wing Radio Frequency Radiation Branch Air Force Research Laboratory

Brooks City-Base, Texas

\section{Kenichi Yamazaki}

Central Research Institute of Electric

Power Industry

Yokosuka Research Laboratory

Kanagawa, Japan 


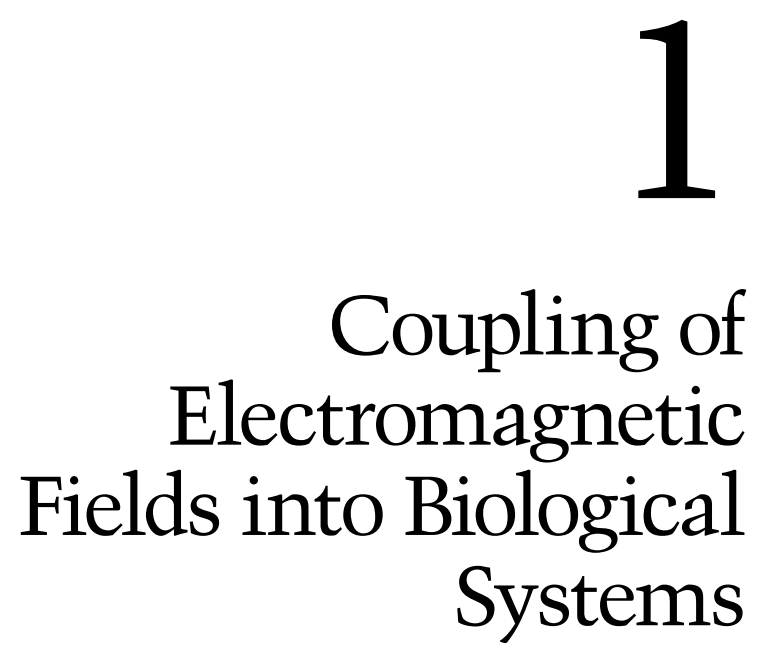

1.1 Introduction 2

1.2 Physical Laws Governing

Electromagnetic Phenomena .................................... 3

1.3 Electromagnetic Properties of Tissue...................... 5

1.4 Electromagnetic Phenomena at Tissue Interfaces 6

1.5 Static Electric and Magnetic Fields........................ 7

1.6 Time-Varying Electromagnetic Fields .................. 9

1.7 Low-Frequency and Quasistatic Electric and Magnetic Fields 10

1.8 Propagation of Electromagnetic Energy from Antennas

Quasistatic Fields of a Dipole Antenna • Near Field of a Dipole Antenna - Far Field of a Dipole Antenna

1.9 Coupling of Quasistatic Electric and Magnetic Fields 16 Quasistatic Electric Field Coupling • Quasistatic Magnetic Field Coupling - Combined Quasistatic Electric and Magnetic Fields - Summary of Quasistatic and Low-Frequency Field Coupling

1.10 Radiofrequency Fields and Energy Deposition. .20 Radiofrequency Reflection and Transmission at Planar Interfaces - Radiofrequency Field Coupling to Bodies with Curvature - Orientation and Polarization Dependence - Radiofrequency Coupling in the Near Field 
1.11 Radiofrequency Dosimetry and Energy Absorption in Anatomical Models.... .25

Coupling from Handheld Mobile

Phones - Specific Absorption Rate in Childlike

Head Models • Fields from Body-Worn

Devices - Whole-Body Exposure from Cell-Phone

Base Stations - Summary of Radiofrequency Field

Coupling

1.12 Coupling of Short and Ultra-Wideband

Pulses into the Human Body

Induced Electromagnetic Pulse Fields in Biological

Bodies - Gaussian Electromagnetic Pulse

Inside a Planar Biological Medium - Gaussian

Electromagnetic Pulse inside Spherical Head

Models - Constant Conductivity Spherical Model

for Electromagnetic Pulse Propagation - Induced

Ultra-Wideband Field and Current - Summary of

Short and Ultra-Wideband Pulse Coupling

1.13 Coupling of Millimeter and Terahertz Waves ... 59

Transmitted and Reflected Millimeter

Waves and Terahertz Fields, and Energy

Deposition - Summary of Millimeter-Wave

Coupling

References. .63

\subsection{Introduction}

Electromagnetic fields or energies of frequencies that range from $0 \mathrm{~Hz}$ to $1 \mathrm{THz}(1 \mathrm{THz}=$ $10^{12} \mathrm{~Hz}$ ) have wavelengths longer than $1 \mu \mathrm{m}$ in air. Furthermore, at wavelengths close to the micrometer limit of the spectrum, electromagnetic energy behaves as infrared radiation. These wavelengths produce photons (or quanta) of low energy; therefore, under ordinary circumstances, the energy in a photon is too low to break chemical bonds, excite electrons, or produce ionization of biological molecules. Consequently, they are often referred to as low-energy or nonionizing radiation. Electromagnetic energies propagate through a material medium (including biological medium) at a constant speed in that medium. For example, they propagate through air or vacuum at the speed of light, that is, at $2.998 \times 10^{8} \mathrm{~m} / \mathrm{s}$. Moreover, electromagnetic energies with wavelengths longer than $10 \mathrm{~m}$ (frequencies lower than $30 \mathrm{MHz}$ ) have interaction properties that differ greatly from those of wavelengths that are approximately equal to or less than the physical dimensions of a human body.

Although living organisms thrive in a natural electromagnetic environment, they are increasingly subjected to a myriad of human-made nonionizing radiation in the form of electromagnetic fields and waves for telecommunication uses, industrial and medical applications, and many other purposes. For example, the radiofrequency (RF) band of $300-30,000 \mathrm{kHz}$ (or $0.3-30 \mathrm{MHz}$ with wavelengths from $1000 \mathrm{~m}$ to $10 \mathrm{~m}$ ) is used in medicine for ablating, coagulating, and cauterizing tissue. Recently, nanosecond-pulsed electric fields were shown to induce long-lasting plasma membrane permeabilization changes. They are providing new insights into the nature of pulsed electric field induced 
opening of conductance pores and into molecular mechanisms that underlie biological effects. The technological breakthrough at millimeter-wave $(\mathrm{mmW})$ and terahertz frequencies has stimulated new applications not only in biology and medicine but also in environmental studies, material science, telecommunication, and security screening in the form of whole-body image scans. Besides their primary intended roles, these fields and waves produce other effects that may influence the vital activities of a biological system. The changes produced depend on many physical and biological factors. They may or may not be grossly apparent and observable soon after exposure of the living organism.

The biological effects of electromagnetic fields and waves have been a subject of scientific research since the discovery of electromagnetic radiation and its first use in therapeutic applications more than 100 years ago. Since then, our knowledge regarding its effects on health has increased tremendously. Nevertheless, they have become the focus of much attention because of the expansion and distribution of electric power at 50 and $60 \mathrm{~Hz}$ in the extremely-low-frequency (ELF) spectrum (between $3 \mathrm{~Hz}$ and $3 \mathrm{kHz}$ ) and because of the accelerated use of $\mathrm{RF}$ radiation $(300 \mathrm{MHz}-6 \mathrm{GHz}$ and beyond) in wireless communication in recent decades. A notable reason for the increased attention on the subject is the uncertainty and lack of understanding of the mechanism of interaction of electromagnetic fields and waves with biological systems. Although ELF fields and $\mathrm{RF}$ radiation are all part of the same known electromagnetic spectrum, the mode of coupling into biological tissues and mechanism of interaction can be quite different for the two. This chapter discusses the coupling of electromagnetic fields and waves into biological systems. Clearly, regardless of the mechanism of interaction fields must be coupled into the system and energy must be transferred, absorbed, or deposited in the biological system in order for the system to respond in some manner. Thus, to gain a greater knowledge of biological responses, the electric, magnetic, or electromagnetic field that is effective in exerting its influence must be quantified and correlated with the observed effect.

This chapter is intended to provide a common understanding of essential interactions and field coupling phenomena to facilitate better appreciation and understanding of their importance in research on biological effects and in scientific, industrial, and medical applications. For further information, the reader is referred to the book series on Advances in Electromagnetic Fields in Living Systems edited by Lin (1994, 2009).

\subsection{Physical Laws Governing Electromagnetic Phenomena}

Electromagnetic phenomena consist of electric and magnetic fields that change with space and time. Their spatial variation is dictated by the electromagnetic properties of the material medium, that is, electrical permittivity and magnetic permeability. The physical interactions of electromagnetic fields with biological systems are defined by laws describing their characteristics and behavior in biological systems and other material media. These mathematical expressions are commonly known as Maxwell's equations (Maxwell 1904). Maxwell's equations are laws that define the relationship 
between spatially and temporally averaged electric and magnetic fields. They are relevant in regions or volumes whose dimensions are larger than atomic dimensions. The time intervals of observation are typically long enough to allow the averaging of atomic fluctuations. Solutions to Maxwell's equations, with appropriate boundary conditions, prescribe the behavior of electric and magnetic fields in material media. These equations are valid for linear or nonlinear, isotropic or anisotropic, and homogeneous or inhomogeneous media in the frequency range from $0 \mathrm{~Hz}$ to $1 \mathrm{THz}$.

Maxwell's four equations may be specified in either integral or differential form. They are specified in the integral form since these lend to simple physical interpretations. The differential equations may be derived by using Stokes' and divergence theorems from vector analysis (Jordan and Balmain 1968):

$$
\begin{gathered}
\oint \mathbf{E} \times \mathrm{d} l=-\int_{s}(\partial \mathbf{B} / \partial t) \times \mathrm{d} s \\
\oint \mathbf{H} \times \mathrm{d} l=\int_{s}[\mathbf{J}+(\partial \mathbf{D} / \partial t)] \times \mathrm{d} s \\
\oint \mathbf{D} \times \mathrm{d} s=\int_{v} \rho \mathrm{d} v \\
\oint \mathbf{B} \times \mathrm{d} s=0
\end{gathered}
$$

where

$$
\begin{aligned}
& \mathbf{E}=\text { electric field strength (volt/meter) } \\
& \mathbf{H}=\text { magnetic field strength (ampere/meter) } \\
& \mathbf{D}=\text { electric flux density (coulomb/square meter) } \\
& \mathbf{B}=\text { magnetic flux density (tesla) } \\
& \mathbf{J}=\text { conduction current density (ampere/square meter) } \\
& \rho=\text { electric charge density (coulomb/cubic meter) } \\
& v=\text { volume } \\
& s=\text { surface }
\end{aligned}
$$

It can be seen from the right-hand side of Equations 1.2 and 1.3 that the sources of electromagnetic fields and waves are charges and currents.

Moreover, according to Equation 1.1, also known as Faraday's law, the total voltage induced in an arbitrary closed path is equal to the time rate of decrease of magnetic flux through the area bounded by the closed path. Therefore, a time-varying magnetic field generates an electric field. There is no restriction on the nature of the medium.

Equation 1.2, the Ampere-Maxwell law, states that the sum or line integral of magnetic field strength around a closed path is equal to the total current enclosed by the path. The total current may consist of two types of currents: (1) conduction current with density J and (2) displacement current with density $\partial \mathbf{D} / \partial t$. Thus, Ampere's law implies that a magnetic field can be produced only by the flow of current or movement of charges. The displacement current was introduced by James Clerk Maxwell to join the separate laws that govern electricity and magnetism into a unified electromagnetic theory. It also 
led to the postulate that electromagnetic waves can transport energy and the hypothesis that light is an electromagnetic wave.

Equation 1.3 is Gauss' electric law, which states that the net outward flow of electric flux through a closed surface is equal to the charge contained in the volume enclosed by the surface. Likewise, Gauss' law for magnetic fields (Equation 1.4) states that the net outward flow of magnetic flux through a closed surface is zero. Therefore, magnetic flux lines are always continuous and they form closed loops.

The auxiliary equations that bridge the fields and flux densities produced by a given current or charge distribution are

$$
\begin{aligned}
& \mathbf{D}=\varepsilon \mathbf{E} \\
& \mathbf{B}=\mu \mathbf{H} \\
& \mathbf{J}=\sigma \mathbf{E}
\end{aligned}
$$

Free space or vacuum is a medium in which the permittivity, $\varepsilon$, is given by

$$
\varepsilon_{0}=8.854 \times 10^{-12}=1 /(36 \pi) \times 10^{-9} \mathrm{~F} / \mathrm{m}
$$

Free space permeability, $\mu$, is given by

$$
\mu_{0}=4 \pi \times 10^{7} \mathrm{H} / \mathrm{m}
$$

Finally, the electrical conductivity for free space is $\sigma=0$. For all other linear, isotropic, and homogeneous media, it is the convention to introduce the following dimensionless ratios:

$$
\begin{gathered}
\varepsilon_{\mathrm{r}}=\varepsilon / \varepsilon_{0} \\
\mu_{\mathrm{r}}=\mu / \mu_{0}
\end{gathered}
$$

Equations 1.10 and 1.11 give the relative dielectric constant and relative permeability, respectively. Living matters generally have relative permeability equal to that of free space, with the exception of cells, molecules, or organisms endowed with ferromagnetic particles. However, the relative dielectric constants show characteristic dependence on frequency and material medium.

\subsection{Electromagnetic Properties of Tissue}

Typically, dielectric constants decrease and conductivities increase with increasing frequency (Figure 1.1). Biological materials exhibit very high dielectric constants, especially at low frequencies, compared to other homogeneous solids and liquids. This is because biological tissues are composed of macromolecules, cells, and other membrane-bound substances. Mobile counterions are associated with charges on cell membranes, and 


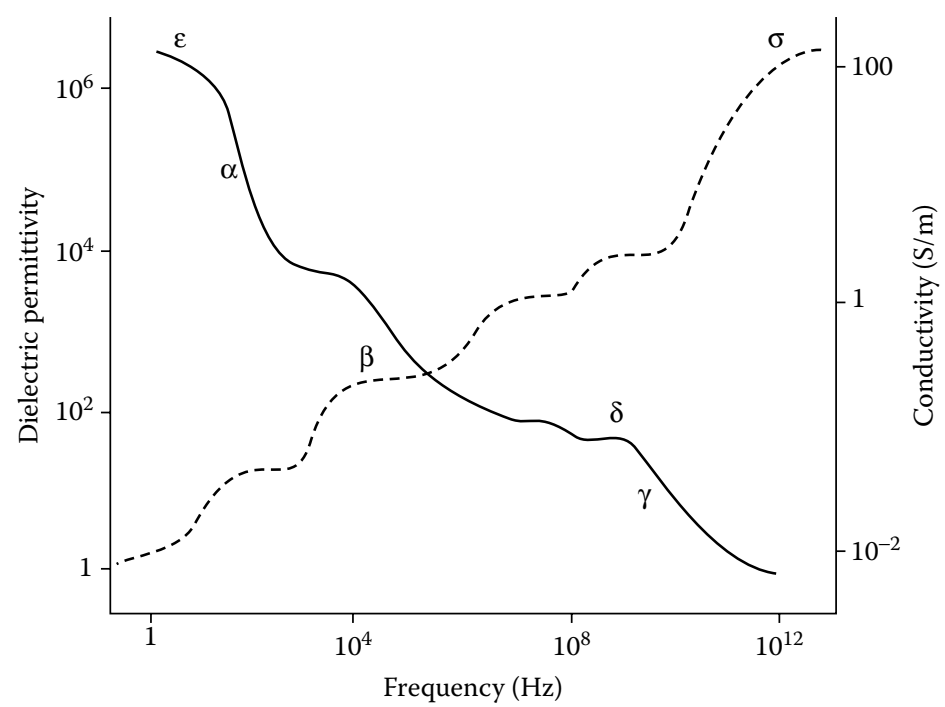

FIGURE 1.1 Dielectric permittivity and electrical conductivity of muscle-like biological materials as a function of frequency.

thus membrane capacitance dominates the behavior of the dielectric constant at low frequencies. This frequency dependence is a result of the dramatic change that membrane capacitance undergoes as the frequency increases at extremely low frequencies $(<3 \mathrm{kHz})$. An applied electric field causes charges to accumulate at the boundaries separating tissue regions of different dielectric properties, like intra- and extracellular spaces. The conductivity of biological tissues behaves in a similar manner. Cell membranes have a relatively high capacitance at low frequencies. They become progressively short-circuited for frequencies above $1 \mathrm{kHz}$, facilitating the participation of intracellular fluid in electric current conduction. This causes conductivity to increase with increase in frequency.

As frequency increases, insufficient time is allowed during each cycle to permit complete charging of cell membranes. The total charges per cycle must decrease, along with membrane capacitance, with increase in frequency. This behavior gives rise to a decrease in the dielectric constant between $10 \mathrm{kHz}$ and $30 \mathrm{MHz}$. For still higher frequencies, the change in membrane capacitance stabilizes until the rotational and vibrational properties of polar molecules of water become significant. At these frequencies, the rotation of water molecules is accompanied by viscous loss, which principally accounts for the mechanism of increased conductivity.

\subsection{Electromagnetic Phenomena at Tissue Interfaces}

Electromagnetic phenomena require that certain boundary conditions are satisfied at a boundary surface, where the tissue permittivity changes abruptly. These conditions may be derived by applying Maxwell's equations (Equations 1.1 through 1.4) to infinitesimal regions containing these interfaces. These four boundary conditions are summarized as 
follows for fields that are tangential (or parallel; labeled t) or normal (or perpendicular; labeled $\mathrm{n}$ ) to the interface:

1. The tangential components of electric field strength are continuous across the boundary $\left(\mathbf{E}_{\mathrm{t} 1}=\mathbf{E}_{\mathrm{t} 2}\right)$. Electric fields inside are the same as those outside.

2. The normal components of electric flux densities may differ by an amount equal to the surface charge density $\left(\mathbf{D}_{\mathrm{n} 1}-\mathbf{D}_{\mathrm{n} 2}=\rho\right)$. The surface charge on the boundary is zero for dielectric or nonconducting materials.

3. The tangential components of magnetic field strength may differ by an amount equal to the surface current density $\left(\mathbf{H}_{\mathrm{t} 1}-\mathbf{H}_{\mathrm{t} 2}=\mathbf{J}\right)$. In all cases, except for perfect conductors, the surface current density is vanishingly small. Thus, for dielectric or nonconducting materials, the tangential components of magnetic field strength are continuous across the boundary $\left(\mathbf{H}_{\mathrm{t} 1}=\mathbf{H}_{\mathrm{t} 2}\right)$. The magnetic fields inside are the same as those outside.

4. The normal components of magnetic flux density are continuous across the boundary $\left(\mathbf{B}_{\mathrm{n} 1}=\mathbf{B}_{\mathrm{n} 2}\right)$. The internal magnetic flux density is the same as the magnetic flux density outside.

However, if the field is neither perpendicular nor parallel to the interface, a combination of the aforementioned boundary conditions prevails. Also, the orientation of the total electric field at the surface of a boundary separating two media can be derived from the perpendicular $\left(\mathbf{E}_{\mathrm{n} 1}\right)$ and parallel $\left(\mathbf{E}_{\mathrm{t} 1}\right)$ components. The ratio of these components is equal to the tangent of the angle between them.

$$
\tan \theta=\mathbf{E}_{\mathrm{n} 1} / \mathbf{E}_{\mathrm{t} 1}
$$

Thus, the orientation of the total electric field can be determined by knowing its perpendicular $\left(\mathbf{E}_{\mathrm{n} 1}\right)$ and parallel $\left(\mathbf{E}_{\mathrm{t} 1}\right)$ components.

\subsection{Static Electric and Magnetic Fields}

The conservation of charge gives rise to an equation of continuity in the differential form for current flow such that

$$
\nabla \cdot \mathbf{J}(\mathbf{r}, t)+\frac{\partial \rho(\mathbf{r}, t)}{\partial t}=0
$$

For static (direct current or zero-frequency) electric fields that do not vary with time and a semi-infinite dielectric layer, Equation 1.13 reduces to

$$
\mathbf{J}_{\mathrm{n} 1}=\mathbf{J}_{\mathrm{n} 2} \text { or } \sigma_{1} \mathbf{E}_{\mathrm{n} 1}=\sigma_{2} \mathbf{E}_{\mathrm{n} 2}
$$

Thus, the current densities across an interface are equal to each other. Maxwell's equations (Equations 1.3 and 1.4) in the differential form are given by

$$
\nabla \cdot \mathbf{D}=\rho
$$




$$
\nabla \cdot \mathbf{B}=0
$$

Since magnetic permeability is the same as that of free space for most biological materials $\left(\mu_{\mathrm{r}}=1\right)$, the magnetic fields $(\mathbf{B}$ and $\mathbf{H})$ inside are the same as those outside, in total or by component. For example, Equation 1.16 for an infinite layer of biological material becomes $\mathbf{B}_{\mathrm{n} 1}-\mathbf{B}_{\mathrm{n} 2}=0$. Thus, the normal components of magnetic flux density $\mathbf{B}$ inside and outside are equal to each other. Likewise, $\mathbf{H}_{\mathrm{n} 1}-\mathbf{H}_{\mathrm{n} 2}=0$ since $\mathbf{B}=\mu \mathbf{H}$ and $\mu$ is the same for most biological materials.

As mentioned earlier, the tangential components of electric fields inside are the same as those outside $\left(\mathbf{E}_{\mathrm{t} 1}=\mathbf{E}_{\mathrm{t} 2}\right)$. However, the normal components of electric flux densities inside and outside differ by an amount equal to surface charge density, that is, $\mathbf{D}_{\mathrm{n} 1}-\mathbf{D}_{\mathrm{n} 2}=\rho$. For a highly conducting medium, the electric field inside is essentially zero: $\mathbf{D}_{\mathrm{n} 2}=\varepsilon_{2} \mathbf{E}_{\mathrm{n} 2}=0$ and $\mathbf{E}_{\mathrm{t} 1}=\mathbf{E}_{\mathrm{t} 2}=0$. Note that if $\sigma_{2}$ in Equation 1.14 approaches infinity as in a perfectly conducting medium, then $E_{\mathrm{n} 2}=0$. Thus, static electric fields at the surface of a good conductor must be perpendicular to the boundary since $\mathbf{D}_{\mathrm{n} 1}=\rho$ is the only component that remains. Figure 1.2 shows the electric distribution when a long cylindrical conductor is placed in an initially uniform static electric field; the placing of the cylinder changes the field immediately outside to a nonuniform field. The electric fields terminate normally on charges on the conducting surface. In a similar manner, static electric fields in air at the skin surface or at the interface between air and biological tissue are mostly perpendicular because a large $\tan \theta$ requires $\theta$ to be nearly $90^{\circ}$ in Equation 1.12.

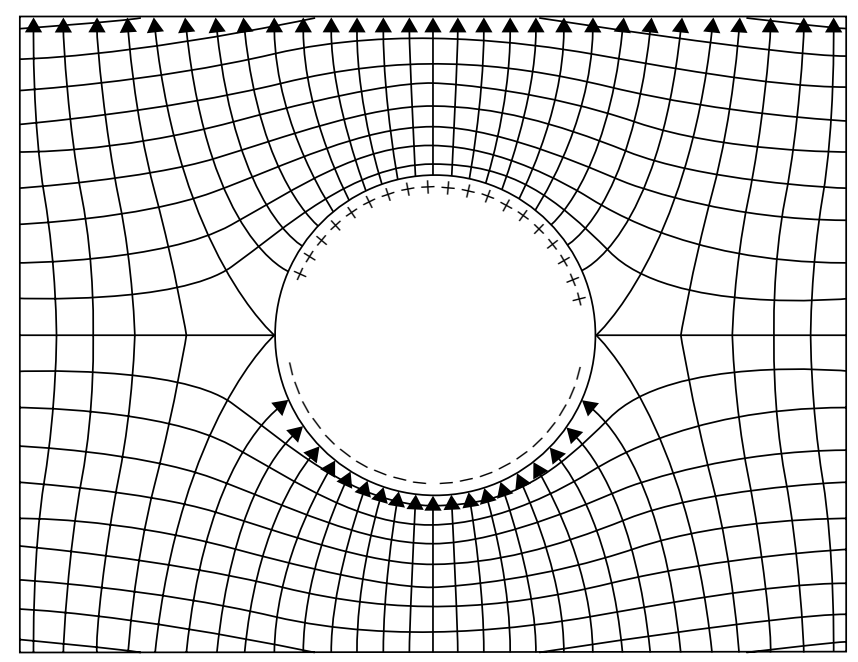

FIGURE 1.2 Electric field distribution when a long cylindrical conductor is placed in an initially uniform static electric field: The field immediately outside is nonuniform and perpendicular to the conducting surface. (From Maxwell, J. C. 1904. Treatise on Electricity. 3rd ed. New York: Oxford University Press. With permission.) 
The orientation of total electric fields in two media separated by a boundary can be expressed as in Equation 1.12 for media 1 and 2, such that

$$
\begin{aligned}
& \tan \theta_{1}=\mathbf{E}_{\mathrm{n} 1} / \mathbf{E}_{\mathrm{t} 1} \\
& \tan \theta_{2}=\mathbf{E}_{\mathrm{n} 2} / \mathbf{E}_{\mathrm{t} 2}
\end{aligned}
$$

Combining Equations 1.14, 1.17, and 1.18, for $\mathbf{E}_{\mathrm{t} 1}=\mathbf{E}_{\mathrm{t} 2}$ we obtain

$$
\tan \theta_{1}=\left(\sigma_{2} / \sigma_{1}\right)\left(\mathbf{E}_{\mathrm{n} 2} / \mathbf{E}_{\mathrm{t} 1}\right)=\left(\sigma_{2} / \sigma_{1}\right)\left(\mathbf{E}_{\mathrm{n} 2} / \mathbf{E}_{\mathrm{t} 2}\right)=\left(\sigma_{2} / \sigma_{1}\right) \tan \theta_{2}
$$

Thus, the orientation of static electric fields in dielectric media is determined by the relative electrical conductivity of the media. If medium 1 is air with low conductivity, $\sigma_{1}=10^{-12} \mathrm{~S} / \mathrm{m}$, and medium 2 is tissue medium with conductivity $\sigma_{2}=1.0 \mathrm{~S} / \mathrm{m}$, from Equation 1.19, $\tan \theta_{1}=10^{12} \tan \theta_{2}$; this large factor indicates that the electric field in air would be almost perpendicular to the skin surface, as mentioned earlier. This is true even for smaller angles inside tissues that render the tissue electric field nearly parallel to the boundary. In fact, this is the case for any internal field orientation due to the huge difference in conductivity values.

Moreover, the strength of the inside field is much lower than that of the outside field as can be seen from Equation 1.14, that is, $\mathbf{E}_{\mathrm{n} 2}=\sigma_{1} / \sigma_{2} \mathbf{E}_{\mathrm{n} 1}$. When a long cylindrical biological body is placed in an initially uniform static electric field, it changes the field immediately outside it to a nonuniform field by superposing the uniform field and fields resulting from the rearrangement of dielectrics inside the biological cylinder. The net effect is to produce a uniform but reduced inside field that has the same direction as the uniform outside field (except near the boundary, which is distorted). In general, the electric field inside the body is reduced in strength by a factor that is inversely proportional to conductivity and is independent of body size.

\subsection{Time-Varying Electromagnetic Fields}

The interaction of time-varying electromagnetic fields with biological systems is a function of the configuration of the electromagnetic energy source and the field's frequency or wavelength. They impact the penetration and deposition of electromagnetic power, induction of electric and magnetic fields, and absorption of electromagnetic energy in biological tissue. Moreover, when considering electromagnetic interactions it is necessary to account for the frequency or wavelength and its relationship with the physical dimension and geometry of the source and the biological body. This may be seen by examining the propagation of electromagnetic energy through antennas into space or a material medium. However, a description of the interaction at frequencies where the wavelength is long and the time variation is slow is provided first. 


\subsection{Low-Frequency and Quasistatic Electric and Magnetic Fields}

At low frequencies, where the wavelength is at least an order of magnitude longer than the dimensions of the human body or the biological object, and when the field varies slowly with time, electromagnetic interactions inside the body become quasistatic and the electric and magnetic field components become decoupled (Lin 2000; Lin, Guy, and Johnson 1973). For example, at $100 \mathrm{~Hz}$ and $100 \mathrm{kHz}$ the wavelengths in air are $3000 \mathrm{~km}$ and $300 \mathrm{~m}$, respectively, which are at least 150 times longer than a body having a height of $2 \mathrm{~m}$. The induced field inside can be obtained by combining two independent static electric and magnetic formulations of the electromagnetic field problem.

If time variation is represented by $e^{-j \omega t}$, where $\mathrm{j}=(-1)^{-1 / 2}$ and $\omega=2 \pi \mathrm{f}$ are the complex variable and the radian frequency, respectively, Equations 1.13 and 1.15 applied to two infinite layers separated by an interface take the following form:

$$
\begin{gathered}
\sigma_{1} \mathbf{E}_{\mathrm{n} 1}-\sigma_{2} \mathbf{E}_{\mathrm{n} 2}=-j \omega \rho \\
\varepsilon_{1} \mathbf{E}_{\mathrm{n} 1}-\varepsilon_{2} \mathbf{E}_{\mathrm{n} 2}=\rho
\end{gathered}
$$

Combining these two equations, we obtain

$$
\mathbf{E}_{\mathrm{n} 1}=\left(\sigma_{2}+\mathrm{j} \omega \varepsilon_{2}\right) /\left(\sigma_{1}+\mathrm{j} \omega \varepsilon_{1}\right) \mathbf{E}_{\mathrm{n} 2}
$$

where $\varepsilon_{1}$ and $\varepsilon_{2}$ are the permittivities of media 1 and 2 , respectively. For an air and tissue interface with $\varepsilon_{1}=\varepsilon_{0}$ and $\varepsilon_{2}=10^{6} \varepsilon_{0}$, and $\sigma_{1}=10^{-12} \mathrm{~S} / \mathrm{m}$ and $\sigma_{2}=0.1 \mathrm{~S} / \mathrm{m}$, respectively, at $60 \mathrm{~Hz}$ we obtain

$$
\mathbf{E}_{\mathrm{n} 1} \cong\left(\sigma_{2}\right) /\left(\mathrm{j} \omega \varepsilon_{0}\right) \mathbf{E}_{\mathrm{n} 2}=-\mathrm{j} 3 \times 10^{7} \mathbf{E}_{\mathrm{n} 2}
$$

Thus, the $60 \mathrm{~Hz}$ ELF electric field inside can be reduced by a factor of 30 million from the field outside. The ELF electric field interaction is dominated by conductivity, which is considerably higher than the contributions from dielectric permittivity. The orientation of the outside electric field becomes distorted in its vicinity and is essentially perpendicular to the surface of the air-tissue interface, a situation similar to static electric fields.

Similarly, the magnetically induced electric field inside the body is identical to the quasistatic solution of Equation 1.1:

$$
\oint \mathbf{E} \cdot \mathrm{d} l=\int_{s}(\partial \mathbf{B} / \partial t) \cdot \mathrm{d} s
$$

For example, if the body is approximated by an infinitely long cylinder or a finite sphere, an externally applied uniform magnetic field will induce in any cross-sectional plane of the cylinder or the circumference of the sphere an electric field whose magnitude is given by

$$
\mathbf{E}=\omega \mathbf{B} r / 2=(\pi f r \mu) \mathbf{H}
$$

where $f=\omega /(2 \pi)$ is the frequency, $\mu$ is the permeability, $r$ is the radial distance, B is the magnetic flux density, and $\mathbf{H}$ is the strength of the magnetic field component. Thus, 
the magnetic field produces an internal electric field that varies directly with distance away from the center and in proportion to frequency. Note that there is no induced electric field at the center, but it reaches a maximum at the body surface. For a $60 \mathrm{~Hz}$ uniform magnetic flux density of $0.4 \mu \mathrm{T}\left(\mathrm{T}=\mathrm{Wb} / \mathrm{m}^{2}\right)$ oriented along the long axis of the human body with 0.12 and $0.20 \mathrm{~m}$ as radii of the head and trunk, respectively, the induced electric fields along the circular path subscribing the head and trunk are 9.04 and $15.07 \mu \mathrm{V} / \mathrm{m}$, respectively, at the surface.

The electric fields inside circulate in closed loops and form eddy currents in a medium with finite conductivity $\sigma$ such that the eddy current density is

$$
\mathbf{J}=\sigma \mathbf{E}=(\sigma \pi f r \mu) \mathrm{H} \phi
$$

The eddy current's magnitude increases with distance from the center of the body and this current encircles the magnetic field that produces it (Figure 1.3)

When applying Equations 1.24 and 1.25 to estimate fields induced or current inside a biological object (animal, human, or tissue preparation), any significant deviations from homogeneity or circular symmetry must be taken into account. Equations 1.24 and 1.25 should be applied to each region inside the body having a different conductivity, which behaves as a unit with its own body center and radius or an equivalent radius. However, due to the presence of opposing induced field orientations and current paths at internal interfaces, the highest field and current densities tend to occur with the large dimensions associated with outer layers of a body or tissue preparation so long as the conductivities are not grossly different and the regions are not separated by nonconducting materials.

The circulating eddy currents induced inside a prolate homogeneous spheroidal model of the human body immersed in a uniform horizontal or vertical magnetic field are shown in Figure 1.3. The induced electric fields are polarization dependent in that they are always in a plane perpendicular to the magnetic field. The eddy current loop is the largest when the dimension of the homogeneous region is the largest.

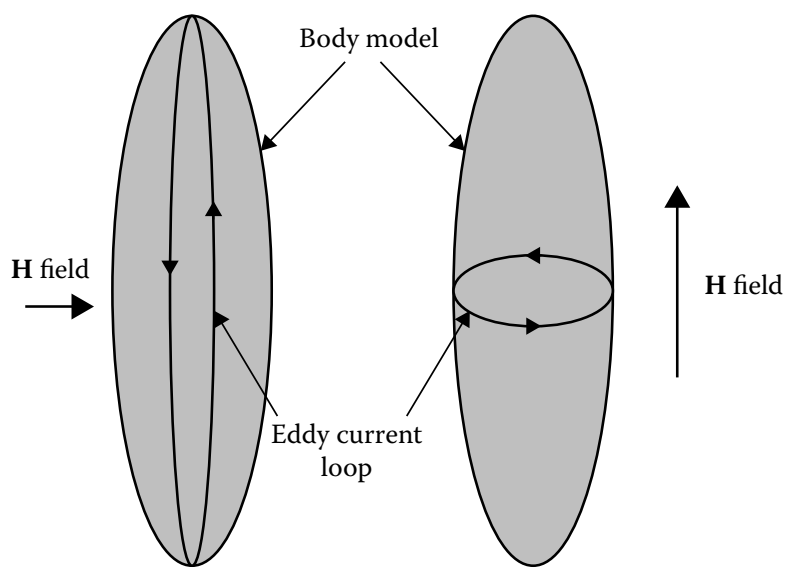

FIGURE 1.3 Circulating eddy currents induced inside a prolate homogeneous model of the human body immersed in a uniform horizontal or vertical magnetic field. 


\subsection{Propagation of Electromagnetic Energy from Antennas}

Electromagnetic energy at all frequencies can be radiated into space or a material medium through the use of antennas. The spatial distribution of electromagnetic energy from an antenna is directional and varies with distance from the antenna. The short dipole antenna is used in this chapter as a model to examine the coupling and distribution of electromagnetic energy in biological interactions. The dipole antenna is important for both practical and conceptual reasons because any linear antenna may be regarded as a series of dipoles and large antennas of other configurations may be considered as assemblies of many dipoles. Thus, results and observations obtained from a dipole antenna can provide insights into electromagnetic wave propagation associated with antennas, in general.

A dipole antenna of length $\ell$, which is short compared with a wavelength $\lambda \gg \ell$ and long compared with its diameter, is shown in Figure 1.4. For purposes of analytical simplicity, the dipole may be considered as a thin conductor of length $\ell$ carrying a uniform current, $\ell=j \omega q$. It can be shown that the electric and magnetic fields from the dipole have only three components: $\mathbf{E}_{r}, \mathbf{E}_{\theta}$, and $\mathbf{H}_{\phi}$. All other components are zero at all points (Jordan and Balmain 1968). In the spherical coordinate system (Figure 1.5), they are given by

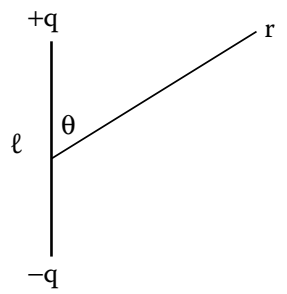

FIGURE 1.4 Elementary dipole antenna of length $\ell$, whose length is short compared with a wavelength $\lambda \gg \ell$ and diameter is small compared with its length.

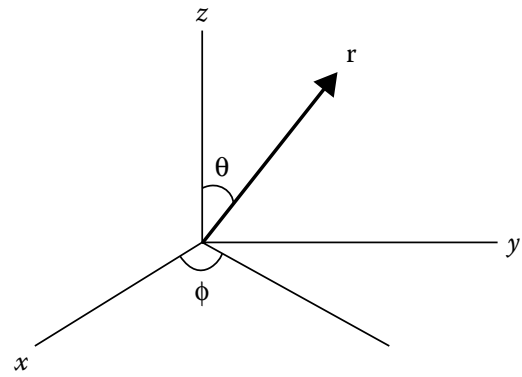

FIGURE 1.5 Spherical coordinate system superimposed on rectangular coordinates. 


$$
\begin{gathered}
\mathrm{E}_{\mathrm{r}}=\left[\mathrm { j } ( \mathrm { q } \ell \omega \beta / \varepsilon ) \mathrm { e } ^ { \mathrm { j } ( \omega t - \beta \mathrm { r } ) } \operatorname { c o s } \theta \left[1 / \mathrm{r}^{2}+1 /\left(\mathrm{j} \beta \mathrm{r}^{3}\right) / 2 \pi\right.\right. \\
\mathbf{E}_{\theta}=\left[\mathrm{j}(q \ell \omega \beta / \varepsilon) \mathrm{e}^{\mathrm{j}(\omega t-\beta \mathrm{r})} \sin \theta\right]\left[\mathrm{j} \beta / \mathrm{r}+1 / \mathrm{r}^{2}+1 /\left(\mathrm{j} \beta \mathrm{r}^{3}\right)\right] / 4 \pi \\
\mathbf{H}_{\phi}=\left[\mathrm{j}(\omega q \ell) \mathrm{e}^{\mathrm{j}(\omega t-\beta \mathrm{r})} \sin \theta\left[\mathrm{j} \beta / \mathrm{r}+1 / \mathrm{r}^{2}\right] / 4 \pi\right.
\end{gathered}
$$

where $\beta=(2 \pi) / \lambda$ is the propagation factor in the medium. This set of three equations (Equations 1.26 through 1.28) prescribes the behavior of electric and magnetic fields at all points from the dipole antenna and has been found to be experimentally consistent.

\subsubsection{Quasistatic Fields of a Dipole Antenna}

If the time variation is slow, $\omega \rightarrow 0$, Equations 1.26 through 1.28 reduce to

$$
\begin{gathered}
\mathbf{E}=\mathrm{E}_{\mathrm{r}} \mathbf{r}+\mathrm{E}_{\theta} \theta \\
\mathbf{E}_{\mathrm{r}}=\left[q \ell /\left(2 \pi \varepsilon r^{3}\right)\right] \cos \theta \\
\mathbf{E}_{\theta}=\left[q \ell /\left(4 \pi \varepsilon r^{3}\right)\right] \sin \theta
\end{gathered}
$$

In Equations 1.29 through 1.31, $q$ is the total number of electric charges and $q \ell$ is the electric dipole moment. The absence of $\omega$ from these equations indicates that for slow time variations associated with electromagnetic energy at long wavelengths, the spread of electric fields from an antenna is quasistatic. Similarly, the quasistatic magnetic field resulting from the dipole antenna's current is given by

$$
\mathbf{H}_{\phi}=\left[\mathbf{I} \ell /\left(4 \pi r^{2}\right)\right] \sin \theta
$$

where $\mathbf{I}=j \omega q$ is the current flowing in a short dipole antenna of length $\ell$. These fields are quasistatic, which means that at low frequencies the transmission of electromagnetic waves into the human body is the same as the coupling of two separate static electric and magnetic fields and the total induced electric field inside the body is given by a vector sum of the two fields from Equations 1.22 and 1.24. Consider the following example: The wavelength of $60 \mathrm{~Hz}$ ELF fields is $5000 \mathrm{~km}$. Therefore, human exposure to these ELF electric and magnetic fields and the interaction of these fields with biological objects are quasistatic in nature. An externally applied uniform electric field gives rise to a uniform induced electric field inside the body, which has the same direction as the applied field but is reduced in strength by a factor inversely proportional to dielectric permittivity and independent of body size (see Equation 1.22). Similarly, the magnetically induced electric field inside the body is identical to that expressed by Equation 1.25, and its magnitude is given by $\mathbf{E}=(\pi f r \mu) \mathbf{H}$, where $r$ is the equivalent radius of the body.

\subsubsection{Near Field of a Dipole Antenna}

Inspection of Equations 1.26 through 1.28 for $\mathbf{E}_{r}, \mathbf{E}_{\theta}$, and $\mathbf{H}_{\phi}$ shows that at points close to the dipole antenna where $r$ is small the $1 / r^{2}$ and $1 / r^{3}$ terms become predominant and the equations simplify to 


$$
\begin{aligned}
& \mathbf{E}_{r}=\left[f q \ell /\left(\varepsilon r^{3}\right)\right] \mathrm{e}^{\mathrm{j}(\omega t-\beta r)} \cos \theta \\
& \mathbf{E}_{\theta}=\left[f q \ell / 2 \varepsilon r^{3}\right] \mathrm{e}^{j(\omega t-\beta r)} \sin \theta \\
& \left.\mathbf{H}_{\phi}=\left[\mathrm{j} f q \ell /\left(2 r^{2}\right)\right] \mathrm{e}^{j(\omega t-\beta} r\right) \sin \theta
\end{aligned}
$$

It can be seen that the maxima and minima of electric and magnetic fields in the near field do not occur at the same point in space. The ratio of electric to magnetic fields (i.e., field impedance) varies from point to point, giving rise to widely divergent field impedances. Also, both the $r$ and $\theta$ components of the electric field are shifted by $90^{\circ}$ in time (in phase quadrature) from the magnetic field. Therefore, the electric and magnetic fields in the near zone are related to each other as in a standing wave.

A standing wave is established when the field reflected by the interface between two different material media is combined with an incident field in the same medium. In the case of normal incidence on a dielectric medium, a portion of the incident field is transmitted into the second medium and continues to propagate in the same direction. For a standing wave, the maxima and minima do not move but stand at the same locations in the first medium; the peaks and nulls of the field always occur at the same points in space at different instants in time.

Variations of magnitude with position in Equations 1.33 through 1.35 are the same as in Equations 1.30 through 1.32. These variations indicate that the near fields of a dipole antenna are quasistatic. This observation extends to all antennas and radiating systems. Also, electromagnetic energy couples with the human body in the same fashion as two separate static electric and magnetic fields do in the near field. The wavelength of $50-60 \mathrm{~Hz}$ ELF fields is about $5000 \mathrm{~km}$ for all practical purposes and exposure to ELF electric and magnetic fields always occurs in the near field. Their interactions are quasistatic in nature, as mentioned in Section 1.7.

The near zone can be divided into two regions: (1) the radiative region and (2) the reactive region. In the radiative region, which is the region closer than $2 \mathbf{D}^{2} / \lambda$, the radiated power varies with distance from the antenna. The space surrounding the antenna where the reactive component predominates is known as the reactive region. The precise extent of the regions varies for different antennas. For most antennas, the transition point between reactive and radiative regions occurs from 0.2 to $0.4 \mathbf{D}^{2} / \lambda$ ( $\operatorname{Lin} 2000$ ). For the short dipole, the reactive component predominates up to a distance of approximately $\lambda /(2 \pi)$ at which the radiative and reactive components are equal to each other. However, the outer limit is of the order of a few wavelengths or less in most cases.

The field represented by the $1 / r^{2}$ term in Equation 1.35 is called the reactive field or inductive field, and it becomes predominant compared with the $1 / r^{3}$ terms in Equations 1.33 and 1.34 at points close to the dipole antenna. It should be noted that at low frequencies wavelengths are long and the induction field may extend to very large distances from the source. The corresponding wavelengths at high frequencies are quite short and the induction field may not exist at all. For example, at $60 \mathrm{~Hz}$ the induction zone may extend to $5000 \mathrm{~km}$ or more, whereas at $300 \mathrm{GHz}$ the wavelength is only $1 \mathrm{~mm}$ and the induction zone is negligible. However, at $900 \mathrm{MHz}$ the wavelength is about $33 \mathrm{~cm}$. Therefore, the corresponding induction zone will include the human body for any near-body communication devices using this frequency. 


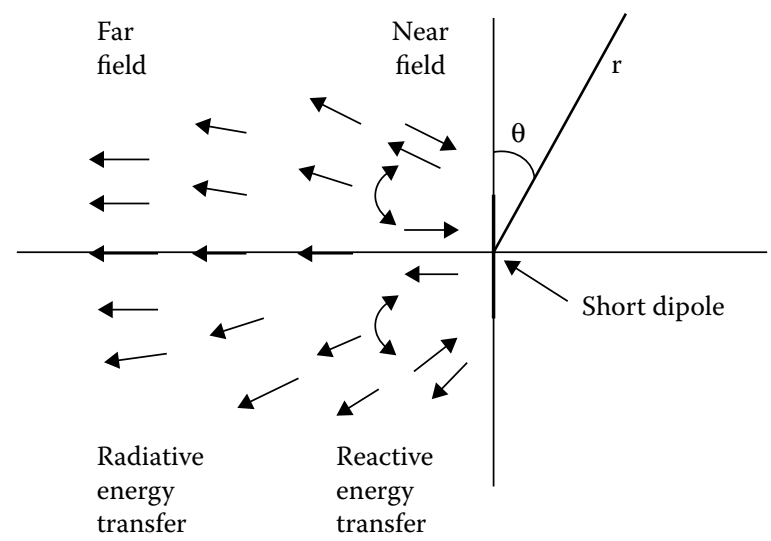

FIGURE 1.6 Flow of electromagnetic energy from a dipole antenna: Arrows represent the direction of energy flow at successive instants in time.

Invoking the Poynting vector shows that the quasistatic and induction terms represent energy that is stored in the field during one quarter of a cycle and is returned to the antenna during the next quarter of the cycle without net or average outward flow. In the near zone, the energy exchange is largely reactive; only the $1 / r$ terms contribute to an average outward flow of energy. The energy transfer characteristics are illustrated in Figure 1.6, in which the arrows represent the direction of energy flow at successive instants in time (Lin 2000). Power density in the near zone is not as uniquely defined as in the far zone, since the electric and magnetic fields and their ratios vary from point to point. Furthermore, the angular distribution actually depends on the distance from the antenna. It is necessary to individually arrive at a quantitative determination of the power density at all points.

\subsubsection{Far Field of a Dipole Antenna}

At points far from the dipole antenna, $r$ is large and terms involving $1 / r^{2}$ and $1 / r^{3}$ in Equations 1.26 through 1.28 can be neglected in comparison with terms involving $1 / \mathrm{r}$. Thus, in the far field only two field components remain, which are given by

$$
\begin{aligned}
& \mathbf{E}_{\theta}=j[\eta \mathbf{I} \beta \ell /(4 \pi r)] e^{j(\omega t-\beta r)} \sin \theta \\
& \mathbf{H}_{\phi}=j\left[(\mathbf{I} \beta \ell /(4 \pi r)] e^{j(\omega t-\beta r)} \sin \theta\right.
\end{aligned}
$$

The wave impedance in the far zone is defined by the ratio $\mathbf{E}_{\theta} / \mathbf{H}_{\phi}$, which is the same as the intrinsic impedance $\eta=(\mu / \varepsilon)^{1 / 2}$ of the medium. Also, $\mathbf{E}_{\theta}$ and $\mathbf{H}_{\phi}$ are in time phase and at right angles to each other. Thus, the electric and magnetic fields in the far field of a dipole are related in the same fashion as in a plane wave. Further, from the Poynting 
vector $(\mathbf{P}=\mathbf{E} \times \mathbf{H})$, one can find the direction and time-average flow of energy per unit area, which is given by

$$
\mathbf{P}=\eta\left\{[\mathbf{I} \beta \boldsymbol{\ell} /(4 \pi \mathrm{r})]^{2}\right\} \mathrm{e}^{\mathrm{j}(\omega} \boldsymbol{t}^{-\beta r)} \sin ^{2} \theta \mathbf{r}
$$

Clearly, energy flow in the far zone is real and uniquely defined. It is outgoing in the radial direction and perpendicular to both $\mathbf{E}_{\theta}$ and $\mathbf{H}_{\phi}$. The electromagnetic energy is hence radiated, and the term radiation zone is synonymous with far field. In the far field, the intensity of radiated energy (power density) decreases as $1 / \mathrm{r}^{2}$ with increase in distance. As in a plane wave, the electric and magnetic fields are outgoing waves with plane wavefronts independent of its source configuration. Also, in the far zone the field strengths decrease as $1 / \mathrm{r}$, and only transverse field components appear.

The distance criterion that is most commonly used to distinguish between near and far zones is that the phase variation of the field from the antenna does not exceed $\lambda / 16$ (Silver 1949). This boundary occurs at a conservative distance of

$$
R=2 D^{2} / \lambda
$$

where $D$ is the largest dimension of the antenna aperture.

\subsection{Coupling of Quasistatic Electric and Magnetic Fields}

As discussed in Section 1.7, for low frequencies where the wavelength is long and time variation is slow, the induced electric and magnetic fields inside a human body are quasistatic in nature. For all practical purposes, exposures to ELF electric and magnetic fields always occur in the near-zone inductive region. Moreover, at ELFs the electric and magnetic field components are decoupled inside a biological body. Indeed, these phenomena arise whenever a biological body or model is small compared to a wavelength. Also, at ELFs the bulk electrical conductivity is on the order of $0.1 \mathrm{~S} / \mathrm{m}$ and the relative dielectric permittivity is about $10^{6}$. The ratio is

$$
\sigma / \omega \varepsilon=3 \times 10^{7} \gg>1
$$

Thus, the conduction current is much greater than the displacement current, and biological materials may be considered as conducting media.

\subsubsection{Quasistatic Electric Field Coupling}

As a model of biological bodies, the induced field inside a spherical model with radius $r$, conductivity $\sigma$, and dielectric permittivity $\varepsilon$ can be considered. For a uniform or constant electric field, $\mathbf{E}_{0}$, polarized in the $x$ direction (see Equations 1.13 and 1.15), we have

$$
\mathbf{E}_{2}=\left(\omega \varepsilon_{0} / \sigma\right) \mathbf{E}_{0} x
$$

Therefore, an applied uniform outside electric field gives rise to a uniform induced electric field inside the body, which has the same direction as the external field but is 
reduced in strength by a factor inversely proportional to the complex permittivity of tissue (Figure 1.7). A surface polarization is set up that generates a uniform electric field inside. Although Equation 1.41 suggests that the induced field is independent of body size, it varies from tissue to tissue as a function of permittivity.

At ELFs, the high tissue dielectric permittivity and conductivity and the behaviors of electric fields at curved boundaries combine to render some very unique phenomena. They conspire to weaken the low-frequency electric fields applied through air by about $10^{-7}$ upon penetration into biological tissues. The same boundary condition distorts the applied uniform electric field in the immediate vicinity of the biological body such that it becomes oriented perpendicular to the surface of the body. Moreover, the surface electric field shows considerable enhancement at each sharp curvature of the body. This phenomenon is illustrated in Figures 1.8 and 1.9 using the distribution of electric field at the surface of a
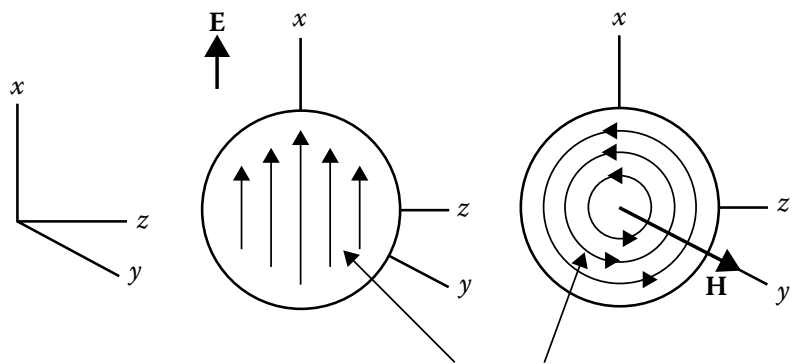

Induced electric field lines

FIGURE 1.7 Coupling of low-frequency electric and magnetic fields into a homogeneous spherical model of biological tissue.

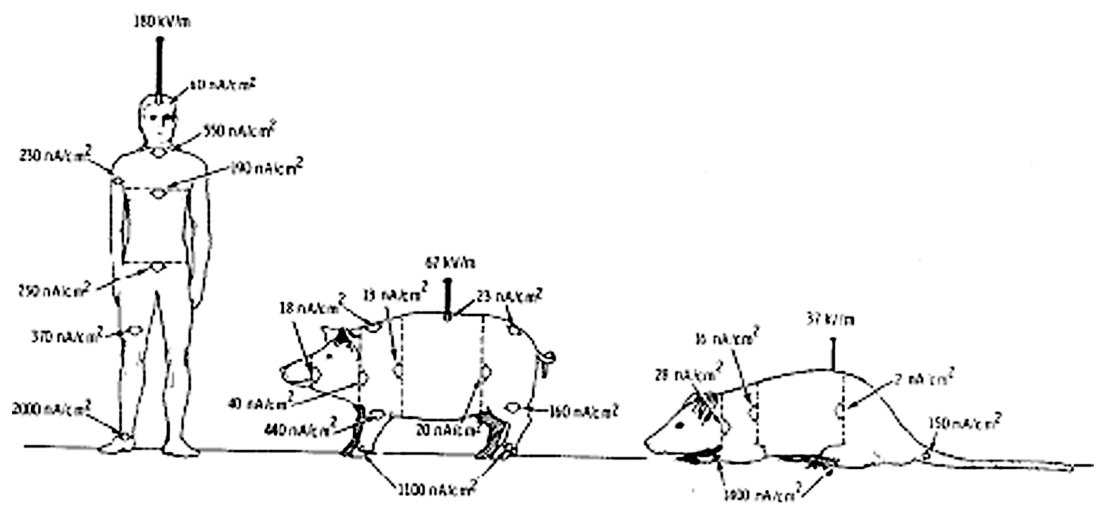

FIGURE 1.8 Current density through selected axial cross sections of human, swine, and murine bodies exposed to a vertical $60 \mathrm{~Hz} 10-\mathrm{kV} / \mathrm{m}$ electric field: Relative body sizes are not to scale. Average axial current densities are estimated values over each cross section. Current densities perpendicular to the surface of the bodies shown for man and pig are from calculations. Surface electric fields are measured values for man and pig, whereas those for rats are estimated. (From Kaune, W. T., and R. D. Phillips. 1980. Comparison of the coupling of grounded humans, swine, and rats to vertical, $60 \mathrm{~Hz}$ electric fields. Bioelectromagnetics 1:117-29. With permission.) 

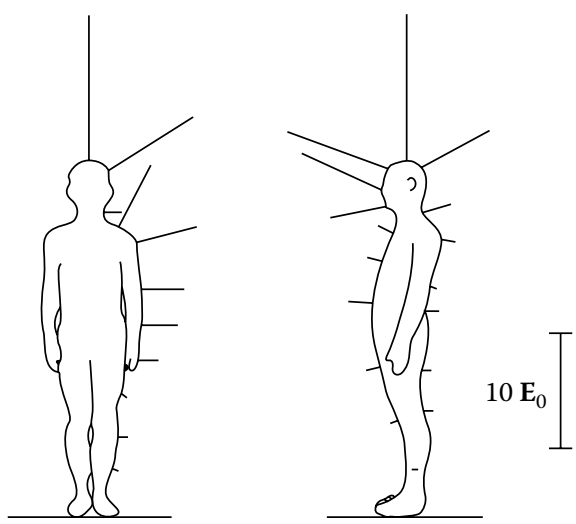

FIGURE 1.9 Extremely-low-frequency electric field distribution measured at the body surface of a human body subject standing under an electric power transmission line: $\mathbf{E}_{0}$ is the electric field strength in air. (Adapted from Shimizu, K., H. Endo, and G. Matsumoto. 1988. Visualization of electric fields around a biological body. IEEE Trans Biomed Eng 35:296-302.)

human subject standing in an ELF field produced under a high-voltage transmission line. The current densities through selected axial cross sections of a grounded human, swine, or murine body exposed to vertical $60 \mathrm{~Hz}$ 10-kV/m electric fields are shown in Figure 1.8. For example, in the case of humans, a current is induced to flow in the vertical direction. Current density is the highest at the narrower cross sections of the neck and leg, which are estimated to be 0.02 and $0.006 \mathrm{~A} / \mathrm{m}^{2}$, respectively. It is noteworthy that the surface electric field is proportional to the height of the body in the field. Although electric current and field distributions within different body components may offer greater quantitative detail, these averaged values clearly demonstrate the significance of body cross sections in determining current distribution (Figure 1.9). Also, the tissue composition of specific body parts contributes to the induced field and current density via their electrical conductivity.

\subsubsection{Quasistatic Magnetic Field Coupling}

As mentioned in Section 1.7, the magnetic permeability of biological materials is approximately the same as that of free space, and the magnetic field everywhere inside a body is equal to that applied externally. However, this is not the case for an induced electric field. For example, a vertically directed uniform magnetic field induces an electric field inside the body that is identical to the quasistatic solution of Equation 1.1, and its magnitude is given by Equation 1.24, that is, $\mathbf{E}=(\omega \mathrm{r} \mu / 2) \mathbf{H}$. The magnetic field produces an electric field inside that varies directly with distance away from the center and in proportion to the frequency and the applied uniform magnetic fields. The induced current density is

$$
\mathbf{J}=\sigma \mathbf{E}=(\sigma \omega \mathrm{r} \mu / 2) \mathbf{H}_{\phi}
$$

Thus, the magnetically induced electric field encircles the magnetic axis and produces an eddy current whose magnitude increases with distance from the center of the body (Figures 1.3 and 1.7). 
It is important to note that when applying Equations 1.24 and 1.42 to estimate induced fields inside a body (animals, humans, or tissue preparations), any significant deviations from homogeneity or circular cylindrical symmetry must be taken into account. Equations 1.24 and 1.42 should be applied to each region inside the body with a different conductivity, which behaves as a unit with its own body center and radius or an equivalent radius. However, due to opposing field orientations and current paths inside the body, the highest field and current densities tend to occur with the large dimensions associated with the outer layers of a body or a tissue preparation as long as the conductivities are not grossly different and the regions are not separated by nonconducting materials.

\subsubsection{Combined Quasistatic Electric and Magnetic Fields}

It is noteworthy that when both fields are present the relative significance of electrically or magnetically induced coupling in humans and animals is a function of body size and the ratio of applied $\mathbf{E}_{1} / \mathbf{H}_{1}$ such that

$$
\mathbf{E}_{\mathrm{e}} / \mathbf{E}_{\mathrm{h}}=\left(2 \varepsilon_{0} / \sigma \mu_{0} \mathrm{r}\right)\left(\mathbf{E}_{1} / \mathbf{H}_{1}\right)
$$

where $\mathbf{E}_{\mathrm{e}}$ and $\mathbf{E}_{\mathrm{h}}$ are the electrically and magnetically induced inside electric fields, respectively. For $\sigma=0.1 \mathrm{~S} / \mathrm{m}$ at $60 \mathrm{~Hz}$,

$$
\mathbf{E}_{\mathrm{e}} / \mathbf{E}_{\mathrm{h}}=20\left(\mathbf{E}_{1} / \mathbf{H}_{1}\right) /\left[(120 \pi)^{2} \mathrm{r}\right]
$$

Accordingly, the magnetically induced field in a human head of radius $10 \mathrm{~cm}$ is a factor of $2(0.6 \pi)$ greater than the electrically induced field for a ratio of applied fields $\mathbf{E}_{1} / \mathbf{H}_{1}=$ $120 \pi$. The electric and magnetic fields induced would be equally significant if the applied electric field strength is $0.6 \pi$ times stronger than the applied magnetic field. The same is true if the size of the body is $1 /(6 \pi)$ of $1 \mathrm{~m}$. Thus, for an isolated cell or an aggregate of cells whose radius is smaller than $1 /(6 \pi)$ of a meter, the electrically induced field is the predominant factor when the ratio of applied fields is $\mathbf{E}_{1} / \mathbf{H}_{1}=120 \pi$. This size dependence illustrates the need for scaling extrapolation results from animals to humans or from cell preparations to whole-body systems. Substantive adjustment is required when comparable induced fields inside different animals are to be obtained by applying external fields at low frequencies.

\subsubsection{Summary of Quasistatic and Low-Frequency Field Coupling}

The coupling and distribution characteristics of low-frequency electric and magnetic fields in biological tissue are summarized in this section. The results apply to frequencies at which the wavelength is long or the largest dimension of the body is small compared with a wavelength, including ELF and quasistatic electric and magnetic fields:

- Exposures to low-frequency electric and magnetic fields occur in the near zone or inductive region of a source.

- Induced electric and magnetic fields inside animals and humans are quasistatic in nature. 
- Electric and magnetic fields are decoupled inside a biological body.

- Magnetic fields inside a body are equal to the field applied externally.

- Electric fields applied through air are weakened by a factor of $10^{-8}-10^{-6}$ upon penetration into biological tissues.

- Electric fields in the immediate vicinity of the biological body are perpendicular to the surface of the body.

- Electric fields are enhanced near the surface at points of sharp curvatures.

- Magnetically induced electric fields encircle the magnetic field axis and produce an eddy current whose magnitude increases with distance from the center of the body.

- Eddy current calculation must be applied to each region inside the body with a different conductivity, which behaves as a unit with its own body center and radius or an equivalent radius.

- The highest induced electric field and eddy current density occur with the large dimensions associated with the outer layers of a body with finite conductivity.

- Relative significance of electrically or magnetically induced coupling in humans and animals is a function of body size and biological properties.

- Conduction current is much greater than displacement current in biological materials at low frequencies.

- Biological materials may be considered as conducting media at low frequencies.

- Biological bodies or models are small compared with a wavelength at low frequencies.

\subsection{Radiofrequency Fields and Energy Deposition}

The coupling of incident RF fields into biological tissues is influenced by the geometry and composition of the exposed object and the frequency and configuration of the source, as noted in Section 1.8. In addition, the width and strength of the incident field differ according to distance from a source and the specific source type, that is, whether the source is handheld, land mobile, base, or broadcast station. As an example, the incident field strength ranges from 3 to $10 \mathrm{~V} / \mathrm{m}\left(24-265 \mathrm{~mW} / \mathrm{m}^{2}\right)$ at typical far-zone distances from a wireless cellular mobile telephone base station. An important distinction in assessing the coupling of RF energy into biological systems is the determination of whether the exposure is taking place in the near zone or the far zone of a given source. The $2 D^{2} / \lambda$ distance is approximately $6 \mathrm{~cm}$ for a $10-\mathrm{cm}$ RF antenna operating at $900 \mathrm{MHz}$ in free space. Clearly, both near-zone inductive and far-zone field interactions are encountered in the vicinity of RF mobile telecommunication services. In the near field, quasistatic interactions prevail and the aforementioned discussions can be applied to understand and estimate induced fields. (See also the subsequent section (Section 1.10.4) on "RF Coupling in the Near Zone" for further information and discussions.)

In the far zone of an antenna, the radiated RF energy propagates as plane waves. The interaction of RF radiation with biological systems is independent of the source configuration as can be seen from Equations 1.36 through 1.38. The electric and magnetic fields are uniquely defined and are related through a constant factor $\eta$, the intrinsic 
impedance of the medium. Therefore, the determination of electric field behavior is sufficient to characterize the interaction. Accordingly, the following discussion will begin with plane-wave RF field interactions.

For all practical and environmental purposes, human exposure to ELF electric and magnetic fields always take place in the near-zone inductive region regardless of distance from the source of such fields. An equivalent plane electromagnetic wave at ELF would be two separate and independent quasistatic electric and magnetic fields with a constant field impedance equal to that of free space, $\eta_{0}=120 \pi \Omega$. As previously indicated, at ELFs the electric and magnetic field components are decoupled inside a biological body.

\subsubsection{Radiofrequency Reflection and Transmission at Planar Interfaces}

At boundaries separating regions of different biological materials, RF energy is reflected or transmitted (Figure 1.10). For a plane wave impinging normally from a medium of intrinsic impedance $\eta_{1}$ on a flat medium of intrinsic impedance $\eta_{2}$, the reflection coefficient, $R$ is given by

$$
\begin{gathered}
R=\left(\eta_{2}-\eta_{1}\right) /\left(\eta_{2}+\eta_{1}\right) \\
T=\left(2 \eta_{2}\right) /\left(\eta_{2}+\eta_{1}\right)
\end{gathered}
$$

The transmission coefficient $T$ provides a measure of RF energy coupling, and $T$ and $R$ are related as $T=1+R$. The fraction of incident power reflected by the discontinuity is $R^{2}$ and the transmitted fraction is $T^{2}=\left(1-R^{2}\right)$. For very similar tissues, where $\eta_{1}$ is approximately the same as $\eta_{2}$, there is minimal reflection and maximum transmission. As the transmitted field propagates in the tissue medium, RF energy is extracted from the field and deposited in the medium, resulting in a progressive reduction of power density of the field as it advances in the tissue. This reduction is quantified by penetration depth, which is the distance through which the power density decreases by a factor of $\mathrm{e}^{-2}$. Table 1.1 gives the calculated penetration depth and transmission coefficient for air-tissue interfaces, using typical dielectric permittivity for tissues with high water content, such as muscle and most organs, and tissues with low water content, including bone and fat.
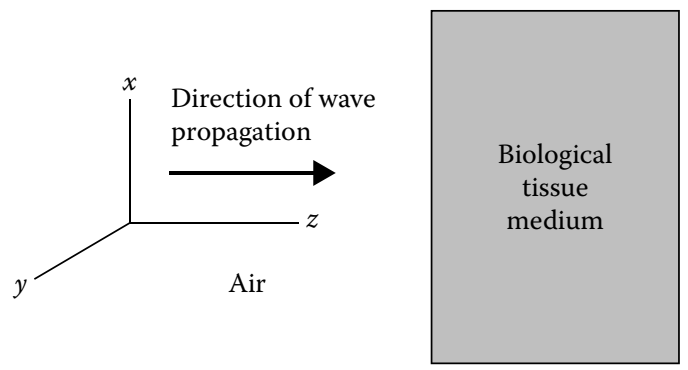

FIGURE 1.10 Plane wave impinging on a tissue layer. 
TABLE 1.1 Propagation Characteristics of RF Plane Waves in Biological Tissues Having Low and High Water $\left(\mathrm{H}_{2} \mathrm{O}\right)$ Contents as a Function of Frequency at $37^{\circ} \mathrm{C}$

\begin{tabular}{lcccccccc}
\hline $\begin{array}{l}\text { Frequency } \\
(\mathrm{MHz})\end{array}$ & \multicolumn{2}{c}{$\begin{array}{c}\text { Dielectric } \\
\text { Constant }\end{array}$} & \multicolumn{2}{c}{$\begin{array}{c}\text { Conductivity } \\
(\mathrm{S} / \mathrm{m})\end{array}$} & \multicolumn{2}{c}{$\begin{array}{c}\text { Penetration Depth } \\
(\mathrm{cm})\end{array}$} & \multicolumn{2}{c}{$\begin{array}{c}\text { Transmission } \\
\text { Coefficient }(T)\end{array}$} \\
\hline $\mathrm{H}_{2} \mathrm{O}$ & High & Low & High & Low & High & Low & High & Low \\
27 & 113 & 20.0 & 0.61 & 0.03 & 14.3 & 77.0 & 0.14 & 0.56 \\
40 & 97 & 14.6 & 0.69 & 0.03 & 11.2 & 58.8 & 0.17 & 0.62 \\
433 & 53 & 5.6 & 1.43 & 0.08 & 3.6 & 18.3 & 0.36 & 0.82 \\
915 & 51 & 5.6 & 1.60 & 0.10 & 2.5 & 12.8 & 0.40 & 0.83 \\
2,450 & 47 & 5.5 & 2.21 & 0.16 & 1.7 & 8.1 & 0.43 & 0.84 \\
5,800 & 43 & 5.1 & 4.73 & 0.26 & 0.8 & 4.7 & 0.44 & 0.85 \\
10,000 & 40 & 4.5 & 10.3 & 0.44 & 0.3 & 2.6 & 0.45 & 0.87 \\
\hline
\end{tabular}

Clearly, the coupling of RF energy from air into planar tissue is greater for low-water-content tissue compared to high-water-content tissue. It is greater for higher RFs than for lower ones and ranges from about $15 \%$ to $80 \%$. The data given in Table 1.1 show that the penetration depth for low-water-content tissues such as bone and fat is about five times greater than that for high-water-content tissues and that it is also frequency dependent. The transmission coefficient for air-tissue interfaces nearly doubles for low-water-content tissues compared with high-water-content tissues. Moreover, the transmission coefficient for tissue-tissue interfaces is generally larger than that for airtissue interfaces, whereas the reflection coefficient shows just the opposite trend. The reflection coefficient varies from a low of 5\% for muscle-blood interfaces to a high of about $50 \%$ for bone-muscle interfaces (Lin and Bernardi 2007).

In a layered tissue structure having different dielectric permittivities, the coupling behavior can be very complex. Multiple reflections can occur between tissue interfaces. The transmitted field will combine with the reflected field to form standing waves in each tissue layer. The peaks of the standing waves can result in greater coupling of RF energy into the tissue layer. The standing-wave phenomenon becomes especially pronounced if the thickness of each layer is greater than the penetration depth for that tissue layer and is approximately one-half wavelength or longer at RF. This dependence of standing-wave oscillation peaks on layer thickness is a manifestation of layer resonance, which can enhance power transmission.

\subsubsection{Radiofrequency Field Coupling to Bodies with Curvature}

A plane-wave RF field in the far zone can overcome exponential losses and produce enhanced coupling at great depths in bodies with curved surfaces. Typically, if the largest dimension of the body is comparable to the wavelength of the impinging RF field, energy deposition and distribution will be influenced by the surface curvature of the whole body or the body part and tissue composition. In particular, the ratio of geometric variables and wavelength affects the characteristics of RF energy coupling. An example of this phenomenon is given in Figure 1.11, where absorbed energy distributions inside a 9-cm-radius homogeneous spherical model of the brain are shown. Note that the distributions have 

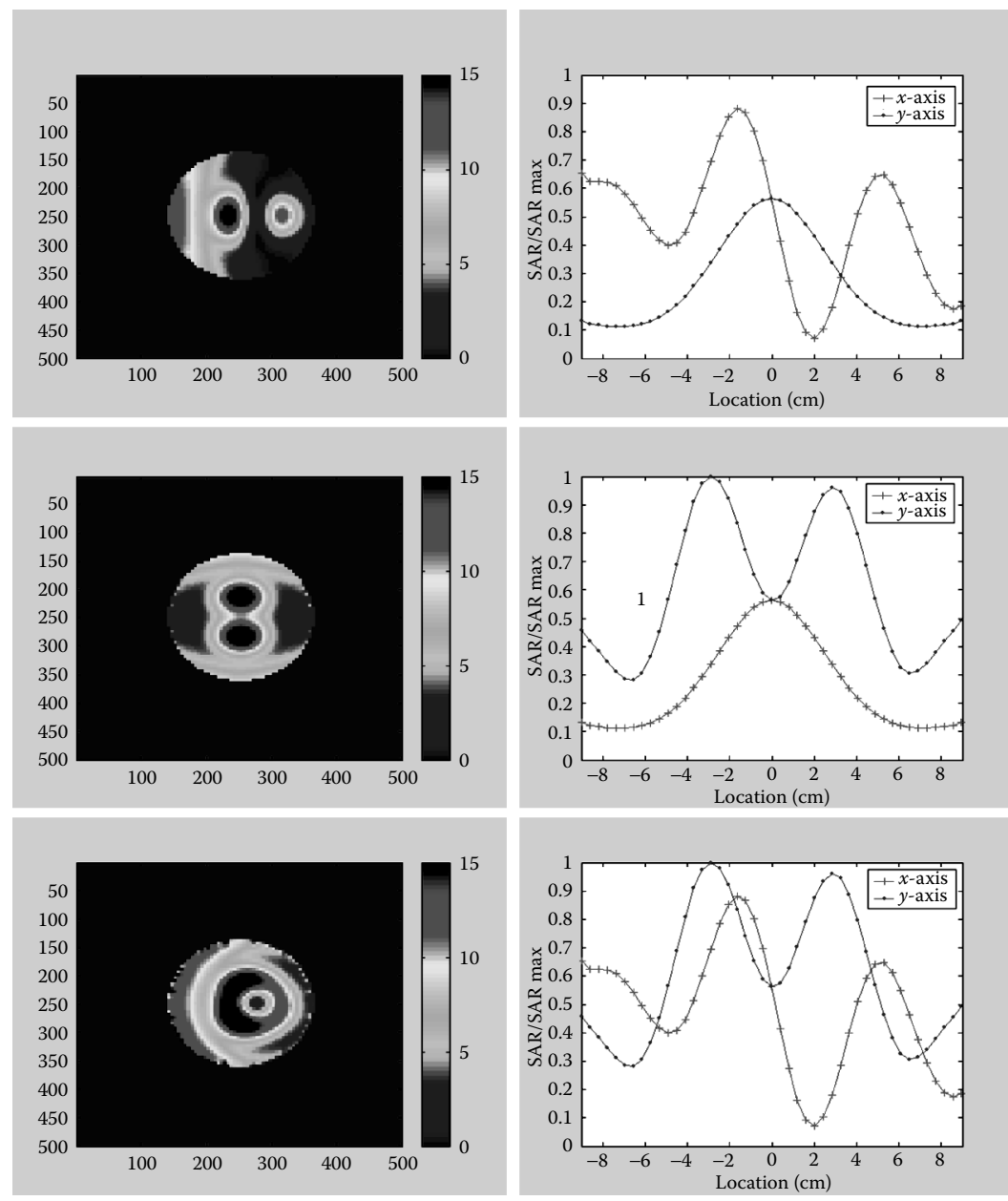

FIGURE 1.11 (See color insert.) Specific absorption rate distribution inside a brain sphere under plane-wave exposure: The three columns represent values for the $x y, y z$, and $z x$ planes, respectively. The corresponding line distributions along (red) the direction and transverse to the direction (blue) of propagation are shown at the bottom (diameter $=18 \mathrm{~cm}$ and frequency $=$ $400 \mathrm{MHz}$ ). The direction of wave propagation is along the $x$ axis.

peaks deep inside the brain sphere and local peaks may be several times greater than those due to exponential losses in planar homogeneous models. The enhancement is a result of refraction of the incident plane wave into the brain sphere by the curved tissue surface and the standing wave (or geometric resonance phenomenon). In addition, the top and bottom or left and right sides of the model have equal magnitudes because of spherical symmetry of the brain model and uniform transverse fields of the incident plane wave. It is noted that RF coupling is weaker and penetration depth is shorter for nonuniform incident fields, especially for incident radiation of limited beam width (Lin and Bernardi 2007). 
The coupling of plane RF radiation into more complex models of the head structure in which a spherical core of the brain is surrounded by five concentric shells of other tissues shows that the effect of skin, fat, skull, dura, and cerebrospinal fluid on specific absorption rate (SAR) distribution is to increase SAR in the skin. Absorptions in fat and skull are the lowest among tissue layers (Lin 1986). Moreover, the peak and average SAR values may be several times greater than the values for homogeneous models. The enhancement is apparently due to resonant coupling of plane-wave RF into the brain sphere by the outer tissue layers.

Although a spherical model can provide some results that are relatively close to those obtained with more realistic head models, the SAR values obtained with spherical or simplified head models that do not include the ear tend to be greater than those obtained with head models that include the ear. A hand holding a handset absorbs a significant amount of the cell phone's output power (see Section 1.11.1).

\subsubsection{Orientation and Polarization Dependence}

For elongated bodies such as a human body or the prolate spheroid, shown in Figure 1.3, for which the height-to-width ratio is large, the coupling of RF energy is influenced by the orientation of the electric field vector (polarization) with respect to the body. The three principal polarizations of the impinging plane wave to be distinguished are as follows: (1) E-polarization, in which the electric vector is parallel to the long axis of the body; (2) H-polarization, in which the magnetic vector is parallel to the long axis of the body; and (3) K-polarization, in which neither the electric vector nor the magnetic vector is parallel to the long axis of the body. The frequency at which the highest (resonant) absorption occurs is a function of both polarization and the exposed subject. In general, the shorter the subject the higher the resonance frequency and vice versa. Further, E-polarization couples RF energy most efficiently into the body in a plane-wave field for frequencies up to and slightly above the resonance region, where the body dimension and wavelength are approximately equal.

For RFs well below resonance, such that the ratio of long body dimension $(L)$ to free space wavelength $(\lambda)$ is less than 0.2 , the average SAR is characterized by an $f^{2}$ dependence. The SAR goes through the resonance region in which $0.2<L / \lambda<1.0$. Specifically, the SAR rapidly increases to a maximum near $L / \lambda=0.4$ and then falls off as $1 / f$. At frequencies for which $L / \lambda>1.0$, whole-body absorption decreases slightly but approaches asymptotically about one-half of the incident power, that is, 1-power reflection coefficient is transmitted into biological tissue. The resonances are not nearly as well defined for $\mathrm{H}$-polarization as for E-polarization. The average SAR for $\mathrm{H}$-polarization gradually reaches a plateau throughout the RF spectrum (Lin and Gandhi 1996).

\subsubsection{Radiofrequency Coupling in the Near Field}

The accelerated use of RF radiation for wireless communication in recent years has generated considerable attention on the amount of RF energy coupled into human bodies and on the potential biological effects of radiation. The antenna of a cellular mobile telephone is typically located next to the user's head, thereby creating an exposure situation 
in the near zone of the RF antenna. As mentioned in Section 1.8.2, the distance to the near field is on the order of a few wavelengths or less in most antenna systems. Even for an elementary dipole, the reactive near-field distance is approximately $\lambda /(2 \pi)$, which is about $5 \mathrm{~cm}$ in air at $900 \mathrm{MHz}$ or $2 \mathrm{~cm}$ at $2400 \mathrm{MHz}, 900$ and $2400 \mathrm{MHz}$ being the typical frequencies used for wireless mobile communication.

As discussed in Section 1.8.2, the near fields of an antenna are quasistatic (see Equations 1.30 through 1.35). In contrast to a plane wave in the far field, near-field RF electric and magnetic fields are in time quadrature and their magnitudes vary with location or distance. Wave impedance is no longer the same as intrinsic impedance and varies from point to point in the near zone. The maxima of electric and magnetic fields also do not occur at the same location in space. These are precisely the characteristics of a standing wave, and thus $\mathrm{RF}$ radiation in the near field behaves like a standing-wave field. The RF energy will be transferred back and forth between the radiating antenna and the human body.

Typical wireless RF antennas for personal communication are tiny compared to the size of a human head (Balanis 2008). The beam width in the near field is also smaller than the head. As shown in Figure 1.6, the field is diverging in the near zone. In addition to the reactive induction field, there is a radiative component that is outgoing and is proportional to the product of the electric and magnetic field components. Since RF currents produce magnetic fields and time-varying magnetic fields generate electric fields in tissue, RF coupling in biological tissues can be properly expressed in terms of induced electric fields.

\subsection{Radiofrequency Dosimetry and Energy Absorption in Anatomical Models}

Electromagnetic fields must be coupled into tissues and energy must be absorbed or deposited in the biological systems in order for biological system to respond in some manner. Thus to establish any biological response, the electric, magnetic, or electromagnetic field that is effective in exerting its influence must be quantified and correlated with the observed effect. The commonly employed metrics or dosimetric quantities include incident field, induced field, SAR, and SA in biological systems or tissue media. The metric SAR (in watt per kilogram) is a derived quantity and is defined as the time derivative of the incremental energy absorbed by (or dissipated in) an incremental mass contained in a volume of a given density (NCRP 1981). This definition allows SAR to be used as a metric for RF in both near and far fields. Specific absorption (in joules per kilogram) is the total amount of energy deposited or absorbed and is given by the integral of SAR over a finite interval of time. Information on SA and SAR is of interest because it can serve as an index for the extrapolation of experimental results from cell to animal, animal to animal, and animal to human exposures. It is also useful in analyzing relationships among various observed biological effects in different experimental models and subjects. Indeed, SAR has been adopted worldwide as the dosimetric quantity in regulations established for human exposure to cellular mobile telephone RF fields.

The induced field is of primary interest because it relates the RF field to specific responses of the body, facilitates an understanding of biological phenomena, and is independent of the mechanisms of interaction. Once the induced field is known, 
quantities such as SAR can be derived by a simple conversion formula. For example, from an induced electric field $\mathbf{E}$ in volts per meter one can calculate SAR:

$$
\mathrm{SAR}=\sigma \mathrm{E}^{2} / \rho_{\mathrm{m}}
$$

where $\sigma$ is the bulk electrical conductivity and $\rho_{\mathrm{m}}$ is the mass density (kilogram per cubic meter) of tissue, respectively. At present, the smallest isotropic implantable electric field probe available with sufficient sensitivity for practical use is about $1 \mathrm{~mm}$ in diameter and is quite expensive. Consequently, a common practice in experimental dosimetry relies on the temperature elevation produced under a short-duration $(<30$ seconds) high-intensity exposure condition. (The short duration is insufficient for significant convective or conductive heat contribution to temperature rises in tissues.) The intensity is sufficient to produce a measurable temperature elevation. In this case, the time rate of initial rises in temperature (slope) can be related to SAR through a secondary procedure, such that

$$
\mathrm{SAR}=c \Delta T / \Delta t
$$

where $\Delta T$ is the temperature increment (in degree Celsius), $c$ is the specific heat capacity of tissue (joules per kilogram degree Celsius), and $\Delta t$ is the time duration over which $\Delta T$ is measured. It is important to distinguish the use of SAR and its derivation from temperature-based measurements. The quantity of SAR is merely a metric for energy deposition or absorption and it should not be construed to imply any mechanism of interaction, thermal or otherwise. However, it is a quantity that pertains to a macroscopic phenomenon by virtue of the use of bulk electrical conductivity and mass density in its derivation in Equation 1.46 and the use of specific heat capacity of tissue in Equation 1.47.

It is important to note the use of bulk electrical conductivity, specific heat capacity, and mass density (kilogram per cubic meter) of tissue in the derivation of SAR from electric field strength and temperature elevation. The use of these factors in the derivation of SAR implies that a volume of tissue mass must be selected over which SAR is determined. In common usage, 1 or $10 \mathrm{~g}$ of tissue in the form of a cubic volume are specified. It is obvious that the numerical value of SAR would be the same regardless of what mass or volume is chosen if the induced field and power deposition are uniform. A variance arises when the absorption is nonuniform or when tissues with differing properties are included within the same volume of averaging mass. In principle, a 10-g averaging mass can underestimate SARs of nonuniform fields by up to a factor of 10 compared with a 1-g averaging mass. It is emphasized here that recent advances suggest spatial resolutions comparable to $0.01 \mathrm{~g}$ or less are routinely obtained using available computational algorithms and resources to provide higher spatial precision in SAR determination.

\subsubsection{Coupling from Handheld Mobile Phones}

A topic of considerable current interest is the coupling of RF energy from handheld cellular mobile telephones and other personal communication systems into the human head or body. Cell phones are designed to operate in close proximity with the user and are typically located next to the user's head. Aside from the intended purpose of 
radiation into air to enable wireless communication, RF energy from these devices is coupled into the user's head, neck, or hand situated in the near zone of the radiating system. A variety of experimental and computational methods have been used to quantify induced fields and SARs, and to assess the health and safety risks posed by these wireless communication devices (Lin and Bernardi 2007; Bernardi et al. 2009; see also Chapter 5 of this book). It is noteworthy that SAR has been adopted as the metric in guidelines for human exposure to cellular mobile telephone RF fields and compliance testing of cell phones worldwide (FCC 1996; ICNIRP 1998; IEEE 2005; NCRP 1986). A 1997 Federal Communications Commission (FCC) bulletin provides acceptable methods for determining compliance limits through the use of computational and experimental models.

Experimental measurement of SAR distributions in anatomically shaped models of the human head employs isotropic electric probes in plastic skulls filled with brainequivalent liquid dielectric phantom materials (Balzano, Garay, and Steel 1978; Balzano, Garay, and Manning 1995; Cleveland and Athey 1989; Gandhi et al. 1999; Kuster and Balzano 1992; Schmid, Egger, and Kuster 1996). These efforts are compounded by the variability of electric and magnetic fields in the near zone of the antenna and also by the wide array of possible device positions and tilt angles of the antenna during normal use. Thus, an intended use position and an angle of $30^{\circ}$ are often adopted in measurements for a given device. Nevertheless, a survey of the reported measurements in homogeneous models of head phantoms showed that the measured SARs vary depending on the specific antenna configuration and placement of the antenna next to the head (Table 1.2). Other contributing factors include difficulties associated with performing accurate and reliable SAR measurements when the source is in close proximity to the head. Measurements made in a homogeneous brain liquid phantom using five commercially available telephone models showed a difference of two between 1-g and 10-g SAR metrics (Kuster and Balzano 1997). In general, the 10-g SAR metric tends to provide more conservative estimates.

TABLE 1.2 Representative Measured 1-g SARs in Homogeneous Head Phantoms Exposed to Wireless Communication Devices (600-mW Output Power)

\begin{tabular}{lcccc}
\hline \multicolumn{5}{c}{ Maximum SAR } \\
\hline Frequency $(\mathrm{MHz})$ & $($ Brain) $(\mathrm{W} / \mathrm{kg})$ & Distance $(\mathrm{cm})$ & Antenna Type & Reference \\
\hline 815 & 1.0 & $1.0-2.0$ & $1 / 4 \lambda$ & $\begin{array}{c}\text { Cleveland and } \\
\text { Athey }(1989)\end{array}$ \\
855 & 2.1 & $1.0-2.0$ & $1 / 2 \lambda$ & \\
835 & 0.63 & 1.0 & $1 / 4 \lambda$ & $\begin{array}{c}\text { Anderson and } \\
\text { Joyner }(1995)\end{array}$ \\
835 & $0.44-0.83$ & 1.0 & $1 / 2 \lambda$ & $1 / 4 \lambda$ \\
900 & $1.8($ Ear) & 2.5 & & $\begin{array}{c}\text { Balzano, Garay, and } \\
\text { Manning (1995) }\end{array}$ \\
& 3.6 & 2.5 & $1 / 2 \lambda$ & $\begin{array}{c}\text { Kuster and Balzano } \\
\text { (1992) }\end{array}$ \\
\hline
\end{tabular}

Source: Lin, J. C. 2000. Mechanisms of field coupling into biological systems at ELF and RF frequencies. In Advances in Electromagnetic Fields in Living Systems. vol. 3, ed. J. C. Lin, 1-38. New York: Kluwer/ Plenum. 
To standardize SAR measurements for compliance testing of cell phones against applicable exposure guidelines and to minimize variability in measured results, a specific anthropomorphic mannequin (SAM) is proposed for laboratory settings (IEEE Standard 1528; IEEE 2003). The simplified physical model of the human head consists of a lossless plastic shell and ear spacer. Inside the shell, there is a homogeneous fluid having the dielectric properties of average head tissue at the test frequency. Because current technology does not allow reliable measurement of SAR in small, complex geometric structures such as a simulated pinna, SAM uses a thin lossless ear spacer on the model to mimic the energy reaching the brain and to reduce the measurement uncertainty. It is designed to provide conservative SAR values averaged over 1 and $10 \mathrm{~g}$ of tissue mass for a majority of individuals during normal use of wireless handsets. The SAM has also been adopted by the U.S. FCC (1997), European Committee for Electrotechnical Standardization (CENELEC 2003; EN 50361), and Association of Radio Industries and Businesses in Japan (ARIB 2002; ARIB STD-T56). The extent to which SAM is truly conservative has been a subject of study for several investigators; they used computational dosimetry that compared the SAR in SAM with that in realistic anatomical models of the human head (Gandhi and Kang 2002; Christ et al. 2005; Kainz et al. 2005; Beard et al. 2006).

There are many advantages to numerical modeling in SAR determination. These include a more realistic representation of the human anatomy and the telephone as well as the device's position relative to the user. The ability to vary these parameters to examine the dosimetric interaction of RF radiation from cellular telephone operation with the entire human body or its parts such as the head and the hand is also important (Lin and Gandhi 1996; Lin and Bernardi 2007).

Regarding the aforementioned aspect, the most widely applied numerical method is the finite-difference time-domain (FDTD) algorithm. This method, first proposed by Yee (1966), is based on the substitution of each partial derivative in Maxwell's equations in time domain with its finite difference representation. This substitution leads to a set of six equations for which each field component is evaluated at a point of time as a function of the adjacent components evaluated in preceding points of time. The space and time variables are divided into discrete increments in which the electromagnetic field is assumed constant. This leads to the definition of a unit cell (referred to as Yee's cell) in which the electromagnetic field is assumed constant. To improve the method's precision without increasing the complexity of the formulas, the electric field and magnetic field components are placed in different positions within the Yee's cell and evaluated at half-time steps.

A stability condition is imposed to ensure convergence of the solution. This condition is usually referred to as the Courant condition, and it limits the time step $(\Delta t)$ as a function of the space steps $(\Delta x, \Delta y$, and $\Delta z)$ according to

$$
\Delta t \leq \frac{1}{v \sqrt{\frac{1}{\Delta x^{2}}+\frac{1}{\Delta y^{2}}+\frac{1}{\Delta z^{2}}}}
$$


where $v$ represents the speed of wave propagation in vacuum. Similarly, to ensure that the solution is correct an accuracy condition must be satisfied:

$$
\max (\Delta x, \Delta y, \Delta z)<<\lambda_{\min }
$$

where $\lambda_{\min }$ is the minimum wavelength in a tissue medium with the highest dielectric permittivity. Finally, to limit the computational domain for finite resources, absorbing boundary conditions (ABCs) are employed. Several ABCs have been proposed over the years (e.g., Mur's ABC, Higdon ABC, and retarded time). At present, the ABCs most often used are those proposed by Berenger (1994), known as perfectly matched layer (PML). For more details, the reader is referred to recent books on the subject like the ones by Kunz and Luebbers (1993) and Taflove (1995).

Several three-dimensional (3-D) models of the human body have been developed and utilized for SAR computations. These have proved very useful since they offer more realistic representations of the human anatomy. The most frequently used highresolution human model is from the "Visible Human" (VH) project of the National Library of Medicine (Ackerman 1998). The VH is a 3-D digital image library representing an adult human male and female. The for both the male and female include photographic images obtained through cryosectioning of human cadavers and digital images obtained through computed tomography (CT) and magnetic resonance imaging (MRI) of the same cadavers. The male data set, the first to be constructed, consists of 1871 digital axial images obtained at $1.0-\mathrm{mm}$ intervals with a pixel resolution of $1 \mathrm{~mm}$, whereas the female data set contains 5189 digital axial images obtained with a finer spatial grid of $0.33 \mathrm{~mm}$. The male images have been segmented at the Air Force Research Laboratory, Brooks Air Force Base, Texas (Mason et al. 2000). The final segmented model, made freely available to the scientific community (ftp://starview. brooks.af.mil), consists of $586 \times 340 \times 1878$ voxels with a resolution of $1 \times 1 \times 1 \mathrm{~mm}^{3}$. The model is segmented into about 40 different tissue types with frequency-specific tissue permittivity, such as those given in Table 1.3, which are used to represent each tissue type in the inhomogeneous model of the human body. The model is widely used to study head exposure to RF fields from cell phones (Bernardi et al. 2001a,b; Gjonaj et al. 2002; Wang et al. 2004; Pisa et al. 2005) and for other dosimetric calculations (Lin and Wang 2010; Wang et al. 2007, 2008). In fact, the model is now included in many commercially available electromagnetic simulation tools with capabilities for dosimetric calculations.

Cell phones can be modeled simply as half-wavelength dipoles or as quarterwavelength monopoles over a box; but more complex and realistic phone geometries also have been considered. The half-wavelength dipole is used as a first approximation (Dimbylow 1993; Chen and Wang 1994; Martens et al. 1995; Bernardi, Cavagnaro, and Pisa 1996). A better model is a quarter-wavelength monopole over a box (Toftgard, Hornsleth, and Andersen 1993; Jensen and Rahmat-Samii 1995; Nikita et al. 2000). These antenna configurations can be regarded as rough models of the retractable antenna, which was used in nearly all of the early cell phone handsets. Helical antennas for more compact terminals require the use of graded mesh in numerical models to 
TABLE 1.3 Permittivity (Conductivity and Dielectric Constant) of Biological Tissues for the RF Used by Mobile Telephones $(800-900 \mathrm{MHz}$ )

\begin{tabular}{lcc}
\hline Tissue Type & Dielectric Constant & Conductivity (S/m) \\
\hline Air & 1.0 & 0.0 \\
Fat & 11 & 0.17 \\
Bone & 21 & 0.33 \\
Skin & 35 & 0.6 \\
Muscle & 50 & 1.08 \\
Lung & 12 & 0.24 \\
Brain & 41 & 0.86 \\
Cerebrospinal fluid & 78 & 1.97 \\
Blood & 55 & 1.86 \\
Eye & 67 & 1.97 \\
Vitreous humor & 67 & 1.68 \\
Cornea/sclera & 51 & 1.13 \\
Lens & 45 & 0.75 \\
\hline
\end{tabular}

Source: Lin, J. C. 2000. Mechanisms of field coupling into biological systems at ELF and RF frequencies. In Advances in Electromagnetic Fields in Living Systems. vol. 3., ed. J. C. Lin, 1-38. New York: Kluwer/Plenum. With permission.

obtain good agreement with experimental results (Bernardi et al. 2001a,b). Planar integrated antennas for dual-band operation can be mounted on the top, side, or back of the phone (Jensen and Rahmat-Samii 1995; Rowley and Waterhouse 1999; Bernardi et al. 2000; Li et al. 2000). Since commercial phones are not planar, conformal graded mesh is needed in the numerical models (Pisa et al. 2005). For a planar antenna-equipped cell phone, an important consideration is the influence of the hand holding the handset. In this case, the hand has a detuning effect on the antenna resonant frequency and this causes a reduction in the bandwidth, which becomes evident when the hand masks the antenna.

The model adopted for the phone case and internal circuit structures of the phone can also influence computed absorption by the user's head. Earlier models had plastic-coated metal boxes as phone cases. To achieve better modeling of the shape of cell phones, computer-aided design (CAD) files (Tinniswood, Furse, and Gandhi 1998) and topometric sensors (Schiavoni et al. 2000) are used. Moreover, in most studies, the internal structures (printed circuit board [PCB], battery, keypad, and buttons) of the phone are modeled simply using a homogeneous perfect conductor. Further, CAD files are used to model the internal structures of the phone (Chavannes et al. 2003).

In the remainder of this section, computed results under a variety of exposure conditions are discussed. Table 1.4 shows a comparison between the 1-g SARs obtained for two phone models: (1) CAD-derived phone models and (2) idealized box models (Gandhi et al. 1999). Note the comparable values of SAR and power absorbed in the head and hand of a user obtained from realistic and idealized phone models. It is observed that using an incorrect model for the phone case could lead to overestimating the SAR value averaged over 1 or $10 \mathrm{~g}$ by as much as three times (Pisa et al. 2005). 
TABLE 1.4 Comparison between CAD-Derived Phone Models and Their Equivalent PlasticCovered Metal-Box Models

\begin{tabular}{llccc}
\hline & & $\begin{array}{c}\text { Power Absorbed } \\
\text { in the Head } \\
\text { (Percentage) }\end{array}$ & $\begin{array}{c}\text { Power Absorbed in } \\
\text { the Hand } \\
\text { (Percentage) }\end{array}$ \\
\hline $\begin{array}{l}835 \mathrm{MHz} \text { phone } \\
\text { (radiated power: }\end{array}$ & CAD model & 2.17 & 23.8 & 17.2 \\
$\begin{array}{l}\text { (SAR } \mathrm{mW} \text { ) } \\
\text { Plastic-covered } \\
\text { metal-box model }\end{array}$ & 2.39 & 26.7 & 17.8 \\
$\begin{array}{l}\text { (radiated power: } \\
125 \mathrm{~mW} \text { ) }\end{array}$ & $\begin{array}{c}\text { CAD model } \\
\text { Plastic-covered } \\
\text { metal-box model }\end{array}$ & 0.78 & 32.1 & 17.9 \\
\hline
\end{tabular}

Source: Gandhi, O. P., G. Lazzi, A. Tinniswood, and Q. S. Yu. 1999. Bioelectromagnetics 20 (Suppl 4): S93-S101.

TABLE 1.5 SAR Data for a Monopole (Covered by a Rubber Slab) over a Metallic PCB Covered by a Plastic Box

\begin{tabular}{lccccc}
\hline & \multicolumn{2}{c}{ Cheek Position } & & \multicolumn{2}{c}{ Tilted Position } \\
\cline { 2 - 3 } \cline { 5 - 6 } Frequency & $\mathrm{SAR}_{1 \mathrm{~g}}(\mathrm{~W} / \mathrm{kg})$ & $\mathrm{SAR}_{10 \mathrm{~g}}(\mathrm{~W} / \mathrm{kg})$ & & $\mathrm{SAR}_{1 \mathrm{~g}}(\mathrm{~W} / \mathrm{kg})$ & $\mathrm{SAR}_{10 \mathrm{~g}}(\mathrm{~W} / \mathrm{kg})$ \\
\hline $835 \mathrm{MHz}$ & 7.5 & 5.2 & 5.0 & 13.1 & 3.4 \\
$1900 \mathrm{MHz}$ & 9.2 & 5.3 & & 13 \\
\hline
\end{tabular}

Source: Kainz, W., A. Christ, T. Kellom, S. Seidman, N. Nikoloski, B. Beard, and N. Kuster. 2005. Phys Med Biol 50:3423-45. With permission.

Common or intended headphone positions have been used to compute SAR distributions in the head. For example, the SARs computed for 1 and $10 \mathrm{~g}$ of tissue at $835 \mathrm{MHz}$ and $1900 \mathrm{MHz}$, for $1.0 \mathrm{~W}$ of radiated power, are presented in Table 1.5. The computation was done using the cell phone model of a generic monopole, which was covered by a rubber slab (71 mm long for $835 \mathrm{MHz}$ and $36 \mathrm{~mm}$ long for $1900 \mathrm{MHz}$ ) and placed on a flat metallic plate $(40 \times 1 \times 100 \mathrm{~mm})$, representing the PCB, inside a plastic box $(42 \times 21 \times 102 \mathrm{~mm})$. Apart from the typical observation of higher SARs (approximately double) for the higher operating frequency and the conservative estimation of 10 -g averaged SAR values, it is observed in a comparison between "cheek" or "tilt" headphone positions that the cheek position always gives the higher maximum SAR.

A representative list of computed SAR values for cellular telephones operating at 900 and $1800 \mathrm{MHz}$ is given in Table 1.6. For the two types of simulated antenna configurations, the results do not exhibit any consistent effect of antenna model on induced SAR. However, the peak SAR tends to be lower for longer antennas compared with shorter antennas at the higher frequency. It is interesting to note that some computed data based on head and neck anatomy have recorded values for 1-g SARs that are twice as high as those computed for 10-g SARs (Gandhi et al. 1999; Kainz et al. 2005).

It is significant from the energy coupling perspective that $40 \%-50 \%$ of the radiated RF power is absorbed by the human body (Table 1.6); this was reported earlier by other investigators also (Tinniswood, Furse, and Gandhi 1998; Gandhi et al. 1999). 


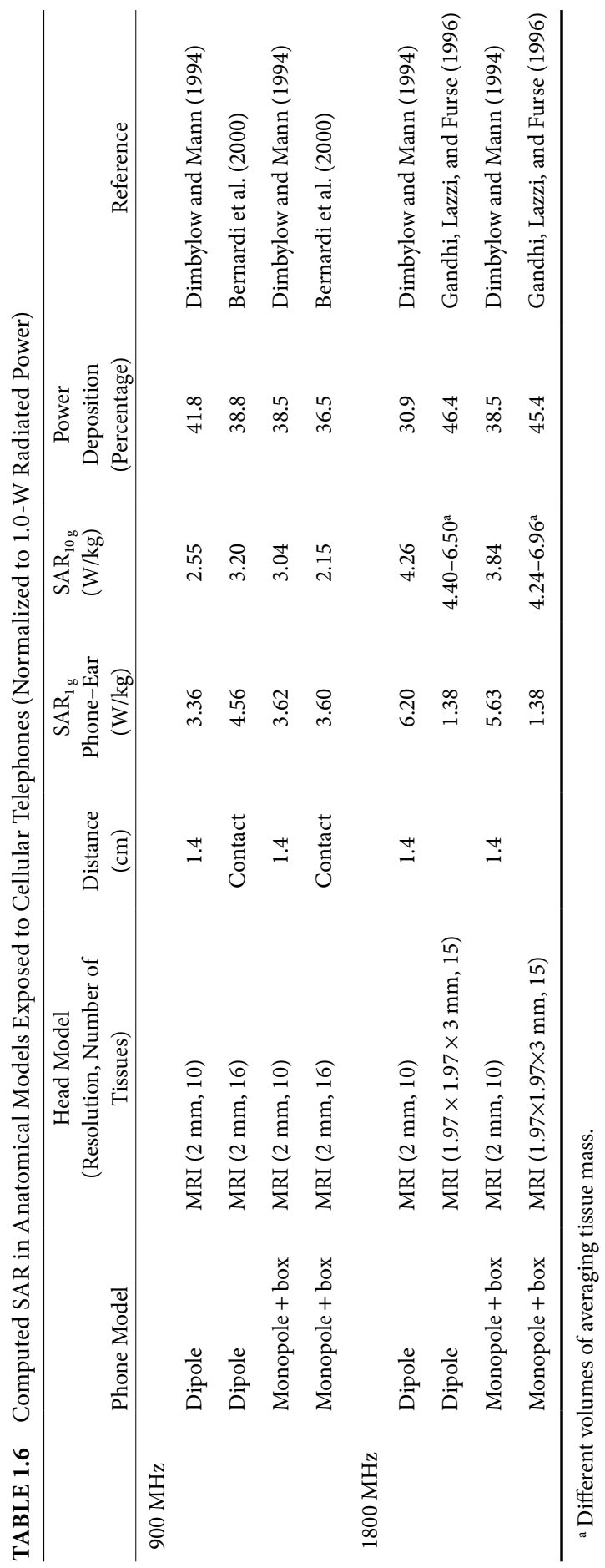


The bulk of power deposition is on the side of the head nearest to the radiating structure of the cellular telephone. The SAR distribution follows an exponential trend away from the side closest to the antenna and is considerably lower elsewhere in the head. The maximum SAR value and its distribution in the head are related to the distance of the radiating element from the skin surface and the electric current distribution on the antenna. For example, the highest SAR is typically located near the feed point of the antenna. Also, the peak SAR is lower for longer antennas compared to shorter antennas, coinciding with the fact that the high-current region for the longer antenna is higher up along the antenna and thus further away from the surface of the head.

At the feed point of the antenna, the current density is the highest, which gives rise to a strong magnetic field. Because of the dominance of quasistatic magnetic field coupling in the near zone of the antenna, the induced electric field is closely correlated with the current distribution on the antenna. The maximum SAR and its distribution in the head follow an eddy current course. Since eddy currents circulating in the ear (pinna) and the head must go through a narrow appendage, the current density and SAR value are the highest at this location. Also, RF electric and magnetic fields are decoupled in the near zone. The effect of the electric field is weaker since the dielectric permittivity of muscle and brain tissues is relatively high at these frequencies. Thus, inductive coupling of the magnetic field generated by the antenna current dominates power deposition in the near zone of a cellular telephone antenna.

Questions have been raised concerning the intercomparability of computational results using different models of the human head and the mobile telephone transceiver. A comparative dosimetry study under the acronym of CEPHOS-cell phones standards-was conducted between 1997 and 1999 by investigators from 15 organizations in Europe. The project compared different implementations of the FDTD scheme and differences in the modeling of the antenna and the head on SAR (Nikita et al. 2000). The project found that the computed results were not sensitive to the ABCs used, although the variability in peak local SAR and 10 -g averaged SAR was mostly related to the modeling of the head and antenna. For example, the uncertainty related to antenna modeling in predicting the averaged SAR values was on the order of $12 \%-15 \%$. The position of peak local SAR was independent of both antenna modeling and the ABCs. The total uncertainty in computing the power absorbed by the head was mainly related to the model for the head. It concluded that large differences in numerical results could be obtained from different numerical models of the head or antenna structure. Another study evaluated SARs in 14 head models based on different anatomical characteristics, although the comparison among the different head models focused on differences in modeling the pinna (Kainz et al. 2005).

As an illustration, Figure 1.12 presents the SAR distribution inside the head with a cell phone on the right side of a male user with an output power of $0.25 \mathrm{~W}$. The computer modeling employed a basic voxel size of $1 \mathrm{~mm} \times 1 \mathrm{~mm} \times 1 \mathrm{~mm}$ and the voxel size was reduced to $0.2 \mathrm{~mm}$ in head regions close to the phone. In this case, the 0 - $\mathrm{dB}$ SAR equals $1.25 \mathrm{~W} / \mathrm{kg}$. It can be seen that most of the power deposition is on the side of the head nearest to the radiating structure of the cellular telephone. The SARs are considerably lower elsewhere in the head. 


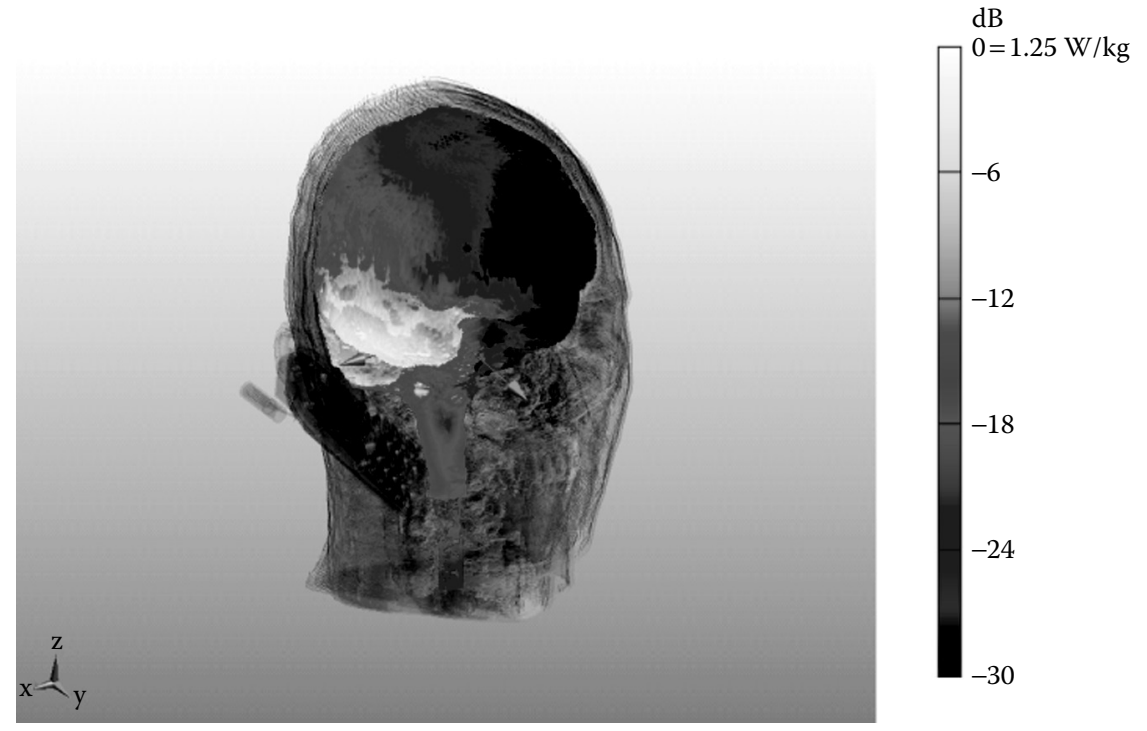

FIGURE 1.12 (See color insert.) Distribution of specific absorption rate inside the head of a young European male irradiated by a $902 \mathrm{MHz}$ cellular mobile telephone: Radiofrequency radiation is absorbed mostly in the temporal cortical region of the brain; $0 \mathrm{~dB}$ in the scale equals 1.25 W/kg. (Courtesy of Myoung Soo Kwon, University of Turku, Finland.)

\subsubsection{Specific Absorption Rate in Childlike Head Models}

A major topic of interest is the exposure of children to cell phone radiation, that is, whether the exposure of a child's head produces SAR levels and distributions that are different from those produced in adults (Lin 2003). There are many variables and aspects of the problem that are often uncontrolled, poorly accounted for, or misunderstood. Some of the differences are attributable to different age and anatomical features of the child model, variable phone orientations, separation between the head and phone, parameters used for SAR normalization (output power or antenna current), and procedures adopted for SAR averaging. The choice of different averaging procedures, starting with the same absorption profile, could lead to average SARs not being significantly different for a marked increase with decreasing head sizes. One of the major causes for increased absorption in a child's head is the decreased distance of separation between the cell phone antenna and the head due to the thinness of ear. Moreover, how results are presented, such as the extent of brain tissue involvement in children, can vary depending on whether it is based on the conventional definition of penetration depth (i.e., $\mathrm{e}^{-2}$ depth) or on the quantity of impacted brain tissue relative to the head size. The paucity of data on age-dependent tissue conductivity and permittivity is also a limitation.

Some computed results normalized to radiated power showed that the peak 1-g SARs for both the head and the brain tissues increase monotonically with reduction in head size (Gandhi, Lazzi, and Furse 1996; Gandhi and Kang 2002). In particular, the peak 1-g SAR for brain tissues in child head models could be up to $60 \%$ higher at $1900 \mathrm{MHz}$ 
and $20 \%$ higher at $835 \mathrm{MHz}$ compared with the values for adult head models. It was suggested that the shielding effect of the pinna was larger at the higher frequency of $1900 \mathrm{MHz}$. Moreover, the observation was reported as the result of a greater extent of absorbed energy distribution for the child models at both 835 and $1900 \mathrm{MHz}$ and the thinner ears of the smaller models, resulting in the antenna being located closer to the brain region. In contrast, Schoenborn, Burkhardt, and Kuster (1998) concluded that there were no differences in the absolute extent of SAR distribution between adult and child head models. It should be noted that the SAR values were normalized to the antenna feed-point current in this case. The effect of normalization by antenna current versus output power was demonstrated in a paper by Wang and Fujiwara (2003).

The influence of anatomical features on SAR was studied by Hadjem et al. (2005). The result of their study supported the conclusion that since the brain is closer to the cell phone in the heads of children, the absorption of RF energy in child brain models is slightly higher than that in adults. Another study showed that 1- and 10-g average SAR values are significantly influenced by the SAR averaging procedure. Adoption of a fixed-cube averaging procedure yielded a marked increase in average SAR with decreasing head size (Bit-Babik et al. 2005). However, the results of the study conducted by Beard et al. (2006) showed that for $1900 \mathrm{MHz}$ cell phones, the peak 1- and 10-g SAR values in the head, pinna, and average tissue of the adult model are consistently higher than those in the child model, whether normalized to the antenna current or radiated power for the cheek and tilt positions. Nevertheless, a majority of the SARs were higher in the child than in the adult model, especially for the $835 \mathrm{MHz}$ phone in tilt position when normalized to the antenna current.

\subsubsection{Fields from Body-Worn Devices}

A hands-free wireless device for a cell phone including the headset can be positioned at different body locations. Evaluation of SAR for these body-worn devices can be accomplished numerically in a similar manner as described in Section 1.11.1 using a heterogeneous model that correctly reproduces the human anatomy. In addition, for the regulatory compliance of body-worn devices several flat phantoms have been suggested for use in experimental SAR measurement (FCC 1997; Christ et al. 2006; IEC 2010), because the part of the body where the phone is located is often flat.

It has been shown that when the cell phone is placed with the antenna close to the body, SARs in the chest can be up to 2.1 times higher at $835 \mathrm{MHz}$ and up to 5.8 times higher at $1900 \mathrm{MHz}$ compared to the SARs found when the antennas are placed away from the body (Gandhi and Kang 2002). This is expected since turning the phone with the antenna away from the body can cause the antenna to be $12-16 \mathrm{~mm}$ closer to the body than in the opposite placement. Moreover, both the peak 1- and 10-g SARs are reduced monotonically with increasing separation between the cell phone and the body.

\subsubsection{Whole-Body Exposure from Cell-Phone Base Stations}

Most public and some occupational exposures to the electromagnetic field radiated by wireless communication base stations take place in the far field of the antenna and involve the whole body. The coupling of fields from a fixed base station may be assessed 
by assuming a plane wave incident on a human body. Specifically, FDTD calculations of SAR distribution in anatomical models of children and adults exposed to uniform RF field of both vertical and horizontal polarizations have been conducted at frequencies in the cellular mobile communication band (900-3000 MHz).

In a study by Piuzzi et al. (2011), five different human body models were developed; all were obtained by appropriately scaling the segmented VH data set along the three spatial directions while maintaining a $2-\mathrm{mm}$ resolution. They include an adult model and two 13-year-old and two 7-year-old child models. The two different child phantoms are a child-scaled and a childlike model. The former was obtained by uniformly scaling the $\mathrm{VH}$ phantom, whereas the latter was derived by applying a nonuniform scaling, which yielded a more realistic gross anatomy.

The original VH phantom, having a height of $188 \mathrm{~cm}$ and a mass of $103 \mathrm{~kg}$, was scaled in its axial cross sections, 0.91 scaling factor for shoulder-to-shoulder $(x$-direction) scaling and 0.83 for front-to-back ( $y$-direction) scaling, to arrive at a final mass of about $80 \mathrm{~kg}$, approximating the weight of a standard-mass adult male (CDC 2009). The two child-scaled models were obtained using appropriate scaling factors along the three directions of the $\mathrm{VH}$ model to reproduce a $50 \%$ mass-for-age and staturefor-age for this age group (WHO 2000). The final 13-year-old child model (0.71 scaling factor in the axial sections and 0.83 in the vertical direction) has a height of $156 \mathrm{~cm}$ and mass of $44 \mathrm{~kg}$, whereas the 7-year-old child model (0.59 scaling factor in the axial sections and 0.67 in the vertical direction) is $125 \mathrm{~cm}$ tall with a body mass of $24 \mathrm{~kg}$.

The scaling procedure, however, does not produce anatomically correct child models, particularly with reference to the proportion between the head and the trunk. Indeed, it is well-known that proportionally children tend to have larger heads than adults. For this reason, two childlike models have also been derived by applying different scaling factors to different body segments. In this case, the body is divided into four major segments: (1) trunk (feet to neck), (2) lower face (chin to the center of the mouth), (3) upper face (center of the mouth to the center of the eyes), and (4) head (above the center of the eyes). The scaling factors for the trunk were kept the same as those adopted for the child-scaled models, whereas scaling factors for the face and head were based on anthropometric data (Leslie and Farkas 1981).

The adult model and the two scaled child models are shown in Figure 1.13. It can be seen that the scaled child models appear different from normal children because of their rather small heads. Anatomical realism is accomplished in the childlike model shown in Figure 1.14, which gives a comparison between the 7-year-old child-scaled and childlike models. Note that although childlike models have realistic body shapes, they do not ensure that the internal organs and other structures are accurately reproduced.

Figure 1.15 presents the computed whole-body average SAR $\left(\mathrm{SAR}_{\mathrm{WB}}\right)$ for the adult and the two scaled child models for two different polarizations using the FDTD algorithm. Note that child-scaled and childlike models of a given age essentially yield the same $\mathrm{SAR}_{\mathrm{WB}}$ values. It can be seen that for a given incident power density $\left(10 \mathrm{~W} / \mathrm{m}^{2}\right)$, $\mathrm{SAR}_{\mathrm{WB}}$ is higher for smaller body model sizes. Also, while $\mathrm{SAR}_{\mathrm{WB}}$ decreases as a function of frequency for all body sizes, its variation with frequency differs for horizontal and vertical polarizations; the horizontal polarization produces a higher absorption above 

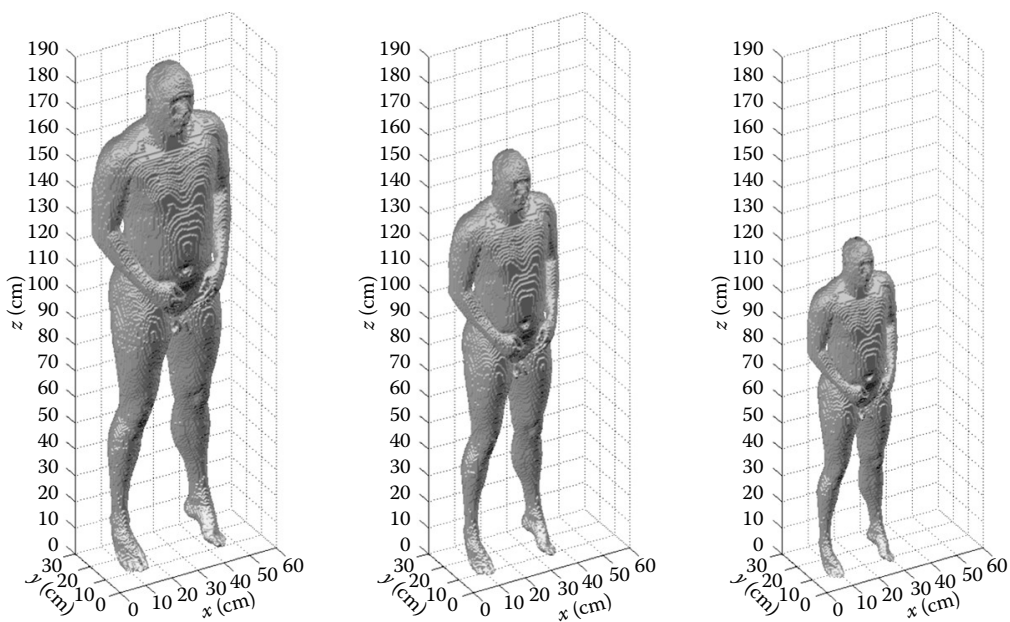

FIGURE 1.13 (See color insert.) Adult (left), 13-year-old child-scaled (middle), and 7-year-old child-scaled (right) phantom body models. (From Piuzzi, E., P. Bernardi, M. Cavagnaro, S. Pisa, and J. C. Lin. 2011. Analysis of adult and child exposure to uniform plane waves at mobile communication systems frequencies (900 MHz-3 GHz). IEEE Trans Electromagn Compat 53:38-47. With permission.)
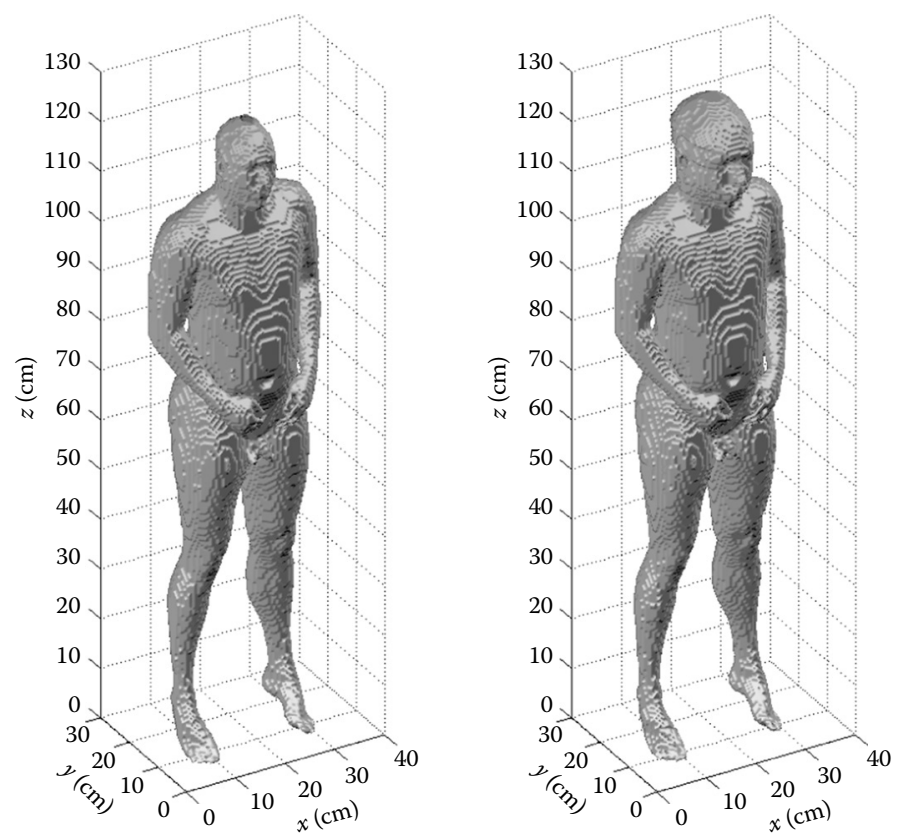

FIGURE 1.14 (See color insert.) Comparison of 7-year-old child-scaled (left) and childlike (right) body models. (From Piuzzi, E., P. Bernardi, M. Cavagnaro, S. Pisa, and J. C. Lin. 2011. Analysis of adult and child exposure to uniform plane waves at mobile communication systems frequencies (900 MHz-3 GHz). IEEE Trans Electromagn Compat 53:38-47. With permission.) 


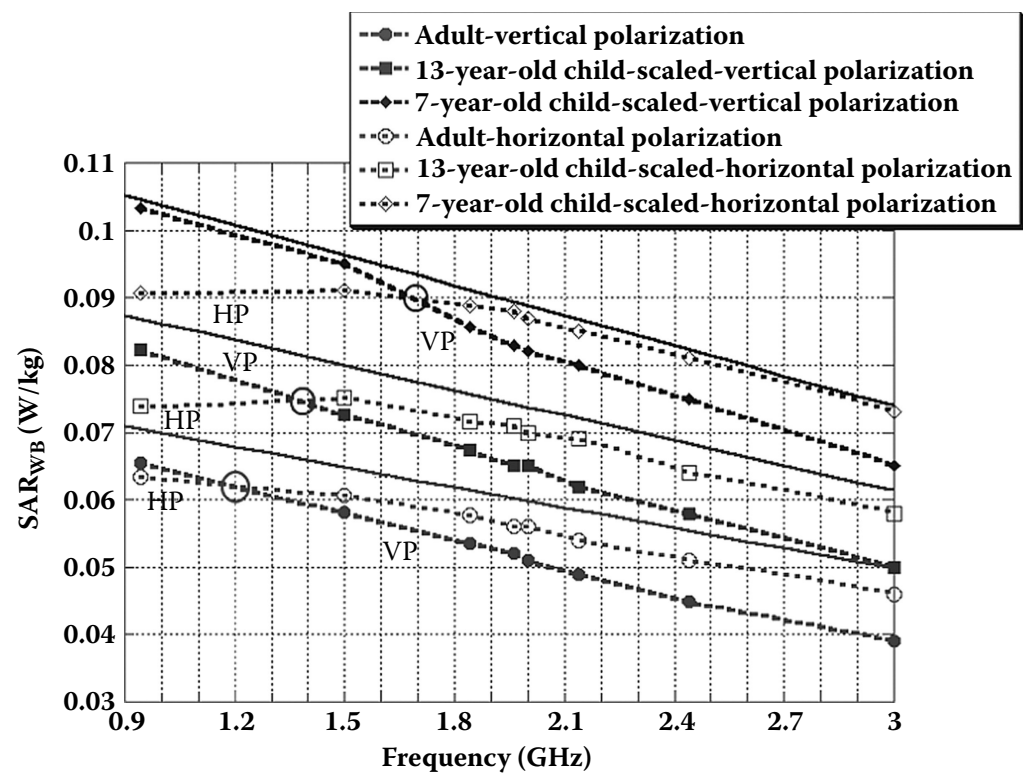

FIGURE 1.15 Whole-body-averaged specific absorption rate (SAR) for $10-\mathrm{W} / \mathrm{m}^{2}$ incident power density, showing fitted curves for $\mathrm{SAR}_{\mathrm{WB} \text { max }}$ over the frequency range of $900-3000 \mathrm{MHz}$. (From Piuzzi, E., P. Bernardi, M. Cavagnaro, S. Pisa, and J. C. Lin. 2011. Analysis of adult and child exposure to uniform plane waves at mobile communication systems frequencies $(900 \mathrm{MHz}-3 \mathrm{GHz}$ ). IEEE Trans Electromagn Compat 53:38-47. With permission.)

a transition frequency (see the circles in Figure 1.15). It is interesting to note that the transition frequency is inversely proportional to model size. Moreover, Figure 1.15 suggests that the variation of maximum $S_{A B}$ with frequency is approximately linear for the three body models over the cellular mobile communication frequency range of $900-$ $3000 \mathrm{MHz}$ if both polarizations are taken into account. Thus, the variation of $\mathrm{SAR}_{\mathrm{WB} \text { max }}$ with frequency may be approximated by a linear curve-fitting procedure, that is,

$$
\mathrm{SAR}_{\mathrm{WBmax}}=(3.1-0.39 \cdot f) \cdot\left(\frac{S}{M}\right) \cdot P_{\mathrm{inc}}
$$

where $f$ is the frequency in $\mathrm{GHz}, P_{\text {inc }}$ is incident power density in $\mathrm{W} / \mathrm{m}^{2}$, and $S / M$ is the surface-to-mass ratio expressed in the units of square meter per kilogram. A set of fitted curves are plotted in Figure 1.15. A nearly constant ratio can be seen among the fitted lines for the three body models over this frequency band. Indeed, a nearly constant ratio can be seen among the fitted lines for the three body models over the frequency range of interest. 


\subsubsection{Summary of Radiofrequency Field Coupling}

The coupling of RF radiation into biological tissues is influenced by the frequency and configuration of the source and the size and composition of the exposed body, as well as the body's distance from the source. Both near-field inductive and far-field radiative interactions contribute significantly to fields induced inside the body. This is also the case for cell phones and other wireless RF personal telecommunication systems. The field coupling characteristics for the far field and the near field are summarized in Sections 1.11.5.1 and 1.11.5.2.

\subsubsection{Field Coupling in the Far Field}

Field coupling characteristics for the far field are summarized as follows:

- Coupling is characterized by plane-wave RF field interaction.

- Interaction is independent of the source configuration.

- Electric and magnetic fields of the outgoing wave are in time phase with each other.

- Electric and magnetic fields are uniquely defined and are related through the intrinsic impedance of the medium ( $120 \pi \Omega$ in air).

- Determination of electric field behavior is sufficient to characterize the interaction.

- Coupling of RF power from air into planar tissue models ranges from about $20 \%$ to $60 \%$ at wireless communication frequencies.

- Enhanced coupling can occur at greater depths in bodies with curved surfaces from the refraction of RF fields.

- Enhanced RF energy deposition can occur because of geometrically resonant absorption by the head at $400-1500 \mathrm{MHz}$. The SAR maxima or hot spots may occur inside the head.

- The coupling of RF energy depends on electric field polarization for elongated bodies whose height-to-width ratio is large.

- Integrated SAR (or average SAR) in the head or the body is similar for both homogeneous and inhomogeneous models.

\subsubsection{Field Coupling in the Near Field}

Field coupling characteristics for the near field are summarized as follows:

- Radiofrequency electric and magnetic fields are decoupled, independent, and nonuniform.

- Antenna beam width is divergent and small compared with the dimensions of the human head for cellular mobile telephones.

- Wave impedance varies from point to point in the near field.

- The RF electric and magnetic fields are in time quadrature $\left(90^{\circ}\right.$ out of time phase).

- Maxima of electric and magnetic fields occur at different locations as in a standing-wave field. 
- The RF energies are transferred back and forth between the source (antenna) and the body.

- The SAR level inside the body is influenced by direct interaction of the source with the body.

- The electric field effect is weaker since dielectric permittivity of tissue is relatively high.

- Inductive coupling of wireless antenna current-generated magnetic field dominates field coupling and power deposition or energy absorption.

- The SAR value varies with specific antenna configuration and the placement of the antenna next to the head or body.

- Anatomy of the head and tissue inhomogeneity can influence the maximum value and distribution of SAR in the head of a mobile telephone user.

- Integrated SAR (or average SAR) in the body is similar for both homogeneous and inhomogeneous models.

- A large fraction (40\%-50\%) of the radiated RF power is absorbed by the human body (including the head).

- The bulk of power deposition occurs on the side of the head nearest to the radiating structure of the cellular telephone.

- The SAR distribution follows an exponential trend away from the antenna side and it is considerably lower elsewhere in the head of a cell phone user.

- Maximum SAR and its distribution in the head are functions of distance of the radiating element from the skin surface and the current distribution on the antenna.

- For a comparable level of electric field to be induced inside the body or a model by an ELF magnetic field, the applied ELF electric field must be $120 \pi$ times greater than the electric field of a plane wave.

\subsection{Coupling of Short and Ultra-Wideband Pulses into the Human Body}

The biological effects of electromagnetic pulses (EMPs) of varying durations have long been investigated in research laboratories. Electromagnetic pulses with electric fields ranging from 20 to $500 \mathrm{kV} / \mathrm{m}$ or higher and with frequency spectra of $0-80 \mathrm{MHz}$ are produced by nuclear EMP simulators. They are used to assess the electromagnetic compatibility or immunity of electronic instruments and systems under the conditions of a nuclear burst. However, it is only during recent years that the benefit and capability of the large bandwidth provided by very short or ultrawideband (UWB) EMPs are being considered for imaging, sensing, and communication applications (Di Benedetto et al. 2006; Lin et al. 2008). Examples of applications include biomedical imaging; vehicular radar; ground-penetrating radars (GPRs); through-wall sensing; and communications systems such as handheld transceivers, sensor networks, and wireless personal area networks (WPAN), using nanosecondwide pulses. 
The U.S. FCC defines an UWB device or system as any device or system in which the fractional bandwidth is greater than $0.20 \%$ or occupies $0.5 \mathrm{GHz}$ of spectrum (FCC 2002). This definition requires that UWB systems with a center frequency greater than $2.5 \mathrm{GHz}$ must have a $-10-\mathrm{dB}$ bandwidth of at least $500 \mathrm{MHz}$, whereas UWB systems operating with a center frequency below $2.5 \mathrm{GHz}$ must have a fractional bandwidth of at least $0.20 \%$. The FCC based its definition of a UWB device on the -10 - $\mathrm{dB}$ bandwidth rather than the $-20-\mathrm{dB}$ bandwidth used earlier, because UWB devices operate so close to the noise floor that in many cases it is not possible to measure the $-20-\mathrm{dB}$ bandwidth. The FCC also requires that the bandwidth be determined using the antenna that is designed to be used with a UWB device. The emission limits for UWB systems are specified in terms of effective isotropic radiated power (EIRP) measured in $\mathrm{dBm}$ ( $\mathrm{dB}$ referenced to $1 \mathrm{~mW}$ ) with $1 \mathrm{MHz}$ resolution bandwidth. The highest value is -41.3 $\mathrm{dBm} / \mathrm{MHz}$, which corresponds to $75 \mathrm{nW} / \mathrm{MHz}$. A UWB system offers a powerful combination of low power, high throughput, wide range, and good inherent security, using nanosecond pulses.

Earlier investigations of induced fields and power depositions and their interactions with biological systems relied on mathematical analyses of canonical shapes of dielectric equivalent bodies using models such as planar tissue layers and spherical objects (Lin 1975, 1976a; Lin, Wu, and Lam 1975; Lin and Lam 1976) The well-known effect of microwave hearing from pulse-induced thermoelastic pressure in the human head has been investigated both analytically (Lin 1976b,c; 1977a,b,c; 1978; 1980; 1990) and numerically (Lin and Wang 2010; Watanabe et al. 2000); detailed information can be found in the works mentioned in the reference list and it is not discussed here. Section 1.12.1 presents induced fields and power depositions for EMP and UWB fields in models of biological bodies. Note that a significant difference between UWB and EMP is the duration of the pulse; also, their shapes may be different. A typical UWB has a duration measured between the half values in a few nanoseconds. A typical EMP has a duration ranging from $400 \mathrm{~ns}$ to a few microseconds at half strength.

\subsubsection{Induced Electromagnetic Pulse Fields in Biological Bodies}

Propagation of EMP in a biological medium has been the subject of a number of investigations. The case of plane-wave EMP incidence on an air-tissue interface has been considered for Gaussian, biphasic, and multiphase pulses (Lin 1975; Lin 1976a,b,c; Lin, $\mathrm{Wu}$, and Lam 1975). The problem is formulated analytically in the frequency domain using frequency-dependent complex dielectric permittivity. A steady state transfer function is obtained for the biological medium from the transmission characteristics of a coherent plane wave incident on the medium. The temporal and spatial dependence of the induced EMP field is derived from inverse Fourier transform. Results of these calculations have provided insights into the behavior of transient EMPs in biological materials.

A typical time-domain EMP with peak amplitude of $50 \mathrm{kV} / \mathrm{m}$ and half-strength time duration of about $1 \mu \mathrm{s}$ is shown in Figure 1.16. The points in the figure represent an average, measured EMP in the time domain that is not exceeded by a significant amount in 


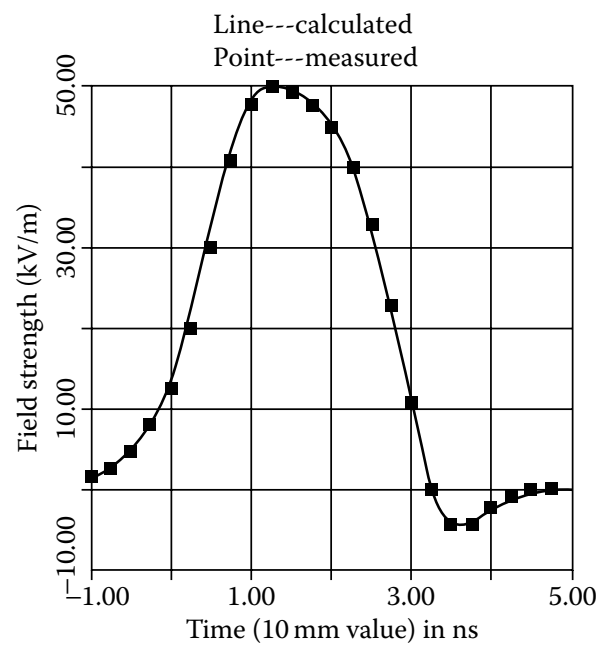

FIGURE 1.16 A typical electromagnetic pulse waveform. (From Lin, J. C., C. L. Wu, and C. K. Lam. 1975. Transmission of electromagnetic pulse into the head. Proc IEEE 63:1726-7. With permission.)

time or in strength (Lin, Wu, and Lam 1975). This EMP waveform can be characterized by a triple exponential time function such as

$$
\mathrm{e}_{0}(t)=\left[A / \mathrm{g}\left(\tau_{\mathrm{r}}\right)\right][\exp (-\alpha t)-\mathrm{a} \exp (-\beta t)-(1-\mathrm{a}) \exp (-\gamma t)]
$$

with

$$
g\left(\tau_{r}\right)=\exp \left(-\alpha \tau_{r}\right)-a \exp \left(-\beta \tau_{r}\right)-(1-a) \exp \left(-\gamma \tau_{r}\right)
$$

where a, $\alpha, \beta$, and $\gamma$ are constants; $\tau_{\mathrm{r}}$ is the pulse rise time or the time taken for the pulse to reach its maximum strength; $A$ is the peak electric field strength of the pulse and exp is the exponential function. The solid line is computed from Equation 1.51a using the values $A=50 \mathrm{kV} / \mathrm{m}, \mathrm{a}=0.1497, \alpha=1.20 \times 10^{6}, \beta=0.11 \alpha, \gamma=253.62 \alpha$ and $\tau_{\mathrm{r}}=17.78 \mathrm{~ns}$.

From the Fourier analysis given in Section 1.12.2, the transmitted pulse inside a homogeneous spherical model of the head is related to the time rate of change of the incident EMP. Representative results using an effective average conductivity of $0.2 \mathrm{~S} / \mathrm{m}$ for the brain are shown in Figures 1.17 and 1.18. The sphere of radius $10 \mathrm{~cm}$ (Figure 1.17) simulates an adult human-size head and the spherical model of radius $3.5 \mathrm{~cm}$ (Figure 1.18) mimics the head of an animal such as an adult cat or a rhesus monkey. The incident pulse is normalized to $1 \mathrm{~V} / \mathrm{m}$. The transmitted pulse along the $z$ axis is shown since the maxima of transmitted EMP amplitude always occur along the direction of propagation and through the center of the sphere. It can be seen that the transmitted pulse amplitude is the highest at the leading surface of the spherical head for both cases and decreases monotonically with increasing distance into the model. 


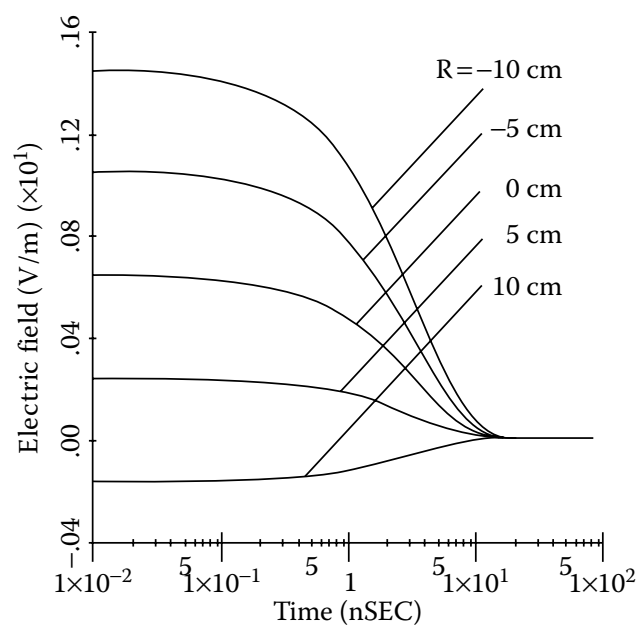

FIGURE 1.17 Transmitted electromagnetic pulse inside a sphere of radius $10 \mathrm{~cm}$ representing an adult human-size head as a function of time and location. (From Lin, J. C., C. L. Wu, and C. K. Lam. 1975. Transmission of electromagnetic pulse into the head. Proc IEEE 63:1726-7. With permission.)

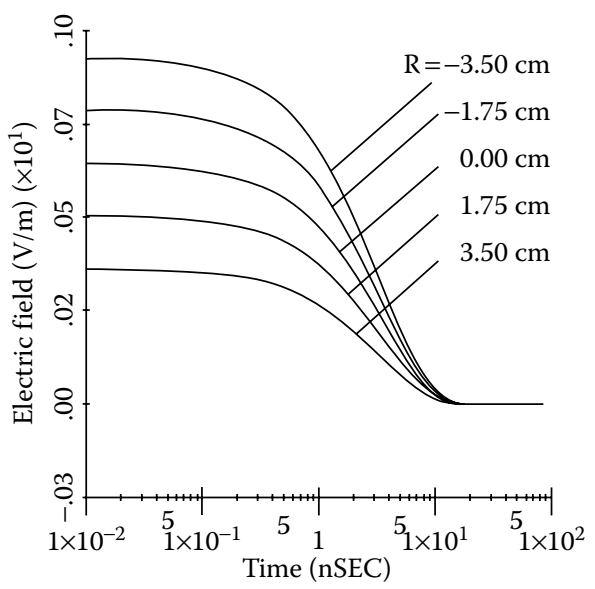

FIGURE 1.18 Transmitted electromagnetic pulse inside a sphere of radius $3.5 \mathrm{~cm}$ representing a small animal head as a function of time and location. (From Lin, J. C., C. L. Wu, and C. K. Lam. 1975. Transmission of electromagnetic pulse into the head. Proc IEEE 63:1726-7. With permission.)

The magnitude of the transmitted pulses in both humans and animals for the typical EMP shown in Figure 1.16 with peak electric field strength of $50 \mathrm{kV} / \mathrm{m}$ is shown in Figures 1.17 and 1.18. It can be seen that the peak transmitted pulse amplitude in a spherical head of radius $10 \mathrm{~cm}$ is $735 \mathrm{~V} / \mathrm{m}$, obtained from Figure 1.17, and occurs at the leading surface. For a brain sphere of radius $3.5 \mathrm{~cm}$, the corresponding transmitted pulse amplitude is $472 \mathrm{~V} / \mathrm{m}$ (see Figure 1.18). 


\subsubsection{Gaussian Electromagnetic Pulse inside a Planar Biological Medium}

The ratio of the transmitted electric field at a given depth in a biological medium and the incident field at $z=0$ is defined as the steady state transfer function. It characterizes the steady state transfer properties of the medium as a function of frequency. For a planar biological medium with complex dielectric permittivity

$$
\varepsilon^{\star}=\varepsilon^{\prime}-\varepsilon^{\prime \prime}
$$

or

$$
\varepsilon^{\star}(\omega)=\varepsilon_{\mathrm{h}}+\left(\varepsilon_{1}-\varepsilon_{\mathrm{h}}\right) /\left(1+j \omega / \omega_{\mathrm{s}}\right)
$$

where the indices $h$ and 1 characterize the complex dielectric permittivity values far above and below the critical frequency $\omega_{\mathrm{s}}$, respectively. The values for $\omega_{\mathrm{s}}, \varepsilon_{\mathrm{l}}$, and $\varepsilon_{\mathrm{h}}$ in the frequency range of interest are approximated by $160 \pi, 2.55 \times 10^{6}$, and $0.2 \times 10^{6}$, respectively, for musclelike tissues. The behaviors of $\varepsilon^{\prime}$ and $\varepsilon^{\prime \prime}$ as a function of frequency are shown in Figure 1.19. The steady state transfer function is given by

$$
\mathbf{H}(z, \omega)=\mathbf{E}_{t}(z, \omega) / \mathrm{E}_{0}(0, \omega)=2 /\left[1+\left(\varepsilon^{\star}\right)^{0.5}\right] \exp (-\alpha z) \exp (-\mathrm{j} \beta z)
$$

At the surface where $z=0$, the transfer function is equal to $T$, the transmission coefficient. As the frequency is reduced such that $\omega \ll \omega_{s}$, it becomes

$$
T=2 /\left[1+\left(\varepsilon^{\star}\right)^{0.5}\right] \sim 0.00125\left[l+\mathrm{j} 0.922\left(\omega / \omega_{\mathrm{s}}\right)\right]
$$

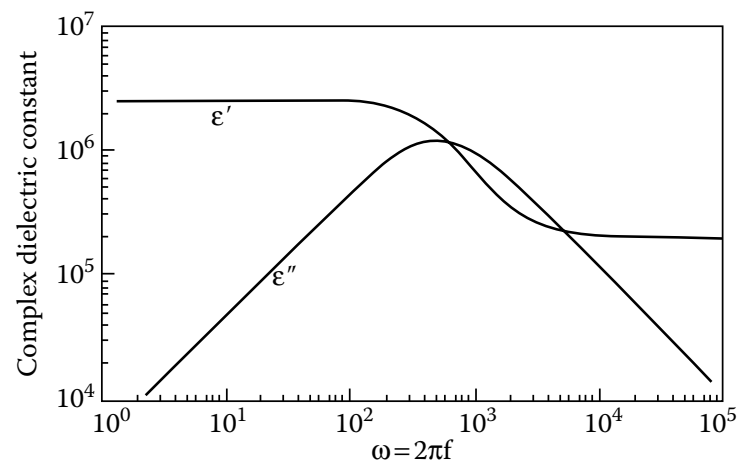

FIGURE 1.19 Complex dielectric constant or permittivity of muscle at low frequencies. (From Lin, J. C. 1975. Interaction of electromagnetic transient radiation with biological materials. IEEE Trans Electromagn Compat 17:93-7. With permission.) 


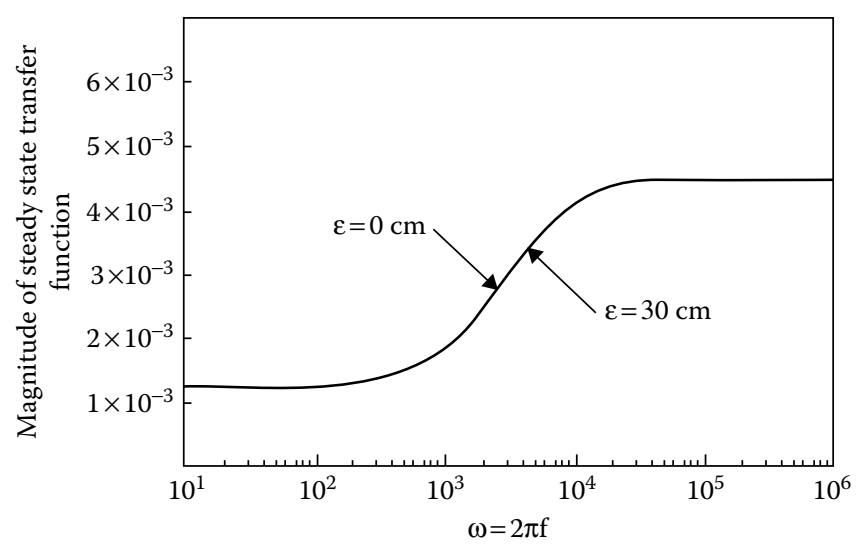

FIGURE 1.20 Magnitudes of steady state transfer functions for muscle materials at different depths as a function of frequency. (From Lin, J. C. 1975. Interaction of electromagnetic transient radiation with biological materials. IEEE Trans Electromagn Compat 17:93-7. With permission.)

It follows that for $\omega \rightarrow 0, T \rightarrow 0.00125$. Therefore, a negligible amount of the incident wave is transmitted into the muscle. This means that the incident wave experiences almost total reflection. In the high-frequency range, that is, $\omega \gg \omega_{s}$

$$
T \sim 0.00447 /\left(1-\mathrm{j} 5.88 \omega_{\mathrm{s}} / \omega\right)
$$

Again, the transmitted wave has small, constant amplitude and is independent of position. The magnitude of the steady state transfer function given by Equation 1.54 at different depths is shown in Figure 1.20 as a function of frequency for muscle material (Lin 1975). Note that the curve starts as a constant as the frequency is increased from zero. The upper limit of the low-frequency range is around $10 \mathrm{~Hz}$. The constant value of 0.00125 is equal to that predicted by the asymptotic analysis given by Equation 1.52 The high-frequency range begins at $10 \mathrm{kHz}$. The magnitude of $\mathbf{H}(z, \omega)$ in this case is 0.00447 as obtained earlier in the limit of $\omega_{s} / \omega \rightarrow 0$. In addition, within the distances considered, $\mathbf{H}(z, \omega)$ is independent of location inside the muscle layer. This evidently is a consequence of the assumed complex dielectric permittivity.

For a Gaussian EMP (Figure 1.21), the incident electric field is

$$
\mathrm{e}_{0}(t)=\mathrm{e}_{0}(0) \exp \left[-t^{2} /\left(2 \tau^{2}\right)\right]
$$

where $\tau$ is the pulse width and exp is the exponential function. The Fourier transform of Equation 1.57 is (see Figure 1.22 for representative spectral distributions of the incident Gaussian electric pulses).

$$
\mathbf{E}_{0}(z, \omega)=(2 \pi)^{1 / 2} \tau \mathrm{e}_{0}(0) \exp \left(-\omega^{2} \tau^{2} / 2\right) \exp (-\mathrm{j} \omega z / c)
$$




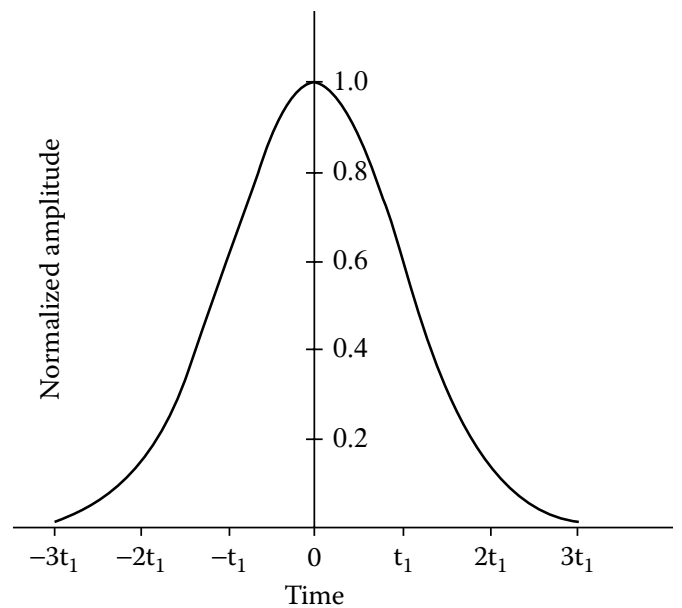

FIGURE 1.21 Incident Gaussian pulse. (From Lin, J. C. 1976a. Electromagnetic pulse interaction with mammalian craniel structures. IEEE Trans on Biomed Eng 23:61-5. With permission.)

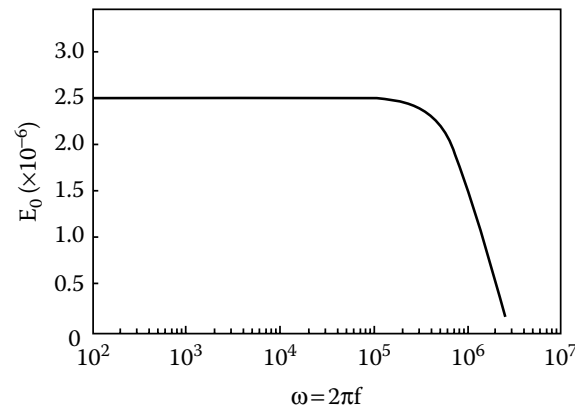

(a)

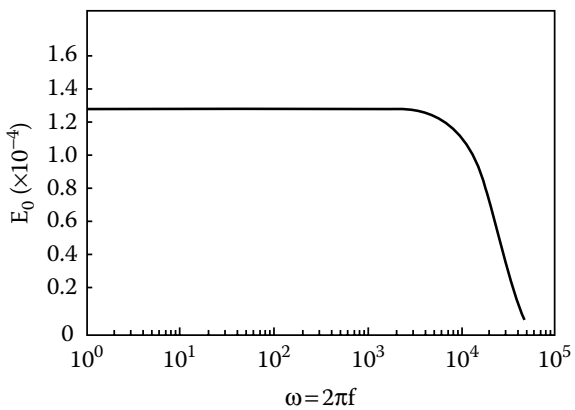

(b)

FIGURE 1.22 Spectral distribution of the incident Gaussian electric pulses at (a) $\tau=1 \mu$ s and (b) $\tau=50 \mu \mathrm{s}$. (From Lin, J. C. 1975. Interaction of electromagnetic transient radiation with biological materials. IEEE Trans Electromagn Compat 17:93-7. With permission.)

where $c$ is the speed of propagation. Combining Equations 1.54 and 1.58, one can obtain the frequency-domain transmitted field:

$$
\mathbf{E}_{t}(z, \omega)=\mathbf{E}_{0}(z, \omega) \mathbf{H}(z, \omega)
$$

The instantaneous EMP inside the biological medium is given by the inverse Fourier transform of Equation 1.59. The transmitted EMP waveforms in a muscle medium are shown in Figures 1.23 and 1.24 as a function of distance from the air-muscle interface for $\tau=1 \mu$ s and $50 \mu$ s, respectively (Lin 1975). It can be seen from Figures 1.23 and 1.24 that within the distances of interest, the transmitted pulse characteristics are independent of location. For an incident pulse of given strength, the transmitted pulse strength 


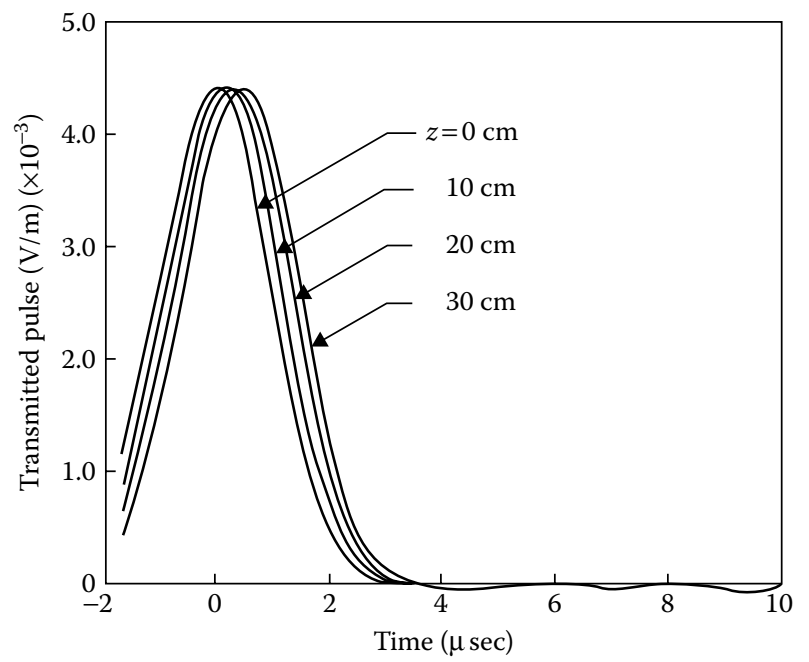

FIGURE 1.23 Transmitted waveforms of Gaussian incident pulse as a function of time and depth, $z$ for $\tau=1 \mu$ s. (From Lin, J. C. 1975. Interaction of electromagnetic transient radiation with biological materials. IEEE Trans Electromagn Compat 17:93-7. With permission.)

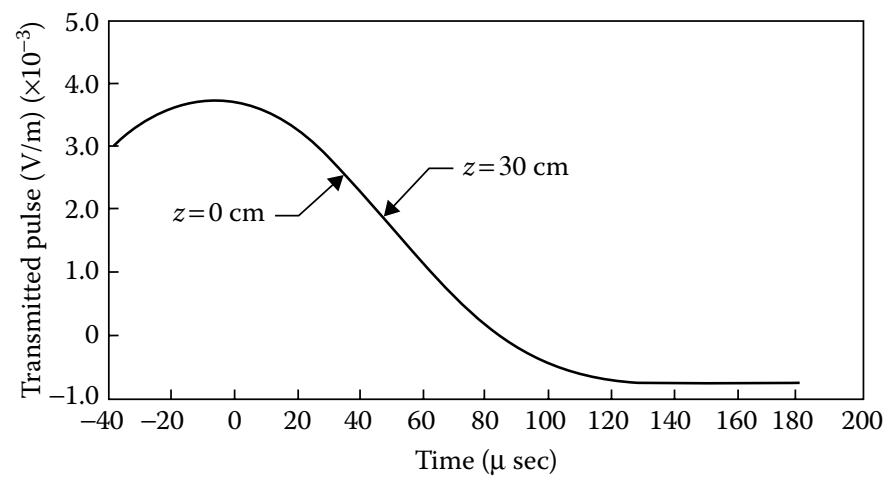

FIGURE 1.24 Transmitted waveforms of Gaussian incident pulse as a function of time and depth, $z$ for $\tau=50 \mu$ s. (From Lin, J. C. 1975. Interaction of electromagnetic transient radiation with biological materials. IEEE Trans Electromagn Compat 17:93-7. With permission.)

is related inversely to the pulse width, that is, the narrower the pulse the higher the transmitted pulse strength. Moreover, Figures 1.23 and 1.24 suggest that pulse widths that are sufficiently narrow will not be altered by the medium. However, at $\tau=50 \mu \mathrm{s}$, the broadening is about $20 \%$ of the initial width, that is, a considerable amount of broadening occurs. The transmitted pulse strengths in both cases are at least two orders of magnitude smaller (Figure 1.25). It should also be noted that although the incident pulse is entirely positive, the transmitted pulse has both positive and negative components. The positive part, however, dominates. 


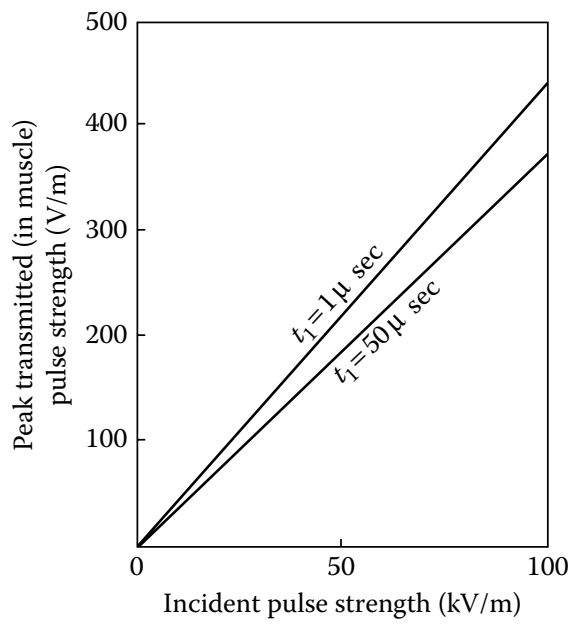

FIGURE 1.25 Peak transmitted pulse amplitude as a function of incident pulse magnitude. (From Lin, J. C. 1975. Interaction of electromagnetic transient radiation with biological materials. IEEE Trans Electromagn Compat 17:93-7. With permission.)

For an incident pulse of $50 \mathrm{kV} / \mathrm{m}$, with a pulse width of $1 \mu$ s the transmitted pulse strength reaches $221 \mathrm{~V} / \mathrm{m}$, whereas for the $50-\mu$ s pulse the strength is $188 \mathrm{~V} / \mathrm{m}$. This is primarily due to the fact that the shorter pulse has a higher spectral content relative to the longer one and the higher frequencies are more readily transmitted into the muscle medium (Figure 1.25).

\subsubsection{Gaussian Electromagnetic Pulse inside Spherical Head Models}

The formulation mentioned in Section 1.12.2 can also be applied to study the characteristics of Gaussian EMP in spherical models that approximate human and laboratory animal cranial structures.

For simplicity, the model is assumed to be homogeneous and to consist only of brain matter. In this case, the steady state transfer function, $\mathbf{H}(r, \omega)$ of the brain sphere over the applicable frequency range in spherical coordinates (Lin 1976a) is

$$
\mathbf{H}(r, \omega)=\left(3 / \varepsilon^{*}\right) \mathbf{x}-\mathrm{j}(\omega r / 2 c)(\cos \theta \mathbf{x}-\sin \theta \cos \theta \mathbf{z})
$$

The first term of Equation 1.60 is uniform and is induced by the electric component of the incident field. It is in the same direction as the incident plane-wave EMP field. The second term occurs due to the magnetic component of the incident field, and its contribution varies with the radius of the sphere. The latter are eddy currents, which form continuous loops (see Section 1.9).

Results obtained for the permittivity of brain materials characterized by the dispersion formula (Equation 1.53), with values of $\varepsilon_{l}, \varepsilon_{h}$, and $\omega_{s}$ equal to $6.6 \times 10^{6}, 35$, and $6 \pi \times 10^{4}$, 
respectively, and an incident Gaussian pulse (see Equation 1.57 and Figure 1.21) are shown in Figure 1.26 for an adult human-size head model and in Figure 1.27 for a smaller animal such as a cat or a rhesus monkey. The incident pulse amplitude is normalized to $1 \mathrm{~V} / \mathrm{m}$. Since the maxima of transmitted EMP amplitude always occur along the direction of propagation and through the center of the spheres, the transmitted EMP along the $z$ axis is shown

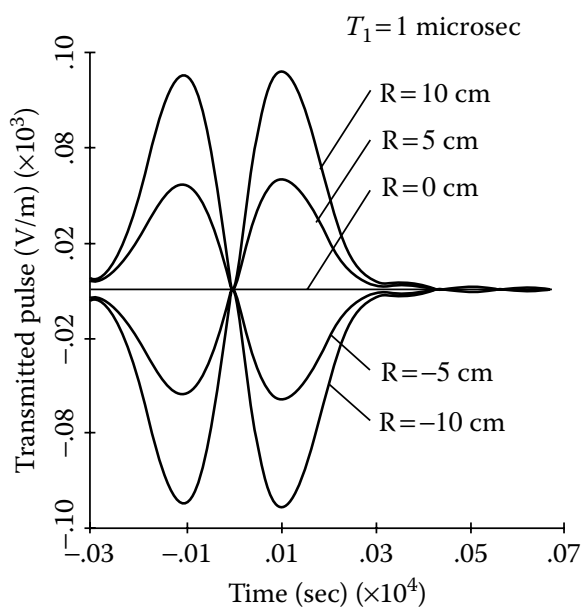

FIGURE 1.26 Transmitted pulse in a human-size head model exposed to a $1 \mu$ s-wide Gaussian electromagnetic pulse using the complex permittivity of Equation 1.53 for brain matter. (From Lin, J. C. 1976a. Electromagnetic pulse interaction with mammalian craniel structures. IEEE Trans on Biomed Eng 23:61-5. With permission.)

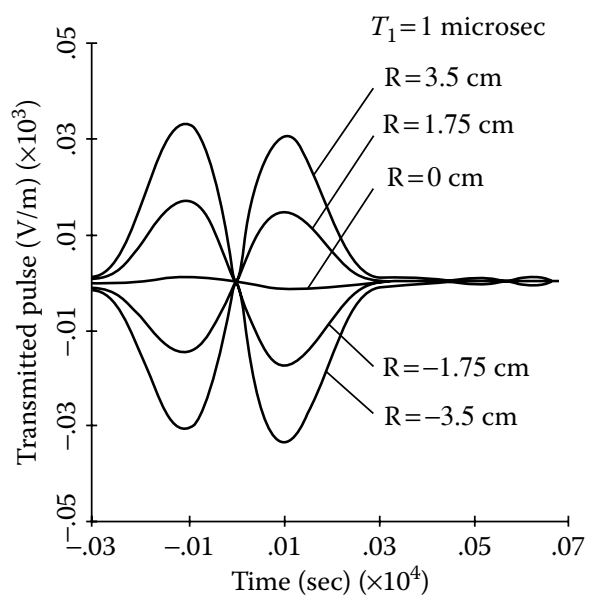

FIGURE 1.27 Transmitted pulse in a small animal-size head model exposed to a 1- $\mu$ s Gaussian electromagnetic pulse using a complex permittivity for brain matter. (From Lin, J. C. 1976a. Electromagnetic pulse interaction with mammalian cranial structures. IEEE Trans on Biomed Eng 23:61-5. With permission.) 
in each of these figures. It can be seen from these figures that for all cases the transmitted amplitude is the highest at the leading surface of the spherical head and that it decreases initially with increasing distances into the model. The pulse amplitude approaches a zero or a minimum near the center of the sphere, then increases with increasing radial distance, and finally reaches a maximum at the trailing surface. However, the peak amplitude at the trailing surface is slightly lower than that at the leading surface. Hence, the transmitted pulse amplitude is asymmetric about the center of the spherical model.

Similar to planar tissue models, the transmitted pulse has both positive and negative components. In fact, the positive portion of the transmitted EMP is almost equal to the negative portion at any given location inside the spherical head. The widths of the positive and negative pulses are about the same as the width of the incident pulse. Although not shown, the principal difference between a $1-\mu$ s and a $10-\mu$ s pulse is that the transmitted EMP for the former is approximately 10 times higher than for the latter. Therefore, the transmitted EMP amplitude is inversely proportional to the pulse width, which is the principal distinguishing characteristic for the two Gaussian pulses, thereby illustrating the dependence of the transmitted EMP on pulse widths.

To appreciate the magnitude of the transmitted EMPs in both humans and animals, it is possible to consider a typical EMP with a peak electric field strength of $50 \mathrm{kV} / \mathrm{m}$. Figure 1.26 indicates that a peak transmitted pulse amplitude is only $4.75 \mathrm{~V} / \mathrm{m}$ at the leading surface of a sphere of radius $10 \mathrm{~cm}$ and the transmitted amplitude one quarter of the way into the sphere is $2.5 \mathrm{~V} / \mathrm{m}$. These are relatively small values.

\subsubsection{Constant Conductivity Spherical Model for Electromagnetic Pulse Propagation}

The imaginary part of the complex dielectric permittivity is related to the conductivity as $\varepsilon^{\prime \prime}=\sigma / \omega \varepsilon_{0}$, where $\varepsilon_{0}$ is the permittivity of vacuum. Since $\varepsilon^{\prime}$ is small compared to $\varepsilon^{\prime \prime}$ for brain matter at lower frequencies, $\varepsilon^{\prime}$ may be neglected. This is similar to neglecting the displacement current. Furthermore, if we assume a constant conductivity for the brain, Equation 1.60 becomes

$$
\mathbf{H}(r, \omega)=\mathrm{j} \omega\left[3 \varepsilon_{0} / \sigma \mathbf{x}-r /(2 c)(\cos \theta \mathbf{x}-\sin \theta \cos \theta \mathbf{z})\right]
$$

On substituting Equation 1.61 into Equation 1.59 and then taking the inverse Fourier transform, the induced electric field can be calculated:

$$
\mathbf{e}_{t}(r, t)=\left[\left(3 \varepsilon_{0} / \sigma\right) \mathbf{x}-r /(2 c)(\cos \theta \mathbf{x}-\sin \theta \cos \theta \mathbf{z})\right]\left(\mathrm{de}_{0} / \mathrm{d} t\right)
$$

Note that this result is in a closed form and can be evaluated exactly. It is seen that for the case of frequency-independent $\sigma$ the induced pulse inside a spherical model of the brain is a function of the time rate of change of the incident EMP. After substituting the explicit expression of Equation 1.57 for $\mathrm{e}_{0}$ in Equation 1.62 and performing the indicated differentiation, we obtain

$$
\mathbf{e}_{t}(r, t)=-\mathrm{e}_{0}(0)\left(t / 2 \tau^{2}\right) \exp \left[-t^{2} /\left(2 \tau^{2}\right)\right]\left[\left(3 \varepsilon_{0} / \sigma\right) \mathbf{x}-r /(2 c)(\cos \theta \mathbf{x}-\sin \theta \cos \theta \mathbf{z})\right]
$$


An average conductivity of $0.2 \mathrm{~S} / \mathrm{m}$ is typical of the conductivities of brain matter in the frequency range of $10 \mathrm{~Hz}$ to $10 \mathrm{MHz}$ (Lin 1976a). The computed results for the constant conductivity model are shown in Figures 1.28 and 1.29. Compared to Figures 1.26 and 1.27, it is clear that constant conductivity gives both larger and wider pulses inside the spherical model. For example, an impinging EMP with peak electric field strength of $50 \mathrm{kV} / \mathrm{m}$ produces a peak transmitted pulse amplitude of $6.7 \mathrm{~V} / \mathrm{m}$ in a spherical head of $10-\mathrm{cm}$ radius for a $1-\mu$ s pulse. This represents a $50 \%$ increase of the transmitted field strength under the constant conductivity model. Another significant difference between the two approximations (constant conductivity and complex permittivity) is that for the constant conductivity model the transmitted pulse amplitude decreases monotonically with increasing distance from the leading surface of the sphere. Therefore, it is clearly not symmetrical.

A common feature of the two approximations is that the transmitted pulses are oscillatory and have positive and negative contributions, although the incident Gaussian pulse is entirely positive. This clearly indicates that the transmitted pulse depends on the time derivative of the impinging EMP. It suggests that the constant conductivity model can be used to estimate maximum transmission. However, it may not be as accurate for spatial distribution predictions of the transmitted field strength. In any event, the coupling is quite small-less than $10 \mathrm{~V} / \mathrm{m}$ is coupled into a spherical head of $3.5-$ or $10-\mathrm{cm}$ radius for a $50-\mathrm{kV} / \mathrm{m}$ pulse varying in width from 1 to $10 \mu \mathrm{s}$. It is significant to note that for both approaches the transmitted EMP at $1 \mu \mathrm{s}$ is approximately 10 times higher than that for the $10-\mu$ s pulse, which is also the case for smaller spheres. Therefore, a short pulse is more readily transmitted into biological objects than a long one.

$$
T_{1}=1 \text { microsec }
$$

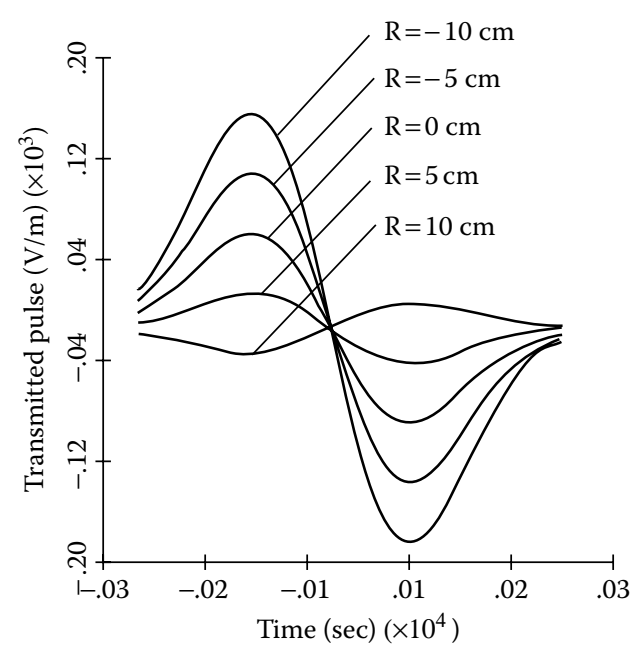

FIGURE 1.28 Transmitted pulse in a human-size head model exposed to a 1- $\mu$ s Gaussian electromagnetic pulse using constant brain conductivity: Note that the scale of the graph is adjusted for clarity. (From Lin, J. C. 1976a. Electromagnetic pulse interaction with mammalian cranial structures. IEEE Trans on Biomed Eng 23:61-5. With permission.) 


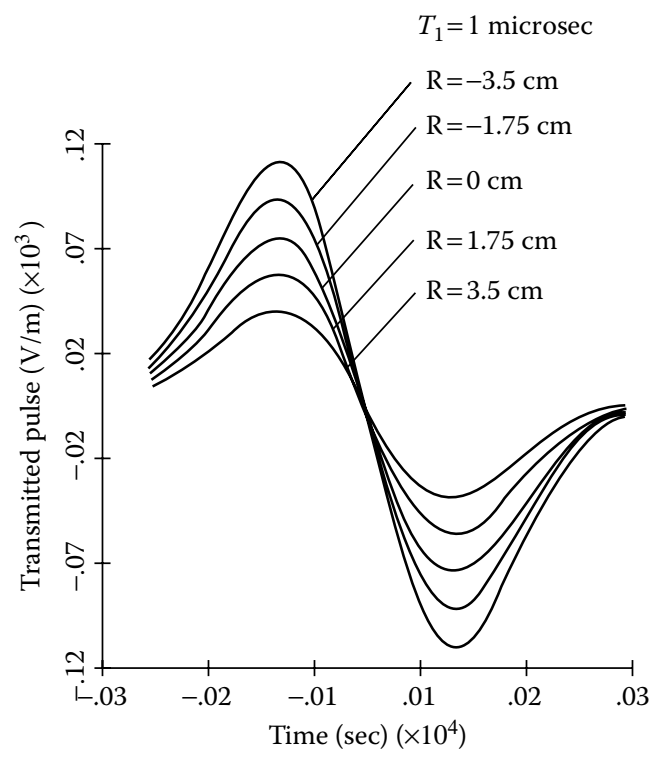

FIGURE 1.29 Transmitted pulse in a small animal-size head model exposed to 1- $\mu$ s Gaussian electromagnetic pulse using constant brain conductivity: Note that the scale of the graph is adjusted for clarity. (From Lin, J. C. 1976a. Electromagnetic pulse interaction with mammalian cranial structures. IEEE Trans on Biomed Eng 23:61-5. With permission.)

\subsubsection{Induced Ultra-Wideband Field and Current}

A representative UWB pulse with peak amplitude of $1.1 \mathrm{~V} / \mathrm{m}$ in time domain is shown in Figure 1.30. It is interesting to note that the pulse has a rise time of about $0.2 \mathrm{~ns}$ and total time duration of about $7.0 \mathrm{~ns}$. The spectrum of the pulse is shown in Figure 1.31. Most of the energy in the pulse is concentrated in the $200-900 \mathrm{MHz}$ band with the peak energy being about $500 \mathrm{MHz}$.

The frequency-dependent FDTD [(FD $\left.{ }^{2} \mathrm{TD}\right]$ formulation is used to calculate the coupling of a UWB pulse into the heterogeneous model of the human body. From the calculated internal fields, the vertical currents passing through the various layers of the standing body are then calculated: This requires calculation of frequency-dependent permittivity properties of various tissues characterized by the Debye equation (Equation 1.64) with two relaxation constants (Gandhi, Gao, and Chen 1992, 1993; Furse, Chen, and Gandhi 1994):

$$
\varepsilon *(\omega)=\varepsilon_{0}\left[\varepsilon_{\infty}+\frac{\varepsilon_{s 1}-\varepsilon_{\infty}}{1+j \omega \tau_{1}}+\frac{\varepsilon_{s 2}-\varepsilon_{\infty}}{1+j \omega \tau_{2}}\right]
$$

where $\varepsilon_{\infty}$ is the high-frequency permittivity; the static (zero-frequency) dielectric permittivity is given by

$$
\varepsilon_{s}=\varepsilon_{s 1}+\varepsilon_{s 2}-\varepsilon_{\infty}
$$




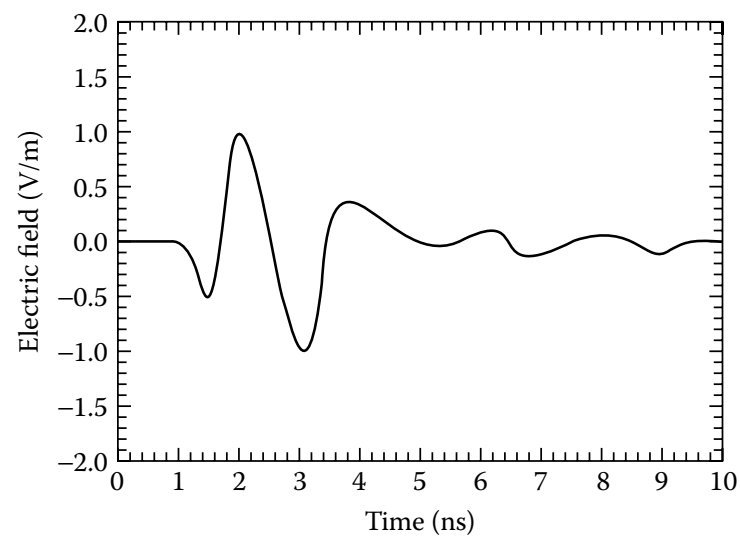

FIGURE 1.30 A representative ultra-wideband pulse with peak incident electric field $=1.1 \mathrm{~V} / \mathrm{m}$. (From Lin, J. C., and P. Bernardi. 2007. Computer methods for predicting field intensity and temperature change. In Bioengineering and Biophysical Aspects of Electromagnetic Fields, ed. F. Barnes and B. Greenebaum, Chapter 10, 293-380. Boca Raton, FL: CRC Press. With permission.)

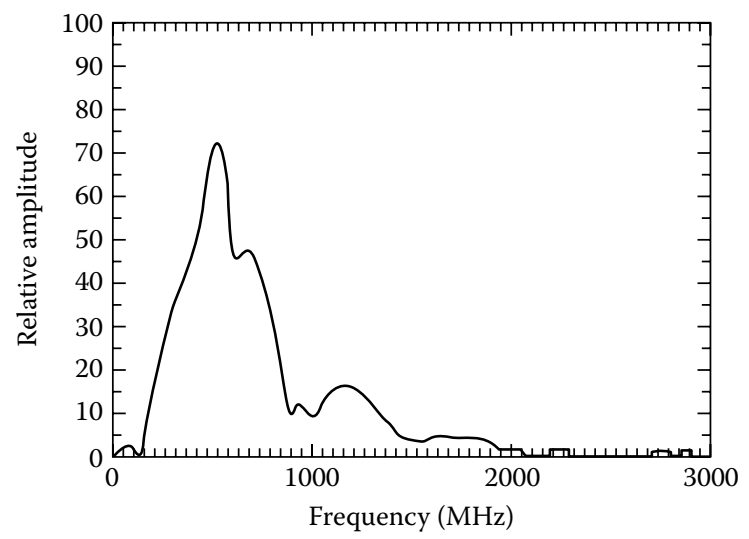

FIGURE 1.31 Spectrum of the ultra-wideband pulse of Figure 1.30. (From Lin, J. C., and P. Bernardi. 2007. Computer methods for predicting field intensity and temperature change. In Bioengineering and Biophysical Aspects of Electromagnetic Fields, ed. F. Barnes and B. Greenebaum, Chapter 10, 293-380. Boca Raton, FL: CRC Press. With permission.)

For the results shown here, the measured properties of biological tissues (muscle, fat, bone, blood, intestine, cartilage, lung, kidney, pancreas, spleen, lung, heart, brain/ nerve, skin, and eye) were obtained from the literature. Optimized values for $\varepsilon_{\mathrm{s} 1}, \varepsilon_{\mathrm{s} 2}$, $\varepsilon_{\infty}, \tau_{1}$, and $\tau_{2}$ in Equation 1.64 were obtained by nonlinear least-squares matching with the measured data for fat and muscle (Table 1.7), with $\tau_{1}$ and $\tau_{2}$ being the average of the optimized values for fat and muscle, respectively. All other tissues have properties falling between these two types of tissues. The averaging was done to facilitate volume 
TABLE 1.7 Debye Constants for Tissues with $\tau_{1}=46.2 \times 10^{-9} \mathrm{~s}$ and $\tau_{2}=0.91 \times$ $10^{-10} \mathrm{~s}$, Which Are the Average of the Optimized Values for Fat and Muscle

\begin{tabular}{lrrr}
\hline Tissue & $\varepsilon_{\infty}$ & \multicolumn{1}{c}{$\varepsilon_{s 1}$} & $\varepsilon_{s 2}$ \\
\hline Muscle & 40.0 & 3948.0 & 59.09 \\
Bone/cartilage & 3.4 & 312.8 & 7.11 \\
Blood & 35.0 & 3563.0 & 66.43 \\
Intestine & 39.0 & 4724.0 & 66.09 \\
Liver & 36.3 & 2864.0 & 57.12 \\
Kidney & 35.0 & 3332.0 & 67.12 \\
Pancreas/spleen & 10.0 & 3793.0 & 73.91 \\
1/3 Lung & 10.0 & 1224.0 & 13.06 \\
Heart & 38.5 & 4309.0 & 54.58 \\
Brain/nerve & 32.5 & 2064.0 & 56.86 \\
Skin & 23.0 & 3399.0 & 55.59 \\
Eye & 40.0 & 2191.0 & 56.99 \\
\hline
\end{tabular}

Source: Lin, J. C., and P. Bernardi. 2007. Computer methods for predicting field intensity and temperature change. In Bioengineering and Biophysical Aspects of Electromagnetic Fields, ed. F. Barnes and B. Greenebaum, Chapter 10, 293-380. Boca Raton, FL: CRC Press. With permission.

averaging of tissue properties in cells of the heterogeneous human model. Having $\tau_{1}$ and $\tau_{2}$ constant for all tissues allowed linear (volume) averaging of the $\varepsilon$ values for each tissue in a given cell so as to calculate $\varepsilon$ values for that cell.

The internal fields and vertical currents passing through various layers of the body are calculated, by using the following equation:

$$
\mathbf{I}_{z}(t)=\delta^{2} \sum_{i, j} \frac{\partial \mathbf{D}_{z}}{\partial t}
$$

where $\delta$ is the cell size $(1.31 \mathrm{~cm})$, and the summation is carried out for all cells in a given layer. The layer-averaged absorbed energy density or SA and the total energy $W$ absorbed by the whole body can be calculated using the following equations:

$$
\begin{gathered}
\left.\mathrm{SA}\right|_{\text {layer } k}=\frac{\delta t}{N_{k}} \sum_{i, j, t} \frac{\mathrm{E}(i, j, k, t)}{\rho(i, j, k)} \cdot \frac{\partial \mathrm{D}(i, j, k, t)}{\partial t} \\
W=\delta t \cdot \delta^{3} \sum_{i, j, k, t} \mathrm{E}(i, j, k, t) \cdot \frac{\partial \mathrm{D}(i, j, k, t)}{\partial t}
\end{gathered}
$$

In Equations 1.67 and $1.68, \delta t$ is the time step $(\delta / 2 c=0.02813 \mathrm{~ns})$ used for time-domain calculations, $N_{k}$ is the number of cells in layer $k$ of the body, and $\rho(i, j, k)$ is the mass density in kilogram per cubic meter for each cell in the corresponding layers. 
The results to follow (Figures 1.32 and 1.33) assume the incident fields to be vertically polarized, since this polarization is known to result in the strongest coupling for a standing person. Also, a uniform UWB plane-wave illumination of the whole body is assumed for incident fields. The (FD) ${ }^{2} \mathrm{TD}$ procedure is used to calculate the temporal variations of total vertical currents for various sections of the body for both the shoewearing grounded and ungrounded exposure conditions of the model. Current variations for some representative sections such as those through the eyes and the bladder

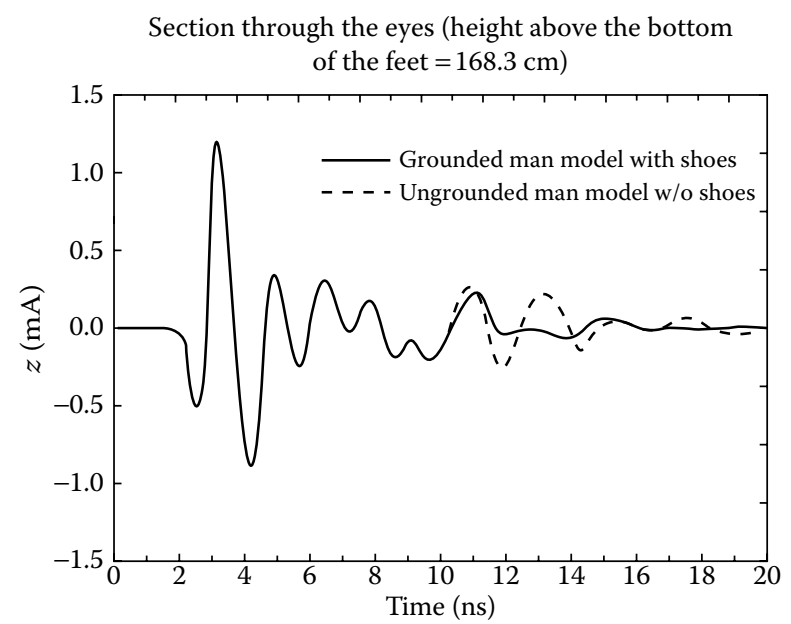

(a)

Section through the bladder (height above the bottom of the feet $=91.0 \mathrm{~cm}$ )

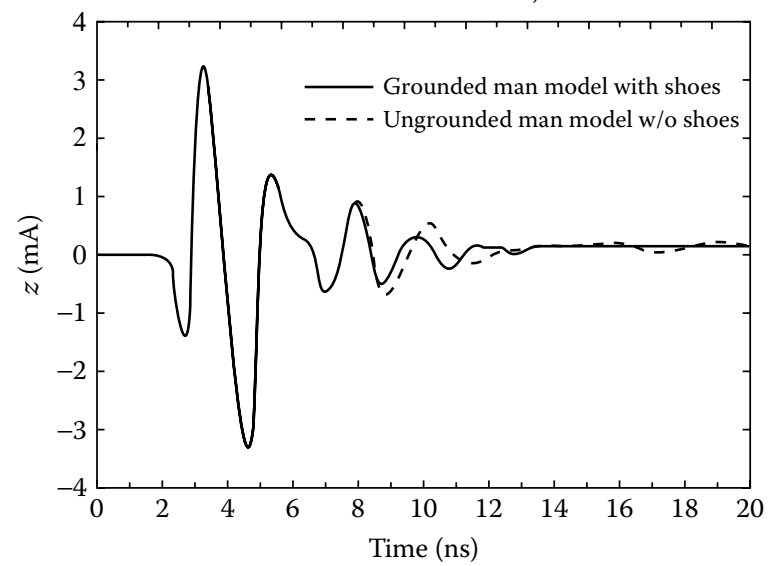

(b)

FIGURE 1.32 Currents induced in various sections of the body of a human male for shoewearing grounded and ungrounded conditions of exposure: $\mathbf{E}_{\text {peak }}=1.1 \mathrm{~V} / \mathrm{m}$. (From Lin, J. C., and P. Bernardi. 2007. Computer methods for predicting field intensity and temperature change. In Bioengineering and Biophysical Aspects of Electromagnetic Fields, ed. F. Barnes and B. Greenebaum, Chapter 10, 293-380. Boca Raton, FL: CRC Press. With permission.) 


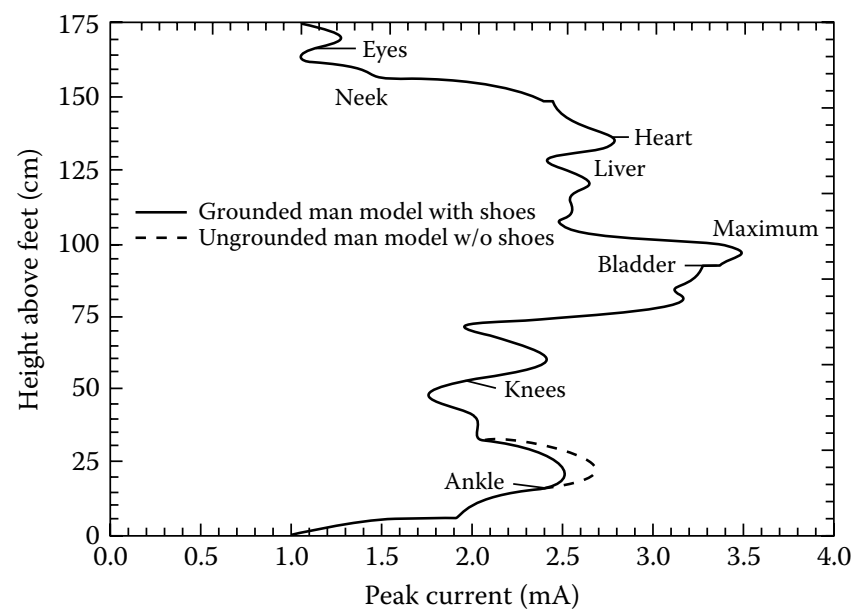

FIGURE 1.33 Peak currents induced for various sections of the body of a human male for shoewearing grounded and ungrounded conditions: $\mathbf{E}_{\text {peak }}=1.1 \mathrm{~V} / \mathrm{m}$. (From Lin, J. C., and P. Bernardi. 2007. Computer methods for predicting field intensity and temperature change. In Bioengineering and Biophysical Aspects of Electromagnetic Fields, ed. F. Barnes and B. Greenebaum, Chapter 10, 293-380. Boca Raton, FL: CRC Press. With permission.)

are given in Figures 1.32 and 1.33. The calculated peak currents for the various sections are about $1.1-3.2 \mathrm{~mA} /(\mathrm{V} / \mathrm{m})$. It is interesting to note that there is very little difference in the induced currents irrespective of whether the model is grounded or not. This is due to the fact that most of the energy in the pulse is at frequencies in excess of $300 \mathrm{MHz}$, where the effect of the ground plane on the induced currents or the SARs is minimal.

In Figure 1.33, the peak current for each section of the body is shown with a section resolution of $1.31 \mathrm{~cm}$. The maximum peak sectional current of $3.5 \mathrm{~mA}$, which is equal to $3.2 \mathrm{~mA} /(\mathrm{V} / \mathrm{m})$, occurs at a height of $96.3 \mathrm{~cm}$ above the bottom of the feet. Similar results were observed for calculations using isolated and grounded models of the human body for plane-wave exposures at frequencies of 350-700 $\mathrm{MHz}$, where the highest induced currents on the order of 3.0-3.2 $\mathrm{mA} /(\mathrm{V} / \mathrm{m})$ were calculated for sections of the body that are at heights of $85-100 \mathrm{~cm}$ relative to the feet.

The SA and the total absorbed energy for exposure to the UWB pulse can be calculated using Equations 1.67 and 1.68. The total energies absorbed by the body exposed to a single pulse of the type shown in Figure 1.30 are 2.0 and $1.91 \mathrm{pJ}$ (picojoules) for isolated and shoe-wearing grounded conditions, respectively (Lin and Bernardi 2007).

\subsubsection{Summary of Short and Ultra-Wideband Pulse Coupling}

It should be mentioned that in general the interaction of pulsed fields with biological systems can be very different from exposure of systems to continuous wave fields. In addition to EMP and UWB, pulse operations are commonly found in radar and MRI systems. A distinguishing feature in these cases is that they all involve radiation of 
sequences of a large number of pulses. Experimental and theoretical studies have shown that auditory responses can be evoked when the head of human subjects is irradiated with radarlike pulses (Lin 1978, 1980, 1990). Auditory responses to RF pulses have been reported and predicted for humans undergoing MRI procedures (Lin and Wang 2010; Roschmann 1991). However, this topic will not be covered in this chapter; the interested reader can find additional details on this topic in the cited reference works.

The topics of high power electromagnetic pulses are discussed include two types of pulses for a variety of exposure conditions. Specifically, EMPs with electric field strengths up to $500 \mathrm{KV} / \mathrm{m}$ or higher and pulse widths in the microseconds range with frequency spectra up to $80 \mathrm{MHz}$ and short nanosecond UWB pulses have spectra that extend up to $10,000 \mathrm{MHz}$. Their salient features in field coupling into biological matrials are summarized below.

\subsubsection{Gaussian Pulse in Planar Medium}

- The transmitted Gaussian pulse characteristics are independent of location inside a planar tissue layer.

- For an incident pulse of given strength, the transmitted pulse strength is related inversely to the pulse width, that is, the narrower the pulse the higher the transmitted pulse strength.

- Pulse widths that are sufficiently narrow will not be altered by the medium. However, at $\tau=50 \mu$ s the broadening is about $20 \%$ of the initial width, that is, a considerable amount of broadening occurs.

- The transmitted pulse strengths are at least two orders of magnitude smaller than incident pulse.

- For an incident pulse of $50 \mathrm{kV} / \mathrm{m}$ with a pulse width of $1 \mu \mathrm{s}$, the transmitted pulse strength will reach $221 \mathrm{~V} / \mathrm{m}$, whereas that for the $50-\mu$ s pulse will reach $188 \mathrm{~V} / \mathrm{m}$.

- Shorter pulses have higher spectral content relative to longer ones and the higher frequency components are more readily transmitted into the biological medium and have greater strength than the lower frequency components.

\subsubsection{Gaussian Pulse in a Spherical Body}

For dispersive permittivity:

- The maxima of transmitted EMP amplitude always occur along the direction of propagation and through the center of the brain sphere.

- Transmitted pulse amplitude is asymmetric about the center of the spherical model.

- Pulse amplitude approaches a zero or minimum value near the center.

- Width of the transmitted EMP is about the same as the width of the incident pulse.

- Transmitted EMP amplitude is inversely proportional to pulse width.

- Peak transmitted pulse amplitude is relatively small, about four orders of magnitude lower than the incident value. 
For constant conductivity:

- Since $\varepsilon^{\prime}$ is small compared to $\varepsilon^{\prime \prime}$ for brain matter at low frequencies, $\varepsilon^{\prime}$ may be neglected to obtain a frequency-independent $\sigma$. This will result in a closed-form solution for the induced pulse inside the model.

- Induced pulse inside a spherical model of the brain is a function of the time rate of change of incident EMP.

- Transmitted pulse amplitude decreases monotonically with increasing distance from the leading (exposed) surface of the sphere, and it is clearly not symmetrical.

\section{Common Features of These Two Approximation}

- The transmitted pulse is oscillatory and has positive and negative contributions, although the incident Gaussian pulse may be entirely positive.

- The transmitted pulse depends on the time derivative of the impinging EMP.

- The constant conductivity model may be used to estimate maximum transmission. However, it may not be as accurate for spatial distribution predictions of the transmitted field strength.

- Field coupling is quite small-less than $10 \mathrm{~V} / \mathrm{m}$ is coupled into a spherical head of radius 3.5 or $10 \mathrm{~cm}$ for a $50-\mathrm{kV} / \mathrm{m}$ pulse varying in width from 1 to $10 \mu \mathrm{s}$.

- The transmitted EMP amplitude for the $1-\mu$ s pulse is approximately 10 times higher than that for the $10-\mu$ s pulse, which is also the case for smaller spheres. Therefore, a shorter pulse is more readily transmitted into biological objects than a longer pulse.

\subsubsection{Typical Electromagnetic Pulse Waveform in a Spherical Head Model}

The salient features include:

- The maxima of transmitted EMP amplitude always occur along the direction of propagation and through the center of the sphere.

- Transmitted pulse amplitude is highest at the leading surface of the spherical head and it decreases monotonically with increasing distance into the model.

- The magnitude of transmitted pulses is directly proportional to the size of the model. For a typical EMP with a peak electric field strength of $50 \mathrm{kV} / \mathrm{m}$, peak transmitted pulse amplitude in a spherical head of radius $10 \mathrm{~cm}$ is $735 \mathrm{~V} / \mathrm{m}$ and it occurs at the leading surface. For a brain sphere of radius $3.5 \mathrm{~cm}$, the corresponding transmitted pulse amplitude is $472 \mathrm{~V} / \mathrm{m}$.

\subsubsection{Ultra-Wideband Pulse-Induced Current Inside the Body}

The salient features include:

- For a pulse with a rise time of $0.2 \mathrm{~ns}$ and total time duration of $7.0 \mathrm{~ns}$, the spectrum shows that most of the energy in the pulse is concentrated in the $200-900 \mathrm{MHz}$ band with the peak energy occurring at about $500 \mathrm{MHz}$. 
- There is very little difference in induced current irrespective of whether the model is grounded or not. This is due to the fact that most of the energy in the pulse is at frequencies in excess of $300 \mathrm{MHz}$, where the effect of the ground plane on the induced current or SAR is minimal.

\subsection{Coupling of Millimeter and Terahertz Waves}

Recent advances in $\mathrm{mmW}$ and terahertz technologies have motivated a wide range of telecommunication, industrial, medical, and scientific applications including security screening in the form of whole-body image scanning for concealed objects. Some of the specific applications involve manufacturing inspection, biomarker and trace chemical detection, automated vehicular guidance for cars on highways, advanced radars to obtain better forecasts of localized and intense rainfall, and high-capacity short-range wireless data communications.

In general, mmW occupies the frequencies from 30 to $300 \mathrm{GHz}$ with corresponding wavelengths of 1-10 mm, whereas terahertz waves span the frequencies of 100-1000 $\mathrm{GHz}$ (or $1 \mathrm{THz}$ ) with wavelengths of $0.3-3.0 \mathrm{~mm}$. Clearly, there is an overlap between $\mathrm{mmW}$ and terahertz waves. Studies on $\mathrm{mmW}$ interactions aimed toward biomedical applications began about 30 years ago (Gandhi 1983; Pakhomov et al. 1998). However, recent advances in device technology at terahertz frequencies have spearheaded considerable interest in terahertz applications in biology and medicine among others, as mentioned earlier. A comprehensive review of the research conducted on biological effects of $\mathrm{mmW}$, especially from the former Soviet Union, showed that at intensities of $100 \mathrm{~W} / \mathrm{m}^{2}$ or less $\mathrm{mmW}$ can affect cell growth and proliferation, enzyme activity, genetic status, functions of excitable membranes, peripheral receptors, and other biological systems (Pakhomov et al. 1998). Nevertheless, there is a paucity of investigations on transmitted fields and induced energy deposition in biological tissues at these frequency bands. The discussions of this section (1.13) focus specifically on the coupling of mmWs into biological tissues. For a complete discussion on the biological interactions of terahertz radiation, readers are referred to Chapter 7 of this book.

\subsubsection{Transmitted and Reflected Millimeter Waves and Terahertz Fields, and Energy Deposition}

In principle, at the frequencies of millimeter and terahertz waves the induced fields and energy deposition in biological medium can be determined in much the same manner as for RF if the permittivities of relevant tissues at these frequencies is known. Although some earlier extrapolations were conducted based on Debye formulas and using complex dielectric permittivity of the skin at low frequencies, some measurements for skin within the $\mathrm{mmW}$ range have recently become available for humans (Alekseev and Ziskin 2007) and rodents (Alekseev et al. 2008). Data for human skin in the frequency range of $37-74 \mathrm{GHz}$ are shown in Figure 1.34, in which the measured results are compared to earlier extrapolations. It can be seen that the recent data tend to be lower. More importantly, at $\mathrm{mmW}$ frequencies the permittivity of skin is governed by cutaneous free water contents. 


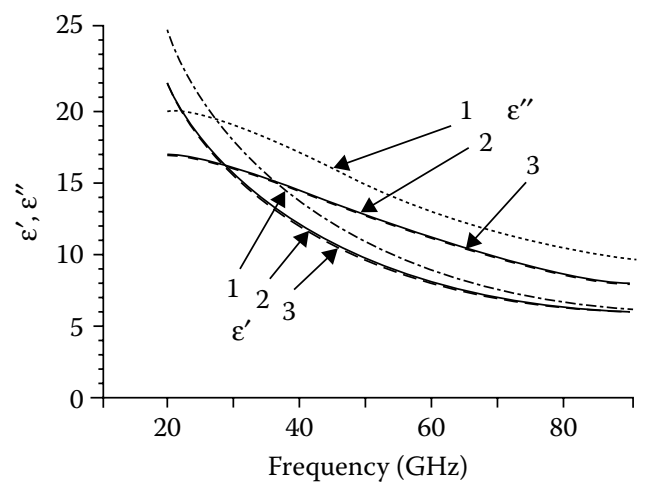

FIGURE 1.34 Measured skin permittivity (solid lines 2) for $\varepsilon^{\prime}$ and $\varepsilon^{\prime \prime}$ of human (forearm) skin: Shown in the figure for comparison are dotted (1) and dashed (3) lines representing predicted frequency dependence of skin permittivity calculated by Gandhi and Riazi (1986) and Gabriel, Lau, and Gabriel (1996), respectively. (From Alekseev, S. I., and M. C. Ziskin. 2007. Human skin permittivity determined by millimeter wave reflection measurements. Bioelectromagnetics 28:331-9. With permission.)

It should be noted that skin tissue is not homogeneous but consists of a multilayer of stratum corneum (SC), epidermis, and dermis. Moreover, it is differentiated according to body location; for example, forearm and palm skins have thin and thick SC, respectively. Similar to Equation 1.53, it has been shown that the $\mathrm{mmW}$ permittivity of different skin layers may be described by the Debye equation with a single relaxation time such that (Alekseev, Gordiienko, and Ziskin 2008):

$$
\varepsilon_{\mathrm{l}}^{*}(\omega)=\varepsilon_{h i}+\left(\varepsilon_{l i}-\varepsilon_{h i}\right) /(1+\mathrm{j} \omega \tau)+\sigma_{i} / \mathrm{j} \omega \varepsilon_{0}
$$

The values of $\tau, \varepsilon_{h i}=\varepsilon_{\infty}, \Delta \varepsilon_{\mathrm{t}}=\varepsilon_{l i}-\varepsilon_{h i}$, and $\sigma_{i}$ for each of the skin layers in the models are given in Table 1.8.

Some measured power reflection coefficients $\left(R^{2}\right)$ for human forearm and palm skins are given in Figure 1.35. The fraction of transmitted power $\left(T^{2}\right)$ is found from $T^{2}=1-$ $R^{2}$ (see Equations 1.44 and 1.45). It can be seen that reflections from the palm are lower than those from the forearm (Alekseev and Ziskin 2007). The disjuncture for the two sets of data obtained in the two frequency ranges of $37-53$ and $54-74 \mathrm{GHz}$ is the result of measuring the magnitude of reflection with two waveguides of different sizes and different cutoff wavelengths. Given the trend toward lower values for higher frequencies, perhaps the gap may be bridged by extrapolating between the two segments; in each case, there would be a reasonable continuum of reflection as a function of frequency. Thus, the reflection coefficients for frequencies from 37 to $74 \mathrm{GHz}$ decreased from $60 \%$ to $45 \%$ and from $40 \%$ to $20 \%$ for skin on the forearm and the palm, respectively.

Calculations of $\mathrm{mmW}$ power transmission coefficients for skin on the forearm showed an increase from 55\% to $65 \%$ between 30 and $90 \mathrm{GHz}$ (Alexseev et al. 2008). A thick SC in the palm causes an increase in transmission as a result of layer matching phenomenon at higher $\mathrm{mmW}$ frequencies. The transmitted power density profile, 
TABLE 1.8 Permittivity Parameters for the Debye Equation and Dimensions of Skin Models Used in Calculations

\begin{tabular}{|c|c|c|c|c|c|c|}
\hline & \multirow[b]{2}{*}{ Parameter } & \multicolumn{3}{|c|}{ Forearm Model } & \multicolumn{2}{|c|}{ Palm Model } \\
\hline & & 1 & 2 & 3 & 2 & 3 \\
\hline \multirow[t]{4}{*}{ SC } & $\varepsilon_{\infty}$ & - & 2.96 & 2.96 & 3.63 & 3.63 \\
\hline & $\Delta \varepsilon$ & - & - & - & 9.7 & 9.5 \\
\hline & $d(\mathrm{~mm})$ & - & 0.015 & 0.015 & 0.43 & 0.42 \\
\hline & $\sigma(\mathrm{S} / \mathrm{m})$ & - & 0 & 0 & 0 & 0 \\
\hline \multirow[t]{5}{*}{$E^{-}+D$} & $\varepsilon_{\infty}$ & 4.0 & 4.0 & 4.0 & 4.52 & 4.52 \\
\hline & $\Delta \varepsilon$ & 32.0 & 32.6 & 32.4 & 27.2 & 26.4 \\
\hline & $d(\mathrm{~mm})$ & $\infty$ & $\infty$ & 1.45 & $\infty$ & 1.85 \\
\hline & $\sigma(\mathrm{S} / \mathrm{m})$ & 1.4 & 1.4 & 1.4 & 1.4 & 1.4 \\
\hline & $\tau \times 10^{12}(\mathrm{~S})$ & 6.9 & 6.9 & 6.9 & 6.9 & 6.9 \\
\hline
\end{tabular}

Source: Alekseev, S. I., O. C. Gordiienko, and M. C. Ziskin. 2008. Reflection and penetration depth of millimeter waves in murine skin. Bioelectromagnetics 29:340-4.

Note: SC stands for stratum corneum, E + D stands for the rest of the epidermis plus the dermis, and $d$ stands for layer thickness. Model 1-homogeneous skin with $\mathrm{E}+\mathrm{D}$; model 2SC and E + D; model 3-SC, E + D, and fat.

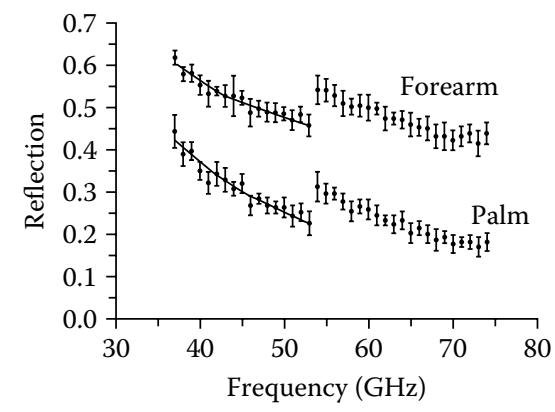

FIGURE 1.35 Measured power reflection coefficient in the forearm and palm as a function of millimeter-wave frequency: Solid lines are for three-layered skin model containing the stratum corneum and epidermis plus dermis. Error bars represent SD; $\mathrm{n}=12$. (From Alekseev, S. I., and M. C. Ziskin. 2007. Human skin permittivity determined by millimeter wave reflection measurements. Bioelectromagnetics 28:331-9. With permission.)

penetration depth $\delta$ (distance to $\mathrm{e}^{-1}$ of surface values of the $\mathrm{mmW}$ field), and SAR in human skin calculated for homogeneous and multilayer planar models are shown in Figures 1.36 through 1.38 .

The transmitted power density profiles at 42 and $61 \mathrm{GHz}$ in skin model 3 (Table 1.8; $\mathrm{SC}, \mathrm{E}+\mathrm{D}$, and fat) are illustrated in Figure 1.36. It can be seen that exposure at $61 \mathrm{GHz}$ produces a higher power density in the epidermis, whereas exposure at the lower $42 \mathrm{GHz}$ is more intense in the dermis. The frequency dependence of power deposition is further illustrated by differences in the penetration depths calculated for the forearm and palm 


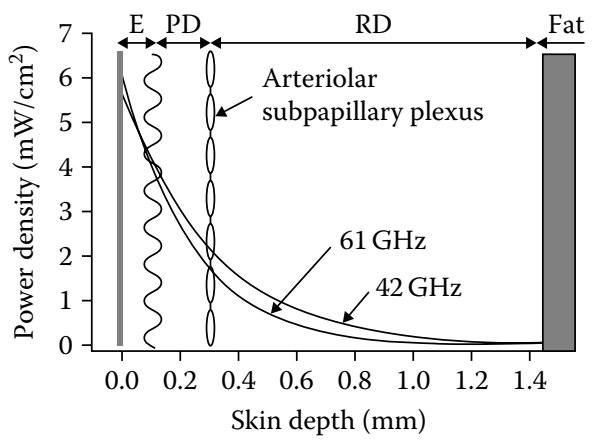

FIGURE 1.36 Power density profile for millimeter wave at 42 and $61 \mathrm{GHz}$ within the forearm skin, which was calculated using model 3 for an incident power density of $100 \mathrm{~W} / \mathrm{m}^{2}\left(10 \mathrm{~mW} / \mathrm{cm}^{2}\right)$ : The first thin layer shows the stratum corneum. The boundary between the epidermis $(\mathrm{E})$ and dermis (at a depth of $0.1 \mathrm{~mm}$ ) is marked by a wavy line. The arteriolar subpapillary plexus is located at the boundary between the papillary dermis (PD) and reticular dermis (RD); at a depth of $0.3 \mathrm{~mm}$. (From Alekseev, S. I., A. A. Radzievsky, M. K. Logani, and M. C. Ziskin. 2008. Millimeter wave dosimetry of human skin. Bioelectromagnetics 29:65-70. With permission.)

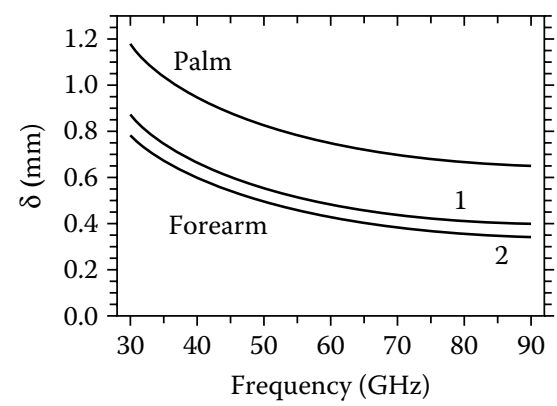

FIGURE 1.37 Penetration depth $(\delta)$ of plane millimeter-wave field in human skin: Calculations were performed using model 2. Curves land 2 were obtained for the forearm skin using permittivity values given in Table 1.8 and those given by Gandhi and Riazi (1986), respectively. (From Alekseev, S. I., A. A. Radzievsky, M. K. Logani, and M. C. Ziskin. 2008. Millimeter wave dosimetry of human skin. Bioelectromagnetics 29:65-70. With permission.)

skins, as shown in Figure 1.37 for both model 2 and model 3 (Table 1.8). As shown in the figure, these calculations yielded a higher penetration depth of $\mathrm{mmW}$ into the palm skin than the forearm skin, again demonstrating the influence of a matching thicker SC layer. Moreover, $\mathrm{mmW}$ can go through the human skin deep enough (e.g., $\delta=0.65 \mathrm{~mm}$ at $42 \mathrm{GHz}$ ) to affect most skin structures located in the epidermis and dermis. Note that a slightly higher penetration depth in the forearm skin with a thin SC was obtained by Alekseev et al. (2008) than the depths reported earlier by Gandhi and Riazi (1986), using assumed permittivity values for skin.

The dependence of SAR on mmW frequency at different depths calculated for the homogeneous skin model is illustrated in Figure 1.38. As expected, the highest SAR values occur at the skin surface. In general, SAR increases with $\mathrm{mmW}$ frequency. 


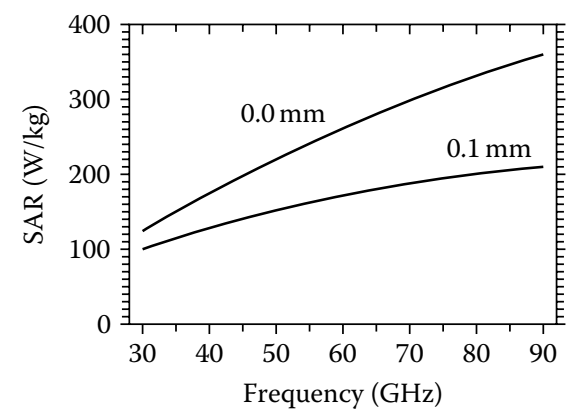

(a)

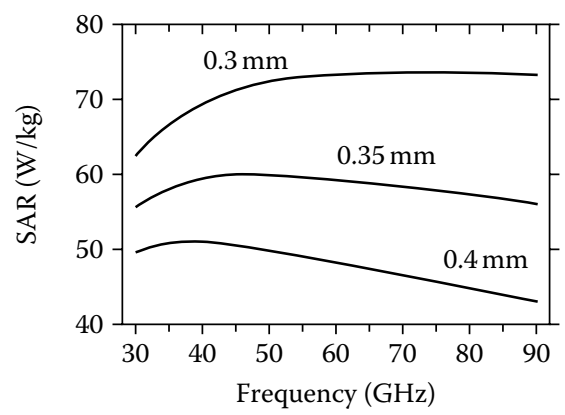

(b)

FIGURE 1.38 Specific absorption rates (SARs) at different depths in skin calculated for an incident power density of $100 \mathrm{~W} / \mathrm{m}^{2}$ : (a) SARs at 0.0 - and 0.1-mm depths; (b) SARs at 0.30-, 0.35-, and 0.40-mm depths. (From Alekseev, S. I., A. A. Radzievsky, M. K. Logani, and M. C. Ziskin. 2008. Millimeter wave dosimetry of human skin. Bioelectromagnetics 29:65-70. With permission.)

However, at the highest frequencies the SAR in the deeper regions of the skin is lower because of the reduced penetration depth at these frequencies.

\subsubsection{Summary of Millimeter-Wave Coupling}

There is a paucity of data on permittivity and coupling, such as data on reflection, transmission, and induced energy deposition, into biological tissues in the $\mathrm{mmW}$ frequency band. Available information for $30-90 \mathrm{GHz}$ indicates that the behavior of relative permittivity follows the behavior of the lower RF frequencies. Specifically,

- Real and imaginary parts of permittivity for skin decrease from 20 to 6 and from 20 to 12 , respectively.

- Power reflection coefficients for frequencies from 37 to $74 \mathrm{GHz}$ decrease from $60 \%$ to $45 \%$ and from $40 \%$ to $20 \%$ for skin on the forearm and palm, respectively.

- Power transmission coefficient for skin on the forearm showed an increase from $55 \%$ to $65 \%$, respectively, between 30 and $90 \mathrm{GHz}$.

- Penetration depth $(\delta)$ of a plane-wave field decreases from 0.8 to $0.4 \mathrm{~mm}$ and from 1.2 to $0.7 \mathrm{~mm}$ for skin on the forearm and palm, respectively, between 30 and 90 $\mathrm{GHz}$.

- The SAR increases with mmW frequency. However, at the highest frequencies the SAR in the deeper regions of the skin is lower because of the reduced penetration depth at these frequencies.

\section{References}

Ackerman, M. J. 1998. The Visible Human Project. Proc IEEE 86:504-11.

Alekseev, S. I., and M. C. Ziskin. 2007. Human skin permittivity determined by millimeter wave reflection measurements. Bioelectromagnetics 28:331-9.

Alekseev, S. I., A. A. Radzievsky, M. K. Logani, and M. C. Ziskin. 2008. Millimeter wave dosimetry of human skin. Bioelectromagnetics 29:65-70. 
Alekseev, S. I., O. C. Gordiienko, and M. C. Ziskin. 2008. Reflection and penetration depth of millimeter waves in murine skin. Bioelectromagnetics 29:340-4.

Anderson, V., and K. H. Joyner. 1995. Specific absorption rate levels measured in a phantom head exposed to radio frequency transmissions from analog hand-held mobile phones. Bioelectromagnetics 16:60-9.

ARIB. 2002. Specific Absorption Rate (SAR) Estimation for Cellular Phone. Tokyo, Japan: Association of Radio Industries and Businesses STD-T56.

Balanis, C., ed. 2008. Modern Antenna Handbook. Hoboken, NJ: Wiley.

Balzano, Q., O. Garay, and F. R. Steel. 1978. Energy deposition in simulated human operators of $800 \mathrm{MHz}$ portable transmitters. IEEE Trans Vehicular Tech 27:174-81.

Balzano, Q., O. Garay, and T. J. Manning. 1995. Electromagnetic energy exposure of simulated users of portable cellular telephones. IEEE Trans Vehicular Tech 44:390-403.

Beard, B. B., W. Kainz, T. Onishi, T. Iyama, S. Watanabe, O. Fujiwara, J. Wang, G. BitBabik, A. Faraone, J. Wiart, A. Christ, et al. 2006. Comparisons of computed mobile phone induced SAR in the SAM phantom to that in anatomically correct models of the human head. IEEE Trans Electromagn Compat 48:397-407.

Berenger, J. P. 1994. A perfectly matched layer for the absorption of electromagnetic waves. J Comput Phys 114:185-200.

Bernardi, P., M. Cavagnaro, and S. Pisa. 1996. Evaluation of the SAR distribution in the human head for cellular phones used in a partially closed environment. IEEE Trans Electromagn Compat 38:357-66.

Bernardi, P., M. Cavagnaro, S. Pisa, and E. Piuzzi. 2000. Specific absorption rate and temperature increases in the head of a cellular phone user. IEEE Trans Microwave Theory Tech 48(7):1118-26.

Bernardi, P., M. Cavagnaro, S. Pisa, and E. Piuzzi. 2001a. A graded-mesh FDTD code for the study of human exposure to cellular phones equipped with helical antennas. ACES J 16:90-6.

Bernardi, P., M. Cavagnaro, S. Pisa, and E. Piuzzi. 2001b. Power absorption and temperature elevations induced in the human head by a dual-band monopole-helix antenna phone. IEEE Trans Microwave Theory Tech 49:2539-46.

Bernardi, P., M. Cavagnaro, S. Pisa, E. Piuzzi, and J. C. Lin. 2009. Dosimetry and temperature aspects of mobile phone exposures. In Advances in Electromagnetic Fields in Living Systems, vol. 5, pp 221-76. New York: Springer.

Bit-Babik, G., A. W. Guy, C. K. Chou, A. Faraone, M. Kanda, A. Gessner, J. Wang, and O. Fujiwara. 2005. Simulation of exposure and SAR estimation for adult and child heads exposed to radiofrequency energy from portable communication devices. Radiat Res 163:580-90.

CDC. 2009. Centers for Disease Control and Prevention (CDC) Growth Charts (online). Available at: http://www.cdc.gov/growthcharts/:Atlanta.

CENELEC. 2003. Basic Standard for the Measurement of Specific Absorption Rate Related to Human Exposure to Electromagnetic Fields from Mobile Phones (300 MHz-3 GHz), EN 50361. Brussels, Belgium: CENELEC.

Chavannes, N., R. Tay, N. Nikoloski, and N. Kuster. 2003. Suitability of FDTD-based TCAD tools for RF design of mobile phones. IEEE Antennas and Propagation Magazine 45:52-66. 
Chen, H. Y., and H. H. Wang. 1994. Current and SAR induced in a human head model by the electromagnetic field irradiated from a cellular telephone. IEEE Trans Microwave Theory Tech 42:2249-54.

Christ, A., N. Chavannes, N. Nikoloski, H. U. Gerber, K. Poković, and N. Kuster. 2005. A numerical and experimental comparison of human head phantoms for compliance testing of mobile telephone equipment. Bioelectromagnetics 26:125-37.

Christ, A., T. Samaras, A. Klingenbock, and N. Kuster. 2006. Characterization of the electromagnetic near-field absorption in layered biological tissue in the frequency range from $30 \mathrm{MHz}$ to $6000 \mathrm{MHz}$. Phys Med Biol 51:4951-65.

Cleveland Jr., R. F., and T. W. Athey. 1989. Specific absorption rate (SAR) in models of the human head exposed to hand-held UHF portable radios. Bioelectromagnetics 10:173-86.

Di Benedetto, M. G., T. Kaiser, A. F. Molisch, I. Oppermann, C. Politano, and D. Porcino. 2006. UWB Communication Systems: A Comprehensive Overview. New York, NY: Hindawi.

Dimbylow, P. J. 1993. FDTD calculations of SAR for a dipole closely coupled to the head at $900 \mathrm{MHz}$ and $1.9 \mathrm{GHz}$. Phys Med Biol 38:361-8.

Dimbylow, P. J., and S. M. Mann. 1994. SAR calculations in an anatomically realistic model of the head for mobile communication transceivers at $900 \mathrm{MHz}-1.8 \mathrm{GHz}$. Phys Med Biol 39:1537-53.

FCC. 1996. Guidelines for Evaluating the Environmental Effects of Radiofrequency Radiation, FCC 96-326. Washington, DC.

FCC. 1997. Evaluating Compliance With FCC Guidelines for Human Exposure to Radiofrequency Electromagnetic Fields, OET Bulletin No. 65. http://www.fcc.gov/oet/ rfsafety/sar.html (last accessed July 22, 2010).

FCC. 2002. Revision of Part 15 of the Commission's Rules Regarding Ultra-Wideband Transmission Systems, FCC 02-48. Washington, DC.

Furse, C. M., J. Y. Chen, and O. P. Gandhi. 1994. A frequency-dependent finite-difference time-domain method for induced current and SAR calculations for a heterogeneous model of the human body. IEEE Trans Electromagn Compat 36(2):128-33.

Gabriel, S., R. W. Lau, and C. Gabriel. 1996. The dielectric properties of biological tissues. 2. Measurement in the frequency range of $10 \mathrm{~Hz}$ to $20 \mathrm{GHz}$. Phys Med Biol 41:2251-69.

Gandhi, O. P. 1983. Some basic properties of biological tissues for potential biomedical applications of millimeter-waves. J Microwave Power 18:295-304.

Gandhi, O. P., and G. Kang. 2002. Some present problems and a proposed experimental phantom for SAR compliance testing of cellular telephones at 835 and $1900 \mathrm{MHz}$. Phys Med Biol 47:1501-18.

Gandhi, O. P., and A. Riazi. 1986. Absorption of millimeter waves by human beings and its biological implications. IEEE Trans Microwave Theory Tech 34:228-35.

Gandhi, O. P., B. Q. Gao, and J. Y. Chen. 1992. A frequency-dependent finite-difference time-domain formulation for induced current calculations in human beings. Bioelectromagnetics 13(6):543-55. 
Gandhi, O. P., B. Q. Gao, and J. Y. Chen. 1993. A frequency-dependent finite-difference time-domain formulation for general dispersive media. IEEE Trans Microwave Theory Tech 41(4):658-65.

Gandhi, O. P., G. Lazzi, and C. M. Furse. 1996. Electromagnetic absorption in the human head and neck for mobile telephones at 835 and $1900 \mathrm{MHz}$. IEEE Trans Microwave Theory Tech 44:1884-97.

Gandhi, O. P., G. Lazzi, A. Tinniswood, and Q. S. Yu. 1999. Comparison of numerical and experimental methods for determination of SAR and radiation patterns of handheld wireless telephones. Bioelectromagnetics 20(Suppl 4):S93-S101.

Gjonaj, E., M. Bartsch, M. Clemens, and S. Schupp. 2002. High-resolution human anatomy models for advanced electromagnetic field computations. IEEE Trans Magn 38:357-60.

Hadjem, A., D. Lautru, C. Dale, M. F. Wong, V. H. Hanna, and J. Wiart. 2005. Study of specific absorption rate (SAR) induced in two child head models and in adult heads using mobile phones. IEEE Trans Microwave Theory Tech 53(1):4-11.

ICNIRP. 1998. Guidelines for limiting exposure to time-varying electric, magnetic, and electromagnetic fields (up to $300 \mathrm{GHz}$ ). Health Phys 74(4):494-522.

IEC. 2010. Human exposure to radio frequency fields from hand-held and body-mounted wireless communication devices - Human models, instrumentation, and procedures, IEC 62209-2 Ed. 1.0. Geneva, Switzerland: International Electrotechnical Commission.

IEEE. 2003. IEEE Recommended Practice for Determining the Peak Spatial-Average Specific Absorption Rate (SAR) in the Human Head from Wireless Communications Devices: Measurement Techniques, Standard 1528. New York, NY: IEEE.

IEEE. 2005. IEEE Standard for Safety Levels With Respect to Human Exposure to Radio 628 Frequency Electromagnetic Fields, $3 \mathrm{kHz}$ to $300 \mathrm{GHz}$, IEEE Standard 629 C95.1. New York, NY.

Jensen, M. A., and Y. Rahmat-Samii. 1995. EM interaction of handset antennas and a human in personal communications. Proc IEEE 83:7-17.

Jordan, E. C., and K. G. Balmain. 1968. Electromagnetic Waves and Radiating Systems. New York: Prentice-Hall.

Kainz, W., A. Christ, T. Kellom, S. Seidman, N. Nikoloski, B. Beard, and N. Kuster. 2005. Dosimetric comparison of the specific anthropomorphic mannequin (SAM) to 14 anatomical head models using a novel definition for the mobile phone positioning. Phys Med Biol 50:3423-45.

Kaune, W. T., and R. D. Phillips. 1980. Comparison of the coupling of grounded humans, swine, and rats to vertical, $60 \mathrm{~Hz}$ electric fields. Bioelectromagnetics 1:117-29.

Kunz, K. S., and R. J. Luebbers. 1993. The Finite Difference Time Domain Method for Electromagnetics. Boca Raton, FL: CRC Press.

Kuster, N., and Q. Balzano.1992. Energy absorption mechanism by biological bodies in the near field of dipole antennas above $300 \mathrm{MHz}$. IEEE Trans Vehicular Tech 41:17-23.

Kuster, N., and Q. Balzano. 1997. Experimental and numerical dosimetry. In Mobile Communications Safety, ed. N. Kuster, Q. Balzano, and J. C. Lin, 13-64. London, UK: Chapman and Hall.

Leslie, G., and M. D. Farkas. 1981. Anthropometry of the Head and Face. New York: Elsevier. 
Li, L. W., M. S. Leong, P. S. Kooi, and T. S. Yeo. 2000. Specific absorption rates in human head due to handset antennas: A comparative study using FDTD method. J Electromag Waves Appl 14:987-1000.

Lin, J. C. 1975. Interaction of electromagnetic transient radiation with biological materials. IEEE Trans Electromagn Compat 17:93-7.

Lin, J. C. 1976a. Electromagnetic pulse interaction with mammalian cranial structures. IEEE Trans on Biomed Eng 23:61-5.

Lin, J. C. 1976b. Microwave auditory effect-A comparison of some possible transduction mechanisms. J Microwave Power 11:77-81.

Lin, J. C. 1976 c. Microwave-induced hearing sensation: Some preliminary theoretical observations. J Microwave Power 11:295-8.

Lin, J. C. 1977a. On microwave-induced hearing sensation. IEEE Trans Microwave Theory Tech 25:605-13.

Lin, J. C. 1977b. Further studies on the microwave auditory effects. IEEE Trans Microwave Theory Tech 25:936-41.

Lin, J. C. 1977c. Calculations of frequencies and threshold of microwave-induced auditory signals. Radio Sci 12(SS-1):237-52.

Lin, J. C. 1978. Microwave Auditory Effects and Applications. Springfield, IL: Charles C. Thomas.

Lin, J. C. 1980. The microwave auditory phenomenon. Proc IEEE 68:67-73.

Lin, J. C. 1986. Computer methods for field intensity predictions. In CRC Handbook of Biological Effects of Electromagnetic Fields, ed. C. Polk and E. Postow, 273-313. Boca Raton, FL: CRC Press.

Lin, J. C. 1990. Auditory perception of pulsed microwave radiation. In Biological Effects and Medical Applications of Electromagnetic Fields, ed. O. P. Gandhi, Chapter 12, 277-318. New York: Prentice-Hall.

Lin, J. C., ed. 1994. Advances in Electromagnetic Fields in Living Systems. vol. 1. New York: Plenum Press.

Lin, J. C. 2000. Mechanisms of field coupling into biological systems at ELF and RF frequencies. In Advances in Electromagnetic Fields in Living Systems. vol. 3., ed. J. C. Lin, 1-38. New York: Kluwer/Plenum.

Lin, J. C. 2003. Risk to children from cellular telephone radiation. IEEE Microwave Magazine 4:20-6.

Lin, J. C. 2009. Health effects of cell phone radiation. In Advances in Electromagnetic Fields in Living Systems. vol. 5., ed. J. C. Lin, 1-277. New York: Springer.

Lin, J. C., and C. K. Lam. 1976. Coupling of Gaussian electromagnetic pulse into musclebone model of biological structure. J Microwave Power 11:67-75.

Lin, J. C., and P. Bernardi. 2007. Computer methods for predicting field intensity and temperature change. In Bioengineering and Biophysical Aspects of Electromagnetic Fields, ed. F. Barnes and B. Greenebaum, Chapter 10, 293-380. Boca Raton, FL: CRC Press.

Lin, J. C., and O. P. Gandhi. 1996. Computational methods for predicting field intensity. In Handbook of Biological Effects of Electromagnetic Fields, ed. C. Polk and E. Postow, 337-402. Boca Raton, FL: CRC Press.

Lin, J. C., and Z. W. Wang. 2010. Acoustic pressure waves induced in human heads by RF pulses from high-field MRI scanners. Health Phys 98(4):603-13. 
Lin, J. C., A. W. Guy, and C. C. Johnson. 1973. Power deposition in a spherical model of man exposed to 1-20 MHz EM fields. IEEE Trans Microwave Theory Tech MTT 21:791-7.

Lin, J. C., C. L. Wu, and C. K. Lam. 1975. Transmission of electromagnetic pulse into the head. Proc IEEE 63:1726-7.

Lin, J. C., P. Bernardi, S. Pisa, M. Cavagnaro, and E. Piuzzi. 2008. Antennas for biological experiments. In Modern Antenna Handbook, ed. C. Balanis, pp. 1429-60. Hoboken, NJ: Wiley.

Mason, P. A., W. D. Hurt, T. J. Walters, J. A. D’Andrea, P. Gajsek, K. L. Ryan, D. A. Nelson, K. I. Smith, and J. M. Ziriax. 2000. Effects of frequency, permittivity, and voxel size on predicted specific absorption rate values in biological tissue during electromagnetic-field exposure. IEEE Trans Microwave Theory Tech 48(11):2050-8.

Maxwell, J. C. 1904. Treatise on Electricity. 3rd ed. New York: Oxford University Press.

Martens, L., J. Moerloose, D. Zutter, J. Poorter, and C. Wagter. 1995. Calculation of the electromagnetic fields induced in the head of an operator of a cordless telephone. Radio Sci 30:283-90.

NCRP. 1981. Radiofrequency Electromagnetic Fields: Properties, Quantities and Units, Biophysical Interaction, and Measurements, Report 67. National Council on Radiation Protection and Measurements: Bethesda, MD.

NCRP. 1986. Biological Effects and Exposure Criteria for Radiofrequency Electromagnetic Fields, NCRP Report No. 86. National Council on Radiation Protection and Measurement: Bethesda, MD.

Nikita, K. S., M. Cavagnaro, P. Bernardi, N. K. Uzunoglu, S. Pisa, E. Piuzzi, J. N. Sahalos, G. I. Krikelas, J. A. Vaul, P. S. Excell, G. Cerri, et al. 2000a. A study of uncertainties in modeling antenna performance and power absorption in the head of a cellular phone user. IEEE Trans Microwave Theory Tech 48:2676-85.

Pakhomov, A. G., Y. Akyel, O. N. Pakhomova, B. E. Stuck, and M. R. Murphy. 1998. Current state and implications of research on biological effects of millimeter waves: A review of the literature. Bioelectromagnetics 19:393-413.

Pisa, A., M. Cavagnaro, V. Lopresto, E. Piuzzi, G. A. Lovisolo, and P. Bernardi. 2005. A procedure to develop realistic numerical models of cellular phones for an accurate evaluation of SAR distribution in the human head. IEEE Trans Microwave Theory Tech 53(4):1256-65.

Piuzzi, E., P. Bernardi, M. Cavagnaro, S. Pisa, and J. C. Lin. 2011. Analysis of adult and child exposure to uniform plane waves at mobile communication systems frequencies (900 MHz-3 GHz). IEEE Trans Electromagn Compat 53:38-47.

Roschmann, P. 1991. Human auditory system response to pulsed radiofrequency energy in RF coils for magnetic resonance at 2.4 to $179 \mathrm{MHz}$. Magn Reson Med 21:197-215.

Rowley, J. T., and R. B. Waterhouse. 1999. Performance of shorted patch antennas for mobile communication handsets at $1800 \mathrm{MHz}$. IEEE Trans Microwave Theory Tech 47:815-22.

Silver, S. 1949. Electromagnetic Theory. New York: McGraw-Hill.

Schiavoni, A., P. Bertotto, G. Richiardi, and P. Bielli. 2000. SAR generated by commercial cellular phones-phone modeling, head modeling, and measurements. IEEE Trans Microwave Theory Tech 48:2064-71. 
Schmid, T., O. Egger, and N. Kuster. 1996. Automated E-field scanning system for dosimetric assessments. IEEE Trans Microwave Theory Tech 44:105-13.

Schoenborn, F., M. Burkhardt, and N. Kuster. 1998. Differences in energy absorption between heads of adults and children in the near field of sources. Health Phys 74:160-8.

Shimizu, K., H. Endo, and G. Matsumoto. 1988. Visualization of electric fields around a biological body. IEEE Trans Biomed Eng 35:296-302.

Taflove, T. 1995. Computational Electrodynamics: The Finite-Difference Time-Domain Method. Norwood, MA: Artech House.

Tinniswood, A. D., C. M. Furse, and O. P. Gandhi. 1998. Computation of SAR distributions for two anatomically based models of the human head using CAD files of commercial telephone and the parallel FDTD code. IEEE Trans Antenna Propag 46:829-33.

Toftgard, J., S. N. Hornsleth, and J. B. Andersen. 1993. Effects on portable antennas of the presence of a person. IEEE Trans Antennas Propag 41(6):739-46.

Wang, J., and O. Fujiwara. 2003. Comparison and evaluation of electromagnetic absorption characteristics in realistic children for $900 \mathrm{MHz}$ mobile telephones. IEEE Trans Microwave Theory Tech 51:966-71.

Wang, J., O. Fujiwara, S. Watanabe, and Y. Yamanaka. 2004. Computation with a parallel FDTD system of human-body effect on electromagnetic absorption for portable telephones. IEEE Trans Microwave Theory Tech 52:53-8.

Wang, Z. W., J. C. Lin, J. T. Vaughan, and C. M. Collins. 2008. On consideration of physiological response in numerical models of temperature during MRI of the human head. J Magn Reson Imaging 28:1303-8.

Wang, Z. W., J. C. Lin, W. H. Mao, W. Z. Liu, M. B. Smith, and C. M. Collins. 2007. SAR and temperature: Simulations and comparison to regulatory limits for MRI. J Magn Reson Imaging 26:437-41.

Watanabe, Y., T. Tanaka, M. Taki, and S. Watanabe. 2000. FDTD analysis of microwave hearing effect. IEEE Trans Microwave Theory Tech 48:2126-32.

WHO. 2000. Obesity: Preventing and Managing the Global Epidemic. Report of a WHO consultation, WHO Tech. Rep. Series 894. Geneva, Switzerland: World Health Organization.

Yee, K. 1966. Numerical solution of initial boundary value problems involving Maxwell's equations in isotropic media. IEEE Trans Antennas Propag 14: 302-7. 



\author{
R. P. Joshi \\ K. H. Schoenbach
}

\section{2}

\section{Pulsed Electric Fields in Biological Cells and Membranes}

2.1 Introduction 71

2.2 Interaction of Electric Fields with Living Cells 73

2.3 Modeling Electric Fields in Cells 76 Continuum Mean-Field Approaches for Spherical Cells - Continuum Approaches Applied to More Complicated Cell Geometries - Models of Electric Field Interactions in Tissues and Nerves

2.4 Temperature Changes Due to Electric Pulsing. .94

2.5 Role of the Biological Dielectric Properties........ 97

2.6 Extracting Parameters from Cell Suspensions 102

References. 105

\title{
2.1 Introduction
}

The interaction of electric and magnetic fields with biological systems has been a persistent scientific interest over the past two decades (Blank 1995; Polk and Postow 1996). Applications and mechanistic understanding have continued to receive growing attention in this arena due to issues relating to therapies, health risks and hazards, biosensing, and medical delivery technologies. Biological sensory systems can detect very weak steady fields. For example, variations as small as a few percentage of the Earth's magnetic field $\left(5 \times 10^{-5} \mathrm{~T}\right)$ can be detected (Lohmann and Lohmann 1996), while sharks and rays sense extremely weak electric fields in seawater. However, the effects of weak fields are difficult to understand and any possible role in triggering or coordinating coupled biophysical mechanisms in organized cell systems is challenging to analyze. Thus, molecular changes or signaling induced by low fields can be difficult to extract and discern from other sources of change such as natural biochemical pathways. By 
contrast, the application of high-intensity electric fields does not present such difficulties. Short exposures of cells and tissues to strong fields represent an important subset of high voltage external excitation. The fields involved are larger in the order of magnitude and so can readily pass the conceptual tests based on signal-to-noise ratio (SNR) criteria in spite of the short exposure times.

Models of electric field interactions with biological cells predict that pulses with durations shorter than the charging time of the outer membrane can penetrate and affect intracellular structures. Experimental studies in which human cells were exposed to pulsed electric fields of up to $300 \mathrm{kV} / \mathrm{cm}$ amplitude, with durations as short as $10 \mathrm{~ns}$, have confirmed this hypothesis. The observed effects include the breaching of intracellular granule membranes without permanent damage to the cell membrane, abrupt rises in intracellular free calcium levels, enhanced expression of genes, cytochrome $c$ release, and electroporation for gene transfer and drug delivery. At increased electric fields, the application of nanosecond pulses induces apoptosis (programmed cell death) in biological cells, an effect that has been shown to be beneficial in reducing the growth of tumors (Nuccitelli et al. 2006). Such nanosecond electrical pulses have been shown to successfully treat melanoma tumors by using needle arrays as pulse delivery systems. Besides treating tumors, possible applications of the intracellular electrical effects are enhancing gene delivery to the nucleus, controlling calcium release and cell functions, and cell immobilization. Reducing the pulse duration of intense electric field pulses even further into the subnanosecond range will allow for the use of wideband antennas to deliver the electromagnetic (EM) fields into tissue with a spatial resolution in the centimeter range.

This chapter primarily examines the concepts of electric field interactions in cells, though some treatment of tissues has also been included. The focus is on ultrashort $(\sim 10-100 \mathrm{~ns})$, pulsed high-intensity fields $(\sim 100 \mathrm{kV} / \mathrm{cm})$. A theoretical basis for evaluating field strengths and penetrating into single cells is given, together with modeling results as appropriate. Continuum approaches are discussed for electric field evaluations that can then form the basis for subsequent methods of molecular dynamics or structural bioinformatics for analyzing cellular electrical responses. The time-dependent and spatially dependent electric field $E(r, t)$ can then yield important parameters such as the specific absorption rate (SAR) and total absorbed dose (AD). For example, from the knowledge of the local density $(\rho)$ and the electrical conductivity $(\sigma)$, the SAR can be obtained as $\sigma E(r, t)^{2} / \rho$. The present discussions have an important bearing on microdosimetry, that is, the quantitative evaluation of the electric fields and voltages across the cell membrane leading to energy absorption. Microdosimetry can also be extremely useful in developing more effective Micro-Second Pulsed Electric Field ( $\mu$ sPEF) waveforms and in designing innovative pulse applicators. Here, both simple and complicated cell models are discussed. The role of the dielectric properties of biological cells is also outlined because these parameters affect the development and magnitude of electric field generation within the biosystem. It is argued that the frequency-dependent dielectric response can be used in designing shorter pulses that would have greater bioeffects. Finally, some of the experimental techniques pertinent to the measurement of dielectric properties are briefly considered. 


\subsection{Interaction of Electric Fields with Living Cells}

Since the late 1950s, the interaction of living cells with EM fields has been the subject of an enormous ongoing research effort. An extremely wide variety of phenomena have being investigated. These range from cellular effects due to weak low-frequency EM fields associated with transmission lines and household wiring (Davis et al. 1992) to electrical trauma arising from exposure to strong electric fields (Lee and Kolodney 1987; Gaylor, Prakah-Asante, and Lee 1988; Bhatt, Gaylor, and Lee 1990; Lee, Canaday, and Hammer 1993; Block et al. 1995). Even in the case of household wiring, a large spectrum of situations is of interest, from commonly occurring domestic and industrial incidents involving low-frequency main voltage to much rarer cases of radio-frequency shock resulting from contact with very high-voltage radio masts (Hocking et al. 1994). The field of electric field interactions with biotissues is vast and includes aspects such as animal navigation, endogenous fields and currents, drug delivery and other medical interventions, human health hazards from environmental and occupational EM fields (Blank and Findl 1987; Reilly 1992; Wiltschko and Wiltschko 1995; Polk and Postow 1996) to cellular electromanipulation (Buescher and Schoenbach 2003), and cancer therapy (Nuccitelli et al. 2006).

The influence of both EM and static magnetic fields on cells, tissues, plants, and animals has been studied (Berg 1993). Exposure to EM fields has been reported to increase the risk of certain types of cancer, such as leukemia, cancer of the central nervous system, and lymphoma (Wertheimer and Leeper 1979; Savitz et al. 1988). The effects of extremely low-frequency (ELF) EM fields on biological systems have also been evaluated by various groups (Blank 1993; Saunders, Sienkiewicz, and Kowalczuk 1991). Different targets including proliferation, enzyme reactions, biopolymer syntheses, and membrane transport have been investigated with respect to their alteration by EM energy (Berg 1995; Goodman, Greenbaum, and Marron 1995). For example, proliferation yield increased more than $25 \%$ over the control when a $0.5 \mathrm{mT}, 50 \mathrm{~Hz}$ EM field was added to the yeast Saccharomyces cerelisiae (Mehedintu and Berg 1997). With Corynebacterium glutamicum, it was found that an amplitude of $3.4 \mathrm{mT}$ at a frequency of $15 \mathrm{~Hz}$ increased the Adenosine Triphosphate (ATP) level more than $20 \%$ compared with the control after 8 hours of continuous exposure (Lei and Berg 1998). In another report, the colony-forming efficiency increased 40\%$70 \%$ over the control when adding a $1.1 \mathrm{mT}, 60 \mathrm{~Hz}$ EM field to JB6 cells (West et al. 1994).

The earliest report of bioeffects arising from the direct application of voltage using contact techniques (as opposed to contactless exposure using EM radiation) was in 1958. Exposure of the nodes of Ranvier to electric fields was seen to lead to some type of "electrical breakdown" (Stampfli 1958). Almost a decade later, damaging effects of strong electric fields on microorganisms were reported suggesting nonthermal membrane interactions (Sale and Hamilton 1967, 1968). Subsequent experiments showed that strong electric field pulses caused molecular transport across a biological membrane (Neumann and Rosenheck 1972). Artificial planar bilayer membrane measurements provided strong support for the transient aqueous pore hypothesis (Abidor et al. 1979; Pastushenko and Chizmadzhev 1982; Melikov 2001). This evidence invoked the structural rearrangement of membrane molecules with charging. The prominent 
observable effect was the rapid increase of electrical conductivity. The conductivity change was attributed to pore formation in the lipid bilayer membrane. Further evidence for chemical transport through membranes involved experiments with red blood cells (Sukhorukov, Mussauer, and Zimmermann 1998; Kinosita and Tsong 1977; Teissie and Tsong 1981; Serpersu, Kinosita and Tsong 1985). Erythrocytes were also used to demonstrate that DNA delivery into a cell is associated with dielectric breakdown of the cell membrane (Auer, Brandner, and Bodemer 1976).

A large body of work has also been carried out on the interaction of small spheroidal cells with external fields, motivated by application to cardiac defibrillation. The literature included investigation of cell excitation (Tung and Borderies 1992; Krassowska and Neu 1994; Fishler et al. 1996; Cheng, Tung, and Sobie 1999) and membrane electroporation (O’Neill and Tung 1991; DeBruin and Krassowska 1998, 1999).

Applications of cellular electric stimulation can roughly be divided into two broad groups. On the one hand, electric fields can be used as tools to modify various properties and responses of cells such as increases in membrane permeability for introducing various molecules and drugs into cells (Neumann et al. 1982; Mir et al. 1995; Sersa et al. 1995; Mouneimne et al. 1990; Raffy and Teissié 1995), fusion of cells (Zimmermann 1982; Sowers 1987), physical separation of different cell types (Arnold 2001), the killing of nonhealthy cells, and neuromuscular manipulation for therapy (Wang et al. 2002). The other aspect of electric pulsing is geared toward its utility to characterize various properties of biological cells or their constituents, both in suspensions and in tissues. Among the most important approaches in such characterization is the evaluation of cellular responses to electric fields at different frequencies. By varying the frequency of the field, values of the measured parameters form a spectrum. Values of frequencydependent bulk dielectric permittivity of suspensions or tissues, cellular angular velocity in rotating electric fields, and the dielectrophoretic spectrum evaluation are some of the important aspects that can be assessed (Foster and Schwan 1989; Führ, Glaser, and Hagedorn 1985), which would otherwise be difficult to measure. The characterization techniques rely on the fact that dielectric properties of biological systems typically display extremely high dielectric permittivity at low frequencies and fall off in distinct steps with increasing frequency. These frequency-dependent changes permit identification and investigation of a number of completely different underlying processes.

The basic mechanism underlying a majority of these methods and field induced biophenomena is the induction of a potential difference across the membrane by the external electric field. The seat of the electric field-driven bioresponses tends to be membranes because these sheaths represent nonconducting barriers that can easily be charged by external voltage pulsing. Consequently, large electric fields can be created across membranes that can then drive a host of bioeffects. The membranes are crucial not only because high electric fields can be created in these regions but also because important biological processes (e.g., irreversible apoptosis) are launched from these sites. For example, the extrinsic apoptotic pathway (Green 2000; Peter and Krammer 2003) involves the clustering of molecules such as the tumor necrosis factor-related apoptosis-inducing ligand (TRAIL), Fas-associated protein with death domain (FADD), and procaspase-8 leading to the formation of the death-inducing production. This in turn sets into motion a series of biochemical reactions that eventually lead to apoptosis 
(Song, Joshi, and Beebe 2010; Bagci et al. 2006; Budihardjo et al. 1999; Li, P. et al. 1997). The other apoptosis route (known as the intrinsic pathway) involves cytochrome $c$ release from another membrane-the mitochondria, which is an intracellular organelle (Zoratti and Szabo 1995; Marzo et al. 1998).

The classical theory of transmembrane voltage induction was developed in the 1950s by H. P. Schwan and coworkers (Schwan 1957; Pauly and Schwan 1959). Discussions on the increase in permeability (Neumann and Rosenheck 1972) of the plasma membrane of a biological cell-an effect that was termed electroporation-appeared in 1972. The electric fields required to achieve electroporation depend on the duration of the applied pulse because this process involves the gradual charging of the capacitive sheath followed by the molecular rearrangement of the lipids. Typical pulses range from tens of milliseconds with amplitudes of several hundred $\mathrm{V} / \mathrm{cm}$ to pulses of a few microseconds or smaller but requiring fields of several $\mathrm{kV} / \mathrm{cm}$.

More recently, the electrical pulse duration range has been shortened into the nanosecond range. Pulse durations as brief as several nanoseconds and pulse amplitudes as high as $300 \mathrm{kV} / \mathrm{cm}$ are being used (Schoenbach et al. 2008). Conceptually, such short pulse durations offer the possibility of triggering purely electrically driven responses without any thermal heating. In principle, fast processes such as electron transfers between molecules (Kranich et al. 2008), electrophoretic separation and self-organization (Groves, Boxer, and McConnell 1997), or field induced changes in reaction kinetics (De Biase et al. 2009) could also be fashioned. An even newer field of research opens up when the pulse duration is decreased further into the subnanosecond range. This push toward further pulse shortening is driven in part by the possibility of using wideband antennas, rather than direct contact electrodes, to deliver electrical energy and create fields in tissues as discussed in the literature (Kumar et al. 2011). Also conceptually, because the dielectric permittivity of membranes (and the aqueous media) has a nonlocal, time-dependent polarization, ultrafast excitation can effectively sample transient permittivities that are different from the steadystate values. This provides for electrical-based value selection of the dielectric parameters.

The influence of short pulses has been shown to reach into the cell interior (Schoenbach, Beebe, and Buescher 2001). This can perhaps be better understood through the following simple argument. Consider a spherical shell with a concentric inner organelle as shown in Figure 2.1. We assume for simplicity that the conductivities of both membranes are zero and that current continuity applies across line ABCDE shown in Figure 2.1. For long-duration, slow-rising pulses, a near quasisteady state prevails, and the current density, $J$, is nearly given by $J=\sigma E+\varepsilon \mathrm{d} E / \mathrm{d} t \sim \sigma E$, where $E, \sigma$, and $\varepsilon$ refer to the local electric field, conductivity, and permittivity, respectively. Because $\sigma$ for the membranes is nearly zero, there is virtually no current through them. Choosing the cell center as the reference voltage, the node potentials are then roughly as follows: $V_{\mathrm{D}} \sim 0$ and $V_{\mathrm{B}} \sim V_{\mathrm{C}}$. Also, the negligible membrane current, under the quasisteady state forces is $V_{\mathrm{CD}} \sim V_{\mathrm{FG}} \sim 0$. Thus, the potential across the inner membrane can be expected to be very modest, at best. This implies that membrane poration and other electrical effects would not be strong across cellular substructures and inner organelles, and the outer membrane would shield the cell at almost all times. Fast-rising, ultrashort pulses, on the other hand, would force a large nonequilibrium transient and create substantially large values of $V_{\mathrm{CD}}$ and $V_{\mathrm{FG}}$ across the inner membranes. This would allow fairly large potentials $(\sim 1 \mathrm{~V}$ or so, a value 


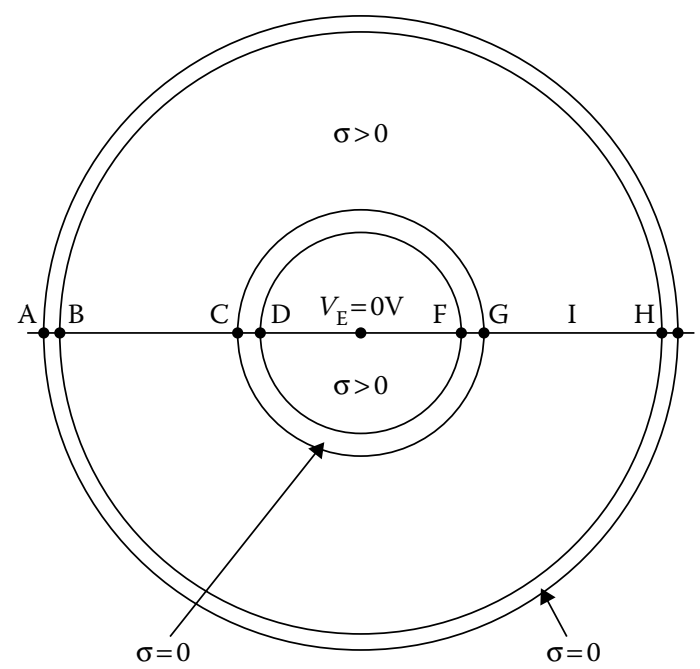

FIGURE 2.1 Rough voltage analysis in the quasisteady state for a model double-shelled cell in response to a slow rising, long pulse. (After Joshi, R. P., Q. Hu, K. H. Schoenbach, and S. J. Beebe. 2004. Phys Rev E 69:051901.)

close to the electroporation threshold) to be developed across the membranes of inner organelles (e.g., the mitochondria and the endoplasmic reticulum).

It becomes possible to initiate a host of intracellular bioeffects through the use of such ultrashort pulsing. High-intensity, nanosecond, pulsed electric fields (nsPEFs) have been shown to be versatile nonthermal tools capable of producing cellular electroporation (Schoenbach et al. 2004), intracellular calcium release (Vernier et al. 2003; Beebe et al. 2004; Joshi et al. 2007), shrinkage of tumors (Nuccitelli et al. 2006) and cellular apoptosis (Beebe et al. 2003), temporary blockage of action potential propagation in nerves (Joshi et al. 2008), activation of platelets, and release of growth factors for accelerated wound healing (Schoenbach et al. 2007).

\subsection{Modeling Electric Fields in Cells}

In the classical theory of voltage induction across cell membranes by time-varying harmonic fields, both the cytoplasm and the extracellular medium were described as purely conductive (i.e., having nonzero conductivity but zero dielectric permittivity), whereas the membrane was treated as a lossy dielectric (i.e., having both nonzero conductivity and permittivity). This led to the description for voltage induction as a first-order process characterized by the following time constant $\tau_{\mathrm{m}}$ (Neumann et al. 1982):

$$
\tau_{\mathrm{m}}=R C_{\mathrm{m}} /\left[\left\{2 \lambda_{\mathrm{i}} \lambda_{\mathrm{c}} /\left(\lambda_{\mathrm{i}}+2 \lambda_{\mathrm{c}}\right)\right\}+\left\{R \lambda_{\mathrm{m}} / d\right\}\right]
$$

with $\lambda_{\mathrm{i}}, \lambda_{\mathrm{m}}$, and $\lambda_{\mathrm{e}}$ being the conductivities of the cytoplasm, cell membrane, and extracellular medium, respectively; $R$ the spherical cell radius; $d$ the membrane thickness 
$(\sim 5-7 \mathrm{~nm})$; and $C_{\mathrm{m}}$ the membrane capacitance, with $\varepsilon_{\mathrm{m}}$ denoting the dielectric permittivity of the membrane. The transmembrane voltage could then be derived from Equation 2.1 for an external alternating current field. In the frequency domain, the transmembrane voltage $\Delta \Phi$ could be expressed as follows (Grosse and Schwan 1992):

$$
\Delta \Phi=1.5 E R \cos (\theta) /\left[1+i \omega \tau_{\mathrm{m}}\right]
$$

with $E$ being the amplitude of the external electric field, $\theta$ the polar angle measured with respect to the direction of the field, and $\omega$ the angular frequency of the field. In Equation 2.2, $\Delta \Phi$ is complex and its absolute value gives the amplitude of the transmembrane voltage.

Several other methods have been used to calculate the transmembrane potential (TMP) with varying degrees of sophistication. One class of approaches uses the analytic method, which usually assumes that a cell's shape is regular, such as an ellipsoid or a sphere. Hence, strictly speaking, this is only an estimation of the actual TMP induced in real cells due to this geometric approximation. Other methods use the finite-element scheme (FES) with a better geometric cell model to achieve greater accuracy (Fear and Stuchly 1998; Miller and Henriquez 1988; Bartsch, Baumann, and Grebe 1997) The FES has been widely applied to solve Maxwell equations (Jin 1993; Douglas, Santos, and Sheen 2000) because it is stable and good at modeling complex structures. However, it needs special effort to build a nonuniform mesh for a cell with a thin membrane. Because the ratio between the cell diameter $(\sim 5 \mu \mathrm{m})$ and membrane thickness $(\sim 5 \mathrm{~nm})$ is above 1000 , it is not easy to achieve a good three-dimensional (3D) mesh.

The boundary element method (BEM) is suitable for homogeneous media and can model regions with rapidly changing variables with better accuracy than the FES (Becker 1992; Kythe 1995). Meanwhile, meshes are only on the boundaries, that is, a two-dimensional surface boundary for a closed 3D region. Thus, it is easier to obtain meshes for the BEM than for the FES. An FES-BEM coupling method has been introduced and studied (Hsiao 1990; Johnson and Nedelec 1980). The coupling method has been applied in dealing with unbounded EM problems to take advantages of both the FES and BEM.

\subsubsection{Continuum Mean-Field Approaches for Spherical Cells}

Modeling the various cellular effects and biochemical outcomes produced by the application of ultrashort pulsing is very complex and multifaceted. At the purely electrical level, the applied voltage dynamically creates electric fields that pass through the various cellular structures and sets up a TMP across all membranes. This then creates the electrical driver for a range of biophenomena including structural rearrangement of the membrane lipid bilayer (i.e., electroporation (Mir et al. 1995; Teissie et al. 1999; Neumann, Kakorin, and Toensig 1999; Barnett and Weaver 1991)), intercellular ionic flows, changes in chemical composition, modulation of conductivities, and even the serial triggering of biochemical reactions (the apoptotic machinery) that could lead to cell death.

Analysis of the cellular response to electrical pulses requires, as an essential first step, the evaluation of voltage and current distributions within cells and their time-dependent 
evolution. The problem of coupling harmonic electric fields to biological cells and the transmembrane voltage induction was first studied in the early 80s (Schwan 1983). Later contributions (Foster and Schwan 1995 and Foster 2000) extended this study to radiofrequency (RF) field exposures of spherical cells. Essentially, the potential induced by an external field across the membrane of a spherical cell in suspension, $\Delta \Phi$, could be calculated using the following simple analytical expression (Schwan 1983):

$$
\Delta \Phi=1.5 E r \cos (\theta) /\left[1+r\left\{G_{\mathrm{m}}+i \omega C_{\mathrm{m}}\right\}\left(\rho_{\mathrm{i}}+\rho_{\mathrm{a}} / 2\right)\right]
$$

where $r$ is the radius of the cell, $E$ the external electric field value, $\omega$ the angular frequency of the external excitation, $\theta$ the angle at any location on the membrane with respect to the applied field direction, $C_{\mathrm{m}}$ the membrane capacitance per unit area, $G_{\mathrm{m}}$ the membrane conductance per area, and $\rho_{\mathrm{i}}$ and $\rho_{\mathrm{a}}$ the resistivities of cell interior and exterior electrolyte, respectively. Results for the TMP arise in a straightforward manner if one divides the cell into concentric shells to represent the interior cytoplasm, the membrane sheath, the outer extracellular region, etc. The Laplace equation is then solved for each region to obtain the potential distribution and transmembrane voltages. The potential in various regions has the following general form:

$$
\Phi(r, \theta)=-A_{\mathrm{i}} r \cos (\theta)+B_{\mathrm{i}} r^{3} \cos (\theta) / r^{2}
$$

For the outermost region, $A_{\mathrm{i}}=E$ and for the inner most region, $B_{\mathrm{i}}=0$.

Other model analyses for sinusoidal electric excitations have been reported, including the studies by the University of Ljubljana group (Kotnik and Miklavcic 2000b). Some of their more recent investigations (Kotnik and Miklavcic 2006) probed the conditions under which induced voltages at organelle membranes could exceed those established at the cell plasma membrane. It was shown that upon a suitable choice of the cell electrical parameters, potentials could be induced across subcellular membranes that would exceed those across the outer cell membrane. Frequency-domain analyses yielded insights into the dependence of the voltage induction on the electric and geometric parameters. Particularly, they showed that if the organelle interior was electrically more conductive than the cytosol or if the organelle membrane had a lower dielectric permittivity than the cell membrane, then transmembrane voltages across the organelle membranes could exceed those across the outer cell membrane. Time-domain analysis was then used to determine the temporal evolution of TMPs by pulses with rise times and durations in the nanosecond range. For example, the temporal response of the membrane voltages to an applied fast-rising $(1 \mathrm{~ns})$ trapezoidal electric field (Kotnik and Miklavcic 2006) showed that for the first $117 \mathrm{~ns}$ the voltage across a 3- $\mu \mathrm{m}$ diameter spherical organelle would exceed that across the plasma membrane for a $10-\mu \mathrm{m}$ cell.

Although insightful, the primary drawback of analytical approaches is their inability to address the dynamic changes in cell parameters in a self-consistent manner. For example, application of a transmembrane voltage over time leads to increases in the membrane conductance due to localized electroporation. Consequently, the $G_{\mathrm{m}}$ and $C_{\mathrm{m}}$ parameters of Equation 2.3 change, and one can no longer obtain analytical, closed-form solutions. Under such conditions (as are routinely encountered under intense field, short-duration 
pulsing), an alternate approach for modeling is to represent the electrical characteristics of cells by a distributed equivalent circuit, and then perform node analyses to obtain the potentials in the time domain. Distributed circuit solvers such as SPICE are not, however, suited to the present problem for the following reasons: (1) In such circuit solvers, values of the circuit parameters (e.g., resistors, capacitors, etc.) cannot be made time dependent; (2) the stochastic nature of pore formation cannot be included; (3) the geometric dependence of $R$ and $C$ makes it inconvenient to run simulations with dynamic variations in shapes and sizes as pores form; and (4) soft thresholds inherent in the pore formation process are difficult to implement. For example, some degree of poration can start at voltages lower than the $1-\mathrm{V}$ threshold generally assumed but it would then take more time. Also, given biological variability, orientational effects, etc., all pores need not have a rigid threshold value. (5) Finally, it is difficult to use circuit models to simulate the gradual resealing of pores. The resealing process always takes a long time (microseconds or longer), and the long-lived, diffusion-driven currents cannot be modeled by circuit simulators.

A simple lumped circuit representation with inclusion of the dynamic changes in cell parameters has been discussed in the literature (e.g., Vasilkoski et al. 2006). In this model for spherical cells, the transmembrane voltage is governed by the following relations:

$$
\begin{gathered}
\left(C_{\mathrm{m}}+C_{\mathrm{e}}\right) \mathrm{d}(\Delta \Phi) / \mathrm{d} t=C_{\mathrm{e}} \mathrm{d} V_{\text {app }} / \mathrm{d} t+V_{\text {app }} / R_{\mathrm{e}}-\Delta \Phi\left[G_{\mathrm{m}}(n)+1 / R_{\mathrm{e}}\right], \text { for } t \leq t_{\text {pulse }} \\
\left(C_{\mathrm{m}}+C_{\mathrm{e}}\right) \mathrm{d}(\Delta \Phi) / \mathrm{d} t=G_{\mathrm{m}}(n) \mathrm{d}(\Delta \Phi) / \mathrm{d} t, \text { for } t>t_{\text {pulse }}
\end{gathered}
$$

where $C_{\mathrm{m}}$ and $C_{\mathrm{e}}$ denote the capacitance of the membrane and the electrolyte, respectively, and $R_{\mathrm{e}}$ is the resistance of the aqueous medium surrounding the cell. Equation $2.5 \mathrm{~b}$ allows a straightforward calculation of the transmembrane voltage $\Delta \Phi$ if the cell parameters are constant. However, quite generally, the membrane conductance $G_{\mathrm{m}}(n)$ can change nonlinearly as a function of time due to the applied voltage. Consequently, the pore conductance becomes a function of time and is spatially nonuniform as dictated by the pore-size distribution $n(r, \theta, t)$ over the membrane surface. Under such conditions, the biophysical mechanisms of electroporation and evaluations of the pore densities at biological membranes need to be taken into account, and these are described through the Smoluchowski equation (Abidor et al. 1979). Details of this aspect, including pore growth and evolution of the radial distributions are discussed later in this section.

A purely numerical approach was discussed (Joshi, Hu, and Schoenbach 2004) for calculating the time-dependent potentials and current flows throughout the cell. It was based on a time domain nodal analysis involving a dynamic, distributed circuit representation of a cell and its membrane structures. The entire cell can be broken up into segments, and each segment represented by a parallel combination of a resistor $(R)$ and capacitor $(C)$. For computational efficiency, azimuthal symmetry of spherical cells can be used to map the three-dimensional structure into the $r$ and $\Phi$ coordinates of a spherical system. This method is different from the Legendre polynomial models often used for the electrostatic cases (Joshi et al. 2001) because it allows for dynamic flows and also lends itself to the inclusion of membrane poration effects. For such analyses, volume and shape changes of cells can be ignored because the external applied pulse is too short for cells to deform much during the time interval. Application of Kirchhoff's current law at each node then yields a set of $\mathrm{N}$ coupled linear equations in the $\mathrm{N}$ node voltages. 
Data on the dielectric constants and conductivities of cell membranes and cytoplasm, as well as other organelles (e.g., nuclear membrane and nucleoplasm), required for theoretical analyses have been obtained using dielectric spectroscopy of cells (Ermolina et al. 2001; Feldman, Ermolina, and Hayashi 2003). Aspects pertaining to dielectric spectroscopy are briefly in Section 2.6. The availability of cell parameters enables such distributed circuit computations. Typical values for the plasma membrane of mammalian cells (e.g., B- or T-lymphocyte cells) are the relative permittivities on the order of 10 and conductivities of approximately $10^{-5} \mathrm{~S} / \mathrm{m}$. For the cytoplasm, the relative permittivity is approximately that of water (i.e., 80), and the conductivity is typically one-fifth that of seawater (i.e., $\sim 1 \mathrm{~S} / \mathrm{m}$ ).

As already mentioned, the discretized membrane conductances in Figure 2.2 are dynamic and can change nonlinearly with voltage based on possible electroporation effects. This aspect can be incorporated into the simulations by using the Smoluchowski equation to account for pore formation, growth, and dynamics (Joshi et al. 2001; Joshi et al. 2004; Joshi et al. 2002). Details of the formation energy and pore dynamics have been discussed at length in the literature (Weaver and Minter 1981; Weaver and Chizmadzhev 1996; Glaser et al. 1988; Pastushenko and Chizmadzhev 1983; Winterhalter and Helfrich 1987; Neu and Krassowska 2006). Hence, only a brief discussion of this biophysical process is presented in this chapter. The basic underlying concept of using a diffusive motion across an energy landscape was originally developed in 1916 (Smoluchowski 1916). The evolution of the pore density follows a diffusive

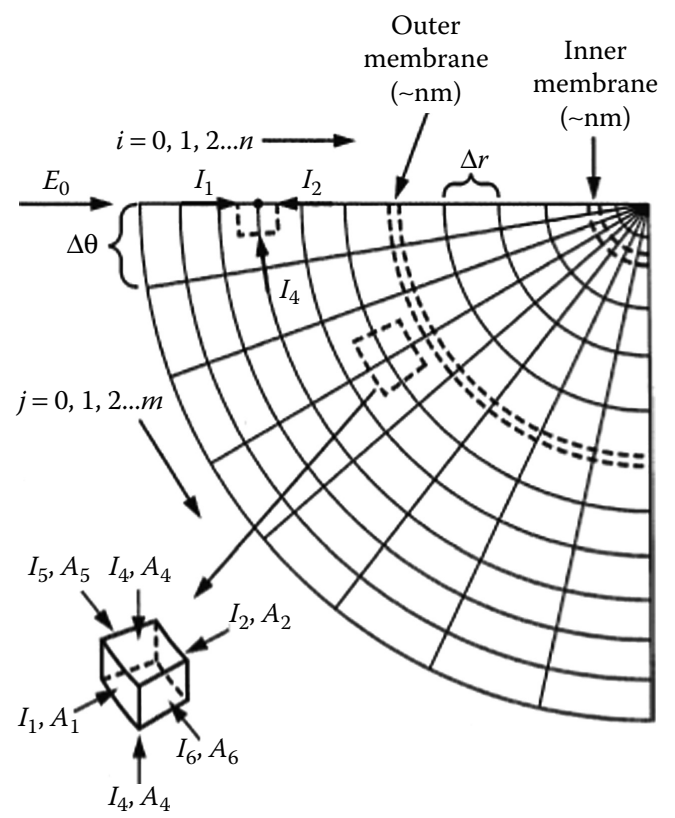

FIGURE 2.2 Schematic of one quarter of the model used to represent a cell for distributed electrical calculations. The dotted box shows a typical element with current flows. (After Joshi, R. P., Q. Hu, K. H. Schoenbach, and S. J. Beebe. 2004. Phys Rev E 69:051901.) 
Brownian motion across the energy landscape. Continuum Smoluchowski theory yields the pore density distribution function $n(r, t)$, with $r$ being the pore radius (Pastushenko and Chizmadzhev 1983; Joshi and Schienbach 2000; Freeman, Wang, and Weaver 1994):

$$
\partial n(r, \theta, t) / \partial t-\{D /(k T)\} \partial[n(r, \theta, t)\{\partial E(r, t) / \partial r\}] / \partial r-D \partial^{2} n(r, \theta, t) / \partial r^{2}=S(r, t)
$$

where $S(r, t)$ is the source (i.e., pore formation) term, $D$ is a pore diffusion constant, $k$ the Boltzmann constant, $T$ the absolute temperature, and $E(r, t)$ the pore formation energy. In Equation (2.6), $n(r, t)$ denotes the pore density, with $n(r, t) \mathrm{d} r$ representing the pore number of membrane pores lying within a radial spacing of $r$ and $r+\mathrm{d} r$ at time $t$. The pore energy $E(r, t)$ is a sum of mechanical and electrical contributions. The mechanical contribution, $E_{\mathrm{m}}(r)$, includes the edge energy per unit length $\gamma$ and the surface tension $\mathrm{T}$ of the membrane-water interface, while the electrical contribution results from the radial displacement of the lipid cylindrical walls of the pore by the aqueous electrolyte. Physically, the radial displacement of the pore walls is facilitated by the electric field-driven Maxwell stress tensor created at the membrane-electrolyte interface. Thus, traditionally, $E(r, t)=$ $2 \pi \gamma-\pi r^{2} \mathrm{~T}-\pi\{\Delta \Phi(\theta, t)\}^{2} r^{2}$. More sophisticated expressions for this pore energy $E(r)$ have also been discussed in the literature (Vasilkoski et al. 2006; Joshi et al. 2002).

The development of membrane pores is a time- and position-dependent sequence of events. Pore formation and dynamics influence both the membrane conductivity and permittivity and both can be taken into account at each time step. Solution for $n(r, \theta, t)$ from Equation 2.6 then yields the dynamic conductance and capacitance of the membrane as a function of time and angular position.

Results for the temporal development of membrane voltage across the outer and inner cell membranes obtained using the distributed approach for a double-shelled model (Joshi et al. 2004) are shown in Figure 2.3a and b. Two trapezoidal pulses, one having a 300 -ns duration and the other having an 11-ns pulse, were chosen. For the 300-ns pulse, membrane voltage across the inner organelle membrane exceeds that of the outer one during the first 75 ns. For the 11-ns pulse, on the other hand, the membrane voltage across the inner organelle exceeds that across the outer membrane for the entire pulse duration. This clearly shows that by reducing the pulse width, it becomes possible to charge inner membranes to higher potentials and thus trigger intracellular effects. Most conventional electroporation pulses have fairly long durations (typically in the microsecond to millisecond range) and do not afford strong internal charging. Furthermore, the use of extremely high electric field magnitudes is crucial in the present context because only such strong excitation can drive bioeffects despite the short duration of the external forcing function.

Traditionally, the neglect of the intracellular regions for potential bioelectric phenomena can be attributed to the notion that intracellular organelles are generally shielded from external electric fields by the outer membranes (Adair 1991; Foster and Schwan 1986). This is certainly true for low-frequency voltage excitations or for timescales exceeding the cell membrane charging times that are roughly in the 1-ms range (Krassowska and Neu 1994; Beeler and Reuter 1977). However, results of electric field penetration with high-frequency pulsing (similar conceptually to the results of Figure $2.3 \mathrm{~b}$ ) have also been reported by other groups using a continuum model. As an 


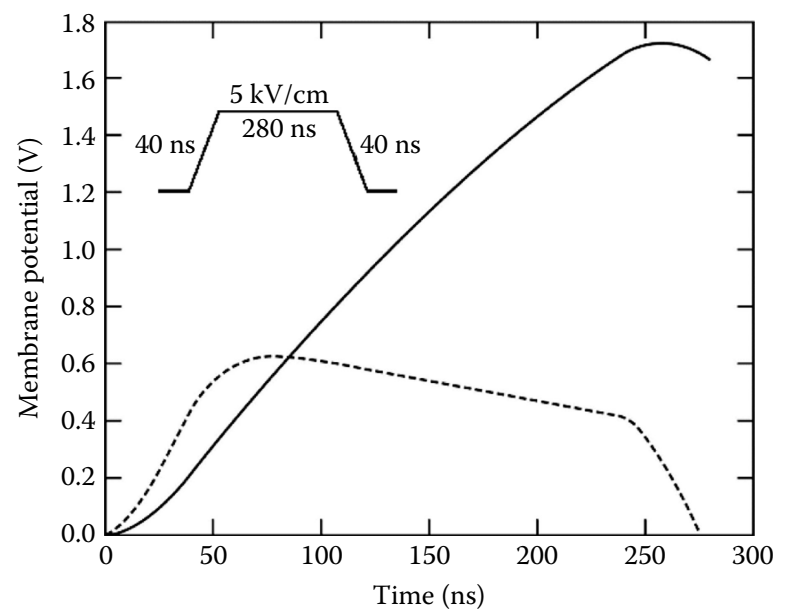

(a)

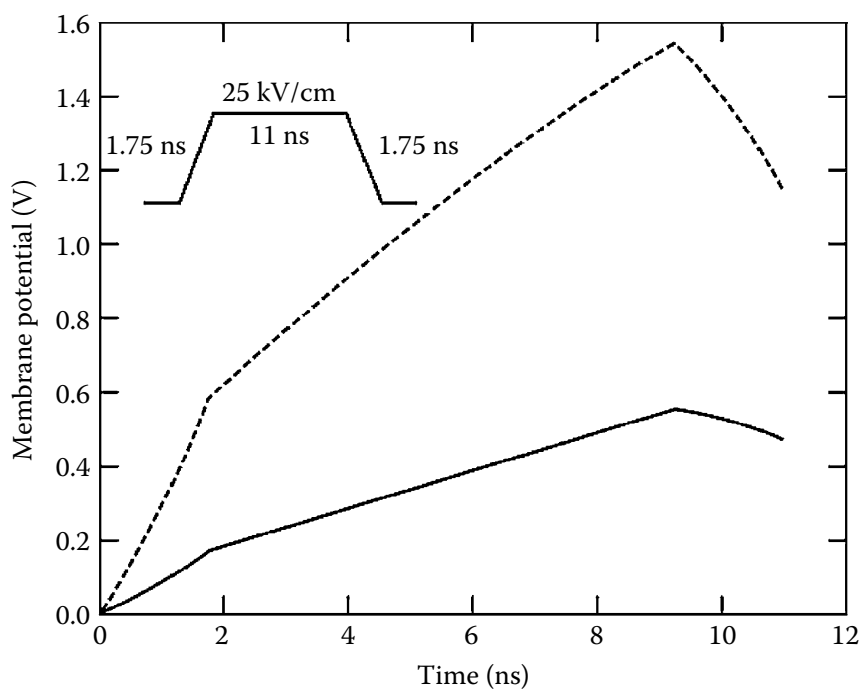

(b)

FIGURE 2.3 (a) Membrane voltage across plasma membrane (solid line) and inner (mitochondrial) membrane (dotted line) for a 300-ns trapezoidal pulse. (b) The same for an 11-ns long pulse. The thickness of the inner membrane was assumed to be half of that of the plasma membrane. (From Joshi, R. P., Q. Hu, K. H. Schoenbach, and S. J. Beebe. 2004. Phys Rev E 69:051901. With permission.)

example, transmembrane voltages (Kotnik and Miclavcic 2006) are shown in Figure 2.4. Here, the conductivity of the organelle interior was assumed to be higher than that of the cytosol and the membrane permittivity lower than that of the plasma membrane. A similar result also emerged if the organelle membrane had lower dielectric permittivities than the cellular membrane. 


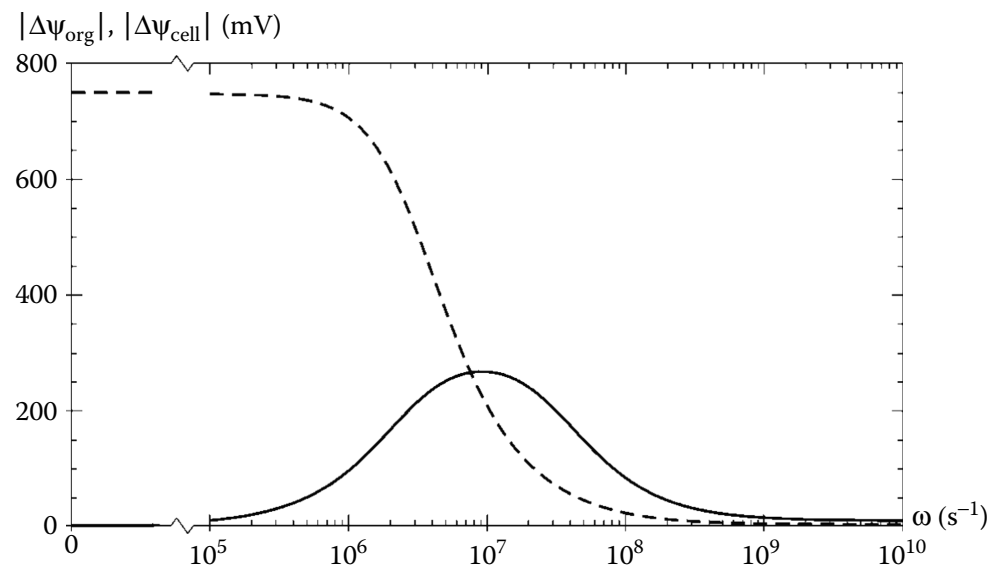

FIGURE 2.4 The frequency dependence of the voltages induced across the cell membrane (dashed line) and an organelle membrane (solid line) in an alternating field for a cell where the conductivity of the organelle interior was increased and the capacitance of the organelle membrane was decreased with respect to their default values. (After Kotnik, T., and D. Miclavcic. 2006. Biophys J 90:480-91.)
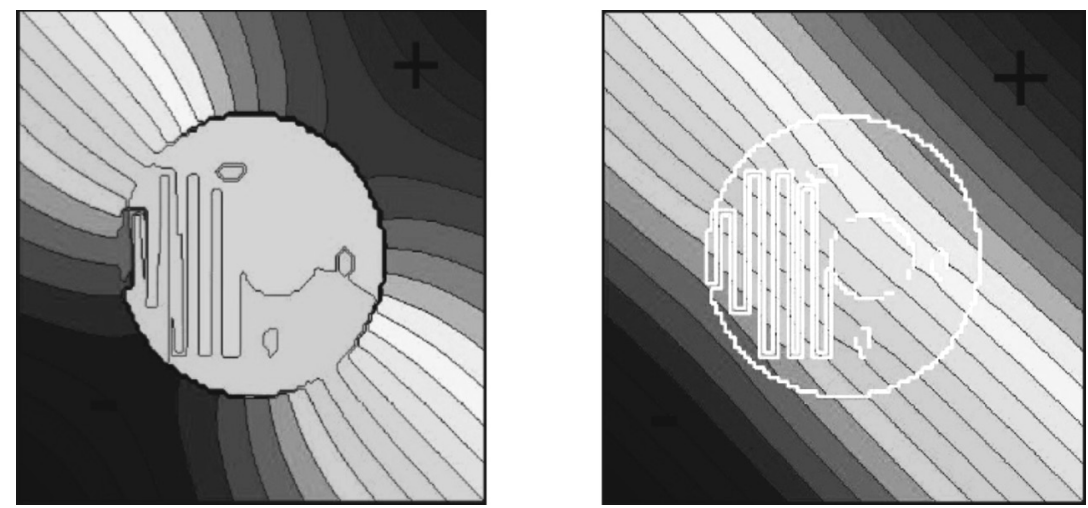

FIGURE 2.5 The effect of $7-\mu$ s long pulses with $1.1 \mathrm{kV} / \mathrm{cm}$ field amplitude (left) and that with $60 \mathrm{~ns}$ at $60 \mathrm{kV} / \mathrm{cm}$ amplitude (right) on cells. The electrical parameters were chosen such that the electrical energy for both cases is identical. (From Gowrishankar, T. R., A. T. Esser, Z. Vasilkoski, K. C. Smith, and J. C. Weaver. 2006. Biochem Biophys Res Comm 341:1266-76. With permission.)

With the onset of poration, the membrane can no longer be regarded as a linear, passive circuit element but instead becomes "active" with variable resistivity and variable permeability. Modeling of cells with active membranes has also been the topic of publications by the team headed by James Weaver at MIT. Weaver's group has focused on a lattice model (Smith et al. 2006; Stewart, Gowrishankar, and Weaver 2006; Weaver 2003). The Smoluchowski equation has been applied for the voltage-dependent description of the nonlinear membrane resistance and pore development. Figure 2.5 shows the results of such a continuum model (Gowrishankar et al. 2006). Here, the poration of 
the plasma membrane and subcellular membranes was compared for pulses of $7 \mu$ s and 60 -ns duration. The electric field of the pulses was adjusted such that the energy density in both cases was identical. For 60-ns long pulses (left), the cell membranes, plasma and internal, are fully exposed to the applied $60 \mathrm{kV} / \mathrm{cm}$ pulse, clearly demonstrating that nanosecond pulses allow us to affect subcellular membrane potentials. When the cell was exposed to a long pulse ( $7-\mu$ s duration) at $1.1 \mathrm{kV} / \mathrm{cm}$, only effects on the plasma membrane were seen. Thus, the cell interior is shown to be shielded for longer pulsing.

Long pulses cause the creation of membrane pores large enough for large molecules to pass, whereas the application of short, nanosecond pulses was found to cause the creation of a high-density "nanopores" with radii in the range 0.8-1.5 $\mathrm{nm}$ (Schoenbach et al. 2007; Vasilkoski et al. 2006; Joshi, Song, and Sridhara 2009). This effect has been termed supraporation. As an example, we focus on membrane electroporation in response to a trapezoidal $560 \mathrm{kV} / \mathrm{cm}$ pulse with an $\mathrm{ON}$ time of $1 \mathrm{~ns}$ and rise and fall times of $0.1 \mathrm{~ns}$, in keeping with typical experimental waveforms generated in our laboratory. The pore distribution $n(r)$ per unit area was evaluated as a function of radius $r$ using the Smoluchowski equation.

Figure 2.6 illustrates some of the characteristics associated with the intense, nanosecond pulsing. Pore distributions as a result of the electroporation process with and without a constant surface tension are shown. Conventionally, a constant tension $\Gamma_{0}$ of $10^{-3} \mathrm{~J} / \mathrm{m}^{2}$ has been used, while a heuristic model (Isambert 1998) described the tension

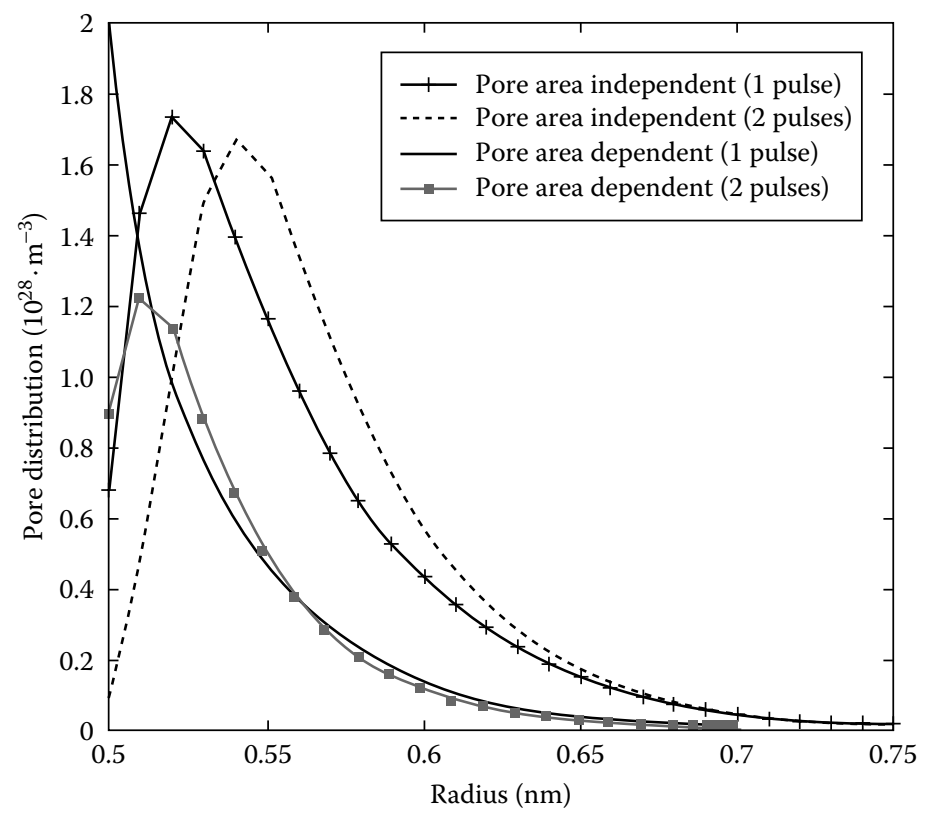

FIGURE 2.6 Simulation results of the pore distribution function $n(r)$ for one and two pulses using constant and pore area-dependent tension. A $560 \mathrm{kV} / \mathrm{cm}$ average external field was used. (After Joshi, R. P., J. Song, and V. Sridhara. 2009. IEEE Trans Dielectr and Electr Insula 16:1243-50.) 
$\Gamma(r)$ as $\Gamma(r)=\Gamma_{0}\left[1-r^{2} / r_{\infty}{ }^{2}\right]$, with $r_{\infty}$ being a constant parameter. The varying tension is perhaps more physical as it accounts for changes in the mechanical properties of cells due to structural rearrangements and poration. The curves of Figure 2.6 reveal a distinct shift toward larger pore radii for the fixed tension $\Gamma_{0}$. For the two-pulse cases, an "idle time" of $0.6 \mathrm{~ns}$ was used to separate the second voltage pulse from the first. The most probable pore radius with the dynamic tension is predicted to be less than $0.55 \mathrm{~nm}$, and thus essentially small nanopores are predicted. This result indirectly agrees with several experimental observations of negligible propidium iodide (PI) dye uptake following nanosecond pulsing (Chen et al. 2004; Pakhomov et al. 2007; Kennedy et al. 2008). A possible explanation is that the pore population formed by the ultrashort pulsing has diameters smaller than the dye dimensions, and hence disallow dye uptake by the cells. The predictions of Figure 2.6 also support the hypothesis that the pore diameters upon ultrashort pulsing are small, so that the membrane is mainly permeable only to small ions (or even nanoparticles), but not large molecules.

\subsubsection{Continuum Approaches Applied to More Complicated Cell Geometries}

Most analyses and calculations of the TMP resulting from external pulsing have assumed spherical cells. In biology, examples of such spherical shapes are vesicles, protoplasts, murine myeloma cells (Gimsa and Wachner 1999), and some bacteria such as Streptococcus (Batzing 2002). However, most other cells are nonspherical in nature. As it turns out though, many cells deviating from the spherical shape do exhibit rotational symmetry and can be represented either as prolate or oblate spheroids fairly accurately. For example, mammalian red blood cells are close to an oblate spheroidal shape, while retina photoreceptor cells (Radu et al. 2005), many bacteria such as Escherichia coli and Pseudomonas (Gimsa and Wachner 1999), and yeasts (Asencor et al. 1993) roughly have a prolate spheroidal geometry. This makes a compelling case, at least from the practical standpoint, to examine bioelectric pulsing effects in such spheroidal cell shapes for more realistic analyses and predictions. There have only been a few reports in the literature on analyses for irregularly shaped cells (e.g., Pucihar et al. 2006) using finite-element approaches to solve for the TMP, though electroporation was not explicitly considered. Their method allows for arbitrarily shaped cellular geometries. A few other studies for spheroidal cells (Kotnik and Miklavcic 2000a; Maswiwat et al. 2007) did not consider electroporation in a self-consistent manner.

An evaluation of the TMP and possible membrane electroporation in spheroidal cells arising from an ultrashort, high-intensity pulse has been reported (Hu and Joshi 2009). That study coupled the Laplace equation with the Smoluchowski theory of pore formation and used double-shell models. Though the treatment of pores using the Smoluchowski theory remains the same as for spherical cells, calculations for the potential are slightly different.

The schematic shown in Figure 2.7 represents a double-shelled prolate spheroidal cell suspended in a medium. Because the spheroids have rotational symmetry in the $x-y$ plane, the arbitrarily oriented, externally applied electric field $E_{0}(t)$ can be assumed 


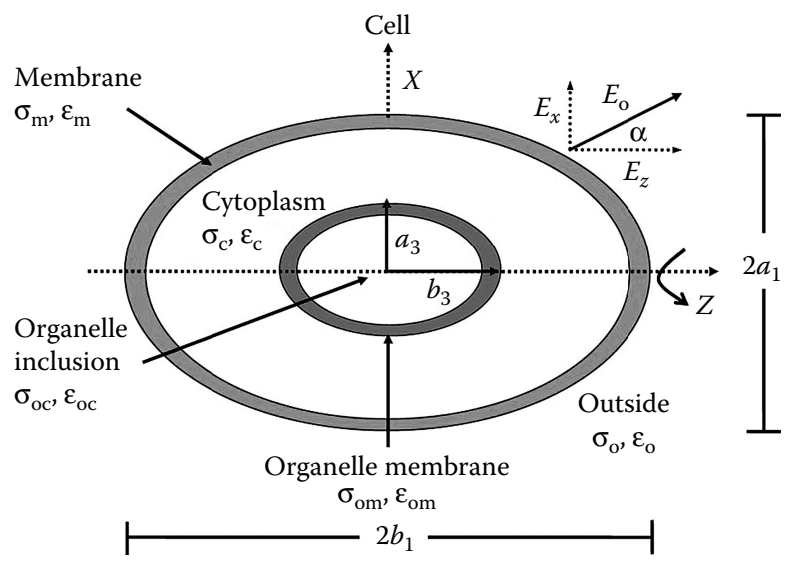

FIGURE 2.7 Schematic of the spheroidal cell model for the transmembrane potential calculations under an arbitrary external electric field orientation. (After Hu, Q., and R. P. Joshi. 2009. IEEE Trans Biomedical Eng 56:1617-26.)

to be in the $x$ - $z$ plane. In Figure 2.7, $\alpha$ is the angle between $E_{0}(t)$ and the rotational axis $z$. The cell shown is characterized by an outer shell (plasma membrane) and an inner shell (nuclear envelope). Each shell is represented by two spheroids and assumes that the four spheroids share the same foci. The outer shell has an outer equatorial radius $a_{1}$, an inner equatorial radius $a_{2}$, an outer polar radius $b_{1}$, and an inner polar radius $b_{2}$. The focal distance $c$ can then be found as $c=\sqrt{b_{1}^{2}-a_{1}^{2}}=\sqrt{b_{2}^{2}-a_{2}^{2}}$ for prolate $\left(b_{i}>a_{i}\right)$ spheroids of the outer shell. Thickness at the polar points is $h_{\mathrm{o}}=b_{1}-b_{2}$. The inner shell has an outer equatorial radius $a_{3}$, an inner equatorial radius $a_{4}$, an outer polar radius $b_{3}$, and an inner polar radius $b_{4}$. Thickness at the polar points is $h_{\mathrm{i}}=b_{3}-b_{4}$. The outer region has an assigned conductivity $\alpha_{o}$ and permittivity $\varepsilon_{0}$, while the corresponding parameters for the cell membrane are $\alpha_{\mathrm{m}}$ and $\varepsilon_{\mathrm{m}}$. In Figure $2.7, \sigma_{\mathrm{c}}$ and $\varepsilon_{\mathrm{c}}$ are the conductivity and permittivity of the cytoplasm; $\sigma_{\mathrm{om}}$ and $\varepsilon_{\mathrm{om}}$ are the conductivity and permittivity of the organelle membrane; and $\sigma_{\mathrm{oc}}$ and $\varepsilon_{\mathrm{oc}}$ are the conductivity and permittivity of the organelle cytoplasm.

Because the external electrical field $E_{0}(t)$ can be decomposed into its $E_{x}(t)$ and $E_{z}(t)$ components, the overall TMP $\Delta \Phi$ can be obtained by summing the separate contributions from $E_{x}(t)$ and $E_{z}(t)$. The Laplace equation for prolate spheroids is as follows:

$$
\begin{aligned}
\nabla^{2} \Phi= & \frac{1}{c^{2}\left(u^{2}-v^{2}\right)}\left\{\frac{\partial}{\partial u}\left[\left(u^{2}-1\right) \frac{\partial \Phi}{\partial u}\right]+\frac{\partial}{\partial v}\left[\left(1-v^{2}\right) \frac{\partial \Phi}{\partial v}\right]\right\} \\
& +\frac{1}{c^{2}\left(u^{2}-1\right)\left(1-v^{2}\right)} \frac{\partial^{2} \Phi}{\partial \varphi^{2}}=0
\end{aligned}
$$

Here, the usual prolate spheroidal coordinates $(u, v, \Phi)$ have been used. In Equation $2.7, u=\cosh (\xi), v=\cos (\theta)$ with $\xi>0$ and the polar angle is $\theta \in[0, \pi]$. The azimuthal angle is $\varphi \in[0,2 \pi]$. Because the prolate spheroid is symmetrical with regards to the azimuthal 
angle $\varphi$, the last term in Equation 2.7 can be omitted when only $E_{z}$ is present in the system. Hence, one gets the following:

$$
\nabla^{2} \Phi=\frac{\partial}{\partial u}\left[\left(u^{2}-1\right) \frac{\partial \Phi}{\partial u}\right]+\frac{\partial}{\partial v}\left[\left(1-v^{2}\right) \frac{\partial \Phi}{\partial v}\right]=0
$$

By separation of variables, Equation 2.8 can be easily solved to yield the following:

$$
\begin{gathered}
\phi_{\mathrm{oc}}^{z}(u, v, t)=A_{\mathrm{oc}}(t) u v, \quad u \leq u_{4} \\
\phi_{\mathrm{om}}^{z}(u, v, t)=v\left[A_{\mathrm{om}}(t) u+B_{\mathrm{om}}(t)\left(\frac{u}{2} \ln \frac{u+1}{u-1}-1\right)\right], \quad u_{4}<u<u_{3} \\
\phi_{\mathrm{c}}^{z}(u, v, t)=v\left[A_{\mathrm{c}}(t) u+B_{\mathrm{c}}(t)\left(\frac{u}{2} \ln \frac{u+1}{u-1}-1\right)\right], \quad u_{3}<u<u_{2} \\
\phi_{\mathrm{m}}^{z}(u, v, t)=v\left[A_{\mathrm{m}}(t) u+B_{\mathrm{m}}(t)\left(\frac{u}{2} \ln \frac{u+1}{u-1}-1\right)\right], \quad u_{2}<u<u_{1} \\
\phi_{\mathrm{o}}^{z}(u, v, t)=v\left[-c_{\mathrm{o}} E_{z}(t) u+B_{\mathrm{o}}(t)\left(\frac{u}{2} \ln \frac{u+1}{u-1}-1\right)\right], \quad u>u_{1}
\end{gathered}
$$

Here $\phi_{\mathrm{oc}}^{z}(u, v, t), \phi_{\mathrm{om}}^{z}(u, v, t), \phi_{\mathrm{c}}^{z}(u, v, t), \phi_{\mathrm{m}}^{z}(u, v, t)$, and $\phi_{\mathrm{o}}^{z}(u, v, t)$ are the potentials induced by $E_{z}$ at the organelle cytoplasm, the organelle membrane, the cellular cytoplasm, the plasma membrane, and the environment, respectively. Also, $E_{z}(t)=E_{0}(t) \cos (\alpha)$ and $u_{i}=b_{i} / c, i=1,2,3,4$.

Finally, $A_{\mathrm{oc}}(t), A_{\mathrm{om}}(t), A_{\mathrm{c}}(t), A_{\mathrm{m}}(t), B_{\mathrm{om}}(t), B_{\mathrm{c}}(t), B_{\mathrm{m}}(t)$, and $B_{\mathrm{o}}(t)$ are the coefficients that can be determined by applying matching boundary conditions at the interfaces of the five regions. The TMP $\Delta \phi^{z}$ induced by an electric field $E_{z}$ can be obtained as follows:

$$
\begin{gathered}
\Delta \phi_{\mathrm{m}}^{z}=\phi_{\mathrm{m}}^{z}\left(u_{2}, v, \varphi, t\right)-\phi_{\mathrm{m}}^{z}\left(u_{1}, v, \varphi, t\right) \\
\Delta \phi_{\mathrm{om}}^{z}=\phi_{\mathrm{om}}^{z}\left(u_{4}, v, \varphi, t\right)-\phi_{\mathrm{om}}^{z}\left(u_{3}, v, \varphi, t\right)
\end{gathered}
$$

In order to obtain the TMP induced by an electric field $E_{x}$ alone, one can express $\Phi(u, v, \varphi)=U(u) V(v) F(\varphi)$. Algebraic manipulation then leads to the following final solution:

$$
\begin{gathered}
\phi_{\mathrm{oc}}^{x}(u, v, \varphi, t)=S_{\mathrm{oc}}(t) \sqrt{u^{2}-1} \sqrt{1-v^{2}} \cos \varphi, \quad u \leq u_{4} \\
\phi_{\mathrm{om}}^{x}(u, v, \varphi, t)=\left[S_{\mathrm{om}}(t)+T_{\mathrm{om}}(t)\left(\frac{1}{2} \ln \frac{u+1}{u-1}-\frac{u}{u^{2}-1}\right)\right] \times \sqrt{u^{2}-1} \sqrt{1-v^{2}} \cos \varphi, \quad u_{4}<u \leq u_{3} \\
\phi_{\mathrm{c}}^{x}(u, v, \varphi, t)=\left[S_{\mathrm{c}}(t)+T_{\mathrm{c}}(t)\left(\frac{1}{2} \ln \frac{u+1}{u-1}-\frac{u}{u^{2}-1}\right)\right] \times \sqrt{u^{2}-1} \sqrt{1-v^{2}} \cos \varphi, \quad u_{3}<u \leq u_{2}
\end{gathered}
$$


$\phi_{\mathrm{m}}^{x}(u, v, \varphi, t)=\left[S_{\mathrm{m}}(t)+T_{\mathrm{m}}(t)\left(\frac{1}{2} \ln \frac{u+1}{u-1}-\frac{u}{u^{2}-1}\right)\right] \times \sqrt{u^{2}-1} \sqrt{1-v^{2}} \cos \varphi, \quad u_{2}<u \leq u_{1}$

$\phi_{\mathrm{o}}^{x}(u, v, \varphi, t)=\left[-c_{\mathrm{o}} E_{x}(t)+T_{\mathrm{o}}(t)\left(\frac{1}{2} \ln \frac{u+1}{u-1}-\frac{u}{u^{2}-1}\right)\right] \times \sqrt{u^{2}-1} \sqrt{1-v^{2}} \cos \varphi, \quad u>u_{1}$

where $\phi_{\mathrm{oc}}^{x}(u, v, t), \phi_{\mathrm{om}}^{x}(u, v, t), \phi_{\mathrm{c}}^{x}(u, v, t), \phi_{\mathrm{m}}^{x}(u, v, t)$, and $\phi_{\mathrm{o}}^{x}(u, v, t)$ are the potentials induced by $E_{x}(t)$ at organelle cytoplasm, organelle membrane, cellular cytoplasm, the plasma membrane, and outer region, respectively. Here $E_{x}(t)=E_{0}(t) \sin (\alpha)$, while $S_{\mathrm{oc}}(t)$, $S_{\mathrm{om}}(t), S_{\mathrm{c}}(t), S_{\mathrm{m}}(t), T_{\mathrm{om}}(t), T_{\mathrm{c}}(t), T_{\mathrm{m}}(t)$, and $T_{\mathrm{o}}(t)$ are the time-varying coefficients that can be determined by applying matching boundary conditions at the interfaces of the five regions. Invoking continuity in potential and current density at the membrane interfaces leads to the following boundary conditions:

$$
\begin{gathered}
\left.\phi_{\mathrm{o}}(u, v, \varphi, t)\right|_{u_{1}}=\left.\phi_{\mathrm{m}}(u, v, \varphi, t)\right|_{u_{1}} \\
\left.\phi_{\mathrm{m}}(u, v, \varphi, t)\right|_{u_{2}}=\left.\phi_{\mathrm{c}}(u, v, \varphi, t)\right|_{u_{2}} \\
\left.\phi_{\mathrm{c}}(u, v, \varphi, t)\right|_{u_{3}}=\left.\phi_{\mathrm{om}}(u, v, \varphi, t)\right|_{u_{3}} \\
\left.\phi_{\mathrm{om}}(u, v, \varphi, t)\right|_{u_{4}}=\left.\phi_{\mathrm{oc}}(u, v, \varphi, t)\right|_{u_{4}} \\
\sigma_{\mathrm{o}} E_{u}^{\mathrm{o}}(t)+\left.\varepsilon_{\mathrm{o}} \frac{\partial E_{u}^{\mathrm{o}}(t)}{\partial t}\right|_{u_{1}}=\sigma_{\mathrm{m}}(t) E_{u}^{\mathrm{m}}(t)+\left.\varepsilon_{\mathrm{m}}(t) \frac{\partial E_{u}^{\mathrm{m}}(t)}{\partial t}\right|_{u_{1}} \\
\sigma_{\mathrm{m}}(t) E_{u}^{\mathrm{m}}(t)+\left.\varepsilon_{\mathrm{m}}(t) \frac{\partial E_{u}^{\mathrm{m}}(t)}{\partial t}\right|_{u_{2}}=\sigma_{\mathrm{c}} E_{u}^{\mathrm{c}}(t)+\left.\varepsilon_{\mathrm{c}} \frac{\partial E_{u}^{\mathrm{c}}(t)}{\partial t}\right|_{u_{2}} \\
\sigma_{\mathrm{c}} E_{u}^{\mathrm{c}}(t)+\left.\varepsilon_{\mathrm{c}} \frac{\partial E_{u}^{\mathrm{c}}(t)}{\partial t}\right|_{u_{3}}=\sigma_{\mathrm{om}}(t) E_{u}^{\mathrm{om}}(t)+\left.\varepsilon_{\mathrm{om}}(t) \frac{\partial E_{u}^{\mathrm{om}}(t)}{\partial t}\right|_{u_{3}} \\
\sigma_{\mathrm{om}}(t) E_{u}^{\mathrm{om}}(t)+\left.\varepsilon_{\mathrm{om}}(t) \frac{\partial E_{u}^{\mathrm{om}}(t)}{\partial t}\right|_{u_{4}}=\sigma_{\mathrm{oc}} E_{u}^{\mathrm{oc}}(t)+\left.\varepsilon_{\mathrm{oc}} \frac{\partial E_{u}^{\mathrm{oc}}(t)}{\partial t}\right|_{u_{4}}
\end{gathered}
$$

Here $E_{u}=-\partial \phi / \partial u$ is the electric field along the $u$ direction.

The TMP, $\Phi$, for an oblate spheroidal cell can similarly be obtained by solving the Laplace equation in oblate spheroid coordinates with respect to $E_{x}(t)$ and $E_{z}(t)$. This leads to the following:

$$
\phi_{\mathrm{oc}}^{x}(u, v, \varphi, t)=I_{\mathrm{oc}}(t) \sqrt{u^{2}+1} \sqrt{1-v^{2}} \cos \varphi, \quad u \leq u_{4}
$$




$$
\begin{aligned}
& \phi_{\mathrm{om}}^{x}(u, v, \varphi, t)=\left[I_{\mathrm{om}}(t)+J_{\mathrm{om}}(t)\left(\cot ^{-1}(u)-\frac{u}{u^{2}+1}\right)\right] \times \sqrt{u^{2}+1} \sqrt{1-v^{2}} \cos \varphi, \quad u_{4}<u \leq u_{3} \\
& \phi_{\mathrm{c}}^{x}(u, v, \varphi, t)=\left[I_{\mathrm{c}}(t)+J_{\mathrm{c}}(t)\left(\cot ^{-1}(u)-\frac{u}{u^{2}+1}\right)\right] \times \sqrt{u^{2}+1} \sqrt{1-v^{2}} \cos \varphi, \quad u_{3}<u \leq u_{2} \quad \\
& \phi_{\mathrm{m}}^{x}(u, v, \varphi, t)=\left[I_{\mathrm{m}}(t)+J_{\mathrm{m}}(t)\left(\cot ^{-1}(u)-\frac{u}{u^{2}+1}\right)\right] \times \sqrt{u^{2}+1} \sqrt{1-v^{2}} \cos \varphi, \quad u_{2}<u \leq u_{1} \\
& \phi_{\mathrm{o}}^{x}(u, v, \varphi, t)=\left[-c E_{x}+J_{\mathrm{o}}(t)\left(\cot ^{-1}(u)-\frac{u}{u^{2}+1}\right)\right] \times \sqrt{u^{2}+1} \sqrt{1-v^{2}} \cos \varphi, \quad u>u_{1}
\end{aligned}
$$

Here, $I_{\mathrm{oc}}(t), I_{\mathrm{om}}(t), I_{\mathrm{c}}(t), I_{\mathrm{m}}(t), J_{\mathrm{om}}(t), J_{\mathrm{c}}(t), J_{\mathrm{m}}(t)$, and $J_{\mathrm{o}}(t)$ are the coefficients that can be solved by the Equation (2.12).

Using the analyses described earlier, it was shown that the response of prolate spheroids is faster than that of the sphere, with the outer membrane reaching its steady-state value in about $2 \mu \mathrm{s}$. The simulation result also shows that the TMP across an inner organelle could exceed the value across the plasma membrane at least over the first $0.4 \mu \mathrm{s}$ or so, indicating a possibility of intracellular electromanipulation. The TMP induced by pulsed external voltages is predicted to be higher in oblate spheroids in comparison to both spherical and prolate spheroidal cells and to occur sooner. This results from the flattening of the surface area in this oblate spheroidal geometry. The result suggests that field-assisted drug delivery and molecular/ionic uptake for chemotherapy could work best for flatter-shaped cells. Hence, external fields could conceivably be applied, in addition to an electroporation pulse, to first orient the cells for optimal uptake.

A representative result, in this context, is shown in Figure 2.8 for a double-shelled prolate spheroidal cell (Hu and Joshi 2009). The outer shell was taken to have an equatorial radius $a_{1}$, an inner equatorial radius $a_{2}$, an outer polar radius $b_{1}$, an inner polar radius $b_{2}$, and focal distance $c=\left(b_{1}^{2}-a_{1}^{2}\right)^{1 / 2}=\left(b_{2}^{2}-a_{2}^{2}\right)^{1 / 2}$. Similarly, the inner shell had an equatorial radius $a_{3}$, an inner equatorial radius $a_{4}$, an outer polar radius $b_{3}$, and an inner polar radius $b_{4}$. Using $a_{1}: b_{1}=3: 5$ with $b_{1}=5 \mu \mathrm{m}$ and $b_{3}=4 \mu \mathrm{m}$, predictions of the TMP versus time are shown in Figure 2.8 at polar angle $\theta=15^{\circ}$ for both fixed membrane conductivity (i.e., without considering electroporation) and variable membrane conductivity. The electrical excitation pulse was trapezoidal with rise, fall, and $\mathrm{ON}$ times of $1.5,1.5$, and $10 \mathrm{~ns}$, respectively, with a maximum intensity of $100 \mathrm{kV} / \mathrm{cm}$. With variable membrane conductivity, the TMP goes to zero quickly because the membrane acts as a "leaky capacitor." On the other hand, the TMP for membrane with constant conductivity takes a much longer time to fall back to zero because of the larger time constant.

Numerical microdosimetry techniques based on the finite-element method (FEM) have been utilized to evaluate the electric field and potential distributions on the realistic-shaped cells (Wang, Alfadhl, and Chen 2008). It was found that the shape and orientation of cells can strongly affect the field strength and distribution. 


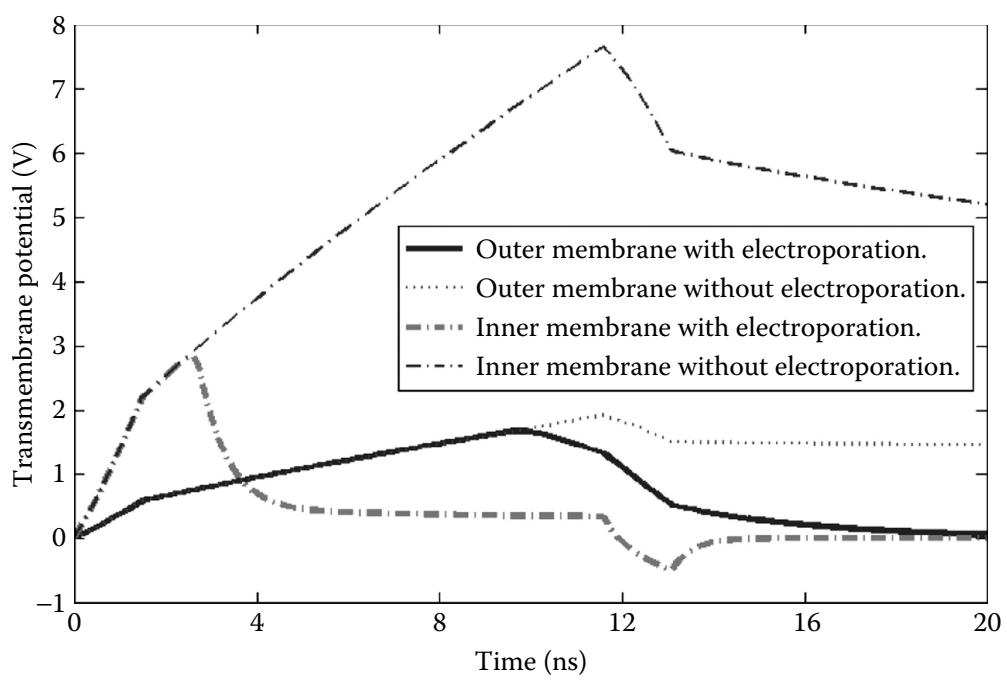

FIGURE 2.8 Simulation results for a double-shelled prolate spheroidal cell $\left(a_{1}: b_{1}=3: 5\right)$ with $b_{1}=5 \mu \mathrm{m}$ and $b_{3}=4 \mu \mathrm{m}$. A $100 \mathrm{kV} / \mathrm{cm}$, trapezoidal external pulse with rise, fall, and ON times of 1.5, 1.5, and $10 \mathrm{~ns}$, respectively, was used. (After Hu, Q., and R. P. Johsi. 2009. IEEE Trans Biomedical Eng 56:1617-26.)

\subsubsection{Models of Electric Field Interactions in Tissues and Nerves}

Some work has been reported in quantifying electric fields in tissues, particularly the skin (Weaver 2000; Weaver, Vaughan, and Chizmadzhev 1999). Human skin provides a formidable barrier against dessication, mechanical injury, and the entry of infectious microorganisms and toxic chemicals. The stratum corneum (SC) is the skin's outermost $\sim 20$ - $\mu \mathrm{m}$-thick layer and responsible for the barrier function (Elias and Menon 1991). It provides an effective "brick wall" (Michaels, Chasekaran, and Shaw 1975) with multilamellar lipid bilayer ("mortar") surrounding keratin-filled corneocytes ("bricks") that present a large barrier to ionic and molecular transport. A simplified brick wall model was used to probe skin site electroporation (Weaver 2000) and the creation of microconduits. For example, the dark, solid lines in Figure 2.9 represent five to six lipid bilayer membranes between corneocytes, with aqueous pathways (light regions in center and right parts) created by electrical pulses. The hatched regions indicate keratin matrix within corneocytes. A single, local transport region is depicted, showing only one-third of the 15-16 corneocyte layers of the SC. Experiments (Ilic et al. 1999) using an electrically insulating mask with a $200-\mu \mathrm{m}$ diameter hole have demonstrated the creation of a single microconduit of about the same size.

Electric field calculations for cylindrical geometries are quite important from the standpoint of analyzing field penetration and bioeffects in nerves. Most reports on nerve stimulation (McNeal 1976; Rattay 1990; Reilly 1998) have focused on other issues such as plasma membrane potentials, nerve excitation thresholds, influence of different 


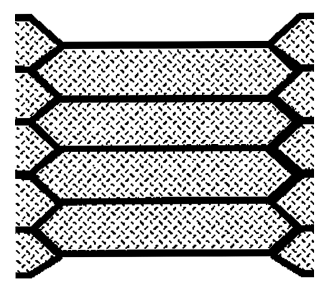

(a)

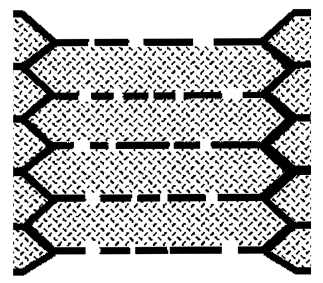

(b)

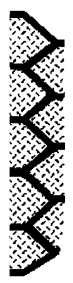

(c)

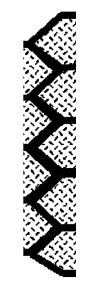

FIGURE 2.9 Simulation results for electric field effects obtained from a brick-wall skin model. (a) Prior to aqueous pathway creation. (b) After electrical pulses, and (c) minimum size microconduit ( $-50-\mu \mathrm{m}$ diameter), caused by removal of most of a single stack of corneocytes after keratin matrix disruption. (After Weaver, J. C. 2000. IEEE Trans Plasma Sci 28:24-33.)

electrode geometries and waveshapes on evoking action potentials, and even possibilities of electrical conduction blocks. Almost no attention has been given to quantify possible electric field penetration into nerve cells. For example, the commonly used distributed circuit analyses (McNeal 1976; Rattay 1990) represent the cell interior by a single effective resistance and do not attempt to probe spatial variations of the internal fields. To gauge the electric field distributions and effects on intracellular regions, more rigorous calculations (e.g., Clark and Plonsey 1968) need to be performed.

One possible approach for gauging the field strengths in nerves due to current source stimulations is to solve the Poisson's equation in cylindrical geometries. The cylindrical geometry is usually taken to adequately represent a simplified geometry for a nerve. For cylindrical geometries, the potential can conveniently be expressed in terms of a Fourier-Bessel series (Schnabel and Struijk 2001; Joshi and Song 2010). This potential is the solution of Poisson's equation satisfying the following: $\left.\boldsymbol{\nabla}^{2} \varphi+\left(\varepsilon_{0} / \sigma_{o}\right) \boldsymbol{\nabla}^{2}[\partial \varphi / \partial t)\right]$ $=I_{\mathrm{s}} / \sigma_{\mathrm{o}}$, with $I_{\mathrm{s}}$ denoting the source current density at coordinates $\left(r=r_{\mathrm{s}}, \theta=0, z=0\right)$. For a cylindrical neuron with axis along the $z$-direction, inner radius $a$ and membrane thickness $d$ (i.e., an outer neuron radius $b=a+d$ ) as shown in Figure 2.10, the timedependent potentials $\varphi(r, \theta, z, t)$ can be expressed as follows:

$$
\begin{gathered}
\varphi_{\mathrm{i}}(r, \theta, z, t)=\int_{0}^{\infty} \sum_{n=0}^{\infty} P_{n}(k, t) I_{n}(k r) \cos (n \theta) \cos (k z) \mathrm{d} k, \quad \text { for } 0 \leq r \leq a \\
\varphi_{\mathrm{m}}(r, \theta, z, t)=\int_{0}^{\infty} \sum_{n=0}^{\infty}\left[Q_{n}(k, t) I_{n}(k r)+R_{n}(k, t) K_{n}(k r)\right] \cos (n \theta) \cos (k z) \mathrm{d} k, \quad \text { for } \quad a \leq r \leq b \\
\varphi_{\mathrm{o}}(r, \theta, z, t)=\int_{0}^{\infty} \sum_{n=0}^{\infty} S_{n}(k, t) K_{n}(k r) \cos (n \theta) \cos (k z) \mathrm{d} k+T(t) /\left[4 \pi \sigma_{\mathrm{o}}\left|r-r_{\mathrm{s}}\right|\right], \text { for } \quad b \leq r
\end{gathered}
$$

In Equation 14(a through c), $I_{n}(k r)$ and $K_{n}(k r)$ are the modified Bessel functions of the first and second kind of order $n, r_{s}$ is the location of the current source, $\sigma_{o}$ is the conductivity of the outer region (i.e., $r \geq b$ ), while $P_{n}(k, t), Q_{n}(k, t), R_{n}(k, t), S_{n}(k, t)$, and $T(t)$ are time-dependent coefficients that need to be determined from suitable matching conditions and total current continuity considerations. Matching the voltages as well as 


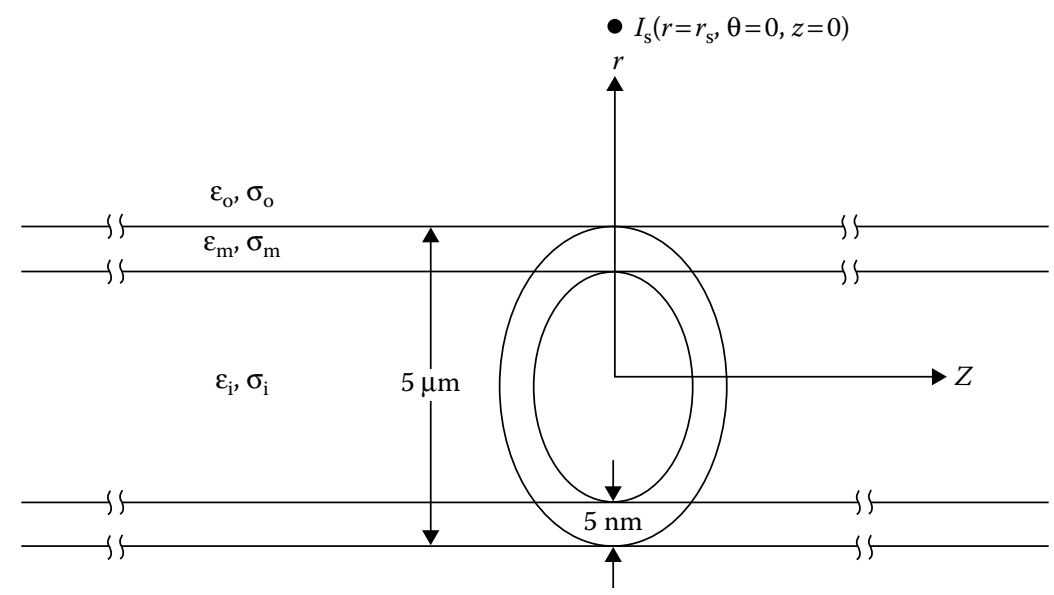

FIGURE 2.10 Schematic (not to scale) of the nerve geometry. An external source is placed at $r=r_{\mathrm{s}}, \theta=0, z=0$ in the vicinity of a cylindrical nerve with its axis parallel to the $z$-direction.

the normal currents at $r=a$ and $r=b$ leads to a complete solution. Very briefly, the final equations are as follows:

$$
\begin{aligned}
& \sigma_{\mathrm{m}} Q_{0}(k, t) I_{0}{ }^{\prime}(k b)+\sigma_{\mathrm{m}} R_{0}(k, t) K_{0}{ }^{\prime}(k b)+\varepsilon_{\mathrm{m}}\left\{\mathrm{d} Q_{0}(k, t) / \mathrm{d} t\right\} I_{0}{ }^{\prime}(k b)+\varepsilon_{\mathrm{m}}\left\{\mathrm{d} R_{0}(k, t) / \mathrm{d} t\right\} K_{0}{ }^{\prime}(k b)= \\
& \sigma_{0}\left[Q_{0}(k, t) I_{0}(k b) / K_{0}(k b)+R_{0}(k, t)-T /\left(2 \pi^{2} \sigma_{\mathrm{o}}\right)\right] I_{0}(k b)\left[K_{0}\left(k r_{\mathrm{s}}\right) / K_{0}(k b)\right] K_{0}{ }^{\prime}(k b)+\varepsilon_{\mathrm{o}}\left\{\mathrm { d } \left[Q_{0}(k, t)\right.\right. \\
& \left.\left.I_{0}(k b) / K_{0}(k b)+R_{0}(k, t)-T /\left(2 \pi^{2} \sigma_{o}\right)\right] I_{0}(k b)\left[K_{0}\left(k r_{s}\right) / K_{0}(k b)\right] / \mathrm{d} t\right\} K_{0}{ }^{c}(k b)+\left\{T /\left(2 \pi^{2}\right)\right\} I_{0}{ }^{\prime}(k b) \\
& K_{0}\left(k r_{\mathrm{s}}\right)+\left\{\varepsilon_{\mathrm{o}}(\mathrm{d} T / \mathrm{d} t) /\left(2 \pi^{2} \sigma_{0}\right)\right\} I_{0}{ }^{\prime}(k b) K_{0}\left(k r_{\mathrm{s}}\right) \\
& \sigma_{\mathrm{m}} Q_{n}(k, t) I_{n}{ }^{\prime}(k b)+\sigma_{\mathrm{m}} R_{n}(k, t) K_{n}{ }^{\prime}(k b)+\varepsilon_{\mathrm{m}}\left\{\mathrm{d} Q_{n}(k, t) / \mathrm{d} t\right\} I_{n}{ }^{\prime}(k b)+\varepsilon_{\mathrm{m}}\left\{\mathrm{d} R_{n}(k, t) / \mathrm{d} t\right\} K_{n}{ }^{\prime}(k b)= \\
& \sigma_{0}\left[Q_{n}(k, t) I_{n}(k b) / K_{n}(k b)+R_{n}(k, t)-T /\left(\pi^{2} \sigma_{\mathrm{o}}\right)\right] I_{n}(k b)\left[K_{n}\left(k r_{\mathrm{s}}\right) / K_{n}(k b)\right] K_{n}{ }^{\prime}(k b)+\varepsilon_{\mathrm{o}}\left\{\mathrm { d } \left[Q_{n}(k, t)\right.\right. \\
& \left.\left.I_{n}(k b) / K_{n}(k b)+R_{n}(k, t)-T /\left(\pi^{2} \sigma_{\mathrm{o}}\right)\right] I_{n}(k b)\left[K_{n}\left(k r_{\mathrm{s}}\right) / K_{n}(k b)\right] / \mathrm{d} t\right\} K_{n}{ }^{\prime}(k b)+\left\{T / \pi^{2}\right\} I_{n}{ }^{\prime}(k b) K_{n}\left(k r_{\mathrm{s}}\right) \\
& +\left\{\varepsilon_{\mathrm{o}}(\mathrm{d} T / \mathrm{d} t) /\left(\pi^{2} \sigma_{0}\right)\right\} I_{n}{ }^{\prime}(k b) K_{n}\left(k r_{\mathrm{s}}\right)
\end{aligned}
$$

Equation 2.15a and b enables the explicit calculation of $Q_{n}(k, t)$ and $R_{n}(k, t)$ for all orders of $n$, in terms of $T(t)$. Using the relation between $T(t)$ and the excitation current, $T(t)+\left(\varepsilon_{\mathrm{o}} / \sigma_{\mathrm{o}}\right) \mathrm{d} T(t) / \mathrm{d} t=I_{\mathrm{s}}(t)$, the function $T(t)$ can be obtained. Hence, the timedependent voltages $\varphi_{\mathrm{i}}(r, \theta, z, t), \varphi_{\mathrm{m}}(r, \theta, z, t)$, and $\varphi_{\mathrm{o}}(r, \theta, z, t)$ can all be fully determined.

Magnitudes of the axial electric field inside the cylindrical neuron structure (i.e., at $r=0$ ) at four different $z$-locations are shown in Figure 2.11. The results shown were in response to a trapezoidal current pulse with a $100 \mathrm{~ns}$ rise time, $700 \mathrm{~ns}$ ON time, and a $100 \mathrm{~ns}$ fall time. The longitudinal neuron axis was located $1 \mathrm{~mm}$ from the external pulsing source. These $z$-locations corresponded to positions 5, 10, 15, and $20 \mathrm{~mm}$ downstream from the stimulation electrode. In the coordinate system chosen, $z=0$ corresponds to a plane normal to the neuron and passing through the excitation source. As seen from Figure 2.11, the axial electric field values all increase during the pulse $\mathrm{ON}$ time. At the earliest times, the role of the displacement current is the strongest, and so the electric field variation is strong. Later, the displacement current contributions 


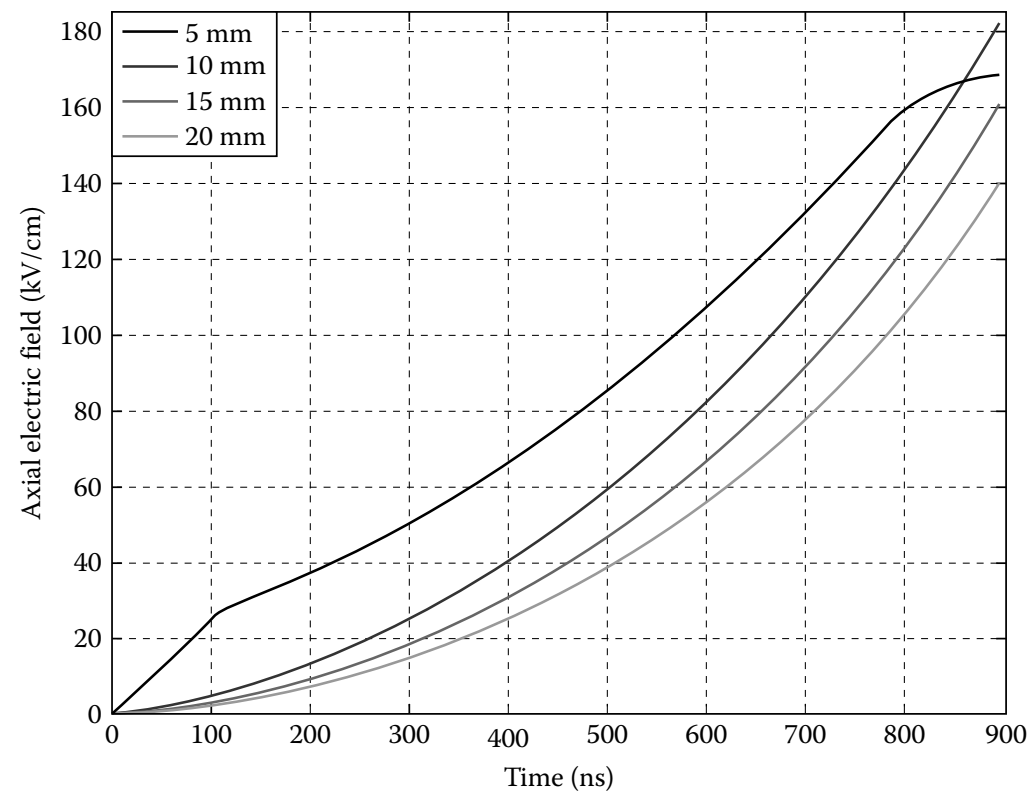

FIGURE 2.11 (See color insert.) Calculated axial electric fields at the center of the $2.5-\mu \mathrm{m}$ radius neuron for the trapezoidal external pulse at locations of 5, 10, 15, and $20 \mathrm{~mm}$ downstream. The excitation source was taken to be $1 \mathrm{~mm}$ from the nerve axis.

begin to decrease and the distributed voltages are progressively governed by the resistive conduction currents. Electric fields as high as $160 \mathrm{kV} / \mathrm{cm}$ are predicted, corresponding to the transmembrane voltage shifts on the order of $80 \mathrm{mV}$ within $1 \mu$ s for a 5 -nm-thick membrane. In this nanosecond pulsing context, it has already been reported that electric fields of about $20-80 \mathrm{kV} / \mathrm{cm}$ are sufficient to cause calcium release from internal stores within cells (Beebe et al. 2004; Vernier et al. 2008). Thus, the $160 \mathrm{kV} / \mathrm{cm}$ fields threading the internal organelles can be expected to cause similar bioeffects. A quick train of multiple pulses would add tunability based on the number within a short burst.

The higher electric fields predicted in Figure 2.11, slightly further along the nerve axis (e.g., at $10 \mathrm{~mm}$ vs. $5 \mathrm{~mm}$ ), emerge from the stronger role of conduction currents at later times. This is easily visualized in terms of a simple distributed circuit representation consisting of a ladder network for the nerve. More current paths from the vicinity of the excitation source merge on to the axial corridor with increasing distance. Consequently, the longitudinal current increases. For a given resistivity, the axial potential drop and the electric field are also enhanced. It might be mentioned that internal electric fields and TMP changes within the cell can be expected to remain in effect beyond the 1- $\mu$ s timescales shown in Figure 2.11. A longer time estimate of $\sim 1 \mathrm{~ms}$ for internal current flows and electrical membrane discharging is based on typical time constants for nerves.

Finally, it may be mentioned that the results of Figure 2.11 seem roughly consistent with two recent reports. In one experiment, both muscle twitch facilitation and inhibition were demonstrated in rat experiments by using nanosecond pulsing 
(Pakhomov et al. 2006). The facilitation could be the result of calcium release from internal stores followed by biochemical reactions involving troponin (Wexler, Ding, and Binder-Macleod 1997). Inhibition at higher fields, on the other hand, could be due to localized nerve membrane poration. With localized poration, the outer membrane could have the effect of attenuating voltage waves traveling down the neurons. More recently, Despa et al. (2009) showed electromuscular effects linked to direct nerve excitation by external electric fields. A common theme in their observations seems to be that neuromuscular bioresponses can be stimulated or modulated by direct electrical pulsing at remote sites.

\subsection{Temperature Changes Due to Electric Pulsing}

In dealing with in vivo biological tissues exposed to an electric field, it is important to consider the SAR and AD because these quantities influence the biological outcomes. More importantly, perhaps, the temperature rise associated with Joule heating is an important indicator because most internal processes are energy driven. The severity of this temperature rise is dependent on the electric pulse input parameters and tissue electrical properties. In some cases, such as electrochemotherapy, pulse parameters are deliberately chosen to ensure negligible changes in temperature with complete reversibility.

Electrochemotherapy incorporates electroporation with chemotherapeutic drugs such as bleomycin and has been shown to be an effective technique in treating tumors in vivo (Gothelf, Mir, and Gehl 2003; Heller, Gilbert, and Jaroszeski 1999). Clinical electroporation pulse parameters are typically short $(-100 \mu \mathrm{s})$, with fields in the $1-2 \mathrm{kV} / \mathrm{cm}$ range with pulse repetition rates of $1 \mathrm{~Hz}$ (Belehradek et al. 1993; Rols et al. 2000). Due to the relatively short-pulse durations of these "electroporation pulses," the resulting temperature increases and associated thermal damage to the tissue are generally negligible.

For large molecule electroporation such as electrogene and DNA transfer, longer pulses are required to utilize electrophoretic effects (Rols and Teissie 1998; Mir et al. 1999). Pulse parameters in such cases may result in noticeable tissue heating. An in vivo study of electrogene transfer conducted on rat liver cells (Suzuki et al. 1998) has shown that the highest rate of gene transfer occurs at locations on the border between the necrotized and surviving cells. It has also been shown (Muramatsu, Nakamura, and Park 1998) that the amount of gene transfer is strongly tied to the amount of heat generated, below the threshold of tissue damage. Another innovative application of electroporation has been proposed (Davalos, Mir, and Rubinsky 2005), in which irreversible electroporation (requiring longer, more intense pulses than reversible electroporation) is used to induce nonthermal killing of cancerous tissue. In that study, a numerical study was conducted exploring electroporation parameters that maximized irreversible electroporation (while minimizing thermal damage) in order to isolate cell killing by electroporation from cell killing by heating.

The temperature distribution in the context of in vivo electroporation is affected by several factors such as the presence of a blood vessel, external convective influences, metabolic heat generation, perfusion influences, and electrode geometry or spacing. Given the experimental difficulties of obtaining both spatially and temporally resolved data on heating, these issues have often been probed through simulations. For example, Davalos, Rubinsky, and Mir (2003) have implemented one- and two-dimensional 
finite-element models in order to gain useful insights into the role played by various factors on the temperature. Others have explored the thermal effects of various geometric, biological, and electroporation pulse parameters (Becker and Kuznetsov 2006) including the blood vessel presence and size, plate electrode configuration, and pulse duration and frequency. Using a three-dimensional transient finite volume model of in vivo parallel plate electroporation of liver tissue in the presence of a blood vessel, the target region tissue was found to have lower thermal damage. This was attributed to heat conduction away from the region by the blood vessel. Increase in the plate spacing increased the thermal damage as the volume to surface area ratio increased. The results of dividing a long pulse into shorter pulses of equal intensity and frequency showed that increasing the time over which energy can be introduced into the system resulted in lower maximum target region temperatures and significantly decreased thermal dose values. Similarly, it was found that decreasing pulse frequency resulted in lower thermal damage and lower maximum temperatures.

Some important parameters from a practical standpoint are the exposure duration, SAR, AD, pulse shape and width, and the repetition rate. Although the specific effects and bioresponses are complicated to predict and quantify, the thermal effects can be obtained from the pulse duration and SAR. Mathematically, the SAR (in $\mathrm{W} / \mathrm{Kg}$ ) can be obtained from the expression $\sigma E^{2} / \rho$, where $E$ is the local electric field created by the external excitation, $\sigma$ the conductivity of the biomaterial, and $\rho$ the material density; while the $\mathrm{AD}$ is the product of the SAR, the pulse duration, and number of pulses. Experimental data on cell subject to the ultrashort, high-intensity pulses have shown temperature increases of less than $3^{\circ} \mathrm{C}$ when subjected to a single $\sim 330 \mathrm{kV} / \mathrm{cm}$ pulse of 10-ns duration. However, trains of 20-400 pulses were shown to deliver sufficient energy to cause temperature increases in excess of $6^{\circ} \mathrm{C}$, as shown in Figure 2.12.

Calculations on slightly longer 60 -ns electric pulses having a $60 \mathrm{kV} / \mathrm{cm}$ intensity show peak temperature increases of about $3^{\circ} \mathrm{C}$. Applying the distributed circuit model discussed in Section 2.3.1 to a spherical cell of radius $10 \mu \mathrm{m}$ consisting of cytoplasm $\left(\varepsilon=60 \varepsilon_{0}\right.$, $\sigma=0.13 \mathrm{~S} / \mathrm{m})$, outer medium $\left(\varepsilon=80 \varepsilon_{0}, \sigma=0.6 \mathrm{~S} / \mathrm{m}\right)$, and a 5 -nm-thick plasma membrane $\left(\varepsilon=8 \varepsilon_{0}, \sigma=5.3 \times 10^{-6} \mathrm{~S} / \mathrm{m}\right)$ yielded the curves as shown in Figure 2.13. Changes in local conductivity due to membrane poration were included and though not shown here, large increases by orders of magnitude were predicted over time. For example, the highest conductivity was predicted to be about $0.007 \mathrm{~S} / \mathrm{m}$. The power density values are shown in Figure 2.13 as a function of time for five different angular locations. The initial power dissipation is negligible because the membrane acts as a virtual open circuit with the displacement current being the dominant mechanism. Later ( $\sim 40 \mathrm{~ns}$ and beyond), the conduction current develops as pores begin to form and increase both in numbers and radial size. Due to the nonlinear increases in conductance and pore densities over time, the power density attains a peak and then begins to reduce. The decrease can be associated with a collapse of the local TMP as the pores effectively produce an electrical "short."

Based on the power density, changes in temperature as a function of time and angular location were obtained. Values for the density $\rho_{\mathrm{M}}$ and membrane specific heat $c_{\mathrm{M}}$ were chosen to be $10^{3} \mathrm{~kg} / \mathrm{m}^{3}$ and $2 \times 10^{3} \mathrm{~J} / \mathrm{kg} / \mathrm{Kelvin}$, respectively, in keeping with reports in the literature (Croce et al. 2010). Figure 2.14 shows the temperature profile. Interestingly, the largest temperature increase, which occurs at the poles (i.e., $\theta=0^{\circ}$ ), is predicted to 


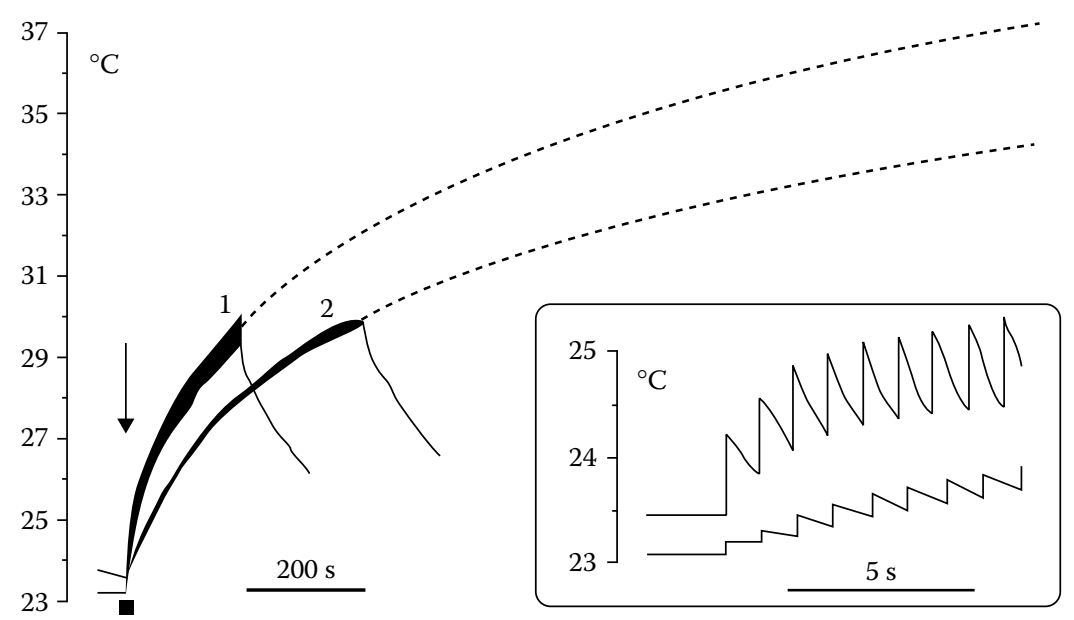

FIGURE 2.12 Heating of the medium in an electroporation cuvette by a train of 200 pulses at 360 $\mathrm{kV} / \mathrm{cm}$ (1) or 400 pulses at $150 \mathrm{kV} / \mathrm{cm}$ (2). The pulse repetition rate was $1 \mathrm{~Hz}$ in both measurements. Arrow indicates the onset of exposure. Dashed lines are the best-fit approximations of heating dynamics. The portion of the graph identified by a small rectangle under the curves is expanded in the inset, to show heating and cooling by individual nanosecond pulses in the train. (After Pakhomov, A. G., A. Phinney, J. Ashmore, K. Walker III, J. F. Kolb, S. Kono, K. H. Schoenbach, and M. R. Murphy. 2004. IEEE Trans Plasma Sci 32:1579-86.)

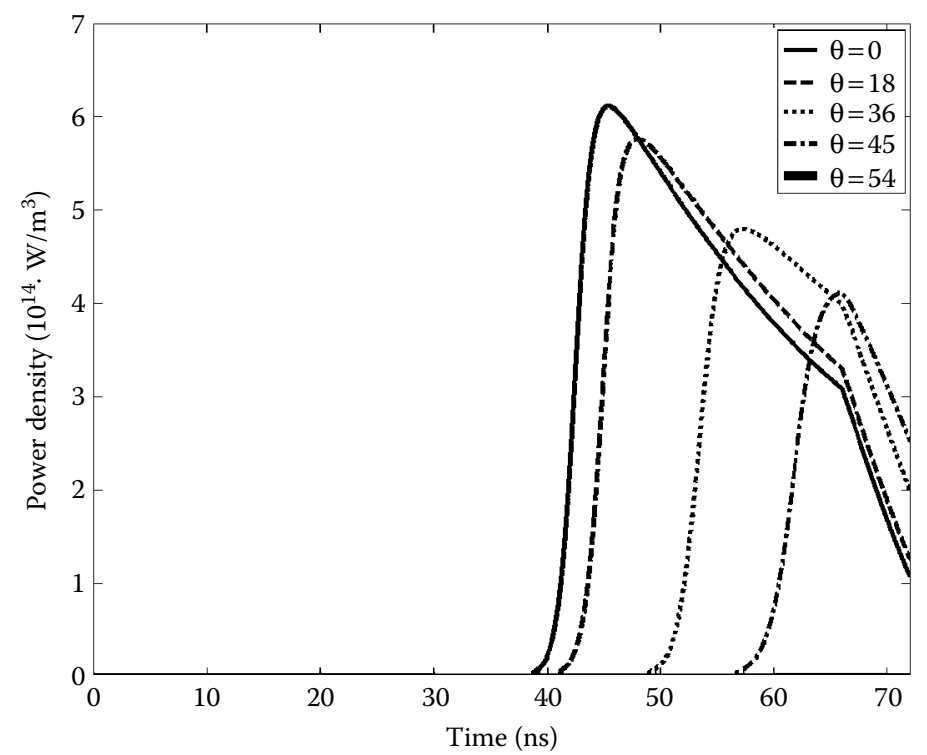

FIGURE 2.13 Calculated dissipated power density at the membrane as a function of time. Five different angular locations are shown. 


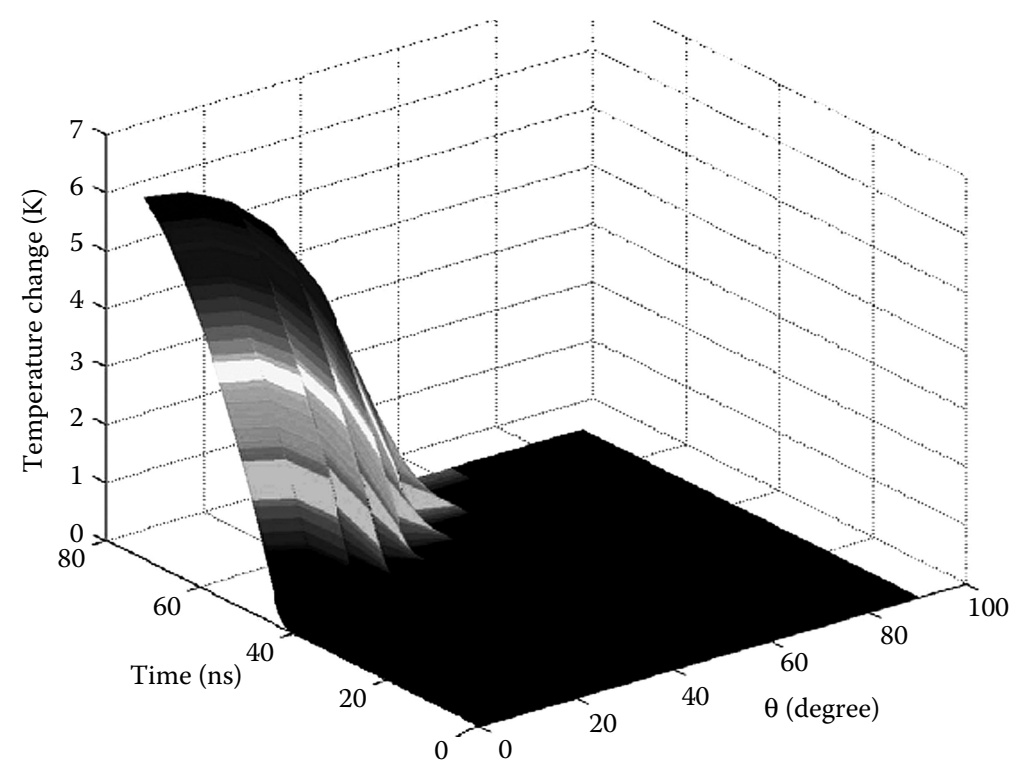

FIGURE 2.14 Simulation results for the membrane temperature change as a function of time at different angular locations.

have a value of about $6 \mathrm{~K}$. However, a fairly rapid fall off is seen in Figure 2.14 away from the poles, with near negligible temperature changes predicted for much of the cells. So, while a near thermalized situation seems to prevail in an averaged sense, localized heating could be a factor.

It may also be mentioned that treatment of cells and tissues by strong electric fields can also be used as a minimally invasive surgical procedure to ablate undesirable tissue without the use of adjuvant drugs. The electric pulses required are larger in magnitude and duration than those for reversible electroporation. Furthermore, it has been shown (Davalos, Mir, and Rubinsky 2005) that localized regions can be ablated by electric pulses prior to the onset of any deleterious thermal effects. In particular, treated areas in the livers of male Sprague-Dawley rats (Edd et al. 2006) exhibited microvascular occlusion, endothelial cell necrosis, and diapedeses, resulting in ischemic damage to parenchyma, while preserving the large blood vessel architecture. Mathematical analysis supported the hypothesis that this damage was primarily nonthermal in nature and that sharp borders between affected and unaffected regions corresponded to electric fields of 300-500 V/cm from 20-ms pulses.

\subsection{Role of the Biological Dielectric Properties}

Bioeffects driven by external pulsed electric fields primarily depend on two factors: (1) the strength of the applied voltage and (2) the pulse duration. Thus, for example, at a fixed electric field, the cellular response to a $100 \mathrm{~ns}$ pulse can be very different from that of a $1 \mathrm{~ns}$ duration pulse. The difference persists even when the care is taken to adjust the 
electric fields at the two different pulse durations to ensure that a constant total energy is maintained (Schoenbach et al. 2009). The difference arises from dielectric effects, and in the ultrafast pulsing context, it is associated with the finite times required for the reorientation of the dipoles within the system. For example, water (a polar material that surrounds the cell membrane) has a dielectric relaxation time of about 8 ps (Buchner, Barthel, and Stauber 1999). This time is roughly the duration taken to displace the bonded charges within the molecular structures (e.g., polar water or the lipidic cell membranes) by an external electric field. Similarly, there are other relaxation values that would be associated with the displacement of lipidic molecules within membranes. As a result, any applied electrical disturbance that occurs on timescales much greater than $\sim 1 \mathrm{~ns}$ (as is the case for most conventional stimulations) will effectively see conditions prevailing at near steady state. These include "quasistatic" dielectric constants and well-established screening layers adjacent to the membrane comprising of charges and counterions. However, in the case of electric pulses that are shorter (e.g., in the subnanosecond regime), there will not be sufficient time for charge accumulation or the complete reorientation of the dipoles within either the aqueous medium or the membrane hydrocarbons. Consequently, the effective dielectric constants seen by the driving electric field, as well as the ratio of these parameters at the interfaces, will be different. In short, by using ultrafast pulsing, it becomes technologically possible to effectively select electrical parameters of the biosystem, different from the steadystate values. Biophenomena can then be driven under a distinctive and abnormal set of parameters.

Several comprehensive and critical reviews of the dielectric properties of tissues and biological materials have been given (Foster and Schwan 1989). The central idea is to measure and evaluate the permittivities and conductivities of biotissues (and surrounding aqueous media). Using the notion of Fourier transforms and signal decomposition, time-dependent quantities (such as charge kinetics and dipole movements) can most conveniently be expressed in the frequency domain for linear responses. In the simplest model, the polarization $D$ of a sample/tissue will relax toward a steady state as a firstorder process with a relaxation time $\tau$ as follows:

$$
D(t)=D_{\infty}+\left[D(0)-D_{\infty}\right]\left[1-\mathrm{e}^{-t / \tau}\right]
$$

where $D_{\infty}$ is the "instantaneous" response that might arise if the system could respond extremely fast with no lag time, while $D(0)$ is the steady-state response after a long time. The time constant $\tau$ given in Equation 2.16 depends on the process and can be as short as subnanoseconds (e.g., reorientation of short molecules) to seconds (for counterion redistributions). As a result, the dielectric constant (a measure of electrical screening) is then expressed in the frequency domain as follows:

$$
\varepsilon=\varepsilon_{\infty}+\left[\varepsilon(0)-\varepsilon_{\infty}\right] /[1+j \omega \tau]-j \sigma_{0} /\left(\omega \varepsilon_{0}\right)
$$

where $\varepsilon_{\infty}$ and $\varepsilon(0)$ denote the dielectric constants at very large and near-zero frequencies, respectively, and $\sigma_{0}$ is the static conductivity. Thus experimentally, the principal objective is to obtain data on the $\varepsilon(0), \varepsilon_{\infty}$, and $\sigma_{0}$ parameters. 
Depending on the processes involved and the inherent physics, the dielectric behavior can be more complicated than the single time constant description. This could occur if, for example, the kinetics are not of first order, or if there happen to be multiple relaxation processes. In such cases, the polarization needs to be expressed as a series involving various time constants as the following:

$$
\begin{gathered}
D(t)=D_{\infty}+D_{1}\left[1-\mathrm{e}^{-t / \tau 1}\right]+D_{2}\left[1-\mathrm{e}^{-t / \tau 2}\right]+\ldots \\
\varepsilon=\varepsilon_{\infty}+\Delta \varepsilon_{1} /\left[1+j \omega \tau_{1}\right]+\Delta \varepsilon_{2} /\left[1+j \omega \tau_{2}\right]+\ldots
\end{gathered}
$$

where $\tau_{1}, \tau_{2}$, etc., are the various time constants associated with the various relaxation processes. An empirical function in-line with Equation 2.17 that has been widely used (Cole and Cole 1941) is as follows:

$$
\varepsilon=\varepsilon_{\infty}+\left[\varepsilon(0)-\varepsilon_{\infty}\right] /\left[1+(j \omega \tau)^{1-\alpha}\right]-j \sigma_{0} /\left(\omega \varepsilon_{0}\right)
$$

The most important mechanisms that influence the dielectric properties are the following: (1) the interfacial polarization (the Maxwell-Wagner effects), (2) dipolar orientation, and (3) ionic diffusion (or counterion polarization). Of these, the first arises in the context of mixtures and suspensions, and several reviews have already appeared on this subject (e.g., Hanai 1968; Dukhin and Shilov 1974; Irimajiri, Hanai, and Inouye 1979). The time constants are roughly the product of the resistance $(R)$ and capacitance $(C)$ of the interfacial region, and the values are in the radio-frequency range. The orientational contributions to dielectric relaxation have also been studied, and the applications to protein solutions have been reported (Debye 1929; Takashima 1969). The counterion polarization has time constants that roughly depend on $L^{2} / D$, where $L$ represents the diffusion length and $D$ the diffusion coefficient. Typical values lie in the audio-frequency regime.

These concepts can be applied to predict the TMP development across cell membranes especially under ultrashort, pulsing conditions. For a spherical cell, the potentials developed in response to an ultrafast external electric field $E_{0}(t)$ are roughly solutions of the Laplace equation. In the case of a simple, spherical single-shell model with three regions consisting of the intracellular region of radius $a$, an extracellular region starting from $r>b$, and membrane thickness $b-a$, the expressions are as follows:

$$
\begin{gathered}
\Phi_{\mathrm{E}}(r, \theta)=\left(-E_{0} r+\mathrm{A} / r^{2}\right) \cos (\theta), \text { for } r>b \\
\Phi_{\mathrm{M}}(r, \theta)=\left(-\mathrm{B} r+\mathrm{C} / r^{2}\right) \cos (\theta), \text { for } b>r>a \\
\Phi_{\mathrm{I}}(r, \theta)=(-\mathrm{K} r) \cos (\theta), \text { for } a>r
\end{gathered}
$$

In Equation 2.19(a through c), A, B, C, and $\mathrm{K}$ are constants that need to be determined by matching the potential and electric fields at the boundaries $r=a$ and $r=b$, while $\Phi_{\mathrm{E}}$, $\Phi_{\mathrm{M}}$, and $\Phi_{\mathrm{I}}$ denote the voltages within the intracellular, membrane, and extracellular regions. Algebraic manipulation finally leads to the following:

$$
\Delta \Phi_{\mathrm{M}}=\mathrm{B}(b-a)-\mathrm{C}\left(1 / b^{2}-1 / a^{2}\right)
$$


with

$$
\begin{gathered}
\mathrm{A}=\left(E_{0}-\mathrm{B}\right) b^{3}+\mathrm{C} \\
\mathrm{B}=3 \varepsilon_{\mathrm{E}} E_{0} b^{3}\left(\varepsilon_{\mathrm{I}}+2 \varepsilon_{\mathrm{M}}\right) /\left[b^{3}\left(\varepsilon_{\mathrm{M}}+2 \varepsilon_{\mathrm{E}}\right)\left(\varepsilon_{\mathrm{I}}+2 \varepsilon_{\mathrm{M}}\right)+2 a^{3}\left(\varepsilon_{\mathrm{M}}-\varepsilon_{\mathrm{E}}\right)\left(\varepsilon_{\mathrm{I}}-\varepsilon_{\mathrm{M}}\right)\right] \\
\mathrm{C}=3 \varepsilon_{\mathrm{E}} E_{0} a^{3} b^{3}\left(\varepsilon_{\mathrm{I}}-\varepsilon_{\mathrm{M}}\right) /\left[b^{3}\left(\varepsilon_{\mathrm{M}}+2 \varepsilon_{\mathrm{E}}\right)\left(\varepsilon_{\mathrm{I}}+2 \varepsilon_{\mathrm{M}}\right)+2 a^{3}\left(\varepsilon_{\mathrm{M}}-\varepsilon_{\mathrm{E}}\right)\left(\varepsilon_{\mathrm{I}}-\varepsilon_{\mathrm{M}}\right)\right]
\end{gathered}
$$

The dielectric constants $\varepsilon_{\mathrm{I}}, \varepsilon_{\mathrm{E}}$, and $\varepsilon_{\mathrm{M}}$ for the intracellular, extracellular regions, and the membrane, respectively, are given by the following general expression:

$$
\varepsilon_{\mathrm{k}}=\varepsilon_{\infty \mathrm{k}}+\left[\varepsilon_{\mathrm{k}}(0)-\varepsilon_{\infty \mathrm{k}}\right] /\left[1+j \omega \tau_{\mathrm{k}}\right]-j \sigma_{0 \mathrm{k}} /\left(\omega \varepsilon_{0}\right)
$$

with $\mathrm{k}$ representing one of the three regions. Clearly, for a very short pulse (say having subnanosecond duration), a large number of frequency components would contribute, and hence, the response would be collectively dictated by dielectric constants at a correspondingly large number of frequencies. Table 2.1 lists some of the parameters that have been reported for simple cell.

A quick calculation reveals the role played by pulse duration and presents a case for shorter pulse widths. To incorporate the nonlocality in polarization with time through the frequency-dependent permittivities, the pulse waveforms can be converted into the frequency domain through a discrete Fourier transform (DFT). The pulses' sampling time and the total observation time window have to be chosen to allow proper time signal reconstruction. These values were set equal to $0.01 \mathrm{~ns}$ and $1 \mu \mathrm{s}$, respectively. Then for each frequency sample of the signal spectrum, the Laplace equation was solved based on Equation 2.20 ( $\mathrm{d}$ through g) to yield the TMP values. Then, an inverse DFT was applied to reconstruct the temporal behavior.

The magnitude of the TMP (with both the real and imaginary parts) is shown in Figure 2.15 as a function of frequency for a rectangular 2-ns electric pulse having a maximum amplitude of $100 \mathrm{kV} / \mathrm{cm}$. As might be expected, the highest TMP is created by a lower frequency excitation. The relative permittivity of the cell membrane, including both the real and imaginary parts, at these frequencies is shown in Figure 2.16. A much lower value of about 4 is seen at the frequencies beyond $1 \mathrm{GHz}$. More interestingly, Figure 2.17 shows the TMP values for pulses of various durations ranging from $8 \mathrm{~ns}$ down to $0.8 \mathrm{~ns}$, as a function of time. Rectangular electric field waveforms were used

TABLE 2.1 Dielectric Model Parameters for a Cell

\begin{tabular}{llccc}
\hline Cell Compartment & $\varepsilon(0)$ & $\varepsilon_{\infty}$ & $T(\mathrm{~ns})$ & $\sigma_{0}(\mathrm{~S} / \mathrm{m})$ \\
\hline $\begin{array}{l}\text { Intracellular } \\
\text { medium }\end{array}$ & 67 & 5 & 0.00889 & 0.55 \\
Cell membrane & 11.7 & 4 & 0.88493 & $1.1 \times 10^{-7}$ \\
$\begin{array}{l}\text { Extracellular } \\
\text { medium }\end{array}$ & 67 & 5 & 0.00889 & 0.55 \\
\hline
\end{tabular}

Source: After Merla, C., M. Liberti, F. Apollonio, and G. d'Inzeo. 2009. Bioelectromagnetics 30:286-98. 


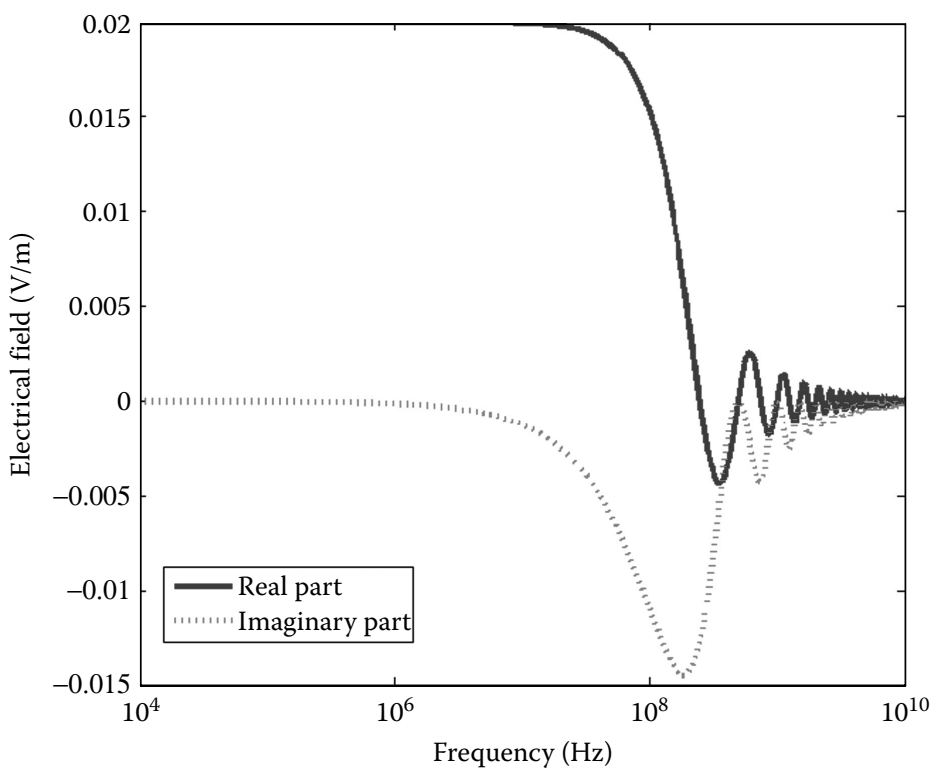

FIGURE 2.15 A nanosecond rectangular pulse with a $10^{7} \mathrm{~V} / \mathrm{m}$ electric field, transformed into the frequency domain.

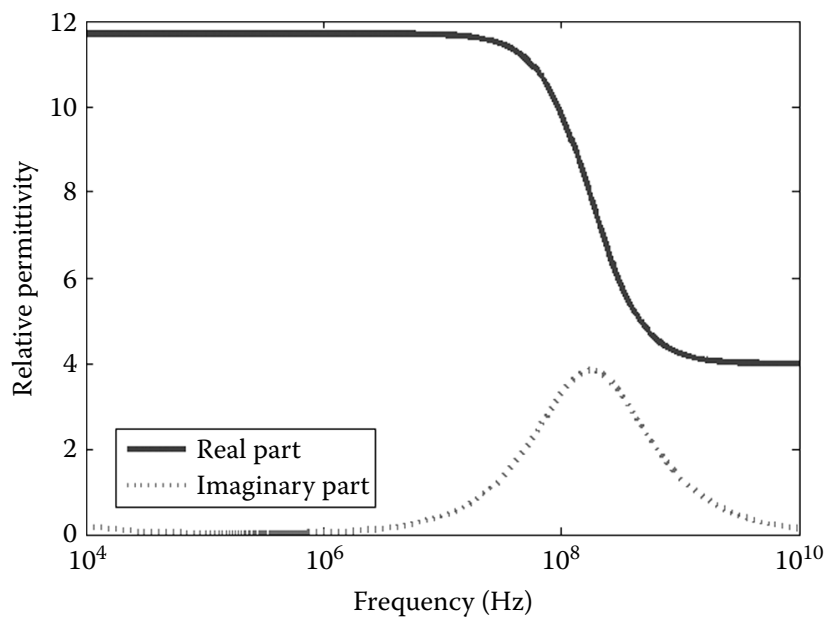

FIGURE 2.16 Relative permittivity of cell membrane as a function of frequency.

in all cases, and the field amplitude was adjusted each time to ensure a constant total energy. As seen from Figure 2.17, the highest TMP is predicted for the subnanosecond pulse duration, with progressive decreases for the longer 2-ns and 4-ns pulses. Physically, this occurs because for shorter pulses, the frequency spectrum of the waveform shifts to a higher range. Because the dielectric constant at higher frequencies decreases, the effective charging time constant of the membrane $(\sim \varepsilon / \sigma)$ is reduced. This enables quicker 


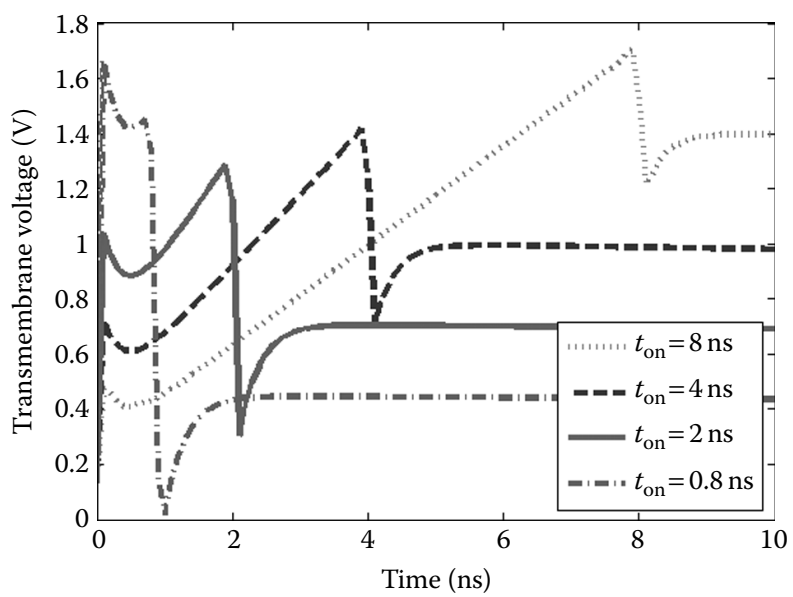

FIGURE 2.17 Transmembrane potential (TMP) induced by pulses of various durations but the same total energy.

membrane charging. The plot of Figure 2.17 also shows that for a given energy input, the more efficient way to create TMPs to drive bioeffects is through the use of shorter (i.e., subnanosecond) pulses. However, because the peak electric fields needed from the voltage supply are larger, special pulsed power techniques and equipment (e.g., Kolb, Susumu, and Schoenbach 2006) would be needed to exploit this temporal regime. It may also be pointed out that even though the 8 -ns pulse seems to have about the same peak voltage as the 0.8 -ns pulse, electroporation was ignored here. However, in actual practice, due to some poration during the longer 8-ns time frame, the peak voltage might not be as high. For the shorter 0.8 -ns pulse though, not much poration might have occurred, and so a higher TMP would be presented to the membrane, leading to stronger bioeffects at the same external energy.

\subsection{Extracting Parameters from Cell Suspensions}

Though the earlier discussion has focused on the dielectric properties of cell membranes, actual experimental measurements simply record the response of cell suspensions. Hence, it becomes necessary to carefully extract the requisite parameters from global measurements and also to select the appropriate technique for data generation. Dielectric spectroscopy is an appropriate and powerful tool to investigate the dielectric properties of cells and organelles, and it has a long history of over 75 years (Debye 1929; Onsager 1936; Kirkwood 1939; Oncley 1938). For example, such studies led to the deduction that erythrocytes were composed of a poorly conducting envelope enclosing a conducting electrolyte (Hoeber 1910) and measurements of membrane thicknesses (e.g., Fricke 1925). Foundations for the understanding of the dispersion of a biological cell suspended in an electrolyte were laid over 50 years ago (Maxwell 1891; Wagner 1914; Schwan 1957).

More recently, it has been shown that the application of the time domain dielectric spectroscopy (TDDS) can be very successful in obtaining new information on the 
structural and dynamic parameters of biological tissues (Cole 1975; Cole, Mashimo, and Winsor 1980; Feldman and Fedotov 1987; Hager 1994). Basically, TDDS is based on transmission line theory in the time domain that aids in the study of heterogeneities in coaxial lines according to the change of the shape of a test signal. A rapidly increasing voltage step $V(t)$ is applied to the line and recorded, along with the reflected voltage $R(t)$ that is returned from the sample and delayed by the cable propagation time. Any cable or instrument artifacts are separated from the sample response due to the propagation delay, thus making them easy to identify and control. The entire frequency spectrum is captured at once, thus eliminating drift and distortion between frequencies. The complex permittivity is then obtained from these single measurements. An important challenge of this technique though is to correct for electrode polarization effects. Electrode polarization, which is due to the formation of an electric double layer at the interface between electrode and conductive biological sample, complicates the determination of dielectric spectra of cell suspensions and tissues at $\alpha$ - and $\beta$-dispersion range. Recent studies have attempted to address this aspect (Bordi, Cametti, and Gili 2001).

For cell suspensions, the dielectric permittivity $\varepsilon_{\text {mix }}$ can be expressed by the wellknown Maxwell-Wagner relationship:

$$
\varepsilon_{\text {mix }}=\varepsilon_{1}\left[\left(2 \varepsilon_{1}+\varepsilon_{\mathrm{c}}\right)-2 p\left(\varepsilon_{1}-\varepsilon_{\mathrm{c}}\right)\right] /\left[\left(2 \varepsilon_{1}+\varepsilon_{\mathrm{c}}\right)+p\left(\varepsilon_{1}-\varepsilon_{\mathrm{c}}\right)\right]
$$

where $p$ is the volume fraction of the suspended cells, $\varepsilon_{1}$ represents the dielectric permittivity of the liquid phase, and $\varepsilon_{\mathrm{c}}$ is the cell permittivity. The latter, in turn, can be expressed in terms of multishell models comprising of concentric membranes that enclose conducting media (Ermolina et al. 2001). A crude schematic of this is shown in Figure 2.18.

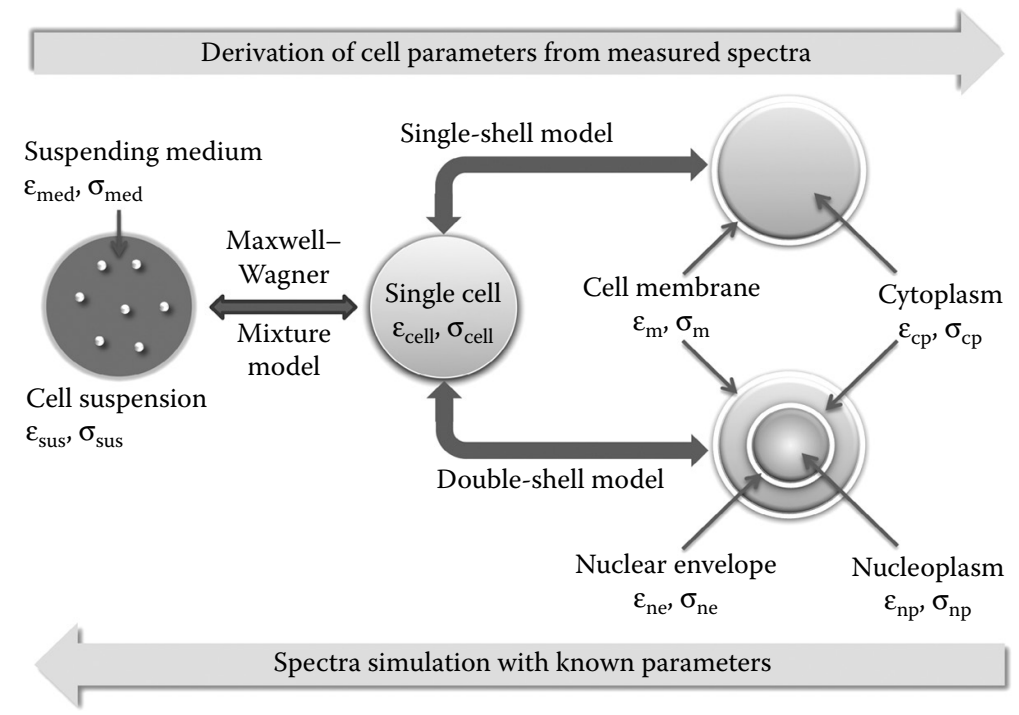

FIGURE 2.18 Overview of dielectric models and the relationship from cell suspension to cellular structures. 
An additional utility of such dielectric parameter measurements is in medical diagnostics. This is linked to the fact that electrical properties (e.g., permittivity and conductivity) of cells and tissues are distinct for each type. This occurs due to variability in moisture content, size, distributions of molecular dipole moments, density, and texture (Gabriel, Lau, and Gabriel 1996). For example, fat has a lower permittivity and conductivity than muscle. Even for the same tissue, a pathological change may result in different electric properties (Semenov et al. 2002). Hence, electrical properties can be used not only to differentiate the anatomical structure but also to differentially diagnose malignant versus normal tissue, providing "functional" information.

Another application can be toward real-time monitoring of treatment responses. For example, high-field electric pulsing can lead to nonlinear cell membrane effects such as electroporation (Schoenbach et al. 2004). This alters the effective conductivity of cells and allows the passage of molecules (including drugs and chemotherapeutic agents) into cells. Loading molecules into cells should then result in a strong contrast of conductivity within the tissue. In practice, four types of cells could exist in such treated tissue: normal, malignant, normal loaded with delivered molecules, and malignant cells loaded with delivered molecules. The average dielectric properties of the tissues should be different depending on the percentage compositions of the four cell types in the tissue. Presumably, tissue with a dominant percentage of tumor cells should have a higher dielectric permittivity than the tissue in which normal cells dominate. Such difference can be $10 \%$ to 10 -fold depending on the tissue composition (Lazebnik et al. 2007). The drug-loaded tissue should have a dielectric permittivity in between this range. Hence, the dielectric properties can be a good marker to monitor targeted drug delivery and to ascertain whether the agents introduced do indeed have the intended consequence at the desired rate. As a simple example, the data in Figure 2.19 show changes in the

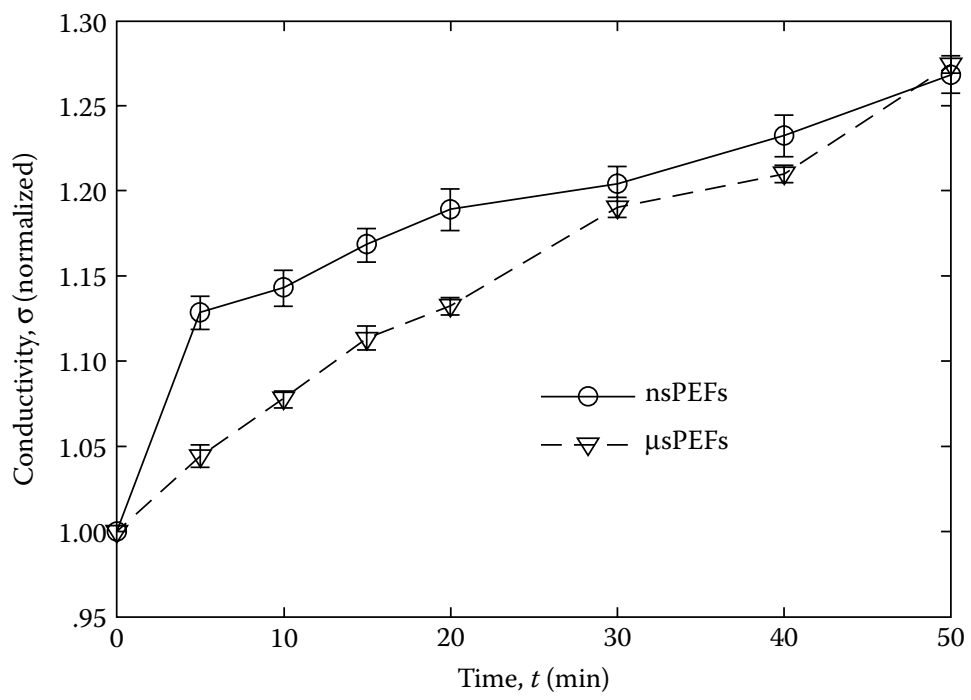

FIGURE 2.19 Low-frequency cell suspension conductivity after pulsed electric field exposures. 
low-frequency cell suspension conductivity following two electroporating electrical pulses (of nanosecond and microsecond durations) as a function of time. For both nanosecond- and microsecond-pulsed electric field exposures, low-frequency suspension conductivity monotonically increases. These increases are likely associated with the formation of membrane pores.

\section{References}

Abidor, I. G., V. B. Arakelyan, L. V. Chernomordik, Y. A. Chizmadzhev, V. F. Pastushenko, and M. R. Tarasevich. 1979. Electric breakdown of bilayer membranes: The main experimental facts and their qualitative discussion. Bioelectrochem Bioenerget 6:37-52.

Adair, R. K. 1991. Constraints on biological effects of weak extremely-low-frequency electromagnetic fields. Phys Rev A 43:1039-48.

Arnold, W. M. 2001. Positioning and levitation media for the separation of biological cells. IEEE Trans Ind Appl 37:1468-75.

Asencor, F. J., C. Santamaria, F. J. Iglesias, and A. Dominguez. 1993. Dielectric energy of orientation in dead and living cells of Schizosaccharomyces pombe: Fitting of experimental results to a theoretical model. Biophys J 64:1626-31.

Auer, D., G. Brandner, and W. Bodemer. 1976. Dielectric breakdown of the red blood cell membrane and uptake of SV 40 DNA and mammalian cell RNA. Naturwiss 63:391.

Bagci, E. Z., Y. Vodovotz, T. R. Billiar, G. B. Ermentrout, and I. Bahar. 2006. Bistability in apoptosis: Roles of Bax, Bcl-2, and mitochondrial permeability transition pores. Biophys J 90:1546-59.

Barnett, A., and J. C. Weaver. 1991. A unified, quantitative theory of reversible electrical breakdown and rupture. Bioelectrochem Bioenerg 25:163-82.

Bartsch, G., M. Baumann, and R. Grebe. 1997. Numerical calculation of electric fields surrounding biological cells in three dimensions. Bioelectrochem Bioenerg 42:221-6.

Batzing, B. L. 2002. Microbiology: An introduction. Pacific Grove: Thompson Learning.

Becker, A. A. 1992. The Boundary Element Method in Engineering. New York: McGraw-Hill.

Becker, S. M., and A. V. Kuznetsov. 2006. Numerical modeling of in vivo plate electroporation thermal dose assessment. Trans ASME 128:76-84.

Beebe, S. J., P. F. Blackmore, J. White, R. P. Joshi, and K. H. Schoenbach. 2004. Nanosecond pulsed electric fields modulate cell function through intracellular signal transduction mechanisms. Physiol Meas 25:1077-93.

Beebe, S. J., P. M. Fox, L. J. Rec, E. L. Willis, and K. H. Schoenbach. 2003. Nanosecond, high intensity pulsed electric fields induce apoptosis in human cells. FASEB J 17:1493-5.

Beeler, G. W., and H. Reuter. 1977. Reconstruction of the action potential of ventricular myocardial fibres. J Physiol 268:177-210.

Belehradek, M., C. Domenge, B. Luboinski, S. Orlowski, J. Belehradek, and L. M. Mir. 1993. Electrochemotherapy, a new antitumor treatment: first clinical phases I-II trial. Cancer 72:3694-700.

Berg, H. 1993. Electrostimulation of cell metabolism by low frequency electric and electromagnetic fields. Bioelectrochem Bioenerg 31:1-25. 
Berg, H. 1995. Possibilities and problems of low frequency weak electro- magnetic fields in cell biology. Bioelectrochem Bioenerg 38:153-9.

Barnett, A., and J. C. Weaver. 1991. A unified, quantitative theory of reversible electrical breakdown and rupture. Bioelectrochem Bioenerg 25:163-82.

Bhatt, D. L., D. C. Gaylor, and R. C. Lee. 1990. Rhabdomolysis due to pulsed electric fields. Plast Reconstr Surg 86:1-11.

Blank, M. 1993. Biological effects of electromagnetic fields. Bioelectrochem Bioenerg 32:203-10.

Blank, M., ed. 1995. Electromagnetic Fields: Biological Interactions and Mechanisms. New York: American Chemical Society.

Blank, M., and E. Findl. 1987. Mechanistic Approaches to Interactions of Electromagnetic Fields with Living Systems. New York: Plenum Press.

Block, T. A., J. N. Aarsvold, K. L. Matthews, R. A. Mintzer, P. River, M. Capelli-Schellpfeffer, R. L. Wollmann, S. Tripathi, C.-T. Chen, and R. C. Lee. 1995. Nonthermally mediated muscle injury and necrosis in electrical trauma. J Burn Care Rehab 16:581-8.

Bordi, F., C. Cametti, and T. Gili. 2001. Reduction of the contribution of electrode polarization effects in the radiowave dielectric measurements of highly conductive biological cell suspensions. Bioelectrochemistry 54:53-61.

Buchner, R., J. Barthel, and J. Stauber. 1999. The dielectric relaxation of water between 0 degrees and 35 degrees C. Chem Phys Lett 306:57-63.

Budihardjo, I., H. Oliver, M. Lutter, X. Luo, and X. Wang. 1999. Biochemical pathways of caspase activation during apoptosis. Annu Rev Cell Dev Biol 15:269-90.

Buescher, E. S., and K. H. Schoenbach. 2003. Effects of submicrosecond, high intensity pulsed electric fields on living cells-intracellular electromanipulation. IEEE Trans Diel Electr Ins 10:788-94.

Chen, N., K. H. Schoenbach, J. F. Kolb, R. J. Swanson, A. L. Garner, J. Yang, R. P. Joshi, and S. J. Beebe. 2004. Leukemic cell intracellular responses to nanosecond electric fields. Biochem Biophys Res Comm 317:421-7.

Cheng, D. K. L., L. Tung, and E. A. Sobie. 1999. Nonuniform responses of transmembrane potential during electric field stimulation of single cardiac cells. Am J Physiol H351-62.

Clark, J., and R. Plonsey. 1968. The extracellular potential field of the single active nerve fiber in a volume conductor. Biophys J 8:842-64.

Cole, R. H. 1975. Evaluation of dielectric behavior by time domain spectroscopy. I. Dielectric response by real time analysis. J Phys Chem 79:1459-69.

Cole, K. S., and R. H. Cole. 1941. Dispersion and absorption in dielectrics I. Alternating current characteristics. J Chem Phys 9:341-51.

Cole, R. H., S. Mashimo, and P. Winsor IV. 1980. Evaluation of dielectric behavior by time domain spectroscopy. 3. Precision difference methods. J Phys Chem 84:786-93.

Croce, R. P., A. De Vita, V. Pierro, and I. M. Pinto. 2010. A thermal model for pulsed EM field exposure effects in cells at nonthermal levels. IEEE Trans Plasma Sci 38:149-55.

Davalos, R. V., L. M. Mir, and B. Rubinsky. 2005. Tissue ablation with irreversible electroporation. Ann Biomed Eng 33:223-31.

Davalos, R. V., B. Rubinsky, and L. M. Mir. 2003. Theoretical analysis on the thermal effects during in vivo tissue electroporation. Bioelectrochemistry 61:99-107. 
Davis, J. D., W. R. Bennett, J. V. Brady, R. L. Brent, L. Gordis, W. E. Gordon, S. W. Greenhouse, R. J. Reiter, G. S. Stein, C. Susskind, and D. Trichopoulos. 1992. Health effects of low-frequency electric and magnetic fields. Oak Ridge Associated Universities Panel Report ORAU 92/F8.

De Biase, P. M., D. A. Paggi, F. Doctorovich, P. Hildebrandt, D. A. Estrin, D. H. Murgida, and M. A. Marti. 2009. Molecular basis for the electric field modulation of cytochrome $c$ structure and function. J Am Chem Soc 131:16248-56.

DeBruin, K. A., and W. Krassowska. 1998. Electroporation and shock-induced transmembrane potential in a cardiac fiber during defibrillation strength shocks. Ann Biomed Eng 26:584-96.

DeBruin, K. A., and W. Krassowska. 1999. Modelling electroporation in a single cell. I. Effects of field strength and rest potential. Biophys J 77:1213-24.

Debye, P. 1929. Polar Molecules. New York: Chemical Catalog Co.

Despa, F., S. Basati, Z. D. Zhang, J. D’Andrea, J. P. Reilly, E. N. Bodnar, and R. C. Lee. 2009. Electromuscular incapacitation results from stimulation of spinal reflexes. Bioelectromagnetics 30:411-21.

Douglas Jr., J, J. E. Santos, and D. Sheen. 2000. A nonconforming mixed finite element method for Maxwell's equations. Math Models Methods Appl Sci 10:593-613.

Dukhin, S. S., and V. N. Shilov. 1974. Dielectric Phenomena and the Double Layer in Dispersive Systems. New York: John Wiley and Sons.

Edd, J. F., L. Horowitz, R. V. Davalos, L. M. Mir, and B. Rubinsky. 2006. in vivo results of a new focal tissue ablation technique: irreversible electroporation. IEEE Trans Biomed Engr 53:1409-15.

Elias, P. M., and G. K. Menon. 1991. Structural and lipid biochemical correlates of the epidermal permeability. Adv Lipid Res 24:1-26.

Ermolina, I., Y. Polevaya, Y. Feldman, B. Ginzburg, and M. Schlesinger. 2001. Study of normal and malignant white blood cells by time domain dielectric spectroscopy. IEEE Trans Dielectr Electr Ins 8:253-61.

Fear, E. C., and M. A. Stuchly. 1998. Biological cells with gap junctions in low-frequency electric fields. IEEE Trans Biomed Eng 45:856-66.

Feldman, Y., I. Ermolina, and Y. Hayashi. 2003. Time domain dielectric spectroscopy study of biological systems. IEEE Trans Diel Electr Ins 10:728-53.

Feldman, Y. D., and V. D. Fedotov. 1987. Investigation of rotational diffusion of globular proteins by time domain spectroscopy. Russ J Phys Chem 61:1045-51.

Fishler, M. G., E. A. Sobie, N. V. Thakor, and L. Tung. 1996. Mechanisms of cardiac cell excitation with premature monophasic and biphasic field stimuli: A model study. Biophys J 70:1347-62.

Foster, K. R. 2000. Thermal and nonthermal mechanisms of interaction of radio-frequency energy with biological systems. IEEE Trans Plasma Sci 28:15-23.

Foster, K. R., and H. P. Schwan. 1986. Dielectric properties of tissues. In Handbook of Biological Effects of Electromagnetic Fields, ed. C. Polk and E. Postow, 25-102. Boca Raton: CRC Press.

Foster, K. R., and H. P. Schwan. 1989. Dielectric properties of tissues and biological materials: A critical review. Crit Rev Biomed Eng 17:25-104. 
Foster, K. R., and H. P. Schwan. 1995. Dielectric properties of tissues-A review. In Handbook of Biological Effects of Electromagnetic Radiation, ed. C. Polk and E. Postow, 26-102. Boca Raton, Florida: CRC Press.

Freeman, S. A., M. A. Wang, and J. C. Weaver. 1994. Theory of electroporation of planar bilayer membranes: Predictions of the aqueous area, change in capacitance, and pore-pore separation. Biophys J 67:42-56.

Fricke, H. 1925. The electric capacity of suspensions with special reference to blood. J Gen Physiol 9:137-52.

Führ, G., R. Glaser, and R. Hagedorn. 1985. Rotation of dielectrics in a rotating electric high-frequency field: Model experiments and theoretical explanation of the rotation effect of living cells. Biophys J 49:395-402.

Gabriel, S. S., R. W. Lau, and C. Gabriel. 1996. The dielectric properties of biological tissues. 2. Measurements in the frequency range $10 \mathrm{~Hz}$ to $20 \mathrm{GHz}$. Phys Med Biol 41:2251-69.

Gaylor, D. C., K. Prakah-Asante, and R. C. Lee. 1988. Significance of cell size and tissue structure in electrical trauma. J Theor Biol 133:223-37.

Gimsa, J., and D. Wachner. 1999. A polarization model overcoming the geometric restrictions of the Laplace solution for spheroidal cells: Obtaining new equations for fieldinduced forces and transmembrane potential. Biophys J 77:1316-26.

Glaser, R. W., S. L. Leikin, L. V. Chernomordik, V. F. Pastushenko, and A. I. Sokirko. 1988. Reversible electrical breakdown of lipid bilayers: formation and evolution of pores. Biochimica et Biophysica Acta 940:275-87.

Goodman, E. M., B. Greenbaum, and M. T. Marron. 1995. Effects of electromagnetic fields on molecules and cells. Int Rev Cytology 158:279-338.

Gothelf, A., M. Mir, and J. Gehl. 2003. Electrochemotherapy: results of cancer treatment using enhanced delivery of Bleomycin by electroporation. Cancer Treat Rev 29:371-87.

Gowrishankar, T. R., A. T. Esser, Z. Vasilkoski, K. C. Smith, and J. C. Weaver. 2006. Microdosimetry for conventional and supra-electroporation in cells with organelles. Biochem Biophys Res Comm 341:1266-76.

Green, D. R. 2000. Apoptotic pathways: Paper wraps stone blunts scissors. Cell 102:1-4.

Grosse, C., and H. P. Schwan. 1992. Cellular membrane potentials induced by alternating fields. Biophys J 63:1632-42.

Groves, J. T., S. G. Boxer, and H. M. McConnell. 1997. Electric field induced reorganization of two-component supported bilayer membranes. Proc Natl Acad Sci USA 94:13390-5.

Hager III, N. 1994. Broadband time domain reflectometry dielectric spectroscopy using variable time scale sampling. Rev Sci Instrum 65:887-91.

Hanai, T. 1968. Electrical properties of emulsions. In Emulsion Science, ed. P. Sherman, Chap. 5, 354-478. New York: Academic Press.

Heller, R., R. Gilbert, and M. J. Jaroszeski. (1999). Clinical applications of electrochemotherapy. Adv Drug Delivery Rev 35:119-29.

Hocking, B., K. Joyner, H. Newman, and R. Aldred. 1994. Case report: Radiofrequency shock and burn. Med J Aust 161:683-5.

Hoeber, R. 1910. Eine methode die elektrische leitf ahigkeit im inner von zellen zu messen. Arch Ges Physiol 133:237-59. 
Hsiao, G. C. 1990. The coupling of boundary element and finite element methods. $Z$ Angew Math Mech 70:493-503.

$\mathrm{Hu}, \mathrm{Q}$., and R. P. Joshi. 2009. Analysis of intense, subnanosecond electrical pulse-induced transmembrane voltage in spheroidal cells with arbitrary orientation. IEEE Trans Biomedical Eng 56:1617-26.

Ilic L., T. R. Gowrishankar, T. E. Vaughan, T. O. Herndon, and J. C. Weaver. 1999. Spatially constrained skin electroporation with sodium thiosulfate and urea creates transdermal microconduits. J Controlled Release 61:185-202.

Irimajiri, A., T. Hanai, and A. Inouye. 1979. A dielectric theory of "multi-stratified shell" model with its application to a lymphoma cell. J Theor Biol 78:251-69.

Isambert, H. 1998. Understanding the electroporation of cells and artificial bilayer membranes. Phys Rev Lett 80:3404-7.

Jin, J. 1993. Finite Element Method in Electromagnetics. New York: Wiley.

Johnson, C., and J. C. Nedelec. 1980. Coupling of boundary integral and finite element methods. Math Comput 35:1063-1080.

Joshi, R. P., Q. Hu, R. Aly, K. H. Schoenbach, and H. P. Hjalmarson. 2001. Self-consistent simulations of electroporation dynamics in biological cells subjected to ultrafast electrical pulses. Phys Rev E 64:011913.

Joshi, R. P., Q. Hu, and K. H. Schoenbach. 2004. Modeling studies of cell response to ultrashort, high-intensity electric fields - implications for intracellular manipulation. IEEE Trans Plasma Sci 32:1677-86.

Joshi, R. P., Q. Hu, K. H. Schoenbach, and S. J. Beebe. 2004. Energy-landscape-model analysis for irreversibility and its pulse-width dependence in cells subjected to a high-intensity ultrashort electric pulse. Phys Rev E 69:051901.

Joshi, R. P., Q. Hu, K. H. Schoenbach, and H. P. Hjalmarson. 2002. Improved energy model for membrane electroporation in biological cells subjected to electrical pulses. Phys Rev E 65:041920.

Joshi, R. P., A. Mishra, J. Song, A. Pakhomov, and K. H. Schoenbach. 2008. Simulation studies of ultrashort, high-intensity electric pulse induced action potential block in whole-animal nerves. IEEE Trans Biomed Eng 55:1391-8.

Joshi, R. P., A. Nguyen, V. Sridhara, Q. Hu, R. Nuccitelli, and K. H. Schoenbach. 2007. Simulations of intra-cellular calcium release dynamics in response to a highintensity, ultra-short electric pulse. Phys Rev E 75:041920.

Joshi, R. P., and K. H. Schoenbach. 2000. Electroporation dynamics in biological cells subjected to ultrafast electrical pulses: A numerical simulation study. Phys Rev E 62:1025-33.

Joshi, R. P., and J. Song. 2010. Model analysis of electric fields induced by high-voltage pulsing in cylindrical nerves. IEEE Trans Plasma Sci 38:2894-900.

Joshi, R. P., J. Song, and V. Sridhara. 2009. Aspects of lipid membrane bio-responses to subnanosecond, ultrahigh voltage pulsing. IEEE Trans Dielectr and Electr Insula 16:1243-50.

Kennedy, S. M., Z. Ji, J. C. Hedstrom, J. H. Booske, and S. C. Hagness. 2008. Quantification of electroporative uptake kinetics and electric field heterogeneity effects in cells. Biophys J 94:5018-27.

Kinosita Jr, K., and T. Y. Tsong. 1977. Formation and resealing of pores of controlled sizes in human erythrocyte membrane. Nature 268:438-41.

Kirkwood, J. G. 1939. The dielectric polarization of polar liquids. J Chem Phys 7:911-9. 
Kolb, J. F., K. Susumu, and K. H. Schoenbach. 2006. Nanosecond pulsed electric field generators for the study of subcellular effects. Bioelectromagnetics 27:172-87.

Kotnik, T., and D. Miklavcic. 2000a. Analytical description of transmembrane voltage induced by electric fields on spheroidal cells. Biophys J 79:670-9.

Kotnik, T., and D. Miklavcic. 2000b. Second-order model of membrane electric field induced by alternating external electric fields. IEEE Trans Biomed Engr 47:1074-81.

Kotnik, T., and D. Miclavcic. 2006. Theoretical evaluation of voltage inducement on internal membranes of biological cells exposed to electric fields. Biophys J 90:480-91.

Kranich, A., H. K. Ly, P. Hildebrandt, and D. H. Murgida. 2008. Direct observation of the gating step in protein electron transfer: electric-field-controlled protein dynamics. J Am Chem Soc 130:9844-8.

Krassowska, W., and J. C. Neu. 1994. Response of a single cell to an external electric field. Biophys J 66:1768-76.

Kythe, P. K. 1995. An Introduction to Boundary Element Methods. Boca Raton, FL: CRC.

Lazebnik, M., D. Popovi, L. MacCartney, C. B. Watkins, M. J. Lindstron, J. Harter, S. Sewall, T. Ogilvie, A. Magliocco, T. M. Breslin, W. Temple et al. 2007. A large-scale study of ultrawideband microwave dielectric properties of normal, benign and malignant breast tissues obtained from cancer surgeries. Phys Med Biol 52:6093-115.

Lee, R. C., D. J. Canaday, and S. M. Hammer. 1993. Transient and stable ionic permeabilization of isolated skeletal muscle cells after electrical shock. J Burn Care Rehab 14:528-40.

Lei, C., and H. Berg. 1998. Electromagnetic window effects on proliferation rate of Corynebacterium glutamicum. Bioelectrochem Bioenerg 45:261-5.

Li, P., D. Nijhawan, I. Budihardjo, S. M. Srinivasula, M. Ahmad, E. S. Alnemri, and X. Wang. 1997. Cytochrome $c$ and dATP-dependent formation of Apaf-1/caspase-9 complex initiates an apoptotic protease cascade. Cell 91:479-89.

Lohmann, K. J., and C. M. F. Lohmann. 1996. Detection of magnetic field intensity by sea turtles. Nature 380:59-61.

Marzo, I., C. Brenner, N. Zamzami, J. M. Jürgensmeier, S. A. Susin, H. L. A. Vieira, M. C. Prévost, Z. Xie, S. Matsuyama, J. C. Reed, and G. Kroemer. 1998. Bax and adenine nucleotide translocator cooperate in the mitochondrial control of apoptosis. Science 281:2027-31.

Maswiwat, K., D. Wachner, R. Warnke, and J. Gima. 2007. Simplified equations for the transmembrane potential induced in ellipsoidal cells of rotational symmetry. J Phys D 40:914-923.

Maxwell, J. C. 1891. Treatise of Electricity and Magnetism. Oxford: Clarendon Press.

McNeal, D. R. 1976. Analysis of a model for excitation of myelinated nerve. IEEE Trans Biomed Engr 23:329-37.

Mehedintu, M., and H. Berg. 1997. Proliferation response of yeast Saccharomyces cerelisiae on electromagnetic field parameters. Bioelectrochem Bioenerg 43:67-70.

Melikov, K. C., V. A. Frolov, A. Shcherbakov, A. V. Samsonov, and Y. A. Chizmadzhev. 2001. Voltage-induced nonconductive prepores and metastable pores in unmodified planar bilayer. Biophys J 80:1829-36.

Merla, C., M. Liberti, F. Apollonio, and G. d'Inzeo. 2009. Quantitative assessment of dielectric parameters for membrane lipid bi-layers from RF permittivity measurements. Bioelectromagnetics 30:286-98. 
Michaels, A. S., S. K. Chasekaran, and J. E. Shaw. 1975. Drug permeation through human skin: Theory and in vitro experimental measurements. AIChEJ 21:985-96.

Miller, C. E., and C. S. Henriquez. 1988. Three-dimensional finite element solution for biopotentials: Erythrocyte in an applied field. IEEE Trans Biomed Eng 35:712-8.

Mir, L. M., M. F. Bureau, J. Gehl, T. Nakada, I. Sasagawa, H. Suzuki, and N. Aoyama. 1999. High efficiency gene transfer into skeletal muscle mediated by electric pulses. Proc Natl Acad Sci USA 96:4262-7.

Mir, L. M., S. Orlowski, J. Belehradek Jr, J. Teissié, M. P. Rols, G. Sersa, D. Miklavcic, R. Gilbert, and R. Heller. 1995. Biomedical applications of electric pulses with special emphasis on antitumor electrochemotherapy. Bioelectrochem Bioenerg 38:203-7.

Mouneimne, Y., P. F. Tosi, R. Barhoumi, and C. Nicolau. 1990. Electroinsertion of full length recombinant CD4 into red blood cell membrane. Biochim Biophys Acta 1027:53-58.

Muramatsu, T., A. Nakamura, and H. Park. 1998. in vivo electroporation: a powerful and convenient means of nontrivial gene transfer to tissues of living animals. Int J Mol Med 1:55-62.

Neu, J. C., and W. Krassowska. 2006. Singular perturbation analysis of the pore creation transient. Phys Rev E 74:031917.

Neumann, E., S. Kakorin, and K. Toensig. 1999. Fundamentals of electroporative delivery of drugs and genes. Bioelectrochem Bioenerg 48:3-16.

Neumann, E., and K. Rosenheck. 1972. Permeability changes induced by electric impulses in vesicular membranes. J Membrane Biol 10:279-90.

Neumann, E., M. Schaefer-Ridder, Y. Wang, and P. H. Hofschneider. 1982. Gene transfer into mouse lyoma cells by electroporation in high electric fields. EMBO J 1:841-5.

Nuccitelli, R., U. Pliquett, X. Chen, W. Ford, J. Swanson, S. J. Beebe, J. F. Kolb, and K. H. Schoenbach. 2006. Nanosecond pulsed electric fields cause melanomas to selfdestruct. Biochem Biophys Res Commun 343:351-60.

Oncley, J. L. 1938. Studies of the dielectric properties of protein solution I. Carboxyhaemoglobin. J Am Chem Soc 60:1115-23.

O'Neill, R. J., and L. Tung. 1991. Cell-attached patch clamp study of the electropermeabilization of amphibian cardiac cells. Biophys J 59:1028-39.

Onsager, L. 1936. Electric moments of molecules in liquids. J Am Chem Soc 58:1486-93.

Pakhomov, A. G., J. K. Kolb, R. P. Joshi, K. H. Schoenbach, T. Dayton, J. Comeaux, J. Ashmore, and C. Beason. 2006. Neuromuscular disruption with ultrashort electrical pulses. Proc SPIE-Int Soc Opt Eng 6219:621903-10.

Pakhomov, A. G., A. Phinney, J. Ashmore, K. Walker III, J. F. Kolb, S. Kono, K. H. Schoenbach, and M. R. Murphy. 2004. Characterization of the cytotoxic effect of high-intensity, 10-ns duration electrical pulses. IEEE Trans Plasma Sci 32:1579-86.

Pakhomov, A. G., R. Shevin, J. A. White, J. F. Kolb, O. N. Pakhomova, R. P. Joshi, and K. H. Schoenbach. 2007. Membrane permeabilization and cell damage by ultrashort electric field shocks. Arch Biochem Biophys 465:109-118.

Pastushenko, V. F., and Y. A. Chizmadzhev. 1982. Stabilization of conducting pores in BLM by electric current. Gen Physiol Biophys 1:43-52.

Pastushenko, V. F., and Y. A. Chizmadzhev. 1983. Electrical breakdown of lipid vesicles. Biofizika 28:1036-9. 
Pauly, H., and H. P. Schwan. 1959. Über die Impedanz einer Suspension von kugelförmigen Teilchen mit einer Schale. Z Naturforsch 14B:125-131.

Peter, M. E., and P. H. Krammer. 2003. The CD95(APO-1/Fas) DISC and beyond. Cell Death Differ 10:26-35.

Polk, C., and E. Postow. 1996. CRC Handbook of Biological Effects of Electromagnetic Fields. 2nd ed. Boca Raton: CRC Press.

Pucihar, G., T. Kotnik, B. Valic, and D. Miklavcic. 2006. Numerical determination of transmembrane voltage induced on irregularly shaped cell. Ann Biomed Eng 34:642-52.

Radu, M., M. Ionescu, N. Irimescu, K. Iliescu, R. Pologea-Moraru, and E. Kovacs. 2005. Orientation behavior of retinal photoreceptors in alternating electric fields. Biophys $J$ 89:3548-54.

Raffy, S., and J. Teissié. 1995. Insertion of glycophorin A, a transmembraneous protein, in lipid bilayers can be mediated by electropermeabilization. Eur J Biochem 230:722-32.

Rattay, F. 1990. Electrical Nerve Stimulation: Theory, Experiments and Applications. New York: Springer.

Reilly, J. P. 1992. Electrical Stimulation and Electropathology. Cambridge: Cambridge University Press.

Reilly, J. P. 1998. Applied Bioelectricity. New York: Springer-Verlag.

Rols, M. P., J. M. Bachaud, P. Giraud, C. Chevreau, H. Roche, and J. Teissie. 2000. Electrochemotherapy of cutaneous metastases in malignant melanoma. Melanoma Res 10:468-74.

Rols, M. P., and J. Teissie. 1998. Electropermeabilization of mammalian cells to macromolecules: control by pulse duration. Biophys J 75:1415-23.

Sale, A. J. H., and W. A. Hamilton. 1967. Effects of high electric fields on microorganisms: II. killing of bacteria and yeasts. Biochem Biophys Acta 148:781-8.

Sale, A. J. H., and W. A. Hamilton. 1968. Effects of high electric fields on microorganisms: III. lysis of erythrocytes and protoplasts. Biochim Biophys Acta 163:37-43.

Saunders, R. D., Z. J. Sienkiewicz, and C. I. Kowalczuk. 1991. Biological effects of electromagnetic fields and radiation. J Radiol Prot 11:27-42.

Savitz, D. A., H. A. Wachtel, S. Barnes, E. M. John, and J. C. Tvrdik. 1988. Case control study of childhood cancer and exposure to $60 \mathrm{~Hz}$ magnetic fields. Am J Epidemiol 128:21-38.

Schnabel, V., and J. J. Struijk. 2001. Evaluation of the cable model for electrical simulation of unmyelinated nerve fibers. IEEE Trans Biomed Engr 48:1027-33.

Schoenbach, K. H., S. J. Beebe, and E. S. Buescher. 2001. Intracellular effect of ultrashort electrical pulses. J Bioelectromagnetics 22:440-8.

Schoenbach, K. H., B. Hargrave, R. P. Joshi, J. F. Kolb, C. Osgood, R. Nuccitelli, A. Pakhomov, R. J. Swanson, M. Stacey, J. A. White, S. Xiao et al. 2007. Bioelectric effects of intense nanosecond pulses. IEEE Trans Diel Electr Insul 14:1088-119.

Schoenbach, K. H., R. P. Joshi, S. J. Beebe, and C. E. Baum. 2009. A scaling law for membrane permeabilization with nanopulses. IEEE Trans Diel Electr Insul 16:1224-35.

Schoenbach, K. H., R. P. Joshi, J. Kolb, N. Chen, M. Stacey, P. Blackmore, E. S. Buescher, and S. J. Beebe. 2004. Ultrashort electrical pulses open a new gateway into biological cells. Proc IEEE 92:1122-37. 
Schoenbach, K. H., S. Xiao, R. P. Joshi, J. T. Camp, T. Heeren, J. F. Kolb, and S. J. Beebe. 2008. The effect of intense subnanosecond electrical pulses on biological cells. IEEE Trans Plasma Sci 36:414-24.

Schwan, H. P. 1957. Electrical properties in tissue and cell suspensions. Adv Biol Med Phys 5:147-209.

Schwan, H. P. 1983. Biophysics of the interaction of electromagnetic energy with cells and membranes. In Biological Effects and Dosimetry of Nonionizing Radiation, ed. M. Grandolfo, S. M. Michaelson, and A. Rindl, 213-31. New York: Plenum Press.

Semenov, S. Y., R. H. Svenson, V. G. Posukh, A. G. Nazarov, Yu E. Sizov, A. E. Bulyshev, A. E. Souvorov, W. Chen, J. Kasell, and G. P. Tatsis. 2002. Dielectric spectroscopy of canine myocardium during ischemia and hypoxia at frequency spectrum from 100 $\mathrm{kHz}$ to $6 \mathrm{GHz}$. IEEE Trans Med Imaging 21:703-07.

Serpersu, E. H., K. Kinosita Jr, and T. Y. Tsong. 1985. Reversible and irreversible modification of erythrocyte membrane permeability by electric field. Biochem Biophys Acta 812:779-85.

Sersa, G., M. Cemažar, and D. Miklavcic. 1995. Antitumor effectiveness of electrochemotherapy with cis-diamminedichloroplatinum(II) in mice. Cancer Res 55:3450-5.

Smith, K. C., T. R. Gowrishankar, A. T. Esser, D. A. Stewart, and J. C. Weaver. 2006. The spatially distributed dynamic transmembrane voltage of cells and organelles due to 10 ns pulses: meshed transport networks. IEEE Trans Plasma Sci 34:1394-404.

Smoluchowski, M. 1916. Drei vortrage uber diffusion, Brownsche bewegung und koagulation von kolloidteilchen. Physik Zeits 17:557-85.

Song, J., R. P. Joshi, and S. J. Beebe. 2010. Cellular apoptosis by nanosecond, high-intensity electric pulses: model evaluation of the pulsing threshold and extrinsic pathway. Bioelectrochemistry 79:179-86.

Sowers, A. E. 1987. Cell Fusion. New York: Plenum.

Stampfli, R. 1958. Reversible electrical breakdown of the excitable membrane of a Ranvier node. An Acad Brasil Ciens 30:57-63.

Stewart, D. A., T. R. Gowrishankar, and J. C. Weaver. 2006. Three dimensional transport lattice model for describing action potentials in axons stimulated by external electrodes. Bioelectrochemistry 69:88-93.

Sukhorukov, V. L., H. Mussauer, and U. Zimmermann. 1998. The effect of electrical deformation forces on the electropermeabilization of erythrocyte membranes in low- and high-conductivity media. J Membr Biol 163:235-45.

Suzuki, T., B. Shin, K. Fujikura, T. Matsuzaki, and K. Takata. 1998. Direct gene transfer into rat liver cells by electroporation. FEBS Lett 425:436-40.

Takashima, S. 1969. Dielectric properties of proteins. Dielectric relaxation. In Physical Principles and Techniques of Protein Chemistry. New York: Academic Press.

Teissie, J., N. Eynard, B. Gabriel, and M. P. Rols. 1999. Electropermeabilization of cell membranes. Adv Drug Deliv Rev 35:3-19.

Teissie, J., and T. Y. Tsong. 1981. Electric field induced transient pores in phospholipid bilayer vesicles. Biochemistry 20:1548-54.

Tung, L., and J. R. Borderies. 1992. Analysis of electric field stimulation of single cardiac muscle cells. Biophys J 63:371-86. 
Vasilkoski, Z., A. T. Esser, T. R. Gowrishankar, and J. C. Weaver. 2006. Membrane electroporation: the absolute rate equation and nanosecond time scale pore creation. Phys Rev E 74:021904.

Vernier, P. T., Y. Sun, M. T. Chen, M. A. Gundersen, and G. L. Craviso. 2008. Nanosecond electric pulse-induced calcium entry into chromaffin cells. Bioelectrochemistry 73:1-4.

Vernier, P. T., Y. Sun, L. Marcu, S. Salemi, C. M. Craft, and M. A. Gundersen. 2003 Calcium bursts induced by nanosecond electric pulses. Biochem Biophys Res Commun 310:286-95.

Wagner, K. W. 1914. Erklärung der dielectrischen nachwirkungsvorgänge auf grund maxwellscher vorstellungen. Arch Electrotech 2:371-87.

Wang, Z., Y. Alfadhl, and X. Chen. 2008. Numerical microdosimetry of complex shaped cell models in electroporation. IEEE Antennas Propag Soc, AP-S Int Symp (Digest) 4320-23.

Wang, R. Y., Y. R. Yang, M. W. Tsai, W. Y. J. Wang, and R. C. Chan. 2002. Effects of functional electric stimulation on upper limb motor function and shoulder range of motion in hemiplegic patients. Am J Phys Med Rehabil 81:283-90.

Weaver, J. C. 2000. Electroporation of cells and tissues. IEEE Trans Plasma Sci 28:24-33.

Weaver, J. C. 2003. Electroporation of biological membranes from multicellular to nanoscales. IEEE Trans Diel Electr Ins 10:754-68.

Weaver, J. C., and Y. Chizmadzhev. 1996. Theory of electroporation: A review. Bioelectrochem Bioenerg 41:135-60.

Weaver, J. C., and R. A. Mintzer. 1981. Decreased bilayer stability due to transmembrane potentials. Phys Lett A 86:57-9.

Weaver, J. C., T. E. Vaughan, and Y. Chizmadzhev. 1999. Theory of electrical creation of aqueous pathways across skin transport barriers. Adv Drug Deliv Rev 35:21-39.

Wertheimer, N., and E. Leeper. 1979. Electrical wiring configurations and childhood cancer. Am J Epidemid 109:273-84.

West, R. W., W. G. Hinson, D. B. Lyle, and M. L. Swicord. 1994. Enhancement of anchorageindependent growth in JB6 cells exposed to $60 \mathrm{Hertz}$ magnetic fields. Bioelectrochem Bioenerg 34:39-43.

Wexler, A. S., J. Ding, and S. A. Binder-Macleod. 1997. A mathematical model that predicts skeletal muscle force. IEEE Trans Biomed Engr 44:337-48.

Wiltschko, W., and R. Wiltschko. 1995. Magnetic Orientation in Animals. Berlin: Springer Verlag.

Winterhalter, M., and W. Helfrich. 1987. Effect of voltage on pores in membranes. Phys Rev A 36:5874-6.

Zimmermann, U. 1982. Electric field mediated fusion and related electrical phenomena. Biochim Biophys Acta 694:227-77.

Zoratti, M., and I. Szabo. 1995. The mitochondrial permeability transition. Biochim Biophys Acta 1241:139-76. 


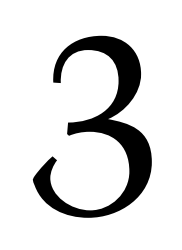

\section{Static, Low- Frequency, and Pulsed Magnetic Fields in Biological Systems}

3.1 Introduction 115

3.2 Mechanisms of Biological Effects and

Biomagnetic Phenomena of Magnetic Fields ... 116

3.3 Experimental Studies on Magnetic

Field Effects.

In Vivo Studies - Tissue, Molecular, and

Cellular Studies

3.4 Response of Plants and Microorganisms to Magnetic Fields

Plant Growth, Response, and

Magnetotropism - Magnetotaxis - Antibacterial Effects

3.5 Medical Applications

Transcranial Magnetic Stimulation and Induced

Current - Magnetoencephalography - Magnetic

Resonance Imaging - Magnetic Orientation for

Tissue Engineering - Treatments of Pain, Cancer, and Other Diseases

Shoogo Ueno

Hideyuki Okano
3.6 Conclusion

Acknowledgment

References.

\subsection{Introduction}

It is presumed that life on Earth has evolved in the presence of natural and ubiquitous magnetic fields, that is, the geomagnetic field and magnetic storms. It is not surprising that biological systems can respond to a wide range of magnetic fields including 
static magnetic fields (SMFs), low-frequency magnetic fields, and pulsed magnetic fields. Regarding the magnetic sensing mechanism of biological systems, it has been reported that animals can detect small changes in the geomagnetic field by two distinct mechanisms: (1) using the mineral magnetite as the primary sensor and (2) using magnetically sensitive chemical reactions. Several models have been proposed to account for the effects of magnetic fields on biological sensing and reactions.

In modern society, there are many sources of electromagnetic fields (EMFs) or time-varying magnetic fields. Humans are exposed daily to human-made and naturally originated fields. During the past decade, questions about whether the exposure to EMFs may be linked with adverse health effects have been raised. Although the interaction of nonthermal EMFs, in particular low frequencies up to $300 \mathrm{~Hz}$, with biological systems has been investigated, there is no biophysical mechanism that can explain many of the observed biological effects of low and moderate levels of magnetic fields $(<1 \mathrm{~T})$. Proposed mechanisms include effects on electric currents, direct forces on biomagnetic materials, effects on free radicals, ion cyclotron resonance (ICR), charge transfer processes, stochastic resonance, and a dynamical systems/Larmor procession model, etc (see the review by Shigemitsu 2006; Pilla 2007).

Recent developments in medical instrumentation such as magnetic resonance imaging (MRI) and transcranial magnetic stimulation (TMS) have raised questions as to whether strong (in the tesla [T] range) magnetic fields influence human health. Medical applications of SMFs of moderate intensities (1-200 mT) for pain reduction and tissue healing have also been studied for many years. Therapeutic applications of permanent magnets and other electromagnetic devices have recently been expanded to various areas such as treatments of pain and diseases like rheumatoid arthritis and cancer.

The objective of this chapter is to describe and shed light on some of the more recent information on the biological effects and medical applications of magnetic fields. A discussion of possible implications of these effects on biological systems is also provided. This chapter consists of four sections. Each section contains a comprehensive review of a recent topic of interest. Short summaries of the mechanism of magnetic field action on biological systems and the biomagnetic phenomena are described in Section 3.2, which includes a brief review of well-known mechanisms that are discussed before mentioning the interactions of weak SMFs with biological systems including magnetic sensing and sensing using magnetite as the primary sensor. Section 3.3 reviews and summarizes more recent in vivo and in vitro experimental results of the effects of magnetic fields including TMS and MRI fields. Section 3.4 covers special topics including the response of plants to magnetic fields. In parallel with a comprehensive review on biological effects, the development and medical applications of biomagnetic phenomena are introduced in Section 3.5, and they are reviewed with emphasis on the applications that are currently under investigation.

\subsection{Mechanisms of Biological Effects and Biomagnetic Phenomena of Magnetic Fields}

Table 3.1 shows three types of well-known mechanisms of the biological effects of magnetic fields including SMFs and time-varying magnetic fields (Ueno and Shigemitsu 2007). As shown in Table 3.1, there are two basic mechanisms of SMFs: (1) magnetic 
TABLE 3.1 Well-Known Mechanisms of the Biological Effects of Magnetic Fields

1. Time-Varying Magnetic Field

$$
\begin{array}{ll}
\text { Eddy currents } J=-\sigma \frac{B}{t} & \text { Nerve stimulation } \\
\text { Heat SAR }=\sigma \frac{E^{2}}{\rho} & \text { Thermal effects }
\end{array}
$$

2. Static Magnetic Fields

a. Homogeneous magnetic field magnetic torque

$$
T=-\frac{1}{2 \mu_{0}} B^{2} \Delta x \sin 2 \theta
$$

b. Inhomogeneous magnetic field magnetic force Parting of water by magnetic fields (Moses effect)

$$
F=\frac{x}{\mu_{0}}(\operatorname{grad} B) B
$$

3. Multiplication of magnetic fields and other energy

Yield effect of cage product and escape product

Photochemical reactions with radical pairs

Singlet-triplet intersystem crossing

Source: Ueno, S., and T. Shigemitsu. 2007. Biological effects of static magnetic fields. Handbook of Biological Effects of Electromagnetic Fields: Bioengineering and Biophysical Aspects of Electromagnetic Fields. 3rd ed. F. S. Barnes, and B. Greenebaum, 203. Boca Raton, FL: CRC Press. With permission.

torques on objects and (2) mechanical force effects. A radical pair mechanism is also proposed. When biological materials or systems are exposed to a spatially homogeneous SMF, they tend to rotate to a stable direction, which is determined by the anisotropy of magnetic susceptibility of the materials and magnetic torque $T$ acting on the materials, as described by the following equation:

$$
T=-\frac{1}{2 \mu_{0}} B^{2} \Delta x \sin 2 \theta
$$

where $B$ is the magnetic flux density, $\Delta x$ is the anisotropy of magnetic susceptibility of the materials, $\theta$ is the angle between magnetic field (magnetic flux) direction and the long axis of the materials, and $\mu_{0}$ is the magnetic permeability of vacuum. The magnetic orientation of diamagnetic materials such as fibrin and collagen can be observed and explained by this principle (Torbet, Fryssinet, and Hudry-Clergeon 1981).

Next, when biological materials or systems are exposed to a spatially inhomogeneous SMF, the materials or systems tend to move along the direction of the steepest gradient of magnetic force. The magnetic force $F$ acting on materials is proportional to the product of magnetic flux density $B$, gradient of magnetic flux density $\operatorname{grad} B$, and magnetic susceptibility $x$ of the materials, as described by the following equation:

$$
F=\frac{x}{\mu_{0}}(\operatorname{grad} B) B
$$


where $\mu_{0}$ is the magnetic permeability of vacuum. Dramatic demonstrations of these forces can be seen when water is parted by an SMF of 4-8 T with a gradient of $50 \mathrm{~T} / \mathrm{m}$ (Ueno and Iwasaka 1994a,b). Magnetic levitation of diamagnetic materials such as wood and other organic materials is realized in magnetic fields $\geq 20 \mathrm{~T}$ (Beaugnon and Tournier 1991). This phenomenon can also be explained by the principles of magnetic force: the "force product" of maximum magnetic density and its maximum gradient. Moreover, if magnetic forces are strong enough the repulsion of diamagnetic objects can balance gravity, and objects levitated in this way can be held in stable equilibrium, apparently violating Earnshaw's theorem (Berryy and Geimz 1997). A variety of objects (one of which was a living frog) was levitated in a magnetic force of about $1400 \mathrm{~T}^{2} / \mathrm{m}$ in $16 \mathrm{~T}$.

The magnetic field effect observed with radical pair recombination is one of the well-known mechanisms by which magnetic fields interact with biological systems. Throughout the past decades there have been several experimental results describing the effects of magnetic fields on radical pair recombination.

As reviewed recently by Ueno and Shigemitsu (2007), several biophysical and biochemical effects can be expected when biological systems are simultaneously exposed to SMFs and other forms of energy such as light and radiation (Ueno and Harada 1986; McLauchlan and Steiner 1991). Photochemical reactions produced by a radical pair intermediate are expected to show SMF effects that arise from an electron Zeeman interaction; an electron-nuclear hyperfine interaction (Fermi-contact interaction); or a hyperfine interaction mechanism, such as an electron-exchange interaction in a radical pair intermediate (Hata 1976; Tanimoto et al. 1976, 1989; Schulten 1982a; Nagakura and Molin 1992; Natarajan and Grissom 1997; Nagakura, Hayashi, and Azumi 1998; Hayashi 2004).

Biological free radicals are most commonly oxygen or nitrogen based with an unpaired electron, leading to them being known by the terms reactive oxygen species (ROS), such as superoxide anion $\left(\mathrm{O}_{2}^{-}\right)$, hydroxyl radical $(\mathrm{OH} \bullet)$, and singlet oxygen $\left({ }^{1} \mathrm{O}_{2}\right)$, or reactive nitrogen species (RNS), such as nitric oxide (NO) (Engström 2007). The ROS and RNS play significant roles in immunological defense (Nathan 1992), intracellular signaling (Lander 1997), and intercellular communication (Thannickal and Fanburg 2000) of biological systems. It is assumed that exposure to SMF can change the lifetime of radical pairs and the yields of cage products (the yield of recombination reaction of the radical pair in solvent cage) and escape products (the dissociated radicals outside the solvent cage). If an SMF affects cells through the radical pair mechanism, an SMF influences the spin of electrons in free radicals, which may lead to changes in chemical reaction kinetics and alter cellular function (Brocklehurst and McLauchlan 1996). The dependence of a reaction yield on an external magnetic field strength is called a MARY spectrum (MARY stands for magnetically affected reaction yield) (Hayashi 2004).

Free radical reactions are ubiquitous in biology, and recent developments of the radical pair mechanism of low-field effects $(\leq 1 \mathrm{mT})$, including the effects of SMFs and EMFs (Schulten 1982b; Scaiano, Cozens, and McLean 1994; Brocklehurst and McLauchlan 1996; Timmel et al. 1998; Eveson et al. 2000; Brocklehurst 2002; Wang and Ritz 2006), and the consideration of detailed biochemical and biophysical systems (Cintolesi et al. 2003; Liu et al. 2005; Efimova and Hore 2008) make this mechanism a prime candidate for studying the effects down to the geomagnetic field strength, as recently reviewed by 
Engström (2007). Several models describing the influence of magnetic field on kinetics of enzymatic reactions that involve free radical-dependent chemistry have been elaborated. These models demonstrate that even subtle alterations in radical pair recombination kinetics induced by weak magnetic fields might lead to measurable effects on enzyme activity (Eichwald and Walleczek 1996; Izmaylov, Tully, and Frisch 2009). Study of the effects of magnetic field on radical pair reactions that may have biological consequences is important (Brocklehurst 2002). According to theoretical estimates, the physical transduction step induced by low fields is not vulnerable to thermal perturbations (Walleczek 1995; Adair 1999). Regarding the spin states of radicals, the radical pair theory predicts that an applied magnetic field perturbs the interconversion of the singlet and triplet states, resulting in an increase in the proportion of the triplet state and thus the free radical concentration (Brocklehurst 2002).

Therefore, knowing the effects of magnetic fields on free radical reactions is particularly important when considering human health and the relation of immunological and neurodegenerative diseases and stress response. Several attempts have been made to explore the parameters of free radical reactions occurring when living organisms, cells, and biochemicals are exposed to magnetic fields.

Regarding magnetic spin effects in radical enzymatic reactions involving at least one ROS, the Grissom research group (Harkins and Grissom 1994) showed experimentally that the activity of the $\mathrm{B}_{12}$-dependent enzyme ethanolamine ammonia lyase changes with exposure to SMFs of $100 \mathrm{mT}$. This finding is the first recorded SMF effect on an enzyme-catalyzed reaction with known radical pair intermediates in a cell-free solution. It is a milestone in biomagnetism research on free radicals. Extensive experiments have also been carried out with the heme enzymes horseradish peroxidase (HRP) and cytochrome $\mathrm{C}$ oxidase. The Grissom research group reported that the rate of HRP increases by $20 \%$ at fields as low as $1 \mathrm{mT}$ (Taraban et al. 1997). However, a recent detailed reinvestigation by the Woodward research group (Jones, Scrutton, and Woodward 2006) demonstrated that the reported effects of SMF on HRP were not observed up to $75 \mathrm{mT}$. Instead, the same research group studied the radical recombination reaction of radicals generated from the photolysis of 2-hydroxy-4'-(2-hydroxyethoxy)-2-methylpropiophenone $(\alpha-H P)$ on a microsecond timescale using time-resolved mid-infrared spectroscopy (Vink and Woodward 2004). The reaction was found to exhibit opposite biphasic magnetic field dependencies at 2 and $21 \mathrm{mT}$, and the effect at low fields was the first such observation for neutral free radicals in isotropic solution.

Mohtat et al. (1998) examined the behavior of radical pairs derived by hydrogen abstraction of the triplet state of benzophenone and some of its derivatives from bovine serum albumin, human serum albumin, and calf thymus deoxyribonucleic acid (DNA). The SMF strength was as high as $150 \mathrm{mT}$ with durations as long as 10 milliseconds. The results of this study indicated that radical pair behavior is sensitive to magnetic fields, and this effect can be interpreted by using the theory of free radical recombination.

The Hore research group researched the weak SMF effects on free radical recombination reactions (Till et al. 1998; Timmel et al. 1998). Using the triplet state of benzophenone as a convenient source of pairs, the Hore research group examined the effects of weak (1-2 $\mathrm{mT})$ SMF on radical recombination reactions in micelles 
(Eveson et al. 2000). They found that the concentration of free radicals escaping from the micelle was not only affected by, but also depended on the conditions surrounding the radical pair. The Hore research group demonstrated that the yield of ${ }^{1} \mathrm{O}_{2}$ sensitized by chemically modified, carotenoid-less (quinine-depleted) bacterial photosynthetic reaction centers on the R-26 mutant of Rhodobacter sphaeroides and, as a consequence, the stability of the reaction center protein is strongly affected by an SMF of even a few milliteslas: a $50 \%$ reduction for fields of $20-100 \mathrm{mT}$ and a $10 \%$ reduction for fields of $1 \mathrm{mT}$ (Liu et al. 2005).

Ritz, Dommer, and Phillips (2002) reviewed the physiological basis of animal magnetoreception and suggested a possible link between photoreception and magnetoreception from their findings in behavioral and theoretical studies. Migratory birds have the ability to sense the geomagnetic field and use it as a source of compass information. The candidates for a biophysical mechanism of this magnetoreception capability are magnetite and magnetically sensitive chemical reactions in animals. Ritz, Adem, and Schulten (2000) postulated the possibility that magnetoreception involves radical pair processes as a biophysical mechanism. The radical pair mechanism theory has been developed, which provides insight into the magnetic compass of living organisms using the geomagnetic field. A blue-light photoreceptor, cryptochrome (CRY), is the most promising magnetoreceptor candidate based on the radical pair mechanism. The "radical pair/ cryptochrome hypothesis" has propelled a great deal of research activities in recent years (Winklhofer 2010; see also Section 3.3.1.1). The hypothesis is that the chemical compounds in a radical pair are highly sensitive to the spatial orientation of magnetic field lines. Ritz et al. (2010) considered the potential consequences of such optimally devised radical pairs on oxidative stress levels in cells and suggested possible transduction pathways and neural processing strategies for magnetic stimuli detected by photoreceptors such as CRY.

There are several reports that strong SMF effects play significant roles in endogenous and exogenous ROS generation. In contrast, only a few reports on endogenous RNS (NO) generation in biological systems have been described. However, there are safety concerns that the mechanisms of SMF might induce potentiation of endogenous ROS-/ RNS-induced apoptosis or necrosis or both. Regarding the physical effects of magnetic force, it has been reported that the force product of $400 \mathrm{~T}^{2} / \mathrm{m}$ in $8 \mathrm{~T}$ affected the dynamic movement of paramagnetic oxygen bubbles and restrained the evaporation of dissolved oxygen molecules (molar magnetic susceptibility $=3449 \times 10^{-6} \mathrm{~cm}^{3} / \mathrm{mol}$ ) from a reaction mixture such as that required for the decomposition of $\mathrm{H}_{2} \mathrm{O}_{2}$ (Ueno and Iwasaka 1996a). Consequently, the dissolved oxygen levels in solution might be increased. Moreover, it was recently reported that greater enhancement of chemical reaction rate occurs in solution resulting from the magnetic force of $44 \mathrm{~T}^{2} / \mathrm{m}$ in $0.63 \mathrm{~T}$, which attracts oxygen molecules in the air (when the solution depth was $\leq 2.6 \mathrm{~mm}$ and the duration of exposure was $\geq 150$ minutes) (Aoyagi et al. 2006). This effect is considered to be further enhanced in aqueous solutions containing paramagnetic metallic complexes such as stable Cu (II) complexes and heme Fe (III) complexes (Sakurai et al. 2000). Therefore, when examining the effects of strong gradient SMF on free radical reactions, the magnetic force (or force product) acting on the oxygen molecules should be considered. 
In nature, biological systems are considered to be one of the nonlinear systems most sensitive to various external magnetic fields, including SMFs and EMFs (Zhadin 2001), and it has been estimated that some of these responses are mediated through free radical reactions. In particular, millitesla-level SMFs have field strengths that must be subjected to increased investigation because SMF therapy could be useful in treating vascular and circulatory diseases, including ischemic pain and hypertension, primarily due to the modulation of blood flow or blood pressure or both partly through free radical reactions such as an NO-mediated pathway (Okano 2008a,b). However, recent studies have implicated ROS/RNS in the pathogenesis of vascular dysfunction and hypertension and the activation of the sympathetic nervous system (SNS) (Campese et al. 2004; Zhao et al. 2006), although the lifetimes of ROS and RNS in biological systems are extremely short, for example, nanoseconds to microseconds for ROS (Gorman and Rodgers 1992) and a few seconds for NO (one RNS) (Blanchard et al. 1997). Since NO exerts a tonic inhibition of central SNS activity (Ye, Nosrati, and Campese 1997), increased production of ROS could inactivate the neuronal isoform of nitric oxide synthase (nNOS) and subsequently result in activation of the SNS (Campese et al. 2004). In addition, ROS/RNS are generated after ischemia/reperfusion from intracellular oxidases present in the myocardium and in infiltrating leukocytes (Becker 2004). Karogodina, Sergeeva, and Stass (2009) suggest that SMF effect due to a radical pair involving $\mathrm{NO}$ in a biological system requires either a rather strong SMF in the tesla range or an internal enhancer of SMF. Although oxidative stress may be important in hypertension as well as in other cardiovascular disorders (Hirooka, 2011), the underlying mechanisms of SMF-induced ROS/RNS generation have not been clarified.

There is no established effect of repetitive exposures to SMF on a cumulative disorder (Schenck 2005). Nonetheless, there are safety concerns regarding MRI for diagnostic imaging and magnetic levitation for transportation using high-intensity SMF in the tesla range (Prato et al. 2010). Based on advanced studies of SMF effects on oxidative stress reactions, the potentially hazardous effect of SMF on living organisms is that exposure to SMF can increase the activity, concentration, and lifetime of paramagnetic free radicals, which might cause oxidative stress, genetic mutation, and/or apoptosis (Mohtat et al. 1998; Zhang et al. 2003; Okano 2008a; Dini 2010). In particular, SMF exposure initiates an iron-mediated process that increases free radical formation in brain cells, leading to the breaking of DNA strands and cell death.

Although iron ions are important components of normally functioning organisms, in some cases iron ions can be toxic to organisms (Dobson 2007). Because of its redox potential, Fe (II) generally has the potential to do more damage than oxidized Fe (III). For this reason, iron ions are primarily stored as Fe (III) within ferritin in organisms. The association of abnormal accumulation of metal ions with specific neurodegenerative disorders, such as Alzheimer's, Parkinson's, and Huntington's diseases, has been known for over 50 years (Goodman 1953). Various forms of metal ions may play a significant role in the biochemical processes that lead to the progression of neurodegenerative diseases (Dobson 2007). Although there is much speculation about this role, the primary mechanism is thought to be the result of oxidative stress, that is, free radical generation 
via Fenton reaction, which is the iron-catalyzed oxidation of hydrogen peroxide $\left(\mathrm{H}_{2} \mathrm{O}_{2}\right)$ (Conner and Menzies 1995; Markesbery 1997; Koppenol 2001).

In addition to the radical pair mechanism, one of the other established physical mechanisms is "magnetic induction" (van Rongen et al. 2007). This mechanism originates from the following two types of interaction:

1. Electrodynamic interactions with moving electrolytes: An SMF exerts Lorentz forces on moving ionic charge carriers and thereby gives rise to induced electric fields and currents. This interaction is the basis of magnetically induced potentials associated with flowing blood, which have been theoretically analyzed by Kinouchi, Yamaguchi, and Tenforde (1996). The authors suggest that the sinoatrial node of the heart that controls cardiac pacing is the region most sensitive to current and calculate that for a field of $5 \mathrm{~T}$ the current density in this region is about $100 \mathrm{~mA} / \mathrm{m}^{2}$, which is around $10 \%$ of the maximum endogenous current from cardiac electrical activity and rises to around $20 \%$ for $10 \mathrm{~T}$. A detailed assessment of the effects of electric fields on cardiac function using computational models of cardiac electrophysiology indicates that whereas fields up to $8 \mathrm{~T}$ are unlikely to affect the heart rate and rhythm, this is not necessarily true for higher fields (Holden 2005). More recently, however, using a uniform SMF of 0.2 T, Kainz et al. (2010) successfully demonstrated the experimental and theoretical validation of a magnetohydrodynamic (MHD) solver for blood flow analysis. The measured voltage value probably induced by MHD signal was $245 \mu \mathrm{V}$. The computational MHD results can then be correlated with the actual measurements. The authors hope to develop a MHD based biomarker to non-invasively estimate the blood flow for the evaluation of heart failure.

2. Induced electric fields and currents: Time-varying magnetic fields induce electric currents in living tissues in accordance with Faraday's law of induction. Electric currents may also be induced by movement in an SMF. In particular, motion along a field gradient or rotational motion produces a change in flux linkage, which induces an electric current, in contrast to linear motion of the body within a uniform SMF. For linear movement in a gradient field, the magnitudes of induced currents and associated electric fields increase with velocity of the movement and amplitude of the gradient. Calculations suggest that such induced electric fields are substantial during normal movement around or within fields of 2-3 T (Crozier and Liu 2005) and account for the numerous reports of vertigo, nausea, and increased levels of magnetic phosphenes experienced by patients, volunteers, and workers moving in such fields (Schenck et al. 1992; Chakeres and de Vocht 2005; de Vocht, Stevens, et al. 2006; de Vocht, van Drooge, et al. 2006). Glover and Bowtell (2008) measured in situ surface electric fields induced by typical human body movements such as walking or turning in the "fringe" magnetic fields of a whole-body 3-T MRI scanner. These values were $0.15,0.077$, and $0.015 \mathrm{~V} / \mathrm{m}$ for the upper abdomen, head, and across the tongue, respectively. A peak electric field of $0.3 \mathrm{~V} / \mathrm{m}$ was measured for the chest. The speed of movements was not specified in this study. In a body moving at a constant speed of $0.5 \mathrm{~m} / \mathrm{s}$ into a 4-T magnet, Crozier and Liu (2005) estimated the maximum induced electric 
field strength to be $2 \mathrm{~V} / \mathrm{m}$, which is equal to the apparent threshold for peripheral nerve stimulation in the frequency range from $10 \mathrm{~Hz}$ to $1 \mathrm{kHz}$ (ICNIRP 1998). It should be noted, however, that frequencies associated with body movements are likely to be $\leq 10 \mathrm{~Hz}$, the frequency below which more negative resting membrane potential decreases the electrical excitability of neurons due to the slow inactivation of voltage-gated sodium ion channels (Bezanilla 2002). Head translational and rotational frequencies during walking, for example, vary from 0.4 to $4.0 \mathrm{~Hz}$ (Grossman et al. 1988; Pozzo et al. 1990; MacDougall and Moore 2005).

Biomagnetic phenomena for magnetic fields having different intensities and frequencies are shown in Figure 3.1 (Ueno and M. Iwasaka 1996b; Ueno and Shigemitsu 2007). It is important to consider the intensities and frequencies of magnetic fields involved in biomagnetic phenomena when discussing the relationship between magnetism and living organisms. Regarding the effects of magnetism on living organisms, it should

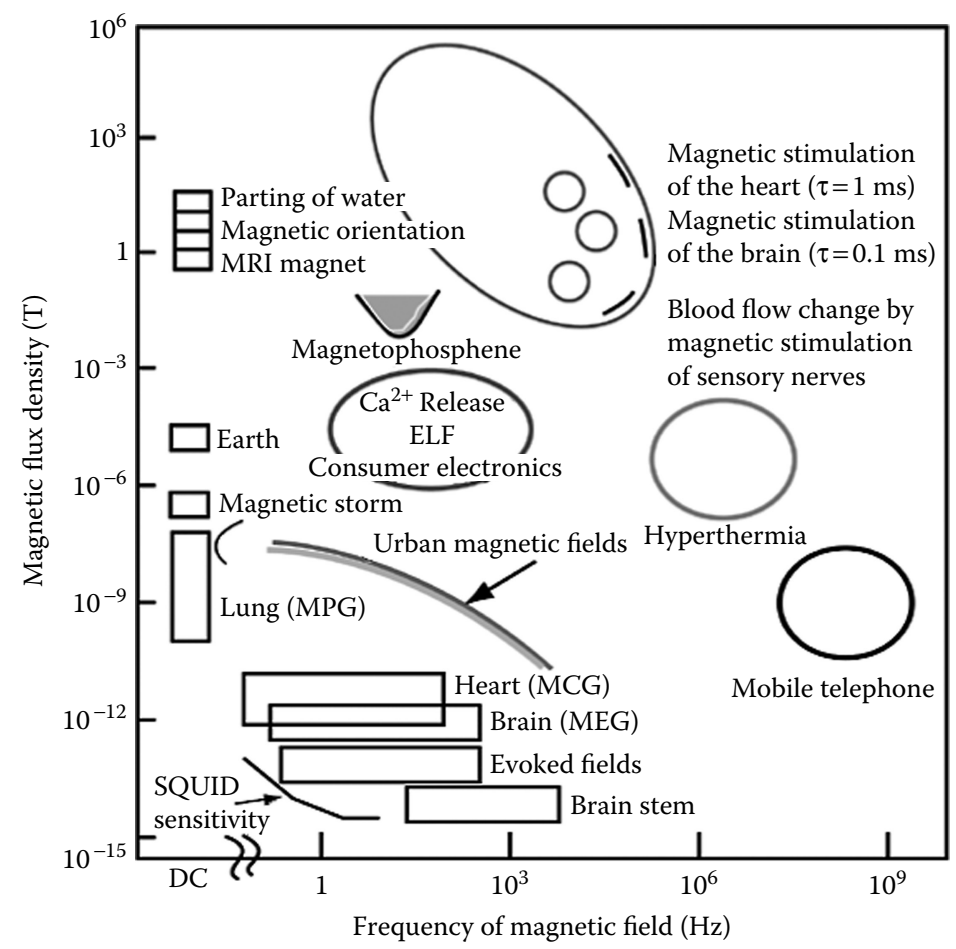

FIGURE 3.1 Biomagnetic phenomena for medical and therapeutic applications. MPG, magnetopneumography; MCG, magnetocardiography; MEG, magnetoencephalography. (From Ueno, S., and M. Iwasaka. 1996b. Magnetic nerve stimulation and effects of magnetic fields on biological, phyisical, and chemical processes. Biological Effects of Magnetic and Electromagnetic Fields, ed. S. Ueno, 1. New York: Springer; Ueno, S., and T. Shigemitsu. 2007. Biological effects and static magnetic fields. Handbook of Biological Effects of Electromagnetic Fields: Bioengineering and Biophysical Aspects of Electromagnetic Fields. 3rd ed. F. S. Barnes, and B. Greenebaum, 203. Boca Raton, FL: CRC Press. With permission.) 
be realized that the actions of SMFs and time-varying magnetic fields differ from each other in terms of the mechanisms fundamental to each.

Studies on the biological effects of magnetic fields have resulted in significant developments in the medical applications of SMF as well as EMF, after the development of high-strength superconducting magnets. The three mainstays of such medical applications are TMS, MRI, and the measurement of biomagnetic fields with a superconducting quantum interference device (SQUID). These techniques have also contributed much to the amazing progress made in understanding brain functions. The TMS locally stimulates the human cerebral cortex with millimeter-order spatial resolution from a figure-eight coil placed on the skull. A three-dimensional (3D) imaging of the brain neuron function has been enabled by the utilization of SQUID in magnetoencephalography (MEG), functional MRI (fMRI), and current-distribution MRI. Results from TMS and imaging studies indicate potential applications of biomagnetics in brain science and clinical neuropsychiatry.

The technique of locally applying magnetic stimulation by a strong pulsed magnetic field on the order of $1 \mathrm{~T}$ transcranially to the brain is called TMS. When a strong electric current is applied to a figure-eight coil placed over the head for 150 milliseconds a pulsed magnetic field on the order of $1 \mathrm{~T}$ is produced; this field generates eddy currents in the brain, which excite the nervous system or stimulate excitable tissues. The first study of magnetic stimulation of the human brain was conducted by Barker, Jalionus, and Freeston (1985) who utilized single coils for the purpose; hence, localized magnetic stimulation of a targeted portion of the human brain was impossible in this study. The focal and vectorial magnetic stimulation of a human cortex using a figure-eight coil was developed by the following groups of researchers: Ueno et al. (1978); Ueno, Lovsund, and Oberg (1986); Ueno, Matsuda, and Fujiki (1989, 1990); Ueno, Matsuda, and Hiwaki (1990, 1991); and Ueno, Tashiro, and Harada (1988); this enabled the stimulation of the motor cortex of a human brain at $5-\mathrm{mm}$ resolution. Localized magnetic stimulation contributed to the creation of functional maps of the motor cortex related to hand and foot areas. An optimal direction of probe placement for the targeting of stimulating currents, which induce neural excitation in each functional area of the cortex, based on functional maps was observed, which is the socalled vectorial feature. Variations in the functional maps of the cortex with changes in orientation of the stimulating current were observed as well. It is a proven fact that the vectorial feature allows studies that reflect both functional and anatomical organizations of neural fibers in the brain. Localized magnetic nerve stimulation of the brain is suitable for investigations of brain function and construction without causing damage to any tissues.

\subsection{Experimental Studies on Magnetic Field Effects}

Recent advance of biological science and technology can help us understand magnetic field effects more clearly. In this major section consisting of In Vivo Studies (3.3.1), and Tissue, Molecular, and Cellular Studies (3.3.2), we provide an overview of a variety of studies on magnetic field effects to explore the uderlying mechanisms and the recent preclinical applications. 


\subsubsection{In Vivo Studies}

The use of animal models to determine the magnetic field effects has prompted a variety of methodologies and provided important evidence, as shown in the following sections: Biological Sensing and Magnetite (3.3.1.1); Behavior and Recognition (3.3.1.2); Occurance of Analgesia (3.3.1.3); Reproduction and Development (3.3.1.4); Circulatory System Effects (3.3.1.5); Neuroendocrine, Visual, and Neurophysical Systems (3.3.1.6); Musculoskeletal System (3.3.1.7); Skeletal System (3.3.1.8); In vivo Genotoxicity (3.3.1.9).

\subsubsection{Biological Sensing and Magnetite}

The geomagnetic field has been used by some bacteria and animals for navigation purposes, although it is a quasistatic and quasiuniform magnetic field ranging about 25-65 $\mu \mathrm{T}$ in unperturbed space. Ritz, Adem, and Schulten (2000) postulated the possibility that magnetoreception involves radical pair processes as a biophysical mechanism. They first considered a system of radical pairs as a model for the magnetic sensory organ and evaluated the influence of the geomagnetic field on this radical pair system. European robins (Erithacus rubecula) were used in this study, and the results showed a disruption of magnetic orientation in robins when they were exposed to a vertically aligned broadband field of $0.1-10.0 \mathrm{MHz}$ and $0.085 \mathrm{mT}$ or the single frequency of $7 \mathrm{MHz}$ and $0.47 \mathrm{mT}$ together with the geomagnetic field (Ritz et al. 2004). The disorientation observed was found to depend on the angle between the $7 \mathrm{MHz}$ oscillating field and the geomagnetic field. The robins oriented in the migratory direction when the oscillating field was parallel to the geomagnetic field. The authors suggested a magnetic compass based on a radical pair mechanism, due to the resonance effect on singlet-triplet (S-T) transitions in oscillating fields.

It has been reported that chemical magnetoreception via the radical pair mechanism requires in principle the following sequence of three events: (1) generation of a radical pair species with correlated electron spins, either in an S or a T state; (2) coherent evolution of the radical pair between the near-degenerate $S$ and $T$ spin states; and (3) different reaction pathways of the $S$ and $T$ radical pairs. The frequency of $S-T$ interconversion in the radical pair and hence the relative yields of reaction products and/or the lifetime of the radical pair depend on the strength of any applied magnetic field. Magnetic field effects in radical pairs are thus kinetic rather than thermodynamic in origin and may be detected for magnetic fields whose electron Zeeman energies are much smaller than the average thermal energy per molecule, $k_{\mathrm{B}} T$. The radical pair lifetime needs to be in the microseconds range (Cintolesi et al. 2003) if a magnetic field of $50 \mu \mathrm{T}$ is expected to have a significant effect on it.

Henbest et al. (2008) demonstrated a magnetic field effect on the photochemical yield of a flavin-tryptophan radical pair in Escherichia coli photolyase. This result provides proof for the principle that photolyases, and most likely CRYs by extension, have the fundamental properties required to form the basis of a magnetic compass. Gegear et al. $(2008,2010)$ suggested that animal CRY mediates light-dependent magnetoreception through an unconventional photochemical mechanism using Drosophila transgenesis, and CRY-dependent magnetosensitivity does not require a functioning circadian clock. 
Yoshii, Ahmad, and Helfrich-Förster (2009) demonstrated that Drosophila's circadian clock is sensitive to magnetic fields and that this sensitivity depends on the light activation of CRY and the applied field strength, consistent with the radical pair mechanism. The CRY is widespread throughout biological systems and has been suggested as the receptor for magnetic compass orientation in migratory birds. The results establish the circadian clock of Drosophila as a model system for CRY-dependent magnetic sensitivity. Furthermore, given that CRY occurs in multiple tissues of Drosophila, including those potentially implicated in flying orientation, future studies may yield insights that could be applicable to the magnetic compass of migratory birds and even to potential magnetic field effects in humans. As another CRY-related mechanism, Zaporozhan and Ponomarenko (2010) hypothesized that CRY is a transcriptional repressor of the major circadian complex CLOCK/BMAL1 (reviewed by Langmesser et al. 2008) and, therefore, magnetic fields via some modulation of CRY function can influence circadian gene expression and modify the activity of the transcription factor nuclear factor- $\kappa \mathrm{B}$ (NF- $\mathrm{KB}$ )- and glucocorticoids-dependent signaling pathways.

The radical pair mechanism imposes some constraints that should be satisfied by a viable chemical magnetoreceptor (Rodgers and Hore 2009). For this purpose, computer simulations have been performed on a collection of radical pairs undergoing restricted rigid body rotation, coherent anisotropic spin evolution, electron spin relaxation, and spin-selective recombination reactions. Lau et al. (2010) showed that perfect molecular ordering and complete immobilization, as in a crystal at low temperatures, are not requirements for efficient radical pair magnetoreception. It is acceptable to have radical pairs that are uniaxially ordered with a moderate order parameter, which undergo medium-amplitude orientational fluctuations with respect to the director and whose motional correlation time is longer than about a quarter of their lifetime. The authors assumed that a magnetosensitive CRY specifically bound to a visual receptor protein, rhodopsin, would share its orientational ordering and motional restriction and hence could have the properties necessary for efficient magnetoreception. Hill and Ritz (2010) modeled the effect of molecular disorder on the performance of a radical pair compass and estimated the minimum number of radical pairs required in a receptor cell to achieve a certain resolution in terms of directional and intensity variations of a magnetic field.

More recently, Phillips, Jorge, and Muheim (2010) suggested that the effects of light on magnetic compass orientation are mediated by a light-dependent magnetoreception mechanism or instead are due to input from a non-light-dependent (e.g., magnetite-based) magnetoreception mechanism that secondarily interacts with other light-dependent processes.

Regarding magnetite-based magnetoreception, an experimental study in European robins, E. rubecula, suggested that the interactions between the magnetite receptors in the beak and the visual system occurring at higher levels in the brain do not involve the magnetoreception system based on radical pair process in the right eye, but rather they involve other, nonlateralized components of the visual system (Wiltschko et al. 2010). Winklhofer and Kirschvink (2010) proposed a magnetite hypothesis and focused on "magnetic torque transducer models," where elongated magnetic structures like a chain of magnetosomes respond to a magnetic field by restricted rotational motion about an elastic pivot. These authors theoretically analyzed interactions of magnetosomes 
with cytoskeletal filaments and thermal fluctuations to derive constraints for possible transduction mechanisms involving mechanosensory elements such as ion channels.

Nishimura et al. (2010) investigated the behavioral responses of a diurnal agamid lizard (Pogona vitticeps) to a sinusoidal extremely low frequency electric and magnetic field (ELF-EMF; 6 and $8 \mathrm{~Hz}, 2.6 \mu \mathrm{T}, 10 \mathrm{~V} / \mathrm{m}$ ). Lizards in the EMF group were exposed to ELF-EMF for 12 hours a day (during the daytime). The number of tail lifts was monitored beginning 3 days before exposure and ending after 5 days of exposure. The average number of tail lifts per individual per day was found to be greater in the EMF group than in the control group. The authors confirmed the reproducibility of this response by a crossover trial. These results suggest that at least some lizards are able to perceive ELF-EMF. Furthermore, when the parietal eye of the lizards was covered with a small round aluminum cap, which could block light, the tail-lifting response to ELF-EMF disappeared. These experiments suggest that lizards can perceive EMF and the parietal eye may be involved in light-dependent magnetoreceptive responses.

\subsubsection{Behavior and Recognition}

Scientific interest in behavioral changes in organisms has led to the development of a psychology of learning that studies the effects of various external stimuli in living organisms. Several investigations have been conducted for studying the effects of magnetic fields on behavior and the central nervous system of organisms using techniques developed specifically in these research fields. Behavioral research directed toward the effects of magnetic exposure on living organisms mainly addresses two questions: (1) whether magnetic fields are sensed and avoided and (2) whether magnetic fields have any influence on the functions of learning and memory. Magnetic field experiments addressing both the questions have been conducted using several indicators, such as open-field behavior, operant behavior, and spontaneous motor activity, as tools of observation and measurement. This section focuses on recent advances and new aspects in magnetic field effects and mechanisms on biological systems (see also Section 3.3.1.1).

Regarding SMF effects on the functions of learning and memory, Ammari et al. (2008) investigated the behavioral effects of an SMF (128 mT, $1 \mathrm{~h}$ /day for 5 days) in male rats. The authors concluded that SMF exposure altered emotional behaviors in the plus maze and led to cognitive impairments, or at least substantial attention disorders, in the Morris water maze.

Fu et al. (2008) investigated the short- and long-term effects of ELF-EMF on spatial recognition memory in mice by using a two-trial recognition Y-maze that is based on the innate tendency of rodents to explore novel environments. Mice were exposed to an EMF ( $25 \mathrm{~Hz}, 0.6 \mathrm{mT}$; or $50 \mathrm{~Hz}, 1.1 \mathrm{mT}$ ) for either 7 days (short term) or 25 days (long term) and then tested in the Y-maze. The results indicated that neither short- nor longterm exposures to EMF affected the locomotor activity of mice in the Y-maze. However, long-term exposure to a $50 \mathrm{~Hz}$ EMF reduced recognition of the novel arm. These findings suggest that ELF-EMF impairs spatial recognition memory in the Y-maze depending on the field strength or the duration of exposure or both.

In contrast, Liu et al. (2008) suggested that ELF-EMF (50 Hz, $2 \mathrm{mT}$ ) improves learning and memory function. In this study, authors examined the changes in spatial learning and memory in rats using the Morris water maze test after 4 weeks of daily exposure 
to ELF-EMF for either 1 hour or 4 hours. The authors found that ELF-EMF reduced the latency to find a hidden platform and improved long-term memory of former location of the platform without affecting short-term memory and motor activity. These findings indicate that ELF-EMF exerts a positive effect on the acquisition and maintenance of spatial memory.

Shin et al. (2007) demonstrated that exposure to ELF-EMF $(60 \mathrm{~Hz}, 2.4 \mathrm{mT}, 1 \mathrm{~h} /$ day for 1 or 7 days) enhanced dopamine levels in rat striatum. The authors examined the role of dopaminergic receptors in ELF-EMF-induced behavioral changes. They suggested that ELF-EMF-induced behavioral responses are, at least in part, mediated by the activation of dopamine $\mathrm{D}_{1}$-like receptors.

Burda et al. (2009) showed that the ELF-EMF generated by high-voltage power lines disrupts the alignment of bodies of animals with the geomagnetic field. Body orientation of cattle and roe deer was random on pastures under or near power lines. Moreover, cattle exposed to various magnetic fields directly beneath or in the vicinity of power lines trending in various magnetic directions exhibited distinct patterns of alignment. The disturbing effect of ELF-EMF on body alignment diminished with distance of the body from the conductors. The authors provided evidence for magnetic sensation in large mammals as well as evidence for an overt behavioral reaction to weak ELF-EMF in vertebrates.

In human studies, de Vocht et al. (2007) investigated the impact of exposure to "stray" magnetic field from a whole-body 7-T MRI scanner on neurobehavioral performance and cognition. Twenty-seven volunteers completed four sessions, which comprised 1.6-T (twice), 0.8-T, and negligible exposure sessions. During three sessions, the volunteers were instructed to complete a series of standardized head movements to generate additional time-varying fields ( 300 and $150 \mathrm{mT} / \mathrm{s}$ ). The results suggested that there are effects on visual perception and hand-eye coordination; but these were found to be weak and variable between studies. The magnitude of these effects may depend on the magnitude of time-varying fields and not so much on SMF. Although this study did not include exposure above 1.6 T, it suggested that the use of strong magnetic fields is not a significant confounder in fMRI studies of cognitive function.

Cavin et al. (2007) examined an initial characterization of the metallic taste effect in some workers when moving around a 7-T MRI scanner. This study indicated that $50 \%$ of subjects perceive a metallic taste for head shaking with a period of 1.5 seconds (magnetic field in an anterior-posterior direction) causing a $\mathrm{d} B / \mathrm{d} t$ of $2.3 \pm 0.3 \mathrm{~T} / \mathrm{s}$. Presence of dental fillings is not a requirement for the sensation of metallic taste.

Schlamann et al. (2010) assessed potential cognitive deficits under the influence of an SMF at various field strengths that some other studies had already reported. Mostly no MRI sequences were performed. All subjects underwent one MRI examination for 63 minutes at $1.5 \mathrm{~T}$ and one at $7 \mathrm{~T}$ within an interval of 10-30 days. The order of the examinations was randomized. Subjects were referred to six standardized neuropsychological tests strictly focused on attention immediately before and after each MRI examination. Only six subtests revealed significant differences between pre- and post-MRI conditions. In these tests, the subjects achieved better results in post-MRI testing than in pre-MRI testing. The other tests revealed no significant results. The improvement in post-MRI testing is only explicable in terms of learning effects. The authors concluded 
that MRI examinations, even in ultra-high-field scanners, do not seem to have any persisting influence on the attention networks of human cognition immediately after exposure.

\subsubsection{Occurrence of Analgesia}

Specific SMFs, ELF-EMFs, and pulsed EMFs (PEMFs) have been shown to induce analgesia (antinociception) in snails, rodents, and healthy human volunteers. In particular, the effects of ELF-EMFs on analgesia have been reviewed in detail by Prato, Thomas, and Cook (2005). However, evidence concerning the effects of magnetic fields on nociceptive or pain sensation processes is contradictory in the literature probably due to differences in species, characteristics of the magnetic fields, and duration of the exposure.

Prato et al. (2005) showed that repeated daily exposures for 1 hour to an ambient magnetic field-shielded environment induce analgesia (antinociception) in mice. The exposures were carried out in the dark during the mid-light phase of the diurnal cycle. However, when the mice were exposed in the presence of visible light (400-750 nm) the analgesic effects of shielding were eliminated. The Prato research group later showed that the analgesic effect of magnetic field shielding in mice is light-intensity and wavelength dependent (Prato et al. 2009). Introduction of red light (peak wavelength at $635 \mathrm{~nm}$ ) had little or no effect, presumably because mice do not have photoreceptors sensitive to red light above $600 \mathrm{~nm}$ in their eyes. By contrast, introduction of ultraviolet light (peak wavelength at $405 \mathrm{~nm}$ ) abolished this effect, presumably because mice do have ultraviolet A receptors. Blue light exposures (peak wavelength at $465 \mathrm{~nm}$ ) of different intensities demonstrated that the effect has an intensity threshold of $12 \%$ of the blue light in the housing facility.

Sándor et al. (2007) examined the action of acute exposure to a gradient SMF ( $B_{\max }$ of $389 \mathrm{mT}, 39 \mathrm{~T} / \mathrm{m}$, for 30 minutes) on acute visceral and somatic chemonociception and inflammatory mechanical hyperalgesia in mice. The SMF exposure significantly diminished the number of acetic acid- or $\mathrm{MgSO}_{4}$-induced abdominal contractions (acute visceral nociception), the number of formalin-evoked paw lickings and liftings in both phase I (acute somatic nociception) and phase II (acute inflammatory nociception), and mechanical hyperalgesia evoked by intraplantar (i.pl.) injection of carrageenan as well as a capsaicin receptor TRPV1 agonist (resiniferatoxin). Selective inactivation of capsaicin-sensitive sensory fibers by high-dose resiniferatoxin pretreatment decreased nocifensive behaviors in phase II of the formalin test to a similar extent, suggesting that pro-inflammatory neuropeptides such as substance $\mathrm{P}$ and calcitonin gene-related peptide released from these fibers are involved in this inflammatory reaction. They found that significant inhibitory effects of SMF on formalin-induced nociception and carrageenan-evoked hyperalgesia were absent in resiniferatoxin-pretreated mice. The findings indicate that capsaicin-sensitive nerves are involved in the SMF-induced antinociceptive action. The same research group reported that analgesic effect is induced by whole-body exposure to the same gradient SMF as in the previous study in mice (László et al. 2007). As a result, the authors found a magnet arrangement recipe that achieves an analgesic effect of over $80 \%$ in the writhing test. The same research group further reported that SMF ( $B_{\max }$ of $330 \mathrm{mT}, 13.2 \mathrm{~T} / \mathrm{m}$, for 30 minutes) increased thermal pain threshold (TPT) and reduced within-block thermal habituation in healthy 
human volunteers, leaving pain perception unchanged (Kovács-Bálint et al., 2011). They speculated that SMF-induced peripheral neuronal or circulatory mechanisms may be involved in the observed TPT increase by setting the pain fiber adaptation potential to higher levels.

Many studies have demonstrated that ELF-EMFs may be involved in hyperalgesia. Jeong, Choi, Moon, et al. (2005) suggested that an ELF-EMF $(60 \mathrm{~Hz}, 2.5 \mathrm{mT})$ can produce hyperalgesia and such a response can be regulated by the benzodiazepine system in rats. Diazepam (a benzodiazepine receptor agonist), flumazenil (a benzodiazepine receptor antagonist), or both were used with EMF exposure. When testing the pain threshold of rats using hot plate tests, EMF or diazepam was found to induce hyperalgesic effects with a reduction in latency. These effects were blocked by pretreatment with flumazenil. When the rats were exposed simultaneously to EMF and diazepam, the latency tended to decrease without statistical significance. The induction of hyperalgesia by coexposure to EMF and diazepam was also blocked by flumazenil. However, pretreatment by $\gamma$-aminobutyric acid (GABA) receptor antagonists such as bicuculline (a GABA antago- $^{-}$ nist) or phaclofen (a $\mathrm{GABA}_{\mathrm{B}}$ antagonist) did not antagonize the hyperalgesic effect of EMF. These results suggest that the benzodiazepine system may be involved in EMFinduced hyperalgesia. The same research group later suggested that the ELF-EMF might cause $\mathrm{Ca}^{2+}$-dependent NOS activation, which then induces hyperalgesia with an increase in NO synthesis in mice (Jeong et al. 2006). They concluded that ELF-EMF may produce hyperalgesia by modulating NO synthesis via $\mathrm{Ca}^{2+}$-dependent NOS.

Bao et al. (2006) investigated the analgesic effect of an ELF-EMF (55.6 Hz, 8.1 mT) in rats. The authors found that tail-flick latencies (TFLs) increased significantly after exposure ( $6 \mathrm{~h} /$ day for 4 days). The analgesic effects seemed to decrease gradually when the rats were exposed daily for another 10 days. The levels of TFL decreased in 1 day when the rats were removed after a 4-day exposure. The concentrations of hypothalamus $\beta$-endorphin, substance $\mathrm{P}$, and brain stem serotonin (5-HT) were found significantly increased on day 4 . However, no differences were found when the rats were exposed for another 10 days, and there were no significant increases when rats were removed after the fourth day of exposure and tested for nociception on days 5 and 7 with no changes in biochemical markers at 7 days. These results suggest that ELF-EMFs have an analgesic effect, but only on days 3 and 4 . The authors speculated that the EMF effect may be associated with increases in endogenous $\beta$-endorphin, substance $\mathrm{P}$, and 5-HT.

Mert, Gunay, and Ocal (2010) examined the efficacy of PEMF $(1,10,20$, and $40 \mathrm{~Hz}$; $1.5 \mathrm{mT} ; 1 \mathrm{~h} /$ day for 4 weeks) on diabetic neuropathy in streptozotocin (STZ)-induced acute ( 3 days) and chronic (6 weeks) diabetic rats. After STZ administration, blood glucose levels elevated and body weights decreased in the studied rats. Although PEMF treatment did not affect changes in body weight, the blood glucose levels of PEMFtreated diabetic rats exhibited a decrease during the treatments. Diabetic animals displayed marked decreases in mechanical thresholds and thermal latencies. Whereas treatment with PEMF partially restored the mechanical threshold and thermal latency in acute diabetic rats, PEMF had a corrective effect only on the mechanical threshold of chronic diabetic rats. These results suggested that treatment with PEMF can potentially ameliorate the painful symptoms of diabetes, such as hyperalgesia and allodynia, by partially preventing hyperglycemia. 


\subsubsection{Reproduction and Development}

There is a series of reports concerning the effects of many different kinds of magnetic fields on reproduction and development. In particular, in the last 10 years the effects of strong SMFs, including MRI fields (uniform SMFs, gradient magnetic fields, and radiofrequency $[\mathrm{RF}]$ pulse fields), on fertility, the developing embryo, and the fetus have been investigated in great detail (Ueno and Shigemitsu 2007). In contrast, more recently, reports on the effects of extremely weak magnetic fields or removal of the geomagnetic field have been increasing.

The Persinger research group (Whissell and Persinger 2007) investigated the interaction between NO modulation and perinatal weak magnetic fields. Dams were exposed from 2 days before to 14 days after birth to one of six magnetic field conditions $(7 \mathrm{~Hz}$; $1 \mathrm{nT}, 5 \mathrm{nT}, 10 \mathrm{nT}, 50 \mathrm{nT}, 500 \mathrm{nT}$, and sham) and given one among water, NO precursor L-arginine, or the NOS inhibitor N-methylarginine (NMA). At weaning (day 22), their offsprings were placed in the open field for observation. Rats given 50- or 500-nT field + water were hyperactive and showed increased rearing and bodyweight. These effects were attenuated or absent in groups given 50- or 500-nT field + NMA. The behavior of groups given sham field + L-arginine was similar to that of animals given 50 - or 500 -nT field + water. Results suggested a critical developmental role for $\mathrm{NO}$ and the involvement of NO in magnetic field effects. The same research group further examined the effects of extremely weak magnetic fields $(0.5 \mathrm{~Hz}, 5-10 \mathrm{nT}$, for 8 days; McDonald and Persinger 2009). Adult rats were perinatally exposed to magnetic field or sham conditions while their mothers drank tap water containing the NOS inhibitor L-arginine methyl ester (L-NAME) or tap water alone. One week after birth the rats were rendered hypoxic for 1 minute or they served as controls. Exposure to either the magnetic field or the NOS inhibitor reduced the numbers of neurons within the bed nucleus of the stria terminalis by about $25 \%$, whereas exposure to either the hypoxia or the magnetic field resulted in comparable decreases in cell numbers within the ventromedial nucleus (dorsomedial part). Males had 15\% fewer neurons in these nuclei compared with females. The results of for the interactions involving perinatal exposure to the magnetic field were comparable to the magnitudes of those associated with one minute of hypoxia for one week postnatally. These results showed the sensitivity of specific structures of the developing brain to interactions between subtle environmental variables.

The same research group investigated the effects of weak magnetic fields $(7 \mathrm{~Hz}$, $\leq 1.2 \mu \mathrm{T}$ ) on blood chemistry, cerebral sizes, and hippocampal cytomorphology in adult male and female albino Wistar rats (St-Pierre, Mazzuchin, and Persinger 2008). Rats had been exposed during their entire prenatal development period to any of the following magnetic fields: very low (5-20 nT), low (30-50 nT), medium (90-580 nT), or high (590 nT to $1.2 \mu \mathrm{T}$ ). Adult rats that had been exposed prenatally to the physiologically patterned magnetic fields at low and medium intensities exhibited peak elevations of aminotransaminase, glucose, and uric acid. Numbers of cytometric anomalies were also found to be significantly elevated within regions of the hippocampus known for neuronal neogenesis in adults. The results suggested that a common factor in cellular adhesion or plasticity might be permanently altered by prenatal exposure to a narrow intensity of a series of physiologically patterned magnetic fields. 
Novikov, Sheiman, and Fesenko (2008) studied the effects of weak static (direct current $[D C]$ ) and alternating (alternating current $[A C]$ ) magnetic fields, as well as combined (AC-DC) collinear magnetic fields, on the intensity of morphogenesis processes in the planarian Dugesia tigrina. The authors found that combined magnetic fields (e.g., $42-\mu \mathrm{T} \mathrm{DC}+3.7 \mathrm{~Hz} 100-\mathrm{nT} \mathrm{AC}$ ) produced a stimulating effect on the fission and regeneration of planarians. The exposure to "zero" field also induced significant stimulating effects comparable with those produced by combined magnetic fields in the effective range. In contrast, AC fields alone produced inhibitory effect. The authors speculated that the effects observed may be of great importance in analyzing the mechanisms of action of weak and superweak fields on living organisms.

From another aspect, Muehsam and Pilla (2009a,b) proposed a Lorenz model for weak magnetic field bioeffects, suggesting that weak, exogenous AC-DC magnetic fields can act on an ion or ligand bound in a molecular cleft, based on the assumption that the receptor molecule is able to detect the Larmor trajectory of an ion or ligand within the binding site. To date, however, there is insufficient direct experimental evidence pertaining to this model. Further studies are required to decipher and better understand the mechanisms of the bioeffects of DC magnetic fields (SMF).

Zhang et al. (2004) applied Drosophila to the study of learning and memory to investigate the removal effect of the geomagnetic field for successive generations of living organisms. Using the operant visual learning/memory paradigm in a flight simulator, this study revealed that wild-type flies raised in a hypomagnetic field environment continuously for 10 successive generations became gradually impaired in visual conditioning learning and memory formation; finally, the tenth generation flies became morphs of nonlearners and completely amnesiac. The control experiments show that the impairment cannot be ascribed to any apparent sensorimotor problems in Drosophila. The reverse shift from hypomagnetic field to natural geomagnetic field learning and memory ability following six consecutive generations. Thus, these findings demonstrate conclusively that some serious, but reversible, learning and memory impairments may occur in living organisms as a result of a prolonged separation from the geomagnetic field over many consecutive generations.

Hung et al. (2010) investigated the effects of SMFs of up to $200 \mathrm{mT}$ on the developmental and aging processes of Caenorhabditis elegans. Treatment with a 200-mT SMF reduced the development time from L 2 to L3 stage by $20 \%$, from L 3 to L 4 by $23 \%$, and from $\mathrm{L} 4$ to young adult by $31 \%$. After SMF exposure, the average life span was reduced from 31 days to 24 days for wild-type nematodes. The upregulation of the clock genes clk-1, lim-7, daf-2, unc-3, and age-1 after SMF treatment was verified by quantitative realtime Reverse Transcription-Polymerase Chain Reaction (RT-PCR). Apparently, induction of gene expression is selective and dose dependent. The total developmental time was significantly reduced for the lin-4, lin-14, lin-41, and lin-7 mutants, but not for the let-7, clk-1, unc-3, and age-1 mutants. Life span analyses revealed that the let-7, unc-3, and age-1 mutants were not affected by SMF exposure. The authors and, thereafter, Lee, Hung, and Huang (2010) suggest that the SMF accelerated nematode development and shortened nematode life span through pathways associated with the let-7, clk-1, unc-3, and age-1 mutants. 
Peric-Mataruga et al. (2008) investigated the effects of an SMF of $320 \mathrm{mT}$ on the pupae of yellow mealworm, Tenebrio molitor. The values of morphometric parameters of neurosecretory neurons and corpora allata were significantly increased after SMF exposure. The SMF influenced characteristics of protocerebral neurosecretory neurons and corpora allata in the late T. molitor pupae.

Saito, Suzuki, and Suzuki (2006) reported that an acute SMF exposure of $400 \mathrm{mT}$ had teratogenic effects on developing fetuses. Fetuses were exposed to the SMF in utero for 6 minutes for 1 day from 7.5 to 14.5 days of pregnancy. Various types of malformations were observed, including polydactylism, abdominal fissure, fused rib, vestigial thirteenth rib, lumbar rib, brain hernia, and curled tail, whereas only a low incidence $(\leq 2.8 \%)$ of curled tail was detected in the control group. These deformations were apparently caused by SMF exposure, but the effects did not reflect the so-called exposure period specificity.

Monfared, Jorsaraei, and Abdi (2009) investigated the protective effects of vitamins $\mathrm{E}$ and $\mathrm{C}$ against 1.5-T SMF in MRI on spermatogenesis parameters. Results showed that compared with sham exposure SMF could reduce germ cell count and sperm duct diameter; although vitamins $\mathrm{C}$ and $\mathrm{E}$ could modify the reduction in germ cell count, they did not show any protective effect on sperm duct diameter reduction. The authors conclude that the protective effects of vitamins $\mathrm{C}$ and $\mathrm{E}$ are different and depend on the type of effects. It seems that the modifying effects of vitamins are additive, but vitamin $\mathrm{E}$ plays a more important role than vitamin $\mathrm{C}$ against the SMF on spermatogenesis parameters in clinical MRI.

Eguchi et al. (2006) examined cleavage and survival of Xenopus embryos exposed to 8-T SMF. The authors investigated fertilized Xenopus embryos exposed to SMF both in a static chamber and in a rotating culture system. The results of these investigations showed that the strong SMF changed the third cleavage furrow from the usual horizontal one to a perpendicular one. However, when the direction of gravity was randomized by exposing the embryos to SMF in a rotating culture system the third cleavage furrow was formed horizontally; this finding suggested that the observed distortion of the third cleavage furrow in SMF-exposed embryos was accomplished by altering gravity effects, which were elicited by diamagnetic force due to a high-gradient magnetic field. The results also showed that the SMF did not damage survival. The findings of the investigation are that SMF and altering gravity cause distortion of the third cleavage furrow and that the effects of exposing cleavage embryos to SMF were transient and did not affect postcleavage development. The authors also suggest that strong SMF is not hazardous to the cleavage and blastula-gastrula transition of developing embryonic cells.

Gonet, Kosik-Bogacka, and Kuźna-Grygiel (2009) investigated the effects of an ELFEMF $(50 \mathrm{~Hz}, 2.0 \mathrm{mT})$ on the oviposition and development of Drosophila melanogaster. The results showed that ELF-EMF exposure of $D$. melanogaster females of generation $\mathrm{P}$ and all the development stages of generation $\mathrm{F} 1$ weakened the oviposition of these insects in their subsequent generations.

Goodman et al. (2009) examined the effects of an ELF-EMF $(60 \mathrm{~Hz}, 8 \mu \mathrm{T}, 1$ hour twice daily for 15 days) on the regeneration of the planaria Dugesia dorotocephala. The ELF-EMF exposure during the initial 3 days postsurgery caused a significant increase 
in regeneration for both heads and tails, especially tails. The first appearance of eyes occurred at day 7 posttransection in the tail portions exposed to the ELF-EMF. In the sham control tail samples, the initial appearance of eyes occurred 48 hours later. Concurrently, ELF-EMF-exposed heads and tails exhibited an elevation in the levels of heat shock protein 70 (HSP70) protein, activation of an extracellular signal-regulated kinase (ERK) cascade, and increase in the serum response factor (SRF)-serum response element (SRE) binding. The authors concluded that this is accompanied by an increase in HSP70 protein levels, activation of specific kinases, and upregulation of transcription factors that are generally associated with repair processes.

Blackman (2006) raised the question of whether early exposure to EMFs can leave an imprint on an organism that alters further development of the organism and results in negative health consequences. This possibility deserves further experimental scrutiny.

\subsubsection{Circulatory System Effects}

Effects of magnetic fields including SMFs, ELF-EMFs, and PEMFs on the circulatory system have been reviewed in experimental animals (Tenforde 2005; Saunders 2005; McKay, Prato, and Thomas 2007; Ohkubo et al. 2007; Robertson et al. 2007; McNamee et al. 2009; Ohkubo and Okano 2010) and in humans (Chakeres and de Vocht 2005; Crozier and Liu 2005; van Rongen 2005; McKay, Prato, and Thomas 2007; Robertson et al. 2007; McNamee et al. 2009). There are many therapeutic applications for locally increased blood flow. It is suggested that magnetic fields have the potential to modify microcirculatory perfusion. A recent review regarding the effects of magnetic fields on microcirculation and microvasculature by McKay, Prato, and Thomas (2007) reported that nearly half of the cited experiments (10/27) are related to vasodilatory effect, increased blood flow, or increased blood pressure. Conversely, three of the total 27 studies reported a decrease in blood perfusion or pressure. Four studies reported no effect. The remaining 10 studies found that magnetic fields could trigger either vasodilation or vasoconstriction depending on the initial tone of the vessel. In terms of cellular effects of magnetic fields related to perfusion, four of a total of 19 studies reported an increase in $\mathrm{NO}$ activity from magnetic field exposures (one of these studies used a model with an altered vessel state prior to exposure), one study found a biphasic effect, and five found no effect. Nine studies reported vascular development effects (seven reported increased angiogenesis, and two reported decreased angiogenesis). Other cellular effects were reported in three studies.

For a human study with MRI, Gupta, Weeks, and Richie (2008) presented a mathematical approach of computing the elevation of the T-wave of an electrocardiography (ECG) signal in an MRI bore caused by the flow of blood in the aorta. The elevation was computed mathematically using the equations of magnetofluid dynamics (MFD). A method was developed to measure MFD-induced voltage based on discretization of the aortic arch and measurement of the blood flow profile in the aorta. The results were compared to the ECG signals measured in humans in the bore of a 1.5-T imaging magnet (1.5-T SMF alone). The computed ECG signals at the 12 leads were very similar to the measured values.

McNamee et al. $(2010,2011)$ suggested that ELF-EMFs $(60 \mathrm{~Hz}, 0.2$ and $1.8 \mathrm{mT})$ did not affect perfusion, heart rate, or mean arterial pressure. The decrease in perfusion and 
heart rate trends over time appears to be associated with a combination of inactivity (resulting in decreased body temperatures) and reduced physiological arousal. In contrast, Nishimura et al. (2011) suggested that repeated exposure to an ELF-EMF (6 and $8 \mathrm{~Hz}, 1 \mu \mathrm{T}, 10 \mathrm{~V} / \mathrm{m}$, for at least two 10 - to 15 -minute sessions per week, over a period of 4 weeks) has a blood pressure-lowering effect on humans with mild-to-moderate hypertension.

Robertson et al. (2007) reviewed several mechanisms of protection, such as HSPs, opioids, collateral blood flow, and NO induction, and the evidence supporting the use of ELF-EMF as a means of providing protection in each of these mechanisms. Although there are few studies demonstrating direct protection with ELF-EMF therapies, there are many published reports indicating that ELF-EMF may be able to influence some of the biochemical systems with protective applications.

McKay et al. (2010) investigated the acute effect of a PEMF $(72 \mathrm{~Hz}, 225 \mu \mathrm{T}, 6.7 \mathrm{mV} / \mathrm{m}$, for 30 and 60 minutes) on blood flow in the skeletal microvasculature of a male Sprague Dawley rat model. Acetylcholine $(0.1,1.0$, and $10.0 \mathrm{mM}$ ) was used to perturb normal blood flow and to delineate the differential effects of PEMF, based on the degree of vessel dilation. The authors found that there were no significant effects of PEMF on peak blood flow, heart rate, and myogenic activity, but a small attenuation effect on anestheticinduced respiratory depression was noted.

\subsubsection{Neuroendocrine, Visual, and Neurophysiological Systems}

Neuroendocrine, visual, and neurophysiological systems also are considered to be related to the aforementioned magnetic perception (3.3.1.1), analgesia (3.3.1.3), and circulatory system (3.3.1.5). Zhang et al. (2007) examined the effects of long-term exposures to a near-zero magnetic environment on the noradrenergic activities in the brain stem of golden hamsters. Both the content of norepinephrine (NE) and the density of NE-immunopositive neurons in tissue decreased significantly after the treatment, and the effects were found to be progressive with time. These variations may contribute substantially to the behavioral and mood disorders reported in other studies that occur when animals are shielded from the geomagnetic field.

Jeong, Choi, Choi, et al. (2005) investigated the effect of an ELF-EMF (60 Hz, $2.5 \mathrm{mT}$ ) on convulsions in rats. The authors determined the onset and duration of convulsions induced by exposure to bicuculline alone or by coexposure to ELF-EMF and bicuculline. Coexposure to ELF-EMF and bicuculline decreased GABA levels in the cortex, hippocampus, and hypothalamus, whereas exposure to ELF-EMF alone reduced the levels of GABA only in the hippocampus. These results suggest that ELF-EMF may modulate bicuculline-induced convulsions due to GABA neurotransmissions in rat brains.

Varró et al. (2009) examined the effects of ELF-EMF $(50 \mathrm{~Hz}, 250-500 \mu \mathrm{T})$ on synaptic efficacy in the central nervous system. Electrophysiological investigation was carried out ex vivo in rat neocortical and hippocampal slices, and basic synaptic functions, shortterm plasticity (STP) and long-term plasticity (LTP), and seizure susceptibility were tested. The most pronounced effect was a decrease in basic synaptic functions in both slices treated directly ex vivo $(50 \mathrm{~Hz}, 250-320 \mu \mathrm{T}$, for 1 hour $)$ observed as a diminution in the amplitude of evoked potentials. In contrast, whole-body exposure to ELF-EMF 
(50 Hz, $500 \mu \mathrm{T}$, for a single session of 15 hours and total of 60 hours, i.e., $15 \mathrm{~h}$ /day for 4 days) induced enhanced STP and LTP in hippocampal slices and increased seizure susceptibility in neocortical slices. The authors concluded that although ELF-EMF exposure exerts significant effects on synaptic activity, the overall changes may strongly depend on the synaptic structure and neuronal network of the affected region together with the specific spatial parameters and constancy of ELF-EMF.

Walker et al. (2007) tested the effect of PEMF (2 Hz;0.03, 0.3 and $3 \mathrm{mT} ; 1,10$, and $100 \mu \mathrm{V} / \mathrm{cm} ; 4 \mathrm{~h} /$ day for 5 days) on nerve crush injury in a rat model. However, no difference was found in recovery of the toe-spread function between any PEMF treatments compared with the sham exposure.

Yang et al. (2010) investigated the neuroprotective effects of repetitive or rapid-rate transcranial magnetic stimulation (rTMS; $0.5 \mathrm{~Hz}, 250 \mathrm{~V} / \mathrm{m}$ ) on 6-hydroxydopamine (6-OHDA)-induced rats with Parkinson's disease (PD) (see also Section 3.5.1). Rapidrate transcranial magnetic stimulation was given to rats with $\mathrm{PD}$ induced by 6-OHDA daily for 4 weeks to examine its protective effects. Rotational test showed that rTMS significantly attenuated apomorphine-induced turns in rats with PD. Tissue processing showed that rTMS alleviated 6-OHDA-induced loss of dopaminergic neurons in rat substantia nigra. Furthermore, rTMS decreased the levels of cyclooxygenase-2 (COX-2) and tumor necrosis factor (TNF)- $\alpha$ in rat substantia nigra and prevented the fall of dopamine in the striatum of rats with PD.

Smith, Jackson, and Rorden (2009) investigated whether rTMS (28 Hz) could disrupt frontal eye fields (FEFs) while participants of the study performed an auditory localization task. Participants were stimulated for 176 milliseconds at $28 \mathrm{~Hz}$ (five pulses), and stimulation began 32 milliseconds before the onset of the central cue. In each trial a visual cue directed a participant's attention to the probable laterality of an auditory target, and the participant had to decide whether the subsequent target sound came from an upper or a lower speaker. In the absence of TMS, individuals could respond faster to targets that occurred on the cued side (valid trials) than when the target appeared contralaterally to the cued side (invalid side). The presence of TMS interfered with this effect, such that the costs associated with ipsilateral invalidly cued targets were substantially reduced. These results suggest that the eye-movement system is needed for normal auditory attention.

Hamidi, Tononi, and Postle (2008) conducted a functional neuroimaging study for investigating which specific regions of the frontal and the posterior parietal cortices contribute to the retention of information in spatial working memory using rTMS (10 Hz, produced motor-evoked potential $[\mathrm{MEP}] \geq 50 \mu \mathrm{V})$. They assessed the necessity for short-term retention of spatial information of brain areas identified by previous functional imaging studies: dorsolateral prefrontal cortex (DLPFC), FEFs, superior parietal lobule (SPL), and intraparietal sulcus (IPS). The administration of rTMS spanned the 3-second delay period of a spatial delayed-recognition task. The postcentral gyrus (PCG) was included to control any regionally nonspecific effects of rTMS. The only regionally specific effect was a significant decrease in reaction time when rTMS was applied to SPL. Additionally, rTMS lowered accuracy to a greater extent when applied to the left hemisphere than to the right and was more disruptive when applied contralaterally versus ipsilaterally to the 
visual field in which the memory probe was presented. Although it seems paradoxical, the finding of rTMS-induced improvement in task performance has a precedent and is consistent with the idea that regions associated with spatial sensory-motor processing make necessary contributions to the short-term retention of this information.

The same research group further investigated the neurophysiological effects of rTMS $(10 \mathrm{~Hz})$ delivered during the delay period of a visual working memory task by simultaneously recording brain activity with electroencephalography (EEG) (Hamidi et al. 2009). Subjects performed visual working memory tests for locations or for shapes, and in half the trials rTMS was delivered to the SPL or a control brain area. The wide range of individual differences in the effects of rTMS on task accuracy, from improvement to impairment, was predicted by individual differences in the effect of rTMS on power in the $\alpha$-band of the EEG $(10 \mathrm{~Hz})$ : A decrease in $\alpha$-band power corresponded with improved performance, whereas an increase in $\alpha$-band power corresponded with the opposite. The EEG effect was localized to cortical sources encompassing the FEFs and the IPS, and was specific to task (location, but not object memory) and to the rTMS target (SPL, not control area). Furthermore, for the same task condition rTMS-induced changes in cross-frequency phase synchrony between $\alpha$ - and $\gamma$-band $(>40 \mathrm{~Hz}$ ) oscillations predicted changes in behavior. These results suggest that $\alpha$-band oscillations play an active role in cognitive processes and do not simply reflect the absence of processing. Furthermore, this study shows that the complex effects of rTMS on behavior can result from biasing endogenous patterns of network-level oscillations.

Regarding rTMS influence on concurrent task performance, Johnson, Hamidi, and Postle (2010) revealed the effects of rTMS on power in the $\alpha$-band and on $\alpha$ : $\gamma$-phase synchrony, each predicting its effect on behavior in a combined rTMS/EEG study that investigated a complex set of relations between rTMS, EEG activity, and behavioral performance. These findings suggest that rTMS influences performance by biasing endogenous task-related oscillatory dynamics, rather than by creating a "virtual lesion." To further differentiate between these two alternatives, the authors compared the effects of a $10 \mathrm{~Hz}$ rTMS on neural activity with the results of an experiment in which rTMS was replaced with a $10 \mathrm{~Hz}$ luminance flicker. The authors reasoned that the $10 \mathrm{~Hz}$ flicker would produce widespread entrainment of neural activity to the flicker frequency and comparison of these EEG results with those from the rTMS study would shed light on whether the latter also reflected entrainment to an exogenous stimulus. Results revealed pronounced evidence for "entrainment noise," produced by $10 \mathrm{~Hz}$ flicker-increased oscillatory power and intertrial coherence (ITC) at the driving frequency, and increased $\alpha: \gamma$-phase synchronization that were nonetheless largely uncorrelated with behavior. This contrasts markedly with $10 \mathrm{~Hz}$ rTMS for which the only evidence for stimulationinduced noise, elevated ITC at $30 \mathrm{~Hz}$, differed qualitatively from the flicker results. The authors suggested that simultaneous recording of the EEG offers an important means of directly testing assumptions about how rTMS exerts its effects on behavior.

\subsubsection{Musculoskeletal System}

Abdelmelek et al. (2006) investigated the noradrenergic system and skeletal muscle $\mathrm{HSP} 72$ response to an SMF ( $\leq 128 \mathrm{mT}, 1 \mathrm{~h}$ /day for 5 days) in male rats. At thermoneutrality 
$\left(25^{\circ} \mathrm{C}\right)$, the SMF exposure induced an increase in NE content in gastrocnemius muscle at $128 \mathrm{mT}(+25 \%)$ but had no effect at $67 \mathrm{mT}(+1 \%)$, indicating a stimulatory effect of SMF on the noradrenergic system activity. However, the SMF did not induce significant increase in HSP72 levels in gastrocnemius muscles $(+29 \%)$. The results indicate that noradrenergic systems in gastrocnemius muscles are affected by SMF exposure. Furthermore, the same research group showed that SMF (128 mT, $1 \mathrm{~h}$ /day for 5-15 consecutive days) effects on glucose and lipid metabolism are time dependent (Lahbib et al. 2010).

Jimena et al. (2009) investigated the effect of an ELF-EMF ( $60 \mathrm{~Hz}, 0.7 \mathrm{mT}$, for 2 hours) on oxidative damage and skeletal muscle injury prompted by mepivacaine injection in the anterior tibial muscle of Wistar rats. Results revealed that mepivacaine induces oxidative stress, EMF prevents the harmful effects induced by mepivacaine, and EMF facilitates the regeneration process of skeletal muscle. In conclusion, the results showed the ability of EMFs to modify skeletal muscle response to mepivacaine.

Sakuraba et al. (2005) assessed the potential application and effectiveness of functional magnetic stimulation (FMS) for preventing skeletal muscle atrophy in adult rats. Functional magnetic stimulation using magnetic stimulator was performed to the soleus muscle of rats by placing a round magnetic coil on the back of the third to fifth lumbar vertebral levels (L3-L5) at $1 \mathrm{~T}$ and $20 \mathrm{~Hz}, 60 \mathrm{~min} / \mathrm{d}$ for $\leq 10$ days. An RT-PCR was applied to evaluate relative amounts of messenger ribonucleic acid (mRNA) specific to four myosin heavy chain (MHC) isoforms in soleus muscle during contractile activity by FMS. Ten-day unloading by hind limb suspension induced a drastic decrease in MHCI $\beta$ and MHCIIa mRNA expressions, although expressions of MHCIIb and MHCIId $(x)$ mRNA were not decreased. The FMS resuscitated the downregulation of mRNA levels of MHCI $\beta$ and MHCIIa. These results suggest that FMS on acute atrophied muscles is useful for preventing muscle atrophy.

Raux et al. (2010) examined the hypothesis that modulating supplementary motor area (SMA) activity using rTMS $(5$ and $10 \mathrm{~Hz})$ alters primary motor cortex $\left(\mathrm{M1}_{\mathrm{DIA}}\right)$ excitability in humans. The amplitude and latency of diaphragm MEPs $\left(\mathrm{MEP}_{\mathrm{DIA}}\right)$, evoked through single pulse $\mathrm{M} 1_{\text {DIA }}$ stimulation before and up to 16 minutes after SMA stimulation, were taken as indicators of $\mathrm{Ml}_{\mathrm{DIA}}$ excitability. The $\mathrm{MEP}$ from the first dorsal interosseous (FDI) muscle $\left(\mathrm{MEP}_{\mathrm{FDI}}\right)$ served as a control. Four SMA-conditioning sessions were performed in random order at 1-week intervals. Two aimed at increasing the SMA activity ( 5 and $10 \mathrm{~Hz}$, both at 110\% of FDI active motor threshold [aMT] and referred to as $5 \mathrm{~Hz}$ and $10 \mathrm{~Hz}$, respectively) and two aimed at decreasing it ( $1 \mathrm{~Hz}$ either at $110 \%$ of FDI aMT or at resting motor threshold [rMT]). The $5 \mathrm{~Hz}$ significantly increased $\mathrm{MEP}_{\text {DIA }}$ and $\mathrm{MEP}_{\mathrm{FDI}}$ amplitudes within a maximum of 11-16 minutes poststimulation. The $10 \mathrm{~Hz}$ increased $\mathrm{MEP}_{\mathrm{FDI}}$ amplitude with a similar time course, but it did not increase $\mathrm{MEP}_{\mathrm{DIA}}$ amplitude. Both aMT and rMT failed to decrease $\mathrm{MEP}_{\mathrm{DIA}}$ and $\mathrm{MEP}_{\mathrm{FDI}}$ amplitudes. The $\mathrm{MEP}_{\mathrm{DIA}}$ and $\mathrm{MEP}_{\mathrm{FDI}}$ latencies were unaffected by rTMS. These results demonstrate that $5 \mathrm{~Hz}$ rTMS over the SMA can increase the excitability of $\mathrm{M}_{\text {DIA }}$. These observations are consistent with the hypothesis of a functional connection between SMA and $\mathrm{M} 1_{\text {DIA }}$.

\subsubsection{Skeletal System}

Aydin and Bezer (2011) ascertained the effect that an SMF device (22-26 mT) implanted in rabbit femur had on fracture healing. Results verified that an intramedullary implant 
with an SMF improves bone healing in the first two weeks radiologically and that the configuration difference in magnetic poles has an effect on bone quality. However, the $\mathrm{SMF}$ was found to have no significant effects on bone mineral density (BMD) values. Xu et al. (2011) investigated the effects of a gradient SMF ( $B_{\max }$ of $180 \mathrm{mT}$ for 6 weeks) on osteoporosis of the lumbar vertebrae in ovariectomized rats. A small disc magnet was implanted to the right side of spinous process of the third lumbar vertebra. The results suggested that the SMF increased the BMD values of osteoporotic lumbar vertebrae in ovariectomized rats.

It has been reported that PEMF stimulation may be clinically beneficial during fracture healing and for a wide range of bone disorders. Shen and Zhao (2010) examined the effects of a PEMF $(15 \mathrm{~Hz}, 0.8 \mathrm{mT}$ ) on disuse osteoporosis (DOP) rats. Eighty 4-month-old female Sprague Dawley rats were randomly divided into intact (INT) group, DOP group, calcitonin-treated (CT) group, and PEMF-stimulated group. The right hind limbs of all the rats were immobilized by tibia-tail fixation except for those rats in the INT group. Rats in the CT group were injected with calcitonin (2 IU/kg i.p., once a day), and rats in the PEMF group were irradiated with PEMF immediately following operation. The BMD, serum transforming growth factor- $\beta 1$ (TGF- $\beta 1$ ) concentration, and interleukin-6 (IL-6) concentration of the proximal femur were measured 1, 2, 4, and 8 weeks after treatment. Compared with the CT and DOP groups, the BMD and serum TGF- $\beta 1$ concentration in the PEMF group significantly increased after 8 weeks. The IL- 6 concentration in the DOP group was elevated significantly after operation. The PEMF group showed significantly lower IL-6 levels than the DOP group. The results demonstrated that PEMF stimulation can efficiently suppress bone mass loss. Therefore, the authors concluded that PEMF exposure may affect bone remodeling process by promoting TGF- $\beta 1$ secretion and inhibiting IL- 6 expression.

Benazzo et al. (2008) evaluated PEMF (75 Hz, $1.5 \mathrm{mT}, 0.07 \mathrm{mV} / \mathrm{cm}, 6 \mathrm{~h} /$ day for 6 months) effects on the integration of osteochondral autografts in sheep. At one month, the osteogenic activity at the transplant-host subchondral bone interface was found to be increased in PEMF-treated animals compared with the controls. Articular cartilage was healthy in both controls and stimulated animals. At 6 months, complete resorption was observed in four control grafts only. Cystlike resorption areas were more frequent within the grafts of sham-treated animals versus those of PEMF-treated ones. The average volume of the cysts was not significantly different between the two groups; nevertheless, analysis of the variance of volumes demonstrated a significant difference. The histological score showed no significant differences between controls and stimulated animals, but the percentage of surface covered by fibrous tissue was found to be higher in the control group than in the stimulated one. The IL-1 and TNF- $\alpha$ concentrations in the synovial fluid were significantly lower and TGF- $\beta 1$ concentration was significantly higher in PEMF-treated animals compared with the controls. One month after osteochondral graft implantation the authors observed larger bone formation in PEMF-treated grafts, which favors early graft stabilization. In the long term, PEMF exposure limited bone resorption in subchondral bone; furthermore, the cytokine profile in the synovial fluid was indicative of a more favorable articular environment for the graft. 


\subsubsection{In Vivo Genotoxicity}

Knowledge about the relationship between exposure to magnetic fields and generation of free radicals in in vivo systems and in the cells is increasing. Regarding the role of ROS and RNS in genotoxicity induced by magnetic fields, it has been reviewed that free radical reactions are modulated by exposure to various magnetic fields of different intensities and frequency ranges and the duration of such exposures: many fields result in excitatory effects, some in inhibitory action, and others have no effect (Engström 2007; Simkó 2007; Okano 2008a,b). The magnetic fields influence (increase/decrease/ maintain) endogenous and exogenous ROS/RNS in in vivo, in vitro, and cell-free systems. However, magnetic field effects on pathogenesis and clinical relevance via ROS/ RNS have not been resolved. The underlying mechanisms of the modulation of ROS/ RNS induced by magnetic fields have not been clarified, although the relationship between oxidative stress and hypertension and/or ischemia-reperfusion injury has been implicated (see also Section 3.3.2.3).

Falone et al. (2008) examined whether the aging process can increase susceptibility toward widely present ELF-EMF-mediated prooxidative challenges. Female Sprague Dawley rats were continuously exposed to an ELF-EMF $(50 \mathrm{~Hz}, 0.1 \mathrm{mT})$ for 10 days. The results indicated that ELF-EMF exposure significantly affects antioxidative capability both in young and aged animals, although in opposite ways. Exposed young individuals enhanced their neurotrophic signaling and antioxidative enzymatic defense against a possible ELF-EMF-mediated increase in ROS. In contrast, aged animals underwent a significant decrease in the major antioxidant enzymatic activities. The authors suggested that ELF-EMF acts as a risk factor for the occurrence of oxidative stress-based nervous system pathologies associated with aging.

Yokus et al. (2008) investigated the genotoxic effect of an ELF-EMF $(50 \mathrm{~Hz}, 100$ and $500 \mu \mathrm{T}, 2 \mathrm{~h}$ /day for 10 months) on oxidative DNA base modifications. In rat leukocytes, levels of 8-hydroxyguanine (8-OH-Gua), 2,6-diamino-4-hydroxy-5-formamidopyrimidine (FapyGua), and 4,6-diamino-5-formamidopyrimidine (FapyAde) were measured following exposure to ELF-EMF. Levels of FapyAde, FapyGua, and 8OHdG in DNA were significantly increased by $100-\mu \mathrm{T}$ but not by $500-\mu \mathrm{T}$ ELF-EMF compared with the cage-control and the sham groups. The authors indicated that ELF-EMF generates oxidatively induced DNA base modifications, which are mutagenic in mammalian cells, such as FapyGua, FapyAde, and 8-OH-Gua. These findings support the hypothesis that chronic exposure to $50 \mathrm{~Hz}$ EMFs may be potentially genotoxic. However, the intensity of ELF-EMF has an important influence on the extent of DNA damage.

Torres-Duran et al. (2007) examined the effect of an ELF-EMF $(60 \mathrm{~Hz}, 2.4 \mathrm{mT}$, for 2 hours) on serum and liver lipid levels in rats. The results showed that ELF-EMF increases the serum values of high-density lipoprotein cholesterol and the liver content of lipoperoxides (measured as thiobarbituric acid reactive substances [TBARSs]) and decreases total cholesterol in the liver. The same research group further evaluated the effects of acute exposure to an ELF-EMF ( $60 \mathrm{~Hz}, 2.4 \mathrm{mT}$, for 2 hours), the application of movement restraint, and the combination of both on antioxidant systems in the plasma, liver, kidney, and heart of rats (Martinez-Samano 2010). Immediately after treatment, reduced glutathione (GSH), catalase (CAT), superoxide dismutase (SOD), and TBARS 
levels were measured in tissues. The GSH concentration was significantly lower in the heart of all experimental animals compared with the control group; furthermore, the decrease was higher in the liver of restrained animals. Superoxide dismutase activity was found to be lower in the plasma of restrained and EMF-exposed animals compared with unrestrained rats. There were no significant differences in CAT activity and TBARS levels among all the experimental groups versus the control group. The authors suggest that EMF might alter the metabolism of free radicals, decreasing SOD activity in plasma and GSH content in heart and kidney, but does not induce lipid peroxidation. Oxidative stress induced by movement restraint was stronger than that produced by EMF.

Goraca, Ciejka, and Piechota (2010) examined the effects of an ELF-EMF ( $40 \mathrm{~Hz}$, $7 \mathrm{mT}, 60 \mathrm{~min} /$ day for 2 weeks) on ROS generation in rat heart tissue. The results of the study indicated that the ELF-EMF caused significant increase in TBARS and $\mathrm{H}_{2} \mathrm{O}_{2}$ concentrations and decrease in the concentrations of GSH and total free - $\mathrm{SH}$ groups in heart homogenates. Moreover, the exposure to ELF-EMF resulted in a decrease in plasma antioxidant capacity.

Negishi et al. (2008) evaluated the hypothesis that power-frequency EMF $(50 \mathrm{~Hz}$, $\leq 350 \mu \mathrm{T}, 22 \mathrm{~h} /$ day, $7 \mathrm{~d}$ /week for 30 weeks) is a significant risk factor for the occurrence of hematopoietic neoplasia. The results showed that there was no observed sexual difference in the cumulative proportions of mice with 7,12-dimethylbenz(a)anthracene (DMBA)induced leukemia and no relationship between EMF exposure and the occurrence of neoplasia. These data provide no evidence to support the aforementioned hypothesis.

Emre et al. (2011) investigated the effect of an ELF-EMF (consecutive four pulse trains of $1,10,20$, and $40 \mathrm{~Hz}, 1.5 \mathrm{mT}, 0.6 \mathrm{~V} / \mathrm{m}$ ) on oxidative stress in rat liver tissue. The results showed significant increase in the levels of oxidative stress indicators, for example, plasma ALT, AST, alkaline phosphatase (ALP) albumin, bilirubin, total protein, malondialdehyde (MDA), and SOD activity, and indicated significantly higher apoptotic cell percentage but lower necrotic cell percentage in the ELF-EMF-exposed group compared with both the unexposed and the sham control groups. However, DNA ladder analyses did not differentiate between the groups. Although the effect of ELF-EMF on apoptosis is not a clearly established issue, the release of extracellular calcium ion has been considered by the authors as a critical step in the process.

Raggi et al. (2008) studied the effects of the relationship between EMF and oxidative stress in humans. The authors reported the effects of an ELF-EMF $(1-100 \mathrm{~Hz}, 1-100 \mu \mathrm{T})$ therapy device on oxidative status in humans. This device supplies complex magnetic signals with specific choices of frequency, intensity, and shape that are based on Liboff's ICR hypothesis. Thirty-two healthy volunteers were treated using the therapy device. A quantitative determination of oxidative stress was obtained at three time points by measuring MDA concentrations in peripheral blood before and after the cycle and one month following completion of the cycle. A highly significant reduction in mean MDA (53.8\%) was found at the end of treatment. One month later the mean MDA values were found to have risen again, but there was still a significant overall reduction of $15.6 \%$ compared with the original values.

Reddy et al. (2010) investigated the genotoxic effect of a PEMF $(<1000 \mathrm{~Hz}, 1 \mathrm{mT}$, for eight weeks) on mice. Positive control animals exposed to $1 \mathrm{~Gy} \gamma$-radiation were also included in the study. The extent of genotoxicity and cytotoxicity was assessed from 
the incidence of micronuclei (MN) and percent polychromatic erythrocytes (PCEs) in the blood and bone marrow, respectively. The data indicated that values of both indices in PEMF-exposed mice were not significantly different from those observed in shamexposed animals. In contrast, positive control mice exhibited significantly increased MN levels and decreased PCEs in both tissues. The results suggested that PEMF did not induce significantly increased genotoxicity and cytotoxicity in experimental mice.

\subsubsection{Tissue, Molecular, and Cellular Studies}

These so-called “in-vitro" studies on magnetic field effects, designed primarily to explore the underlying mechanisms, are reviewed in the following sections: Cell Growth, Proliferation, and Differentiation (3.3.2.1); Cell Membrane and Metabolic Activity (3.3.2.2); In Vitro Genotoxicity (3.3.2.3).

\subsubsection{Cell Growth, Proliferation, and Differentiation}

Regarding the effect of SMF on angiogenesis, Wang et al. (2009) investigated the effects of gradient SMF ( $B_{\max }$ of $400 \mathrm{mT}, 2.09 \mathrm{~T} / \mathrm{m}$, for 11 days) on angiogenesis both in vitro and in vivo and suggested that gradient SMF might inhibit or prevent the formation of new blood vessels and could be helpful for the treatment of some diseases relevant to tumor angiogenesis (see also Section 3.5.5). In contrast to the inhibition angiogenesis by gradient SMF, Okano et al. (2006) and Okano, Tomita, and Ikada $(2007,2008)$ reported that gradient SMF promoted angiogenesis $\left(B_{\max }\right.$ of $120 \mathrm{mT}, 28 \mathrm{~T} / \mathrm{m}$, for 10 days) in vitro. Therefore, the effects and mechanisms of gradient SMF on angiogenesis have not yet been determined.

Dini et al. (2009) investigated the morphofunctional influence of an SMF (6 mT, for 72 hours) during differentiation induced by 12-O-tetradecanoyl-13-phorbol acetate (TPA, $50 \mathrm{ng} / \mathrm{ml}$ ) in human leukaemia U937 cells. The cell morphology of U937 cells was investigated by optic and electron microscopy. Specific antibodies or molecules or both were used to label CD11c, CD14, phosphatidylserine, and F-actin and to investigate the distribution and activity of lysosomes, mitochondria, and smooth endoplasmic reticulum (SER). The ion $\left[\mathrm{Ca}^{2+}\right]_{\mathrm{i}}$ was evaluated with a spectrophotometer. The degree of differentiation in SMF-exposed cells was lower than that of nonexposed cells in a timedependent manner. The SMF-exposed cells showed cell shape and F-actin modification, inhibition of cell attachment, appearance of membrane roughness and large blebs, and impaired expression of specific macrophagic markers on the cell surface. The intracellular localization of SER and lysosomes was only partially affected by exposure. A significant localization of mitochondria with an intact membrane potential at the cell periphery in nonexposed, TPA-stimulated cells was observed; conversely, in the presence of SMF mitochondria were mainly localized near the nucleus. In no case did SMF exposure affect cell viability. The authors suggested that the sharp intracellular increase of $\left[\mathrm{Ca}^{2+}\right]_{\mathrm{i}}$ could be one of the causes of the aforementioned changes.

The Dini research group (Dini and Panzarini 2010) further reported that an exposure to 6-mT SMF affects fluid-phase endocytosis and phagocytosis in monocyte/macrophages in a differentiation degree-dependent manner. The phagocytosis index and rate of phagocytosis decreased under SMF exposure, whereas the number of latex particles 
bound to the plasma membrane of TPA-differentiated U937 and THP-1 cells increased. Conversely, the rate of phagocytosis of apoptotic cells increased under SMF exposure, whereas the number of apoptotic cells bound to the plasma membrane of isolated human Kupffer cells, RAW 264.7 macrophages, and TPA-differentiated THP-1 and U937 cells decreased. In nondifferentiated U937 and THP-1 cells, SMF exposure enhanced the number of cell surface-bound apoptotic cells and latex beads.

Kim et al. (2005) studied the effects of various SMFs (1-10 mT) on the adsorption of specific recombinant fibronectin (FN) peptide (hFNIII9-10) on the titanium surface in human osteosarcoma TE-85 cells. The authors found significant increases in cell attachment and proliferation, especially in cells exposed to a field of $1 \mathrm{mT}$. However, SMFs of up to $10 \mathrm{mT}$ did not influence $\mathrm{FN}$ adsorption on the titanium surface.

Ventura et al. (2005) showed that the exposure of pluripotent mouse embryonic stem (ES) cells to an ELF-EMF $(50 \mathrm{~Hz}, 0.8 \mathrm{mT})$ triggered the expression of GATA-4 and $\mathrm{Nkx}-2.5$, acting as cardiac lineage-promoting genes in different animal species, as well as humans. The ELF-EMF also enhanced prodynorphin gene expression and the synthesis and secretion of dynorphin B, an endorphin playing a major role in cardiogenesis. These effects occurred at the transcriptional level and ultimately resulted in a remarkable increase in the yield of ES-derived cardiomyocytes. These results demonstrate the potential use of ELF-EMFs in modifying the gene program of cardiac differentiation in ES cells without the aid of gene transfer technologies and suggest that this development may pave the way for novel approaches in tissue engineering and cell therapy.

Mannerling et al. (2010) reported the influence of ELF-EMFs $(50 \mathrm{~Hz}, 0.025-0.10 \mathrm{mT}$, for 1 hour) on different cellular parameters in human leukaemia cells (K562). The positive control heat treatment $\left(42^{\circ} \mathrm{C}\right.$, for 1 hour) did not affect either cell proliferation or superoxide radical anion production but caused accumulation of cells in the $G_{2}$ phase and increased the stress protein HSP70. The EMF exposure $(0.10 \mathrm{mT}$, for one hour) did not affect either cell cycle kinetics or proliferation. Both vertical and horizontal EMF exposures for one hour caused significantly and transiently increased HSP70 levels (more than twofold) at several flux densities, compared with the sham controls and the heat treatment. This exposure also increased (by 30\%-40\%) the levels of the superoxide radical anion, which was comparable to the positive control phorbol myristate acetate. Addition of free radical scavengers (melatonin or 1,10-phenantroline) inhibited the EMF-induced increase in HSP70. In conclusion, an early response to EMF in K562 cells seems to be the presence of an increased amount of oxygen radicals, leading to HSP70 induction. Furthermore, the results suggest that the effective flux density threshold is $\leq 0.025 \mathrm{mT}$, and also that it is the EMF and not the induced electric field that is the active field.

Jansen et al. (2010) investigated the effect of a PEMF (15 Hz, $100 \mu \mathrm{T}, 5$-millisecond bursts with 5-microsecond pulses) on human bone marrow-derived stromal cell (BMSC) metabolism and investigated, specifically, whether the PEMF can stimulate the osteogenic differentiation of BMSC. The authors suggest that PEMF exposure enhances mineralization and induces differentiation at the expense of proliferation. The osteogenic stimulus of PEMF was confirmed by the upregulation of several osteogenic marker genes in the PEMF-treated group, which preceded the deposition of mineral itself. 
These findings indicated that PEMF can directly stimulate osteoprogenitor cells toward osteogenic differentiation.

Sun et al. (2009) investigated the effect of a PEMF (15 Hz, $1.8 \mathrm{mT}, 8 \mathrm{~h} /$ day for 8 days) on the proliferation and differentiation potential of human bone marrow mesenchymal stem cells (BMMSCs). Results showed that more viable BMMSCs were obtained in the PEMF-exposed cultures. Although the growth rates of BMMSCs during the exponential growth phase were not significantly affected, $20 \%-60 \%$ higher cell densities were achieved during the exponentially expanding stage. Many newly divided cells appeared from 12 to 16 hours after PEMF treatment. These results suggested that PEMF could enhance BMMSC proliferation during the exponential phase and that this possibly resulted from the shortening of the lag phase. In addition, the PEMF-exposed BMMSCs showed multilineage differentiation potential similar to the control group.

Tsai et al. (2009) examined the effect of a PEMF $(7.5 \mathrm{~Hz}, 0.13 \mathrm{mT}, 2 \mathrm{mV} / \mathrm{cm})$ on the proliferation and osteogenic differentiation of human mesenchymal stem cells (hMSCs). The production of ALP was significantly enhanced at day 7 with PEMF treatment in both basal and osteogenic cultures compared with the controls. The expressions of other early osteogenic genes, including Runx $2 / \mathrm{Cbfa} 1$ and ALP, were also partially modulated by PEMF, indicating that osteogenesis in hMSC was associated with the specific PEMF. Based on ALP and alizarin red S staining, the accumulation of ALP protein produced by the hMSC as well as calcium deposits reached their highest levels at day 28 . The results indicated that findings on PEMF stimulation provide insights into the development of PEMF as an effective technology in regenerative medicine.

\subsubsection{Cell Membrane and Metabolic Activity}

Shen, Chao, and Du (2007) examined the effects of an SMF (125 mT) on two types of voltage-gated potassium channel (VGPC) currents, I and $\mathrm{I}(\mathrm{K}, \mathrm{V})$, in rat trigeminal root ganglion (TRG) neurons by whole-cell patch-clamp experiments. The results demonstrated that SMFs could influence the inactivation kinetics of these two VGPC currents by altering the inactivation rate and velocity. No significant change was observed in activation properties. These findings support the hypothesis that biological membranes would be deformed and physiological characteristics of ion channels on the membrane would be influenced in moderate-intensity SMFs.

Martino, Perea, et al. (2010) examined the response of human umbilical vein endothelial cells (HUVECs) to weak SMFs (60 and $120 \mu \mathrm{T}$ ). The authors investigated proliferation, viability, and the expression of functional parameters such as endothelial NOS (eNOS), NO, and also the gene expression of vascular endothelial growth factor (VEGF). An SMF of $120 \mu \mathrm{T}$ increased HUVEC proliferation by $40 \%$ in 48 hours, compared with the group shielded in a micrometal cylinder $(0.2-0.7 \mu \mathrm{T})$. The HUVECs showed sensitivity to both magnetic field intensity and exposure time; HUVECs responded to SMFs as low as $60 \mu \mathrm{T}$. However, both SMF exposures for 1 hour had no effect on proliferation. The HUVEC functionality increased after SMF exposure, which upregulated eNOS expression. The SMF had no effect on VEGF gene expression, which indicated there was no increased potential for tumor development by the overexpression of VEGF. Although SMF increased eNOS-positive HUVECs, NO concentration remained unaltered throughout the culture period. The authors assumed that weak SMFs may open 
new avenues of research in vascular therapies by promoting endothelial cell growth and by enhancing the healing response of endothelium.

Furthermore, the same authors (Martino, Portelli, et al. 2010) showed that reduction of the geomagnetic field inhibits the growth rates of model cancer cell lines. In particular, a reduced field of $0.2-0.5 \mu \mathrm{T}$ led to a decrease in the viable number of fibrosarcoma cells (HT1080) compared with the geomagnetic field level of $45 \mu \mathrm{T}$. The authors speculated that although the underlying mechanism is unclear, this change as assessed by cell number is not a result of apoptosis, necrosis, or cell cycle alterations.

Potenza et al. (2010) described the effects of a gradient SMF ( $B_{\max }$ of $300 \mathrm{mT}$; for 4, 24,48 , and 72 hours) on cell growth and mitochondrial DNA integrity of HUVEC. The SMF was shown to be significant after 4 hours of exposure, inducing damage at both the nuclear and mitochondrial levels, reducing mitochondrial content, and increasing ROS levels. Twenty-four hours of exposure increased mitochondrial DNA content as well as expression of one of the main genes related to mitochondrial biogenesis. No significant differences between exposed and sham cultures were found after exposures of 48 and 72 hours. The results suggested that a $300-\mathrm{mT}$ SMF does not cause permanent DNA damage in HUVECs and that it stimulates a transient mitochondrial biogenesis.

Sullivan, Balin, and Allen (2011) reported that SMF (230-250 mT) exposure stimulates ROS production in human fetal lung cells (WI-38) during the first 18-hour period when cells are attaching to the culture vessel. These results support the hypothesis that increased ROS formation may account for SMF effects on cell attachment. However, SMF decreases growth in WI-38 cells long after the increase in ROS has abated, suggesting that other mechanisms account for SMF effects on cell growth.

Chiu et al. (2007) tested the effects of an SMF (400 mT) on osteoblasts differentiation in MG63 osteoblast-like cells. The results showed that the SMF increased ALP activity and extracellular matrix release in MG63 cells. In contrast, SMF-exposed cells exhibited significant increase in fluorescence anisotropy at 6 hours, with significant reduction in the proliferation effects of growth factors noted at 24 hours. The authors suggested that one of the possible mechanisms by which SMF affects osteoblastic maturation is increasing the membrane rigidity and reducing the proliferation-promoting effects of growth factors at the membrane domain. The same research group also investigated the effects of an SMF (400 mT, for 5 days) on the differentiation of MG63 cells cultured on the surface of poly-L-lactide (PLLA) substrates by MTT assay (Feng et al. 2010). The SMF-exposed cells exhibited decreased MTT values after 1 and 3 days of culture. In addition, SMF promoted the expression of extracellular matrix in MG63 cells on the PLLA substrate. After 1 day, the ALP-specific activity of SMF-exposed MG63 cells was significantly increased by 1.5 -fold. These results showed that MG63 cells seeded on a PLLA disc and treated with SMF had a more differentiated phenotype.

Wang et al. (2009) investigated the effect of SMF (230-280 mT) on cellular signaling pathways in human embryonic cells. The SMF-mediated responses were manifest at the cellular level as morphological changes and biochemical markers indicative of pre-oligodendrocyte differentiation. This study provides a framework describing how SMF exposure is transduced from a plausible molecular biosensor (lipid membranes) to cell-level responses that include differentiation toward neural lineages. In addition, the authors suggested that SMF might be involved in new relationships between 
gangliosides, time-dependent regulation of IL- 6 signaling by glycosphingolipids, and the fate of embryonic cells. Furthermore, Wang et al. (2010) reported that SMF exposure elicited qualitatively similar responses as ZM241385, a PD drug candidate.

Khodarahmi, Mobasheri, and Firouzi (2010) examined the effect of a homogeneous SMF of $2.1 \mathrm{~T}$ on viability and morphology of rat astrocytes; but the study showed that the SMF exposure for 72 hours did not significantly affect the viability and morphological properties. Hirai et al. (2006) screened genes responsive to brief exposure to an SMF (100 $\mathrm{mT}$, for $15 \mathrm{~min}$ ) in cultured rat hippocampal neurons. The authors cloned and identified Ntan1 (amidohydrolase for N-terminal asparagine) as an SMF-responsive gene in rat brain. Ntan1 is an essential component of a protein degradation signal, which is a destabilizing $\mathrm{N}$-terminal residue of a protein, in the $\mathrm{N}$-end rule. Northern blot analysis showed that Ntan 1 mRNA level was increased by about threefold 3 hours after exposure. The SMF also increased the transcriptional activity of Ntan1 promoter. The SMF induced degradation of microtubule-associated protein 2 (MAP2) without affecting cell morphology and viability, which was prevented by a selective inhibitor of $26 \mathrm{~S}$ proteasome in hippocampal neurons. Overexpression of Ntan1 using recombinant Ntan1 adenovirus vector resulted in a marked decrease in MAP2 expression in hippocampal neurons. The results suggest that brief exposures to SMF leads to the induction of Ntan1 responsible for MAP2 degradation through the ubiquitin-proteasome pathway in rat hippocampal neurons.

Yang et al. (2010) explored the role of calmodulin in the effects of an SMF (400 mT) on osteoblastic MG63 cells. The role of calmodulin antagonist W-7 was used to evaluate alterations in osteoblastic proliferation and differentiation after exposure. The results showed that SMF increased ALP activity and phosphodiesterase 1C gene expression in MG63 cells. Addition of W-7 significantly inhibited the SMF-induced cellular response. The authors suggested that one possible mechanism by which SMF affects osteoblastic maturation is a calmodulin-dependent mechanotransduction pathway.

Sakurai, Terashima, and Miyakoshi (2008) investigated whether exposure to an inhomogeneous strong SMF of $10 \mathrm{~T}$ affects prostaglandin $\mathrm{E}_{2}\left(\mathrm{PGE}_{2}\right)$ secretion from a mouse osteoblastic cell line, MC3T3-E1. The authors also investigated the $\mathrm{PGE}_{2}$ synthesizing enzyme, COX-2, and translocation of NF- $\kappa B$, which is involved in the induction of COX-2 expression. An inhomogeneous SMF exposure was performed at the 10 T-exposure position, at which the magnetic flux density was found to be the highest, and at the $6 \mathrm{~T}$-exposure position, at which the magnetic gradient was found to be the highest $(41.7 \mathrm{~T} / \mathrm{m})$. The secretion of $\mathrm{PGE}_{2}$ was not affected by exposure at the $10 \mathrm{~T}$-exposure position compared with sham exposure, but it was enhanced at the 6 T-exposure position (by about 1.5-fold). Similarly, COX-2 expression and NF- $\kappa$ B translocation were not enhanced at the $10 \mathrm{~T}$-exposure position, but they increased at the 6 T-exposure position (by about twofold and two- to threefold, respectively). These findings suggested that exposure to a high magnetic gradient induced the secretion of $\mathrm{PGE}_{2}$ and the expression of the COX-2 protein, which was mediated through increased translocation of NF- $\kappa \mathrm{B}$.

The same research group (Sakurai, Terashima, and Miyakoshi 2009) further evaluated the effects of a strong SMF (10 T) on glucose-metabolic pathways. Hamster-derived insulin-secreting cells (HIT-T15) were cultured under exposure to sham and SMF 
conditions (3-10 T, 0-41.7 T/m) for 0.5 and 1 hours. Exposure to an SMF with a high magnetic gradient for 1 hour significantly increased insulin secretion and insulin-1 mRNA expression. Exposure to an SMF with a high magnetic flux density for 0.5 hour significantly enhanced responsiveness to glucose stimulation. The SMF did not affect insulin content, cell proliferation, or cell number. These results suggest that MRI systems with higher magnetic flux densities might not cause cell proliferative or functional damages on insulin-secreting cells.

Regarding the effects of ELF-EMF on glucose-metabolic pathways, the same research group investigated the effects of an ELF-EMF $(60 \mathrm{~Hz}, 5 \mathrm{mT})$ on $\beta$-cell survival and function in HIT-T15 cells (Sakurai et al. 2008). The authors found that exposure to ELFEMF for 5 days without glucose increased cell number, exposure for 2 days without glucose and for 5 days with $100-\mathrm{mg} / \mathrm{dL}$ glucose increased insulin secretion to the culture medium, and exposure for 2 and 5 days with 40 - and 100-mg/dL glucose increased intracellular insulin concentration. The authors speculated that the increase in cell number under apoptotic culture conditions by exposure to ELF-EMF could lead to new therapeutic concepts in the treatment of diabetes.

Gaetani et al. (2009) assessed the hypothesis that exposure of human cardiospheres (CSs) and cardiosphere-derived cells (CDCs) to an ELF-EMF, tuned at $\mathrm{Ca}^{2+}$ - ICR, may drive their differentiation toward a cardiac-specific phenotype.

The exposure parameters were calculated based on the following equation:

$$
f=\frac{q \cdot B_{\mathrm{DC}}}{m \cdot 2 \pi}
$$

where $q$ and $m$ are charge and mass of an ion, respectively; $B_{\mathrm{DC}}$ is the flux density of the applied SMF, and $f$ is the frequency of the superimposed EMF.

Since at resonance conditions the maximum possible extent of energy is believed to be transferred to the system, the intensity of the EMF applied was in the microtesla range. Under these conditions, the amount of heating due to "Joule effect" is negligible and all the effects reported after cell exposure must be related to cyclotron exposure.

In this study, CSs and CDCs were exposed for 3 or 5 days to both an SMF $(10 \mu \mathrm{T})$ and an ELF-EMF $(7 \mathrm{~Hz}, 2.5 \mu \mathrm{T})$, close to the ICR frequency corresponding to the chargeto-mass ratio of $\mathrm{Ca}^{2+}$. A significant increase in the expression of cardiac markers was observed after 5-day exposure to $\mathrm{Ca}^{2+}$-ICR in both human CSs and CDCs, as was evidenced at the transcriptional, translational, and phenotypical levels. The mobilization of $\mathrm{Ca}^{2+}$ among intracellular storages was observed. These results suggest that ELF-EMF tuned at $\mathrm{Ca}^{2+}$-ICR could be used to drive cardiac-specific differentiation in adult cardiac progenitor cells without any pharmacological or genetic manipulation of the cells that are used for therapeutic purposes.

Soda et al. (2008) examined the effect of an ELF-EMF $(60 \mathrm{~Hz}, 3 \mathrm{mT})$ on differentiation of mouse osteoblast-like MC3T3-E1 cells together with the addition of insulin-like growth factor I (IGF-I). The pharmacological results suggested that ELF-EMF-induced collagen synthesis was mediated through the p38 mitogen-activated protein kinase (p38 MAPK) pathway and that suppression of the phosphatidylinositol 3-kinase (PI3K) pathway allowed the acceleration of collagen synthesis. 
Vianale et al. (2008) investigated the effect of an ELF-EMF $(50 \mathrm{~Hz}, 1 \mathrm{mT})$ on keratinocyte proliferation and production of chemokines, regulated on activation, normal $\mathrm{T}$ cell expressed and secreted (RANTES), monocyte chemoattractant protein (MCP)-1, macrophage inflammatory protein (MIP)- $1 \alpha$, and IL- 8 in the human keratinocyte cell line HaCaT. Significantly increased growth rates were observed after 48 hours of EMF exposure compared with control cells, whereas no difference in cell viabilities was detected. Gene expression and release of RANTES, MCP-1, MIP-1 $\alpha$, and IL- 8 were significantly reduced after 72 hours of exposure. The NF- $\mathrm{KB}$ levels became almost undetectable after only 1 hour of exposure, and they were inversely correlated with cell density. The authors suggested that ELF-EMF modulates chemokine production and keratinocyte growth through inhibition of the NF- $\mathrm{KB}$ signaling pathway and thus may inhibit inflammatory processes. They postulated that ELF-EMF could represent an additional therapeutic approach in the treatment of skin injury. The same research group further revealed that the exposure of HaCaT cells to ELF-EMF increased inducible NOS (iNOS) and eNOS expression levels (Patruno et al. 2010). These ELF-EMF-dependent increased expression levels were paralleled by increased NOS activities and increased NO production. In addition, higher levels of activator protein 1 (AP-1) expression as well as a higher cell proliferation rate were associated with ELF-EMF exposure. In contrast, ELF-EMF exposure decreased COX-2 expression, $\mathrm{PGE}_{2}$ production, catalase activity, and $\mathrm{O}_{2}{ }^{-}$production. The authors postulated that the ability of ELF-EMFs to upregulate NOS activities, and thus nitrogen intermediates as well as cell proliferation, and to downregulate COX-2 expression and the downstream intermediate $\mathrm{PGE}_{2}$, highlights the potential therapeutic role of ELF-EMFs in wound healing processes.

Ke et al. (2008) investigated the effect of an ELF-EMF $(50 \mathrm{~Hz}, 0.4 \mathrm{mT})$ on clustering of epidermal growth factor (EGF) receptors and ras protein activation in Chinese hamster lung (CHL) cells. The results showed that, compared with sham-exposed cells, 5 minutes of exposure slightly induced EGF receptor clustering and 15 minutes of exposure significantly enhanced receptor clustering. Ras protein was also activated after exposure. Exposure to a "noise" EMF (30-90 Hz), at the same intensity and durations, did not significantly affect EGF receptor clustering and ras protein activation. However, by superimposing the noise EMF, the EGF receptor clustering and ras activation induced by $50 \mathrm{~Hz}$ EMF were found to be inhibited. These results suggest that membrane receptors are one of the most important targets where a $50 \mathrm{~Hz}$ EMF interacts with cells and that ras protein participates in the signal transduction process of the $50 \mathrm{~Hz}$ EMF. Furthermore, a noise EMF can inhibit the effects caused by a $50 \mathrm{~Hz}$ EMF. The same research group also suggested that membrane receptors are one of the main targets where an ELF-EMF interacts with cells and that the intensity threshold in the case of EGF receptors is between 0.05 and $0.1 \mathrm{mT}$ (Sun et al. 2008). An incoherent EMF could completely inhibit the effects induced by an ELF-EMF of equal or lower intensity.

Salerno et al. (2009) investigated the influence of an ELF-EMF $(50 \mathrm{~Hz}, 0.5 \mathrm{mT})$ or an SMF $(0.5 \mathrm{mT})$ on subsets of human $\mathrm{CD}^{+} \mathrm{T}$ cells. The $\mathrm{CD} 4^{+} \mathrm{T}$ cells can be divided into different subsets on the basis of surface marker expression, such as CD45, and T cells can be divided into naive $\left(\mathrm{CD} 45 \mathrm{RA}^{+}\right)$and memory $\left(\mathrm{CD} 45 \mathrm{RA}^{-}\right)$cells. The authors found that the $\mathrm{CD}^{+} \mathrm{CD} 45 \mathrm{RA}^{-} \mathrm{T}$ subset was more sensitive after 2 hours of exposure to either of the two magnetic fields. Decreases in the release/content of interferon (IFN)- $\gamma$, in cell proliferation 
and in intracellular free calcium concentrations, were observed in $\mathrm{CD}^{+}{ }^{+} \mathrm{CD} 45 \mathrm{RA}^{-} \mathrm{T}$ cells compared with $\mathrm{CD}^{+} \mathrm{CD} 45 \mathrm{RA}^{+} \mathrm{T}$ cells in either of the magnetic fields. The results suggested that exposure to either magnetic field induces a delay in the response to stimulants and that modifications are rapidly reversible, at least after a short exposure.

Blackman (2006) commented that because calcium ion release is a measure of neurotransmitter activity, calcium ion release can be chosen as a simple method for studying EMF effects on cells. Dose-response curves show responses at two levels of exposure with a flat response to doses between these two dose peaks. These two levels are known as intensity windows.

Zhang et al. (2010) studied the effects of an ELF-EMF $(50 \mathrm{~Hz}, 0.8 \mathrm{mT})$ on intracellular calcium ion $\left(\left[\mathrm{Ca}^{2+}\right]_{\mathrm{i}}\right)$. Osteoblastic cells were used as a model both to test the hypothesis that ELF-EMFs can alter the concentrations of $\left[\mathrm{Ca}^{2+}\right]_{\mathrm{i}}$ and to examine the predicted window effect. The results demonstrated that ELF-EMFs can induce the uptake of $\left[\mathrm{Ca}^{2+}\right]_{\mathrm{i}}$ as the empirical evidence of the specified window effects of $\left[\mathrm{Ca}^{2+}\right]_{i}$ in osteoblasts.

Morabito et al. (2010) examined the effects of short-term exposure to ELF-EMF $(50 \mathrm{~Hz}, 0.1$ and $1.0 \mathrm{mT}$, for 30 minutes) on muscle cell differentiation and function in $\mathrm{C} 2 \mathrm{C} 12$ cells. The focus was on markers of oxidative stress and $\mathrm{Ca}^{2+}$ handling. The data revealed that an ELF-EMF of $1.0 \mathrm{mT}$ induced ROS production in myoblasts and myotubes with a concomitant decrease in mitochondrial membrane potential; activated the cellular detoxification system, increasing catalase and GSH peroxidase (GPx) activities; and altered intracellular $\mathrm{Ca}^{2+}$ homeostasis, increasing the spontaneous activity of myotubes and enhancing cellular reactivity to a depolarizing agent $(\mathrm{KCl})$ or an agonist (caffeine) of intracellular store $\mathrm{Ca}^{2+}$ channels. In conclusion, the results suggested a possible link between exposure to an ELF-EMF and modification of the cellular redox state, which in turn could increase the level of intracellular $\mathrm{Ca}^{2+}$ and thus modulate the metabolic activity of $\mathrm{C} 2 \mathrm{C} 12$ cells.

For PEMF effects, Li et al. (2006) compared the effects of ultrasound (US) on osteoblast proliferation with those of a PEMF $(7.5 \mathrm{~Hz}, 2 \mathrm{mV} / \mathrm{cm})$ by using different signal transduction pathway inhibitors. The cells were stimulated for 15 minutes under US or for 2 hours under PEMF exposure. The results showed that there are different transduction pathways for US and PEMF stimulation that lead to an upgrade of osteoblast proliferation, although their pathways all lead to an increase in cytocolic $\mathrm{Ca}^{2+}$ and the activation of calmodulin. These findings offered a biochemical mechanism to support the process of US- and PEMF-induced enhanced healing of bone fractures.

Martino et al. (2008) investigated the effects of a repetitive pulse burst PEMF $(15 \mathrm{~Hz}$, $0.9 \mathrm{mT} / 10 \mu \mathrm{s}, 9 \mathrm{mV} / \mathrm{cm}$ ) on the cellular activity of SaOS-2 osteoblast-like cells. The PEMF stimulation did not affect metabolic activity and cell number. However, the ALP activity of SaOS-2 cells and mineral nodule formation increased significantly after PEMF stimulation. These observations suggested that PEMF does not affect cellular metabolism; however, it may play a role in the enhancement of SaOS-2 cell mineralization.

\subsubsection{In Vitro Genotoxicity}

It has been known for several years that oxidative stress damages lipids, proteins, and nucleic acids, resulting in cell membrane and synaptic disorganization, neural cell signaling dysfunction following apoptotic or necrotic events, or both (Cui et al. 2004). 
In this context, the relationship between magnetic fields and oxidative stress has also been investigated in vitro (see also Section 3.3.1.9).

Hao et al. (2011) investigated whether SMFs ( $8.8 \mathrm{mT}$, for 12 hours) can enhance the killing effect of adriamycin (ADM) in human leukemia cells (K562). The authors showed that the 8.8-mT SMF enhanced the cytotoxity potency of ADM on K562 cells and suggested that the decrease in P-glycoprotein expression may be one reason underlying this effect.

Sarvestani et al. (2010) evaluated the influence of an SMF (15 mT, for 5 hours) on the progression of cell cycle in rat BMSCs. The cells were divided into two groups. One group was exposed to SMF alone, whereas the other group was exposed to X-rays before SMF exposure. The population of cells did not show any significant difference in the first group, but the second group exposed to acute radiation before SMF exposure showed a significant increase in the number of cells in the $G_{2} / M$ phase. The SMF intensified the effects of X-ray exposure, whereas SMF alone did not have any detectable influence on cell cycle.

Schwenzer et al. (2007) reported that there was no effect of an SMF alone (3.0 T, for 2 hours) on clonogenic ability, proliferation, and cell cycle in eugenic human lung fibroblasts (Hel 299).

Kimura et al. (2008) examined the effect of 3- or 5-T SMF on gene expression in the experimental model metazoan Caenorhabditis elegans. In addition, transient induction of $h p s 12$ family genes was observed after SMF exposure. The small-hps gene $h p s 16$ was also induced but to a much lesser extent, and the lacZ-stained population of $h p s 16-1::$ lac $Z$ transgenic worms did not significantly increase after SMF exposure with or without a second stressor, mild heat shock. Several genes encoding apoptotic cell death activators and secreted surface proteins were upregulated after ionizing radiation (IR), but they were not induced by SMF. The RT-PCR analyses for 12 of these genes confirmed the expression differences between worms exposed to SMF and those exposed to IR. In contrast to IR, exposure to high SMFs did not induce DNA double-strand breaks or germ line cell apoptosis during meiosis. These results suggest that the response of $C$. elegans to high SMFs is unique and capable of adjustment during long exposure, and that this treatment may be less hazardous than other invasive treatments and drugs.

Lupke et al. (2006) examined the cell-activating capacity of an ELF-EMF $(50 \mathrm{~Hz}$, $1.0 \mathrm{mT}$ ) on human umbilical cord blood-derived monocytes. The results confirmed the previous findings of cell-activating capacity of ELF-EMFs in human monocytes, which was detected as an increased ROS release. Furthermore, gene expression profiling (human UniGene RZPD-2 cDNA array) was performed to achieve a comprehensive view of the genes involved in the cell activation process after 45 minutes of exposure. These results indicated alteration of 986 genes involved in metabolism, cellular physiological processes, signal transduction, and immune response. Significant regulations could be analyzed for five genes (expression greater than twofold or less than 0.5 -fold): (1) IL 15RA (receptor $\alpha$ chain); (2) EPS15R (epidermal growth factor receptor pathway substrate 15-like 1); (3) DNMT3A (hypothetical protein MGC16121); (4) DNMT3A (DNA [cytosine-5] methyltransferase $3 \alpha$ ); and (5) one gene with no match to known genes, DKFZP586J1624. Real-time RT-PCR analysis of the expression kinetics of IL15RA, and IL10RA during the 45-minute exposure indicated the regulation of cell activation via 
the alternative pathway, whereas the delayed gene expression of FOS, IL2RA, and the melatonin-synthesizing enzyme HIOMT suggested the suppression of inflammatory processes. Accordingly, the authors suggested that ELF-EMF activates human monocytes via the alternative pathway. The same research group further demonstrated that ELF-EMFs ( $50 \mathrm{~Hz}, 0.05-1.0 \mathrm{mT}$, for 48 hours) significantly elevated phagocytic activity, free radical release, and IL-1 $\beta$ production and concluded that ELF-EMFs did not induce any genotoxic effect (Frahm et al. 2006).

Simkó (2007) hypothesized that ELF-EMFs can modify the activity of an organism by changing ROS levels leading to oxidative stress, because free radicals can interact with DNA resulting in single-strand breaks. The author reviewed several studies on oxidative stress, as well as the changes in oxidant and antioxidant content after ELF-EMF exposure. Finally, the author concluded that modulations of the oxidant and antioxidant levels through ELF-EMF exposure can play a causal role in cancer development. However, the mechanisms of ELF-EMF-induced genotoxicity are not yet clear.

Koyama et al. (2008) investigated the effects of an ELF-EMF (60 Hz, $5 \mathrm{mT}$, for 24 hours) on cell functions. The number of apurinic/apyrimidinic (AP) sites in human glioma A172 cells was measured following ELF-EMF exposure. The cells were exposed to an ELF-EMF, to genotoxic agents, to methyl methane sulfonate (MMS), and to hydrogen peroxide $\left(\mathrm{H}_{2} \mathrm{O}_{2}\right)$ alone, or to an ELF-EMF with the genotoxic agents. There was no difference in the number of AP sites between ELF-EMF-exposed cells and sham controls. With MMS or $\mathrm{H}_{2} \mathrm{O}_{2}$ alone, the number of AP sites increased with longer treatment times. The exposure to ELF-EMF in combination with genotoxic agents increased AP-site levels compared with exposure to genotoxic agents alone. These results suggested that the number of AP sites induced by MMS or $\mathrm{H}_{2} \mathrm{O}_{2}$ is enhanced by exposure to ELF-EMFs. The authors postulated that ELF-EMFs can enhance the activity or lengthen the lifetime of radical pairs.

Di Loreto et al. (2009) investigated the effects of ELF-EMF (50 Hz, 0.1 and $1 \mathrm{mT}$ ) on oxidative damage. The authors showed that ELF-EMFs increased cell viability and reduced the levels of apoptotic death in rat neuronal primary cultures, with no significant effects on the main antioxidative defenses. Linear regression analysis suggested a positive correlation between reduced GSH and ROS levels in 1-mT EMF-exposed cells. They assumed that GSH plays an important role in antioxidant defense toward the ELF-EMF-induced redox challenge. Moreover, the GSH-based cellular response was achieved together with an overexpression of a brain-derived neurotrophic factor as well as with the IL-1 $\beta$-dependent regulation of prosurvival signaling pathways after ELFEMF exposure.

Focke et al. (2010) confirmed that intermittent exposure of human primary fibroblasts to an ELF-EMF $(50 \mathrm{~Hz}, 1 \mathrm{mT})$ induces a slight but significant increase in DNA fragmentation in the comet assay. The results showed that EMF-induced responses are dependent on cell proliferation, suggesting that processes of DNA replication rather than the DNA itself may be affected. Consistently, the effects correlated with a reduction in actively replicating cells and a concomitant increase in apoptotic cells in exposed cultures, whereas a combined formamidopyrimidine glycosylase (Fpg)-Comet test failed to produce evidence for oxidative DNA base damage. Hence, ELF-EMF-induced effects are reproducible under specific conditions and can be explained by minor disturbances 
in S-phase processes and occasional triggering of apoptosis rather than by the generation of DNA damage.

Jimenez-Garcia et al. (2010) found that the application of an ELF-EMF $(120 \mathrm{~Hz}$, $4.5 \mathrm{mT}$ ) inhibits chemically induced preneoplastic lesions in the rat liver through the reduction of cell proliferation, without altering the apoptosis process. Monache et al. (2008) investigated the effect of an ELF-EMF (50 Hz, $1 \mathrm{mT}$, for 12 hours) on the activation of angiogenesis in cultured umbilical HUVECs. The EMF exposure increased the degree of endothelial cell proliferation and tubule formation and accelerated the process of wound healing. The EMF exposure induced a major reorganization of fibers and of focal adhesion complexes, and increased phosphorylation as well as the overall expression of VEGF receptor 2 (KDR/Flk-1). The authors suggested that EMF may modulate in vitro some endothelial functions correlated to angiogenesis through signal transduction pathways dependent on VEGF.

The same research group also reported that the exposure of human spermatozoa to an ELF-EMF ( $50 \mathrm{~Hz}, 5 \mathrm{mT}$ ) improves sperm motility (Iorio et al. 2011). The ELF-EMF exposure resulted in a progressive and significant increase in mitochondrial membrane potential and in the levels of $\mathrm{ATP}, \mathrm{ADP}$, and $\mathrm{NAD}^{+}$measured as sperm kinematic parameters. No significant effects were detected on other parameters such as ATP to ADP ratio and energy charge. When carbamoyl cyanide $\mathrm{m}$-chlorophenylhydrazone (CICCP) was applied to inhibit the oxidative phosphorylation in the mitochondria, the values of energy parameters and motility in the sperm incubated in the presence of glucose and exposed to ELF-EMF did not change, indicating that glycolysis was not involved in mediating the ELF-EMF stimulatory effect on motility. In contrast, when pyruvate and lactate were provided instead of glucose, the energy status and motility increased significantly in ELF-EMF-treated sperm. Under these culture conditions, the inhibition of glycolytic metabolism by 2-deoxy-D-glucose (DOG) again resulted in increased values of energy and kinematic parameters, indicating that gluconeogenesis was not involved in producing glucose for use in glycolysis. The authors concluded that the key role in mediating the stimulatory effects exerted by ELF-EMF on human sperm motility is played by mitochondrial oxidative phosphorylation rather than glycolysis.

Garip and Akan (2010) investigated the effect of an ELF-EMF $(50 \mathrm{~Hz}, 1 \mathrm{mT})$ on the number of apoptotic cells in human leukemia cells (K562) that are or are not induced with oxidative stress and the correlation with HSP70 levels. One sample was treated with $\mathrm{H}_{2} \mathrm{O}_{2}$ and the other was left untreated. Exposure to ELF-EMF alone for 3 hours caused a decrease in the number of apoptotic cells and a slight increase in viability. However, it increased the number of apoptotic cells. In cells treated with $\mathrm{H}_{2} \mathrm{O}_{2}, \mathrm{HSP} 70$ and ROS levels were increased by exposure to ELF-EMF. These results showed that the effect of ELF-EMF on biological systems depends on the status of the cell: Whereas in cells not exposed to oxidative stress ELF-EMF is able to decrease the number of apoptotic cells by inducing an increase in HSP levels, it increases the number of apoptotic cells in oxidative stress-induced cells.

Polaniak et al. (2010) examined the effects of vitamin E, an ELF-EMF ( $400 \mathrm{~Hz}, 0.11 \mathrm{mT}$, $130 \mathrm{~V} / \mathrm{m}$, for 16 minutes), and their combination in different time intervals of exposure of vitamin E on the AT478 murine squamous cell carcinoma line. Following vitamin E treatment, activity of antioxidant enzymes increased compared with the control group. 
Application of ELF-EMF alone or with vitamin E increased both SOD isoenzymes and GPx activities compared with the control group. The results suggested that ELF-EMF alters antioxidative activities of vitamin E in AT478 tumor cells.

Kaszuba-Zwoinska et al. (2010) investigated whether a PEMF can affect proliferation and death of cancer cells. The U937 human lymphoid cell line was exposed to a PEMF (50 Hz, $45 \mathrm{mT}$ three times for 3 hours per each stimulation with 24-hour intervals) during 4 days of cultivation. The PEMF potentiated density-induced death, both apoptosis and necrosis. Puromycin, a telomerase inhibitor, was used as a cell death inducer at a concentration of $100 \mu \mathrm{g} / \mathrm{mL}$. By contrast, combined interference of three doses of puromycin and three times of PEMF stimulation resulted in a reduction of apoptosis by $24.7 \%$ and necrosis by $13 \%$ compared with the same doses of puromycin alone. The PEMF protects U937 cells against puromycin-induced cell death. The PEMF effects on the human lymphoid cell line depend on cell density. Increased cell density induced cell death and, in contrast, prevented cell death induced by puromycin.

\subsection{Response of Plants and Microorganisms to Magnetic Fields}

These topics are promising research areas for applications to crop science, biotechnology, control of microbial growth and so forth. Recent studies on the effects of magnetic fields on plant growth response are reviewed in Section 3.4.1. Recently proposed mechanisms of magnetotaxis are described in Section 3.4.2. Recent results of antibacterial effects by magnetic fields are addressed in Section 3.4.3.

\subsubsection{Plant Growth, Response, and Magnetotropism}

Several magnetic treatments have been reported for improving germination parameters and biomass accumulation in various plants. The effects are assumed to be due to increased photosynthetic efficiency (Shine, Guruprasad, and Anand 2011), enhanced activities of hydrolyzing enzymes (Vashisth and Nagarajan 2010), reduced oxidative stress (Hajnorouzi et al. 2011), and increased osmotic pressure (Cakmak, Dumlupinar, and Erdal, 2010).

Vashisth and Nagarajan (2008) examined the effect of an SMF ( $\leq 250 \mathrm{mT})$ on the seeds of chickpea (Cicer arietinum). Results showed that the SMF enhanced seed performance significantly in terms of laboratory germination, speed of germination, seedling length, and seedling dry weight compared with the unexposed control. However, the response varied with field strength and duration of exposure without showing any particular trend. Among the various combinations of field strengths and durations of exposure, $50 \mathrm{mT}$ for 2 hours, $100 \mathrm{mT}$ for 1 hour, and $150 \mathrm{mT}$ for 2 hours gave the best results. Exposure of seeds to these three SMFs improved seed coat membrane integrity as it reduced the electrical conductivity of seed leachate. In soil, seeds exposed to these three treatments produced significantly increased seedling dry weights of 1-month-old plants. The root characteristics of the plants showed dramatic increase in root length, root surface area, and root volume. Such improved functional root parameters suggested that 
magnetically treated chickpea seeds may perform better under rainfed (unirrigated) conditions where there is a restrictive soil moisture regime.

The same research group (Vashisth and Nagarajan 2010) further investigated the effect of SMFs ( $\leq 250 \mathrm{mT}$ ) on the seeds of sunflower (Helianthus annuus). The treatment of sunflower seeds in these SMFs increased the speed of germination, seedling length, and seedling dry weight under laboratory germination tests. Of the various treatments, exposure to 50 and $200 \mathrm{mT}$ for 2 hours yielded the peak performance. The SMF exposure improved seed coat membrane integrity and reduced cellular leakage and electrical conductivity. Treated seeds when planted in soil resulted in statistically higher seedling dry weight, longer root length, higher root surface area, and higher root volume in 1 -month-old seedlings. In germinating seeds, enzyme activities of $\alpha$-amylase, dehydrogenase, and protease were significantly higher in treated seeds in contrast to controls. The higher enzyme activity in SMF-treated sunflower seeds was assumed the reason behind fast germination and early vigor of seedlings.

Cakmak, Dumlupinar, and Erdal (2010) reported the effects of different intensities of SMFs (4 and $7 \mathrm{mT}$ ) on seed germination and seedling growth of bean and wheat seeds in different media having 0-, 2-, 6-, and 10-atm osmotic pressure prepared with sucrose or salt. The germination percentages of the treated seeds were compared with those of untreated seeds germinated in different osmotic pressures during 7-day incubation. The application of both SMFs promoted the germination ratios of bean and wheat seeds, regardless of increase in osmotic pressure of sucrose or salt. Growth data measured on the seventh day showed that the treated plants grew faster than control. After 7 days of incubation, the mean length of treated seedlings was found to be statistically higher than that of control plants in all the media considered. The greatest germination and growth rates in both plants were found in the 7-mT-exposed group. Effects of SMF on germination and growth increased positively with increasing osmotic pressure or salt stress compared with their respective controls. In contrast, SMF caused an increase in the dry biomass accumulation of roots and shoots of both bean and wheat seedlings; however, this effect was found statistically significant in all conditions for wheat but not for bean, in general.

Huang and Wang (2008) assessed the effect of ELF-EMFs on the early growth of plants using mung beans. Sinusoidal pulsed-width modulation (SPWM) voltages with different fundamental frequencies $(10-60 \mathrm{~Hz})$ were set for this purpose. The results indicated that the ELF-EMF induced by a 20 - or $60 \mathrm{~Hz}$ SPWM voltage has an enhancing effect on the early growth of mung beans, whereas the ELF-EMF induced by SPWM voltages of other frequencies $(30-50 \mathrm{~Hz}$ ) have an inhibitory effect especially at $50 \mathrm{~Hz}$.

Zaporozhan and Ponomarenko (2010) proposed a theory that magnetic fields induce definite genetic effects due to the existence of magnetic field-sensitive transcription factor repressors capable of regulating the biological activity of organisms through epigenetic mechanisms. These substances are proteins of the CRY/photolyase family. Valuable information regarding the magnetosensitivity of CRY and its biological responses to weak magnetic fields was obtained with the plant model Arabidopsis thaliana (Ahmad et al. 2007). If plants for which magnetic responses have no apparent function are sensitive to external magnetic fields, it is conceivable that other CRY-containing species are also sensitive to such fields (Harris et al. 2009). The most recent scheme concerning magnetic field effects on CRY activity is that the FADH-access cavity of the helical domain is 
predicted to be a functionally active site of the CRY molecule (Solov'yov, Chandler, and Schulten 2007). The unpaired electron spins $\left(S_{1}\right.$ and $\left.S_{2}\right)$ precess about a local magnetic field produced by the addition of the external magnetic field $B$ with contributions $I_{1}$ and $\mathrm{I}_{2}$ from the nuclear spins on the two radicals. The spin precession continuously alters the relative spin orientation, causing singlet (antiparallel) to triplet (parallel) interconversion, which underlies the magnetic field effect. Electron back-transfer from tryptophan to FADH quenches CRY's active state. However, this back-transfer can take place only when the electron spins are in the singlet state, and this dependence on spin allows the external magnetic field, $B$, to affect CRY activation.

\subsubsection{Magnetotaxis}

Magnetotactic bacteria are microorganisms that orient and migrate along magnetic field lines. Magnetotactic bacteria contain membrane-bound intracellular iron crystals called magnetosomes (Komeili et al. 2006). Magnetosomes comprise a magnetic nanocrystal surrounded by a lipid bilayer membrane. These unique prokaryotic organelles align inside magnetotactic bacterial cells and serve as an intracellular compass allowing the bacteria to navigate along the geomagnetic field in aquatic environments. Polar magnetotactic bacteria in vertical chemical gradients are thought to respond to high oxygen levels by swimming downward into areas with low or no oxygen (toward geomagnetic north in the Northern Hemisphere and geomagnetic south in the Southern Hemisphere). Simmons, Bazylinski, and Edwards (2006) identified populations of polar magnetotactic bacteria in the Northern Hemisphere that respond to high oxygen levels by swimming toward geomagnetic south, which is the opposite of all previously reported magnetotactic behavior. The percentage of magnetotactic bacteria with south polarity in the environment is positively correlated with high redox potential. The coexistence of magnetotactic bacteria with opposing polarities in the same redox environment conflicts with current models of the adaptive value of magnetotaxis.

Two mechanisms of magnetotaxis have been described: (1) Axial magnetotactic cells swim in both directions along magnetic field lines. (2) In contrast, polar magnetotactic cells swim either parallel to the geomagnetic field lines toward the North Pole (north seeking) or antiparallel toward the South Pole (south seeking). Lefèvre et al. (2009) used a magnetospectrophotometry (MSP) assay to characterize both the axial magnetotaxis of Magnetospirillum magneticum strain AMB-1 and the polar magnetotaxis of magneto-ovoid strain MO-1. Two pairs of Helmholtz coils were mounted onto the cuvette holder of a common laboratory spectrophotometer to generate two mutually perpendicular homogeneous magnetic fields parallel or perpendicular to the light beam. The application of magnetic fields allowed measurements of the change in light scattering resulting from cell alignment in a magnetic field or in absorbance due to bacteria swimming across the light beam. The results showed that MSP is a powerful tool for the determination of bacterial magnetism and the analysis of alignment and swimming of magnetotactic bacteria in magnetic fields. Moreover, this assay is useful for characterizing south-seeking derivatives and non-magnetosome-bearing strains obtained from north-seeking MO-1 cultures. These results suggest that oxygen is a determinant factor that controls magnetotactic behavior. 
Cryoelectron tomography of Magnetospirillum strains has revealed that the magnetosome chain is surrounded by a network of filaments that may be composed of MamK given that the filaments are absent in the MamK mutant cells (Scheffel et al. 2006). The process of assembly of MamK filaments is unknown. Pradel et al. (2006) proved the authenticity of MamK filaments and showed that MamK exhibits linear distribution inside Magnetospirillum sp. cells even in the area without magnetosomes. The MamK gene alone is sufficient to direct the synthesis of straight filaments in E. coli, and one extremity of the MamK filaments is located at the cellular pole. By using dual fluorescent labeling of MamK, the authors found that MamK nucleates at multiple sites and assembles into mosaic filaments. Time-lapse experiments revealed that the assembly of MamK filaments is a highly dynamic and kinetically asymmetrical process. MamK bundles might initiate the formation of a new filament or associate to a preexisting filament. These results demonstrated the mechanism of biogenesis of prokaryotic cytoskeletal filaments that are structurally and functionally distinct from the known MreB and ParM filaments. In addition to positioning magnetosomes, other hypothetical functions of the MamK filaments in magnetotaxis include anchoring of magnetosomes and involving in magnetic reception.

The classical model of polar magnetotaxis predicts that the field-parallel migration velocity of magnetotactic bacteria increases monotonically with the strength of an applied magnetic field. Pan et al. (2009) tested this model experimentally on magnetotactic coccoid bacteria that swim along helical trajectories. It turned out that the contribution of the field-parallel migration velocity decreases with increasing field strength (0.1-1.5 mT). This unexpected observation can be explained and reproduced in a mathematical model under the assumption that the magnetosome chain is inclined with respect to the flagellar propulsion axis. The magnetic disadvantage, however, became apparent only in fields stronger than the geomagnetic field, which suggested that magnetotaxis is optimized under geomagnetic field conditions. It is therefore not beneficial for these bacteria to increase their intracellular magnetic dipole moment beyond the value needed to overcome Brownian motion in geomagnetic field conditions.

Magnetotactic bacteria benefit from their ability to form cellular magnetic dipoles by assembling magnetosomes in chains as a means to navigate along geomagnetic field lines on their way to favorable habitats. Faivre et al. (2010) studied the assembly of magnetosomes by ferromagnetic resonance spectroscopy using Magnetospirillum gryphiswaldense cultured in a time-resolved experimental setting. The spectroscopic data showed that magnetic particle growth is not synchronized, the increase in particle number is insufficient to build up cellular magnetic dipoles, and dipoles of assembled magnetosome blocks occur when the first magnetite particles reach a stable single-domain state. The authors assumed that these stable single-domain particles can act as magnetic docks to stabilize the remaining and/or newly nucleated superparamagnetic particles in their adjacencies. The authors postulated that docking is a key mechanism for building the functional cellular magnetic dipole, which in turn is required for magnetotaxis in bacteria.

\subsubsection{Antibacterial Effects}

László and Kutasi (2010) investigated the viability of the microbes Saccharomyces cerevisiae, Bacillus circulans, E. coli, Micrococcus luteus, Pseudomonas fluorescens, Salmonella 
enteritidis, Serratia marcescens, and Staphylococcus aureus; they were tested under SMF exposure for up to 24 hours in either a homogeneous SMF of $159 \mathrm{mT}$ or three types of inhomogeneous SMFs: (1) peak-to-peak magnetic flux density $477 \mathrm{mT}$ with a lateral magnetic gradient of $47.7 \mathrm{~T} / \mathrm{m}$, (2) $12 \mathrm{mT}$ with $1.2 \mathrm{~T} / \mathrm{m}$, or (3) $2.8 \mathrm{mT}$ with $0.3 \mathrm{~T} / \mathrm{m}$. Even the longest period of exposure failed to produce any effect in the growth of bacteria.

Cellini et al. (2008) investigated the influence of an ELF-EMF $(50 \mathrm{~Hz} ; 0.1,0.5$, and $1.0 \mathrm{mT}$; for 20-120 minutes) on the viability of E. coli ATCC 700926. Exposed samples and controls displayed similar total and culturable counts, whereas increased cell viability was observed in exposed samples reincubated for 24 hours outside the solenoid compared with the corresponding controls. An EMF exposure produced significant change in E. coli morphotype with a presence of coccoid cells aggregated in clusters after 24 hours of reincubation outside the solenoid. Atypical lengthened bacterial forms were also observed suggesting a probable alteration during cell division. No changes among DNA fingerprintings and some differences in RNA-AFLP (amplified fragment length polymorphism) analysis were observed for each EMF intensity evaluated. These results indicated that EMF exposure acts as a stressing factor on bacteria, which represents a suitable model to investigate acute and chronic effects related to ELF-EMF exposure.

\subsection{Medical Applications}

Ueno, Sekino, and Ogiue-Ikeda (2006) and Yamaguchi-Sekino, Sekino, and Ueno (2011) reviewed recent advances in biomagnetics and bioimaging techniques such as TMS, MEG, MRI, and magnetic orientation for tissue engineering. In addition to this review, more recent findings related to medical applications are introduced in Sections 3.5.1 through 3.5.5.

\subsubsection{Transcranial Magnetic Stimulation and Induced Current}

Transcranial magnetic stimulation, for example, single- and paired-pulse TMSs and rTMS, is a useful method to examine dynamic brain function without causing any pain, producing a so-called virtual lesion or virtual excitation for a short period. Accordingly, TMS has become increasingly popular and is now a well-established and noninvasive technique in cognitive neuroscience, in particular, in functional, diagnostic, and therapeutic research on the brain. In brain functional research, magnetic stimulation for the temporary blockage or modification of the facultative information process and cognitive process of various sensory systems has been used to identify localization and connecting pathways of brain function. Single- or paired-pulse TMS and rTMS were reviewed by Pascual-Leone et al. (2002) and Fitzgerald, Fountain, and Daskalakis (2006), respectively.

In some cases, applications of TMS disturb brain function temporarily, which results in a virtual lesion in the brain. Zangaladze et al. (1999) showed that the disruption of the function of the occipital cortex with the use of focal TMS interferes with the tactile discrimination of grating orientation. Epstein et al. (2002) used TMS to investigate memory encoding and retrieval, particularly the role of DLPFC in associative memory for visual patterns. TMS was applied on dorsolateral prefrontal cortex of human subjects during 
associative learning task to disturb brain function related to memory and quick retrieval of associative memory. The TMS disrupted associative learning of abstract patterns over the right frontal area, which suggested that the participating cortical networks may be lateralized in accordance with classic concepts of hemispheric specialization. This is the first study to verify the important role of the dorsolateral prefrontal cortex of humans as the retrieval function by TMS method.

Traditionally, stimuli are applied at various scalp positions using a latitude- and longitude-based coordinate system referenced to $\mathrm{Cz}$ (central midline placement of electrodes at specific intervals along the head) in the 10-20 international system at the vertex, while the amplitude of the MEPs generated in contralateral muscles is also measured (Ueno, Matsuda, and Fujiki 1989, 1990). This gives a "map" of the sites on the scalp from which responses can be obtained by each reference muscle. Rothwell et al. (1987) revealed the enormous clinical importance of TMS in motor function evaluation.

Regarding the therapeutic use of TMS, Pascual-Leone et al. (1996) studied the effects of focal rTMS $(10 \mathrm{~Hz}$, produced MEP of greater than or equal to $50 \mu \mathrm{V})$ on the depressive symptoms in 17 patients with medication-resistant depression of psychotic subtype. The study was designed as a multiple crossover, randomized placebo-controlled trial. Sham rTMS and stimulation of different cortical areas were used as controls. Left DLPFC rTMS resulted in significant decrease in scores on the Hamilton depression rating scale (HDRS) and the self-rated Beck questionnaire (BQ). Eleven of the total 17 patients showed pronounced improvement that lasted for about 2 weeks after 5 days of daily rTMS sessions. No patient experienced any significant undesirable side effects. These findings emphasized the role of the left DLPFC in depression and suggested that rTMS of the left DLPFC might become a safe, nonconvulsive alternative to electroconvulsive treatment for depression.

Kujirai et al. (2006) reported that TMS pulses over the hand area of motor cortex activate different subpopulations of synaptic connections if the direction of the induced current in the brain is reversed from posterior-anterior (PA) direction to anteriorposterior (AP) direction. The authors tested whether this also made a difference to the after-effects of paired associative stimulation (PAS: ulnar nerve stimulation followed 25 milliseconds later by a TMS pulse). When 50 pairs of stimuli $(0.1 \mathrm{~Hz})$ were applied using conventional suprathreshold PA-PAS in resting subjects, there was no effect on MEPs in the FDI muscle. In contrast, when the same number of pulses were given while subjects made a small tonic (5\% maximum) contraction, MEP was facilitated and rMT reduced when AP but not PA pulses were used. Subsequent experiments employed subthreshold TMS (95\% of the aMT) during voluntary muscle contraction. Moter-evoked potential facilitation accompanied by reduced AP threshold occurred when PAS was given using AP pulses (AP-Sub-PAS), whereas PAS using PA pulses (PA-Sub-PAS) had no excitatory effect. There was no facilitation if the ulnar nerve stimulus was replaced by digital nerve stimulation. There was a tendency for short-interval intracortical inhibition (SICI) to decrease and intracortical facilitation (ICF) to increase after AP-Sub-PAS. The authors proposed that the increased effectiveness of AP-Sub-PAS over PA-Sub-PAS is due to the fact that AP TMS more readily activates I3 inputs to corticospinal neurons, and hence I3 inputs are an important component of associative plasticity in the human motor cortex. 
Strafella, Ko, and Monchi (2006) examined placebo effects on patients with PD using sham rTMS (placebo-rTMS) alone. The authors measured the changes in striatal [11C] raclopride binding potentials (BP) together with positron emission tomography (PET). Placebo-rTMS induced significant bilateral reduction in $\left[{ }^{11} \mathrm{C}\right]$ raclopride $\mathrm{BP}$ in dorsal and ventral striatum as compared with the baseline condition. This reduction in BP is indicative of an increase in dopamine neurotransmission. The changes in $\left[{ }^{11} \mathrm{C}\right]$ raclopride binding were more evident in the hemisphere contralateral to the more affected side, supporting the hypothesis that the more severe the symptoms the greater the drive for symptom relief and therefore the placebo response. This is the first study addressing the placebo contribution during rTMS. Although the results seemed to confirm earlier evidence that expectation induces dopaminergic placebo effects, they also suggested the importance of placebo-controlled studies in future clinical trials involving brain stimulation techniques.

Kamida et al. (2007) assessed whether paired-pulse TMS-induced MEP can predict surgical prognosis in patients with intractable epilepsy. The MEP of unilateral hand muscles were recorded following paired-pulse TMS of the motor cortex. The authors concluded that paired-pulse TMS-induced MEP may provide predictive value in terms of surgical outcomes in patients with intractable epilepsy.

Jung et al. (2008) investigated the changes in cortical excitability of the human motor cortex induced by rTMS $(10 \mathrm{~Hz}$, produced MEP of greater than or equal to $50 \mu \mathrm{V})$ of different stimulation durations (5 and 1.5 seconds) over the motor hot spot for left FDI muscle. The authors concluded that with different stimulation durations, highfrequency subthreshold rTMS can produce different patterns of long-lasting changes in corticospinal and intracortical excitability in stimulated and unstimulated motor cortex in healthy subjects. The results have important implications for the selection of stimulation parameters other than the frequency of rTMS.

Because DLPFC is a common target for rTMS experiments and therapeutic protocols, Fitzgerald et al. (2009) investigated the optimal method for the localization of DLPFC for use in these studies. Twelve healthy subjects underwent a structural MRI scan, a TMS procedure to establish the location of the motor cortex, and a neuronavigational procedure to assess the relative position of the DLPFC. Several EEG points and a position $5 \mathrm{~cm}$ anterior to motor cortex were established. The DLPFC site used was identified as being approximately halfway between the EEG points F3 and AF3. This point is considerably more anterior than the point identified by measuring $5 \mathrm{~cm}$ anterior to the motor cortex. The authors concluded that EEG points provide a useful way to optimally identify the DLPFC site.

Metaplasticity refers to activity-dependent changes in neural functions that modulate subsequent synaptic plasticity such as long-term potentiation (LTP) and long-term depression (LTD) (Abraham 2008). Using rTMS (20 Hz, produced MEP of greater than or equal to $50 \mu \mathrm{V}$ ), Cohen et al. (2010) investigated whether metaplasticity is dependent on a particular phase of the normal sleep-wake/circadian cycle. The authors suggested that the timing of sessions relative to the sleep-wake/circadian cycle may be a critical factor in the cumulative effect of treatment.

Filipović, Rothwell, and Bhatia (2010) demonstrated that low-frequency ( $\leq 1 \mathrm{~Hz})$ rTMS (LF-rTMS) can reduce excitability in the underlying cortex, promote inhibition, or do 
both. In particular, using LF-rTMS the authors found the impaired inhibitory mechanisms in motor cortex of patients with PD. Previously, Filipović et al. (2009) examined the effects of LF-rTMS (1 Hz, 1800 pulses) on dyskinesia in PD. The stimulator intensity was set individually to just below the aMT. The LF-rTMS was applied twice over the motor cortex for four consecutive days; once real stimuli were used and once sham stimulation was used. Evaluations were done at the baseline and one day after the end of each treatment series. Filipović, Rothwell, and Bhatia (2010) further evaluated the delayed (24-hour) effects of LF-rTMS treatment on physiological measures of excitability of the motor cortex in the same patients. The authors found that LF-rTMS delivered over several consecutive days changes the excitability of the motor cortex by increasing the excitability of inhibitory circuits. The effects persist for at least a day after rTMS.

Shirota et al. (2010) evaluated cerebellar function using TMS to determine whether subclinical cerebellar involvement is present in progressive supranuclear palsy (PSP) patients. The authors studied 11 patients with PSP, 11 patients with PD, and 10 agematched controls. Motor-evoked potentials were recorded from the hand muscle. Cerebellar function was evaluated using suppressive effects of TMS over the cerebellum on MEP elicited by TMS over the contralateral motor cortex, which is called cerebellar inhibition (CBI). Interstimulus intervals of 4-8 milliseconds were used, and the time course of CBI was analyzed. The CBI was found to be reduced in PSP patients. By contrast, the CBI was normal in PD patients in their on state (phases with good response to medication and few symptoms). Although the CBI in their off state (phases with no response to medication and more severe symptoms) should be examined in future studies, the results suggested that Purkinje cells or the dentatothalamocortical pathway assessed by CBI is involved in PSP. These results are compatible with the pathological findings showing severe dentate nucleus degeneration in PSP patients.

Hiscock et al. (2008) reviewed evidence regarding the effect of rTMS on corticospinal pathway excitability and motor function in healthy adults and in people in the aftermath of stroke. After stroke there was a trend for MEP recovery (i.e., presence of MEP) after 10 daily sessions of $3 \mathrm{~Hz}$ rTMS (one study). Motor function in healthy adults might be adversely affected by $1 \mathrm{~Hz}$ rTMS (two studies), whereas combined frequency rTMS was found to have no effect on motor function (one study). The authors concluded that there is as yet insufficient published evidence to guide the dosage of rTMS to the lesioned hemisphere after stroke to improve recovery of a paretic limb. Moreover, apparently there is variability in response to rTMS in healthy adults.

Contralesional dorsal premotor cortex (cPMd) may support residual motor function following stroke. Using TMS-fMRI, Bestmann et al. (2010) performed two complementary experiments to explore how cPMd might perform this role in a group of chronic stroke patients. First, they used paired-coil TMS $(11 \mathrm{~Hz})$ to establish the physiological influence of cPMd on ipsilesional primary motor cortex (iM1) at rest. They found that this influence became less inhibitory/more facilitatory in patients with greater clinical impairment. Second, they applied TMS over cPMd during fMRI $(1.5 \mathrm{~T})$ in these patients to examine the causal influence of cPMd TMS on the whole network of surviving cortical motor areas in both hemispheres and to check whether these influences could change during movement of the affected hand after stroke. The authors confirmed that hand grip-related activation in cPMd was greater in more impaired patients. Furthermore, 
the peak ipsilesional sensorimotor cortex activity shifted posteriorly in more impaired patients. Critical new findings were that concurrent TMS-fMRI results correlated with the level of both clinical impairment and neurophysiological impairment. Specifically, greater clinical and neurophysiological impairment was associated with a stronger facilitating influence of cPMd TMS on blood oxygenation level-dependent (BOLD) signal in posterior parts of ipsilesional sensorimotor cortex during hand grip, corresponding to the posteriorly shifted sensorimotor activity seen in more impaired patients. The cPMd TMS was not found to influence activity in other brain regions in either hemisphere. The authors suggested that this state-dependent influence on ipsilesional sensorimotor regions provides a mechanism by which cPMd supports recovered function after stroke.

Dimyan and Cohen (2010) discussed the use of TMS in the diagnosis, prognosis, and therapy of poststroke motor disability and emphasized that TMS is a useful tool to explore mechanisms of neuroplasticity during spontaneous and treatment-induced recovery of motor function to develop more rational and clinically useful interventions for stroke rehabilitation.

The spatial extent of the effects of TMS on neural tissue is only coarsely understood. One key problem is the realistic calculation of the electric field induced in the brain, which proves difficult due to the complex gyral folding pattern that results in an inhomogeneous conductivity distribution within the skull. Thielscher, Opitz, and Windhoff (2011) estimated the electric field induced in the brain using the finite element method (FEM) together with a high-resolution volume mesh of the human head to better characterize the field induced in cortical gray matter (GM). The volume mesh was constructed from T1-weighted structural MRI to ensure an anatomically accurate modeling of the gyrification pattern. Five tissue types were taken into account, corresponding to skin, skull, cerebrospinal fluid (CSF) including the ventricles, cortical GM, and cortical white matter. The authors characterized the effect of current direction on the electric field distribution in GM. The field strength in GM was increased by up to $51 \%$ when the induced currents were perpendicular to the local gyrus orientation. This effect was mainly restricted to gyral crowns and lips, and did not extend into the sulcal walls. As a result, the focality of the fields induced in GM was increased. The authors speculated that this enhancement effect might in part explain the dependency of stimulation thresholds on coil orientation, which is commonly observed in TMS motor cortex studies. In contrast to the clear-cut effects of the gyrification pattern on induced field strength, current directions were predominantly influenced by the CSF-skull boundary.

In general, exposure to time-varying EMFs results in internal electric $(E)$ fields, body currents, and energy absorption in tissues that depend on the coupling mechanisms and frequency involved. In the case of ELF-EMFs of less than $300 \mathrm{~Hz}$, nonthermal (no heating) effect is expected because the absorbed energy in terms of the local specific absorption rate (SAR; watts per gram) in biological tissues is negligible according to the following equation (Caorsi, Pastorino, and Raffetto 1999):

$$
\text { Local SAR }=\frac{\sigma}{2 \delta}|E|^{2}
$$


where $E$ is the electric field (in volts per meter), $\sigma$ is the electric conductivity (in siemens per meter; approximately $0.2 \mathrm{~S} / \mathrm{m}$ in average tissue conductivity [ICNIRP 1998; Reilly 1998]; approximately $0.1 \mathrm{~S} / \mathrm{m}$ in brain tissue conductivity [Saunders and Jefferys 2002]), and $\delta$ is the human body density (in kilogram per cubic meter; approximately $1000 \mathrm{~kg} / \mathrm{m}^{3}$ in human body [Schult et al. 2010]). For instance, the whole-body average SAR limits of $0.4 \mathrm{~W} / \mathrm{kg}$ for controlled environments and $0.08 \mathrm{~W} / \mathrm{kg}$ for uncontrolled environments estimated by Gandhi (2002) are identical to those mentioned in the International Commission on Non-Ionizing Radiation Protection (ICNIRP) 1998 standard. In contrast, heating abnormal tissue with high-SAR power such as microwave cardiac ablation (MCA) is used to treat heart tissue that allows abnormal electrical conduction by heating it to the point of inactivation (Lin 1999; Rappaport 2004).

Lin (2007) commented that most exposure guidelines are promulgated on a $4-\mathrm{W} / \mathrm{kg}$ SAR to prevent any whole-body exposure from raising the body temperature to $1^{\circ} \mathrm{C}$ above the norm at $37^{\circ} \mathrm{C}$. However, the Lin research group later suggeted that temperature distributions do not always correlate well with SAR distributions and the regulatory limits on local temperature may not be exceeded as readily as those on local SAR (Wang et al. 2007). In modeling temperature increase during MRI of the human head in a head-sized volume coil at up to $3.0-\mathrm{W} / \mathrm{kg}$ head-average SAR, the Lin research group concluded that it may not be necessary to consider thermally induced changes in physiological response (Wang et al. 2008). In addition, Barnes (2006) mentioned that there is a debate regarding whether effects other than the increase in temperature should be used to limit the amount of radiated power.

In the case of EMFs over about $100 \mathrm{kHz}$, it was assumed that "Joule energy" could contribute to the thermal effect through large induced currents. In contrast, at frequencies below $100 \mathrm{kHz}$, it was presumed that electric fields and currents are induced in the body by time-varying external magnetic fields and then the induced $E$ fields in the body may lead to a variation of membrane potentials at the cellular level. Electric excitation of the membrane might be the result of such changes in membrane potentials (ICNIRP 2004).

For TMS and rTMS, a high-intensity, fast magnetic pulse produces a cortical stimulus or excitation through the induction of locally confined eddy currents and induces a rapid depolarization of the nerve cells; therefore, safety guidelines have been reviewed and discussed for avoiding excitation of the central nervous system (e.g., IEEE 2002; Gandhi 2002; Medical Advisory Secretariat 2004; Reilly 2005; Barnes 2006; Blackman 2006, 2009; ARPANSA 2007; Lin 2007; WHO 2007; Wang et al. 2007, 2008; Wood 2008; Rossi et al. 2009; Casali et al. 2010; ICNIRP 2010; Sandrini, Umiltà, and Rusconi 2011).

It has been assumed that the greatest stimulation efficiency occurs if the TMS coil's induced electric field ( $y$ axis) is parallel to cortical columns (Rábago et al. 2009). Multiple target sites within the cortical area of activation were created from the coregistered anatomical and functional images. Electromyography (EMG) feedback from the left FDI was used to assess each target site. The site chosen as the final target site was the one that elicited the best MEP response at the lowest rMT. The TMS-induced MEP response has been used in experiments regarding mirror neurons (Catmur, Walsh, and Heyes 2007). 
More recently, guidelines for limiting exposure to time-varying EMFs ( $1 \mathrm{~Hz}$ to $100 \mathrm{kHz}$ ) have been proposed as a safety standard by ICNIRP (2010). For example, in the ICNIRP 2010 standard, for EMF exposures in the $1 \mathrm{~Hz}$ to $3 \mathrm{kHz}$ range the basic restrictions for induced internal $E$ fields in all tissues of the head and body are 0.8 and $0.4 \mathrm{~V} / \mathrm{m}$ for occupational and general public exposures, respectively. Perception of surface electric charge, direct stimulation of nerve and muscle tissues, and induction of retinal phosphenes are the only well-established adverse effects, and serve as the basis for exposure guidelines (ICNIRP 2010).

Typical values of induced internal $E$ fields in exposures to EMFs are shown in Table 3.2. There are several studies indicating that induced $E$ fields can stimulate excitable tissues that may influence neuronal activity and excitability, and therefore these values should be taken into account in estimations based on the conditions.

Wood (2008) evaluated thresholds for nerve excitation elicited by time-varying magnetic stimulation based on the experimental data from published papers (see Table 3.2). Ueno, Matsuda, and Hiwaki (1991) obtained a threshold of $9.5 \mathrm{~V} / \mathrm{m}$ for frog sciatic nerves at $45^{\circ}$ to the induced $E$ field direction by direct measurements using magnetic stimulation. These authors further suggested that the existence of vectorial characteristics of stimulating currents for neural excitation reflects both the functional and the anatomical organization of neural fibers in the brain.

Estimating threshold values (e.g., threshold intensity for eliciting MEP) is extremely important for standardization of stimulation in double-pulse TMS (Kujirai et al. 1993; Ilic et al. 2002), and good threshold estimates for brain areas would be helpful in therapeutic applications of TMS (Lisanby, Kinnunen, and Crupain 2002). However, the evidence for neurobehavioral effects on brain electrical activity, cognition, sleep, and mood in volunteers exposed to ELF-EMFs is much less clear (ICNIRP 2010).

TABLE 3.2 Typical Values of Induced Internal Electric (E) Fields in Exposures to Time-Varying EMFs

\begin{tabular}{|c|c|c|}
\hline Tissues & Induced $E$ Fields & Authors, Year \\
\hline Central nerve & $10 \mathrm{~V} / \mathrm{m}$ & Reilly $(1998,2002)$ \\
\hline Motor cortex & $25 \mathrm{~V} / \mathrm{m}($ at $50 \mathrm{~Hz}, 3.7 \mathrm{~T})$ & Kowalski, Silny, and Buchner (2002) \\
\hline Motor cortex & $50 \mathrm{~V} / \mathrm{m}$ & Casali et al. (2010) \\
\hline Peripheral nerve & $9.5 \mathrm{~V} / \mathrm{m}$ & Ueno, Matsuda, and Hiwaki (1991) \\
\hline Peripheral nerve & $2 \mathrm{~V} / \mathrm{m}$ (in $10 \mathrm{~Hz}$ to $1 \mathrm{kHz}$ range) & ICNIRP (1998) \\
\hline Peripheral nerve & $6 \mathrm{~V} / \mathrm{m}$ & Reilly $(1998,2002)$ \\
\hline Peripheral nerve & $2 \mathrm{~V} / \mathrm{m}$ & Nyenhuis et al. (2001) \\
\hline Peripheral nerve & $6 \mathrm{~V} / \mathrm{m}$ & Liu, Zhao, and Crozier (2003) \\
\hline Peripheral nerve & $4-6 \mathrm{~V} / \mathrm{m}$ & So, Stuchly, and Nyenhuis (2004) \\
\hline Peripheral nerve & $2 \mathrm{~V} / \mathrm{m}$ & Wood (2008) \\
\hline Retinal phosphenes & $4-16 \mathrm{mV} / \mathrm{m}($ at $20 \mathrm{~Hz}, 5 \mathrm{mT})$ & Taki, Suzuki, and Wake (2003) \\
\hline Retinal phosphenes & $50-100 \mathrm{mV} / \mathrm{m}($ at $20 \mathrm{~Hz}, 5 \mathrm{mT})$ & Saunders and Jefferys (2007) \\
\hline Retinal phosphenes & $56 \mathrm{mV} / \mathrm{m}($ at $20 \mathrm{~Hz})$ & Wood (2008) \\
\hline MEP & $20 \mathrm{mV} / \mathrm{m}$ & Roth, Pell, and Zangen (2010) \\
\hline
\end{tabular}




\subsubsection{Magnetoencephalography}

Magnetoencephalography is a real noninvasive method for measuring magnetic fields as weak as $5 \mathrm{fT}\left(1 \mathrm{fT}=10^{-15} \mathrm{~T}\right)$ generated by neuronal current flow without any external field. The magnetic signals can be detected by SQUIDs arrayed on the scalp. The measurement of MEG can be represented as brain activities with high millisecond-order temporal resolution and high millimeter-order spatial resolution; thus, MEG is useful for investigation of brain function in humans, including higher brain functions such as memory and cognition. Cohen (1968) obtained an MEG for human $\alpha$-waves with the use of a SQUID and a prototype was developed by Cohen, Edelsack, and Zimmerman (1970); as a result of these developments, the use of a whole-head MEG system for spontaneous measurement at multiple points has become practical (Squires 1991; Ahonen et al. 1993; Vrva, Bette, and Burband 1993). In recent years, the whole-head MEG system has been incorporated into brain functional research all over the world and this has led to accelerated progress in research.

Application of forward and inverse problems in MEG analysis is critical to estimate the localization of brain function and disorders. Ueno and Iramina (1991) measured the MEG associated with short memory, cognition, and mental rotation in humans; constructed current-dipole and distributed intracerebral electrical source models; and carried out estimations for the localization of various brain functions during the processing of information. The electrical source of a visually evoked reaction with 150 milliseconds at latency localized in the primary visual cortex was described in a current-dipole model relatively well, whereas a distributed intracerebral electrical source model was more useful in estimating the electrical source incident to a mental rotation with 180 milliseconds or higher at latency. In the distributed electrical source model, a chronological transition of electrical source groups from the occipital lobe area to the posterior temporal lobe area was captured. The MEG is a tool not only for basic brain functional research but also for medical research. Clinical applications of MEG include detection of epileptic spikes, measurement of slow waves associated with brain tumors and cerebrovascular diseases, and detection of cerebroelectric activity of ELFs induced by event-related potentials.

Studies on MEG have revealed enhancement of neural activity of the N1m response of auditory evoked fields in well-trained musicians, reflecting neuroplastic modification of the representation of the auditory cortex (Pantev et al. 1998). In contrast, the amplitude of the $\mathrm{P} 2$ response of auditory evoked potentials is modified by musical experience, with no alteration of N1 (Shahin et al. 2003). Kuriki, Kanda, and Hirata (2006) performed a comprehensive MEG study using stimulation of successive musical instrument tones to examine how the neural activities of different MEG responses were modified in experienced musicians who had commenced musical lessons at the age of 5 years and had continued to practice. The dipole moment of the $\mathrm{P} 2 \mathrm{~m}$ response occurring at $160-180$ milliseconds was significantly enlarged in musicians compared with that in individuals who had not received any musical lessons. The enlargement was found for the dipole moment of N1m occurring at 100-120 milliseconds in a restricted condition but not for the moment of P1m at 50-60 milliseconds. Furthermore, the dipole moment of P2m for successive stimuli, normalized by the moment for the first stimulus, was significantly 
larger for chord tones than for single tones and was significantly larger in the musicians than controls. These results suggest that the $\mathrm{P} 2 \mathrm{~m}$ response is susceptible to modification by musical training during a period of neural maturation, with a short refractory period of neural activity for the auditory input of composite tones. The P2m activity may be specialized to the processing of multifrequency sounds, such as musical timbre consisting of abundant harmonics.

With more recent and advanced applications, Chen, Xiang et al. (2010) investigated noninvasive biomarkers for visuocortical development in healthy children using the MEG system. The latency of M75 and M100 decreased with age. The amplitude ratio of M100 to M75 increased significantly with age. The differences of MEG source images between the left and right occipital cortices for M75 and M145 increased significantly with age. The authors concluded that the latency of M75 and M100 and the amplitude ratio of M100 to M75 are robust biomarkers for the development of visual function in children and speculated that the results form a foundation for quantitative identification of developmental delay and/or abnormalities of visual function in children with brain disorders.

\subsubsection{Magnetic Resonance Imaging}

Magnetic resonance imaging is a method to obtain spatial distribution of nuclear magnetic resonance (NMR) signals using gradient magnetic fields and the Fourier transform (FT). The basic principle of MRI was proposed by Lauterbur (1973). The principles of MRI are described in detail by Callaghan (1993). Briefly, an MRI system consists of a magnet, gradient coils, and RF coils. The MRI utilizes fusion techniques of spatially uniform SMF, spatially gradient magnetic fields, and RF pulse fields. The MRI has become a standard technique for the routine diagnosis of many disease processes, replacing and sometimes surpassing computed tomography (CT). The CT scans expose the body to high doses of X-rays; in contrast to CT, MRI is noninvasive, uses nonionizing radiation, and has a high soft-tissue resolution and discrimination in any imaging plane.

A guideline for exposure of the human body to SMFs set by ICNIRP (2009) suggests $2 \mathrm{~T}$ as the ceiling value for body parts, except for arms and legs, in occupational exposure. In the application of clinical MRI, the current exposure level is confirmed to be less than or equal to $2 \mathrm{~T}$. In SMFs at this strength it is not feasible to obtain resonance images, except for hydrogen atoms. Tomasi and Wang (2007) estimated the magnetic force in the human head during MRI at $4 \mathrm{~T}$ and confirmed that the induced gradient fields increase the magnetic force on tissues. However, even for tissue components with large magnetic susceptibility such as iron-containing proteins, this force is negligible compared with the gravitational force. Therefore, the authors concluded that exposure to uniform SMF at $4 \mathrm{~T}$ does not have a significant risk for tissues in the head.

Glover and Bowtell (2008) showed that current densities above regulatory limits may be generated even for moderate natural movements near a clinical 3-T MRI scanner although the subject did not report any perception of physiological effects. The electric fields induced due to natural movements near the scanner are much higher than those induced by time-varying fringe fields from the gradient coils in a subject standing still next to the scanner while the scanner is operating. 
The MRI of electrical phenomena in living bodies is potentially useful for quantitative evaluations of the biological effects of EMFs and for direct detection of neuronal electrical activities in the brain. Magnetic fields in an object cause a shift in the resonant frequency (Manassen, Shalev, and Navon 1988; Sekino, Matsumoto, et al. 2004) and a change in the phase of MR signals (Joy, Scott, and Henkelman 1989). Spatial distributions of an externally applied magnetic field and electrical current can be estimated from these changes in MR signals. These methods have use in certain medical applications, such as the imaging of current distributions in electrical defibrillation (Yoon et al. 2003).

The fMRI developed by Ogawa, Tank, and Menon (1992) utilizes a technology that reflects various magnetic features of hemoglobin in blood on MR signal patterns. Tomograms of brain function can be obtained from information on localized blood flow in the brain. The fMRI utilizes a BOLD effect of localized blood flow on brain activation for indirect imaging of brain activities. However, no information on electrical conditions in vivo can be obtained with the current MRI and fMRI systems.

Tomatsu et al. (2008) showed the possible brain sites that have activity equivalent to the motor commands with fMRI. The authors hypothesized that short-term temporal patterns of movements or stimuli are reflected in BOLD responses and they searched for regions representing the response. Participants performed two temporal patterns of tapping and/or listened to the same patterns of auditory stimuli in a 3-T fMRI. All patterns were designed to have the same number (11) of events and the same duration but different temporal distributions of events. The 11 events were divided into two parts (10 repetitive taps and one stand-alone tap) and the interval between the two parts was three seconds. The two patterns had reverse order of the two parts. The results revealed that different temporal patterns of auditory stimuli were represented in different temporal features of BOLD responses in the bilateral auditory cortex, whereas different temporal patterns of tapping were reflected in contralateral primary motor cortex and the ipsilateral anterior cerebellum. In bilateral premotor cortex, SMA, visual cortex, and posterior cerebellum, task-related BOLD responses were exhibited, but their responses did not reflect the temporal patterns of the movement and/or stimuli. One possible explanation is that the neuronal activities were similar for the two patterns in these regions. The sensitivity of BOLD response to temporal patterns reflects local differences in functional contributions to the tasks. The authors proposed that this experimental design and analysis may be useful in revealing particular brain regions that participate in multiple functions.

Using fMRI, Postle and Hamidi (2007) demonstrated that short-term retention of the identity or location of visually presented stimuli is disrupted by nonvisual secondary tasks performed in passive listening in the dark to nouns or endogenous generation of saccades, respectively. This indicates that the short-term retention of visual information relies on multiple mental codes, some of them nonvisual. Event-related fMRI reveals the neural correlates of these interference effects to be more complex and more regionally specific than previously described. Although nonspecific dual-task effects produce a generalized decrease in task-evoked fMRI response across many brain regions, the interference-specific effect is a relative increase of activity localized to regions associated with the secondary task in question: left hemisphere perisylvian cortex in the case 
of passive listening distraction and frontal oculomotor regions in the case of saccadic distraction. Within these regions, the neural interference effects are specific to voxels that show delay-period activity on unfilled memory trials. Individual differences were also predicted in the magnitude of the behavioral interference effect. These results indicate that nonvisual processes supported by nonvisual brain areas contribute importantly to visual working memory performance.

Debas et al. (2010) investigated, through fMRI, the neuronal substrates associated with the consolidation process of two motor skills: (1) motor sequence learning (MSL) and (2) motor adaptation (MA). Four groups of healthy young individuals were assigned to either a night/sleep condition, in which they were scanned while practicing a finger sequence learning task or an eight-target adaptation pointing task in the evening (test) and were scanned again 12 hours later in the morning (retest), or a day/awake condition, in which they were scanned on the MSL or the MA tasks in the morning and were rescanned 12 hours later in the evening. As expected and consistent with the behavioral results, the functional data revealed increased test-retest changes in activity in the striatum for the night/sleep group compared with the day/ awake group in the MSL task. By contrast, results of the MA task did not show any difference in test-retest activity between the night/sleep and day/awake groups. When the two MA task groups were combined, however, increased test-retest activity was found in lobule VI of the cerebellar cortex. Together, these findings highlighted the presence of both functional and structural dissociations reflecting the off-line consolidation processes of MSL and MA. The authors suggested that MSL consolidation is sleep dependent and reflected by a differential increase in neural activity within the corticostriatal system, whereas MA consolidation necessitates either a period of daytime or sleep and is associated with increased neuronal activity within the corticocerebellar system.

Impedance-weighted MRI was obtained during applications of external oscillating magnetic fields, which induce impedance-dependent eddy currents in a sample (Ueno and Iriguchi 1998). In another study, spatial distribution of electrical impedance was obtained from electric current distributions by using an iterative algorithm (Khang et al. 2002). The apparent diffusion coefficient reflects electrical conductivity of a tissue, which enables an estimation of anisotropic conductivity of that tissue (Tuch et al. 2001; Sekino, Inoue, and Ueno 2004). This method was applied to the imaging of electrical conductivity in the human brain. Several regions in the white matter, such as the corpus callosum and the internal capsule, exhibited high anisotropy in conductivity. The magnitude and phase of MR signals are affected by tissue permittivity (Sekino et al. 2005). A distinctive signal inhomogeneity arises in the images of objects whose dimension is comparable to the wavelength of the EMF at the resonant frequency. This phenomenon, dielectric resonance, particularly appears in scanners using high SMFs.

The MRI may not be applied widely in brain function research; however, high-quality impedance MRI for impedance and admittance scanning in vivo may lead to the development of a new research field of impedance physiology. It is obvious that information regarding impedance distributions is important for studying magnetic stimulation and MEG inverse problems. 
Sadleir, Grant, and Woo (2010) attempted to theoretically analyze neural function imaging using magnetic resonance electrical impedance tomography (MREIT). The authors found that phase noise in a candidate 17.6-T MRI system should be sufficiently low to detect phase signal differences between active and resting membrane states at resolutions around $1 \mathrm{~mm}^{3}$. The authors further delineated the broad dependencies of signal-to-noise ratio on activity frequency, current application time, and active tissue fractions and outlined strategies that can be used to lower phase noise below that presently observed in conventional MREIT techniques. The authors also proposed the idea of using MREIT as an alternative means of studying neuromodulation.

Once high-quality current-distribution MRI of the detailed distribution of electric source incident to brain neural activities becomes available, comparison of the results of MRI and fMRI will show the relationship between brain neural activities associated with BOLD effects and neural current distributions, which may lead to various new observations of dynamics in brain function localizations. Baumann et al. (2010) mapped the BOLD signal time course in the inferior colliculus (IC), in medial geniculate body (MGB), and in tonotopically defined fields in the auditory cortex of two macaques. The results showed little differences in the BOLD signal time courses within the auditory pathway. However, the authors observed systematic differences in the magnitude of change in the BOLD signal with significantly stronger signal changes in field A1 of the auditory cortex compared with field R. The measured time course of the signal was in good agreement with similar studies in human auditory cortex, but it showed considerable differences with data reported from macaque visual cortex. Consistent with the studies in humans the authors measured a peak in the BOLD response around 4 seconds after the onset of 2 -second broadband noise stimuli, whereas previous studies (Logothetis et al. 2001) recorded from the primary visual cortex of the macaque monkey had reported the earliest peaks to short visual stimuli several seconds later. The comparison of the results with those of previous studies does not support differences in haemodynamic responses within the auditory system between human and nonhuman primates. Furthermore, the authors assumed that the data will aid optimal design of future auditory fMRI studies in nonhuman primates.

Robertson et al. (2010) investigated the dose-response relationship (sham and 100, 200, and $1000 \mu \mathrm{T})$ between an ELF-PEMF $(\leq 300 \mathrm{~Hz})$ and acute thermal pain on the dominant right hand using fMRI. The authors found significant correlations between applied field strength and change in BOLD activity in the anterior cingulate and the ipsilateral insula, indicating that there might be either a dose response or a threshold effect for the applied PEMF.

It is known that fMRI and MEG are sensitive to the frontal and temporal language functions, respectively. Kamada et al. (2007) established combined use of fMRI and MEG to make reliable identification of global language dominance in pathological brain conditions. The authors investigated 117 patients with brain lesions whose language dominance was successfully confirmed by the Wada test. All patients were asked to generate verbs related to acoustically presented nouns (verb generation) for fMRI and to read three-letter words for fMRI and MEG. The fMRI typically showed prominent activations in the inferior and middle frontal gyri, whereas calculated dipoles on MEG typically clustered in the superior temporal region and the fusiform gyrus of the dominant 
hemisphere. A total of 87 patients were further analyzed using useful data from both the combined method and the Wada test. The authors observed a $100 \%$ match of the combined method results with the results of the Wada test, including two patients who showed expressive and receptive language areas dissociated into bilateral hemispheres. The results demonstrated that this noninvasive and repeatable method is not only highly reliable in determining language dominance, but it can also locate the expressive and receptive language areas separately. The authors suggested that the method is a potent alternative to the invasive procedures of the Wada test and is useful in treating patients with brain lesions.

\subsubsection{Magnetic Orientation for Tissue Engineering}

The effects of magnetic fields, in particular, SMFs, on magnetic orientation for tissue engineering have been reported (see the review by Ueno, Sekino, and Ogiue-Ikeda 2006). That is, the magnetic control of living cells using magnetic force may translate into potentially viable tissue and promising medical engineering applications including nerve regeneration. In this case, recognition of the role of diamagnetic, paramagnetic, and ferromagnetic materials in vitro and in vivo may help in unraveling the underlying mechanisms.

Umeno and Ueno (2003) examined the effect of strong SMFs on adherent cells. The previous study by Iwasaka and Ueno (2003) showed that smooth muscle cells cultured in 8- or 14-T superconducting magnets for three days exhibited orientational order parallel to the magnetic field direction. To discuss the process and the mechanism of this orientation, the authors investigated the orientational characteristics of the cell culture with quantitative measurements: an orientational order parameter and the FT analysis. The orientational order parameter indicated the degree of orientation. The value of the parameter was estimated with the FT of microscopic images. The cells cultured under stronger SMFs exhibited stronger ordering, when they were cultured under a strong magnetic gradient force of $400 \mathrm{~T}^{2} / \mathrm{m}$. By contrast, they showed weaker ordering in the control. The ordering was enhanced under uniform, strong magnetic fields, whereas it was not affected or was suppressed by strong gradient forces. The authors suggested that the cells organize themselves to minimize their diamagnetic torsion stresses, which can be induced in the uniform magnetic fields by the membrane's diamagnetic anisotropy.

In contrast to the estimation of torque force induced by a uniform SMF, the intense inhomogeneous SMF acting on diamagnetic materials can generate a strong magnetic force. Qian et al. (2009) have developed a superconducting magnet platform with largegradient high magnetic field (LG-HMF), which can produce three magnetic force fields of $-1360,0$, and $1312 \mathrm{~T}^{2} / \mathrm{m}$ and three corresponding apparent gravity levels of 0,1 , and $2 \mathrm{~g}$ for diamagnetic materials. The authors investigated the effects of different magnetic force fields on osteoblast-like cell (MG-63 and MC3T3-E1) viability, microtubule actin cross-linking factor 1 (MACF1) expression, and MACF1's association with cytoskeleton. Results showed that cell viability increased to different degrees after 24 hours of exposure to 0 or $1 \mathrm{~g}$ conditions, whereas it decreased by about $30 \%$ under $2 \mathrm{~g}$ conditions compared with the control conditions. An increase in MACF1 expression at the RNA or protein level was observed in osteoblast-like cells under the LG-HMF of $-1360 \mathrm{~T}^{2} / \mathrm{m}$ 
( $0 \mathrm{~g}$ ) compared to that under $1312 \mathrm{~T}^{2} / \mathrm{m}$ ( $\left.2 \mathrm{~g}\right)$. Under control conditions, anti-MACF1 staining was scattered in the cytoplasm and partially colocalized with actin filaments or microtubules in most osteoblast-like cells. Under $0 \mathrm{~g}$ conditions, MACF1 labeling was concentrated at the perinuclear region and colocalization was not apparent. The patterns of anti-MACF1 labeling on microtubules varied under LG-HMF environment. In conclusion, LG-HMF affects osteoblast-like cell viability, MACF1 distribution, MACF1 expression, and MACF1's association with cytoskeleton.

Coletti et al. (2007) investigated the SMF effect of $80 \mathrm{mT}$ on mammal skeletal muscle in myogenic rat cell line L6. They found that SMF promoted myogenic cell differentiation and hypertrophy, that is, increased accumulation of actin and myosin and formation of large multinucleated myotubes. The elevated number of nuclei per myotube was derived from increased cell fusion efficiency, with no changes in cell proliferation on SMF exposure. No alterations in myogenin expression, a modulator of myogenesis, occurred on SMF exposure. The SMF induced cells to align in parallel bundles, an orientation that is conserved throughout differentiation. The SMF stimulated the formation of actin stress fiber-like structures. It rescued muscle differentiation in the presence of TNF, a muscle differentiation inhibitor. The authors suggested that SMF promotes myogenic differentiation and cell alignment, without any invasive manipulation. The SMF-enhanced parallel orientation of myotubes is relevant to the tissue engineering of a highly organized tissue such as skeletal muscle. They assumed that SMF rescue of muscle differentiation in the presence of TNF may have important therapeutic implications.

Kim et al. (2008) hypothesized that the application of SMF to neurons will cause neurites to grow in a specific direction. In cultured human SH-SY5Y cells or rat PC12 cells, neurite outgrowth was induced by forskolin, retinoic acid, or nerve growth factor (NGF). When the SMF was applied, the neuronal processes of SH-SY5Y cells tended to be arranged perpendicular to the SMF. In PC12 cells with the application of SMF, the colocalized areas were observed in the axis vertical to the SMF, suggesting the subcellular polarity of the microtubule-actin interaction induced by SMF. These results suggest that SMF can modulate the orientation and direction of neurite formation in cultured neuronal cells. To our knowledge, in the past magnetic orientation was not induced by such a weak magnetic strength as $12 \mathrm{mT}$.

Bone tissue engineering has been investigated as an alternative strategy for autograft transplantation. In the process of tissue engineering, cell seeding into 3D scaffolds is the first step for constructing 3D tissues. Shimizu, Ito, and Honda (2007) have proposed a methodology of cell seeding into 3D porous scaffolds using a magnetic force (generating from a 1-T gradient SMF) and magnetite nanoparticles, referred to as Mag-seeding. The authors applied this Mag-seeding technique to bone tissue engineering using BMSCs and 3D hydroxyapatite (HA) scaffolds. The BMSCs were magnetically labeled with the authors' original magnetite cationic liposomes having a positive surface charge to improve adsorption to cell surface. Magnetically labeled BMSCs were seeded onto a scaffold and a 1-T magnet was placed under the scaffold. By using Mag-seeding, the cells were successfully seeded into the internal space of scaffolds with a high cell density. The cell seeding efficiency into HA scaffolds by Mag-seeding was threefold higher than that 
by control seeding (conventional method; seeding without a magnet). After a 14-day cultivation period using osteogenic induction medium by Mag-seeding, the levels of two representative osteogenic markers (alkaline phosphatase and osteocalcin) were found to be significantly higher than those by control seeding. These results indicate that Magseeding of BMSCs into HA scaffolds is an effective approach to bone tissue engineering.

\subsubsection{Treatments of Pain, Cancer, and Other Diseases}

The clinical effects of SMFs and PEMFs on pain have been well reviewed (Markov 2007; Colbert et al. 2009). Khoromi et al. (2007) assessed the pain-relieving efficacy of SMF (20 mT versus $5 \mathrm{mT}$ ) in a double-blind, randomized, two-phase crossover study in patients with chronic lumbar radicular pain. The effect of $20-\mathrm{mT}$ SMF relative to 5 -mT SMF appeared to increase throughout the week period. The authors proposed that larger, longer-duration, sham-controlled trials with 20-mT magnets should be considered in patients with chronic lumbar radicular pain.

Regarding the effects of PEMF on analgesia, Weintraub and Cole (2004) evaluated a PEMF therapy $(30 \mathrm{~Hz}, 2 \mathrm{mT}, 1 \mathrm{~h}$ /day, five days a week, for nine days) in refractory neuropathic pain in the soles of the feet. These pilot data demonstrated that directing PEMFs to refractory neuropathic pain treatment can provide unexpected short-term analgesic effects in more than $50 \%$ of individuals. The role of placebo is not known and was not tested. The authors stated that the precise mechanism is unclear, but suggested that severe and advanced cases are more magnetically sensitive.

The same research group further investigated in patients with carpal tunnel syndrome (CTS) (Weintraub and Cole 2008). The authors revealed that PEMF + SMF exposure $(20 \mathrm{~Hz}, 5 \mathrm{mT}, 0.5 \mathrm{mV} / \mathrm{cm}, 4 \mathrm{~h} /$ day for 2-10 months) in refractory CTS provides statistically significant short- and long-term pain reduction and mild improvement in objective neuronal functions. They suggested that neuromodulation appears to influence nociceptive- $\mathrm{C}$ and large A-fiber functions, probably through ion/ligand binding.

Shupak et al. (2006) reported the effect of PEMF $(<3 \mathrm{kHz}, \leq 400 \mu \mathrm{T}, 30$ minutes) on pain and anxiety ratings in female rheumatoid arthritis and fibromyalgia patients with a double-blind, randomized, placebo-controlled design. The results provide some initial support for the use of PEMF exposure in reducing pain in chronic pain populations and warrant continued investigation into the use of PEMF exposure for short-term pain relief. Sutbeyaz et al. (2009) further suggested that PEMF therapy $(64 \mathrm{~Hz}, 40 \mu \mathrm{T}, 30 \mathrm{~min}-$ utes per session, twice a day for 3 weeks) might improve function, pain, fatigue, and global status in fibromyalgia patients.

Fernandez, Watson, and Rowbotham (2007) reported that PEMF exposure $(1.25 \mathrm{~Hz}$, 3-ms duration, $60 \mathrm{mT}$, for 30 minutes) had no effect on pain. Weintraub et al. (2009) also confirmed that a PEMF $(25 \mathrm{~Hz}, 31 \mathrm{mT})$ was noneffective in reducing neuropathic pain. However, they proposed future studies with higher dosimetry (300-500 mT), longer duration of exposure, and larger biopsy cohort.

More recent preclinical studies examining the inhibition of angiogenesis by exposure to magnetic fields have turned to the use of magnetic fields in the treatment of cancer (tumor angiogenesis; see also Sections 3.3.1.5 and 3.3.2.1). Brix et al. (2008) evaluated SMF 
effects on capillary flow of red blood cells (RBCs) in unanesthetized Syrian golden hamsters, using a skinfold chamber technique for intravital fluorescence microscopy. Capillary RBC velocities $\left(v_{\mathrm{RBC}}\right)$, capillary diameters $(D)$, arteriolar diameters $\left(D_{\text {art }}\right)$, and functional vessel densities (FVDs) were measured in striated skin muscle at different magnetic flux densities. Exposure above a threshold level of about $500 \mathrm{mT}$ resulted in a significant reduction of $v_{\mathrm{RBC}}$ in capillaries compared with the baseline value. At the maximum field strength of $587 \mathrm{mT}, v_{\mathrm{RBC}}$ was reduced (40\%). Flow reduction was reversible when the field strength was decreased below the threshold level. In contrast, mean values determined at different exposure levels for the parameters $D, D_{\text {art }}$, and FVD did not vary (5\%). Blood flow through capillary networks is affected by SMFs directed perpendicular to the vessels.

The same research group futher analyzed the effects of SMF $(\leq 587 \mathrm{mT})$ on tumor microcirculation (Strieth et al. 2008). In vivo fluorescence microscopy was performed in A-Mel-3 tumors growing in dorsal skinfold chamber preparations of hamsters. Shorttime exposure $(\geq 150 \mathrm{mT})$ resulted in a significant reduction of $v_{\mathrm{RBC}}$ and segmental blood flow in tumor microvessels. At the maximum strength of $587 \mathrm{mT}$, a reversible reduction of $v_{\mathrm{RBC}}(40 \%)$ and of FVD (15\%) was observed. Prolongation of the exposure time (1 minute to 3 hours) resulted in reductions. Microvessel diameters and leukocyte-endothelial cell interactions remained unaffected by SMF exposures. However, in contrast to tumor-free striated muscle controls, exposure at the maximum flux density of $587 \mathrm{mT}$ induced a significant increase in platelet-endothelial cell adherence in a time-dependent manner that was reversible after reducing the strength of the SMF. The authors assumed that these reversible changes may have implications for functional measurements of tumor microcirculation by MRI and new therapeutic strategies using strong SMFs. The same research group futher evaluated the effects of an SMF (586 mT, for 3 hours) on tumor angiogenesis and growth (Strelczyk et al. 2009). Analysis of microcirculatory parameters revealed a significant reduction of FVD, vessel diameters, and RBC velocity in tumors after SMF exposure compared with the control tumors. These changes reflect retarded vessel maturation by antiangiogenesis. The increased edema after SMF exposure indicated an increased tumor microvessel leakiness possibly enhancing drug uptake. The authors concluded that SMF therapy appears to be a promising new anticancer strategy, as an inhibitor of tumor growth and angiogenesis and as a potential sensitizer to chemotherapy.

Ghibelli et al. (2006) examined whether exposure to the SMF of NMR (1 T) generated by an NMR apparatus can affect apoptosis induced on reporter tumor cells of hematopoietic origin. The impressive result was the strong increase (by 1.8-2.5-fold) of damage-induced apoptosis by NMR. This potentiation is due to cytosolic $\mathrm{Ca}^{2+}$ overload consequent to NMR-promoted $\mathrm{Ca}^{2+}$ influx, since it is prevented by intracellular (BAPTA-AM) and extracellular (EGTA) $\mathrm{Ca}^{2+}$ chelation or by inhibition of plasma membrane L-type $\mathrm{Ca}^{2+}$ channels. A 3-day follow-up of treated cultures showed that NMR decreases long-term cell survival, thus increasing the efficiency of cytocidal treatments. Mononuclear white blood cells are not sensitized to apoptosis by NMR, showing that NMR may increase the differential cytotoxicity of antitumor drugs on tumor versus normal cells. The authors suggested that this strong, differential potentiating effect of NMR on tumor cell apoptosis may have important implications, as in fact a possible adjuvant for antitumor therapies. 
Chen, Qi et al. (2010) investigated whether 8.8-mT SMF can enhance the killing potency of cisplatin (DDP) on human leukemic cells (K562). The results showed that SMF enhanced the anticancer effect of DDP on K562 cells, which was correlated with enhanced DNA breakage and drug-induced cell killing. This study suggested the potential of SMF as an adjunctive treatment method for chemotherapy.

Concerning the other therapeutic effects of PEMF, Martiny, Lunde, and Bech (2010) reported that patients with treatment-resistant depression on a PEMF $(55 \mathrm{~Hz}, 1.9 \mathrm{mT}$, $2.2 \mathrm{mV} / \mathrm{cm}$ ) by rTMS showed a clinically and statistically significant better outcome than patients treated with sham PEMF, in which the onset of action was within the first weeks of therapy. Baptista et al. (2009) reported that exposure to PEMF $(72 \mathrm{~Hz}, 200 \mu \mathrm{T}$, $30 \mathrm{~min} /$ day, 5 days a week, for 3 weeks) leads to delayed histological peripheral nerve regeneration and increased oxidative stress without any loss of function recovery. The investigators van Bergen et al. (2009) provide evidence on the effectiveness of a PEMF $(75 \mathrm{~Hz}, 1.5 \mathrm{mT})$ in the management of osteochondral ankle lesions after arthroscopy. Dallari et al. (2009) investigated the effect of a PEMF ( $75 \mathrm{~Hz}, 2 \mathrm{mT}, \geq 6 \mathrm{~h} /$ day, for $\leq 90$ days) in subjects undergoing hip revision using the Wagner SL stem and showed that PEMF treatment aids clinical recovery and bone stock restoration. Ozgüçlü et al. (2010) evaluated the effect of a PEMF $(50 \mathrm{~Hz}, 3 \mathrm{mT})$ on the classical physical treatment of knee osteoarthritis (OA) composed of hot pack, therapeutic US, and terminal isometric exercises. The results showed that PEMF does not have additional effects on the classical physical treatment in reducing the symptoms of knee OA.

\subsection{Conclusion}

Over the last three decades, various studies have been carried out to examine the effects of nonthermal magnetic fields, including TMS and MRI fields, on biological systems. This chapter consisted of two main parts. The first part focused on recent experiments covering topics such as magnetic sensing and behavior, cardiovascular system responses, reproduction and development, genotoxicity, molecular and cellular systems, and free radical and enzyme activity. The second part concentrated on the recent development of diagnostic and therapeutic applications of various magnetic fields together with magnetic orientation for tissue engineering. As shown in this chapter, current studies are more directed toward the development of diagnosis and therapy using biomagnetic phenomena.

With more recent and advanced applications, MEG studies have neurophysiological conditions of neural activities, and brain functions and disorders for mammals, in particular for humans. Moreover, a comparison of the results of MRI and fMRI shows the relationship between brain neural activities associated with BOLD effects and neural current distributions, which may lead to various new observations of dynamics in brain function localizations. The development of new bioimaging technologies such as current-distribution MRI and conductivity MRI, which can directly visualize electrical activities of neurons, enables us to decipher and better understand the dynamics of brain function. These methods have higher temporal resolution (millisecond level) compared with conventional fMRI. The focal and vectorial TMS selectively facilitates or inhibits neuronal activities in targeted regions or hot spots, which is useful for understanding 
the dynamics of brain function. These techniques are leading medicine and biology to a new horizon through the novel applications of magnetism.

The aforementioned studies on magnetic fields with different intensities and frequencies, such as SMFs and PEMFs, provide some therapeutic possibilities for various diseases. In particular, most of the rTMS and PEMF therapies have resulted in improved outcomes in rehabilitative treatment and have shown clear neuroprotective and regenerative effects. In addition, the potentiating effect of magnetic fields on tumor cell apoptosis may have important implications for antitumor therapies. However, the potential therapeutic mechanisms involved have not been proved. Technological advances of superconducting magnets have enabled the study of diamagnetic force on biological macromolecules and cells. The diamagnetic force causes magnetic orientation of macromolecules and cells; this finding has the potential to trigger promising applications in regenerative medicine.

In recent years, an abundance of research papers, review papers, and books has been published describing the possible physical and biological interactions of magnetic fields. These studies provide valuable insight into the phenomenon of biomagnetism and open new avenues for the development of new medical applications. By contrast, knowledge of magnetic field effects on free radical reactions is particularly important when considering human health, the relation between immunological and neurodegenerative diseases, and stress response possibly resulting from apoptotic pathways due to ROS generation. Several attempts have been made to explore the parameters of free radical reactions when living organisms, cells, and biochemicals are exposed to various magnetic fields. However, the effects and mechanisms of magnetic field induced cytotoxic activity or antitumor effect, if any, have not yet been clarified. The influences of these magnetic fields on spin-dependent physiological processes need to be confirmed in further studies.

\section{Acknowledgment}

We thank Professor James C. Lin for his kind invitation to prepare this chapter and for reading and commenting on the chapter.

\section{References}

Abdelmelek, H., A. Molnar, S. Servais, J. M. Cottet-Emard, J. M. Pequignot, R. Favier, and M. Sakly. 2006. Skeletal muscle HSP72 and norepinephrine response to static magnetic field in rat. J Neural Transm 113:821.

Abraham, W. C. 2008. Metaplasticity: Tuning synapses and networks for plasticity. Nat Rev Neurosci 9:387.

Adair, R. K. 1999. Effects of very weak magnetic fields on radical pair reformation. Bioelectromagnetics 20:255.

Ahmad, M., P. Galland, T. Ritz, R. Wiltschko, and W. Wiltschko. 2007. Magnetic intensity affects cryptochrome-dependent responses in Arabidopsis thaliana. Planta 225:615.

Ahonen, A. I., M. S. Hämäläinen, J. E. T. Knnutila, M. J. Kajola, P. P. Laine, O. V. Lounasmaa, L. T. Parkkonen, J. T. Simola, and C. D. Tesch. 1993. 122-Channel SQUID instrument for investigating the magnetic signals from the human brain. Phys Scr T49:198. 
Ammari, M., M. Jeljeli, K. Maaroufi, V. Roy, M. Sakly, and H. Abdelmelek. 2008. Static magnetic field exposure affects behavior and learning in rats. Electromagn Biol Med 27:185.

Aoyagi, S., A. Yano, Y. Yanagida, E. Tanihira, A. Tagawa, and M. Iimoto. 2006. Control of chemical reaction involving dissolved oxygen using magnetic field gradient. Chem Phys 331:137.

ARPANSA. 2007. Exposure limits for electric and magnetic fields $0 \mathrm{~Hz}$ to $3 \mathrm{kHz}$, Public comment draft, Australian radiation protection and nuclear safety agency, Yallambie, Victoria, Australia.

Aydin, N., and M. Bezer. 2011. The effect of an intramedullary implant with a static magnetic field on the healing of the osteotomised rabbit femur. Int Orthop 35:135.

Bao, X., Y. Shi, X. Huo, and T. Song. 2006. A possible involvement of $\beta$-endorphin, substance $\mathrm{P}$, and serotonin in rat analgesia induced by extremely low frequency magnetic field. Bioelectromagnetics 27:467.

Baptista, A. F., B. T. Goes, D. Menezes, F. C. Gomes, J. Zugaib, J. Stipursky, J. R. Gomes, J. T. Oliveira, M. A. Vannier-Santos, and A. M. Martinez. 2009. PEMF fails to enhance nerve regeneration after sciatic nerve crush lesion. J Peripher Nerv Syst 14:285.

Barker, A. T., R. Jalionus, and I. L. Freeston. 1985. Noninvasive magnetic stimulation of the human motor cortex. Lancet 1:1106.

Barnes, F. 2006. Setting standards in the presence of developing scientific understanding. Electromagn Biol Med 25:209.

Baumann, S., T. D. Griffiths, A. Rees, D. Hunter, L. Sun, and A. Thiele. 2010. Characterisation of the BOLD response time course at different levels of the auditory pathway in non-human primates. Neuroimage 50:1099.

Beaugnon, E., and R. Tournier. 1991. Levitation of organic materials. Nature 349:470.

Becker, L. B. 2004. New concepts in reactive oxygen species and cardiovascular reperfusion physiology. Cardiovasc Res 61:461.

Benazzo, F., M. Cadossi, F. Cavani, M. Fini, G. Giavaresi, S. Setti, R. Cadossi, and R. Giardino. 2008. Cartilage repair with osteochondral autografts in sheep: Effect of biophysical stimulation with pulsed electromagnetic fields. J Orthop Res 26:631.

Berryy, M. V., and A. K. Geimz. 1997. Of flying frogs and levitrons. Eur J Phys 18:307.

Bestmann, S., O. Swayne, F. Blankenburg, C. C. Ruff, J. Teo, N. Weiskopf, J. Driver, J. C. Rothwell, and N. S. Ward. 2010. The role of contralesional dorsal premotor cortex after stroke as studied with concurrent TMS-fMRI. J Neurosci 30:11926.

Bezanilla, F. 2002. Voltage sensor movements. J Gen Physiol 120:465.

Blackman, C. F. 2006. Can EMF exposure during development leave an imprint later in life? Electromagn Biol Med 25:217.

Blackman, C. 2009. Cell phone radiation: Evidence from ELF and RF studies supporting more inclusive risk identification and assessment. Pathophysiology 16:205.

Blanchard, B., M. Dendane, J. F. Gallard, C. Houée-Levin, A. Karim, D. Payen, J. M. Launay, and C. Ducrocq. 1997. Oxidation, nitrosation, and nitration of serotonin by nitric oxide-derived nitrogen oxides: Biological implications in the rat vascular system. Nitric Oxide 1:442. 
Brix, G., S. Strieth, D. Strelczyk, M. Dellian, J. Griebel, M. E. Eichhorn, W. Andrā, and M. E. Bellemann. 2008. Static magnetic fields affect capillary flow of red blood cells in striated skin muscle. Microcirculation 15:15.

Brocklehurst, B. 2002. Magnetic fields and radical reactions: Recent developments and their role in nature. Chem Soc Rev 31:301.

Brocklehurst, B., and K. A. McLauchlan. 1996. Free radical mechanism for the effects of environmental electromagnetic fields on biological systems. Int J Radiat Biol 69:3.

Burda, H., S. Begall, J. Červený, J. Neef, and P. Němec. 2009. Extremely low-frequency electromagnetic fields disrupt magnetic alignment of ruminants. Proc Natl Acad Sci U S A 106:5708.

Cakmak, T., R. Dumlupinar, and S. Erdal. 2010. Acceleration of germination and early growth of wheat and bean seedlings grown under various magnetic field and osmotic conditions. Bioelectromagnetics 31:120.

Callaghan, P. T. 1993. Principles of Nuclear Magnetic Resonance Microscopy. Oxford, UK: Oxford University Press.

Campese, V. M., S. Ye, H. Zhong, V. Yanamadala, Z. Ye, and J. Chiu. 2004. Reactive oxygen species stimulate central and peripheral sympathetic nervous system activity. Am J Physiol Heart Circ Physiol 287:H695.

Caorsi, S., M. Pastorino, and M. Raffetto. 1999. Analytic SAR computation in a multilayer elliptic cylinder for bioelectromagnetic applications. Bioelectromagnetics 20:365.

Casali, A. G., S. Casarotto, M. Rosanova, M. Mariotti, and M. Massimini. 2010. General indices to characterize the electrical response of the cerebral cortex to TMS. Neuroimage 49:1459.

Catmur, C., V. Walsh, and C. Heyes. 2007. Sensorimotor learning configures the human mirror system. Curr Biol 17:1527.

Cavin, I. D., P. M. Glover, R. W. Bowtell, and P. A. Gowland. 2007. Thresholds for perceiving metallic taste at high magnetic field. J Magn Reson Imaging 26:1357.

Cellini, L., R. Grande, E. Di Campli, S. Di Bartolomeo, M. Di Giulio, I. Robuffo, O. Trubiani, and M. A. Mariggiò. 2008. Bacterial response to the exposure of $50 \mathrm{~Hz}$ electromagnetic fields. Bioelectromagnetics 29:302.

Chakeres, D. W., and F. de Vocht. 2005. Static magnetic field effects on human subjects related to magnetic resonance imaging systems. Prog Biophys Mol Biol 87:255.

Chen, W. F., H. Qi, R. G. Sun, Y. Liu, K. Zhang, and J. Q. Liu. 2010. Static magnetic fields enhanced the potency of cisplatin on k562 cells. Cancer Biother Radiopharm 25:401.

Chen, Y., J. Xiang, E. G. Kirtman, Y. Wang, R. Kotecha, and Y. Liu. 2010. Neuromagnetic biomarkers of visuocortical development in healthy children. Clin Neurophysiol 121:1555.

Chiu, K. H., K. L. Ou, S. Y. Lee, C. T. Lin, W. J. Chang, C. C. Chen, and H. M. Huang. 2007. Static magnetic fields promote osteoblast-like cells differentiation via increasing the membrane rigidity. Ann Biomed Eng 35:1932.

Cintolesi, F., T. Ritz, C. W. M. Kay, C. R. Timmel, and P. J. Hore. 2003. Anisotropic recombination of an immobilized photoinduced radical pair in a $50-\mu \mathrm{T}$ magnetic field: A model avian photomagnetoreceptor. Chem Phys 294:385. 
Cohen, D. 1968. Magnetoencephalography: Evidence of magnetic fields produced by $\alpha$-rhythm currents. Science 161:784.

Cohen, D., E. A. Edelsack, and J. E. Zimmerman. 1970. Magnetocardiograms taken inside a shielded room with a superconducting point contact magnetometer. Appl Phys Lett 16:278.

Cohen, D. A., C. Freitas, J. M. Tormos, L. Oberman, M. Eldaief, and A. Pascual-Leone. 2010. Enhancing plasticity through repeated rTMS sessions: The benefits of a night of sleep. Clin Neurophysiol 121:2159.

Colbert, A. P., H. Wahbeh, N. Harling, E. Connelly, H. C. Schiffke, C. Forsten, W. L. Gregory et al. 2009. Static magnetic field therapy: A critical review of treatment parameters. Evid Based Complement Alternat Med 6:133.

Coletti, D., L. Teodori, M. C. Albertini, M. Rocchi, A. Pristerà, M. Fini, M. Molinaro, and S. Adamo. 2007. Static magnetic fields enhance skeletal muscle differentiation in vitro by improving myoblast alignment. Cytometry A 71:846.

Connor, R. J., and S. L. Menzies. 1995. Cellular management of iron in the brain. J Neurol Sci 134:33.

Crozier, S., and F. Liu. 2005. Numerical evaluation of the fields induced by body motion in or near high-field MRI scanners. Prog Biophys Mol Biol 87:267.

Cui, J. G., H. Kuroda, N. V. Chandrasekharan, R. P. Pelaez, D. L. Simmons, N. G. Bazan, and W. J. Lukiw. 2004. Cyclooxygenase-3 gene expression in Alzheimer hippocampus and in stressed human neural cells. Neurochem Res 29:1731.

Dallari, D., M. Fini, G. Giavaresi, N. Del Piccolo, C. Stagni, L. Amendola, N. Rani, S. Gnudi, and R. Giardino. 2009. Effects of pulsed electromagnetic stimulation on patients undergoing hip revision prostheses: A randomized prospective doubleblind study. Bioelectromagnetics 30:423.

de Vocht, F., T. Stevens, P. Glover, A. Sunderland, P. Gowland, and H. Kromhout. 2007. Cognitive effects of head-movements in stray fields generated by a 7 Tesla wholebody MRI magnet. Bioelectromagnetics 28:247.

de Vocht, F., T. Stevens, B. van Wendel-de-Joode, H. Engels, and H. Kromhout. 2006a. Acute neurobehavioral effects of exposure to static magnetic fields: Analyses of exposure-response relations. J Magn Reson Imaging 23:291.

de Vocht, F., H. van Drooge, H. Engels, and H. Kromhout. 2006b. Exposure, health complaints and cognitive performance among employees of an MRI scanners manufacturing department. J Magn Reson Imaging 23:197.

Debas, K., J. Carrier, P. Orban, M. Barakat, O. Lungu, G. Vandewalle, A. Hadj Tahar et al. 2010. Brain plasticity related to the consolidation of motor sequence learning and motor adaptation. Proc Natl Acad Sci U S A 107:17839.

Di Loreto, S., S. Falone, V. Caracciolo, P. Sebastiani, A. D’Alessandro, A. Mirabilio, V. Zimmitti, and F. Amicarelli. 2009. Fifty hertz extremely low-frequency magnetic field exposure elicits redox and trophic response in rat-cortical neurons. J Cell Physiol 219:334.

Dimyan, M. A., and L. G. Cohen. 2010. Contribution of transcranial magnetic stimulation to the understanding of functional recovery mechanisms after stroke. Neurorehabil Neural Repair 24:125.

Dini, L. 2010. Phagocytosis of dying cells: Influence of smoking and static magnetic fields. Apoptosis 15:1147. 
Dini, L., M. Dwikat, E. Panzarini, C. Vergallo, and B. Tenuzzo. 2009. Morphofunctional study of 12-O-tetradecanoyl-13-phorbol acetate (TPA)-induced differentiation of U937 cells under exposure to a $6 \mathrm{mT}$ static magnetic field. Bioelectromagnetics 30:352.

Dini, L., and E. Panzarini. 2010. The influence of a $6 \mathrm{mT}$ static magnetic field on apoptotic cell phagocytosis depends on monocyte/macrophage differentiation. Exp Biol Med (Maywood) 235:1432.

Dobson, J. 2007. Magnetic properties of biological material. In Handbook of Biological Effects of Electromagnetic Fields: Bioengineering and Biophysical Aspects of Electromagnetic Fields. 3rd ed. ed. F. S. Barnes and B. Greenebaum, 101. Boca Raton, FL: CRC Press.

Efimova, O., and P. J. Hore. 2008. Role of exchange and dipolar interactions in the radical pair model of the avian magnetic compass. Biophys J 94:1565.

Eguchi, Y., S. Ueno, C. Kaito, K. Sekimizu, and K. Shiokawa. 2006. Cleavage and survival of Xenopus embryos exposed to $8 \mathrm{~T}$ static magnetic fields in a rotating clinostat. Bioelectromagnetics 27:307.

Eichwald, C., and J. Walleczek. 1996. Model for magnetic field effects on radical pair recombination in enzyme kinetics. Biophys $J$ 71:623.

Emre, M., S. Cetiner, S. Zencir, I. Unlukurt, I. Kahraman, and Z. Topcu. 2011. Oxidative stress and apoptosis in relation to exposure to magnetic field. Cell Biochem Biophys 59:71.

Engström, S. 2007. Magnetic field effects on free radical reactions in biology. In Handbook of Biological Effects of Electromagnetic Fields: Bioengineering and Biophysical Aspects of Electromagnetic Fields. 3rd ed. ed. F. S. Barnes, and B. Greenebaum, 157. Boca Raton, FL: CRC Press.

Epstein, C. M., M. Sekino, K. Yamaguchi, S. Kamiya, and S. Ueno. 2002. Asymmetries of prefrontal cortex in human episodic memory: Effects of transcranial magnetic stimulation on learning abstract patterns. Neurosci Lett 320:5.

Eveson, R. W., C. R. Timmel, B. Brocklehurst, P. J. Hore, and K. A. McLauchlan. 2000. The effects of weak magnetic fields on radical recombination reactions in micelles. Int J Radiat Biol 76:1509.

Faivre, D., A. Fischer, I. Garcia-Rubio, G. Mastrogiacomo, and A. U. Gehring. 2010. Development of cellular magnetic dipoles in magnetotactic bacteria. Biophys J 99:1268.

Falone, S., A. Mirabilio, M. C. Carbone, V. Zimmitti, S. Di Loreto, M. A. Mariggio, R. Mancinelli, C. Di Ilio, and F. Amicarelli. 2008. Chronic exposure to $50 \mathrm{~Hz}$ magnetic fields causes a significant weakening of antioxidant defence systems in aged rat brain. Int J Biochem Cell Biol 40:2762.

Feng, S. W., Y. J. Lo, W. J. Chang, C. T. Lin, S. Y. Lee, Y. Abiko, and H. M. Huang. 2010. Static magnetic field exposure promotes differentiation of osteoblastic cells grown on the surface of a poly-L-lactide substrate. Med Biol Eng Comput 48:793.

Fernandez, M. I., P. J. Watson, and D. J. Rowbotham. 2007. Effect of pulsed magnetic field therapy on pain reported by human volunteers in a laboratory model of acute pain. Br J Anaesth 99:266. 
Filipović, S. R., J. C. Rothwell, and K. Bhatia. 2010. Slow (1 Hz) repetitive transcranial magnetic stimulation (rTMS) induces a sustained change in cortical excitability in patients with Parkinson's disease. Clin Neurophysiol 121:1129.

Filipović, S. R., J. C. Rothwell, B. P. van de Warrenburg, and K. Bhatia. 2009. Repetitive transcranial magnetic stimulation for levodopa-induced dyskinesias in Parkinson's disease. Mov Disord 24:246.

Fitzgerald, P. B., S. Fountain, and Z. J. Daskalakis. 2006. A comprehensive review of the effects of rTMS on motor cortical excitability and inhibition. Clin Neurophysiol 117:2584.

Fitzgerald, P. B., J. J. Maller, K. E. Hoy, R. Thomson, and Z. J. Daskalakis. 2009. Exploring the optimal site for the localization of dorsolateral prefrontal cortex in brain stimulation experiments. Brain Stimul 2:234.

Focke, F., D. Schuermann, N. Kuster, and P. Schär. 2010. DNA fragmentation in human fibroblasts under extremely low frequency electromagnetic field exposure. Mutat Res 683:74.

Frahm, J., M. Lantow, M. Lupke, D. G. Weiss, and M. Simkó. 2006. Alteration in cellular functions in mouse macrophages after exposure to $50 \mathrm{~Hz}$ magnetic fields. $J$ Cell Biochem 99:168.

Fu, Y., C. Wang, J. Wang, Y. Lei, and Y. Ma. 2008. Long-term exposure to extremely lowfrequency magnetic fields impairs spatial recognition memory in mice. Clin Exp Pharmacol Physiol 35:797.

Gaetani, R., M. Ledda, L. Barile, I. Chimenti, F. De Carlo, E. Forte, V. Ionta et al. 2009. Differentiation of human adult cardiac stem cells exposed to extremely lowfrequency electromagnetic fields. Cardiovasc Res 82:411.

Gandhi, O. P. 2002. Electromagnetic fields: Human safety issues. Annu Rev Biomed Eng 4:211.

Garip, A. I., and Z. Akan. 2010. Effect of ELF-EMF on number of apoptotic cells; correlation with reactive oxygen species and HSP. Acta Biol Hung 61:158.

Gegear, R. J., A. Casselman, S. Waddell, and S. M. Reppert. 2008. Cryptochrome mediates light-dependent magnetosensitivity in Drosophila. Nature 454:1014.

Gegear, R. J., L. E. Foley, A. Casselman, and S. M. Reppert. 2010. Animal cryptochromes mediate magnetoreception by an unconventional photochemical mechanism. Nature 463:804.

Ghibelli, L., C. Cerella, S. Cordisco, G. Clavarino, S. Marazzi, M. De Nicola, S. Nuccitelli et al. 2006. NMR exposure sensitizes tumor cells to apoptosis. Apoptosis 11:359.

Glover, P. M., and R. Bowtell. 2008. Measurement of electric fields induced in a human subject due to natural movements in static magnetic fields or exposure to alternating magnetic field gradients. Phys Med Biol 53:361.

Gonet, B., D. I. Kosik-Bogacka, and W. Kuźna-Grygiel. 2009. Effects of extremely lowfrequency magnetic fields on the oviposition of Drosophila melanogaster over three generations. Bioelectromagnetics 30:687.

Goodman, L. 1953. Alzheimer's disease-a clinicopathologic analysis of 23 cases with a theory on pathogenesis. J Nerv Ment Dis 118:97. 
Goodman, R., A. Lin-Ye, M. S. Geddis, P. J. Wickramaratne, S. E. Hodge, S. P. Pantazatos, M. Blank, and R. T. Ambron. 2009. Extremely low frequency electromagnetic fields activate the ERK cascade, increase hsp70 protein levels and promote regeneration in Planaria. Int J Radiat Biol 85:851.

Goraca, A., E. Ciejka, and A. Piechota. 2010. Effects of extremely low frequency magnetic field on the parameters of oxidative stress in heart. J Physiol Pharmacol 61:333.

Gorman, A. A., and M. A. Rodgers. 1992. Current perspectives of singlet oxygen detection in biological environments. J Photochem Photobiol B 14:159.

Grossman, G. E., R. J. Leigh, L. A. Abel, D. J. Lanska, and S. E. Thurston. 1988. Frequency and velocity of rotational head perturbations during locomotion. Exp Brain Res $70: 470$.

Gupta, A., A. R. Weeks, and S. M. Richie. 2008. Simulation of elevated T-waves of an ECG inside a static magnetic field (MRI). IEEE Trans Biomed Eng 55:1890.

Hajnorouzi, A., M. Vaezzadeh, F. Ghanati, H. Jamnezhad, and B. Nahidian. 2011. Growth promotion and a decrease of oxidative stress in maize seedlings by a combination of geomagnetic and weak electromagnetic fields. J Plant Physiol published online.

Hamidi, M., H. A. Slagter, G. Tononi, and B. R. Postle. 2009. Repetitive transcranial magnetic stimulation affects behavior by biasing endogenous cortical oscillations. Front Integr Neurosci 3:14.

Hamidi, M., G. Tononi, and B. R. Postle. 2008. Evaluating frontal and parietal contributions to spatial working memory with repetitive transcranial magnetic stimulation. Brain Res 1230:202.

Hao, Q., C. Wenfang, A. Xia, W. Qiang, L. Ying, Z. Kun, and S. Runguang. 2011. Effects of a moderate-intensity static magnetic field and adriamycin on K562 cells. Bioelectromagnetics 32:191.

Harkins, T. T., and C. B. Grissom. 1994. Magnetic field effects on $B_{12}$ ethanolamine ammonia lyase: Evidence for a radical mechanism. Science 263:958.

Harris, S. R., K. B. Henbest, K. Maeda, J. R. Pannell, C. R. Timmel, P. J. Hore, and H. Okamoto. 2009. Effect of magnetic fields on cryptochrome-dependent responses in Arabidopsis thaliana. J R Soc Interface 6:1193.

Hata, N. 1976. The effect of external magnetic field on the photochemical reaction of isoquinoline N-oxide. Chem Lett 5:547.

Hayashi, H. 2004. Introduction to Dynamic Spin Chemistry. Singapore: World Scientific Publishing Co.

Henbest, K. B., K. Maeda, P. J. Hore, M. Joshi, A. Bacher, R. Bittl, S. Weber, C. R. Timmel, and E. Schleicher. 2008. Magnetic-field effect on the photoactivation reaction of Escherichia coli DNA photolyase. Proc Natl Acad Sci USA 105:14395.

Hill, E., and T. Ritz. 2010. Can disordered radical pair systems provide a basis for a magnetic compass in animals? J R Soc Interface 7:S265.

Hirai, T., H. Taniura, Y. Goto, M. Ogura, J. C. Sng, and Y. Yoneda. 2006. Stimulation of ubiquitin-proteasome pathway through the expression of amidohydrolase for $\mathrm{N}$-terminal asparagine (Ntan1) in cultured rat hippocampal neurons exposed to static magnetism. J Neurochem 96:1519.

Hirooka, Y. 2011. Oxidative stress in the cardiovascular center has a pivotal role in the sympathetic activation in hypertension. Hypertens Res 43:407. 
Hiscock, A., S. Miller, J. Rothwell, R. C. Tallis, and V. M. Pomeroy. 2008. Informing dosefinding studies of repetitive transcranial magnetic stimulation to enhance motor function: A qualitative systematic review. Neurorehabil Neural Repair 22:228.

Holden, A. V. 2005. The sensitivity of the heart to static magnetic fields. Prog Biophys Mol Biol 87:289.

Huang, H. H., and S. R. Wang. 2008. The effects of inverter magnetic fields on early seed germination of mung beans. Bioelectromagnetics 29:649.

Hung, Y. C., J. H. Lee, H. M. Chen, and G. S. Huang. 2010. Effects of static magnetic fields on the development and aging of Caenorhabditis elegans. J Exp Biol 213:2079.

IEEE. 2002. IEEE standard for safety levels with respect to human exposure to electromagnetic fields, 0-3 kHz. Rep IEEE Std C95.6. IEEE, New York.

Ilic, T. V., F. MeintzscheI, U. Cleff, D. Ruge, K. R. Kessler, and U. Ziemann. 2002. Short-interval paired-pulse inhibition and facilitation of human motor cortex: The dimension of stimulus intensity. J Physiol 545:153.

International Commission on Non-Ionizing Radiation Protection (ICNIRP). 1998. Guidelines for limiting exposure to time-varying electric, magnetic, and electromagnetic fields (up to $300 \mathrm{GHz}$ ). Health Phys 74:494.

International Commission on Non-Ionizing Radiation Protection (ICNIRP). 2004. ICNIRP statement related to the use of security and similar devices utilizing electromagnetic fields. Health Phys 87:187.

International Commission on Non-Ionizing Radiation Protection (ICNIRP). 2009. Guidelines on limits of exposure to static magnetic fields. Health Phys 96:504.

International Commission on Non-Ionizing Radiation Protection (ICNIRP). 2010. Guidelines for limiting exposure to time-varying electric and magnetic fields $(1 \mathrm{~Hz}-100 \mathrm{kHz})$. Health Phys 99:818.

Iorio, R., S. D. Monache, F. Bennato, C. Di Bartolomeo, R. Scrimaglio, B. Cinque, and R. C. Colonna. 2011. Involvement of mitochondrial activity in mediating ELF-EMF stimulatory effect on human sperm motility. Bioelectromagnetics 32:15.

Iwasaka, M., and S. Ueno. 2003. Detection of intracellular macromolecule behavior under strong magnetic fields by linearly polarized light. Bioelectromagnetics 24:564.

Izmaylov, A. F., J. C. Tully, and M. J. Frisch. 2009. Relativistic interactions in the radical pair model of magnetic field sense in CRY-1 protein of Arabidopsis thaliana. J Phys Chem A 113:12276.

Jansen, J. H., O. P. van der Jagt, B. J. Punt, J. A. Verhaar, J. P. van Leeuwen, H. Weinans, and H. Jahr. 2010. Stimulation of osteogenic differentiation in human osteoprogenitor cells by pulsed electromagnetic fields: an in vitro study. BMC Musculoskelet Disord 11:188.

Jeong, J. H., K. B. Choi, H. J. Choi, H. J. Song, Y. S. Min, S. K. Ko, B. O. Im, and U. D. Sohn. 2005. Extremely low frequency magnetic fields modulate bicuculline-inducedconvulsion in rats. Arch Pharm Res 28:587.

Jeong, J. H., K. B. Choi, N. J. Moon, E. S. Park, and U. D. Sohn. 2005. Benzodiazepine system is involved in hyperalgesia in rats induced by the exposure to extremely low frequency magnetic fields. Arch Pharm Res 28:238.

Jeong, J. H., C. Kum, H. J. Choi, E. S. Park, and U. D. Sohn. 2006. Extremely low frequency magnetic field induces hyperalgesia in mice modulated by nitric oxide synthesis. Life Sci 78:1407. 
Jimena, I., I. Tasset, R. López-Martos, A. J. Rubio, E. Luque, P. Montilla, J. Peña, and I. Túnez. 2009. Effects of magnetic stimulation on oxidative stress and skeletal muscle regeneration induced by mepivacaine in rat. Med Chem 5:44.

Jimenez-Garcia, M. N., J. Arellanes-Robledo, D. I. Aparicio-Bautista, M. A. RodriguezSegura, S. Villa-Trevino, and J. J. Godina-Nava. 2010. Anti-proliferative effect of extremely low frequency electromagnetic field on preneoplastic lesions formation in the rat liver. BMC Cancer 10:159.

Johnson, J. S., M. Hamidi, and B. R. Postle. 2010. Using EEG to explore how rTMS produces its effects on behavior. Brain Topogr 22:281.

Jones, A. R., N. S. Scrutton, and J. R. Woodward. 2006. Magnetic field effects and radical pair mechanisms in enzymes: A reappraisal of the horseradish peroxidase system. $J$ Am Chem Soc 128:8408.

Joy, M., G. Scott, and M. Henkelman. 1989. In vivo detection of applied electric currents by magnetic resonance imaging. Magn Reson Imaging 7:89.

Jung, S. H., J. E. Shin, Y. S. Jeong, and H. I. Shin. 2008. Changes in motor cortical excitability induced by high-frequency repetitive transcranial magnetic stimulation of different stimulation durations. Clin Neurophysiol 119:71.

Kainz, W., J. Guag, S. Benkler, D. Szczerba, E. Neufeld, V. Krauthamer, J. Myklebust, H. Bassen, I. Chang, N. Chavannes, J. H. Kim, M. Sarntinoranont, N. Kuster, 2010. Development and validation of a magnetohydrodynamic solver for blood flow analysis. Phys Med Biol 55:7253.

Kamada, K., Y. Sawamura, F. Takeuchi, S. Kuriki, K. Kawai, A. Morita, and T. Todo. 2007. Expressive and receptive language areas determined by a non-invasive reliable method using functional magnetic resonance imaging and magnetoencephalography. Neurosurgery 60:296.

Kamida, T., M. Fujiki, H. Baba, T. Ono, T. Abe, and H. Kobayashi. 2007. The relationship between paired pulse magnetic MEP and surgical prognosis in patients with intractable epilepsy. Seizure 16:113.

Karogodina, T. Y., S. V. Sergeeva, and D. V. Stass. 2009. Magnetic field effect in the reaction of recombination of nitric oxide and superoxide anion. Appl Magn Reson 36:195.

Kaszuba-Zwoinska, J., K. Wojcik, M. Bereta, A. Ziomber, P. Pierzchalski, E. Rokita, J. Marcinkiewicz, W. Zaraska, and P. Thor. 2010. Pulsating electromagnetic field stimulation prevents cell death of puromycin treated U937 cell line. J Physiol Pharmacol 61:201.

Ke, X. Q., W. J. Sun, D. Q. Lu, Y. T. Fu, and H. Chiang. 2008. $50 \mathrm{~Hz}$ magnetic field induces EGF-receptor clustering and activates RAS. Int J Radiat Biol 84:413.

Khang, H. S., B. I. Lee, S. H. Oh, E. J. Woo, S. Y. Lee, M. H. Cho, O. Kwon, J. R. Yoon, and J. K. Seo. 2002. J-substitution algorithm in magnetic resonance electrical impedance tomography (MREIT): Phantom experiments for static resistivity images. IEEE Trans Med Imaging 21:695.

Khodarahmi, I., H. Mobasheri, and M. Firouzi. 2010. The effect of $2.1 \mathrm{~T}$ static magnetic field on astrocyte viability and morphology. Magn Reson Imaging 28:903.

Khoromi, S., M. R. Blackman, A. Kingman, A. Patsalides, L. A. Matheny, S. Adams, A. A. Pilla, and M. B. Max. 2007. Low intensity permanent magnets in the treatment of chronic lumbar radicular pain. J Pain Symptom Manage 34:434. 
Kim, H. J., I. T. Chang, S. J. Heo, J. Y. Koak, S. K. Kim, and J. H. Jang. 2005. Effect of magnetic field on the fibronectin adsorption, cell attachment and proliferation on titanium surface. Clin Oral Implants Res 16:557.

Kim, S., W. S. Im, L. Kang, S. T. Lee, K. Chu, and B. I. Kim. 2008. The application of magnets directs the orientation of neurite outgrowth in cultured human neuronal cells. J Neurosci Methods 174:91.

Kimura, T., K. Takahashi, Y. Suzuki, Y. Konishi, Y. Ota, C. Mori, T. Ikenaga, T. Takanami, R. Saito, E. Ichiishi, S. Awaji, K. Watanabe, and A. Higashitani. 2008. The effect of high strength static magnetic fields and ionizing radiation on gene expression and DNA damage in Caenorhabditis elegans. Bioelectromagnetics 29:605.

Kinouchi, Y., H. Yamaguchi, and T. S. Tenforde. 1996. Theoretical analysis of magnetic field interactions with aortic blood flow. Bioelectromagnetics 17:21.

Komeili, A., Z. Li, D. K. Newman, and G. J. Jensen. 2006. Magnetosomes are cell membrane invaginations organized by the actin-like protein MamK. Science 311:242.

Koppenol, W. H. 2001. The Haber-Weiss cycle 70 years later. Redox Rep 6:229.

Kovács-Bálint, Z., A. Csathó, J. F. László, P. Juhász, and I. Hernádi. 2011. Exposure to an inhomogeneous static magnetic field increases thermal pain threshold in healthy human volunteers. Bioelectromagnetics 32:131.

Kowalski, T., J. Silny, and H. Buchner. 2002. Current density threshold for the stimulation of neurons in the motor cortex area. Bioelectromagnetics 23:421.

Koyama, S., T. Sakurai, T. Nakahara, and J. Miyakoshi. 2008. Extremely low frequency (ELF) magnetic fields enhance chemically induced formation of apurinic/apyrimidinic (AP) sites in A172 cells. Int J Radiat Biol 84:53.

Kujirai, T., M. D. Cararnia, I. C. Rothwell, B. L. Day, P. O. Thompson, A. Ferbert, S. Wroe, P. Asselman, and C. D. Marsden. 1993. Corticocortical inhibition in human motor cortex. J Physiol 471:501.

Kujirai, K., T. Kujirai, T. Sinkjaer, and J. C. Rothwell. 2006. Associative plasticity in human motor cortex during voluntary muscle contraction. J Neurophysiol 96:1337.

Kuriki, S., S. Kanda, and Y. Hirata. 2006. Effects of musical experience on different components of MEG responses elicited by sequential piano-tones and chords. J Neurosci 26:4046.

Lahbib, A., M. Elferchichi, S. Ghodbane, H. Belguith, S. Chater, M. Sakly, and H. Abdelmelek. 2010. Time-dependent effects of exposure to static magnetic field on glucose and lipid metabolism in rat. Gen Physiol Biophys 29:390.

Lander, H. M. 1997. An essential role for free radicals and derived species in signal transduction. FASEB J 11:118.

Langmesser, S., T. Tallone, A. Bordon, S. Rusconi, and U. Albrecht. 2008. Interaction of circadian clock proteins PER2 and CRY with BMAL1 and CLOCK. BMC Mol Biol 9:41.

László, J., and J. Kutasi. 2010. Static magnetic field exposure fails to affect the viability of different bacteria strains. Bioelectromagnetics 31:220.

László, J., J. Reiczigel, L. Székely, A. Gasparics, I. Bogár, L. Bors, B. Rácz, and K. Gyires. 2007. Optimization of static magnetic field parameters improves analgesic effect in mice. Bioelectromagnetics 28:615.

Lau, J. C., N. Wagner-Rundell, C. T. Rodgers, N. J. Green, and P. J. Hore. 2010. Effects of disorder and motion in a radical pair magnetoreceptor. J $R$ Soc Interface 7:S257. 
Lauterbur, P. C. 1973. Image formation by induced local interactions: Examples employing nuclear magnetic resonance. Nature 242:190.

Lee, C. H., Y. C. Hung, and G. S. Huang. 2010. Static magnetic field accelerates aging and development in nematode. Commun Integr Biol 3:528.

Lefèvre, C. T., T. Song, J. P. Yonnet, and L. F. Wu. 2009. Characterization of bacterial magnetotactic behaviors by using a magnetospectrophotometry assay. Appl Environ Microbiol 75:3835.

Li, J. K., J. C. Lin, H. C. Liu, J. S. Sun, R. C. Ruaan, C. Shih, and W. H. Chang. 2006. Comparison of ultrasound and electromagnetic field effects on osteoblast growth. Ultrasound Med Biol 32:769.

Lin, J. C. 1999. Catheter microwave ablation therapy for cardiac arrhythmias. Bioelectromagnetics (Suppl 4):120.

Lin, J. C. 2007. Dosimetric comparison between different quantities for limiting exposure in the RF band: Rationale and implications for guidelines. Health Phys 92:547.

Lisanby, S. H., L. H. Kinnunen, and M. J. Crupain. 2002. Applications of TMS to therapy in psychiatry. J Clin Neurophysiol 19:344.

Liu, Y., R. Edge, K. Henbest, C. R. Timmel, P. J. Hore, and P. Gast. 2005. Magnetic field effect on singlet oxygen production in a biochemical system. Chem Commun (Issue 2):174.

Liu, T., S. Wang, L. He, and K. Ye. 2008. Chronic exposure to low-intensity magnetic field improves acquisition and maintenance of memory. Neuroreport 19:549.

Liu, F., H. Zhao, and S. Crozier. 2003. On the induced electric field gradients in the human body for magnetic stimulation by gradient coils in MRI. IEEE Trans Biomed Eng 50:804.

Logothetis, N. K., J. Pauls, M. Augath, T. Trinath, and A. Oeltermann. 2001. Neurophysiological investigation of the basis of the fMRI signal. Nature 412:150.

Lupke, M., J. Frahm, M. Lantow, C. Maercker, D. Remondini, F. Bersani, and M. Simkó. 2006. Gene expression analysis of ELF-MF exposed human monocytes indicating the involvement of the alternative activation pathway. Biochim Biophys Acta 1763:402.

MacDougall, H. G., and S. T. Moore. 2005. Functional assessment of head-eye coordination during vehicle operation. Optom Vis Sci 82:706.

Manassen, Y., E. Shalev, and G. Navon. 1988. Mapping of electrical circuits using chemical-shift imaging. J Magn Reson 76:371.

Mannerling, A. C., M. Simkó, K. H. Mild, and M. O. Mattsson. 2010. Effects of $50 \mathrm{~Hz}$ magnetic field exposure on superoxide radical anion formation and HSP70 induction in human K562 cells. Radiat Environ Biophys 49:731.

Markesbery, W. R. 1997. Oxidative stress hypothesis in Alzheimer's disease. Free Radic Biol Med 23:134.

Markov, M. S. 2007. Expanding use of pulsed electromagnetic field therapies. Electromagn Biol Med 26:257.

Martínez-Sámano, J., P. V. Torres-Durán, M. A. Juárez-Oropeza, D. Elías-Viñas, and L. Verdugo-Díaz. 2010. Effects of acute electromagnetic field exposure and movement restraint on antioxidant system in liver, heart, kidney and plasma of Wistar rats: A preliminary report. Int J Radiat Biol 86:1088. 
Martino, C. F., D. Belchenko, V. Ferguson, S. Nielsen-Preiss, and H. J. Qi. 2008. The effects of pulsed electromagnetic fields on the cellular activity of SaOS-2 cells. Bioelectromagnetics 29:125.

Martino, C. F., H. Perea, U. Hopfner, V. L. Ferguson, and E. Wintermantel. 2010. Effects of weak static magnetic fields on endothelial cells. Bioelectromagnetics 31:296.

Martino, C. F., L. Portelli, K. McCabe, M. Hernandez, and F. Barnes. 2010. Reduction of the Earth's magnetic field inhibits growth rates of model cancer cell lines. Bioelectromagnetics 31:649.

Martiny, K., M. Lunde, and P. Bech. 2010. Transcranial low voltage pulsed electromagnetic fields in patients with treatment-resistant depression. Biol Psychiatry 68:163.

McDonald, K. K., and M. A. Persinger. 2009. Altered neuronal densities in sexually dimorphic structures: Comparable effects from perinatal magnetic fields with nitric oxide synthase inhibitors and postnatal hypoxia. Neurosci Lett 450:37.

McKay, J. C., M. Corbacio, K. Tyml, F. S. Prato, and A. W. Thomas. 2010. Extremely low frequency pulsed electromagnetic field designed for antinociception does not affect microvascular responsiveness to the vasodilator acetylcholine. Bioelectromagnetics 31:64.

McKay, J. C., F. S. Prato, and A. W. Thomas. 2007. A literature review: The effects of magnetic field exposure on blood flow and blood vessels in the microvasculature. Bioelectromagnetics 28:81.

McLauchlan, K. A., and U. E. Steiner. 1991. The spin correlated radical pair as a reaction intermediate. Mol Phys 73:241.

McNamee, D. A., M. Corbacio, J. K. Weller, S. Brown, F. S. Prato, A. W. Thomas, and A. G. Legros. 2011. The cardiovascular response to an acute $1800-\mu \mathrm{T}, 60 \mathrm{~Hz}$ magnetic field exposure in humans. Int Arch Occup Environ Health 84:267.

McNamee, D. A., M. Corbacio, J. K. Weller, S. Brown, R. Z. Stodilka, F. S. Prato, Y. Bureau, A. W. Thomas, and A. G. Legros. in press. The response of the human circulatory system to an acute $200-\mu \mathrm{T}, 60 \mathrm{~Hz}$ magnetic field exposure. Int Arch Occup Environ Health.

McNamee, D. A., A. G. Legros, D. R. Krewski, G. Wisenberg, F. S. Prato, and A. W. Thomas. 2009. A literature review: The cardiovascular effects of exposure to extremely low frequency electromagnetic fields. Int Arch Occup Environ Health 82:919.

Medical Advisory Secretariat. 2004. Ontario Ministry of Health and Long-Term Care, Repetitive transcranial magnetic stimulation for the treatment of major depressive disorder: an evidence-based analysis, Ontario Health Technology Assessment Series, Vol. 4, No. 7, Toronto, Canada.

Mert, T., I. Gunay, and I. Ocal. 2010. Neurobiological effects of pulsed magnetic field on diabetes-induced neuropathy. Bioelectromagnetics 31:39.

Mohtat, N., F. L. Cozens, T. Hancock-Chen, J. C. Scaiano, J. McLean, and J. Kim. 1998. Magnetic field effects on the behavior of radicals in protein and DNA environments. Photochem Photobiol 67:111.

Monache, S. D., R. Alessandro, R. Iorio, G. Gualtieri, and R. Colonna. 2008. Extremely low frequency electromagnetic fields (ELF-EMFs) induce in vitro angiogenesis process in human endothelial cells. Bioelectromagnetics 29:640. 
Monfared, A. S., S. G. Jorsaraei, and R. Abdi. 2009. Protective effects of vitamins C and E on spermatogenesis of 1.5 Tesla magnetic field exposed rats. J Magn Reson Imaging 30:1047.

Morabito, C., F. Rovetta, M. Bizzarri, G. Mazzoleni, G. Fanò, and M. A. Mariggiò. 2010. Modulation of redox status and calcium handling by extremely low frequency electromagnetic fields in $\mathrm{C} 2 \mathrm{C} 12$ muscle cells: A real-time, single-cell approach. Free Radic Biol Med 48:579.

Muehsam, D. J., and A. A. Pilla. 2009a. A Lorentz model for weak magnetic field bioeffects: Part I-thermal noise is an essential component of AC/DC effects on bound ion trajectory. Bioelectromagnetics 30:462.

Muehsam, D. J., and A. A. Pilla. 2009b. A Lorentz model for weak magnetic field bioeffects: Part II-secondary transduction mechanisms and measures of reactivity. Bioelectromagnetics 30:476.

Nagakura, S., H. Hayashi, and T. Azumi. 1998. Dynamic Spin Chemistry: Magnetic Controls and Spin Dynamics of Chemical Reactions. 297. New York: Wiley.

Nagakura, S., and Y. Molin. 1992. Magnetic field effects upon photophysical and photochemical phenomena. Chem Phys 162:1.

Natarajan, E., and C. B. Grissom. 1997. Use of magnetic field effects to study coenzyme $\mathrm{B}_{12}$-dependent reactions. Meth Enzymol 281:235.

Nathan, C. 1992. Nitric-oxide as a secretory product of mammalian-cells. FASEB J 6:3051.

Negishi, T., S. Imai, K. Shibuya, I. Nishimura, and T. Shigemitsu. 2008. Lack of promotion effects of $50 \mathrm{~Hz}$ magnetic fields on 7,12-dimethylbenz(a)anthracene-induced malignant lymphoma/lymphatic leukemia in mice. Bioelectromagnetics 29:29.

Nishimura, T., H. Okano, H. Tada, E. Nishimura, K. Sugimoto, K. Mohri, and M. Fukushima. 2010. Lizards respond to an extremely low-frequency electromagnetic field. J Exp Biol 213:1985.

Nishimura, T., H. Tada, X. Guo, T. Murayama, S. Teramukai, H. Okano, J. Yamada, K. Mohri, and M. Fukushima. 2011. A 1- $\mu$ T extremely low-frequency electromagnetic field vs. sham control for mild-to-moderate hypertension: A double-blind, randomized study. Hypertens Res 34:372.

Novikov, V. V., I. M. Sheiman, and E. E. Fesenko. 2008. Effect of weak static and lowfrequency alternating magnetic fields on the fission and regeneration of the planarian Dugesia (Girardia) tigrina. Bioelectromagnetics 29:387.

Nyenhuis, J. A., J. D. Bourland, A. V. Kildishev, and D. J. Schaefer. 2001. Health effects and safety of intense gradient fields. In Magnetic Resonance Procedures: Health Effects and Safety, ed. F. Shellock, 31. Boca Raton, FL: CRC Press.

Ogawa, S., D. W. Tank, and R. Menon. 1992. Intrinsic signal changes accompanying stimulation: Functional brain mapping with magnetic resonance imaging. Proc Natl Acad Sci U S A 89:5951.

Ohkubo, C., H. Okano, A. Ushiyama, and H. Masuda. EMF effects on microcirculatory system. 2007. The Environmentalist 27:395.

Ohkubo, C., and H. Okano. Clinical aspects of static magnetic field effects on circulatory system. 2010. The Environmentalist published online.

Okano, H. 2008a. Effects of static magnetic fields in biology: Role of free radicals. Front Biosci 13:6106. 
Okano, H. 2008b. Effects of static magnetic fields on blood pressure in animals and humans. Curr Hypertens Rev 4:63.

Okano, H., R. Onmori, N. Tomita, and Y. Ikada. 2006. Effects of a moderate-intensity static magnetic field on VEGF-A stimulated endothelial capillary tubule formation in vitro. Bioelectromagnetics 27:628.

Okano, H., N. Tomita, and Y. Ikada. 2007. Effects of $120 \mathrm{mT}$ static magnetic field on TGF$\beta 1$-inhibited endothelial tubular formation in vitro. Bioelectromagnetics 28:497.

Okano, H., N. Tomita, and Y. Ikada. 2008. Spatial gradient effects of $120 \mathrm{mT}$ static magnetic field on endothelial tubular formation in vitro. Bioelectromagnetics 29:233.

Ozgüçlü, E., A. Cetin, M. Cetin, and E. Calp. 2010. Additional effect of pulsed electromagnetic field therapy on knee osteoarthritis treatment: a randomized, placebocontrolled study. Clin Rheumatol 29:927.

Pan, Y., W. Lin, J. Li, W. Wu, L. Tian, C. Deng, Q. Liu, R. Zhu, M. Winklhofer, and N. Petersen. 2009. Reduced efficiency of magnetotaxis in magnetotactic coccoid bacteria in higher than geomagnetic fields. Biophys J 97:986.

Pantev, C., R. Oostenveld, A. Engelien, B. Ross, L. E. Roberts, and M. Hoke. 1998. Increased auditory cortical representation in musicians. Nature 392:811.

Pascual-Leone, A., N. Davey, J. Rothwell, E. M. Wassermann, and B. K. Puri. 2002. Handbook of Transcranial Magnetic Stimulation. London: Hodder Arnold.

Pascual-Leone, A., B. Rubio, F. Pallardó, and M. D. Catalá. 1996. Rapid-rate transcranial magnetic stimulation of left dorsolateral prefrontal cortex in drug-resistant depression. Lancet 348:233.

Patruno, A., P. Amerio, M. Pesce, G. Vianale, S. Di Luzio, A. Tulli, S. Franceschelli, A. Grilli, R. Muraro, and M. Reale. 2010. Extremely low frequency electromagnetic fields modulate expression of inducible nitric oxide synthase, endothelial nitric oxide synthase and cyclooxygenase- 2 in the human keratinocyte cell line HaCat: Potential therapeutic effects in wound healing. Br J Dermatol 162:258.

Peric-Mataruga, V., Z. Prolic, V. Nenadovic, M. Vlahovic, and M. Mrdakovic. 2008. The effect of a static magnetic field on the morphometric characteristics of neurosecretory neurons and corpora allata in the pupae of yellow mealworm Tenebrio molitor (Tenebrionidae). Int J Radiat Biol 84:91.

Pilla, A. A. 2007. Mechanisms and therapeutic applications of time-varying and static magnetic fields. In Handbook of Biological Effects of Electromagnetic Fields: Bioengineering and Biophysical Aspects of Electromagnetic Fields. 3rd ed. ed. F. S. Barnes, and B. Greenbaum, 351. Boca Raton, FL: CRC Press.

Phillips, J. B., P. E. Jorge, and R. Muheim. 2010. Light-dependent magnetic compass orientation in amphibians and insects: Candidate receptors and candidate molecular mechanisms. J R Soc Interface 7:S241.

Polaniak, R., R. J. Bułdak, M. Karoń, K. Birkner, M. Kukla, K. Zwirska-Korczala, and E. Birkner. 2010. Influence of an extremely low frequency magnetic field (ELF-EMF) on antioxidative vitamin E properties in AT478 murine squamous cell carcinoma culture in vitro. Int J Toxicol 29:221.

Postle, B. R., and M. Hamidi. 2007. Nonvisual codes and nonvisual brain areas support visual working memory. Cereb Cortex 17:2151. 
Potenza, L., C. Martinelli, E. Polidori, S. Zeppa, C. Calcabrini, L. Stocchi, P. Sestili, and V. Stocchi. 2010. Effects of a $300 \mathrm{mT}$ static magnetic field on human umbilical vein endothelial cells. Bioelectromagnetics 31:630.

Pozzo, T., A. Berthoz, and L. Lefort. 1990. Head stabilisation during various locomotor tasks in humans. Exp Brain Res 82:97.

Pradel, N., C. L. Santini, A. Bernadac, Y. Fukumori, and L. F. Wu. 2006. Biogenesis of actin-like bacterial cytoskeletal filaments destined for positioning prokaryotic magnetic organelles. Proc Natl Acad Sci U S A 103:17485.

Prato, F. S., D. Desjardins-Holmes, L. D. Keenliside, J. C. McKay, J. A. Robertson, and A. W. Thomas. 2009. Light alters nociceptive effects of magnetic field shielding in mice: Intensity and wavelength considerations. J R Soc Interface 6:17.

Prato, F. S., J. A. Robertson, D. Desjardins, J. Hensel, and A. W. Thomas. 2005. Daily repeated magnetic field shielding induces analgesia in CD-1 mice. Bioelectromagnetics 26:109.

Prato, F. S., A. W. Thomas, and C. M. Cook. 2005. Extremely low frequency magnetic fields (ELFMF) and pain therapy. In Advances in Electromagnetic Fields in Living Systems, Vol. 4., ed. J. C. Lin, 155. New York: Springer Science and Business Media.

Prato, F. S., A. W. Thomas, A. Legros, J. A. Robertson, J. Modolo, R. Z. Stodilka, J. M. Demoor, and W. Huda. 2010. MRI safety not scientifically proven. Science 328:568.

Qian, A. R., L. F. Hu, X. Gao, W. Zhang, S. M. Di, Z. C. Tian, P. F. Yang, D. C. Yin, Y. Y. Weng, and P. Shang. 2009. Large gradient high magnetic field affects the association of MACF1 with actin and microtubule cytoskeleton. Bioelectromagnetics 30:545.

Rábago, C. A., J. L. Lancaster, S. Narayana, W. Zhang, and P. T. Fox. 2009. Automatedparameterization of the motor evoked potential and cortical silent period induced by transcranial magnetic stimulation. Clin Neurophysiol 120:1577.

Raggi, F., G. Vallesi, S. Rufini, S. Gizzi, E. Ercolani, and R. Rossi. 2008. ELF magnetic therapy and oxidative balance. Electromagn Biol Med 27:325.

Rappaport, C. 2004. Cardiac tissue ablation with catheter-based microwave heating. Int J Hyperthermia 20:769.

Raux, M., H. Xie, T. Similowski, and L. Koski. 2010. Facilitatory conditioning of the supplementary motor area in humans enhances the corticophrenic responsiveness to transcranial magnetic stimulation. J Appl Physiol 108:39.

Reddy, S. B., J. Weller, D. Desjardins-Holmes, T. Winters, L. Keenliside, F. S. Prato, T. J. Prihoda, V. Thomas, and A. W. Thomas. 2010. Micronuclei in the blood and bone marrow cells of mice exposed to specific complex time-varying pulsed magnetic fields. Bioelectromagnetics 31:445.

Reilly, J. P. 1998. Applied Bioelectricity: From Electrical Stimulation to Electropathology. New York: Springer-Verlag.

Reilly, J. P. 2002. Neuroelectric mechanisms applied to low frequency electric and magnetic field exposure guidelines-part I: Sinusoidal waveforms. Health Phys 83:341.

Reilly, J. P. 2005. An analysis of differences in the low-frequency electric and magnetic field exposure standards of ices and ICNIRP. Health Phys 89:71.

Ritz, T., S. Adem, and K. Schulten. 2000. A model for photoreceptor-based magnetoreception in birds. Biophys J 78:707. 
Ritz, T., M. Ahmad, H. Mouritsen, R. Wiltschko, and W. Wiltschko. 2010. Photoreceptorbased magnetoreception: Optimal design of receptor molecules, cells, and neuronal processing. J R Soc Interface 7:S135.

Ritz, T., D. H. Dommer, and J. B. Phillips. 2002. Shedding light on vertebrate magnetoreception. Neuron 34:503.

Ritz, T., P. Thalau, J. B. Phillips, R. Wiltschko, and W. Wiltschko. 2004. Resonance effects indicate a radical-pair mechanism for avian magnetic compass. Nature 429:177.

Robertson, J. A., N. Juen, J. Théberge, J. Weller, D. J. Drost, F. S. Prato, and A. W. Thomas. 2010. Evidence for a dose-dependent effect of pulsed magnetic fields on pain processing. Neurosci Lett 482:160.

Robertson, J. A., A. W. Thomas, Y. Bureau, and F. S. Prato. 2007. The influence of extremely low frequency magnetic fields on cytoprotection and repair. Bioelectromagnetics 28:16.

Rodgers, C. T., and P. J. Hore. 2009. Chemical magnetoreception in birds: The radical pair mechanism. Proc Natl Acad Sci U S A 106:353.

Rossi, S., M. Hallett, P. M. Rossini, and A. Pascual-Leone. 2009. Safety, ethical considerations, and application guidelines for the use of transcranial magnetic stimulation in clinical practice and research. Clin Neurophysiol 120:2008.

Roth, Y., G. S. Pell, and A. Zangen. 2010. Motor evoked potential latency, motor threshold and electric field measurements as indices of transcranial magnetic stimulation depth. Clin Neurophysiol 121:255.

Rothwell, J. C., B. L. Day, P. D. Thompson, J. P. Dick, and C. D. Marsden. 1987. Some experiences of techniques for stimulation of the human cerebral motor cortex through the scalp. Neurosurgery 20:156.

Sadleir, R. J., S. C. Grant, and E. J. Woo. 2010. Can high-field MREIT be used to directly detect neural activity? Theoretical considerations. Neuroimage 52:205.

Saito, K., H. Suzuki, and K. Suzuki. 2006. Teratogenic effects of static magnetic field on mouse fetuses. Reprod Toxicol 22:118.

Sakuraba, T., Y. Shimada, S. Takahashi, T. Matsunaga, E. Itoi, and M. Kawatani. 2005. The effect of magnetic stimulation on unloaded soleus muscle of rat: Changes in myosin heavy chain mRNA isoforms. Biomed Res 26:15.

Sakurai, T., S. Terashima, and J. Miyakoshi. 2008. Enhanced secretion of prostaglandin $\mathrm{E}_{2}$ from osteoblasts by exposure to a strong static magnetic field. Bioelectromagnetics 29:277.

Sakurai, T., S. Terashima, and J. Miyakoshi. 2009. Effects of strong static magnetic fields used in magnetic resonance imaging on insulin-secreting cells. Bioelectromagnetics 30:1.

Sakurai, H., H. Yasui, K. Kunitomi, M. Kamatari, N. Kaneko, and A. Nakayama. 2000. Effects of static magnetic field on dissolved oxygen levels in aqueous solutions containing copper (II), iron (II), and heme iron (III) complexes. Pathophysiology 7:93.

Sakurai, T., M. Yoshimoto, S. Koyama, and J. Miyakoshi. 2008. Exposure to extremely low frequency magnetic fields affects insulin-secreting cells. Bioelectromagnetics 29:118.

Salerno, S., C. La Mendola, M. P. La Manna, A. L. Casto, N. Caccamo, and A. Salerno. 2009. Reversible effect of magnetic fields on human lymphocyte activation patterns: Different sensitivity of naive and memory lymphocyte subsets. Radiat Res 172:444. 
Sándor, K., Z. Helyes, K. Gyires, J. Szolcsányi, and J. László. 2007. Static magnetic fieldinduced anti-nociceptive effect and the involvement of capsaicin-sensitive sensory nerves in this mechanism. Life Sci 81:97.

Sandrini, M., C. Umiltà, and E. Rusconi. 2011. The use of transcranial magnetic stimulation in cognitive neuroscience: A new synthesis of methodological issues. Neurosci Biobehav Rev 35:516.

Sarvestani, A. S., P. Abdolmaleki, S. J. Mowla, F. Ghanati, E. Heshmati, Z. Tavasoli, and A. M. Jahromi. 2010. Static magnetic fields aggravate the effects of ionizing radiation on cell cycle progression in bone marrow stem cells. Micron 41:101.

Saunders, R. 2005. Static magnetic fields: Animal studies. Prog Biophys Mol Biol 87:225.

Saunders, R. D., and J. G. Jefferys. 2002. Weak electric field interactions in the central nervous system. Health Phys 83:366.

Saunders, R. D., and J. G. Jefferys. 2007. A neurobiological basis for ELF guidelines. Health Phys 92:596.

Scaiano, J. C., F. L. Cozens, and J. McLean. 1994. Model for the rationalization of magnetic field effects in vivo: Application of the radical-pair mechanism to biological systems. Photochem Photobiol 59:585.

Scheffel, A., M. Gruska, D. Faivre, A. Linaroudis, J. M. Plitzko, and D. Schüler. 2006. An acidic protein aligns magnetosomes along a filamentous structure in magnetotactic bacteria. Nature 440:110.

Schenck, J. F. 2005. Physical interactions of static magnetic fields with living tissues. Prog Biophys Mol Biol 87:185.

Schenck, J. F., C. L. Dumoulin, R. W. Redington, H. Y. Kressel, R. T. Elliott, and I. L. McDougall. 1992. Human exposure to 4.0-Tesla magnetic fields in a whole-body scanner. Med Phys 19:1089.

Schlamann, M., M. A. Voigt, S. Maderwald, A. K. Bitz, O. Kraff, S. C. Ladd, M. E. Ladd, M. Forsting, and H. Wilhelm. 2010. Exposure to high-field MRI does not affect cognitive function. J Magn Reson Imaging 31:1061.

Schult, O. W., L. E. Feinendegen, S. Zaum, W. W. Shreeve, and R. N. Pierson. 2010. Applications of BMI or BSI: Differences and revisions according to age and height. J Obes 2010:647163.

Schulten, K. 1982a. Magnetic field effects in chemistry and biology. Adv Solid State Phys 22:61.

Schulten, K. 1982b. Magnetic field effects in chemistry and biology. In Festkörperprobleme, Vol. 22., ed. J. Treusch. Braunschweig: Vieweg.

Schwenzer, N. F., R. Bantleon, B. Maurer, R. Kehlbach, T. Herberts, C. D. Claussen, and E. Rodegerdts. 2007. In vitro evaluation of magnetic resonance imaging at 3.0 Tesla on clonogenic ability, proliferation, and cell cycle in human embryonic lung fibroblasts. Invest Radiol 42:212.

Sekino, M., Y. Inoue, and S. Ueno. 2004. Magnetic resonance imaging of mean values and anisotropy of electrical conductivity in the human brain. Neurol Clin Neurophysiol 55:1.

Sekino, M., T. Matsumoto, K. Yamaguchi, N. Iriguchi, and S. Ueno. 2004. A method for NMR imaging of a magnetic field generated by electric current. IEEE Trans Magn 40:2188. 
Sekino, M., H. Mihara, N. Iriguchi, and S. Ueno. 2005. Dielectric resonance in magnetic resonance imaging: Signal inhomogeneities in samples of high permittivity. J Appl Phys 97:10R303.

Shahin, A., D. J. Bosnyak, L. J. Trainor, and L. E. Roberts. 2003. Enhancement of neuroplastic P2 and N1c auditory evoked potentials in musicians. J Neurosci 23:5545.

Shen, J. F., Y. L. Chao, and L. Du. 2007. Effects of static magnetic fields on the voltagegated potassium channel currents in trigeminal root ganglion neurons. Neurosci Lett 415:164.

Shen, W. W., and J. H. Zhao. 2010. Pulsed electromagnetic fields stimulation affects BMD and local factor production of rats with disuse osteoporosis. Bioelectromagnetics $31: 113$.

Shigemitsu, T. 2006. Electromagnetic fields, biophysical processes, and proposed biophysical mechanisms. In Electromagnetics in Biology, ed. M. Kato, 193. New York: Springer.

Shimizu, K., A. Ito, and H. Honda. 2007. Mag-seeding of rat bone marrow stromal cells into porous hydroxyapatite scaffolds for bone tissue engineering. J Biosci Bioeng 104:171.

Shin, E. J., J. H. Jeong, H. J. Kim, C. G. Jang, K. Yamada, T. Nabeshima, and H. C. Kim. 2007. Exposure to extremely low frequency magnetic fields enhances locomotor activity via activation of dopamine $\mathrm{D}_{1}$-like receptors in mice. J Pharmacol Sci 105:367.

Shine, M. B., K. N. Guruprasad, and A. Anand. 2011. Enhacement of germination, growth, and photosynthesis in soybean by pre-treatment of seeds with magnetic field. Bioelectricmagnetics published online.

Shirota, Y., M. Hamada, R. Hanajima, Y. Terao, H. Matsumoto, S. Ohminami, S. Tsuji, and Y. Ugawa. 2010. Cerebellar dysfunction in progressive supranuclear palsy: A transcranial magnetic stimulation study. Mov Disord 25:2413.

Shupak, N. M., J. C. McKay, W. R. Nielson, G. B. Rollman, F. S. Prato, and A. W. Thomas. 2006. Exposure to a specific pulsed low-frequency magnetic field: A double-blind placebo-controlled study of effects on pain ratings in rheumatoid arthritis and fibromyalgia patients. Pain Res Manag 11:85.

Simkó, M. 2007. Cell type specific redox status is responsible for diverse electromagnetic field effects. Curr Med Chem 14:1141.

Simmons, S. L., D. A. Bazylinski, and K. J. Edwards. 2006. South-seeking magnetotactic bacteria in the Northern Hemisphere. Science 311:371.

Smith, D. T., S. R. Jackson, and C. Rorden. 2009. Repetitive transcranial magnetic stimulation over frontal eye fields disrupts visually cued auditory attention. Brain Stimul 2:81.

So, P. P. M., M. A. Stuchly, and J. A. Nyenhuis. 2004. Peripheral nerve stimulation by gradient switching fields in magnetic resonance imaging. IEEE Trans Biomed Eng 51:1907.

Soda, A., T. Ikehara, Y. Kinouchi, and K. Yoshizaki. 2008. Effect of exposure to an extremely low frequency-electromagnetic field on the cellular collagen with respect to signaling pathways in osteoblast-like cells. J Med Invest 55:267.

Solov'yov, I. A., D. E. Chandler, and K. Schulten. 2007. Magnetic field effects in Arabidopsis thaliana Cryptochrome-1. Biophys J 92:2711. 
Squires, K. C. 1991. Development of a 37-channel SQUID-based magnetometer for study of the brain and heart. Med Biol Eng Comput 29(Suppl):761.

St-Pierre, L. S., A. Mazzuchin, and M. A. Persinger. 2008. Altered blood chemistry and hippocampal histomorphology in adult rats following prenatal exposure to physiologically-patterned, weak (50-500 nanoTesla range) magnetic fields. Int J Radiat Biol 84:325.

Strafella, A. P., J. H. Ko, and O. Monchi. 2006. Therapeutic application of transcranial magnetic stimulation in Parkinson's disease: The contribution of expectation. Neuroimage 31:1666.

Strelczyk, D., M. E. Eichhorn, S. Luedemann, G. Brix, M. Dellian, A. Berghaus, and S. Strieth. 2009. Static magnetic fields impair angiogenesis and growth of solid tumors in vivo. Cancer Biol Ther 8:1756.

Strieth, S., D. Strelczyk, M. E. Eichhorn, M. Dellian, S. Luedemann, J. Griebel, M. Bellemann, A. Berghaus, and G. Brix. 2008. Static magnetic fields induce blood flow decrease and platelet adherence in tumor microvessels. Cancer Biol Ther 7:814.

Sullivan, K., A. K. Balin, and R. G. Allen. 2011. Effects of static magnetic fields on the growth of various types of human cells. Bioelectromagnetics 32:140.

Sun, W., Y. Gan, Y. Fu, D. Lu, and H. Chiang. 2008. An incoherent magnetic field inhibited EGF receptor clustering and phosphorylation induced by a $50 \mathrm{~Hz}$ magnetic field in cultured FL cells. Cell Physiol Biochem 22:507.

Sun, L. Y., D. K. Hsieh, T. C. Yu, H. T. Chiu, S. F. Lu, G. H. Luo, T. K. Kuo, O. K. Lee, and T. W. Chiou. 2009. Effect of pulsed electromagnetic field on the proliferation and differentiation potential of human bone marrow mesenchymal stem cells. Bioelectromagnetics 30:251.

Sutbeyaz, S. T., N. Sezer, F. Koseoglu, and S. Kibar. 2009. Low-frequency pulsed electromagnetic field therapy in fibromyalgia: A randomized, double-blind, shamcontrolled clinical study. Clin J Pain 25:722.

Taki, M., Y. Suzuki, and K. Wake. 2003. Dosimetry considerations in the head and retina for extremely low frequency electric fields. Radiat Prot Dosimetry 106:349.

Tanimoto, Y., C. Jinda, Y. Fujiwara, M. Itoh, K. Hirai, H. Tomioka, R. Nakagaki, and S. Nagakura. 1989. Laser flash photolysis studies of the magnetic effects on the hydrogen abstraction reaction of 2-naphthylphenylcarbenein micellar solution. J Photochem Photobiol A 47:269.

Tanimoto, Y., H. Hayashi, S. Nagakura, H. Sakurai, and K. Tokumaru. 1976. The external magnetic field effect on the singletsensitized photolysis of dibenzoyl peroxide. Chem Phys Lett 41:267.

Taraban, M. B., T. V. Leshina, M. A. Anderson, and C. B. Grissom. 1997. Magnetic field dependence and the role of electron spin in heme enzymes: Horseradish peroxidase. J Am Chem Soc 119:5768.

Tenforde, T. S. 2005. Magnetcally induced electric fields and currents in the circulatory systems. Prog Biophys Mol Biol 87:279.

Thannickal, V. J., and B. L. Fanburg. 2000. Reactive oxygen species in cell signaling. Am J Physiol Lung Cell Mol Physiol 279:L1005.

Thielscher, A., A. Opitz, and M. Windhoff. 2011. Impact of the gyral geometry on the electric field induced by transcranial magnetic stimulation. Neuroimage 54:234. 
Till, U., C. R. Timmel, B. Brocklehurst, and P. J. Hore. 1998. The influence of very small magnetic fields on radical recombination reactions in the limit of slow recombination. Chem Phys Lett 298:87.

Timmel, C. R., U. Till, B. Brocklehurst, K. A. McLauchlan, and P. J. Hore. 1998. Effects of weak magnetic fields on free radical recombination reactions. Mol Phys 95:71.

Tomasi, D. G., and R. Wang. 2007. Induced magnetic field gradients and forces in the human head in MRI. J Magn Reson Imaging 26:1340.

Tomatsu, S., Y. Someya, Y. W. Sung, S. Ogawa, and S. Kakei. 2008. Temporal feature of BOLD responses varies with temporal patterns of movement. Neurosci Res 62:160.

Torbet, J., M. Fryssinet, and G. Hudry-Clergeon. 1981. Oriented fibrin gels formed by polymerization in strong magnetic fields. Nature 289:91.

Torres-Duran, P. V., A. Ferreira-Hermosillo, M. A. Juarez-Oropeza, D. Elias-Viñas, and L. Verdugo-Diaz. 2007. Effects of whole body exposure to extremely low frequency electromagnetic fields (ELF-EMF) on serum and liver lipid levels, in the rat. Lipids Health Dis 6:31.

Tsai, M. T., W. J. Li, R. S. Tuan, and W. H. Chang. 2009. Modulation of osteogenesis in human mesenchymal stem cells by specific pulsed electromagnetic field stimulation. J Orthop Res 27:1169.

Tuch, D. S., V. J. Wedeen, A. M. Dale, J. S. George, and J. W. Belliveau. 2001. Conductivity tensor mapping of the human brain using diffusion tensor MRI. Proc Natl Acad Sci U S A 98:11697.

Ueno, S., and K. Harada. 1986. Experimental difficulties in observing the effects of magnetic fields on biological and chemical processes. IEEE Trans Magn 22:868.

Ueno, S., and K. Iramina. 1991. Modeling and source localization of MEG activities. Brain Topogr 3:151.

Ueno, S., and N. Iriguchi. 1998. Impedance magnetic resonance imaging: A method for imaging of impedance distributions based on magnetic resonance imaging. J Appl Phys 83:6450.

Ueno, S., and M. Iwasaka. 1994a. Parting of water by magnetic fields. IEEE Trans Magn 30:4698.

Ueno, S., and M. Iwasaka. 1994b. Properties of diamagnetic fluid in high-gradient magnetic fields. J Appl Phys 75:7177.

Ueno, S., and M. Iwasaka. 1996a. Catalytic activity of catalase under strong magnetic fields of up to 8 T. J Appl Phys 79:4705.

Ueno, S., and M. Iwasaka. 1996b. Magnetic nerve stimulation and effects of magnetic fields on biological, physical and chemical processes. In Biological Effects of Magnetic and Electromagnetic Fields, ed. S. Ueno, 1. New York: Springer.

Ueno, S., P. Lovsund, and P. A. Oberg. 1986. Effects of time-varying magnetic fields on action potential in lobster giant axon. Med Biol Eng Comput 24:521.

Ueno, S., T. Matsuda, and M. Fujiki. 1989. Localized stimulation of the human cortex by opposing magnetic fields. In Advances in Biomagnetism, ed. S. J. Williamson, M. Hoke, G. Stroink, and M. Kotani, 529. New York: Plenum Press.

Ueno, S., T. Matsuda, and M. Fujiki. 1990. Functional mapping of the human motor cortex obtained by focal and vectorial magnetic stimulation of the brain. IEEE Trans Magn 26:1539. 
Ueno, S., T. Matsuda, and O. Hiwaki. 1990. Localized stimulation of the human brain and spinal cord by a pair of opposing pulsed magnetic fields. J Appl Phys 67:5838.

Ueno, S., T. Matsuda, and O. Hiwaki. 1991. Estimation of structures of neural fibers in the human brain by vectorial magnetic stimulation. IEEE Trans Magn 27:5387.

Ueno, S., S. Matsumoto, K. Harada, and Y. Oomura. 1978. Capacitative stimulatory effect in magnetic stimulation of nerve tissue. IEEE Trans Magn MAG-14:958.

Ueno, S., M. Sekino, and M. Ogiue-Ikeda. 2006. Bioelectricity and Biomagnetism. New York: Wiley. Published online, 2006.

Ueno, S., and T. Shigemitsu. 2007. Biological effects of static magnetic fields. In Handbook of Biological Effects of Electromagnetic Fields: Bioengineering and Biophysical Aspects of Electromagnetic Fields. 3rd ed. ed. F. S. Barnes, and B. Greenebaum, 203. Boca Raton, FL: CRC Press.

Ueno, S., T. Tashiro, and K. Harada. 1988. Localized stimulation of neural tissues in the brain by means of a paired configuration of time-varying magnetic fields. J Appl Phys 64:5862.

Umeno, A., and S. Ueno. 2003. Quantitative analysis of adherent cell orientation influenced by strong magnetic fields. IEEE Trans Nanobioscience 2:26.

van Bergen, C. J., L. Blankevoort, R. J. de Haan, I. N. Sierevelt, D. E. Meuffels, P. R. d'Hooghe, R. Krips, G. van Damme, and C. N. van Dijk. 2009. Pulsed electromagnetic fields after arthroscopic treatment for osteochondral defects of the talus: Double-blind randomized controlled multicenter trial. BMC Musculoskelet Disord 10:83.

van Rongen, E. 2005. International workshop "effects of static magnetic fields relevant to human health" Repporteurs report: Dosimetry and volunteer studies. Prog Biophys Mol Biol 87:329.

van Rongen, E., R. D. Saunders, E. T. van Deventer, and M. H. Repacholi. 2007. Static fields: Biological effects and mechanisms relevant to exposure limits. Health Phys 92:584.

Varró, P., R. Szemerszky, G. Bárdos, and I. Világi. 2009. Changes in synaptic efficacy and seizure susceptibility in rat brain slices following extremely low-frequency electromagnetic field exposure. Bioelectromagnetics 30:631.

Vashisth, A., and S. Nagarajan. 2008. Exposure of seeds to static magnetic field enhances germination and early growth characteristics in chickpea (Cicer arietinum L.). Bioelectromagnetics 29:571.

Vashisth, A., and S. Nagarajan. 2010. Effect on germination and early growth characteristics in sunflower (Helianthus annuus) seeds exposed to static magnetic field. J Plant Physiol 167:149.

Ventura, C., M. Maioli, Y. Asara, D. Santoni, P. Mesirca, D. Remondini, and F. Bersani. 2005. Turning on stem cell cardiogenesis with extremely low frequency magnetic fields. FASEB J 19:155.

Vianale, G., M. Reale, P. Amerio, M. Stefanachi, S. Di Luzio, and R. Muraro. 2008. Extremely low frequency electromagnetic field enhances human keratinocyte cell growth and decreases proinflammatory chemokine production. Br J Dermatol 158:1189.

Vink, C. B., and J. R. Woodward. 2004. Effect of a weak magnetic field on the reaction between neural free radicals in isotropic solution. J Am Chem Soc 126:16730. 
Vrva, J., K. Bette, and M. Burband. 1993. Whole cortex 64 channel SQUID biomagnetometer system. IEEE Trans AS 3:1878.

Walker, J. L., R. Kryscio, J. Smith, A. Pilla, and B. F. Sisken. 2007. Electromagnetic field treatment of nerve crush injury in a rat model: Effect of signal configuration on functional recovery. Bioelectromagnetics 28:256.

Walleczek, J. 1995. Magnetokinetic effects on radical pairs: A paradigm for magnetic field interactions with biological systems at lower than thermal energy. In Electromagnetic Fields: Biological Interactions and Mechanisms, ed. M. Blank. Washington, DC: Am Chem Soc.

Wang, Z., J. C. Lin, W. Mao, W. Liu, M. B. Smith, and C. M. Collins. 2007. SAR and temperature: Simulations and comparison to regulatory limits for MRI. J Magn Reson Imaging 26:437.

Wang, Z., J. C. Lin, J. T. Vaughan, and C. M. Collins. 2008. Consideration of physiological response in numerical models of temperature during MRI of the human head. J Magn Reson Imaging 28:1303.

Wang, K., and T. Ritz. 2006. Zeeman resonances for radical pair reactions in weak static magnetic fields. Mol Phys 104:1649.

Wang, Z., A. Sarje, P. L. Che, and K. J. Yarema. 2009. Moderate strength (0.23-0.28 T) static magnetic fields (SMF) modulate signaling and differentiation in human embryonic cells. BMC Genomics 10:356.

Wang, Z., P. Yang, H. Xu, A. Qian, L. Hu, and P. Shang. 2009. Inhibitory effects of a gradient static magnetic field on normal angiogenesis. Bioelectromagnetics 30:446.

Wang, Z., P. L. Che, J. Du, B. Ha, and K. J. Yarema. 2010. Static magnetic field exposure reproduces cellular effects of the Parkinson's disease drug candidate ZM241385. PLoS One 5:e13883.

Weintraub, M. I., and S. P. Cole. 2004. Pulsed magnetic field therapy in refractory neuropathic pain secondary to peripheral neuropathy: Electrodiagnostic parameterspilot study. Neurorehabil Neural Repair 18:42.

Weintraub, M. I., and S. P. Cole. 2008. A randomized controlled trial of the effects of a combination of static and dynamic magnetic fields on carpal tunnel syndrome. Pain Med 9:493.

Weintraub, M. I., D. N. Herrmann, A. G. Smith, M. M. Backonja, and S. P. Cole. 2009. Pulsed electromagnetic fields to reduce diabetic neuropathic pain and stimulate neuronal repair: A randomized controlled trial. Arch Phys Med Rehabil 90:1102.

Whissell, P. D., and M. A. Persinger. 2007. Developmental effects of perinatal exposure to extremely weak $7 \mathrm{~Hz}$ magnetic fields and nitric oxide modulation in the Wistar albino rat. Int J Dev Neurosci 25:433.

WHO. 2007. Extremely Low Frequency Fields Environmental Health Criteria Monograph No.238. Switzerland: Geneva.

Wiltschko, R., D. Gehring, S. Denzau, O. Güntürkün, and W. Wiltschko. 2010. Interaction of magnetite-based receptors in the beak with the visual system underlying 'fixed direction' responses in birds. Front Zool 7:24.

Winklhofer, M. 2010. Magnetoreception. J R Soc Interface 7:S131.

Winklhofer, M., and J. L. Kirschvink. 2010. A quantitative assessment of torque-transducer models for magnetoreception. J R Soc Interface 7:S273. 
Wood, A. W. 2008. Extremely low frequency (ELF) electric and magnetic field exposure limits: Rationale for basic restrictions used in the development of an Australian standard. Bioelectromagnetics 29:414.

Xu, S., H. Okano, N. Tomita, and Y. Ikada. 2011. Recovery effects of a $180 \mathrm{mT}$ static magnetic field on bone mineral density of osteoporotic lumbar vertebrae in ovariectomized rats. Evid Based Complement Alternat Med 2011:1.

Yamaguchi-Sekino, S., M. Sekino, and S. Ueno. 2011. Biological effects of electromagnetic fields and recently updated safety guidelines for strong static magnetic fields. Magn Reson Med Sci 10:1.

Yang, J. C., S. Y. Lee, C. A. Chen, C. T. Lin, C. C. Chen, and H. M. Huang. 2010. The role of the calmodulin-dependent pathway in static magnetic field induced mechanotransduction. Bioelectromagnetics 31:255.

Ye, S., S. Nosrati, and V. M. Campese. 1997. Nitric oxide (NO) modulates the neurogenic control of blood pressure in rats with chronic renal failure (CRF). J Clin Invest 99:540.

Yokus, B., M. Z. Akdag, S. Dasdag, D. U. Cakir, and M. Kizil. 2008. Extremely low frequency magnetic fields cause oxidative DNA damage in rats. Int J Radiat Biol 84:789.

Yoon, R. S., T. P. DeMonte, K. F. Hasanov, D. B. Jorgenson, and M. L. G. Joy. 2003. Measurement of thoracic current flow in pigs for the study of defibrillation and cardioversion. IEEE Trans Biomed Eng 50:1167.

Yoshii, T., M. Ahmad, and C. Helfrich-Förster. 2009. Cryptochrome mediates lightdependent magnetosensitivity of Drosophila's circadian clock. PLoS Biol 7:e1000086.

Zangaladze, A., C. M. Epstein, S. T. Grafton, and K. Sathian. 1999. Involvement of visual cortex in tactile discrimination of orientation. Nature 401:587.

Zaporozhan, V., and A. Ponomarenko. 2010. Mechanisms of geomagnetic field influence on gene expression using influenza as a model system: Basics of physical epidemiology. Int J Environ Res Public Health 7:938.

Zhadin, M. N. 2001. Review of Russian literature on biological action of DC and lowfrequency AC magnetic fields. Bioelectromagnetics 22:27.

Zhang, X., J. F. Li, Q. J. Wu, B. Li, and J. C. Jiang. 2007. Effects of hypomagnetic field on noradrenergic activities in the brainstem of golden hamster. Bioelectromagnetics 28:155.

Zhang, B., H. Lu, W. Xi, X. Zhou, S. Xu, K. Zhang, J. Jiang, Y. Li, and A. Guo. 2004. Exposure to hypomagnetic field space for multiple generations causes amnesia in Drosophila melanogaster. Neurosci Lett 371:190.

Zhang, Q. M., M. Tokiwa, T. Doi, T. Nakahara, P. W. Chang, N. Nakamura, M. Hori, J. Miyakoshi, and S. Yonei. 2003. Strong static magnetic field and the induction of mutations through elevated production of reactive oxygen species in Escherichia coli soxR. Int J Radiat Biol 79:281.

Zhang, X., X. Liu, L. Pan, and I. Lee. 2010. Magnetic fields at extremely low-frequency $(50 \mathrm{~Hz}, 0.8 \mathrm{mT})$ can induce the uptake of intracellular calcium levels in osteoblasts. Biochem Biophys Res Commun 396:662.

Zhao, W., S. A. Swanson, J. Ye, X. Li, J. M. Shelton, W. Zhang, and G. D. Thomas. 2006. Reactive oxygen species impair sympathetic vasoregulation in skeletal muscle in angiotensin II-dependent hypertension. Hypertension 48:637. 


\section{4}

\section{Interaction of Extremely Low-Frequency Electromagnetic Fields with Biological Systems}

4.1 Introduction

4.2 EMF and Biological Systems

Electrical Properties of Biological

Tissues - Coupling between EMF and

Biological Systems

4.3 Numerical Dosimetry Approaches

Impedance Method - SPFD Method

4.4 Development of Modeling of the Human

Body

4.5 Coupling and Dosimetry of ELF EMF with Biological Systems

ELF Electric Field • ELF Magnetic

Field - Extremely Low-Frequency

Electromagnetic Field • Validation of Methods

Tsukasa Shigemitsu

Kenichi Yamazaki
4.6 Conclusions

Acknowledgments

References.

\subsection{Introduction}

During the last four decades, research on interaction between extremely low-frequency $(50 / 60 \mathrm{~Hz})$ electromagnetic fields (ELF EMF) and biological systems has increased significantly (Kheifets and Shimkhada 2007; McCormick 2007; the Tenforde 1987; WHO 1984, 1989). In addition to biological research, a major research on possible adverse 
effects of ELF EMF on human health has been completed. There are many review publications on the potential biological and human health effects of ELF EMF (NAS 1996; NIEHS 1998; IARC 2002; ICNIRP 2003). Based on the evaluation of biological and human health effect research, the World Health Organization (WHO) carried out the human health assessment of ELF EMF and published a monograph of the Environmental Health Criteria (EHC) for ELF EMF (up to $100 \mathrm{kHz}$ ) in 2007 (WHO 2007a).

In addition to the review of the successful development in evaluation of biological studies and human health effects, the progress of ELF EMF and intermediate frequency (IF) magnetic field studies in Japan will be reviewed from both experimental and engineering perspectives. Otaka (2001) has briefly reviewed Japanese research efforts on ELF EMF (partly including the study of radio frequency EMF). Historically, from the end of the 1970 s to early 1980s, research on biological and engineering issues had begun in several universities and research organizations across Japan. Some research involved studies on the biological effects of ELF electric field on animals and the distribution of the electric fields and induced currents from a high-voltage transmission line in humans.

Additional research revealed interaction mechanisms between humans or animals and ELF EMF (Barnes 1988, 1992, 2007; Foster 2003). In Japan, research on ELF EMF was carried out mainly by two research groups: one from Hokkaido University and the other from the University of Tokushima. In addition to actual measurement of induced currents and electric fields, they used models of the human body represented by simple structures such as cylinders to estimate induced currents and surface electric fields (Chiba et al. 1984; Shimizu, Endo, and Matsumoto 1988a,b). The results of these studies explained the electric field distribution inside and outside the human body exposed to a high-voltage transmission line. For biological studies using mice, rat, and cats, carefully constructed exposure facilities were successfully developed (Kobayashi et al. 1983; Negishi et al. 2008; Shigemitsu et al. 1981, 1993; Yamazaki et al. 2000a). These facilities helped the research group from Hokkaido University make great contributions to animal-related research (Kato et al. 1986, 1989). For instance, one successful research outcome revealed the impact of various polarized magnetic fields on secretion of melatonin in rats (Kato and Shigemitsu 1997). Dosimetric studies in animals exposed to ELF electric fields were conducted jointly by the United States and Japan at the Central Research Institute of Electric Power Industry (CRIEPI) and the University of Tokushima (Takuma et al. 1990).

In recent years, devices such as the household induction heating cooker, radio frequency identification tag readers, and electronic article surveillance tags for theft prevention are all being increasingly used in households. These devices use the magnetic fields of the ELF and IF regions. There have been concerns on the association between the exposure to IF magnetic fields and human health. So far, no basic research data on the biological effects of IF magnetic fields are available (Shigemitsu et al. 2007). Further studies on the biological effects and health risk assessments of IF magnetic field, ranging from tens of kilohertz to about $100 \mathrm{kHz}$, are needed. Based on Japanese traditional research dedicated to the study of ELF EMF and in order to ensure protection from exposure to IF magnetic field, pioneering research on the biological effects of IF magnetic field started in Japan in the early 2000. The CRIEPI developed in vivo and in vitro exposure facilities for the investigation of toxicological and reproductive effects in 
experimental animals (Nakasono et al. 2008; Shigemitsu et al. 2009). In cellular experiments, microorganisms and cells were used to study the mutagenic effect of IF magnetic fields, using newly built in vitro exposure facilities (Fujita et al. 2007, 2010; Miyakoshi et al. 2007).

As biological studies on animals and cells progressed, dosimetric studies on ELF EMF in Japan excelled (partly including IF magnetic fields) (Kato 2006; Takuma et al. 2006; Suzuki and Taki 2005). Dosimetry can be divided into two categories: experimental and numerical. In experimental dosimetry studies, researchers conducted measurements of induced currents inside inhomogeneous saline models (Yamazaki, Kawamoto, and Shigemitsu 1996; Yamazaki et al. 2000b,c). Here, dosimetry is defined as the evaluation of induced electric fields and current densities in phantom models representing human and animal bodies. In Section 4.2, we review dosimetric intricacies about induced electric fields and current densities inside phantom models exposed to ELF EMF. This comprehensive review provides useful information for understanding the historical development of and recent trends in numerical dosimetry.

In addition to the experimental studies involving animals and dosimetry, this chapter will discuss examples of the state-of-the-art calculations illustrating the induced electric fields and current densities inside biological systems and the assessment of exposure to ELF EMF. This chapter introduces readers to numerical dosimetry and computational bioelectromagnetics through a discussion of dosimetric methods involving macro- and microinteractions with human and animal models. In Section 2, we discuss the electrical properties of tissues in relation to electromagnetic dosimetry and the general properties of coupling between EMF and biological systems. In Section 3, a short summary contains several methods of calculation for estimating the induced electric field inside the model. Before reviewing the results, in Section 4 , we present technological advancements using human and animal models. In Section 5, the discussion branches into three subsections: (1) the interactions between biological tissues and ELF electric fields, ELF electric field dosimetry; (2) the interactions between biological tissues and ELF magnetic fields, ELF magnetic field dosimetry; and (3) the interactions between biological tissues and ELF EMF, ELF EMF dosimetry. Within these frameworks, literature citations are discussed, along with valuable examples of the state-of-the-art calculations applying numerical dosimetry in the field of ELF EMF.

\subsection{EMF and Biological Systems}

\subsubsection{Electrical Properties of Biological Tissues}

In order to understand the effects of EMF on biological tissues, it is necessary to determine the magnitude of induced electric fields and induced current densities in the various parts of the object. The method used for determining the magnitude is called dosimetry. Numerical and experimental dosimetries require information pertaining to the electrical properties of the objects.

The electrical properties of body tissues are usually described in terms of electrical conductivity and the relative dielectric constant called permittivity. These two 
parameters depend strongly on the frequency and type of the tissues. The dielectric constant is represented as $\varepsilon(\mathrm{F} / \mathrm{m})$, and the dielectric constant of free space is $\varepsilon_{0}(=8.854 \times$ $\left.10^{-12} \mathrm{~F} / \mathrm{m}\right)$. So, the relative permittivity is defined as $\varepsilon_{\mathrm{r}}=\varepsilon / \varepsilon_{0}$. The electrical conductivity is represented as $\sigma(\mathrm{S} / \mathrm{m})$. The magnetic permeability of free space is $\mu_{0}\left(=4 \pi \times 10^{-7} \mathrm{H} / \mathrm{m}\right)$. The relative magnetic permeability is defined as $\mu_{\mathrm{r}}=\mu / \mu_{0}$. The entities $\varepsilon_{\mathrm{r}}$ and $\mu_{\mathrm{r}}$ are the relative permittivity and the relative magnetic permeability of a particular medium, respectively. At low frequencies, the tissue conductivity is dominant. At high frequencies, the relative permittivity tends to become dominant. The magnetic permeability of body tissues is almost the same as that in free space and does not change with the frequency.

The relative permittivity $\varepsilon_{\mathrm{r}}$ and the electrical conductivity $\sigma$ of tissues change with frequency. There are relaxation characteristic regions allowing frequency dispersion. An overview of the dielectric properties of biological tissues has been presented in terms of their relaxation mechanisms. The dielectric properties of biological tissues have been categorized according to relaxation regions that are related to the sizes of composing cells and ions. For relative permittivity and electrical conductivity, there are three main relaxation regions: $\alpha, \beta$, and $\gamma$ dispersions for the frequencies ranging from a few hertz to a few gigahertz. They occur (1) at lower frequencies (few kilohertz) - an ionic diffusion process in cellular membrane, which allows the dielectric relaxation in the $\alpha$ region and (2) at higher frequencies $(\sim 10 \mathrm{kHz}$ to $\sim 100 \mathrm{MHz})$ - the $\beta$ dispersion region becomes more evident in response to the relaxation from polarization of cellular membranes and organic macromolecules. The $\alpha$ dispersion is believed to result mainly due to the relaxation phenomenon of an ionic diffusion process at cellular membrane. Although the origin of $\beta$ dispersion is less well understood, it stems due to the blockage of polarization in cellular structural components, including cell membranes, which act as barriers to ion flow. The $\beta$ dispersion is also caused by the polarization of organic polymers and proteins. At gigahertz frequencies, the $\gamma$ dispersion is caused by the polarization of water molecules, both free and bound ones, which are common in biological systems.

In the ELF region, human and animal bodies can be considered as conductive material defined by the relative permittivity and the effective conductivity. There are a number of published papers on the electrical properties of living tissues (Gabriel, Gabriel, and Corthout 1996a; Gabriel, Lau, and Gabriel 1996b,c; Gabriel 2005; Gabriel, Peyman, and Grand 2009; Schwan 1985). Gabriel and coworkers studied electrical properties in more than 30 animals and in humans in the frequency range of $10 \mathrm{~Hz}$ to $20 \mathrm{GHz}$, using an automatic swept-frequency network and impedance analyzers (Gabriel, Gabriel, and Corthout 1996a; Gabriel, Lau, and Gabriel 1996b). Although the electrical properties of the tissues have been studied extensively through experiments, studies on the effective conductivity and the relative permittivity are scarce and the results show wide variations. The reason why there is no accurate data available is that the electrode polarization errors affect the results below $1 \mathrm{kHz}$ and this source of errors may influence the permittivity values below $100 \mathrm{~Hz}$ by a factor of 2 or 3 . From the electrical point of view, the biological tissues below $100 \mathrm{~Hz}$ can be treated as resistance. The permittivity will not play a major role, and the induced current inside the body is evaluated only by conductivity. 


\subsubsection{Coupling between EMF and Biological Systems}

As the wavelength of the EMF in the ELF region (several thousands of kilometers) exceeds the size of any biological object, exposure to electric and magnetic fields needs to be considered separately (Durney and Christensen 2000; Furse, Christensen, and Durney 2009). So, in the ELF region, since exposure to electric and magnetic fields needs to be analyzed separately, induced electric fields and induced currents can add vectorially. Figure 4.1 shows the induced current density in a human model in ELF electric and magnetic fields.

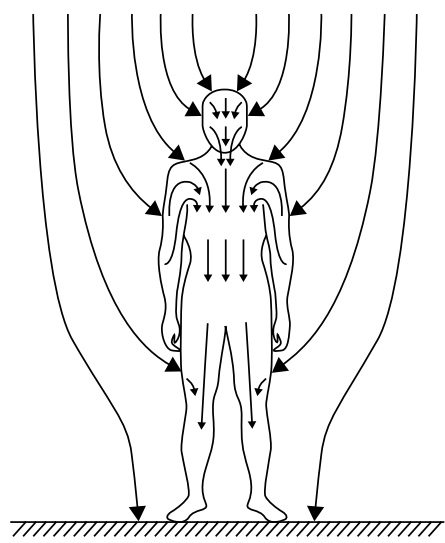

Magnetic field

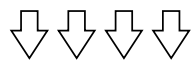

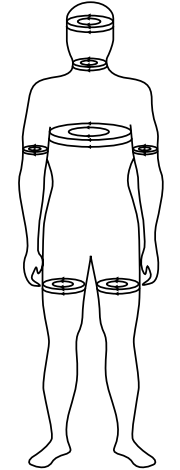

(a) Vertical field

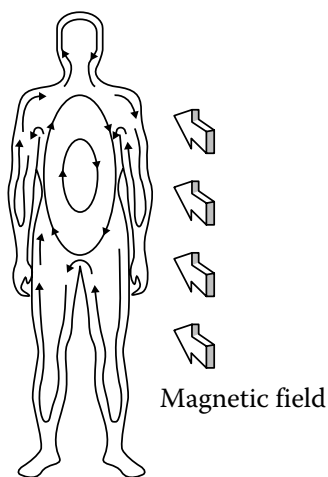

(b) Frontal field

FIGURE 4.1 The induced current density in a human model in extremely low-frequency (ELF) electric and magnetic fields (Reilly p. 346, p. 364, 1998). The figure on top displays perturbations of the electric fields in a person exposed to ELF electric field. Arrows inside the body indicate the direction of intensity of induced internal electric fields and body currents. The bottom figure shows the distributions of internally induced currents in a person exposed to ELF magnetic field. Magnetic field direction is parallel to the body's long axis (lower left) and perpendicular to the front of the body (lower right). 


\subsubsection{Coupling through ELF Electric Fields}

When considering coupling with ELF electric fields, charges are induced on the body surface of an organism. The external electric fields may be disturbed. This disturbance depends on the shape, size, and position of the organism in ELF electric fields. If the human body is in vertical ELF electric fields, electric flux lines enter the body surface perpendicularly and electric currents and electric fields are induced in the conductive human body.

\subsubsection{Coupling through ELF Magnetic Fields}

With regard to coupling of ELF magnetic fields, the magnetic permeability $(\mu)$ of humans and animals is equal to the permeability of free space $\left(\mu_{0}\right)$, and organisms do not disturb the magnetic field. However, electric field is induced by magnetic field based on Faraday's law. As a result, an electric current is induced in organisms exposed to ELF magnetic fields. This induced current flows primarily in a circular path that is perpendicular to the direction of applied ELF magnetic field. In the case of homogeneous materials, the induced current densities are proportional to the radius of the loop, conductivity, frequency, and magnetic flux density. If the magnetic field is oriented from front to back, the largest current densities can be obtained in the largest loop (Figure 4.1).

To summarize, there are major differences in the direction and strength of induced currents inside an organism in relation to ELF electric and magnetic fields. In electric field, the induction between high voltage of object and organisms causes current flow. Vertical electric field on the surface of the organisms situated beneath a transmission line triggers dominant current flow in vertical direction. The current will be greater in a grounded human body under a transmission line than in an ungrounded one. The electric currents induced by magnetic field form closed loops and are called "eddy currents." These eddy currents are zero at the center of the plane perpendicular to the applied magnetic field and grow larger as distance from the center increases.

\subsection{Numerical Dosimetry Approaches}

The state-of-the-art method for numerical computation of EMF inside the human and animal models involves solving the Maxwell equation in anatomical, high-resolution, realistic models based on magnetic resonance imaging (MRI) and computed tomography (CT). Due to the development of computational resources and techniques, numerical simulations have been successfully applied to problems in the field of electromagnetic phenomena including bioelectromagnetics.

Frequently used numerical calculation methods for internal dosimetry in the ELF region are the impedance method (IM) (Orcutt and Gandhi 1988) and scalar potential finite difference (SPFD) method (Dawson and Stuchly 1996). These methods are applied to computational human models constructed by voxels (cubic cells). For calculations, quasistatic approximation is adopted since (1) the conducted current is dominant over the displacement current for the human exposure to magnetic field of frequencies below about $10 \mathrm{MHz}$ and (2) the wavelength of the field is long relative to the human body's dimension. Additional methods that have been applied to bioelectromagnetic 
problems are the quasistatic finite-difference time domain (FDTD) method (Dawson and Stuchly 1996; Hirata et al. 2001; Gustrau et al. 1999), the finite-element method (FEM) (Baraton and Hutlzer 1995), the charge simulation method (CSM) (Yamazaki et al. 2000b), and the surface charge method (Hamada and Kobayashi 2006a; Yamazaki et al. 2000c).

\subsubsection{Impedance Method}

The IM solves circuit network equations involving a human hosting impedance networks (Orcutt and Gandhi 1988). This method is illustrated in the following example (modeling a human). First, a human model is modeled by cubic voxels. The conductivities $(\sigma)$ representing the corresponding tissues are assigned to each voxel. The impedances $(\mathrm{Z})$ and the loop currents (I) are defined at the sides and at the surfaces of the voxels, respectively (Figure 4.2). Here, the impedance $Z$ is a mean value of the impedances of four voxels sharing the same side.

A voltage is induced in each loop by a time-varying magnetic field according to Faraday's law. The voltage induced in a loop of the surface of a voxel ( $x-y$ plane) is represented by

$$
V=-j \omega B_{n} \Delta x \Delta y
$$

where $V$ is induced voltage, $\Delta x \Delta y$ is the length of the side of voxel, $B_{n}$ is the magnetic field component perpendicular to the surface, and $\omega$ is the angular frequency $(=2 \pi f$, where $f$ is the frequency). Using the above-defined impedances and loop currents (unknowns), closed-loop equations are generated. These equations are solved simultaneously to obtain loop currents. The successive overrelaxation (SOR) method is often used to solve the equations. The four obtained loop currents sharing the same side are subsequently added to obtain the total current on each side. The current defined in the center of each voxel can be obtained by averaging the four side currents having the same directions. Then, the current density is obtained by dividing it by the area of the crosssection of the voxel.

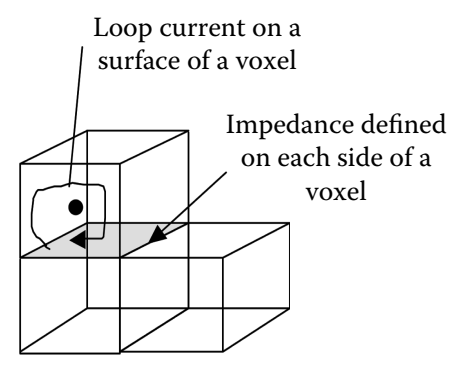

FIGURE 4.2 Voxels and related parameters defined in the impedance method. 


\subsubsection{SPFD Method}

The SPFD method is a finite-difference method that finds electric scalar potentials at nodes (usually apexes) of voxels, which represent human tissues. Following is an example of the application of the method to a human model.

In the ELF region, the secondary magnetic field generated by magnetically induced current in a human body can be ignored since the conductivity of tissues is low and the skin depth is large compared with the size of the human body. Based on the Maxwell equation, the basic equations representing human induced current are

$$
\begin{gathered}
\nabla \times \mathbf{E}=-j \omega \boldsymbol{B}_{0}(\text { from the Maxwell equation }) \\
\nabla \cdot \mathbf{J}=0(\text { current continuity equation }) \\
\mathbf{J}=\sigma \mathbf{E}(\text { Ohm's law })
\end{gathered}
$$

where $\mathbf{E}$ is the intensity of the induced electric field, $\boldsymbol{B}_{0}$ is outer magnetic flux density, $\boldsymbol{\omega}$ is angular frequency, $\sigma$ is conductivity of tissue, and $\mathbf{J}$ is induced current density. The term of displacement current is ignored, and the time derivatives are replaced by $j \omega$.

An outer magnetic vector potential $\boldsymbol{A}_{0}$, which relates to $\boldsymbol{B}_{0}$ as $\boldsymbol{B}_{0}=\nabla \times \boldsymbol{A}_{0}$, is applied to Equation 4.2 and then Equation 4.5 is derived.

$$
\mathrm{E}=-j \omega \mathbf{A}_{0}-\nabla \varphi
$$

where $\varphi$ is the electric scalar potential. By substituting Equations 4.4 and 4.5 into Equation 4.3, Equation 4.6 can be derived.

$$
-\nabla \cdot(\nabla \sigma \varphi)=\nabla \cdot\left(j \omega \sigma \mathbf{A}_{0}\right)
$$

The boundary condition is shown in Equation 4.7, considering that the normal component of induced current on the boundary surface between human and air is zero $\left(\boldsymbol{J}^{\circ} \boldsymbol{n}=0\right)$.

$$
-\mathbf{n} \cdot \nabla \varphi=j \omega \sigma \mathbf{A}_{0} \cdot \mathbf{n}
$$

The electric scalar potential in Equation 4.6 is to be solved using the boundary condition specified in Equation 4.7. Equation 4.7 is to be discretized at a point that eight voxels share. When discretizing Equation 4.6 at point 0 in Figure 4.3,

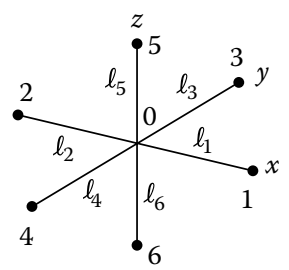

FIGURE 4.3 Points (apexes) and sides of voxels used in the scalar potential finite-difference method. 


$$
\sum_{n=1}^{6} s_{n} \varphi_{n}-\left(\sum_{n=1}^{6} s_{n}\right) \varphi_{0}=j \omega \sum_{n=1}^{6}(-1)^{n} s_{n} \lambda_{n} A_{0 n}
$$

where $\varphi_{n}$ is the electric scalar potential at point $n, A_{0 n}$ is the component of outer vector potential parallel to the side of a voxel that connects points 0 and $n$ at the center of the side, $\ell_{n}$ is the length of side $n$, and $s_{n}$ is the conductance of the side $n . s_{n}$ is expressed as

$$
s_{n}=\bar{\sigma}_{n} a_{n} / \lambda_{n}
$$

where $a_{n}$ is the area of voxel perpendicular to side $n$ and $\bar{\sigma}_{n}$ is the mean value of conductivity of four voxels that share the side. The procedure to calculate the electric field at the center of each voxel is as follows. First-order simultaneous equations whose unknowns are the electric scalar potentials $\varphi_{n}$ are created according to Equation 4.8 for all apexes of voxels corresponding to human tissues. Then, the equations are solved simultaneously to obtain the electric scalar potentials $\varphi_{n}$. To solve the equations, the SOR method and the IM are applied. The electric fields on each side of the voxels are calculated using Equation 4.5. The parameters $x$ and $y$, and components of the electric field at the center of each voxel represent the average of the four electric fields on the sides parallel to each axis. The current density is obtained by dividing it by the area of the voxel's cross-section.

\subsection{Development of Modeling of the Human Body}

Human health research is the driving force behind the development of electromagnetic dosimetry (Bracken 1992; Durney 1980; Guy 1987; Kaune 1992; Tenforde 1992). The human model has been gradually developed, thanks to the development of computer technology. Barnes, McElory, and Charkow (1967) published the first paper on dosimetry, which models human and animal as prolate and oblate spheroidal bodies. They calculated the surface electric fields and induced current densities in the model using the Laplace equation. Since the human body is not electrically homogeneous, the calculation of the induced current densities inside the human body can be performed with help of an anatomically and electrically realistic human body model and through several computational techniques.

In the 1970s, simple prolate, spheroidal, cylindrical human and animal models containing homogeneous tissue with a single conductivity were developed and used for calculations to estimate the electric fields and induced currents when the models were exposed to ELF electric fields from transmission lines. During the 1980s, ELF electric field and magnetic field dosimetry emerged, based on more realistic models in terms of homogeneity related to the anatomical structure of human beings. Many studies on analytical solutions concerning homogeneous shapes in the context of electric field exposure were published (Chen, Chuang, and Lin 1986; Chiba et al. 1984; Deno 1977; Dimbylow 1987; Hart 1992a; Kaune and Forsythe 1985; Kaune and McCreary 1985; Kaune, Kistler, and Miller 1987; Kaune and Forsythe 1988; Kobayashi, Shimizu, and Matsumoto 1987; Polk 1990a, 1992; Polk and Song 1990b; Shiau and Valentino 1981; Shimizu, Endo, and Matsumoto 1988a; Spiegel 1977a, 1981). Figure 4.4 reviews the essential characteristics 

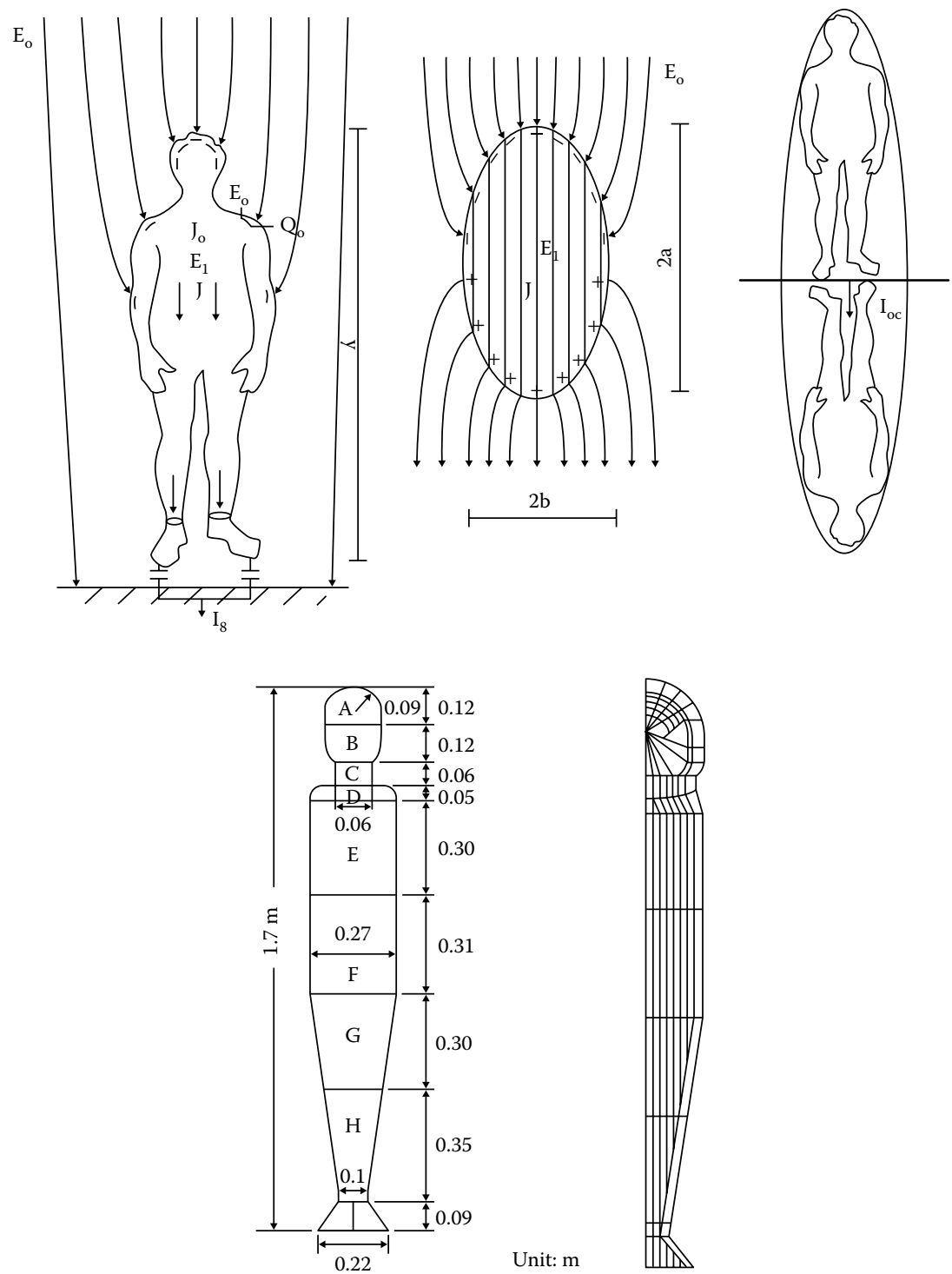

FIGURE 4.4 Examples of the human model in vertical homogeneous electric field. The three figures at the top: (left) realistically shaped model coupled to the ground; (middle) prolate spheroid model consisting of homogeneous human equivalent material; (right) prolate spheroid model short circuited to the ground. (From ICNIRP., ICNIRP., 2003. With permission.) The two figures at the bottom: (left) the axisymmetric human body model separated into nine parts from top to bottom as A, B, C, D, E, F, G, H, and I; (right) division patterns inside and at the vicinity of the axisymmetric human body model for the finite-element analysis. (Courtesy of K. Isaka, A. Chiba, and W. T. Kaune 1998.) 
of the models that are used in experimental and numerical dosimetries in the ELF electric field. The frequently used axisymmetric human body model represents the features of the body, although not perfectly.

After the 1990s, exceedingly realistic and heterogeneous models of the human body with small volume, block, and voxel (volumetric pixel) were developed as MRI technology emerged. Subsequently, MRI and CT scanners allowed researchers to create human models at millimeter-resolution level (Dawson, de Moerloose, and Stuchly 1996; Dawson, Moerloose, and Stuchly 1997a; Dawson, Potter, and Stuchly 2001b; Dawson 1997; Gandhi and Chen 1992; Gandhi 1995). These newly developed models were posed in virtually any position. These human models separate body parts into small volume, block, and voxel of different conductivities. Heterogeneous models consisted of over 30 distinct human organs and tissues. The size of the voxel ranged from 1 to $10 \mathrm{~mm}$ on a side. The conductivities of various organs were assigned the measured values. Data on dielectric properties of tissues (conductivity and relative permittivity) for use in electromagnetic dosimetry were established by measurement by Gabriel, Gabriel, and Corthout (1996a) and Gabriel, Lau, and Gabriel $(1996 \mathrm{~b}, \mathrm{c})$. The conductivity data of organs and tissues at frequencies below $1 \mathrm{MHz}$ have been characterized by Gabriel (2005) and Gabriel, Peyman, and Grand (2009). Gabriel, Lau, and Gabriel (1996b) stated that "it is possible that the dielectric parameters below $1 \mathrm{kHz}$ may be under-corrected. This source of errors may affect the permittivity values below $100 \mathrm{~Hz}$ by up to a factor of two or three." Despite this issue, the dielectric parameters of the biological tissues in the ELF region are generally used in the calculation. It is well known that the individual variability of the dielectric parameters of biological tissues at low frequencies is greater than those at higher frequencies.

The human models for ELF magnetic field exposures have been developed at several universities and organizations. Table 4.1 gives the characteristics of the different MRIbased male and female human models, and Figure 4.5 shows the visualized realistic whole-body male and female human models obtained from the four different organizations. Historically, the University of Utah (USA) (Gandhi and Chen 1992), the University of Victoria (Canada) (Xi, Stuchly, and Gandhi 1994a; Xi and Stuchly 1994b; Dawson, de Moerloose, and Stuchly 1996; Dawson and Stuchly 1996), and the National Radiological Protection Board (NRPB; now, HPA) (UK) (Dimbylow 1998) have developed human models. The Brooks Air Force Base (USA) has developed a male rhesus monkey model and used it for specific absorption rate (SAR) dosimetry calculations (Mason et al. 1999). The National Institute of Information and Communication Technology (NICT, Japan) and the Foundation for Research on Information Technologies in Society (IT'IS, Switzerland) have also developed MRI-based and computer-aided-design (CAD)-based human models (Table 4.1). The University of Victoria developed a phantom model based on MRI scan data (Dawson, Caputa, and Stuchly 1997c). This phantom model was developed from a head and torso model by the Yale Medical School (Zubal et al. 1994). It consisted of 30 organs and tissues, and cubic voxels of $3.6 \times 3.6 \times 3.6 \mathrm{~mm}^{3}$. This model was $1.77 \mathrm{~m}$ in height and weighed $76 \mathrm{~kg}$. Concerning heterogeneous human models, the University of Utah developed a phantom model based on MRI scan data (Furse and Gandhi 1998; Gandhi and Chen 1992). Gandhi and Chen (1992) created a whole-body model at a $2 \times 2 \times 3 \mathrm{~mm}^{3}$ resolution based on MRI scans of an adult male subject who was $1.76 \mathrm{~m}$ tall and weighed $64 \mathrm{~kg}$. The model was scaled up to $71 \mathrm{~kg}$ and segmented into 30 different tissue types. 


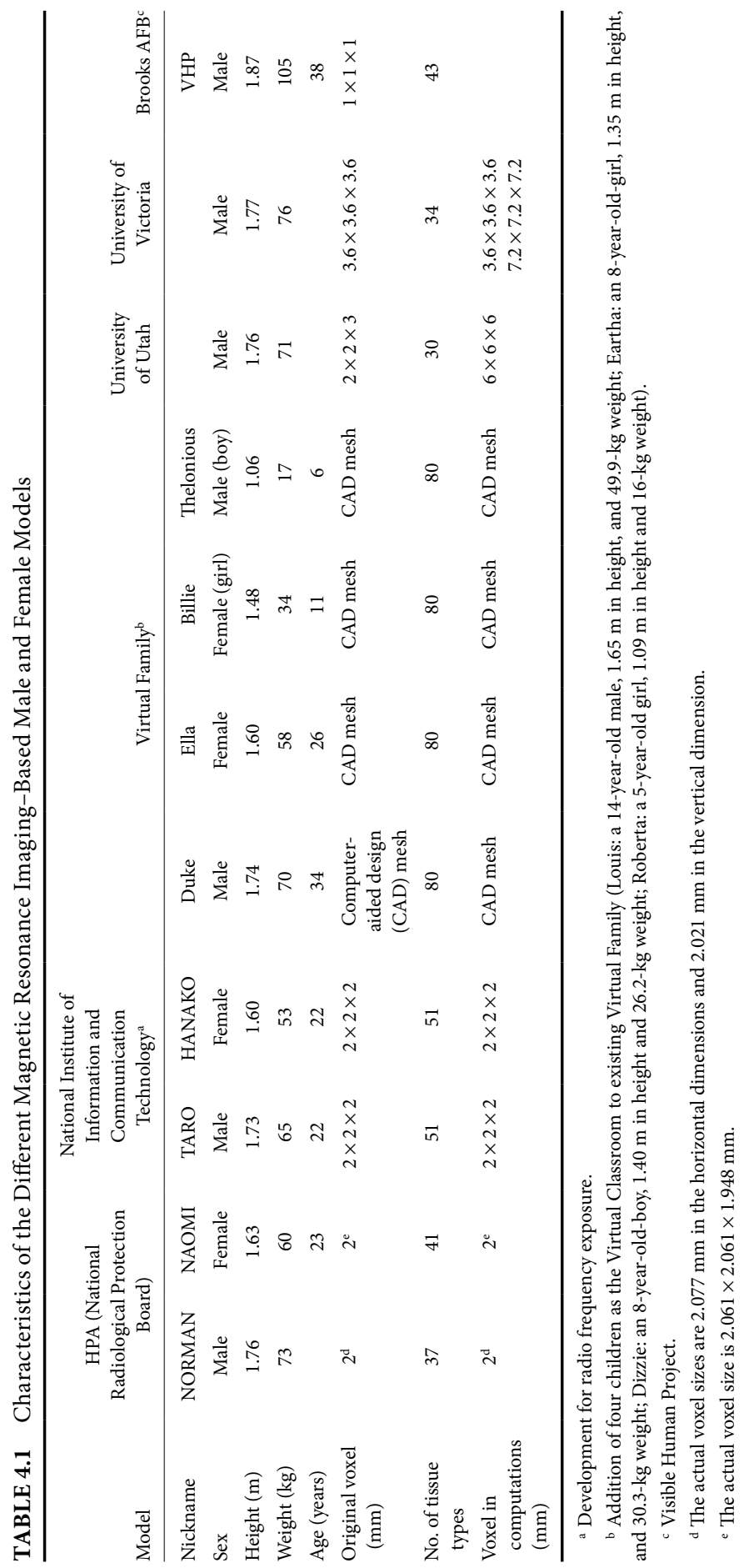



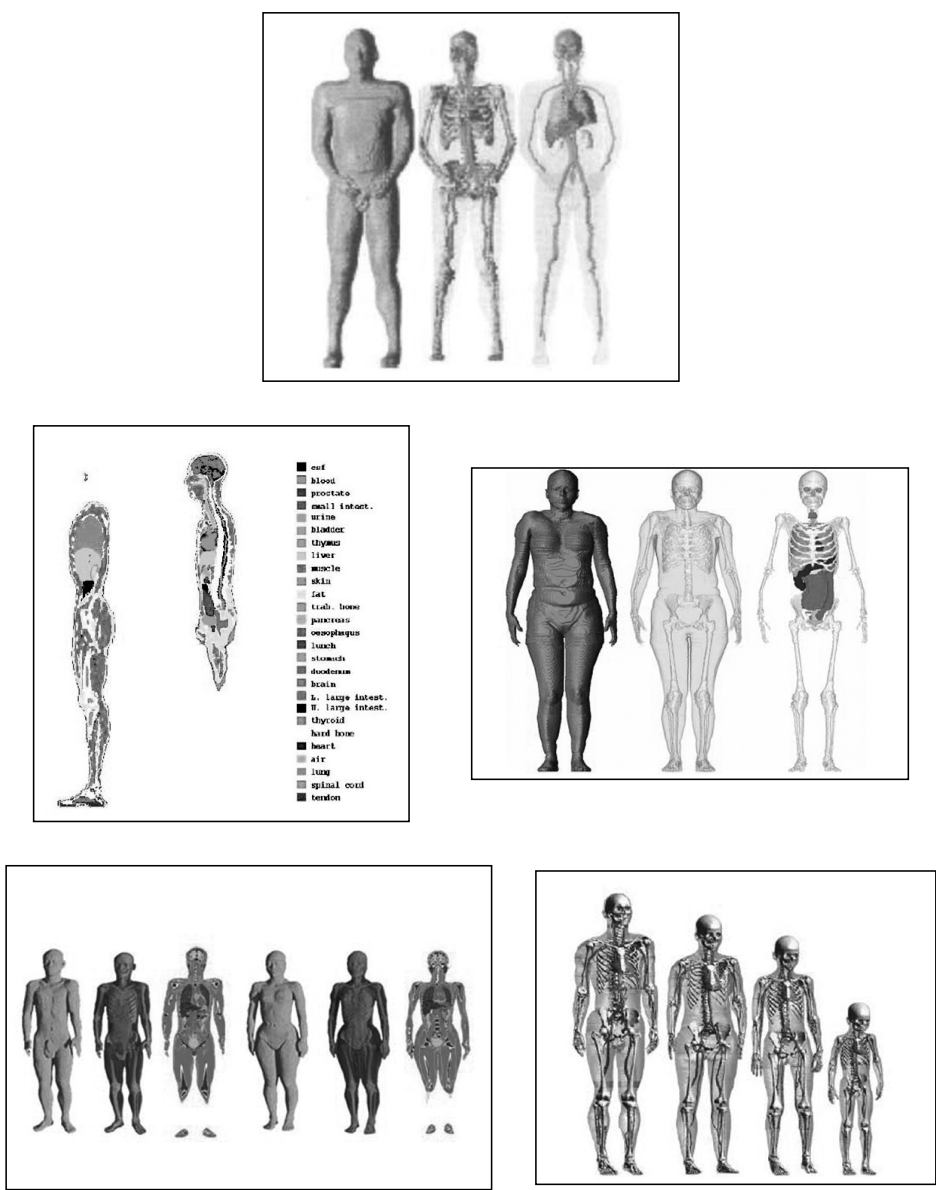

FIGURE 4.5 (See color insert.) The visualized realistic phantom human models. The top figure: the external view, skeleton, major vessels, and organs of the three-dimensional anatomical body phantom model developed by the University of Victoria. (From Stuchly, M. A., and T. W. Dawson. 2000. Interaction of low-frequency electric and magnetic fields with the human body. Proc IEEE 88:641-64. With permission.) The middle two figures: high-resolution three-dimensional image of a male body model called NORMAN (left). The body is divided into over 8 million 2 -mm voxels. The tissue type of each voxel is defined (http://www.hpa.org.uk/Topics/Radiation/ UnderstandingRadiation/InformationSheets/info_EmfModelling/); volume-rendered images of NAOMI (right). (From Dimbylow, P. J. 2005. Development of the female voxel phantom, NAOMI, and its application to calculations of induced current densities and electric fields from applied low frequency magnetic and electric fields. Phys Med Biol 50:1047-70. With permission.) The bottom two figures: the orthogonal section display and bone enhancement image for both Japanese male and female models (left) (courtesy of T. Nagaoka, NICT) and the high-resolution whole-body human models of the Virtual Family developed by the Foundation for Research on Information Technologies in Society (right). (From Christ, A. et al. 2010. The Virtual Family-development of anatomical CAD models of two adults and two children for dosimetric stimulations. Phys Med Biol 55:N23-38. With permission.) 
Dimbylow $(1998,2000)$ has developed a male phantom model called NORMAN at NRPB. Its name is derived from NORmalised MAN. The resolution of that model is $2.077 \times 2.077 \times 2.021 \mathrm{~mm}^{3}$, which is cubical in shape. This model was based on MRI scans of an adult male subject and was segmented into 37 different types. This phantom was standardized at a height of $1.76 \mathrm{~m}$ and weight of $73 \mathrm{~kg}$. NORMAN consisted of a threedimensional, parallel pipe-shaped array of 36 million homogeneous voxels. There were 8.3 million voxels in the body, and the remaining voxels were located in the surrounding. Besides the male phantom model, a female phantom model was also developed. Dimbylow $(2005,2006)$ developed a female phantom model called NAOMI (aNAtOMIcal model) based on a 23 -year-old female subject who was $1.65 \mathrm{~m}$ tall and weighed $58 \mathrm{~kg}$. The resolution was $2 \times 2 \times 2 \mathrm{~mm}^{3}$ voxels and the model was segmented into 41 tissue types. A threedimensional human model based on the photographic data from the Visible Human Project was created by the National Library of Medicine (Ackerman 1998; The Visible Human Project 2009). The original data of the Visible Human Project were obtained from a 38-year-old male cadaver (1.86-m height and 90-kg weight).

Nagaoka et al. $(2002,2004)$ developed realistic, high-resolution, whole-body voxel models of a standing Japanese adult male and female, which were conducive to the dosimetry of radiofrequency EMF. Based on MRI data, these models consisted of cubic voxels of $2 \mathrm{~mm}$ on each side and were segmented into 51 anatomic regions. The male was named TARO (22-year-old, $1.73 \mathrm{~m}$ in height, and a weight of $65.0 \mathrm{~kg}$ ) and the female was named HANAKO (22-year-old, $1.6 \mathrm{~m}$ in height, and a weight of $53.0 \mathrm{~kg}$ ). The MRI $256 \times 256$ axial Pixels were set with a $240-\mathrm{mm}$ field of view (FOV) for the head and 480-mm FOV for other parts of the body. In addition, they developed postured human models based on anatomically realistic voxel models with a standing posture (Nagaoka and Watanabe 2008). Although these models were originally developed for electromagnetic dosimetry in the RF region, the calculation of induced current densities in the ELF region is presented using this whole-body numeric model and a quasistatic FDTD method (Hirata and Fujiwara 2007). This whole-body model is freely available to the scientific community through NICT. As the Virtual Family, the anatomically correct whole-body human models of an adult male, an adult female, and two children were developed for numerical evaluation of electromagnetic exposure (Christ et al. 2010). These Virtual Family models are based on high-resolution MRIs of healthy volunteers and CAD-based three-dimensional models. The family is comprised of an adult man, Duke (34 year old, $1.74 \mathrm{~m}$ in height), an adult woman, Ella (26 year old, $1.6 \mathrm{~m}$ in height), an 11-year-old girl Billie (1.48 $\mathrm{m}$ in height), and a 6 -year-old boy Thelonious ( $1.07 \mathrm{~m}$ in height). The segmentation consists of more than 80 different tissues and organs. The voxel size is in the range of 1 to $5 \mathrm{~mm}^{3}$. All four models from the Virtual Family are also freely available to the scientific community through IT'IS and are being widely applied in several studies on EMF exposure (IT'IS 2010). In addition to the basic models of the Virtual Family, four models were built as the Virtual Classroom.

The refinement of human models over time is a function of the evolution of computer technology, including computer memory, computational time, and computer graphic techniques. Subsequently, numerical methods have been developed to devise human body models based on millimeter-resolution anatomy, using MRI scan data, allowing for the determination of the induced electric fields and current densities attributed to the exposure to ELF EMF. 


\subsection{Coupling and Dosimetry of ELF EMF with Biological Systems}

The dielectric property of tissues, the basic concept of electromagnetic coupling of ELF EMF and biological systems, and several calculation methods and models were briefly introduced in the previous three sections. These aspects are very important for both experimental and numerical dosimetries concerning the estimation of induced currents and internal electric fields inside biological systems, human beings in particular. In this section, the scientific evolution of ELF EMF dosimetry and its application to the human body model are reviewed.

The homogeneous human (or animal) body with a single conductivity was modeled in ELF EMF. The dosimetry involves the coupling between ELF EMF and biological systems and the measurement or calculation of the internal induced currents and electric fields inside the biological systems during and after exposures. In previous dosimetric studies, simple human and animal models were used to calculate the amount of induced electrical quantities. In recent years, advances in computer technology, along with the development of hardware and commercially available software, have made it possible to perform calculations using complex models. Simple geometric models such as the spherical, rotating ellipsoid, oblong, and cylindrical models approximate the human body form; the induced current distributions in the body can be calculated analytically. Information on internal electric field strengths or induced current densities inside the biological systems has been obtained through experiments using a scaled human model or a full-size mannequin. At the ELF region, the electric and magnetic fields can be supposed to be decoupled. So, it is possible to study and compute independently the induced electrical quantities inside the human body.

\subsubsection{ELF Electric Field}

Several steps are involved in the development of dosimetry of the electric field effects. First, the analytical method for estimating the electric fields and body surface charge densities is applied using a simple human model. At the intermediate stage, an anatomically more realistic human model is used for the computation of induced electric fields and induced currents. From the end of the 1990s, computation has been based on MRIbased high-resolution human models with different organ conductivities.

The electric field is disturbed on the surface of a conductive object due to nearby human beings, trees, houses, etc. So, the value of the exposure electric field, called unperturbed electric field, is used as the field strength before the introduction of such objects. In the case of ELF electric field dosimetry, it has been assumed that the electric fields are uniform and are vertically directed to the object such as standing under a transmission line. Depending on the direction of the object to the applied electric field and its strength, the electric fields and induced current densities vary.

\subsubsection{Coupling with Simple and Analytical Models}

Quantification of the electric fields and induced currents in the human body when exposed to transmission lines has been a very important issue in human health assessment. Between 1970 and 1980, dosimetric studies in the ELF region assessed the human 
exposure to electric fields generated by a transmission line (Deno 1977). Deno (1977) presented induced currents in an anatomically detailed human model and developed a simple measuring technique for the external surface electric fields on the human body and human body model standing under the transmission lines. The human body was assumed to act as a conductor at $50 / 60 \mathrm{~Hz}$. It means that the objects like humans and animals perturb electric fields in the vicinity of the transmission lines. This distortion has the enhancement effect of the electric fields in the context of the object's outer surface and the attenuation effect of the electric fields inside the object. The charge distributions and electric fields on the surface of the object are used for assessing the currents and the electric fields inside the body. Deno (1977) also showed that the short-circuit currents (current to ground) induced in the human body are proportional to the square of height of the mannequin model. If the human body is $1.8 \mathrm{~m}$ in height and stands on the ground under the vertical electric fields, short-circuit currents are about $17.5 \mu \mathrm{A} /(\mathrm{kV} / \mathrm{m})$. From the field measurement, Deno (1979) estimated the current densities induced in various parts of the grounded human body under uniform electric fields. The main conclusions are (1) current densities inside the 1.78-m-tall human body standing in an unperturbed electric field of $1 \mathrm{kV} / \mathrm{m}$ are in the range of 0.427 to $3.55 \mathrm{~mA} / \mathrm{m}^{2}$ and (2) the electric fields on the surface are enhanced compared with the unperturbed electric fields and this enhancement factor is 18.3 at the top of the head.

The first approach to the numerical estimation of internal electric fields and current densities in humans was taken using simple models such as spheres and prolate and oblate ellipsoids. The results of these investigations have been reviewed (Kaune and Phillips 1985). Actual measurements have been performed for the short-circuit currents in animal and human models exposed to uniform electric fields. It is worth noting that between 1970 and 1980, many analytical methods addressed experimental and numerical dosimetries through specific simple geometrical models.

As for experimental dosimetry, measurements were limited for only short-circuit currents through body cross-sections (Kaune and Forsyth 1985; Kaune, Kistler, and Miller 1987). Kaune and Forsythe (1985) measured current densities in a homogeneous mannequin model standing on the ground, which was subject to a vertical $60 \mathrm{~Hz}, 10 \mathrm{kV} / \mathrm{m}$ electric fields. For comparison, researchers evaluated the measured current densities induced in a homogeneous human model on the ground versus the measured current densities induced in a grounded hemispheroidal model. The comparison between these two models was positive. It means that the dosimetric evaluation of induced fields and current densities in the human body depends on mathematical modeling. Then, Kaune, Kistler, and Miller (1987) extrapolated the data associated with grounding to the one related to ungrounded exposure conditions.

A number of analytical studies have been reported using a dielectric spherical model. The geometric shapes of many living things such as humans and animals (rat, mice, and baboons) do not represent spherical shapes. Researchers considered ELF electric fields coupling to prolate and oblate dielectric spheroidal models. Researchers used simplistic human models such as prolate dielectric spheroidal objects (Lattarulo and Mastronardi 1981; Shiau and Valentino 1981), circular cylinders (Kaune and McCreary 1985), and simplified body-like shapes in further analysis (Spiegel 1981; Chen, Chuang, and Lin 1986; Dimbylow 1987). Shiau and Valentino (1981) studied the coupling between ELF electric 
fields and biological objects. The object was a dielectric spheroidal model inside two nonspherical spheroids: a prolate dielectric spheroid and an oblate dielectric spheroid. This simple expression was presented for the internal electric field strengths when the dielectric spheroids are exposed to uniform ELF electric fields. The induced internal electric fields, current densities, and absorbed internal powers are calculated for various geometric shapes of the prolate dielectric spheroid and the oblate dielectric spheroid. Their numerical results are illustrated through graphics for various geometric shapes of spheroids. It shows that the relative position of the spheroid with respect to the orientation of the applied electric fields is the extremely important parameter that affects the electric field coupling considerably. In order to clarify the electric field coupling to human beings, Spiegel (1977b) developed a numerical method for predicting currents and normal electric field distributions induced in humans situated in the vicinity of transmission lines. His technique is based on the method of moment (MoM) in which the human body is modeled as a collection of straight cylindrical segments. Its lengths and radii are comparable to the section of the body modeled. Three scenarios are especially considered: a person wearing an insulating outfit standing on the ground beneath transmission lines, an individual having solid ground contact, and a lineman working very close to an energized conductor. DiPlacido, Shih, and Ware (1978) used a CSM to calculate induced currents in grounded and ungrounded human models consisting of 36 spheres. They also presented a theoretical analysis of the proximity effect of operator on electric field meter performance.

Numerical techniques used for mathematical models range from solution of an integral equation by the MoM (Spiegel 1981), the FEM (Chiba et al. 1984; Chiba and Isaka 1997, 1999, 2000; Chiba, Isaka, and Kaune 1998; Isaka et al. 1987), and the Finite Difference Method (FDM) (Chen, Chuang, and Lin 1986; Hart 1990, 1992a; Dimbylow 1987, 1988). In order to investigate the biological effect of $60 \mathrm{~Hz}$ electric fields on experimental animals, Spiegel (1981) developed human and baboon models by using a large number of small cubical blocks. This array of small cubical blocks was arranged to best fit to the object's contour, and to calculate the internal current densities attributed to the grounded and ungrounded models using integral equations. Chen, Chuang, and Lin (1986) developed a numerical method based on a surface-charge integral equation (SCIE) that can be applied to a realistically shaped phantom. This method yielded a mere average local current density. Their research was to quantify the interaction of ELF-LF electric fields with a human body of realistic and homogeneous shape and arbitrary posture, standing in a realistic environment. This numerical method based on an SCIE can calculate the induced electric field on the body surface and inside the body, the induced body current, and the effect of the grounding impedance. Their calculation also applied to the model using guinea pigs. Later on, Chuang and Chen (1989) applied the SCIE method to a three-dimensional heterogeneous biological body. This numerical method based on the combination of a pair of SCIE and an impedance network method was applied to calculate the internal electric fields and currents inside three-dimensional heterogeneous biological bodies with arbitrary grounding impedance induced by ELF-LF electric fields. They confirmed the validity of the method by comparing numerical results and analytical solutions involving conducting concentric spheres.

Kaune and McCreary (1985) used a combined charge-simulation/finite-difference approach to model humans and animals exposed to vertical uniform $60 \mathrm{~Hz}$ electric fields. 
They also developed a detailed model in order to characterize the induced current densities in a homogeneous circular cylinder. They compared the short-circuit currents and induced current density distributions. Based on experiments, Kaune and coworkers examined the electrical interaction between an animal and vertical uniform $60 \mathrm{~Hz}$ electric fields (Kaune and Philips 1980; Kaune and Gillis 1981; Kaune 1981a,b; Kaune and Miller 1984). They determined the electric field strengths at the surface of the body and induced current densities inside the body. They concluded that the electric fields on the surface, as well as the electric fields and current densities inside the body, are all strongly dependent on body shape and its orientation to the electric fields and ground plane. They emphasized that body shape has a major influence on the field experienced by humans or animals exposed to uniform $60 \mathrm{~Hz}$ electric fields. Kaune and Phillips (1980) demonstrated the enhancement of the surface electric fields in three species in a vertical uniform $10 \mathrm{kV} / \mathrm{m}$ electric field. The field at the top of a human, miniature swine, and rat would be about 180,67 , and $37 \mathrm{kV} / \mathrm{m}$, respectively, due to the enhancement effect. The authors summarized the result of electrostatically induced electric fields in miniature swine, rat, and human models exposed to vertical $60 \mathrm{~Hz}$ electric fields. The parameters in that context were total currents (shortcircuit currents), the surface electric fields, and induced currents.

Guy et al. (1982) applied the thermographic method to determine the SAR in animal and human model tissues to help quantify the current distributions in homogeneous bodies of arbitrary shape exposed to $60 \mathrm{~Hz}$ electric fields. They measured SAR for energy deposited in models, applying $57 \mathrm{MHz}$ electric fields generated from resonant cavity. Hart et al. (1989) used a spreadsheet program to calculate the electric fields and current densities produced inside irregular shaped models representing the upper arm and forearm. They calculated the current density distributions produced in human and rat models concerning ELF electric fields (Hart 1990, 1992a).

Amoruso and Lattarulo $(1989,1996)$ applied the diakoptic theory to calculate the induced electric fields in the human body when coupled to high ELF electric field. The diakoptic theory was first formulated for multielement antenna analysis. In their approach, the human body was simulated using a spatial arrangement of 11 interconnected conducting spheroids. The model calculations were based on a $1.70-\mathrm{m}$-tall standard subject placed in a uniform $10 \mathrm{kV} / \mathrm{m}$ vertical electric field. The total current on the grounded person, that is, the short-circuit current, is expressed as the summation of the partial current flows capacitated through 11 elements representing the human body. They compared the current distributions on the grounded human model with those attributed to an ungrounded model. Based on the fundamental approach to the theoretical modeling, they applied diakoptic theory to the dosimetric scaling problem between mice and humans in the context of ELF electric fields. The scaling is discussed in quasi-electrostatic conditions. The 11 elements are replaced with equal amounts of prolate or oblate conducting spheroids.

As part of a basic approach to assess electric field effects near transmission lines, Min et al. (1996) calculated the induced voltage and current in a modeled human and a car close to $60 \mathrm{~Hz}, 765-\mathrm{kV}$ double-circuit transmission line. They concluded that the superposition of phase arrangement was advantageous for reducing the induced voltage in the human. However, considering the corona interference of the transmission line, this superposition of phase arrangement had side effects such as the increase in the electric 
field strength around the transmission line. They also estimated the effective number and position of shield wires to reduce the ground-level field using CSM. Myung, Lee, and Huh (1998) published a research paper on the calculation of the induced currents in the human body around and under $765-\mathrm{kV}$ transmission lines. The short-circuit currents in the body were measured in the range of 0.3 to $6.8 \mathrm{~mA}$. In addition, they calculated and simulated the electric field distribution by applying their algorithm to the Korean case.

A series of research papers on the application of the FEM to the calculation of induced current densities inside grounded and ungrounded human models exposed to $60 \mathrm{~Hz}$ electric fields were published in Japan (Chiba et al. 1984; Chiba, Isaka, and Kaune 1998; Chiba and Isaka 1997, 1998, 1999, 2000, 2004; Isaka et al. 1987; Matsumoto et al. 2004). They presented the experimental and numerical results of induced currents and induced electric fields in a highly simplified human body-shaped model. Chiba and coworkers developed a FEM to estimate the distribution of the current densities in a human model. In the calculations, they assumed that the human model consisted of a biological organism whose conductivity and permittivity were on the same order as those of human body tissues. The first model was a cylinder with a hemisphere, and the second one was a modification of the first one, which resembled a human body much more. These axisymmetric human models were made of wood and insulating material covered with aluminum foil. They had a maximum diameter of $27 \mathrm{~cm}$ and heights ranging from 150 to $180 \mathrm{~cm}$. They also developed a cylinder plus hemisphere human model with the two tissues having different conductivities. Based on the simple wooden model, the electric field strength at the top of the head was 16.0 and 18.3 times higher than the unperturbed electric fields attributed to the 150 - and $180-\mathrm{cm}$-tall models. The induced current densities depended on the transverse section areas and the height of the body part above ground but were independent of conductivities of body tissues. The total induced currents and the induced current densities matched the experimental results well. The current density distributions in a human model standing on an insulating plate were also calculated by the FEM (Chiba and Isaka 2000). Matsumoto et al. (2004) developed a two-step analytical method for the estimation of the induced current densities in a three-dimensional shape model. Two steps were involved in the estimation. The first step consisted of the deviation associated with the induced currents originating from the surface of the human model, measured through the surface charge method. The second step involved the application of these values as the boundary values of the FEM, using an isoparametric hexahedral element.

Takuma et al. (1990) developed a three-dimensional method to calculate the induced currents in human and baboon models using $60 \mathrm{~Hz}$ electric fields. Their calculation program was based on CSM. The animal model consisted of several block parts of simple geometric forms such as a sphere, a cylinder, or a cone. The total calculated induced currents in the baboon model with the postures such as standing upright, positioned on four legs, and sitting on the floor confirmed prior experimental values very well. Techaumnat, Hamada, and Takuma (2000) presented the calculation results of electrostatically induced current in a human model using the Boundary Element Method (BEM). The human model was put together using second-order curved elements and had five distinct internal organs. The conductivity of these internal organs had a considerable impact on the induced currents.

Table 4.2 gives the examples of the calculated and measured current densities in simple homogeneous and heterogeneous human models exposed to ELF electric fields. 
TABLE 4.2 Examples of Calculated and Measured Current Densities in Simple Homogeneous and Heterogeneous Human Models Exposed to Electric Field

\begin{tabular}{|c|c|c|}
\hline Author & Current Density $\left(\mathrm{mA} / \mathrm{m}^{2}\right)$ & Method and Comments \\
\hline $\begin{array}{l}\text { Chen, Chuang, and } \\
\text { Lin (1986) }\end{array}$ & $\begin{array}{l}\text { Calculated current densities agree very } \\
\text { well with the measured values (Kaune } \\
\text { and Forsyth 1985). }\end{array}$ & $\begin{array}{l}60 \mathrm{~Hz}, 10 \mathrm{kV} / \mathrm{m} \text { : surface-charge } \\
\text { integral calculation for } \\
\text { homogeneous grounded } \\
\text { human model }\end{array}$ \\
\hline Chiba et al. (1984) & $\begin{array}{l}\text { Calculated values: } 4.62 \text { for neck and } \\
19.16 \text { for ankle; measured average } \\
\text { values: } 4.66 \text { for neck and } 18.66 \text { for } \\
\text { ankle }\end{array}$ & $\begin{array}{l}60 \mathrm{~Hz}, 10 \mathrm{kV} / \mathrm{m} \text { : finite-element } \\
\text { method (FEM) calculation and } \\
\text { measurement in axisymmetric } \\
\text { human model (170-cm height) }\end{array}$ \\
\hline $\begin{array}{l}\text { Chiba, Isaka, and } \\
\text { Kaune (1998) }\end{array}$ & $\begin{array}{l}\text { Calculated values: } 0.06-0.18 \text { for the } \\
\text { head }\end{array}$ & $\begin{array}{l}60 \mathrm{~Hz}, 1 \mathrm{kV} / \mathrm{m} \text { : FEM calculation } \\
\text { in homogeneous head of } \\
\text { human model }\end{array}$ \\
\hline Chiba and Isaka (1999) & $\begin{array}{l}\text { Calculated average values: } 0.476 \text { for } \\
\text { neck and } 1.864 \text { for feet (for grounded } \\
\text { model); } 0.360 \text { for neck and } 0.947 \text { for } \\
\text { ankle (for } 0.01 \mathrm{~m} \text { above the ground) }\end{array}$ & $\begin{array}{l}60 \mathrm{~Hz}, 1 \mathrm{kV} / \mathrm{m} \text { : FEM calculation } \\
\text { in grounded and ungrounded } \\
\text { human model }\end{array}$ \\
\hline Chiba and Isaka (2000) & $\begin{array}{l}\text { Calculated average values: } 0.334 \text { for } \\
\text { neck and } 0.742 \text { for ankle }\end{array}$ & $\begin{array}{l}60 \mathrm{~Hz}, 1 \mathrm{kV} / \mathrm{m} \text { : FEM calculation } \\
\text { in human model standing on } \\
\text { the insulating plate }\end{array}$ \\
\hline Chuang and Chen (1989) & $\begin{array}{l}\text { Methodological validity was confirmed } \\
\text { by comparison between numerical } \\
\text { results and analytical solutions } \\
\text { attributed to conducting concentric } \\
\text { spheres }\end{array}$ & $\begin{array}{l}60 \mathrm{~Hz}, 10 \mathrm{kV} / \mathrm{m} \text { : surface charge } \\
\text { integral equation/impedance } \\
\text { network calculation in } \\
\text { three-dimensional } \\
\text { heterogeneous head of human } \\
\text { model }\end{array}$ \\
\hline Deno (1977) & $\begin{array}{l}\text { The induced short-circuit current is } \\
\text { expressed as: } I_{\mathrm{sc}}=5.4 \times 10^{-9} h^{2} E ; h \text { is } \\
\text { height of human body; } E \text { is } \\
\text { unperturbed electric field }\end{array}$ & $\begin{array}{l}60 \mathrm{~Hz}, 10 \mathrm{kV} / \mathrm{m} \text { : grounded } \\
\text { model covered with copper foil }\end{array}$ \\
\hline Dimbylow (1987) & $\begin{array}{l}\text { Calculated values: } 0.05-0.09 \text { for neck } \\
\text { and } 0.5-2.23 \text { for ankle }\end{array}$ & $\begin{array}{l}60 \mathrm{~Hz}, 1 \mathrm{kV} / \mathrm{m} \text { : three- } \\
\text { dimensional FDM calculation } \\
\text { in homogeneous shaped, lower } \\
\text { limb of grounded human } \\
\text { model }\end{array}$ \\
\hline Dimbylow (1988) & $\begin{array}{l}\text { Calculated peak values: } \\
(0.142-0.795) \times 10^{-2} \text { in five } \\
\text { different models }\end{array}$ & $\begin{array}{l}60 \mathrm{~Hz} \text { : FDM calculation for } \\
\text { heterogeneous leg and } \\
\text { homogeneous torso of } \\
\text { grounded human model }(1.8 \mathrm{~m} \text {, } \\
70 \mathrm{~kg}) \text { at } 1 \mathrm{~V} / \mathrm{m} \text { applied field }\end{array}$ \\
\hline Furse and Gandhi (1998) & $\begin{array}{l}\text { Calculated peak values: above } 150 \text { for } \\
\text { leg and maximum of } 20 \text { in torso }\end{array}$ & $\begin{array}{l}60 \mathrm{~Hz}, 10 \mathrm{kV} / \mathrm{m} \text { : finite- } \\
\text { difference time domain } \\
\text { calculation in anatomical, } \\
\text { MRI-based, 6-mm resolution, } \\
\text { heterogeneous, grounded } \\
\text { human model }\end{array}$ \\
\hline
\end{tabular}


TABLE 4.2 Examples of Calculated and Measured Current Densities in Simple Homogeneous and Heterogeneous Human Models Exposed to Electric Field (Continued)

\begin{tabular}{|c|c|c|}
\hline Author & Current Density $\left(\mathrm{mA} / \mathrm{m}^{2}\right)$ & Method and Comments \\
\hline Guy et al. (1982) & $\begin{array}{l}\text { Measured average values: }(24-26) \\
\times 10^{-2} \text { for neck (ungrounded model): } \\
33 \times 10^{-3} \text { to } 43 \times 10^{-2} \text { for neck } \\
\text { (grounded model) }\end{array}$ & $\begin{array}{l}60 \mathrm{~Hz}, 1 \mathrm{kV} / \mathrm{m} \text { : Thermographic } \\
\text { measurement of homogeneous } \\
\text { grounded and ungrounded } \\
\text { human model at } 57 \mathrm{MHz} \text { and } \\
\text { extrapolated to } 60 \mathrm{~Hz} \text {. }\end{array}$ \\
\hline Hart (1990) & $\begin{array}{l}\text { Calculated values : } 5.44 \text { for neck and } \\
14.7 \text { for knee of grounded model }\end{array}$ & $\begin{array}{l}60 \mathrm{~Hz}, 10 \mathrm{kV} / \mathrm{m} \text { : Spreadsheet } \\
\text { calculation of grounded block, } \\
\text { homogeneous human model } \\
\text { with irregular shape }\end{array}$ \\
\hline $\begin{array}{l}\text { Kaune and Phillips } \\
\text { (1980) }\end{array}$ & $\begin{array}{l}\text { Measured average values: } 5.5 \text { for neck } \\
\text { and } 20 \text { for feet }\end{array}$ & $\begin{array}{l}60 \mathrm{~Hz}, 10 \mathrm{kV} / \mathrm{m} \text { : grounded } \\
\text { human model }\end{array}$ \\
\hline $\begin{array}{l}\text { Kaune and Forsyth } \\
\text { (1985) }\end{array}$ & $\begin{array}{l}\text { Measured peak values: } 3.75 \text { for neck } \\
\text { and } 3.3 \text { for abdomen (current density } \\
\text { data can be extrapolated to } 1 \mathrm{MHz} \text { ) }\end{array}$ & $\begin{array}{l}60 \mathrm{~Hz}, 10 \mathrm{kV} / \mathrm{m} \text { : homogeneous } \\
\text { grounded saline-filled human } \\
\text { model }\end{array}$ \\
\hline Matsumoto et al. (2004) & $\begin{array}{l}\text { Calculated values: } 2.0 \text { in grounded } \\
\text { model and } 1.1 \text { in ungrounded model } \\
\text { located } 0.01 \mathrm{~m} \text { above the ground }\end{array}$ & $\begin{array}{l}60 \mathrm{~Hz}, 1 \mathrm{kV} / \mathrm{m} \text { : surface charge/ } \\
\text { finite-element calculation in } \\
\text { grounded and ungrounded } \\
\text { human model }\end{array}$ \\
\hline Min et al. (1996) & $\begin{array}{l}\text { Induced current in human model is } \\
\text { less than } 0.5 \mathrm{~mA}\end{array}$ & $\begin{array}{l}\text { 765-kV transmission line: } \\
\text { electrostatic effects on hot-line } \\
\text { workers }\end{array}$ \\
\hline $\begin{array}{l}\text { Motrescu and van } \\
\text { Rienen (2005) }\end{array}$ & $\begin{array}{l}\text { Calculated values: } 0.679 \text { for neck and } \\
3.682 \text { for ankle in grounded model }\end{array}$ & $\begin{array}{l}50 \mathrm{~Hz}, 5.796 \mathrm{kV} / \mathrm{m}(765-\mathrm{kV} \\
\text { transmission line): finite- } \\
\text { integration technique } \\
\text { calculation for human model } \\
\text { with anisotropic muscle tissue }\end{array}$ \\
\hline $\begin{array}{l}\text { Myung, Lee, and } \\
\text { Huh (1998) }\end{array}$ & The short-circuit current: $0.3-6.8 \mathrm{~mA}$ & $\begin{array}{l}\text { 765-kV transmission line: } \\
\text { electrostatic effects on standing } \\
\text { human model }\end{array}$ \\
\hline $\begin{array}{l}\text { Poljak and Rashed } \\
\text { (2002) }\end{array}$ & Calculated value: 0.013 & $\begin{array}{l}60 \mathrm{~Hz}, 1.081 \mathrm{kV} / \mathrm{m}: \mathrm{GB}-\mathrm{BEM} \\
\text { calculation for cylindrical } \\
\text { human body model }\end{array}$ \\
\hline Spiegel (1977b) & $\begin{array}{l}\text { Calculated values: } 3.4 \text { for chest of } \\
\text { grounded man, } 4.7 \text { for chest of } \\
\text { linemen }\end{array}$ & $\begin{array}{l}60 \mathrm{~Hz}, 765-\mathrm{kV} \text { transmission line: } \\
\text { method of moment calculation } \\
\text { in cylindrical segmented } \\
\text { human model }\end{array}$ \\
\hline Spiegel (1981) & $\begin{array}{l}\text { Calculated values: } 1.60 \text { for neck and } 6.7 \\
\text { for ankle (for grounded dummy); } 1.20 \\
\text { for neck and } 1.62 \text { for ankle (in } \\
\text { ungrounded model) }\end{array}$ & $\begin{array}{l}60 \mathrm{~Hz}, 10 \mathrm{kV} / \mathrm{m} \text { : polarization } \\
\text { integral equation calculation } \\
\text { for grounded and ungrounded } \\
\text { human model consisting of } \\
\text { small cubical blocks }\end{array}$ \\
\hline Techaumnat et al. (2000) & $\begin{array}{l}\text { Calculated average values: } 0.0004 \text { for } \\
\text { neck and } 0.001 \text { for leg (for } \\
\text { homogeneous model) }\end{array}$ & $\begin{array}{l}50 \mathrm{~Hz}, 1 \mathrm{~V} / \mathrm{m} \text { : BEM calculation } \\
\text { in human model with } \\
\text { second-order curved elements }\end{array}$ \\
\hline
\end{tabular}




\subsubsection{Coupling with Realistically Shaped Models}

Dimbylow (1987) presented a three-dimensional finite-difference calculation of induced current densities and short-circuit currents in a grounded, homogeneous, realistically shaped human phantom in reference to applied ELF electric fields. He compared the result with Kaune and Forsythes's (1985) published experimental outcomes associated with current density distributions at $60 \mathrm{~Hz}$ in a mannequin with two arms on its sides.

Hirata et al. (2001) calculated the induced electric fields and current densities in a 5 -year-old child's body exposed to vertical $60 \mathrm{~Hz}, 1 \mathrm{kV} / \mathrm{m}$ electric fields in both grounded and isolated conditions and compared them with those related to an adult's body, phantom of the University of Victoria, UVic phantom. They calculated induced electric fields in the adult model using a hybrid method, and in the child, using a quasistatic FDTD method. In this case, the American male and corresponding American child model were used in the calculation. They found that the induced electric fields were lower in the child head than in the adult head. Hirata and Fujiwara (2007) computed the induced current densities in the human models based on Japanese male and female models named TARO and HANAKO, respectively, developed by Nagaoka et al. $(2002,2004)$. The resolution of the model was $2-\mathrm{mm}^{3}$ voxels and was segmented into 51 anatomic regions. Using a quasistatic FDTD method, Hirata and Fujiwara (2007) computed the induced current densities in TARO and HANAKO when exposed to vertical $60 \mathrm{~Hz}, 1 \mathrm{kV} / \mathrm{m}$. Computational results showed that the difference in induced current densities between TARO and HANAKO was less than $20 \%$ depending on the tissues/organs. They pointed out that this difference was reasonable because there was a difference of equal magnitude between NORMAN and NAOMI (Dimbylow 2005). Additionally, they pointed out that the effect of gender and/or race on induced current densities was comparable to or smaller than that caused in the process of computational modeling.

Dawson, Moerloose, and Stuchly (1997a) presented a hybrid ELF electric induction modeling method by combining a quasistatic FDTD method with a frequency-domain SPFD method. Dawson, Caputa, and Stuchly (1998a) used this hybrid numerical method to compute the induced fields in an anatomical, high-resolution human body model exposed to uniform ELF electric fields. This hybrid method was capable of accurately computing the induced fields in a full-body human model that consisted of $1,736,872$ cubic voxels with $3.6-\mathrm{mm}$ edges. A low-resolution model with FDTD was used to calculate the surface charge density on the body. Later, the SPFD code generated the surface charge distribution induced by the external electric field at high resolution. They measured the exposure to $60 \mathrm{~Hz}$ fields, which can be scaled linearly to frequencies up to $100 \mathrm{kHz}$ in consideration of the frequency dependence of tissue conductivity. Dawson et al. (2001a) calculated electric fields in the human body resulting from $60 \mathrm{~Hz}$ contact currents, and they compared electric fields induced in humans and rodents (Dawson et al. 2003). The contact current occurs when a person touches conductive bodies at different potentials, creating a current flow through the body. They used adult and child models for the calculation of contact currents. In order to estimate the electric fields and current densities, they applied the SPFD method. Three paths of contact current were modeled: hand to opposite hand and both feet, hand to hand only, 
and hand to both feet. Dawson, Stuchly, and Kavet (2004a,b) also estimated the electric fields in a heterogeneous human body due to electrostatic discharges by the quasistatic frequency-domain SPFD method.

González, Peratta, and Poljak (2007) and Peratta and Peratta (2010) applied the human exposure assessment to ELF electric fields using the three-dimensional BEM. Based on a realistic anatomically based human body, they assessed human exposure to $60 \mathrm{~Hz}$ electric fields generated by transmission lines and a transformer substation. The human body was modeled through a saline fluid containing a certain conductivity $\sigma$ of $0.5 \mathrm{~S} / \mathrm{m}$. Five different conceptual models were considered: human no arms, human arms open, human arms up, human arms down, and human internal organs. They analyzed the influence factors attributed to the relative position of the arms with respect to the body on the axial distribution of current densities along the body. The total current densities along the torso and legs showed significant increase as arms were raised. When exposed to $60 \mathrm{~Hz}$ electric fields, the neck was protected due to the shielding effect of raised arms. This indicates that the brain was protected by the raised arms. In the case of the transformer substation, the dynamics of current densities were consistent with those obtained in context of a transmission line. They compared the results with the basic restrictions established by the International Commission on Non-Ionizing Radiation Protection (ICNIRP 1998a,b) and found that induced current densities were lower than the work exposure limit, excluding the neck. Motrescu and van Rienen (2005) computed the current induced by ELF electric fields in anisotropic human tissues. In order to compute the induced current densities in the sophisticated human body model standing under a transmission line, they used the algorithm of the Finite-Integration Technique (FIT).

\subsubsection{Coupling with Other Models}

Based on results of long-term biological effect research efforts, it has been suggested that several observed effects are due to induced currents and electric fields triggered in biological systems. Fear and Stuchly (1998) investigated the behavior of biological cells with gap junctions exposed to ELF electric fields. They examined the induced transmembrane potential (TMP) in geometrically complex models of various cell configurations by using the FEM. They showed the FEM approach eases the analysis of TMP in a variety of cell preparations and the examination of parameters such as gap-junction conductivity in geometrically complex cell cluster models. The approach confirms that gap junction-connected cells can be treated as a single similarly shaped cell. The gap influences the potential in the interior of cell configurations, and its effect increases with gap size and conductivity.

Using both FEM and SPFD calculations, Chiu and Stuchly (2005) presented the numerically computed result of two models, a cancellous bone model and a bone marrow stroma cell model, of bone marrow substructures exposed to ELF electric fields. They used bone marrow to clarify the relationship between EMF and childhood leukemia at both cellular and subcellular levels. It is well known that bone marrow is responsible for leukemia. In the cancellous bone model, the enhancement of electric fields was the measure of interest. The enhancement of local electric fields occurred within the cancellous 
bone cavities, but this enhancement was probably unlikely to cause biological effects. The maximum electric field enhancement of $50 \%$ was observed. Since the TMP was a more adequate measure for the stroma cell model, the TMP change was evaluated. According to subsequent calculation, the TMP change across the gap junctions reached over $200 \mu \mathrm{V}$. Table 4.3 lists the examples of the calculated and measured current densities in a realistic human model exposed to ELF electric fields.

TABLE 4.3 Examples of Calculated and Measured Current Densities in Realistic Human Models Exposed to Electric Field

\begin{tabular}{|c|c|c|}
\hline Author & Current Density $\left(\mathrm{mA} / \mathrm{m}^{2}\right)$ & Method and Comments \\
\hline $\begin{array}{l}\text { Dawson, Moerloose, } \\
\text { and Stuchly (1997a) }\end{array}$ & $\begin{array}{l}\text { The validity of the integrated two- } \\
\text { calculation techniques was evaluated } \\
\text { in low resolution. }\end{array}$ & $\begin{array}{l}60 \mathrm{~Hz}, 1 \mathrm{kV} / \mathrm{m} \text { : hybrid method } \\
\text { (scalar potential finite difference } \\
\text { [SPFD]/quasistatic finite- } \\
\text { difference time domain [FDTD]) } \\
\text { calculation for anatomical, } \\
\text { magnetic resonance imaging } \\
\text { (MRI)-based, 3.6-mm resolution, } \\
\text { heterogeneous, grounded and } \\
\text { ungrounded human models }\end{array}$ \\
\hline $\begin{array}{l}\text { Dawson, Caputa, and } \\
\text { Stuchly (1998a) }\end{array}$ & $\begin{array}{l}\text { Calculated peak values are observed in } \\
\text { the head and abdomen; the effects of } \\
\text { conductivity variation and body } \\
\text { position on induced electric fields and } \\
\text { current densities were analyzed. }\end{array}$ & $\begin{array}{l}60 \mathrm{~Hz}, 1 \mathrm{kV} / \mathrm{m} \text { : hybrid method } \\
\text { (SPFD/quasistatic FDTD) } \\
\text { calculation for anatomical, } \\
\text { MRI-based, 3.6-/7.2-mm } \\
\text { resolution, heterogeneous, } \\
\text { grounded and ungrounded } \\
\text { human models }\end{array}$ \\
\hline
\end{tabular}

Dimbylow (2000) Calculated peak values: $0.171 \times 10^{-2}$ in the body of grounded model and $0.474 \times 10^{-2}$ in ungrounded model; $0.100 \times 10^{-3}$ for retina in ungrounded model

González, Peratta, and Poljak (2007)

Calculated peak values: 8-10.2 for neck and 60 for ankle (both in human body with arms up and open)

Hirata et al. (2001)

Hirata and Fujiwara (2007)

Calculated average values across $1-\mathrm{cm}^{2}$ area: 0.25 for neck and 0.517 for legs (in grounded adult); 0.283 for neck and 0.508 for legs (in grounded child)

$50 \mathrm{~Hz}, 1 \mathrm{~V} / \mathrm{m}$ : FDM calculation for anatomical, MRI-based, realistic, 2-mm resolution human model, NORMAN; averaged $1 \mathrm{~cm}^{2}$ for retina.

$60 \mathrm{~Hz}, 10 \mathrm{kV} / \mathrm{m}$ : three-dimensional BEM calculation related to an anatomically realistic human grounded model

$60 \mathrm{~Hz}, 1 \mathrm{kV} / \mathrm{m}$ : hybrid method (SPFD/quasistatic FDTD) calculation on an adult model, and FDTD on a child model

Calculated average values across $1-\mathrm{cm}^{2}$ area: 0.204 for TARO; 0.216 for HANAKO (both for retina)

Potter, Okoniewski, and Stuchky (2000)

The current density and induced fields can be significantly higher than for the uniform electric field exposure on a ground plane.

$60 \mathrm{~Hz}, 1 \mathrm{kV} / \mathrm{m}$ : quasistatic FDTD calculations on anatomical Japanese models, TARO and HANAKO

$60 \mathrm{~Hz}$ : quasistatic FDTD calculation for anatomical, MRI-based, 7.2-mm resolution, heterogeneous, human model in close proximity to power lines 


\subsubsection{ELF Magnetic Field}

During the 1970s, after the development of ELF electric field dosimetric technologies, the calculations focused on induced currents and electric fields in simple spheroidal and prolate human and animal models exposed to ELF magnetic fields by analytical method (Spiegel 1977b; Hart 1992b). Spiegel (1977b) calculated the induced current densities and power absorbed by a prolate spheroid model representing a man when exposed to ELF magnetic fields of transmission lines. Using commercially available spreadsheet software running a FDM, Hart, Evely, and Finch (1993) presented the calculation for electric fields and current density distributions induced in an irregularly shaped inhomogeneous model by ELF magnetic fields.

\subsubsection{Coupling with Simple and Analytical Models}

In the past, it was not clear whether observed biological effects were due to magnetic fields or induced currents inside the test sample. Applying experimental dosimetry, a circular dish system (petri dish) was used to clarify whether observed biological effects were caused directly by magnetic fields or by induced currents. If the results are similar across the petri dish, effects are due to magnetic field, whereas if the results vary across the cross-section of the petri dish, effects are due to induced currents. Subsequently, calculations of induced currents and induced electric fields for several dish systems, including one using an annular ring, have been published (Misakian 1991; Misakian et al. 1993). Polk (1986) examined the effects of DC and ELF magnetic fields on the motion and distribution of counterions on surfaces of cylindrical biological cells.

As far as experimental dosimetry is concerned, there are many reports on measuring magnetically induced electric fields. Experimental dosimetry for measurement of induced electric fields proves difficult due to the need of physical probes in the complex biological tissues. It entails interactions in term of electromagnetic interference between the physical probe and exposure to magnetic fields. Miller and coworkers published a series of papers on experimental dosimetry using a miniaturized physical probe to measure internal electric fields in conductive rat and human models, chicken eggs, and anesthetized rats (Miller 1991a,b, 1994, 1996; Miller and Creim 1997; Robertson-De Mers and Miller 1992). They designed and built a miniaturized electric field probe to provide data on fields induced by $60 \mathrm{~Hz}$ magnetic fields in saline-agar models of a rat and a human. The probe consisted of $200-\mu \mathrm{m}$-diameter silver wires forming $\mathrm{Ag}-\mathrm{AgCl}$ electrodes imbedded in a 17-gauge hypodermic needle. The voltage across the probe electrodes was measured with a lock-in amplifier. The measurement results of this probing agreed roughly with numerical calculations based on the simple cylindrical case. In the subsequent investigation, the electric fields induced in an egg by a $60 \mathrm{~Hz}$ magnetic field at $1 \mathrm{mT}$ were measured. As a result, different conductivities of the yolk and egg modified the induced electric fields. In order to provide data on the electric fields induced in an actual rat by a $60 \mathrm{~Hz}$ magnetic field exposure, Miller and coworkers measured the induced electric fields related to $60 \mathrm{~Hz}$ magnetic fields at $1 \mathrm{mT}$ with a miniaturized electric field probe at $2-\mathrm{mm}$ resolution using rat carcasses. The probe scans were made at three positions in the abdomen for axial, frontal, and lateral exposure. The scanning results showed significant differences from the theoretical calculation based 
on a homogeneous ellipsoidal model of the rat carcasses. Furthermore, they measured the complex electric fields in anesthetized rats using a miniaturized electric field probe. In living animals, the natural endogenous electric fields exist internally and are readily detected as an electrocardiogram waveform. If the magnetic field is applied externally to living animals, an electric field, which is induced by a $60 \mathrm{~Hz}$ magnetic field, is added to endogenous physiological electric fields. Miller and Creim (1997) compared the endogenous electric fields and $60 \mathrm{~Hz}$ magnetically induced electric fields. The induced electric fields were reduced within the body, or in different directions on the body surface. It is well known that the induced electric fields gradually decrease from a maximum at the body surface to zero at the center of the body.

Table 4.4 gives examples of the calculated and measured current densities in a realistic human model exposed to uniform and nonuniform ELF magnetic fields.

Baraton and Hutzler (1995) reported FEM calculations on induced current densities in three different human model configurations, both in uniform and nonuniform magnetic fields. They analyzed induced currents in the human body through either the TRIFOU (three-dimensional eddy current) code or a simple formula. TRIFOU code is the three-dimensional field calculation techniques using the FEM (Bossavit and Vérité 1983). This code solves the Maxwell equation within the body by an iterative process. The inside body is meshed with tetrahedron, while the space around the body remains unmeshed. The boundary conditions on the body surface are factored in using the boundary integrals. Dawson and Stuchly (1997) and Dawson (1997) considered the analytic solution to the problems of electric fields and current densities induced by applied magnetic fields. The analytical solution can be applied to the problem of ELF induction in an equatorially stratified sphere by using axial uniform magnetic fields. This solution is formulated through Green's function.

Gandhi, Deford, and Kanai (1984) introduced the IM for the calculations of current densities inside the human body exposed to ELF magnetic fields. The IM by Gandhi and Chen (1992) has been applied to calculations in an anatomically voxel-based human body model. The human body was based on a semirealistic voxel model. In that calculation, the voxels were differentiated into a three-dimensional network of impedance. Applied ELF magnetic fields inside the human body were researched.

The IM suggested that when representing the biological body as a three-dimensional network of impedance whose individual values are obtained through the conductivities, a system equation was derived using Kirchhoff's law around each loop in this threedimensional network. As an example, using the quasistatic IM, Gandhi et al. (2001) calculated the induced currents in the nominal $2 \times 2 \times 3$ and 6 -mm resolution, anatomically based human body model exposed to $60 \mathrm{~Hz}$ magnetic fields. In the first calculation, the induced electric fields or current densities for the various glands and organs of the body including pineal gland were obtained in the context of exposure to uniform magnetic fields of various orientations and a magnitude of $0.1 \mathrm{mT}(50 \mathrm{~Hz})$ or $1 \mathrm{mT}(60 \mathrm{~Hz})$. These values are the safety guideline levels set by the ICNIRP $(1998 \mathrm{a}, \mathrm{b})$ and the American Conference of Industrial Hygienists (ACGIH 1997). The maximum $1-\mathrm{cm}^{2}$-averaged induced current densities in the brain and spinal cord were within the basic restriction level of $10 \mathrm{~mA} / \mathrm{m}^{2}$ according to the ICNIRP guidelines for occupational exposure. Furthermore, the maximum $1-\mathrm{cm}^{2}$-averaged current densities and electric 
TABLE 4.4 Examples of Calculated and Measured Current Densities in Realistic Human Models Exposed to Uniform and Nonuniform Magnetic Fields

\begin{tabular}{|c|c|c|}
\hline Author & Current Density $\left(\mathrm{mA} / \mathrm{m}^{2}\right)$ & Method and Comments \\
\hline $\begin{array}{l}\text { Baraton and Hutzler } \\
\text { (1995) }\end{array}$ & $\begin{array}{l}\text { Calculated average values: } \\
1.73 \times 10^{-3} \text { for LAT, } 1.53 \times \\
10^{-3} \text { for AP, and } 1.31 \times 10^{-3} \\
\text { for TOP in standing uniform } \\
\text { conductivity human model }\end{array}$ & $\begin{array}{l}50 \mathrm{~Hz}, 1 \mathrm{~A} / \mathrm{m} \text { : TRIFOU/FEM calculations for } \\
\text { human model: (1) standing in an uniform } \\
\text { magnetic field, (2) live-line working, and } \\
\text { (3) using an hair dryer }\end{array}$ \\
\hline $\begin{array}{l}\text { Barchanski et al. } \\
\text { (2006a) }\end{array}$ & $\begin{array}{l}\text { Calculated maximum values } \\
\text { for whole body: } 1.03 \times 10^{-2} \\
\text { for (a); } 4.38 \times 10^{-3} \text { for (b) }\end{array}$ & $\begin{array}{l}50 \mathrm{~Hz} \text {, two electric blankets, (a) } 73.7 \mu \mathrm{T} \text { and } \\
\text { (b) } 78.1 \mu \mathrm{T} \text { : Ex-SPFD calculation for } \\
\text { HUGO model from the Visible Human } \\
\text { Project }\end{array}$ \\
\hline $\begin{array}{l}\text { Barchanski et al. } \\
\text { (2007) }\end{array}$ & $\begin{array}{l}\text { Current density is well below } 2 \\
\text { (ICNIRP limit for basic } \\
\text { restriction of public } \\
\text { exposure) for electric } \\
\text { blankets }\end{array}$ & $\begin{array}{l}\text { Ex-SPFD calculation for anatomical, } \\
\text { MRI-based, realistic, 1-mm resolution } \\
\text { human model for exposure to electric } \\
\text { blankets and for brain model for } \\
\text { transcranial magnetic stimulation }\end{array}$ \\
\hline Caputa et al. (2002) & $\begin{array}{l}\text { The comparisons of induced } \\
\text { electric field for all the organs } \\
\text { show differences of } 1 \% \text { or less } \\
\text { for the majority of tissues } \\
\text { (liver and stomach, etc.) }\end{array}$ & $\begin{array}{l}60 \mathrm{~Hz}, 1 \mu \mathrm{T} \text { AP: SPFD calculations for } \\
\text { realistic human body models; NORMAN } \\
\text { (2- to } 4 \text {-mm resolution), Uvic (1.8-mm } \\
\text { resolution), and AF (2-mm resolution) }\end{array}$ \\
\hline Cheng et al. (1995) & $\begin{array}{l}\text { Calculated maximum values: } \\
8 \text { for the hair dryer and } 10.8 \\
\text { for the electric shaver }\end{array}$ & $\begin{array}{l}60 \mathrm{~Hz} \text {, single dipole from portable appliances } \\
\text { (hair dryer and electric shaver): IM } \\
\text { calculation for MRI-based human head } \\
\text { model, 5-mm voxel }\end{array}$ \\
\hline $\begin{array}{l}\text { Dawson, Caputa, } \\
\text { and Stuchly } \\
(1997 b)\end{array}$ & $\begin{array}{l}\text { Calculated average values for } \\
\text { whole body: } 1.2 \text { with a } \\
\text { maximum of } 32 \text {; for the heart, } \\
0.5 \text { with a maximum of } 2.8\end{array}$ & $\begin{array}{l}60 \mathrm{~Hz}, 1 \mu \mathrm{T}: \text { SPFD calculation for MRI-based } \\
\text { 3.6- and 7.2-mm resolution human body } \\
\text { models }\end{array}$ \\
\hline $\begin{array}{l}\text { Dawson and Stuchly } \\
\text { (1998b) }\end{array}$ & $\begin{array}{l}\text { Calculated average values for } \\
\text { whole body: } 2.78 \times 10^{-3} \text { for } \\
\text { LAT, } 3.37 \times 10^{-3} \text { for AP and } \\
2.34 \times 10^{-3} \text { for TOP }\end{array}$ & $\begin{array}{l}60 \mathrm{~Hz}, 1 \mu \mathrm{T} \text { (AP, LAT, and TOP): SPFD } \\
\text { calculation for anatomically realistic, } \\
\text { MRI-based, 3.6-mm resolution human } \\
\text { model; effects of field orientation }\end{array}$ \\
\hline $\begin{array}{l}\text { Dawson, Caputa, } \\
\text { and Stuchly } \\
(1999 a)\end{array}$ & $\begin{array}{l}\text { Calculated peak values: } 28.4 \\
\text { for whole body (scenario A), } \\
25.0(\mathrm{~B}) \text {, and } 5.9(\mathrm{C})\end{array}$ & $\begin{array}{l}60 \mathrm{~Hz} \text {, three-phase transmission line, each } \\
\text { phase } 500 \text { A: live worker: SPFD calculation } \\
\text { for anatomically heterogeneous, 3.6-mm } \\
\text { voxel conductivity, human body model A: } \\
\text { Worker in a bucket; B: worker in a } \\
\text { conductor-mounted cart; C: worker in an } \\
\text { underground vault }\end{array}$ \\
\hline $\begin{array}{l}\text { Dawson, Caputa, } \\
\text { and Stuchly } \\
\text { (1999b) }\end{array}$ & $\begin{array}{l}\text { Calculated peak values for } \\
\text { whole body at } 1 \mathrm{~A} \text { : } 18.9 \times 10^{-3} \\
\text { for A, } 27.1 \times 10^{-3} \text { for } \mathrm{B} \text {, } \\
26.3 \times 10^{-3} \text { for } \mathrm{C} \text {, and } 20.6 \times \\
10^{-3} \text { for D }\end{array}$ & $\begin{array}{l}60 \mathrm{~Hz}, 230-500 \mathrm{kV} \text {, single-line, live-line } \\
\text { worker: SPFD calculation for anatomical, } \\
\text { voxel-based, heterogeneous conductivity } \\
\text { human model; scenario A: seated worker, } \\
230 \mathrm{kV}, 600 \mathrm{~A} \text {; B: worker with extended } \\
\text { arms seated within a four-conductor } 500 \mathrm{kV} \\
\text { transmission line bundle, each conductor } \\
380 \mathrm{~A}, 500 \mathrm{kV} \text {, each conductor } 380 \mathrm{~A} \text {; C and } \\
\text { D: } 500 \mathrm{kV} \text {, four-conductor, each } 250 \mathrm{~A}\end{array}$ \\
\hline
\end{tabular}

(Continued) 
TABLE 4.4 Examples of Calculated and Measured Current Densities in Realistic Human Models Exposed to Uniform and Nonuniform Magnetic Fields (Continued)

\begin{tabular}{|c|c|c|}
\hline Author & Current Density $\left(\mathrm{mA} / \mathrm{m}^{2}\right)$ & Method and Comments \\
\hline Dimbylow (1998) & $\begin{array}{l}\text { Calculated maximun values } \\
\text { averaged over } 1 \mathrm{~cm}^{2} \text { for retina } \\
\text { at } 50 \mathrm{~Hz} \text { : } 284 \text { for AP, } 551 \text { for } \\
\text { LAT, and } 383 \text { for TOP }\end{array}$ & $\begin{array}{l}50 \mathrm{~Hz}-10 \mathrm{MHz}, 1 \mathrm{~T} \text { : IM and SPFD } \\
\text { calculations for anatomically realistic, 2-mm } \\
\text { resolution voxel human model, NORMAN }\end{array}$ \\
\hline Gandhi et al. (2001) & $\begin{array}{l}\text { Calculated average values for } \\
\text { brain in uniform field: } 0.88 \\
\text { for LAT, } 0.73 \text { for AP, and } 0.57 \\
\text { for TOP; calculated average } \\
\text { values at maximum of } 122 \mu \mathrm{T} \\
\text { in nonuniform field: } 3.0 \text { for } \\
\text { brain }\end{array}$ & $\begin{array}{l}60 \mathrm{~Hz} \text {, uniform } 1 \mathrm{mT} \text {, nonuniform maximum } \\
122 \mu \mathrm{T} \text { (hair dryer) and maximum } 1638 \mu \mathrm{T} \\
\text { (hair clipper): IM calculation for } \\
\text { anatomical, MRI-based, realistic } 2-\times 2-\times \\
3 \text {-mm resolution human model }\end{array}$ \\
\hline Hirata et al. (2009) & $\begin{array}{l}\text { Calculated average values over } \\
1 \mathrm{~cm}^{2} \text { of the nerve tissue: } 2.24 \\
\text { for LAT, } 1.56 \text { for AP, and } 1.21 \\
\text { for TOP (TARO); } 1.67 \text { for }\end{array}$ & $\begin{array}{l}50 \mathrm{~Hz}, 0.1 \mathrm{mT} \text { : quasistatic FDTD calculation } \\
\text { for anatomically based numeric Japanese } \\
\text { male, TARO, and female, HANAKO, } \\
\text { models. }\end{array}$ \\
\hline
\end{tabular}

Iivonen et al. (2005) Maximum values are $72 \times 10^{-3}$

Battery ELF current of a mobile phone FEM/ with FEM and $80 \times 10^{-3}$ with FIT; maximum values of total current density are $101 \times 10^{-3}$ for FEM and $115 \times 10^{-3}$ for FIT

Iivonen and Sarvas (2007)

Calculated maximum values: $83 \times 10^{-3}$ for check and $45 \times 10^{-3}$ for breast pocket

Min and Song

Maximum value is 0.34 in heart

Nishizawa et al. (2004)

The investigation of the accuracy of the equivalent source model of the real electronic appliance and introduction of the magnetic field distribution and induced current density

Nishizawa, Landstorfer, and Kamimura (2007)

The investigation of the accuracy of the equivalent source model of the real electronic appliance and introduction of the magnetic field distribution and induced current density FIT calculation for MRI-based human head model

Equivalent source with magnetic dipoles based on the magnetic field from GSM phone: FEM calculation for human body model, Visible Human and 1-mm resolution head model

$60 \mathrm{~Hz}, 10 \mu \mathrm{T}$ : BME for three human body models with various conductivities

$50 \mathrm{~Hz}$, equivalent source model based on magnetic dipole, nonuniform magnetic filed (hair dryer): SPFD calculation for axially symmetric homogeneous human body model

$50 \mathrm{~Hz}$, coil source model for nonuniform magnetic fields (a drilling machine and hand mixer): SPFD calculation for axially symmetric homogeneous and anatomical human body model 
TABLE 4.4 Examples of Calculated and Measured Current Densities in Realistic Human Models Exposed to Uniform and Nonuniform Magnetic Fields (Continued)

\begin{tabular}{lc}
\hline Author & Current Density $\left(\mathrm{mA} / \mathrm{m}^{2}\right)$ \\
\hline Ruoss et al. (2001) & $\begin{array}{c}\text { Calculated maximum values at } \\
\text { the closest distance of } 1 \mathrm{~cm}: \\
0.04 \text { for kitchen machine and } \\
0.005 \text { for hand blender }\end{array}$ \\
& \\
& Calculated average values: \\
& 0.396 for whole body and \\
Stuchy and Zhao & 0.311 for head (500 kV, \\
(1996) & $1000 \mathrm{~A})$ and 0.829 for whole \\
& body and 0.636 for head \\
& $(25 \mathrm{kV}, 200$ A) \\
Techaumnat, & The induced current density in \\
Hamada, and & an organ varies \\
Takuma (2001) & proportionally to its \\
conductivity. & Calculated maximum values: \\
Tarao, Hayashi, and & $17 \times 10^{-3}$ at $60 \mathrm{~Hz}$ and \\
Isaka (2003) & $3.7 \times 10^{-3}$ at $120 \mathrm{~Hz}$ \\
&
\end{tabular}

Tofani et al. (1995a) Calculated maximum values: $25 \times 10^{-3}$ at $2 \mathrm{~cm}$ distance from hair dryer

Wakeet al. (1998)

Calculated maximum induced current density at retina is about 11

$\mathrm{Xi}$, Stuchly, and

Gandhi (1994a)

Calculated average values in homogeneous man model: $101 \times 10^{-3}$ for AP, $128 \times 10^{-3}$ for LAT, and $91 \times 10^{-3}$ for TOP, rat model: $30 \times 10^{-3}$ for AP, $31 \times 10^{-3}$ for LAT and $5 \times 10^{-3}$ for TOP and mice model: $11.7 \times 10^{-3}$ for AP, $12.3 \times 10^{-3}$ for LAT, and $9.3 \times 10^{-3}$ for TOP

Yamazaki et al. (2000b)
The conductivity value of the organ affects the induced current distribution inside the human body.
Method and Comments

$50 \mathrm{~Hz}$, nonuniform magnetic field from appliance (kitchen machine $(102 \mu \mathrm{T})$ and hand blender $(42 \mu \mathrm{T}))$ : equivalent-source model based on magnetic dipoles: MoM calculation for simple ellipsoidal homogeneous human body model

$60 \mathrm{~Hz}, 25-\mathrm{kV}, 138-\mathrm{kV}$, and $500-\mathrm{kV}$ transmission lines: IM calculation for worker near power lines; heterogeneous human body model; head voxel size 6.65 mm; body $13 \mathrm{~mm}$

$50 \mathrm{~Hz}, 1 \mu \mathrm{T}$ : BEM calculation for homogeneous human mode and human model with internal organs

$60 \mathrm{~Hz}$ with harmonics, hair dryer $(1.2 \mathrm{~kW})$, 3-orthogonal magnetic dipole moment: IM calculation for MRI-based high-resolution human head model

$50 \mathrm{~Hz}$, hair dryer (current carrying coil model): IM calculation in $1.31-\mathrm{cm}$ resolution head and neck model

$20 \mathrm{~Hz}$, nonuniform $5 \mathrm{mT}$ at retina; magnetophosphene: IM calculation for electrical heterogeneous human head model

$60 \mathrm{~Hz}, 1 \mathrm{~T}$ : IM calculation for comparison of homogeneous three-species model (man, rat, and mice), and field orientation: voxel size is $1.31 \mathrm{~cm}$ for man, $1.5 \mathrm{~mm}$ for rat, and $1.0 \mathrm{~mm}$ for mice

$50 \mathrm{~Hz}, 1 \mu \mathrm{T}$ : surface charge calculation for spheroidal and cylindrical human model with several organs

\footnotetext{
AP, applied from front; LAT, applied from side; TOP, applied from above; TRIFOU/FEM, three-dimensional eddy current/finite-element method; ICNIRP, International Commission on Non-Ionizing Radiation Protection; Ex-SPFD, extended scalar potential finite difference; IM, impedance method; MRI, magnetic resonance imaging; FDTD, finite-difference time domain; FIT, Finite-Integration Technique; ELF, extremely low-frequency; MoM, method of moment; GSM, Global System for Mobile Communications; AF, Air Force.
} 
fields in the brain exposed to $1 \mathrm{mT}$ from side to side were $1.67 \mathrm{~mA} / \mathrm{m}^{2}$ and $24.78 \mathrm{mV} / \mathrm{m}$, respectively. From exposure to nonuniform magnetic fields generated from hair dryers and hair clippers, the calculated induced current density distributions are obtained.

Hamada and Kobayashi (2006a, 2008) developed a fast-multipole, surface-chargesimulation method for calculating three-dimensional Laplacian fields in voxel models and applied this method to calculate the induced electric fields in a human head model with $1 \times 1 \times 1 \mathrm{~mm}^{3}$ voxel size exposed to homogeneous ELF magnetic fields. This method treats a surface of a voxel with different outside and inside conductivities as a surface element of the indirect BEM. Their group also developed a generalized equivalent multipole-moment method for calculating three-dimensional Laplacian fields in a multispherical system and applied this method to calculate induced electric fields in a human head model with two eyeballs exposed to ELF magnetic fields. The validity of this method was confirmed by comparing the calculated electric fields with those of the fast-multipole surface-charge-simulation method (Hamada, Yamamoto, and Kobayashi 2006b; Kitano, Hamada, and Kobayashi 2009).

Yamazaki and coworkers published a series of research papers on the magnetically induced currents inside a simple human body model (Yamazaki, Kawamoto, and Shigemitsu 1996; Yamazaki et al. 2000b,c, 2001a). As for experimental dosimetry, they first measured the induced current distributions inside an inhomogeneous saline model caused by ELF magnetic fields and calculated induced values through the CSM (Figure 4.6). After these fundamental approaches, they developed a human body model with several organs (brain, heart, lungs, liver, and intestines). The shapes of other parts were modeled using spheroids and cylinders. Later, a numerical algorithm was developed based on the surface charge method, and the validity of the developed code was compared with an analytical solution. Using the BEM, Min and Song (2006a) analyzed the induced current densities inside a human body model with various conductivities exposed to $60 \mathrm{~Hz}$ magnetic fields. They applied the Korean magnetic field standard to their model and found that the maximum induced current densities inside the human body model satisfied the safe level of ICNIRP. Their human body model had several organs (brain, heart, lung, liver, and intestine) and many other parts, and their shape was spheroidal or cylindrical.

\subsubsection{Coupling with Realistically Shaped Models}

The research group at the University of Victoria applied the IM to calculations in a human body model (Xi, Stuchly, and Gandhi 1994a; Xi and Stuchly 1994b; Stuchly and Zhao 1996; Cheng et al. 1995). From the 1990s, research groups at University of Utah and University of Victoria calculated electric fields and induced currents inside anatomically, MRI-based human body phantom models with voxel resolution of 6 and $7.2 \mathrm{~mm}$ (Gandhi and Chen 1992; Dawson, de Moerloose, and Stuchly 1996). Gandhi and Chen (1992) developed the FDTD method for calculating induced current densities in an anatomically based human body model with $1.31-\mathrm{cm}$ resolution exposed to electric field, magnetic field, and EMF at $60 \mathrm{~Hz}$.

As a step in numerical dosimetry for $60 \mathrm{~Hz}$ magnetic fields, Xi, Stuchly, and Gandhi (1994a) computed the induced electric currents in models of man, rat, and mouse using the IM. All models had realistic shapes, and in the case of rodents, models were based on 


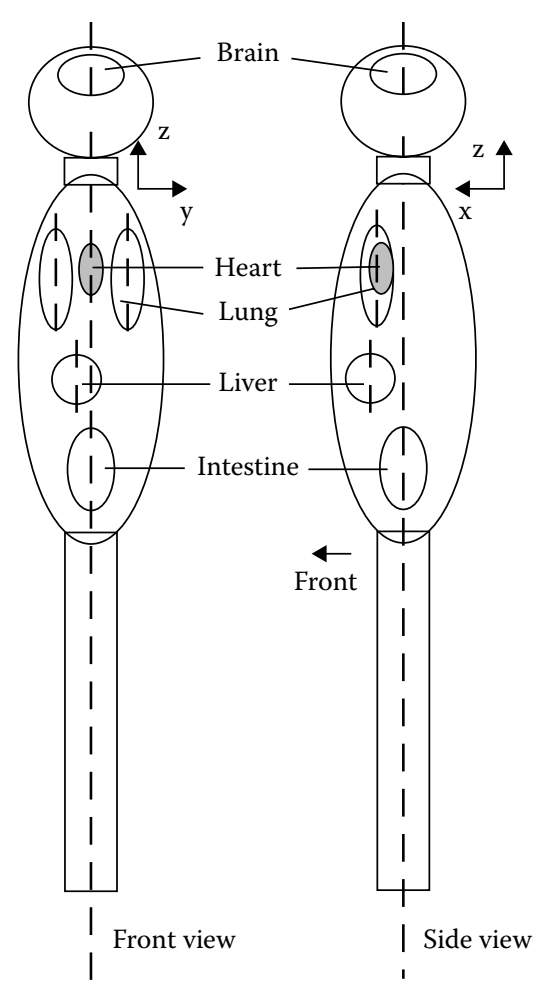

FIGURE 4.6 Example of the simple human model constructed with axisymmetric objects representing five major organs (brain, heart, lung, liver, and intestine) developed by the Central Research Institute of Electric Power Industry. (From Yamazaki, K. et al. 2001a. Investigation magnetically induced current inside the human body: Development tool and effect of organ conductivity. Electr Eng Jpn 134:1-10. With permission.)

MRIs, with homogeneous average tissue conductivity and without their tails. The shapes of models of man were configured for three cases: a homogeneous average tissue conductivity model, a heterogeneous model, and a layered model. All three models showed similar average induced electric fields and currents for any orientation of magnetic fields. When the induced electric fields and currents in homogenous models of man $(70 \mathrm{~kg})$, rat $(0.3 \mathrm{~kg})$, and mouse $(0.02 \mathrm{~kg})$ were compared, the patterns of the induced current densities were quite different from each other. These results illustrated the difficulty associated with scale of different species when estimating and comparing induced electrical values related to biological interactions. The highest current density and electric field estimated for man were equal to approximately $1090 \mu \mathrm{A} / \mathrm{cm}^{2}$ and $152 \mathrm{~V} / \mathrm{m}$ at $1 \mathrm{~T}$.

The SPFD method was introduced in 1996 by Dawson and coworkers (Dawson, de Moerloose, and Stuchly 1996; Dawson and Stuchly 1996; Dawson, Moerloose, and Stuchly 1997a; Dawson, Caputa, and Stuchly 1997b; Dawson and Stuchly 1998b). In this method, the magnetic field sources were characterized as a magnetic vector potential term in the electric field, and the computational domain was discretized with the FEM. 
In addition, the equation for the electric fields was transformed into a scalar potential form, which then was solved using finite differences. The SPFD method was used to model induced electric fields and current densities during the exposure to ELF magnetic fields. First, Stuchly's group at University of Victoria developed and used simple geometric shapes, spheres and ellipsoids, to assess the accuracy of the SPFD method against analytic solutions. They applied detailed calculations to an anatomically MRIbased human model with voxels of 3.6 and $7 \mathrm{~mm}$ exposed to uniform magnetic field.

Dawson and Stuchly (1998c) presented the results of the calculation in terms of the effect of skeletal muscle anisotropy on the induced current densities and electric fields under conditions of $60 \mathrm{~Hz}$ uniform magnetic field exposures. As they mentioned in their research paper, the numerical estimations were based on an isotropic conductivity model for all body components. In reality, the anisotropy of biological tissues, skeletal muscle, should affect the estimation of induced electrical values. The comparison was made between the magnetic induced electrical quantities in the full human body model under several assumptions of anisotropy ratios and those in the isotropic case. The anisotropy of skeletal muscle is the key factor in the numerical modeling of the human body.

In order to compare the dosimetry of a child and that of an adult and to evaluate the accuracy of linear scaling of organ dosimetry between species, Dawson, Caputa, and Stuchly (2002b) evaluated electric fields in high-resolution, anatomically based inhomogeneous models of a human male adult (77-kg weight, $175-\mathrm{cm}$ length, $3.6-\mathrm{mm}$ voxel size). Other models were a male child $(17 \mathrm{~kg}, 110 \mathrm{~cm}, 3.2 \mathrm{~mm})$, a male rat $(591 \mathrm{~g}$, $27.4 \mathrm{~cm}, 0.8 \mathrm{~mm})$, a female rat $(284 \mathrm{~g}, 23.8 \mathrm{~cm}, 0.8 \mathrm{~mm})$, a male mouse $(45 \mathrm{~g}, 11.6 \mathrm{~cm}$, $0.34 \mathrm{~mm})$, and a female mouse $(23 \mathrm{~g}, 8.8 \mathrm{~cm}, 0.35 \mathrm{~mm})$ exposed to $60 \mathrm{~Hz}, 1-\mu \mathrm{T}$ uniform magnetic fields with three different orientations. The calculation included the tissue average, voxel maximum, and 99th percentile values of the electric fields and current densities in organs. Organs included the brain, fat, liver, and lung. The numerical method was based on the quasistatic approximation. The averaged and 99th percentile values of the induced electric fields in the child's brain exposed to $1 \mu \mathrm{T}$ from side to side were $9.38 \mu \mathrm{V} / \mathrm{m}$ and $26.8 \mu \mathrm{V} / \mathrm{m}$, respectively. The conclusions are (1) child-to-adult and mouse-to-rat organ dosimetry shows linear dependence on the geometric scale factor between models and (2) postural and individual organ differences have a significant effect when mouse and human child models are compared. The latter conclusion attracts much attention to scaling-based extrapolation of rodent experimental results versus results of human models.

Stuchly and Gandhi (2000) compared the induced electric fields and current densities during exposure to $60 \mathrm{~Hz}$ electric and magnetic fields on different models from three laboratories. The Utah group used the FDTD method scaled in frequency on 6-mm resolution of their phantom model. The Uvic group used the quasistatic hybrid SPFD on $7.2-\mathrm{mm}$ resolution of their model and the SPFD on $3.6-\mathrm{mm}$ resolution. The averaged electric field in the brain in the phantom model for $60 \mathrm{~Hz}, 1 \mathrm{mT}$ from front to back was $11.5 \mathrm{mV} / \mathrm{m}(\sigma=0.17 \mathrm{~S} / \mathrm{m})$, and $10.6 \mathrm{mV} / \mathrm{m}(\sigma=0.11 \mathrm{~S} / \mathrm{m})$ for the Uvic model with $3.6-\mathrm{mm}$ resolution. They showed that for the average induced electric fields, maximum differences were as low as $60 \%$, and for the average current densities, maximum differences were up to $110 \%$. They concluded that the differences between 
two laboratories could be explained by factors such as the accuracy of the numerical method, dielectric properties, conductivity of the organ, human model differences, and voxel size.

In order to compare the computational efficiency between SPFD and IM, Dimbylow (1998) calculated current densities in a fine-resolution $(2 \mathrm{~mm})$, anatomically realistic voxel phantom called NORMAN with a height of $1.76 \mathrm{~m}$ and a mass of $73 \mathrm{~kg}$ exposed to uniform magnetic fields at frequencies ranging from $50 \mathrm{~Hz}$ to $10 \mathrm{MHz}$. In NORMAN, there were 8.3 million voxels in the body, differentiated into 38 tissue types. Using the same voxel size, the SPFD method required $14 \%$ less memory and was much quicker than IM (1.5-11 times faster). Two calculation methods at $50 \mathrm{~Hz}$ agreed with each other within 2\% for AP (applied from front), 3\% for LAT (applied from the side), and 1\% for TOP (applied from above) orientation.

Barchanski et al. (2005a, 2006a, 2007) extended the classical SPFD approach to the extended SPFD (Ex-SPFD) method. This Ex-SPFD method can treat the arbitrarily shaped, time harmonic, magnetic field source and highly conductive material inside the computational domain. This method was applied to compute the induced currents inside a human body model exposed to electric blankets. Very high resolution was needed to model the human body because of the geometrical complexity. This human model, Hugo model, based on the Visible Human Project represents a 38-year-old male (187- $\mathrm{cm}$ height and 113-kg weight), offers a voxel resolution ranging from $8 \times 8 \times 8 \mathrm{~mm}^{3}$ to $1 \times 1 \times 1 \mathrm{~mm}^{3}$, and has a total of 32 different tissue types. This human model was discretized with 200 million grid cells, which correspond to a resolution of $1.5 \mathrm{~mm}$. The authors proposed a new method for calculating current density distribution within this human model using FIT. This new method may be performed with higher accuracy due to an increased local grid resolution only in the areas of interest in the human voxel model. As an application example, they simulated the current densities induced by an electric blanket with two different configurations that generate magnetic fields of 73.7 and 78.1 $\mu \mathrm{T}$. The induced current densities in the whole body due to the magnetic field originating from two electric blankets are $7.02 \times 10$ and $3.13 \times 10^{-4} \mathrm{~mA} / \mathrm{m}^{2}$. In order to resolve small organs, they refined a local grid for ELF current calculation, which allows one to finely resolve areas of particular interest in three-dimensional human anatomy models (Barchanski et al. 2006b). Eberdt, Brown, and Lazzi (2003) developed two-dimensional, SPICE-linked, multisolution IM for ELF electromagnetic interactions. The IM with high-resolution models has been hampered by the time required to solve the equations. The authors have implemented a multiresolution, two-dimensional mesh generation scheme for the IM to reduce the number of equations. Then, the proposed method can be linked with SPICE (circuit simulator) and can be applied to threedimensional problems.

In order to clear the uncertainty in calculation, Caputa et al. (2002) presented a comparison of anatomically realistic human models and numerical codes in ELF magnetic fields. Using models from the University of Victoria, Uvic model, and NRPB, NORMAN model, an evaluation has been performed for uniform $60 \mathrm{~Hz}$ magnetic fields. The comparison of average $\left(E_{\text {avg }}\right)$, maximum $\left(E_{\max }\right)$, and 99th percentile $\left(E_{99}\right)$ electric fields for all the organs in the models was made. The effect of model size, shape, and resolution with the conductivity values kept constant was also given. These approaches 
hope to confirm the reliability of numerical modeling. The comparisons of induced electric fields for all the organs show differences of $1 \%$ or less for the majority of tissues (e.g., liver, stomach, and muscle). In a few cases, the difference reaches $2 \%-3 \%$ (e.g., skin, spinal cord, and breast). The effects of body model size and shape including anatomy and resolution on the average $\left(E_{\mathrm{h}}\right)$, voxel maximum $\left(E_{\max }\right)$, and 99th percentile $\left(E_{99}\right)$ of the induced electric fields are also investigated.

Hirata et al. (2009) studied the in situ electric fields and induced current densities in anatomically based Japanese adult male and female models, TARO and HANAKO, exposed to $50 \mathrm{~Hz}, 0.1-\mathrm{mT}$ magnetic fields. Using a quasistatic FDTD method, they computed two electrical quantities. As an exposure scenario, three orientations of magnetic fields to the human model standing in free space were considered: AP, LAT, and TOP. The computational results showed that the 99th percentile values of the in situ electric fields and the current densities averaged over an area of $1 \mathrm{~cm}^{2}$ of the nerve tissues in HANAKO were $35 \%$ and $25 \%$ lower, respectively, than those in TARO. They pointed out that the two quantities induced in the Japanese models are smaller than those for European models due to the difference in anatomical modeling. Hirata et al. (2010a) published an interesting paper on the comparison of induced quantities in a human model for ELF magnetic fields. They provided an intercomparison of the in situ electric fields and current densities in an anatomically based Japanese adult male model named TARO for uniform magnetic field exposure at $50 \mathrm{~Hz}$. A total of six Japanese research groups, CRIEPI, NICT, Utsunomiya University (UU), Takamatsu National College of Technology (TNCT), Kyoto University (KU), and Nagoya Institute of Technology (NIT), participated in this research. They used four different computational methods: IM (CRIEPI and NICT), SPFD method (TNCT and UU), fast-multipole surface-difference time-domain method (KU), and quasistatic FDTD method (NIT). The phantom model was standing in free space and was exposed to three orientations of magnetic fields with $0.1 \mathrm{mT}$ : AP, LAT, and TOP. Differences in the maximum and 99th percentile value of the in situ electric fields were less than $30 \%$ and $10 \%$ except for the result of one group. The current density averaged over $1 \mathrm{~cm}^{2}$ for the central nervous system (CNS) tissue is $10 \%$ or less except for the result of one group. It is suggested that the computational uncertainty in the induced current densities and in situ electric fields due to different calculation methods and coding is smaller than that caused by different models and conductivities of tissues. This result is similar to that of Stuchly and Gandhi (2000).

It is very important to calculate the induced current densities and electric fields inside human bodies exposed to ELF magnetic fields generated from electric appliances, transmission lines, and electric power facilities. These sources generate highly nonuniform magnetic fields. For example, workers on the transmission line are exposed to highly nonuniform magnetic fields. Several investigations on nonuniform magnetic fields from domestic and industrial devices have been done (Cheng et al. 1995; Gandhi et al. 2001; Iivonen et al. 2005; Iivonen and Sarvas 2007; Iivonen and Laakso 2009; Nishizawa et al. 2004; Nishizawa, Landstorfer, and Kamimura 2007; Park and Min 2008; Ruoss et al. 2001; Scorretti et al. 2005; Tarao, Hayashi, and Isaka 1997, 1998, 2003; Tofani et al. 1995a,b; Yamazaki and Kawamoto 2001b, 2005, 2007). Tofani et al. (1995b) measured and analyzed the magnetic fields from domestic appliances such as a razor, hair dryer, and drill. Each appliance has high-frequency components. For example, the harmonic 
contents are up to $96 \mathrm{kHz}$ for six razors, up to $3.4 \mathrm{kHz}$ for three hair dryers, and up to $8.6 \mathrm{kHz}$ for three drills. They examined, by IM, the coupling of the highly localized magnetic fields with harmonic components with the induced current densities in the $1.31 \mathrm{~cm}$ resolution human head model. The averaged current densities were from about $2-27 \mu \mathrm{A} / \mathrm{m}^{2}$ for the razor with highest contribution of $8 \mathrm{kHz}$, from 0.3 to $2.5 \mu \mathrm{A} / \mathrm{m}^{2}$ for the hair dryer with $3.4 \mathrm{kHz}$, and from 0.8 to $5.8 \mu \mathrm{A} / \mathrm{m}^{2}$ for the drill with $100 \mathrm{~Hz}$. The application to nonuniform magnetic fields for a transmission line can be found in several papers (Dawson, Caputa, and Stuchly 1999a,b; Stuchly and Zhao 1996). In these cases, the realistic human models based on working configurations are postured around a transmission line, and it is speculated that the highest exposure level occurs for nonuniform magnetic fields such as working close to the conductor.

Nadeem et al. (2004) measured the magnetic fields from a spot welding machine and calculated the induced body currents in a full three-dimensional human model with 3-mm resolution obtained from the Visual Human Project in order to determine whether the ICNIRP basic restriction is exceeded or not. The spot welding machine had magnetic fields of up to $4 \mathrm{mT}$ at the operator position and an almost $50 \mathrm{~Hz}$ waveform. They calculated the induced current densities using IM. The maximum induced current densities at an operator position of $34 \mathrm{~cm}$ away from the machine were below ICNIRP basic restriction. The basic restriction was exceeded if the operator was closer to the machine. By applying the BEM, Park and Min (2008) calculated current densities induced inside a human body model with spheroids or cylinders with several organs. This human body model was simulated for AC arc welder workers. The arc welder of 200-300 A was used for simulation, with $1000 \mathrm{~A}$ applied to the worst case. The condition for calculation was locating the human body model 1-20 cm away from the power cable of the welder. The maximum current densities were calculated on the surface of the heart, and when working within $15 \mathrm{~cm}$, this value may exceed ICNIRP basic restriction. The complex waveforms from the source of pulsed electric and magnetic fields in the working place was reviewed and analyzed (Cooper 2002; Crotti and Giordano 2009; Jokela 2000, 2007).

Dawson, Caputa and Stuchly evaluated the dosimetry for live-line workers exposed to $50 \mathrm{~Hz}$ magnetic fields from high-voltage transmission lines (Dawson, Caputa, and Stuchly 2002a). The dosimetry includes the calculations of the electric fields and current densities induced in tissue and the current densities averaged over $1 \mathrm{~cm}^{2}$. Scenarios include three worker postures for the twin and triple bundles, and four postures for the quadruple bundle. The human models comprising cubic voxels with 3.6-mm edges were derived from the basic upright body posture (Dawson, Moerloose, and Stuchly 1997a). Three to four positions of the worker's body have been simulated for each conductor bundle. The basic restriction of ICNIRP guidelines is $10 \mathrm{~mA} / \mathrm{m}^{2}$ for occupational exposure. From calculation with each scenario, the current densities are exceeded only for some exposure scenarios and a load of $1 \mathrm{kA}$ per conductor. They pointed out that the highest exposure levels very close to the high-voltage transmission line are for nonuniform magnetic fields in occupational scenarios (Stuchly and Dawson 2002) and introduced the simplified method to estimate the induced electrical quantities for four nonuniform magnetic field exposure scenarios by using quasistatic approximation techniques. In two of four configuration scenarios, the conductors were separated by $45.72 \mathrm{~cm}$ with 
carrying of $500 \mathrm{~A}$ (rms) of each conductor. The third scenario was the maintenance condition in an underground vault. Conductors were separated $5.08 \mathrm{~cm}$ on-center, with one cable pair and bus bar for each conductor. The last scenario was the inspection of isophase buses of 700-MW generators. In this case, there were 11 conductors. The lower conductors were located $1.2 \mathrm{~m}$ above ground, and the upper conductors were $3 \mathrm{~m}$ above ground. The conductors were $0.9 \mathrm{~m}$ apart. Depending on the actual exposure scenarios and using the proposed simple method for estimation of induced electric fields, the estimated dose for most organs is conservative.

In the computational approach, the human body model was constructed by discretization into small cells, voxels, with body tissue. Each tissue was generally assigned conductivity and permittivity values of the human tissues. In calculations in the ELF region, conduction current was considered and displacement currents were neglected because of the small magnitude of permittivity. Barchanski et al. (2005b) checked these assumptions using an anatomically realistic human body model, Hugo model, based on the Visible Human representing a 38-year-old male (187-cm height and 113-kg weight) in the frequency range of $10 \mathrm{~Hz}$ to $1 \mathrm{MHz}$. The conclusion is, by FIT, that the impact of displacement currents on the electric fields is independent of model resolution. At $50 \mathrm{~Hz}$, errors are $1 \%$ for the averaged electric fields and $2 \%$ for the maximum electric fields. They emphasized that there existed some uncertainties concerning the model and the dielectric tissue data in the ELF region and the research needed more accurate measurements.

An interesting calculation of induced currents in rat was done by Wake, Tanaka, and Taki (2000). They compared the relationship between the induced current densities in pineal gland and retina of a rat and polarity of magnetic field exposure. Induced currents in two MRI-based numerical rat models with resolutions of up to $0.125 \mathrm{~mm}^{3}$ were calculated by the IM. The authors showed that calculated induced current densities in the whole body were extremely small, $<30 \mu \mathrm{A} / \mathrm{m}^{2}$ in the whole body and $<2 \mu \mathrm{A} / \mathrm{m}^{2}$ in the pineal gland and the retina for both linearly and circularly polarized magnetic fields. Because of the polarization in a vertical plane, both linearly and circularly polarized magnetic fields with the same strength had no significant difference in amplitude or polarization of induced currents in the pineal gland. However, the magnetic fields rotating in the horizontal plane produced the most circularly polarized currents both in the pineal gland and in the retina. Wake et al. (1998) investigated the induced current density distributions in the heterogeneous human head model in magnetophosphenes. Magnetophosphenes is the flickering sensations of light when the human being is exposed to a changing magnetic field. They applied a magnetic field of $20 \mathrm{~Hz}, 5 \mathrm{mT}$ to the center of the retina. The induced current density distributions throughout the head were calculated. The maximum induced current density was estimated to be $11 \mathrm{~mA} / \mathrm{m}^{2}$ in the retina and the induced electric field was $7.3 \times 10^{-3} \mathrm{~V} / \mathrm{m}$. Electrophosphene is also the flickering sensations of light when electric currents pass through the eye to induce currents near the retina. Dosimetric studies for electrophosphenes at ELF electric fields could be done to estimate the current densities and internal electric fields. The thresholds of electrophosphene in the internal electric field in the retina are $200-220 \mathrm{mV} / \mathrm{m}$. The induced current density depends on model conductivity (Carstensen et al. 1985; Lindenblatt and Silny 2002; Taki, Suzuki, and Wake 2003). 


\subsubsection{Coupling with Other Models}

Tenforde (1992) mentioned that an important aspect of understanding the possible effects of ELF EMF on living systems is the analysis of ionic and molecular pathways involved in the interaction of these fields at the cellular and subcellular levels, called microscopic dosimetry. The interactions between the applied magnetic fields and living systems depend on the induced electric fields and currents. So, microdosimetry is used to characterize the induced electric fields and currents at a cellular level when cells are exposed to magnetic fields. There have been several studies on the calculation of the induced electric fields and currents in biological cells exposed to ELF EMF. Hart (1996) showed the current densities and electric field distributions induced in the simplified two-dimensional cell cultures in a petri dish exposed to ELF magnetic fields. In this case, the cells were regarded as conducting squares surrounded by insulating membranes. The gap junctions were considered to connect the interiors of adjacent cells. For vertically applied magnetic fields only, the induced currents and the electric field distributions may deviate from the homogeneous medium model when the cells bind tightly with each other. The presence of a gap junction can produce large transmembrane electric fields or intracellular current densities. Using three-dimensional IM, Stuchly and Xi (1994) computed the average magnetic field induced electric fields and currents of two simplified, more realistically modeled cells placed in a petri dish containing culture medium. They considered a cell monolayer with a random distribution of different cell densities and a cell monolayer with gap junctions. In this study, biological cells were represented by cubes due to the limitations of the computational method. The cell monolayer with random distributions showed that the pattern of the induced current flow for higher cell densities had a limited dependence on the size and shape of the cell container. Perturbations in the spatial distribution of the induced currents were observed when the cells bound with each other in a monolayer. The authors pointed out that the cell densities and cell positions in the culture dish were important factors in determining the induced current distribution. Gap junctions increased the current densities only when their resistance was sufficiently low. The authors emphasized that these results highlighted the complexities involved the determination of the induced current patterns and magnitudes even in a simple preparation of biological cells. This was nearly similar to the results obtained by Hart (1996). These results give insights into macroscopic dosimetry.

Hassan et al. (2003) calculated the induced current perturbations in the vicinity of excitable cells exposed to ELF magnetic fields. In order to calculate the current densities, realistic three-dimensional cell models were used. Three cell morphologies, single spherical adrenal chromaffin cells, single elongated smooth muscle cells, and chromaffin cells, were modeled and calculated using FEM. For a spherical cell, the alterations of the magnitude and spatial distribution of the induced current densities in the immediate vicinity of the cells were obtained. For an elongated cell, cell orientation with respect to the direction of the induced currents highly influenced the induced current densities. These results gave insights into how magnetic field induced electric fields can be applied to target specific cellular processes at the whole tissue level. A simpler model developed by Tarao, Hayashi, and Isaka $(2000,2001)$ was used to calculate the distributions and characteristics of the induced currents in the modeled structure 
by using a semianalytical solution method. In this study, the model was a biological trimedium spherical structure composed of tissues, their membranes, and extra tissues. The results showed that both change in the thickness and electric properties of bimembrane and the difference in the conductivities significantly affected the current passing through the membrane, which resulted in the induced currents.

\subsubsection{Extremely Low-Frequency Electromagnetic Field}

It is possible to treat exposure to electric and magnetic fields separately in the ELF region. If a human stands near and/or under a transmission line, the magnetic fields generate electric fields and electric currents within the human body. The magnitudes of the induced electric fields and the current densities are proportional to the radius of the loop, the electrical conductivity of the tissue, and the rate of change and magnitude of the magnetic flux density. The electric fields generated from the transmission line are vertically polarized, and the current flows in the body from the head, through the torso, to the feet. The EMF at the ELF region has a quasistatic nature, and exposure to electric and magnetic fields is considered separately. In the case of simultaneous exposure to ELF EMF, the induced electrical quantities can be superimposed (Olsen 1996). Exposure to ELF EMF results in no temperature rise and no power absorption inside the body.

Table 4.5 shows the examples of the calculated and measured current densities in simple and anatomical voxel human models exposed to EMF.

Spiegel (1976) calculated the ELF EMF coupling to homogeneous spherical models of human and animals. He suggested that spherical dielectric models of humans or animals could be expected to provide the degree of the right order of magnitude of induced currents, induced electric fields, and power absorption inside models from exposure to the proposed Navy's Sanguine antenna and extra high voltage (EHV)/ultra high voltage (UHV) transmission lines. As an analytical solution, the induced electric fields inside a dielectric sphere are the summation of the quasistatic solution by electric and magnetic fields. The results showed, based on the validity of the spherical models, that neither the Sanguine antenna nor the EHV/UHV transmission lines produced body current or body heat levels to interfere with normal biological function of the human body.

Hart and Marino (1982) published a paper on the calculation of electric and magnetic couplings to a homogeneous, grounded hemiellipsoid model exposed to a high-voltage transmission line. They mentioned that the coupling depends strongly on the conductivity and shape of the model. The calculation of induced electric fields and current densities was conducted for a live-line worker exposed to ELF EMF generated by highvoltage transmission line. This is the scenario for occupational exposure, and basically is nonuniform exposure fields.

Using the FEM, Hoang et al. (2009) computed the induced currents in a heterogeneous human body model exposed to both the electric and magnetic fields generated by a three-phase transmission line. A human body model (named ZOL) composed of 17 different tissues was built by using the segmented data of the Visible Human Project. Furse and Gandhi (1998) used the FDTD method at $10 \mathrm{MHz}$ utilizing the conductivities corresponding to $60 \mathrm{~Hz}$ to calculate the electric fields and currents induced in the $6-\mathrm{mm}$ version of the Utah model and scaled to $60 \mathrm{~Hz}$. 
TABLE 4.5 Examples of Calculated and Measured Current Densities in Human Models Exposed to Electromagnetic Fields

\begin{tabular}{|c|c|c|}
\hline Author & Current Density $\left(\mathrm{mA} / \mathrm{m}^{2}\right)$ & Method and Comments \\
\hline $\begin{array}{l}\text { Bottauscio and Conti } \\
\text { (1999) }\end{array}$ & $\begin{array}{l}\text { Calculated maximum values: } 0.13 \\
\text { in the brain for MF and } 0.17 \text { for } \\
\text { EF beneath power line: } 2.7 \text { for } \\
0.19-0.51 \mathrm{mT} \text { (TOP) during } \\
\text { live-line working on power line }\end{array}$ & $\begin{array}{l}50 \mathrm{~Hz}, 380-\mathrm{kV} \text { transmission line } \\
(2.6-3 \mathrm{kV} / \mathrm{m}, 16-21 \mu \mathrm{T}): \mathrm{BEM} \\
\text { calculation for human body model } \\
\text { with main organs exposed to EMF } \\
\text { beneath a transmission line and } \\
\text { exposed to MF in the proximity of a } \\
\text { conductor of a transmission line }\end{array}$ \\
\hline $\begin{array}{l}\text { Bottauscio, Chiampi, } \\
\text { and Zilberti (2009) }\end{array}$ & $\begin{array}{l}\text { The electric field is the prevalent } \\
\text { source for the induced current } \\
\text { density when the human body } \\
\text { model is placed in position A. }\end{array}$ & $\begin{array}{l}50 \mathrm{~Hz} \text {, three-phase } 380-\mathrm{kV} \\
\text { transmission line, balanced } 1500 \mathrm{~A} \\
\text { (scenario A: human body is placed } \\
2 \mathrm{~m} \text { from the closed phase; } \mathrm{B}: \\
\text { positioned below the central bar): } \\
\text { three-dimensional BEM calculation } \\
\text { for homogeneous axisymmetric } \\
\text { human model (reference human } \\
\text { body in IEC standards) }\end{array}$ \\
\hline $\begin{array}{l}\text { Cech, Leitgeb, and } \\
\text { Pediaditis (2008) }\end{array}$ & $\begin{array}{l}\text { Calculated maximum values: } \\
\text { 10.1-10.3 for simultaneous EF } \\
\text { and MF exposure to grounded } \\
\text { model }\end{array}$ & $\begin{array}{l}50 \mathrm{~Hz}, 5 \mathrm{kV} / \mathrm{m}, 100 \mu \mathrm{T} \text { : FIT } \\
\text { calculation for whole body, } \\
\text { MRI-based, 2-mm voxel resolution, } \\
\text { pregnant woman model }\end{array}$ \\
\hline $\begin{array}{l}\text { Dawson, Caputa, and } \\
\text { Stuchly }(1997 c)\end{array}$ & $\begin{array}{l}\text { Calculated average values: } \\
2.78 \times 10^{-3} \text { for LAT, } 3.37 \times 10^{-3} \\
\text { for AP, and } 2.34 \times 10^{-3} \text { for TOP in } \\
\text { full body; } 4.28 \times 10^{-3} \text { for LAT, } \\
5.22 \times 10^{-3} \text { for AP, and } 3.57 \times 10^{-3} \\
\text { for TOP in muscle }\end{array}$ & $\begin{array}{l}60 \mathrm{~Hz}, 1 \mu \mathrm{T} \text { (AP, LAT, and TOP): } \\
\text { SPFD calculation for anatomical, } \\
\text { MRI-based, } 3.6-\mathrm{mm} \text { resolution } \\
\text { human body model }\end{array}$ \\
\hline Dimbylow (2005) & $\begin{array}{l}\text { Calculated average values in the } \\
\text { retina: } 0.217 \text { in grounded } \\
\text { MAOMI (EF); } 0.13 \text { in } \\
\text { ungrounded }(\mathrm{EF}) ; 2.71 \text { for } \mathrm{AP}, \\
4.89 \text { for LAT, and } 2.79 \text { for TOP }\end{array}$ & $\begin{array}{l}50 \mathrm{~Hz}, 1 \mathrm{kV} / \mathrm{m}, 1 \mathrm{mT} \text { : SPFD } \\
\text { calculation for MRI-based, 2-mm } \\
\text { resolution voxel female model, } \\
\text { NAOMI }\end{array}$ \\
\hline Dimbylow (2006) & $\begin{array}{l}\text { Fetal calculated values averaged } \\
\text { over } 1 \mathrm{~cm}^{2}: 2.21 \text { in the brain at } 26 \\
\text { weeks for AP, } 2.92 \text { for LAT, } 1.89 \\
\text { for TOP, For Naomi; } 2.98 \text { in the } \\
\text { brain for AP, } 4.89 \text { in the retina for } \\
\text { LAT and } 2.79 \text { in the retina for } \\
\text { TOP }\end{array}$ & $\begin{array}{l}50 \mathrm{~Hz}, 1 \mathrm{kV} / \mathrm{m}, 1 \mathrm{mT} \text { : SPFD } \\
\text { calculation for whole-body, } \\
\text { MRI-based, 2-mm resolution hybrid } \\
\text { voxel-mathematical models of the } \\
\text { pregnant female, NAOMI }\end{array}$ \\
\hline Dimbylow (2010) & $\begin{array}{l}\text { Calculated maximum value: } 0.32 \\
\text { for EF, the Virtual Family, female, } \\
\text { Ella, and } 7 \text { for MF TARO }\end{array}$ & $\begin{array}{l}50 \mathrm{~Hz}, 1 \mathrm{kV} / \mathrm{m}, 1 \mathrm{mT} \text { : Models: } \\
\text { NORMAN, NAOMI, TARO, } \\
\text { HANAKO, the Virtual Family: SPFD } \\
\text { and quasistatic potential equation } \\
\text { method calculation for various } \\
\text { postures }\end{array}$ \\
\hline
\end{tabular}

(Continued) 
TABLE 4.5 Examples of Calculated and Measured Current Densities in Human Models Exposed to Electromagnetic Fields (Continued)

\begin{tabular}{|c|c|c|}
\hline Author & Current Density $\left(\mathrm{mA} / \mathrm{m}^{2}\right)$ & Method and Comments \\
\hline Hoang et al. (2009) & $\begin{array}{l}\text { Calculated maximum values: } 9.86 \\
\text { for muscle }\end{array}$ & $\begin{array}{l}50 \mathrm{~Hz} \text {, balanced three-phase } \\
\text { transmission line }(63 \mathrm{kV}, 510 \mathrm{~A}) \text { : } \\
\text { FEM calculation in heterogeneous } \\
\text { human model, named ZOL from the } \\
\text { Visible Human Project }\end{array}$ \\
\hline King and Wu (1995) & $\begin{array}{l}\text { The total axial current, the current } \\
\text { and power densities in the } \\
\text { interior of a human body are } \\
\text { determined; the current and } \\
\text { power densities are so small that } \\
\text { the thermal effects can be ignored }\end{array}$ & $\begin{array}{l}60 \mathrm{~Hz}, \mathrm{EF} \text { and MF of three-phase } \\
\text { transmission line: analytical } \\
\text { calculation for grounded and } \\
\text { ungrounded human model }\end{array}$ \\
\hline $\begin{array}{l}\text { King and Sandler } \\
\text { (1996) }\end{array}$ & $\begin{array}{l}\text { The electric fields do not induce } \\
\text { large currents and fields in a } \\
\text { nerve axon. }\end{array}$ & $\begin{array}{l}\text { Three-phase transmission line; } \\
\text { cylindrical conducting human body } \\
\text { model }\end{array}$ \\
\hline King (1997) & $\begin{array}{l}\text { The current in the body is } \\
\text { significantly increased as the } \\
\text { arms are raised. }\end{array}$ & $\begin{array}{l}60 \mathrm{~Hz}, 220-\mathrm{kV}, 440-\mathrm{kV} \text { transmission } \\
\text { line: analytical solution for } \\
\text { cylindrical conducting human body } \\
\text { model when both arms are raised }\end{array}$ \\
\hline King (1999b) & $\begin{array}{l}\text { The electric field and currents in a } \\
\text { spherical cell and its membrane }\end{array}$ & $\begin{array}{l}60 \mathrm{~Hz} \text {, analytical solution for a } \\
\text { spherical cell }\end{array}$ \\
\hline
\end{tabular}
are determined rigorously in terms of the electric fields induced in the body; in a spherical cell, the interior is effectively shielded by the membrane.
King (2004) The axial currents induced in cylinder is a good approximation of the current induced in an actual body.
Leitgeb and Cech (2008)

Maalej et al. (2009)

Matsumoto et al. (2000)
Total current density is determined by vertical EF and horizontal MF; horizontal MF has an important effect on the total induced current density distributions
$60 \mathrm{~Hz}$, analytical method calculation for spherical, cylindrical, and ellipsoidal human models

$50 \mathrm{~Hz}, 5 \mathrm{kV} / \mathrm{m}, 100 \mu \mathrm{T}$ : FIT calculation for MRI-based, anatomical, 3-mm voxel human model

$60 \mathrm{~Hz}$, live-line worker standing $2 \mathrm{~m}$ away from conductor of doublecircuit $132-\mathrm{kV}$ transmission line (6.2-6.5 kV/m, 63-66.5 $\mu \mathrm{T})$ : FDTD calculation for anatomical, MRIbased human body model from the Visible Human Project

$60 \mathrm{~Hz}, 500 \mathrm{kV}(1 \mathrm{kV} / \mathrm{m}, 1-5 \mu \mathrm{T})$ : FEM calculation for homogeneous grounded and ungrounded prolate spheroid model 
TABLE 4.5 Examples of Calculated and Measured Current Densities in Human Models Exposed to Electromagnetic Fields (Continued)

\begin{tabular}{|c|c|c|}
\hline Author & Current Density $\left(\mathrm{mA} / \mathrm{m}^{2}\right)$ & Method and Comments \\
\hline Min and Song (2006b) & The maximum values: less than 10 & $\begin{array}{l}60 \mathrm{~Hz}, 765-\mathrm{kV} \text { transmission line } \\
\text { worker BEM calculation for } \\
\text { spheroidal or cylindrical human } \\
\text { models with several organs }\end{array}$ \\
\hline Spiegel (1976) & $\begin{array}{l}\text { Calculated maximum values: } 0.1 \\
\text { for EHV transmission line and } \\
0.01 \text { for Sanguine fields }\end{array}$ & $\begin{array}{l}60 \mathrm{~Hz}, \mathrm{EHV} \text { transmission line and } \\
45 / 75 \mathrm{~Hz} \text { Sanguine antenna: } \\
\text { quasistatic analytical solution } \\
\text { calculation in homogeneous } \\
\text { spherical human model }\end{array}$ \\
\hline $\begin{array}{l}\text { Valič, Gajšek, and } \\
\text { Miklavčič (2009) }\end{array}$ & $\begin{array}{l}\text { The implant increased the current } \\
\text { density up to } 9.5 \text { in the model } \\
\text { with implant, } 0.9 \text { in the model } \\
\text { without implant }\end{array}$ & $\begin{array}{l}50 \mathrm{~Hz}, 5 \mathrm{kV} / \mathrm{m}, 100 \mu \mathrm{T} \text { : FDTD } \\
\text { calculation in human female body } \\
\text { model with a conductive implant }\end{array}$ \\
\hline $\begin{array}{l}\text { Xue, Wood, and Dovan } \\
(2004)\end{array}$ & $\begin{array}{l}\text { Calculated maximum values: } 2.37 \\
\text { in fetus and } 14.97 \text { in placenta } \\
(0.3 \mathrm{~m} \text { from a line }) .\end{array}$ & $\begin{array}{l}50 \mathrm{~Hz}, 1000 \text { A carrying power line, } \\
\text { analytical method calculation for } \\
\text { homogeneous axisymmetric } \\
\text { pregnant body model consisting of } \\
\text { cylinder and ellipsoid }\end{array}$ \\
\hline
\end{tabular}

AP, applied from front; LAT, applied from side; TOP, applied from above; SPFD, scalar potential finite difference; MF, magnetic field; EF, electric field; EMF, electromagnetic field; IEC, International Electrotechnical Commission; FIT, Finite-Integration Technique; EHV, extra high voltage.

A series of papers by King and coworkers have been published (King and Wu 1995; King and Sandler 1996; King 1997, 1998a,b, 1999a,b,c, 2004; King and Wu 1998). The induced electric fields and induced currents in the conductive human body model with spherical, ellipsoidal, and cylindrical shapes are determined by analytical method. First, the distribution of EMF of a three-phase transmission line was calculated in analytical form. Under these conditions, the total axial currents and power densities inside a human body model standing on the ground under or near the transmission line were determined. The fields were very low and the currents and power densities so small that the thermal effect could be ignored (King and $\mathrm{Wu}$ 1995). In addition to the transmission line, electric fields and induced currents were calculated for the human body model exposed to a very low-frequency (VLF) (10-30 kHz) transmitter (King and Sandler 1996). In calculating the induced currents at the ELF and VLF regions, it is important to note that all organs of the body are conductors and not dielectrics. Further, the calculation was extended to the case where both arms of the human body model with grounding or ungrounding were raised by an arbitrary angle (King 1997). As a special case, King (1999c) calculated analytically the electric fields induced in the human body model standing on the metal deck of a ship near a vertical antenna (1-30 MHz). King (1999b) provided a quantitative relationship between the electric fields and induced currents in a human body exposed to $60 \mathrm{~Hz}$ high-voltage transmission line and the resulting electric fields and induced currents in the membrane and the interior of a spherical cell. In the case of a spherical cell, the cell is effectively shielded by the membrane compared with 
long nerve cell (King and Wu 1998). King (1999a) analyzed the interaction between currents and electric fields induced in the organs of the human body by external electric and magnetic fields and those involved in the propagation of a signal along a nerve axon. His calculation showed that incident $60 \mathrm{~Hz}$ electric fields near high-voltage transmission line did not induce large enough currents and electric fields in a nerve axon in the leg. The long-term study on the electric fields and currents induced in the organs of the human body exposed to $50-/ 60 \mathrm{~Hz}$ transmission line and 10 - to $30 \mathrm{kHz}$ high-power transmitters are summarized (King 1998b). King's research results are limited by the simplistic human model with electrically homogeneous cylinder.

Bottauscio and Conti (1999) developed a 1.8-m-tall human model with internal organs such as the heart, brain, and liver in order to evaluate the induced currents in complex biological structures after exposure to $50 \mathrm{~Hz}$ electric and magnetic fields. The numerical model is based on BEM. In addition, using three-dimensional BEM, Bottauscio, Chiampi, and Zilberti (2009) evaluated the currents induced inside the reference human body proposed in the International Electrotechnical Commission (IEC) standards exposed to ELF EMF. The validation of the model is performed by comparison with analytical methods and FEM. After the confirmation between the numerical and analytical computations, the authors applied this method to calculate the induced current densities in two conditions: an operator is placed nearby a three-phase $50 \mathrm{~Hz}$, $380-\mathrm{kV}$ line voltage bus bar system and the human body is placed both $2 \mathrm{~m}$ from the closer phase (position A) and below the central bar (position B). The current density contributions due to the electric fields are always predominant, and the presence of the magnetic fields gives rise to an increase in the maximum value of the induced currents of $\sim 20 \%$ in position $\mathrm{A}$ and $\sim 30 \%$ in position $\mathrm{B}$.

Using three-dimensional BEM, Min and Song (2006b) analyzed the induced current densities inside a human model working close to the $60 \mathrm{~Hz}, 765-\mathrm{kV}$ double-circuit transmission line. Their human model consisted of several organs (brain, heart, lung, liver, and intestine) and other various parts, and it was spheroidal or cylindrical. They had two calculations of the human body model located inside and outside the lowest phase of $765-\mathrm{kV}$ transmission line. The maximum induced current densities in all organs were less than $10 \mathrm{~mA} / \mathrm{m}^{2}$ for the human model working outside. However, if the worker stayed inside the multiconductor bundle, the induced current densities inside the human body increased to $20.4 \mathrm{~mA} / \mathrm{m}^{2}$ and exceeded the safe level proposed by ICNIRP. In this case, the worker was recommended to locate his legs at lower subconductors.

Myung et al. (2002) calculated the induced currents of a human head model with $1.27-\mathrm{cm}$ resolution due to $60 \mathrm{~Hz}$ magnetic fields around a $765-\mathrm{kV}$ transmission line using IM. Before the calculation of induced currents, they estimated the three-dimensional magnetic field profile around the transmission line with an analytic solution. The induced current in the human head model with $1.27-\mathrm{cm}$ resolution was estimated. The simulated maximum current density in the human head model was $0.10 \mathrm{~mA} / \mathrm{m}^{2}$ when the head was exposed to the magnetic field of $1.67 \mu \mathrm{T}$. With these results, they found that there is a high eddy current intensity around the eyes of human head.

Matsumoto and coworkers computed the induced currents in the homogeneous spherical and prolate spheroid models exposed to $60 \mathrm{~Hz}$ electric and magnetic fields (Matsumoto, Hayashi, and Isaka 1997, 1999; Matsumoto et al. 2000, 2001). After the 
comparison between the induced current densities in the spherical conductive model of parallel electric and magnetic fields and that of orthogonal fields, they suggested that the total induced current densities were affected by the relationship between the magnitude and the phase difference of the mutually orthogonal electric and magnetic fields. The second calculation using grounded and ungrounded homogeneous prolate spheroid human models exposed to concurrent $60 \mathrm{~Hz}$ vertical, $1 \mathrm{kV} / \mathrm{m}$ electric fields and horizontal 1- to 5- $\mu \mathrm{T}$ magnetic fields with different phase angle between them was made. The horizontal magnetic fields had important effects on the total induced current distributions. This distribution varied with the phase difference. Closing to the grounded condition, the contribution of the horizontal magnetic fields tended to decrease.

Dimbylow (2005) developed a female voxel model named NAOMI with a 2-mm resolution. He applied the NAOMI model to the calculations of induced current densities and electric fields due to exposure to ELF magnetic and electric field. He also pointed out that the induced electric field averaged over a volume of $1 \mathrm{~mm}^{3}$ in nerve tissue might be a more appropriate dose quantity for effects. An expert group from HPA (former NRPB) proposed that the restriction of the induced electric field to less than $100 \mathrm{mV} / \mathrm{m}$ is adequate to protect most adult population and that sensitive individuals should be adequately protected at lower induced electric fields, possibly about a factor of 5 lower, i.e., $20 \mathrm{mV} / \mathrm{m}$ (McKinlay et al. 2004). The induced electric field around $100 \mathrm{mV} / \mathrm{m}$ has been identified for its effects on the nervous system. In Dimbylow's study, comparisons were made between induced electric fields from the NAOMI model and those described in the NRPB Document. A hybrid voxel-mathematical model with a resolution of $2 \mathrm{~mm}$ was developed as a pregnant female model (Dimbylow 2006). Mathematical models with fetus developing at the 8th, 13th, 26th, and 38th week of gestation were converted into voxels and combined with NAOMI. This hybrid voxel-mathematical model was used to calculate the electric fields and induced current densities when exposed to ELF EMF. Using the originally developed pregnant female model named SILVY, Cech, Leitgeb, and Pediaditis $(2007,2008)$ calculated the current densities in a pregnant woman model induced by simultaneous $50 \mathrm{~Hz}$ homogeneous electric and magnetic fields. The SILVY model was based on MRI and CT images of a pregnant woman in the 30th week of gestation. This was an anatomical voxel model of an $89-\mathrm{kg}$ woman. The electric current density distributions with this pregnant model were calculated, and the results were compared with basic restriction recommended by ICNIRP guidelines. In the case of homogeneous electric and magnetic field exposures, both fields induced the electric current densities and resultant values were superimposed by vectorial addition. Under worst case conditions of simultaneous exposure, the basic restrictions exceeded ICNIRP guidelines within the CNS of the mother. However, the basic restrictions are not exceeded in sole field exposure. In addition, the induced current densities within the fetus do not comply with basic restrictions.

In more realistic conditions, Xue, Wood, and Dovan (2004) calculated the induced current densities in the fetus of pregnant workers in $50-\mathrm{Hz}$ high magnetic field exposures. In this case, the body model consisted of a cylinder and an ellipsoid with two outer layers. The hemispherically capped cylinder was used to model the trunk of a pregnant woman and the ellipsoid was used to model a fetus. Two layers were amniotic fluid and placenta. In the calculation, four different exposure scenarios were modeled including a worse case: the body of the pregnant woman was at a distance of $30 \mathrm{~cm}$ from 
the conductor. One was the case of exposure to uniform $10 \mathrm{kV} / \mathrm{m}$ electric fields, and the other three were different magnetic field exposures including nonuniform conditions. This result showed that for pregnant workers, the induced current densities might be greater than the basic restrictions of occupational exposure recommended by ICNIRP, $10 \mathrm{~mA} / \mathrm{m}^{2}$, and the induced current densities for their fetuses might be greater than the basic restrictions of public exposure.

Dimbylow and Findlay (2010) pointed out that the analysis of the interaction of ELF EMF with the body has used adult voxel models in standard standing posture, and changes in posture and size of the body can alter the distribution of the induced current densities. From these speculations, the effects of body posture, anatomical variations, age difference, and pregnancy on the induced current densities from $50 \mathrm{~Hz}$ electric and magnetic fields were considered in an extensive ensemble of voxel models. For the calculations, there were six adult models, eight child models, and seven pregnant female models including NAOMI at various stages of gestation. The SPFD method was used to calculate the induced current densities from uniform $50 \mathrm{~Hz}$ magnetic fields, and the quasistatic potential equation was applied to calculate the induced current densities for $50 \mathrm{~Hz}$ electric fields. The maximum value of $0.32 \mathrm{~mA} / \mathrm{m}^{2}$ for $1 \mathrm{kV} / \mathrm{m}$ occurred in the Virtual Family female, Ella. The authors showed that this value would require an external $6.25 \mathrm{kV} / \mathrm{m}$ to produce basic restriction of $2 \mathrm{~mA} / \mathrm{m}^{2}$ for public exposure. For magnetic fields, the maximum value from all the models was $7.0 \mathrm{~mA} / \mathrm{m}^{2}$ per $\mathrm{mT}$ in TARO. This corresponds to an external $0.286 \mathrm{mT}$ to produce the basic restriction of $2 \mathrm{~mA} / \mathrm{m}^{2}$ for public exposure.

Hart and Gandhi (1998) compared the cardiac-induced endogenous fields and $60 \mathrm{~Hz}$ electric and magnetic field induced exogenous fields in an anatomical human body model. The endogenous fields due to the beating of the heart were computed, and the magnitude of the naturally produced electric field and current densities inside the body were estimated. The endogenous electric field and current densities in most of the tissues in the frequency band of $40-70 \mathrm{~Hz}$ were found to be considerably smaller than those induced in human body by $60 \mathrm{~Hz}$ electric and magnetic fields.

Andreuccetti, Priori, and Zoppetti (2009) proposed the simplified procedure for dosimetric evaluations of ELF sources with complex waveforms. This procedure is based on the use of equivalent field intensity at an arbitrarily chosen reference frequency and allows summarizing into a single-frequency dosimetric evaluation with some approximations. Zoppetti and Andreuccetti (2009a,b) reviewed open problems in assessing compliance the with 2004/40/EC Directive (EU 2004). The problem is the applicability of the numerical technique to check the compliance with exposure limit values at the ELF region. To endorse the 2004/40 EC Directive, current densities must be averaged over a cross-section of $1 \mathrm{~cm}^{2}$ at every point of the exposed tissue, which is introduced by ICNIRP (1998a). The shape of the averaging surface is not specified. According to ICNIRP (1998b), the target tissue for averaging is the tissues of the CNS. This guideline introduces reference levels for external fields and basic restrictions for internal induced quantities and current densities. The current densities can be generally calculated using different types of methods with high-resolution numerical body models cited in this chapter. Zoppetti and Andreuccetti $(2009 \mathrm{a}, \mathrm{b})$ pointed out, as open problems, that neither ICNIRP or the EU Directive define the surface averaging procedure nor indicate how to proceed when the tissue for $1 \mathrm{~cm}^{2}$ averaging does not belong to the CNS. The basic restrictions may permit 
higher current densities in body tissues other than the CNS. The choice of the averaging algorithm is an important source of uncertainty in the quantification of safety guidelines.

For safety standards and guidelines, there are two different documents published by ICNIRP and IEEE (ICNIRP 1998a,b; IEEE 2002). ICNIRP provides basic restrictions on induced current densities based on only the threshold of acute effect in the tissues of the CNS and in the head and trunk of the body. The basic restrictions are $10 \mathrm{~mA} / \mathrm{m}^{2}$ for occupational exposure and $2 \mathrm{~mA} / \mathrm{m}^{2}$ for general public exposure at $50 \mathrm{~Hz}$ (ICNIRP 1998a). The ICNIRP document states that current densities should be averaged over a cross-section of $1 \mathrm{~cm}^{2}$ perpendicular to the current direction. Dimbylow (2008) identified uncertainties and problems in the practical application of the basic restrictions on current densities. For the procedure to average $1 \mathrm{~cm}^{2}$ of the tissues of the CNS, Bahr, Bolz, and Hennes (2007) discussed the accuracy of the calculation method, the variability of models and parameters, and the implication for quantifying guidelines. They computed one voxel value of the in situ electric field for a $5-\mathrm{mm}$ resolution model. Although basic restrictions of existing ICNIRP guidelines are based on the current densities, the IEEE standard used the in situ electric fields over an averaging distance of $5 \mathrm{~mm}$ on an arbitrary direction as a measure. In the latter case, the electrostimulation is identified as the relevant biological effect in the frequency range up to $3 \mathrm{kHz}$.

ICNIRP opened a draft of new guidelines for time-varying electric and magnetic fields up to $100 \mathrm{kHz}$, on July 29, 2009 (ICNIRP 2009). This consultation document was opened until October, 2009 on the ICNIRP Web site, and then the document was withdrawn from the ICNIRP Web site. In the draft guideline, instead of current densities, ICNIRP proposed the in situ electric fields averaged over a cube $\left(5 \times 5 \times 5 \mathrm{~mm}^{3}\right)$ as a measure for basic restrictions. For this reason, the draft guideline mentioned that in the newly prepared guidelines, the physical quantity used to specify the basic restrictions on exposure to EMF is the in situ electric field strength $E$, as it is the electric field that affects nerve cells and other electrically sensitive cells. Because of the relatively large uncertainties in the conductivity of tissues, induced electric field appears to be a more stable dose quantity than the induced current densities. It is also mentioned that a biologically reasonable averaging distance might extend from 1 to $7 \mathrm{~mm}$. The basic restrictions of draft guidelines are $100 \mathrm{mV} / \mathrm{m}$ for occupational exposure and $20 \mathrm{mV} / \mathrm{m}$ for the general public exposure, instead of 10 and $2 \mathrm{~mA} / \mathrm{m}^{2}$, respectively.

In order to provide some insights into the difference between the draft guidelines of the ICNIRP and IEEE standards, Hirata et al. (2010b) compared the in situ electric fields averaged over a volume of $5 \times 5 \times 5 \mathrm{~mm}^{3}$ and a straight line of $5 \mathrm{~mm}$. They used Japanese adult male and female models named TARO and HAMAKO for uniform ELF electric and magnetic field exposures. They used a quasistatic FDTD method for the calculation of the induced electric fields in these anatomical Japanese models. For magnetic field exposure, the human was considered to be standing in free space and three orientations of magnetic fields were assumed: AP, TOP, and LAT with $1 \mathrm{mT}$ at $50 \mathrm{~Hz}$. They compared the computed results with the results of the SPFD method for exposure to magnetic field. For electric field exposure, the human was considered to be standing on the perfect conductor under the vertical electric fields with $1 \mathrm{kV} / \mathrm{m}$ at $50 \mathrm{~Hz}$. They developed three algorithms for calculating the averaged in situ electric fields in nerve tissue. The volumeaveraged electric field in the nerve tissue decreased with the averaging volume. The 99th 
percentile value of the volume-averaged in situ electric field in the nerve tissue was more stable than that of the maximum value for different averaging volume.

The draft ICNIRP guidelines can use the in situ electric field as a measure of the basic restriction (ICNIRP 2009). There is a growing consensus that the in situ electric fields should be used as basic restriction instead of induced current densities. This change requires reevaluation of the rationale for the establishment of basic restrictions from the standpoint of both numerical dosimetry and human health protection. ICNIRP issued new exposure guidelines for time-varying electric and magnetic fields from $1 \mathrm{~Hz}$ to $100 \mathrm{kHz}$ on November 10, 2010 (ICNIRP 2010). In these new exposure guidelines, the physical quantity used to specify the basic restriction is the internal electric field strength. From the results obtained from high-resolution calculations of induced electric field with voxel sizes below $4 \mathrm{~mm}$, it recommends that the determination of induced electric field is the vector summation of the average of the electric field in a small volume $2 \times 2 \times 2 \mathrm{~mm}^{3}$. For specific tissue, the 99th percentile value of the electric field is the relevant value to be compared with the basic restriction.

\subsubsection{Validation of Methods}

Numerical dosimetry in bioelectromagnetics has been developed gradually from using simple human models during the 1970s through about $1-\mathrm{cm}$ anatomical cross-section human models in the 1980 s to 1 - to $10-\mathrm{mm}$ MRI-based and CAD-meshed human models from the end of 1990s until now.

The early human model represented the body by simple objects such as cylinders, spheres, and ellipsoids, and the conductivity of the body was assumed to be homogeneous. The calculations of induced current densities and the electric fields were carried out for these simple human models exposed to a uniform ELF electric or magnetic fields by simple analytical methods. The effects of nonuniform ELF magnetic field sources such as appliances have been also studied. The point is the accuracies of calculation methods and models used in numerical dosimetry. The accuracy of the calculation results has been tested in several ways. Historically, comparisons between the calculated results of the numerical method and measured values in mannequin-like human models were made. The human body model was assumed to be a homogeneous structure, indicating that the conductivity values within models are constant although experimentally obtained conductivity values have considerable uncertainty. The second method compares the solutions in simple models obtained by different methods. The other compares the results of the numerical method to measured values in experimental models using animal and other saline-filled models. From these tests, it can be said that the calculation methods seem to be accurate. On the other hand, the data on biological values such as tissue conductivity are still uncertain in the ELF region. This leads to uncertainty in the assignment of tissue conductivity to cubes or voxels.

It has been led to the method that the Maxwell's equations are solved in term of individual cubes or voxels with assigned conductivities in harmonizing with development of computational algorithms and high-speed computers. These methods have advantages in both modeling a complex shape, anatomical, MRI-based body taking the regional variations in conductivity into consideration and the estimation of the electric fields and 
induced current densities in small voxels of tissues. The numerical calculation method has been progressively developed for numerical dosimetry in bioelectromagnetics. The commonly used methods are as follows: FEM, IM, FDTD, and SPFD. For ELF electric fields, several numerical methods have been used: BEM, Space Charge Method (SCM), FEM, FDM, FDTD, spread sheet, hybrid quasistatic FDTD with SPFD, and MoM. The most computationally efficient method is quasistatic FDTD. This quasistatic FDTD is hybridized with SPFD. For ELF magnetic fields, two calculation methods have mainly been used: IM and SPFD. The IM is a vector, and the SPFD method is scalar. Dimbylow (1998) compared the efficiency of the two methods and indicated that the SPFD method requires less memory for the same size of voxels and is much quicker than IM (1.5-11 times).

For the accuracy of voxel models of human body, Dawson, Potter, and Stuchly (2001b) verified the numerical techniques for the computations of the induced currents in anatomically heterogeneous voxel models of the human body exposed to uniform ELF electric and magnetic fields. For comparison, three numerical methods, the SPFD, the quasi-static FDTD, and the hybrid method, are used for simple body models with different voxel sizes, from 1.8 to $7.2 \mathrm{~mm}$. For uniform magnetic field exposure of $60 \mathrm{~Hz}$, $1 \mu \mathrm{T}$, four human body models were considered: (1) simple homogeneous spheres and ellipsoids, (2) double-layered spheres, (3) a sphere with equatorially varying conductivity, and (4) infinite square base and other infinite right cylinders. For uniform electric field exposure of $60 \mathrm{~Hz}, 1 \mathrm{kV} / \mathrm{m}$, two models, homogeneous and layered spheres, are considered. Errors have been evaluated by comparing induced electric fields in human body models computed by numerical methods with values obtained from analytical solutions. Errors of 1\%-2\% are typical for both electric and magnetic field exposures. However, large errors over $250 \%$ occur in maximum induced electric fields values in a homogeneous sphere with $3.6-\mathrm{mm}$ voxel model. This is the result of singularities introduced by the staircasing smooth surface. These errors are inherent in the voxel human body model and are the greatest at the air-conductive interface of the body. The errors are smaller for interfaces between conductors having different conductivity values. The smaller the conductivity contrast, the smaller the errors. In calculations using the voxel human body model, the error associated with the staircase approximation of a smooth surface becomes a problem for the evaluation of the induced electric fields. Staircase approximation is a common problem when modeling curved structures. The approach to solve this error has not been established until now. Error introduced by staircasing smooth surfaces will always exist while using the voxel human body model. To avoid the inherent error in the voxel human body model, methods that will reduce error should be proposed. The development and application of new numerical methods without using voxel models is one of the options for the future. The differences in calculated data can be explained in terms of the accuracy of applied numerical method, voxel size, human body model size, posture, organ size and shape, and dielectric properties.

\subsection{Conclusion}

This chapter summarizes many state-of-the-art examples of numerical calculation of the electric fields and current densities induced in both very simple human body models and anatomically realistic, high-resolution human body models exposed to ELF 
EMF. This chapter emphasizes the conceptual understanding of the development of the human body model and the coupling between the exposure to ELF EMF and the developed human body model rather than the validation of proposed numerical dosimetry techniques. There are many review publications on the experiments, and numerical dosimetries and interactions of ELF EMF with matter (Kaune 1993; Kavet et al. 2001; Martens 2007; Stuchly and Dawson 2000).

Dosimetry is very important for the design and interpretation of all types of exposure experiment studies and for the establishment of human protection guidelines. Apart from experimental dosimetry, numerical dosimetry provides fundamental information about the external electric and magnetic fields and the internal electrical quantities in living tissues. Earlier, a very simple homogeneous human model was used to estimate the induced electric fields and current densities produced by exposure to ELF EMF. The recent progress in computer technology allowed the development of a more heterogeneous human model with very fine resolution and with CAD mesh. These models have been mainly produced by using MRI scan data of the body. The anatomically realistic human body model consists of various volumes of different conductivities with over 30 organs and tissues of $1-$ to $10-\mathrm{mm}^{3}$ voxels. Two-dimensional simple human body models can be used for the calculation of internal electric fields and current densities in the international standards of IEC in which a simple loop model is proposed for the human body (IEC 2004a, 2007). Three-dimensional models have been developed as an MRI-based human body model.

After publishing EHC 238 in 2007 (WHO 2007a), WHO opened the research recommendations of ELF EMF (WHO 2007b). The research needs are as follows: (1) computational dosimetry relating external electric and magnetic fields to internal electric fields, particularly concerning exposure to combined electric and magnetic fields in different orientations; (2) calculation of induced electric fields in pregnant women and in the fetus; and (3) refinement of microdosimetric models taking into account cellular architecture of neural networks and other complex suborgan systems. The reasons for this research are that (1) for a better understanding of biological effects and for the development of exposure guidelines, more data on internal electric fields for different exposure conditions are needed, (2) it is important to assess possible enhanced induction of electric fields during fetal life in relation to childhood leukemia, and (3) there is a need to further refine microdosimetric models to take into account the cellular architecture of neural networks and other complex suborgan systems identified as being sensitive to induced electric field effects compared with other tissues. In addition to the above issues, the study of electrical properties of tissues is still very important for the qualification of the ELF EMF effect and for the evaluation of possible mechanisms. As suggested in the research agenda, it is necessary to study further the specification of the electrical qualities induced in finer-resolution human body models.

Our scientific understanding of the coupling between ELF EMF and the human body has been gradually improved from the development of experimental and numerical dosimetries. Accurate modeling of a very heterogeneous human body and a human body of complex shape will have a major role in understanding the interactions between the EMF including IF and higher frequency and biological systems. In the future, numerical dosimetry will continue to support research in bioelectromagnetics. 


\section{Acknowledgments}

We thank Dr. Chiyoji Ohkubo, Director, Japan EMF Information Center, for his kind support. We acknowledge Professor Emeritus Dr. Katsuo Isaka of the University of Tokushima for his comments and discussions on this topic. We thank Mr. Thierry Petinaux for his painstaking effort to edit the manuscript. We thank Dr. Soung-Ho Myung of Korea Electrotechnology Research Institute, Changwon, Korea, and Dr. Eugene Rhee of Chuo University, Tokyo, for their kindness.

\section{References}

ACGIH 2000. 1997. TLVs and BEIs: Threshold Limit Values for Chemical Substances and Physical Agents Biological Exposure Indices. 109. Cincinnati, OH: American Conference of Governmental Hygienists.

Ackerman, M. J. 1998. The visible human project. Proc IEEE 86:504-11.

Amoruso, V., and F. Lattarulo. 1989. ELF electric-field induced currents in the human body. Alta Frequenza 68:385-90.

Amoruso, V., and F. Lattarulo. 1996. Diakoptic approach to the electrostatic man-animal scaling problem. Electromagn Biol 15:63-74.

Andreuccetti, D., S. Priori, and N. Zoppetti. 2009. A simplified procedure for dosimetric evaluations on ELF sources with complex waveforms. Radiat Prot Dosimetry 137:201-5.

Bahr, A., T. Bolz, and C. Hennes. 2007. Numerical dosimetry ELF: Accuracy of the method, variability of models and parameters, and the implication for quantifying guidelines. Health Phys 92:521-30.

Baraton, P., and B. Hutzler. 1995. Magnetically induced currents in the human body. IEC Technol Trends Assess 1-45.

Barchanski, A., M. Clemens, H. D. Gersem, T. Steiner, and T. Wdeiland. 2005a. Using domain decomposition techniques for the calculation of low-frequency electric current densities in high-resolution 3D human anatomy models. Int J Comput Math Electr Electron Eng 24:458-67.

Barchanski, A., H. De Gersem, E. Gjonaj, and T. Weiland. 2005b. Impact of the displacement current on low-frequency electromagnetic fields computed using highresolution anatomy models. Phys Med Biol 50:N243-9.

Barchanski, A., M. Clemens, H. De Gersem, and T. Weiland. 2006a. Efficient calculation of current densities in the human body induced by arbitrarily shaped low-frequency magnetic fields. J Comput Phys 214:81-95.

Barchanski, A., T. Steiner, H. De Gersem, M. Clemens, and T. Weikand. 2006b. Local grid refinement for low-frequency current computations in 3-D human anatomy models. IEEE Trans Magn 42:1371-4.

Barchanski, A., M. Clemens, E. Gjonaj, H. De Gersem, and T. Weikand. 2007. Large-scale calculation of low-frequency-induced currents in high-resolution human body models. IEEE Trans Magn 43:1693-6.

Barnes, H. C., A. J. McElory, and J. H. Charkow. 1967. Rational analysis of electric fields in live line working. IEEE Trans PAS 86:482-92. 
Barnes, F. S. 1988. Mechanism of interaction of magnetic fields with biological systems. IEEE Trans Magn 24:2101-4.

Barnes, F. S. 1992. Some engineering models for interactions of electric and magnetic fields with biological systems. Bioelectromagnetics (Suppl 1):67-86.

Barnes, F. S. 2007. Interaction of direct current and extremely low-frequency electric fields with biological materials and systems. In Bioengineering and Biophysical Aspects of Electromagnetic Fields, ed. F. S. Barnes and B. Greenebaum, 115-56. CRC Press: Boca Raton.

Bossavit, A., and J. C. Vérité. 1983. The "TRIFOU” code: Solving the 3-D eddy currents problem by using $\mathrm{H}$ as state variable. IEEE Trans Magn 19:2465-70.

Bottauscio, O., and R. Conti. 1999. Evaluation of induced currents in human models by power frequency electromagnetic fields using a BEM technique. In Electricity and Magnetism in Biology and Medicine, ed. F. Bersani, 315-8. Kluwer Academic/ Plenum Publishers: New York.

Bottauscio, O., M. Chiampi, and L. Zilberti. 2009. Boundary element approaches for the evaluation of human exposure to low frequency electromagnetic fields. IEEE Trans Magn 45:1674-7.

Bracken, T. D. 1992. Experimental macroscopic dosimetry for extremely-low-frequency electric and magnetic fields. Bioelectromagnetics (Suppl 1):15-26.

Caputa, K., P. J. Dimbylow, T. W. Dawson, and M. A. Stuchly. 2002. Modelling fields induced in humans by $50 / 60 \mathrm{~Hz}$ magnetic fields: Reliability of the results and effects of model variations. Phys Med Biol 47:1391-8.

Carstensen, E. L., A. Buettner, V. L. Genberg, and M. A. Miller. 1985. Sensitivity of the human eye to power freqeuncy electric fields. IEEE Trans BME 32:561-5.

Cech, R., N. Leitgeb, and M. Pediaditis. 2007. Fetal exposure to low frequency electric and magnetic fields. Phys Med Biol 52:879-88.

Cech, R., N. Leitgeb, and M. Pediaditis. 2008. Current densities in a pregnant woman model induced by simultaneous ELF electric and magnetic field exposure. Phys Med Biol 53:177-86.

Chen, K. M., H. R. Chuang, and C. J. Lin. 1986. Quantification of interaction between ELF-LF electric fields and human bodies. IEEE Trans BME 33:746-56.

Cheng, J., M. A. Stuchly, C. DeWagter, and L. Martens. 1995. Magnetic field induced currents in a human head from use of portable appliances. Phys Med Biol 40:495-510.

Chiba, A., K. Isaka, Y. Yokoi, M. Nagata, M. Kitagawa, and T. Matsuo. 1984. Application of finite element method to analysis of induced current densities inside human model exposed to $60 \mathrm{~Hz}$ electric fields. IEEE Trans PAS 103:1895-902.

Chiba, A., and K. Isaka. 1997. Current distributions inside the cylindrical model with the internal and the external tissues having different conductivities in the electric fields (in Japanese with English summary). IEEJ Trans B 117:388-94.

Chiba, A., K. Isaka, and W. T. Kaune. 1998. Density distribution of currents inside the brain in the head part of the human model exposed to power frequency electric field (in Japanese with English summary). IEEJ Trans B 118:627-34.

Chiba, A., and K. Isaka. 1998. Current distributions inside a cylindrical model of a human body with and external tissues having different conductivities in electric fields. Electr Eng Jpn 122:19-27. 
Chiba, A., and K. Isaka. 1999. Distribution of induced current density inside an ungrounded human model exposed to electric field (in Japanese with English summary). IEEJ Trans B 119:401-7.

Chiba, A., and K. Isaka. 2000. Current density distribution inside a human model with erect position on the insulating circular plane in the electric field (in Japanese with English summary). IEEJ Trans B 120:630-6.

Chiba, A., and K. Isaka. 2004. Analysis of induced current density inside human model which stands on conductive ground layer in uniform electric field (in Japanese with English summary). IEEJ Trans A 124:1193-7.

Chiu, R. S., and M. A. Stuchly. 2005. Electric fields in bone marrow substructures at power-line frequencies. IEEE Trans BME 52:1103-9.

Christ, A., W. Kainz, E. G. Hahn, K. Honegger, M. Zefferer, E. Neufeld, W. Rascher et al. 2010. The Virtual Family-development of anatomical CAD models of two adults and two children for dosimetric stimulations. Phys Med Biol 55:N23-38.

Chuang, H. R., and K. M. Chen. 1989. A numerical method for the computation of induced currents inside 3-D heterogeneous biological bodies by ELF-LF electric fields. IEEE Trans BME 36:628-34.

Cooper, T. G. 2002. Occupational Exposure to Electric and Magnetic Fields in the Context of the ICNIRP Guidelines. Chilton: National Radiological Protection Board; NRPB-W24.

Crotti, G., and D. Giordani. 2009. Analysis of critical situations in the evaluation of human exposure to magnetic fields with complex waveforms. Radiat Prot Dosimetry 137:227-30.

Dawson, T. W., J. de Moerloose, and M. A. Stuchly. 1996. Comparison of magnetically induced ELF fields in humans computed by FDTD and scalar potential FD codes. Appl Comput Electromagn Soc J 11:63-71.

Dawson, T. W., and M. A. Stuchly. 1996. Analytic validation of a three-dimensional scalarpotential finite-difference code for low-frequency magnetic induction. Appl Comput Electromagn Soc J 11:72-81.

Dawson, T. W., J. D. Moerloose, and M. A. Stuchly. 1997a. Hybrid finite-difference method for high-resolution modeling of low-frequency electric induction in humans. J Comput Phys 136:640-53.

Dawson, T. W., K. Caputa, and M. A. Stuchly. 1997b. A comparison of $60 \mathrm{~Hz}$ uniform magnetic and electric induction in the human body. Phys Med Biol 42:2319-29.

Dawson, T. W., K. Caputa, and M. A. Stuchly. 1997c. Influence of human model resolution on computed currents induced in organs by $60 \mathrm{~Hz}$ magnetic fields. Bioelectromagnetics 18:478-90.

Dawson, T. W., and M. A. Stuchly. 1997. An analytic solution for verification of computer models for low-frequency magnetic induction. Radio Sci 32:343-67.

Dawson, T. W. 1997. Analytic solution for low-frequency magnetic induction in an equatorially stratified sphere: Horizontal excitation. Radio Sci 32:1761-76.

Dawson, T. W., K. Caputa, and M. A. Stuchly. 1998a. High-resolution organ dosimetry for human exposure to low-frequency electric fields. IEEE Trans PWRD 13:366-73.

Dawson, T. W., and M. A. Stuchly. 1998b. High-resolution organ dosimetry for human exposed to low-frequency magnetic fields. IEEE Trans Magn 34:708-18. 
Dawson, T. W., and M. A. Stuchly. 1998c. Effects of skeletal muscle anisotropy on human organ dosimetry under $60 \mathrm{~Hz}$ uniform magnetic field exposure. Phys Med Biol 43:1059-74.

Dawson, T. W., K. Caputa, and M. A. Stuchly. 1999a. Organ dosimetry for human exposure to non-uniform $60 \mathrm{~Hz}$ magnetic fields. IEEE Trans PWRD 14:1234-9.

Dawson, T. W., K. Caputa, and M. A. Stuchly. 1999b. High-resolution magnetic field numerical dosimetry for live-line workers. IEEE Magn 35:1131-4.

Dawson, T. W., K. Caputa, M. A. Stuchly, and R. Kavet. 2001a. Electric fields in the human body resulting from $60 \mathrm{~Hz}$ contact currents. IEEE Trans BME 48:1020-6.

Dawson, T. W., M. Potter, and M. A. Stuchly. 2001b. Evaluation of modeling accuracy of power frequency field interactions with the human body. Appl Comput Electromagn Soc J 16:162-72.

Dawson, T. W., K. Caputa, and M. A. Stuchly. 2002a. Magnetic field exposures for UK liveline workers. Phys Med Biol 47:995-1012.

Dawson, T. W., C. Caputa, and M. A. Stuchly. 2002b. Electric fields induced in humans and rodents by $60 \mathrm{~Hz}$ magnetic fields. Phys Med Biol 47:2561-8.

Dawson, T. W., K. Caputa, M. A. Stuchly, and R. Kavet. 2003. Comparison of electric fields induced in humans and rodents by $60 \mathrm{~Hz}$ contact currents. IEEE Trans BME 50:744-53.

Dawson, T. W., M. A. Stuchly, and R. Kavet. 2004a. Electric fields in the human body due to electrostatic discharges. IEEE Trans BME 51:1460-8.

Dawson, T. W., M. A. Stuchly, and R. Kavet. 2004b. Evaluation of interactions of electric fields due to electrostatic discharge with human tissue. IEEE Trans BME 51:2194-8.

Deno, D. W. 1977. Currents induced in the human body by high voltage transmission line electric field: Measurement and calculation of distribution and dose. IEEE Trans PAS 96:1517-27.

Deno, D. W. 1979. Monitoring of personnel exposed to a $60 \mathrm{~Hz}$ electric field. In Biological Effects of Extremely Low Frequency Electromagnetic Fields, ed. R. D. Phillips, M. F. Gillis, W. T. Kaune and D. D. Mahlum, 93-108. Springfield, VA: Conf-781016, NTIS.

Dimbylow, P. J. 1987. Finite difference calculations of current densities in a homogenous model of a man exposed to extremely low frequency electric fields. Bioelectromagnetics 8:355-75.

Dimbylow, P. J. 1988. The calculation of induced currents and absorbed power in a realistic homogeneous model of the lower leg for applied electric fields from $60 \mathrm{~Hz}$ to $30 \mathrm{MHz}$. Phys Med Biol 33:1453-68.

Dimbylow, P. J. 1998. Induced current densities from low-frequency magnetic fields in a $2 \mathrm{~mm}$ resolution, anatomically realistic model of the body. Phys Med Biol 43:221-30.

Dimbylow, P. J. 2000. Current densities in a $2 \mathrm{~mm}$ resolution anatomically realistic model of the body induced by low frequency electric fields. Phys Med Biol 45:1013-22.

Dimbylow, P. J. 2005. Development of the female voxel phantom, NAOMI, and its application to calculations of induced current densities and electric fields from applied low frequency magnetic and electric fields. Phys Med Biol 50:1047-70.

Dimbylow, P. J. 2006. Development of pregnant female, hybrid voxel-mathematical models and their application to the dosimetry of applied magnetic and electric fields at $50 \mathrm{~Hz}$. Phys Med Biol 51:2383-94. 
Dimbylow, P. J. 2008. Quandaries in the application of the ICNIRP low frequency basic restriction on current density. Phys Med Biol 53:133-45.

Dimbylow, P. J., and R. Findlay. 2010. The effects of body posture, anatomy, age and pregnancy on the calculation of induced current density at $50 \mathrm{~Hz}$. Radiat Prot Dosimetry 139:532-8.

DiPlacido, J., C. H. Shih, and B. J. Ware. 1978. Analysis of the proximity effects in electric field measurements. IEEE Trans PAS 97:2167-77.

Durney, C. H. 1980. Electromagnetic dosimetry for models of humans and animals: A review of theoretical and numerical techniques. Proc IEEE 68:33-40.

Durney, C. H., and D. A. Christensen. 2000. Basic Introduction to Bioelectromagnetics. CRC Press: Boca Raton.

Eberdt, M., P. K. Brown, and G. Lazzi. 2003. Two-dimensional SPICE-linked multiresolution impedance method for low-frequency electromagnetic interactions. IEEE Trans BME 50:881-9.

EU 2004. Directive 2004/40/EC of the European Parliament and of the Council on the minimum health and safety requirements regarding the exposure of workers to the risks arising from physical agents (electromagnetic fields). Official Journal of European Union 184:1-9.

Fear, E. C., and M. A. Stuchly. 1998. Biological cells with gap junctions in low-frequency electric fields. IEEE BME 45:856-66.

Foster, K. R. 2003. Mechanisms of interaction of extremely low frequency electric fields and biological systems. Radiat Prot Dosimetry 106:301-10.

Fujita, A., I. Hirota, Y. Kawahara, and H. Omori. 2007. Development and evaluation of intermediate frequency magnetic field exposure system for studies of in vitro biological effects. Bioelectromagnetics 28:538-45.

Fujita, A., Y. Kawahara, S. Inoue, and H. Omori. 2010. Development of a higher power intermediate frequency magnetic field exposure system for in vitro studies. Bioelectromagnetics 31:156-62.

Furse, C., and O. P. Gandhi. 1998. Calculation of electric fields and currents induced in a millimeter-resolution human model at $60 \mathrm{~Hz}$ using the FDTD method. Bioelectromagnetics 19:293-9.

Furse, C., D. A. Christensen, and C. H. Durney. 2009. Basic Introduction to Bioelectromagnetics. 2nd ed. CRC Press: Boca Raton.

Gabriel, C., S. Gabriel, and E. Corthout. 1996a. The dielectric properties of biological tissues: 1. Literature survey. Phys Med Biol 41:2231-49.

Gabriel, S., R. W. Lau, and C. Gabriel. 1996b. The dielectric properties of biological tissues: 2. Measurements in the frequency range $10 \mathrm{~Hz}-20 \mathrm{GHz}$. Phys Med Biol 41:2251-69.

Gabriel, S., R. W. Lau, and C. Gabriel. 1996c. The dielectric properties of biological tissues: 3. Parametric models for the dielectric spectrum of tissues. Phys Med Biol 41:2271-93.

Gabriel, C. 2005. Dielectric properties of biological tissue: Variation with age. Bioelectromagnetics (Suppl 7):12-8.

Gabriel, C., A. Peyman, and E. H. Grand. 2009. Electrical conductivity of tissue at frequencies below $1 \mathrm{MHz}$. Phys Med Biol 54:4863-78. 
Gandhi, O. P., J. F. Deford, and H. Kanai. 1984. Impedance method for calculation of power deposition patterns in magnetically induced hyperthermia. IEEE Trans BME 31:644-51.

Gandhi, O. P., and J. Y. Chen. 1992. Numerical dosimetry at power-line frequencies using anatomically based models. Bioelectromagnetics (Suppl 1):43-60.

Gandhi, O. P. 1995. Some numerical methods for dosimetry: Extremely low frequencies to microwave frequencies. Radio Sci 30:161-77.

Gandhi, O. P., G. Kang, D. Wu, and G. Lazzi. 2001. Currents induced in anatomic models of the human for uniform and nonuniform power frequency magnetic fields. Bioelectromagnetics 22:112-21.

González, C., P. Peratta, and D. Poljak. 2007. Boundary element modeling of the realistic human body exposed to extremely low frequency (ELF) electric fields: Computational and geometrical aspects. IEEE Trans EMC 49:153-62.

Gustrau, F., A. Bahr, M. Rittweger, S. Goltz, and S. Eggert. 1999. Simulation of induced current densities in the human body at industrial induction heating frequencies. IEEE Trans EMC 41:480-6.

Guy, A. W., S. Davidow, G. Y. Yang, and C. Chou. 1982. Determination of electric current distributions in animals and humans exposed to a uniform $60 \mathrm{~Hz}$ high intensity electric field. Bioelectromagnetics 3:47-71.

Guy, A. W. 1987. Dosimetry associated with exposure to non-ionizing radiation: Very low frequency to microwaves. Health Phys 53:569-84.

Hamada, S., and T. Kobayashi. 2006a. Analysis of electric field induced by ELF magnetic field utilising fast-multiple surface-charge-simulation method for voxel data (in Japanese with English summary). IEEJ Trans A 126:355-62.

Hamada, S., O. Yamamoto, and T. Kobayashi. 2006b. Analysis of electric field induced by ELF magnetic field utilizing generalized equivalent multipole-moment method. Electr Eng Jpn 156:1-14.

Hamada, S., and T. Kobayashi. 2008. Analysis of electric field induced by ELF magnetic field utilizing fast-multipole surface-charge simulation method for voxel data. Electr Eng Jpn 165:1-10.

Hart, R. A., and O. P. Gandhi. 1998. Comparison of cardiac-induced endogenous fields and power frequency induced exogenous fields in an anatomical model of the human body. Phys Med Biol 43:3083-99.

Hart, F. X., and A. A. Marino. 1982. ELF dosages in ellipsoidal models of man due to high voltage transmission line. J Bioelectricity 1:129-54.

Hart, F. X. 1989. Using a spread sheet program to model the interaction of low frequency electric fields with inhomogeneous, irregularly shaped objects. J Bioelectricity 8:201-26.

Hart, F. X. 1990. Use of a spread sheet to calculate the current density distribution produced in human and rat models by low frequency electric fields. Bioelectromagnetics 11:213-28.

Hart, F. X. 1992a. Numerical and analytical methods to determine the current density distributions produced in human and rat models by electric and magnetic fields. Bioelectromagnetics (Suppl 1):27-42. 
Hart, F. X. 1992b. Electric fields induced in rat and human models by $60 \mathrm{~Hz}$ magnetic fields: Comparison of calculated and measured values. Bioelectromagnetics 13:313-6.

Hart, F. X., K. Evely, and C. D. Finch. 1993. Use of a spreadsheet program to calculate the electric field/current density distributions induced in irregularly shaped, inhomogeneous biological structures by low-frequency magnetic fields. Bioelectromagnetics 14:161-72.

Hart, F. X. 1996. Cell culture dosimetry for low-frequency magnetic fields. Bioelectromagnetics 17:48-57.

Hassan, N., I. Chatterjee, N. G. Publicover, and G. L. Graviso. 2003. Numerical study of induced current perturbations in the vicinity of excitable cells exposed to extremely low frequency magnetic fields. Phys Med Biol 48:3277-93.

Hirata, A., K. Caputa, T. W. Dawson, and M. A. Stuchly. 2001. Dosimetry in models of child and adult for low-frequency electric field. IEEE Trans BME 48:1007-12.

Hirata, A., and O. Fujiwara. 2007. Dosimetry in Japanese male and female models for a low-frequency electric field. Phys Med Biol 52:N339-43.

Hirata, A., K. Wake, S. Watanabe, and M. Taki. 2009. In-situ electric field and current density in Japanese male and female models for uniform magnetic field exposures. Radiat Prot Dosimetry 135:272-5.

Hirata, A., K. Yamazaki, S. Hamada, Y. Kamimura, H. Tarao, K. Wake, Y. Suzuki, N. Hayashi, and O. Fujiwara. 2010a. Intercomparison of induced fields in Japanese male model for ELF magnetic field exposures: Effect of different computational methods and codes. Radiat Prot Dosimetry 138:237-44.

Hirata, A., Y. Takano, Y. Kamimura, and O. Fujiwara. 2010b. Effect of the averaging volume and algorithm on the in situ electric field for uniform electric-and magnetic field exposures. Phys Med Biol 55:N243-52.

Hoang, L. H., R. Scorretti, N. Burais, and D. Voyer. 2009. Numerical dosimetry of induced phenomena in the human body by a three-phase power line. IEEE Trans Magn 45:1666-9.

IARC. 2002. Non-ionizing radiation, Part 1: Static and extremely low-frequency (ELF) electric and magnetic fields. In Monographs on the Evaluation of Carcinogenic Risks to Humans, 80. Lyon: Ryon, France.

ICNIRP. 1998a. Guidelines for limiting exposure to time-varying electric, magnetic, and electromagnetic fields (up to $300 \mathrm{GHz}$ ). Health Phys 74:494-522.

ICNIRP. 1998b. Response to questions and comment on the Guidelines for limiting exposure to time-varying electric, magnetic, and electromagnetic fields (up to $300 \mathrm{GHz}$ ). Health Phys 75:438-9.

ICNIRP. 2003. Exposure to Static and Low Frequency Electromagnetic Fields, Biological Effects and Health Consequences $(0-100 \mathrm{kHz})$, ed. R. Matthes, P. Vecchia, A. F. McKinlay, B. Veyret, and J. H. Bernhardt. Oberschleissheim: ICNIRP 13/2003.

ICNIRP. 2009. Draft guidelines for limiting exposure to time-varying electric and magnetic fields ( $1 \mathrm{~Hz}$ to $100 \mathrm{kHz}$ ) (withdrawn from the ICNIRP website in October):1-39.

ICNIRP. 2010. ICNIRP statement-guidelines for limiting exposure to time-varying electric and magnetic fields (1 Hz to $100 \mathrm{kHz}$ ). Health Phys 99:818-36. 
IEC. 2004a. Exposure to electric or magnetic fields in the low and intermediate frequency range-Methods for calculating the current density and internal electric field induced in the human body. Part 2-1: Exposure to magnetic fields $-2 \mathrm{D}$ models. IEC 62226-2-1

IEC. 2007. Exposure to electric or magnetic fields in the low and intermediate frequency range-Methods for calculating the current density and internal electric field induced in the human body. Part 3-1: Exposure to electric fields-Analytical and 2D numerical models. IEC 62226-3-1.

IEEE. 2002. IEEE Standard No.C95.6-2002: IEEE Standard for Safety Levels with Respect to Human Exposure to Electromagnetic Fields, 0 to $3 \mathrm{kHz}$. New York: Institute of Electrical and Electronics Engineers.

Iivonen, S., A. P. Sihvonen, K. Karkkainen, and J. Sarvas. 2005. Numerical assessment of induced ELF currents in the human head due to the battery current of a digital mobile phone. Bioelectromagnetics 26:648-56.

Iivonen, S., and J. Sarvas. 2007. Magnetic-field induced ELF currents in a human body by the use of a GSM phone. IEEE Trans EMC 49:294-301.

Iivonen, S., and I. Laakso. 2009. Computational estimation of magnetically induced electric fields in a rotating head. Phys Med Biol 54:341-51.

Isaka, K., Y. Yokoi, H. Nagata, A. Chiba, Y. Onogi, M. Kitagawa, T. Matsuo, and M. S. A. A. Hamman. 1987. Results of experimental and theoretical analysis of induced currents from a uniform power frequency electric field in humans. In Interaction of Biological Systems with Static and ELF Electric and Magnetic Fields, ed. L. E. Anderson, B. J. Kelman, and R. J. Weigel, 165-76. Richland, WA 99352: Pacific Northwest Laboratory.

IT'IS. 2010. Whole-body human model. http://www.itis.ethz.ch/index/index_humanmodels .html: Foundation for Research on Information Technology in Society (accessed April 28, 2011).

Jokela, K. 2000. Restricting exposure to pulsed and broadband magnetic fields. Health Phys 79:373-88.

Jokela, K. 2007. Assessment of complex EMF exposure situations including inhomogeneous field distributionh. Health Phys 92:531-40.

Kato, M., S. Ohta, T. Kobayashi, and G. Matsumoto. 1986. Response of sensory receptors of the cat's hindlimb to a transient, step-function DC electric fields. Bioelectromagnetics 7:395-404.

Kato, M., S. Ohta, K. Shimizu, Y. Tsuchida, and G. Matsumoto. 1989. Detection-threshold of $50 \mathrm{~Hz}$ electric fields by human subjects. Bioelectromagnetics 10:319-28.

Kato, M., and T. Shigemitsu. 1997. Effects of $50 \mathrm{~Hz}$ magnetic fields on pineal function in the rat. In The Melatonin Hypothesis: Breast Cancer and Use of Electric Power, ed. R. G. Stevens, B. W. Wilson, and L. E. Anderson, 337-76. Battelle Press: Columbus/ Richland.

Kato, M. 2006. Electromagnetics in Biology. Springer Verlag: Tokyo.

Kaune, W. T., and R. D. Phillips. 1980. Comparison of the coupling of grounded humans, swine and rats to vertical, $60 \mathrm{~Hz}$ electric fields. Bioelectormagnetics 1:117-29.

Kaune, W. T., and M. F. Gillis. 1981. General properties of the interaction between animals and ELF electric fields. Bioelectromagnetics 2:1-11. 
Kaune, W. T. 1981a. Interactive effects in $60 \mathrm{~Hz}$ electric field exposure systems. Bioelectromagnetics 2:33-50.

Kaune, W. T. 1981b. Power-frequency electric fields, averaged over the body surfaces of grounded humans and animals. Bioelectromagnetics 2:403-6.

Kaune, W. T., and M. C. Miller. 1984. Short-circuit currents, surface electric fields, and axial current densities for guinea pigs exposed to ELF electric fields. Bioelectromagnetics 5:361-4.

Kaune, W. T., and R. D. Phillips. 1985. Dosimetry for extremely low-frequency electric fields. In Biological Effects and Dosimetry of Static and ELF Electromagnetic Fields, ed. M. Grandolfo, S. M. Michaelson and A. Rindi, 145-65. Plenum Press: New York.

Kaune, W. T., and W. C. Forsyth. 1985. Current densities measured in human models exposed to $60 \mathrm{~Hz}$ electric fields. Bioelectromagnetics 6:13-32.

Kaune, W. T., and F. A. McCreary. 1985. Numerical calculation and measurement of $60 \mathrm{~Hz}$ current densities induced in an upright grounded cylinder. Bioelectromagnetics 6:209-20.

Kaune, W. T., L. M. Kistler, and M. C. Miller. 1987. Comparison of the coupling of grounded and ungrounded humans to vertical $60 \mathrm{~Hz}$ electric fields. In Interaction of Biological Systems with Static and ELF Electric and Magnetic Fields, ed. L. E. Anderson, B. J. Kelman, R. J. Weigel, 185-95. Richland, WA 99352: Pacific Northwest Laboratory.

Kaune, W. T., and W. C. Forsythe. 1988. Current densities induced in swine and rat models by power frequency electric fields. Bioelectromagnetics 9:1-24.

Kaune, W. T. 1992. Macroscopic dosimetry of power-frequency electric and magnetic fields. Bioelectromagnetics (Suppl 1):11-4.

Kaune, W. T. 1993. Introduction to power frequency electric and magnetic fields. Environ Health Perspect 101:73-81.

Kavet, R., M. A. Stuchly, W. H. Bailey, and T. D. Bracken. 2001. Evaluation of biological effects, dosimetric models, and exposure assessment related to ELF electric and magnetic field guidelines. Appl Occup Environ Hyg 16:1118-38.

Kheifets, L., and R. Shimkhada. 2007. Epidemiologic studies of extremely low-frequency electromagnetic fields. In Biological and Medical Aspects of Electromagnetic Fields, ed. F. S. Barnes, and B. Greenebaum, 227-63. CRC Press: Boca Raton.

King, R. W. P., and T. T. Wu. 1995. The complete electromagnetic field of a three-phase transmission line over the earth and its interaction with the human body. J Appl Phys 78:668-83.

King, R. W. P., and S. S. Sandler. 1996. Electric fields and currents induced in organs of the human body when exposed to ELF and VLF electromagnetic fields. Radio Sci 31:1153-67.

King, R. W. P. 1997. Currents and electric fields induced in the human body when the arms are raised. J Appl Phys 81:7116-28.

King, R. W. P. 1998a. The interaction of power-line electromagnetic fields with the human body. IEEE Eng Med Biol (Nov/Dec) 17: 67-78.

King, R. W. P. 1998b. Fields and currents in the organs of the human body when exposed to power lines and VLF transmitters. IEEE Trans BME 45:520-30. 
King, R. W. P., and T. T. Wu. 1998. The electric field induced in cells in the human body when this is exposed to low-frequency electric fields. Phys Rev E 2:2363-9.

King, R. W. P. 1999a. Nerves in a human body exposed to low-frequency electromagnetic fields. IEEE Trans BME 46:1426-31.

King, R. W. P. 1999b. Electric field induced in a spherical cell in the human body when exposed to a 50- to $60 \mathrm{~Hz}$ electromagnetic field. Radio Sci 34:539-47.

King, R. W. P. 1999c. The electric field induced in the human body when exposed to electromagnetic fields at 1-30 MHz on shipboard. IEEE Trans BME 46:747-51.

King, R. W. P. 2004. A review of analytically determined electric fields and currents induced in the human body when exposed to $50-60 \mathrm{~Hz}$ electromagnetic fields. IEEE Trans Antennas Propag 52:1186-92.

Kitano, M., S. Hamada, and T. Kobayashi. 2009. Analytical formula of induced electric fields in a spherical conductor by an dipole magnetic field source. Electr Eng Jpn 166:8-17.

Kobayashi, T., K. Shimizu, G. Matsumoto, F. Nishiyama, H. Nakamura, S. Ohta, and M. Kato. 1983. Uniform DC electric field exposure systems for mice and cats. Bioelectromagnetics 4:303-14.

Kobayashi, T., K. Shimizu, and G. Matsumoto. 1987. Numerical calculation of induced currents in humans and experimental animals exposed to ELF electric fields. In Interaction of Biological Systems with Static and ELF Electric and Magnetic Fields, ed. L. E. Anderson, B. J. Kelman, and R. J. Weigel, 155-64. Richland, WA 99352: Pacific Northwest Laboratory.

Lattarulo, F., and G. Mastronardi. 1981. Equivalence criteria among man and animals in experimental investigations of high voltage power frequency exposure hazards. Appl Math Model 5:92-6.

Leitgeb, N., and R. Cech. 2008. Dosimetric assessment of simultaneous exposure to ELF electric and magnetic fields. IEEE Trans BME 55:671-4.

Lindenblatt, G., and J. Silny. 2002. Electrical phosphenes: On the influence of conductivity inhomogeneities and small-scale structure of the orbita on the current density threshold of excitation. Med Biol Eng Comput 40:354-9.

Maalej, N. M., C. A. Belhadi, T. K. Abdel-Galil, and I. O. Habiballah. 2009. Visible human utilization to render induced electric field and current density images inside the human. Proc IEEE 97:2053-9.

Martens, L. 2007. Different basic dosimetric quantities for the characterization of exposure to low-frequency electric and magnetic fields and the implication for practical exposure conditions and guidelines. Health Phys 92:515-20.

Mason, P. A., J. M. Ziriax, W. D. Hurt, and J. W. D’Andrea. 1999. 3-dimentional models for EMF dosimetry. In Electricity and Magnetism in Biology and Medicine, ed. F. Bersani, 291-4. New York: Kluwer Academic/Plenum.

Matsumoto, T., N. Hayashi, and K. Isaka. 1997. Analysis of induced current density in spherical human model exposed to low frequency and vertical magnetic fields with phase differences (in Japanese with English summary). IEEJ Trans A 117:276-82.

Matsumoto, T., N. Hayashi, and K. Isaka. 1999. Analysis of induced current density in spherical conductive model exposed to mutually parallel and orthogonal low frequency electric and magnetic fields (in Japanese with English summary). IEEJ Trans A 119:1039-44. 
Matsumoto, T., A. Chiba, N. Hayashi, and K. Isaka. 2000. Analysis of induced density in grounded and ungrounded prolate spheroid models in concurrent ELF electric and magnetic fields (in Japanese with English summary). IEEJ Trans A 120:49-55.

Matsumoto, T., A. Chiba, N. Hayashi, and K. Isaka. 2001. Analysis of induced current density in grounded and ungrounded prolate spheroid models in concurrent ELF electric and magnetic fields. Electr Eng Jpn 135:8-15.

Matsumoto, T., A. Chiba, K. Shoukura, H. Ikeda, and K. Isaka. 2004. Analysis of current density induced in an ungrounded human model by the method combining the surface-charge integral equation and finite element method (in Japanese with English summary). IEEJ Trans PE 124:778-84.

McCormick, D. L. 2007. Evaluation of the toxicity and potential oncogenicity of extremely low-frequency magnetic fields in experimental animal model systems. In Biological and Medical Aspects of Electromagnetic Fields, ed. F. S. Barnes, and B. Greenebaum, 21-51. CRC Press: Boca Raton.

McKinlay, A. F., S. G. Allen, R. Cox, P. J. Dimbylow, S. M. Mann, C. R. Muirhead, R. D. Saunders, Z. J. Sienkiewicz, J. W. Stather, and P. Wainwright. 2004. Advice on limiting exposure to electromagnetic fields (0-300 GHz). Doc NRPB 15(3):1-35.

Miller, D. L. 1991a. Miniature-probe measurements of electric fields and currents induced by a $60 \mathrm{~Hz}$ magnetic field in rat and human models. Bioelectromagnetics 12:157-71.

Miller, D. L. 1991b. Electric fields induced in chicken eggs by $60 \mathrm{~Hz}$ magnetic fields and the dosimetric importance of biological membranes. Bioelectromagnetics 12:349-60.

Miller, D. L. 1994. Conductivity differences distort probe measurements of magnetically induced electric fields. Bioelectromagnetics 15:483-7.

Miller, D. L. 1996. Miniature-probe measurements of electric fields induced by $60 \mathrm{~Hz}$ magnetic fields in rats. Bioelectromagnetics 17:167-73.

Miller, D. L., and J. A. Creim. 1997. Comparison of cardiac and $60 \mathrm{~Hz}$ magnetically induced electric fields measured in anesthetized rats. Bioelectromagnetics 18:317-23.

Min, S. W., E. S. Kim, S. H. Myung, B. Y. Lee, and J. K. Park. 1996. Calculation of the induced voltage and current for a human and a car close to $765 \mathrm{kV}$ AC double circuit transmission line (in Korean with English summary). Trans KIEE 45:301-9.

Min, S. W., and K. H. Song. 2006a. Analysis on induced current density inside human body by $60 \mathrm{~Hz}$ ELF magnetic fields (in Korean with English summary). Trans KIEE 55C:76-81.

Min, S. W., and K. H. Song. 2006b. Analysis on current density induced inside body of hot-line worker for $765 \mathrm{kV}$ double circuit transmission line (in Korean with English summary). Trans KIEE 55C:231-8.

Misakian, M. 1991. In vitro exposure parameters with linearly and circularly polarized ELF magnetic fields. Bioelectromagnetics 12:377-81.

Misakian, M., A. R. Sheppard, D. Krause, M. E. Frazier, and D. L. Miller. 1993. Biological, physical, and electrical parameters for in-vitro studies with ELF magnetic and electric fields: A primer. Bioelectromagnetiucs (Suppl 2):1-73.

Miyakoshi, J., E. Horiuchi, T. Nakahara, and T. Sakurai. 2007. Magnetic fields generated by an induction heating (IH) cook top do not cause genotoxicity in vitro. Bioelectromagnetics 28:529-37. 
Motrescu, V. C., and U. van Rienen. 2005. Computation of currents induced by ELF electric fields in anisotropic human tissues using the Finite Integration Techniques (FIT). Adv Radio Sci 6:227-31.

Myung, S. H., J. B. Lee, and C. S. Huh. 1998. Calculation of induced current in the human body around $765 \mathrm{kV}$ transmission lines (in Korean with English summary). KIEES 9:802-12.

Myung, S. H., D. I. Lee, K. Y. Shin, I. S. Han, and J. K. Park. 2002. ELF 3D magnetic field and eddy current calculation of human body around transmission lines (in Korean with English summary). Trans KIEE 51C:485-91.

Nadeem, M., Y. Hamnerius, K. H. Mild, and M. Persson. 2004. Magnetic field from spot welding equipment-is the basic restriction exceeded? Bioelectromagnetics 25:278-84.

Nagaoka, T., K. Sakurai, E. Kunieda, S. Watanabe, H. Honma, T. Suzuki, M. Kawai et al. 2002. The development of whole-body high-resolution voxel models of the average Japanese adult male and female (in Japanese with English summary). Trans Jpn Soc Med Biol Eng 40:239-46.

Nagaoka, T., S. Watanabe, K. Sakurai, E. Kunieda, S. Watanabe, M. Taki, and Y. Yamanaka. 2004. Development of realistic high-resolution whole-body voxel models of Japanese adult males and females of average height and weight, and application of models to radio-frequency electromagnetic-field dosimetry. Phys Med Biol 49:1-15.

Nagaoka, T., and S. Watanabe. 2008. Postured voxel-based human models for electromagnetic dosimetry. Phys Med Biol 53:7047-61.

Nakasono, S., M. Ikehata, M. Dateki, S. Yoshie, T. Shigemitsu, and T. Negishi. 2008. Intermediate frequency magnetic fields do not have mutagenic, co-mutagenic or gene conversion potentials in microbial genotoxicity tests. Mutat Res 649:187-200.

NAS. 1996. Possible Health Effects of Exposure to Residential Electric and Magnetic Fields. National Academy of Sciences. Washington, DC: National Academy Press.

Negishi, T., S. Imai, K. Shibuya, I. Nishimura, and T. Shigemitsu. 2008. Lack of promotion effects of $50 \mathrm{~Hz}$ magnetic fields on 712-Dimethybenz (a) anthrancene-induced malignant lymphoma/lymphatic leukemia in mice. Bioelectromagnetics 29:29-38.

NIEHS. 1998. In Assessment of Health Effects from Exposure to Power-Line Frequency Electric and Magnetic Fields. ed. C. J. Portier, and M. S. Wolfe. Research Triangle Park, NC 27709: National Institute of Environmental Health Sciences of the National Institute of Health. NIH Publ. No.98-3981: Research Triangle Park, NC.

Nishizawa, S., H. O. Ruoss, F. M. Landstorfer, and O. Hashimoto. 2004. Numerical study on an equivalent source model for inhomogeneous magnetic field dosimetry in the low-frequency range. IEEE Trans BME 51:612-6.

Nishizawa, S., F. M. Landstorfer, and Y. Kamimura. 2007. Low-frequency dosimetry of inhomogeneous magnetic fields using the coil source model and the household appliance. IEEE Trans BME 54:497-502.

Olsen, R. G. 1996. Power transmission electromagnetics. Radio Sci Bull 277:29-38.

Orcutt, N., and O. P. Gandhi. 1988. A 3-D impedance method to calculate power deposition in biological bodies subjected to time varying magnetic fields. IEEE Trans Biomed Eng 35:577-83. 
Otaka, Y. 2001. An introduction to bioeffects studies of ELF and RF EMF in Japan. Bioelectromagnetics Newsletter 158:9-10.

Park, J. H., and S. W. Min. 2008. Analyses on current densities induced inside a worker using AC arc welder (in Korean with English summary). Trans KEEE 57:433-8.

Peratta, C. P., and A. Peratta. 2010. Modelling the Human Body Exposure to ELF Electric Fields. WIT Press: Southampton.

Poljak, D., and Y. F. Rashed. 2002. The boundary element modeling of the human body exposed to the ELF electromagnetic fields. Eng Anal Bound Elem 26:871-5.

Polk, C. 1986. Physical mechanisms by which low frequency magnetic fields can affect the distribution of counterions on cylindrical biological cell surfaces. J Biol Phys 14:3-8.

Polk, C. 1990a. Electric fields and surface charges induced by ELF magnetic fields. Bioelectromagnetics 11:189-201.

Polk, C., and J. H. Song. 1990b. Electric fields induced by low frequency magnetic fields in homogeneous biological structures that are surrounded by an electric insulator. Bioelectromagnetics 11:235-49.

Polk, C. 1992. Dosimetry of extremely-low-frequency magnetic fields. Bioelectromagnetics (Suppl 1):209-35.

Potter, M., M. Okoniewski, and M. A. Stuchky. 2000. Low frequency finite difference time domain (FDTD) for modeling of induced fields in humans close to line sources. J Comput Phys 162:82-103.

Reilly, J. P. 1998. Applied Bioelectricity: From Electrostimulation to Electrophathology. 346, 364. New York: Springer-Verlag Inc.

Robertson-De Mers, K. A., and D. L. Miller. 1992. Measurement of magnetically induced electric fields in conductive media near a $60 \mathrm{~Hz}$ current-carrying wire. Bioelectormagnetics 13:209-21.

Ruoss, H. O., W. Spreitzer, S. Nishizawa, S. Messy, and M. Klar. 2001. Efficient determination of current densities induced in the human body from measured low-frequency inhomogeneous magnetic fields. Microw Opt Technol Lett 29:211-3.

Schwan, H. P. 1985. Biophysical principles of the interaction of ELF-fields with living matter. II. Coupling considerations and force. In Biological Effects and Dosimetry of Static and ELF Electromagnetic Fields, ed. M. Grandolfo, S. M. Michaelson, and A. Rndi 243-71. New York and London: Plenum Press.

Scorretti, R., N. Burais, L. Nicolas, and A. Nicolas. 2005. Modeling of induced current into the human body by low-frequency magnetic field from experimental data. IEEE Trans Magn 41:1192-5.

Shiau, Y., and A. P. Valentino. 1981. ELF electric field coupling to dielectric spheroidal models of biological objects. IEEE Trans BME 28:429-37.

Shigemitsu, T., Y. Tsuchida, F. Nishiyama, G. Matsumoto, H. Nakamura, and K. Shimizu. 1981. Temporal variation of the static electric field inside an animal cage. Bioelectromagnetics 2:391-402.

Shigemitsu, T., K. Takeshita, Y. Shiga, and M. Kato. 1993. $50 \mathrm{~Hz}$ magnetic field exposure system for small animals. Bioelectromagnetics 14:107-16.

Shigemitsu, T., K. Yamazaki, S. Nakasono, and M. Kakikawa. 2007. A review of studies of the biological effects of electromagnetic fields in the intermediate frequency range. IEEJ Trans 2:405-12. 
Shigemitsu, T., T. Negishi, K. Yamazaki, Y. Kawahara, A. Haga, K. Kobayashi, and K. Muramatsu. 2009. A newly designed and constructed $20 \mathrm{kHz}$ magnetic field exposure facility for in vivo study. Bioelectromagnetics 30:36-44.

Shimizu, K., H. Endo, and G. Matsumoto. 1988a. Visualization of electric fields around a biological body. IEEE Trans BME 35:296-302.

Shimizu, K., H. Endo, and G. Matsumoto. 1988b. Fundamental study on measurement of ELF electric field at biological body surfaces. IEEE Trans IM 37:779-84.

Spiegel, R. J. 1976. ELF coupling to spherical models of man and animals. IEEE Trans BME 23:387-91.

Spiegel, R. J. 1977a. High-voltage electric field coupling to humans using moment method techniques. IEEE Trans BME 24:466-72.

Spiegel, R. J. 1977b. Magnetic coupling to a prolate spheroid model of man. IEEE Trans PAS 96:208-12.

Spiegel, R. J. 1981. Numerical determination of induced currents in humans and baboons exposed to $60 \mathrm{~Hz}$ electric fields. IEEE Trans EMC 23:382-90.

Stuchly, M. A., and W. Xi. 1994. Modelling induced currents in biological cells exposed to low-frequency magnetic fields. Phys Med Biol 39:1319-30.

Stuchly, M. A., and S. Zhao. 1996. Magnetic field induced currents in the human body in proximity of power lines. IEEE Trans PWRD 11:102-9.

Stuchly, M. A., and O. P. Gandhi. 2000. Inter-laboratory comparison of numerical dosimetry for human exposure to $60 \mathrm{~Hz}$ electric and magnetic fields. Bioelectromagnetics 21:167-74.

Stuchly, M. A., and T. W. Dawson. 2000. Interaction of low-frequency electric and magnetic fields with the human body. Proc IEEE 88:641-64.

Stuchly, M. A., and T. W. Dawson. 2002. Human body exposure to power lines: relation of induced quantities to external magnetic fields. Health Phys 83:333-40.

Suzuki, Y., and M. Taki. 2005. Measurement of magnetic field from an induction heating hob and estimation of induced current density in human body (in Japanese with English summary). IEEJ Trans A 125:427-33.

Taki, M., Y. Suzuki, and K. Wake. 2003. Dosimetry considerations in the head and retina for extremely low frequency electric fields. Radiat Prot Dosimetry 106:349-56.

Takuma, T., T. Kawamoto, K. Isaka, and Y. Yokoi. 1990. A three-dimensional method for calculating currents induced in bodies by extremely low-frequency electric fields. Bioelectromagnetics 11:71-89.

Takuma, T., S. Watanabe, T. Kawamoto, and K. Yamazaki. 2006. A review of studies on the electric field and the current induced in a human body exposed to electromagnetic fields. IEEJ Trans 1:131-9.

Tarao, H., N. Hayashi, and K. Isaka. 1997. Effect of non-uniformity on electric field characteristics inside homogeneous human model induced by non-uniform ambient magnetic field (in Japanese with English summary). IEEJ Trans A 117:317-23.

Tarao, H., N. Hayashi, and K. Isaka. 1998. Characterization of electric fields induced by ELF magnetic fields in biological model of eccentric double-layered sphere (in Japanese with English summary). IEEJ Trans A 118:475-82. 
Tarao, H., N. Hayashi, and K. Isaka. 2000. Analysis of magnetically induced ELF current in biological, spherical model with biomembrane (in Japanese with English summary). IEEJ Trans A 120:135-41.

Tarao, H., N. Hayashi, and K. Isaka. 2001. Analysis of magnetically induced ELF current in biological spherical model with biomembrane. Electr Eng Jpn 135:17-25.

Tarao, H., N. Hayashi, and K. Isaka. 2003. Numerical analysis of induced current in human head exposed to nonuniform magnetic field including harmonics (in Japanese with English summary). IEEJ Trans FM 123:1100-7.

Techaumnat, B., M. Hamada, and T. Takuma. 2000. Calculation of electrostatically induced current in a human body simulated with the curved-element BEM (in Japanese with English summary). IEEJ Trans A 120:810-6.

Techaumnat, B., M. Hamada, and T. Takuma. 2001. Calculation of current in a human body induced by a low frequency magnetic field by the curved-element BEM (in Japanese with English summary). IEEJ Trans A 121:848-53.

Tenforde, T. S. 1987. Interaction of extremely low frequency electric and magnetic fields with humans. Health Phys 53:585-606.

Tenforde, T. S. 1992. Microscopic dosimetry of extremely-low-frequency electric and magnetic fields. Bioelectromagnetics (Suppl 1):61-6.

The Visible Human Project. 2009. National Library of Medicine (US). http://www.nlm .nih.gov/research/visible/visible_human.html (accessed November 16, 2010).

Tofani, S., P. Ossola, G. D’Amore, and O. P. Gandhi. 1995a. Electric field and current density distributions induced in an anatomically-based model of the human head by magnetic fields from a hair dryer. Health Phys 68:71-9.

Tofani, S., L. Anglesio, P. Ossola, and G. d'Amore. 1995b. Spectral analysis of magnetic fields from domestic appliances and corresponding induced current densities in an anatomically based model of the human head. Bioelectromagnetics 16:356-64.

Valič, B., P. Gajšek, and D. Miklavčič. 2009. Current density in a model of a human body with a conductive implant exposed to ELF electric and magnetic fields. Bioelectromagnetics 30:591-9.

Wake, K., T. Tanaka, T. Kawasumi, and M. Taki. 1998. Induced current density distribution in a human related to magnetophosphene (in Japanese with English summary). IEEJ Trans A 118:806-11.

Wake, K., T. Tanaka, and M. Taki. 2000. Analysis of induced currents in a rat exposed to $50 \mathrm{~Hz}$ linearly and circularly polarized magnetic fields. Bioelectromagnetics 21:354-63.

WHO. 1984. Extremely Low Frequency (ELF) Fields. Environmental Health Criteria 35. Geneva: World Health Organization.

WHO. 1989. Magnetic Fields. Environmental Health Criteria 69. Geneva: World Health Organization.

WHO. 2007a. Extremely Low Frequency Fields, Environmental Health Criteria 238. Geneva: World Health Organization.

WHO. 2007b. 2007 WHO Research Agenda for Extremely Low Frequency Fields. http://www .who.int/entity/peh-emf/research/elf_research_agenda_2007.pdf. (accessed April 28, 2011).

Xi, W., M. A. Stuchly, and O. P. Gandhi. 1994a. Induced electric currents in models on man and rodents from $60 \mathrm{~Hz}$ magnetic fields. IEEE Trans BME 41:1018-23. 
Xi, W., and M. A. Stuchly. 1994b. High spatial resolution analysis of electric currents induced in man by ELF magnetic fields. Appl Comput Electromagn Soc J 9:127-34.

Xue, C., A. W. Wood, and T. Dovan. 2004. Induced current density in the foetus of pregnant workers in high magnetic field environments. Aust Phys Eng Sci Med 27:199-206.

Yamazaki, K., T. Kawamoto, and T. Shigemitsu. 1996. Fundamental study of induced current distribution inside the living body caused by ELF magnetic fields (in Japanese with English summary). IEEJ Trans C 116:193-9.

Yamazaki, K., H. Fujinami, T. Shigemitsu, and I. Nishimura. 2000a. Low stray ELF magnetic field exposure system for in vitro study. Bioelectromagnetics 21:76-83.

Yamazaki, K., T. Kawamoto, H. Fujinami, and T. Shigemitsu. 2000b. Investigation of ELF magnetically induced current inside human body-Development of estimation and effect of organ conductivity (in Japanese with English summary). IEEJ Trans A 120:81-7.

Yamazaki, K., T. Kawamoto, H. Fujinami, and T. Shigemitsu. 2000c. Investigation of relationship between ELF magnetic field and induced current inside human bodyComparison of human models under induced current calculation (in Japanese with English summary). IEEJ Trans A 120:369-70.

Yamazaki, K., T. Kawamoto, H. Fujinami, and T. Shigemitsu. 2001a. Investigation of ELF magnetically induced current inside the human body: Development of estimation tool and effect of organ conductivity. Electr Eng Jpn 134:1-10.

Yamazaki, K., and T. Kawamoto. 2001b. Simple estimation of equivalent magnetic dipole moment to characterize ELF magnetic fields generated by electric appliances incorporating harmonics. IEEE Trans EMC 43:240-5.

Yamazaki, K., T. Kawamoto, H. Fujinami, and T. Shigemitsu. 2005. Simplified dosimetry for human exposure to non-uniform ELF magnetic field (in Japanese with English summary). IEEJ Trans A 125:571-6.

Yamazaki, K., T. Kawamoto, H. Fujinami, and T. Shigemitsu. 2007. On the method of investigation of human exposure to nonuniform magnetic field (in Japanese with English summary). IEEJ Trans A 127:239-47.

Zoppetti, N., and D. Andreucetti. 2009a. Influence of the surface averaging procedure of the current density in assessing compliance with the ICNIRP low frequency basic restrictions by means of numerical techniques. Phys Med Biol 54:4835-45.

Zoppetti, N., and D. Andreuccetti. 2009b. Review of open problems in assessing compliance with 2004/40/EC Directive exposure limit values for low-frequency current density by means of numerical techniques. Radiat Prot Dosimetry 137:247-51.

Zubal, I. G., C. R. Harrell, E. O. Smith, Z. Rattner, G. R. Gindi, and P. H. Hoffer. 1994. Computed three dimensional segmented human anatomy. Phys Med Biol 21:299-302. 


\section{5 \\ Mobile \\ Communication Fields in Biological Systems}

Konstantina

S. Nikita

Asimina Kiourti

5.1 Introduction

5.2 Numerical Dosimetry.....

Body Models • Tissue Dielectric

Properties - Computational

Methods - Uncertainty Assessment

5.3 Experimental Dosimetry

Human Phantoms - Measurement Devices and

Equipment • Uncertainty Assessment

5.4 Exposure Assessment

Cellular Phones - Wired and Wireless (Bluetooth)

Hands-Free Devices • Base

Stations - Wi-Fi Systems

5.5 Conclusions

Acknowledgment

References.

\subsection{Introduction}

During the past few years, mobile communication devices operating in the radio frequency (RF) range have evolved and diffused into the market very rapidly. This leads to exposure of the users to RF electromagnetic (EM) radiation emitted by mobile communication equipment such as cellular phones, wired and wireless (Bluetooth) hands-free devices, base stations, Wi-Fi systems, etc., and has caused an increased public concern about the potential health hazards.

The biological effects of the emitted RF EM radiation can be divided into three categories: thermal, athermal, and nonthermal effects (Adey 1993). Thermal effects cause tissue heating since the EM energy absorbed by the human tissues increases the molecular translational and rotational kinetic energy. In the case of athermal effects, even though 
the amount of absorbed EM energy is capable of heating the tissues, the temperature of the tissues does not increase because of the body thermoregulation mechanisms. Finally, nonthermal effects comprise complicated interactions between live cells and ions (calcium, potassium, etc.) and are related to the behavior of big molecules (proteins and DNA).

So far, all recommendations and regulations set by national and international organizations regarding the limits on allowable absorbed power in the body are based on quantitative short-term evaluation of the thermal effects caused by the EM fields (Lin 2000). The two major standards relating to RF radiation have been set up by the Institute of Electrical and Electronic Engineers (IEEE) (IEEE 2005) and the International Commission on Non-Ionizing Radiation Protection (ICNIRP) (ICNIRP 1998). In these standards, basic restrictions are defined, in terms of specific absorption rate (SAR) and induced current density, to limit human exposure to time-varying EM fields. Furthermore, more-readilymeasurable reference levels are provided, in terms of the external electric and magnetic field strength and power density incident on the human body, to practically estimate the exposure and check compliance with the basic restrictions (Lin 2007).

Considerable dosimetric research efforts have been devoted to assess the interactions between the EM radiation emitted by mobile communication devices and the human body (Lin 2009). Dosimetry is necessary to "evaluate the dose," or equivalently, to identify the dose metric that is closely related to the effect of concern. These efforts have been motivated by three factors: (1) the need to evaluate potential health effects and compliance with standards (compliance testing), (2) the need to verify if existing protection standards are still adequate, and (3) the need to assess antenna performance and improve antenna design to minimize the energy absorbed in the human body while maximizing the radiated energy.

The calculation of the SAR prevails in most of the studies (Beard et al. 2006; Christ and Kuster 2005; Virtanen, Keshvari, and Lappalainen 2007). The SAR may be spatially averaged over the total mass of the exposed body or its parts and may be time-averaged over a given time of exposure or even a single pulse or modulation period of the radiation. However, the values of other dosimetric quantities are also calculated in a number of works. For example, the resonance frequencies of biological bodies are examined in the studies of Massoudi et al. (1979), and the currents induced inside the human head are presented in the works of Chen and Wang (1994), while the thermal effects of electromagnetic field exposure are considered in studies by Kriticos and Schwan (1979). Compliance can also be demonstrated by showing that the incident field is below the derived exposure limits defined in terms of the electric and magnetic field strengths. This is a suitable approach for base stations, and tabletop and computer-mounted devices (Bernardi et al. 2000a; Cortel-Carrasco et al. 2006; Lacroux et al. 2008).

Priority was initially given to epidemiological studies related to the possible connection between cellular phone use and brain tumors, parotid and salivary gland tumors, other kinds of head and neck tumors, leukemia, and lymphoma (Christensen et al. 2004, 2005; Hepworth et al. 2006; Kan et al. 2007; Klaeboe, Blaasaas, and Tynes 2007; Lahkola et al. 2007; Lonn et al. 2006; Schoemaker et al. 2005; Schuz et al. 2006; Takebayashi et al. 2006). These studies have not provided any sign that exposure to RF EM fields emitted by cellular phones increases the chance of carcinogenesis. Apart from the epidemiological studies, whose main characteristics are long duration, application to a wide swath. 
of the population, and statistical recording of illnesses and symptoms, research on the biological effects of RF EM radiation emitted by mobile communication devices can also be carried out numerically and experimentally. Selecting the appropriate methods for numerical and experimental dosimetry and performing uncertainty assessment to validate the derived results are of utmost importance.

In this chapter, a review of the numerical tools and experimental methods that can be used to model and assess the interaction of mobile communication devices with the human body is presented. Regarding dosimetry studies on mobile communication devices, the interaction between cellular phones and the user's head has been extensively studied so far. Research assessing the interaction between wired and wireless (Bluetooth) hands-free devices and the user's head and body have also been performed. Furthermore, there is an increased scientific interest in assessing human exposure to base-station antennas and new emerging communication technologies, such as $\mathrm{Wi}-\mathrm{Fi}$, because of the simultaneous and long-lasting exposure of users. The results of recent dosimetry studies related to human exposure to cellular phones, wired and wireless (Bluetooth) hands-free devices, base-station antennas, and Wi-Fi systems are also discussed.

\subsection{Numerical Dosimetry}

Numerical dosimetry was first performed in the 1960s, as a means to assess human exposure to the RF EM fields present, at that time, in the environment, such as fields produced by radio and TV broadcasting antennas and radar systems. The EM source was assumed to be placed far from the exposed human body and was represented by a plane wave (Dimbylow and Gandhi 1991; Gandhi et al. 1992).

Nowadays, numerical dosimetry is widely used to evaluate the interaction between the EM energy emitted by mobile communication devices and the human tissues. This interaction is assessed in terms of the derived dosimetric quantities of SAR and current density and/or the electric and magnetic field induced in the human tissues. Human exposure to both the near- and far-field of the EM source can be examined. Analytical methods can be applied to simplified canonical geometries modeling the human body (or parts of it). On the other hand, numerical methods use body models ranging from very simple homogeneous models to millimeter resolution anatomic models, with the latter providing very fine dosimetric results.

\subsubsection{Body Models}

\subsubsection{Canonical Models}

Basic characteristics of the absorption of RF energy in the human body have been established by homogeneous or heterogeneous (layered) simplified canonical models such as a cube, a sphere, and a spheroid. Those have been systematically summarized (Durney, Massoudi, and Iskander 1986) and used for the rationale of RF safety guidelines.

Whole-body canonical models have largely been used in the literature to model the human body in dosimetry studies. A planar three-layer body model, consisting of a 
low-water-content tissue layer (fat) embedded between two high-water-content tissues (skin and muscle) was proposed by Curto and Ammann (2007; Figure 5.1a). Nine combinations of skin, fat, and muscle tissues with different thicknesses were analyzed to model the absorption in different parts of the body. A similar planar three-layer body model was also used in research by Christ et al. (2006), while in that of Wiart et al. (2005) a multilayer structure composed of skin, hypoderm, muscle, uterus, placenta, amniotic fluid (considered as cephalo spinal fluid; CSF), and fetus (considered as muscle) was analyzed (Figure 5.1b).

Canonical geometries have also been used to model specific parts of the human body. Systems of concentric or eccentric spheres with a maximum number of six layers to model the human head were presented in a number of studies (Cerri, De Leo, and Rosellini 1997; Forgy et al. 1997; Koulouridis and Nikita 2004; Lin and Wang 2005; Lu et al. 1996; Meier et al. 1997; Nikita et al. 2000a,b; Okoniewski and Stuchly 1996). For example, a homogeneous brain tissue and a three-layer spherical human head model consisting of skin, bone, and brain tissues were used in the studies of Koulouridis and Nikita (2004; Figure 5.2a and b, respectively). A rough

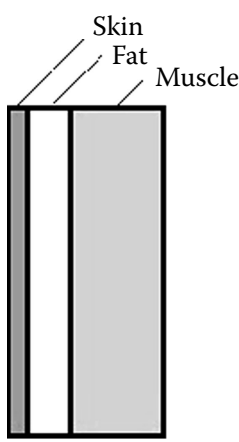

(a)

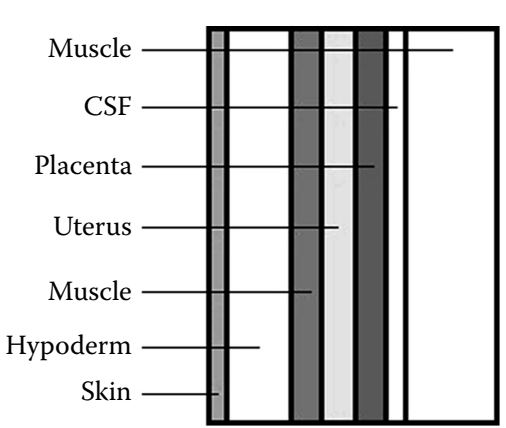

(b)

FIGURE 5.1 Whole-body canonical models used in the research (Data from Curto, S., and M. J. Ammann. 2007. Proc IEEE Antennas and Propag Soc Int Symp, IEEE, 3185-8. Honolulu, HI; Wiart, J. et al. 2005. Modeling of RF head exposure in children. Bioelectromagnetics 26:S19-30.).

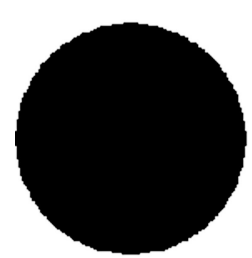

(a)

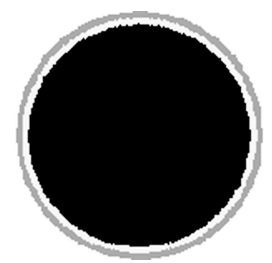

(b)

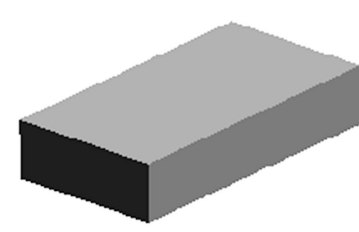

(c)

FIGURE 5.2 Canonical geometries modeling a human (a) homogeneous and (b) three-layer head (Data from Koulouridis, S., and K. S. Nikita. 2004. IEEE Trans Electromagn Compat 46:62-70.)

(c) Torso (Data from Kuhn, S. et al. 2009. Phys Med Biol 54:5493-508.). 
approximation of the human head has also been obtained by using homogeneous or layered dielectric box models (Okoniewski and Stuchly 1996; Pan, Bahrwas, and Wolff 1997). In studies by Kuhn et al. (2009), the human torso was modeled as a rectangular structure filled with standard (IEC 2005) tissue emulating material (Figure 5.2c). Finally, the hand of the operator of a mobile communication device has often been modeled as a homogeneous block model (Toftgard, Hornsleth, and Andersen 1993; Figure 5.3a) or a multilayer block model (Rowley and Waterhouse 1999; Figure 5.3b). Modeling the hand of the operator is important for the evaluation of antenna performance. However, there does not seem to exist strong concern in modeling the hand, perhaps because the main guidelines for exposure allow higher SAR values in this region of the body.

Exposure of children to RF EM radiation has gained considerable attention given their special status during development and growth. In the case of canonical models, it is most commonly assumed that a child model is perfectly proportional to an adult model. As such, children canonical models are obtained through uniform downscaling of the corresponding adult models. For example, in the studies of Koulouridis and Nikita (2004), homogeneous and multilayer spherical children head models were obtained through uniform deformation of spherical adult head models.

Even though heterogeneous models are more representative of actual coupling in human tissue, it has been observed that homogeneous models overestimate the absorbed energy and can be considered as worst-case approximations. This is the reason why, although sophisticated body models have appeared in most recent studies, the homogeneous model case has almost always been present as well (Curto and Ammann 2006; Conil et al. 2008).

The treatment of canonical models is sufficient for computer code checking and allows the use and comparison of different purely numerical techniques to ensure both their validity and applicability in terms of computing time and memory demands. These models are computationally efficient with standard simulation resources and seem to be adequate for obtaining preliminary results regarding radiation hazard purposes. Furthermore, the choice of simple models provides the possibility to easily construct experimental phantoms complying with the numerical ones.

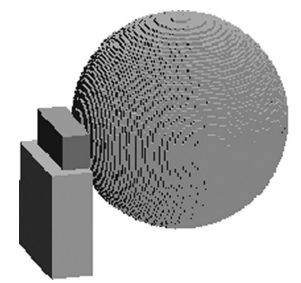

(a)

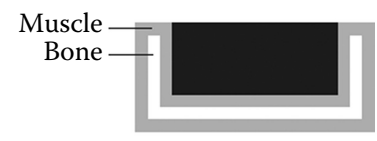

(b)

FIGURE 5.3 Canonical geometries modeling the hand of the operator of a mobile communication device as a (a) homogeneous (Data from Toftgard, J., S. N. Hornsleth, and J. B. Andersen. 1993. IEEE Trans Antennas Propag 41:739-46.) (b) multilayer block model (Data from Rowley, J. T., and R. B. Waterhouse. 1999. IEEE Trans Antennas Propag 47:815-22.). 


\subsubsection{Anatomically Based Models}

In most numerical treatments, the entire human body or parts of it are modeled by cubic cells (voxels). In each cubic cell of the mesh, the tissue properties are considered constant. By assigning the corresponding electric properties to each voxel, one can easily model the anatomical tissues and organs.

In the majority of recent studies, the data for simulating parts of the body are taken from magnetic resonance imaging (MRI) scans (Dimbylow and Gandhi 1991; Dimbylow and Mann 1994; Gandhi, Lazzi, and Furse 1996; Gandhi and Chen 1995; Hombach et al. 1996; Lazzi and Gandhi 1997; Lu et al. 1996; Meier et al. 1997; Schoenborn, Burkhardt, and Kuster 1998), though models based on computed tomography (CT) scans and anatomical images have also been encountered (Bernardi, Cavagnaro, and Pisa 1996). MRI and CT provide gray-scale image data from the head to the feet of the human body as several transverse slices at a designated spacing. The resolution in each slice is in the order of several millimeters. MRI data are generally superior to CT data in identifying interior tissues because of the high contrast images of the soft tissues. In order to be used in numerical dosimetry, these digital data sets must be converted to the so-called segmented version (Chen, Gelb, and Renaut 2003; Wells et al. 1996; Zankl and Wittman 2001). The translation of $3 \mathrm{D}$ data sets obtained by MRI or CT scans into a numerical model is a complex and time-consuming activity that is difficult to perform with only automatic procedures, such as contour recognition algorithms. This process inevitably requires intervention by experts in human anatomy, who are able to distinguish both transitional and marginal regions. Even if software for automatic identification is applied, manual verification or correction is required. It is worth noting that MRI or CT produced in different laboratories inevitably contain differing discretizations. Furthermore, the resolution of the medical imaging techniques is, presently, too high for using their results directly in numerical modeling.

Early examples of anatomically based models include a torso model consisting of 16628 cells, each of side length $12.7 \mathrm{~mm}$, used for SAR prediction (Sullivan, Borup, and Gandhi 1987), as well as a model consisting of approximately 35000 cells, each of side length $10 \mathrm{~mm}$ (as acquired from CT scans of a cancer patient), used for hyperthermia treatment (Sullivan 1990). As computing power increases and computer resources get less expensive, there is a trend to refine the numerical description of the space modeled and move to more detailed anatomical structures. In contemporary models, the highest complexity used for modeling the whole human body is about 50 tissue types and the finest resolution is about $1 \mathrm{~mm}$. For example, in studies by Martinez-Burdalo et al. (2009), a 3D high-resolution head mesh, developed from nuclear magnetic resonance has been resized into a finer grid, without loss of anatomical details. The resulting model has $1 \times 1 \times 1.25 \mathrm{~mm}^{3}$ size cells and includes 18 different biological tissues.

Even though models with resolution on the order of $1 \mathrm{~mm}$ are becoming available, models with 2-3 mm resolution are quite adequate for frequencies of $1-2 \mathrm{GHz}$ (Gandhi et al. 1992). However, special care is required to accurately model organs of particular importance to the human health, such as the eyes, the parotid, the hypophysis gland, etc. (Bernardi, Cavagnaro, and Pisa 1996; Okoniewski and Stuchly 1996). Better resolution is gained by refining the grid locally. However, higher resolution in human head 
modeling has been found to generally increase the observed peak SAR (Okoniewski and Stuchly 1996; Schoenborn, Burkhardt, and Kuster 1998).

\subsection{Adults}

In the recent years, several anatomically realistic models of adults have been developed for use in a wide range of EM dosimetric studies. The Visible Human Project (VHP) (Ackerman 1998) has made available a digital axial anatomical image library at $1 \mathrm{~mm}$ resolution, with associated MRI and CT images, of the body of a human male, named Hugo (Figure 5.4a). Hugo's body model is larger than that of an average man (height $186 \mathrm{~cm}$, weight $90 \mathrm{~kg}$ ) and consists of 38 tissues. Various dosimetric characteristics have been investigated with the VHP man (Gjonaj et al. 2002; Mason et al. 2000; Wang et al. 2004), and the model is now being included in many commercially available EM simulation tools with capabilities for dosimetric evaluation.

Based on the VHP man, several modifications have been suggested. For example, in studies by Bernardi et al. (2000a), a heterogeneous male model has been obtained from a tissue-classified version of the VHP man and has been down-sampled to obtain a final resolution of $5 \mathrm{~mm}$. The body model has a total height of $180 \mathrm{~cm}$ and is composed of 31 different types of tissues/organs. Furthermore, the deviation of Hugo's dimensions (height and weight) from the average values has promoted development of other anatomically realistic models with average height and weight that are specified in International Commission on Radiation Protection (ICRP) and other standards (Dimbylow 2005a; ICRP 1994; Nagaoka et al. 2004).

Several other anatomically based adult models can be found in the literature. In Lacroux et al. (2008), the Zubal adult male model developed by Yale University was used (height $177 \mathrm{~cm}$, weight $78 \mathrm{~kg}$; Figure 5.4b). The model is composed of 31 different tissues and its resolution is $3.6 \mathrm{~mm}$. The Norman model (Dimbylow 1998, 2002) was developed at the National Radiation Protection Board (NRPB) using data obtained from MRI and scaled to the height and mass of the reference man described in ICRP 1994; height $176 \mathrm{~cm}$, weight $73 \mathrm{~kg}$; Figure 5.4c). The voxels have approximately a $2 \mathrm{~mm}$ side and are segmented to 38 tissue types. A male Japanese model (height $172 \mathrm{~cm}$, weight $65 \mathrm{~kg}$ ) was

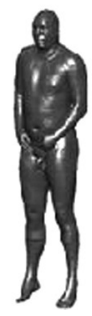

(a)

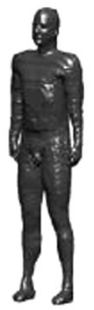

(b)

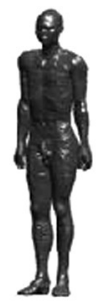

(c)

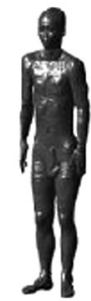

(d)

FIGURE 5.4 Anatomically realistic body models of adults: (a) Hugo. (From Ackerman, M. J. 1988. Proc IEEE, 86, 3. With permission.) (b) Zubal. (From Lacroux, F. et al. 2008. Ann Telecomm, 63, 55. With permission.) (c) Norman. (From Dimbylow, P. 1998. Phys Med Biol, 42, 479; Dimbylow, P. 2002. Phys Med Biol, 47, 2835. With permission.) (d) Japanese Male. (From Nagaoka, T. et al. 2004. Phys Med Biol, 49, 1. With permission.) 
developed by the National Institute of Information and Communication Technology (NICT) (Nagaoka et al. 2004; Figure 5.4d). The model consists of $2 \mathrm{~mm}$ edge voxels and is segmented to 51 tissue types. Examples of body models in postures other than standing can be found in Allen et al. (2003, 2005), Findlay and Dimbylow $(2005,2006)$, and Uusitupa et al. (2010). A review of voxel-based models developed for a range of applications can be found in Caon (2004).

Since the power absorbed in the human head is considered to be the most crucial parameter in assessing the potential hazard caused by the use of mobile communication devices, several research studies have focused on modeling the human head (Bernardi, Cavagnaro, and Pisa 1996; Burkhardt and Kuster 2000; Christ and Kuster 2005; Groot et al. 1997; Hirata, Matsuyama, and Shiozawa 2000; Hombach et al. 1996; Meier et al. 1997; Okoniewski and Stuchly 1996; Schoenborn, Burkhardt, and Kuster 1998; Watanabe et al. 1996). For example, the anatomically correct human head models of Figure $5.5 \mathrm{a}$ and $\mathrm{b}$ are used in the works of Kainz et al. (2005), while those of Figure 5.5c, d, and e are used in the studies of Christ et al. (2010b). Since the human head is a very complex and varied structure, large differences in SAR can be produced depending on the numerical model used.

Anatomic models of the user's hand holding the wireless device can also be found in the literature. A hand with typical handset holding model may be simulated with one tissue approximating the average hand tissues relative permittivity (Figure 5.6a; Watanabe et al. 1996). A homogeneous 3D model has also been used in research by Chavannes et al. (2006) to simulate the user's hand (Figure 5.6b), whereas a realistic model with two tissues (bone and muscle) has been considered in Su et al. (2006; Figure 5.6c).

CAD-based human models have also been developed. The CAD models can easily move and rotate in any direction with 3D CAD software and without any limitation

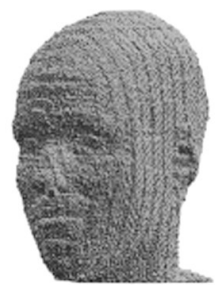

(a)

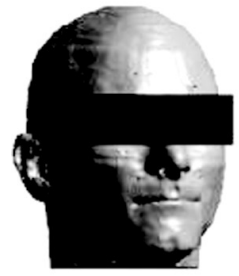

(c)

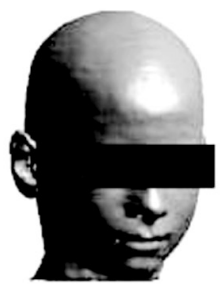

(d)

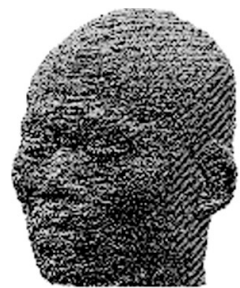

(b)

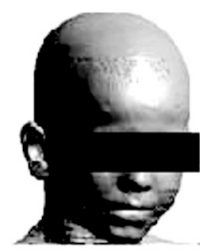

(e)

FIGURE 5.5 (a) European female and (b) Japanese male anatomical head models (From Kainz, W. et al. 2005. Phys Med Biol, 50, 45. With permission.) and (c) Duke, (d) Billie, and (e) Thelonious head models used in the study of (From Christ, A. et al. 2010. Bioelectromagnetics, 31, 12. With permission.) 


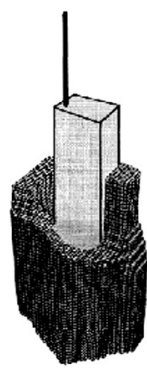

(a)

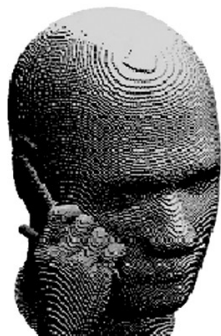

(b)

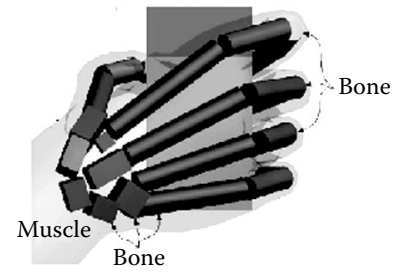

(c)

FIGURE 5.6 Anatomical hand models (a) From Watanabe, S. et al. 1996. IEEE Trans Microw Theory Tech, 44, 83. With permission. (b) From Chavannes, N. et al. 2006. Reliable Prediction of Mobile Phone Performance for Different Daily Usage Patterns Using the FDTD Method. Proc IEEE Int Workshop Antenna Technol, New York. With permission. (c) From Su, C. M. et al. 2006. User's Hand Effects on EMC Internal GSM/DCS Mobile Phone Antenna. Proc IEEE Antennas Propag Soc Int Symp, New Mexico. With permission.

of their spatial resolution (Kainz et al. 2007). The surfaces of the model can be readily deformed, but care must be taken to correctly articulate the joints of the body.

\subsection{Children}

Due to the lack of child MRI or CT images, the first anatomical child models in the literature were based on uniform downscaling of adult models (Dimbylow 2002, 2005b; Findlay and Dimbylow 2006; Gandhi, Lazzi, and Furse 1996; Wang et al. 2006). For example, in ICRP (1994), an adult human model in a sitting position (Findlay and Dimbylow 2005) was scaled to ICRP dimensions for a reference 10 -year-old child when sitting (height $113 \mathrm{~cm}$, weight $32 \mathrm{~kg}$ ), while a 10 -year-old rescaled version of the Norman adult model was presented in studies of Findlay and Dimbylow (2010). Uniform reduction of the geometrical dimensions of adult human heads to represent smaller head models have been reported in studies by Christopoulou, Koulouridis, and Nikita (2009), Gandhi and Kang (2002), Gandhi, Lazzi, and Furse (1996), Hadjem et al. (2005), Koulouridis and Nikita (2004), and Koulouridis, Christopoulou, and Nikita (2005).

However, the uniform-downscaling approach does not take into account the different growth patterns of certain parts of the body. For example, head growth is age dependent. The volume of the brain, the skin, and skull thickness each grow at different rates (Koenig, Donovan, and Pensler 1995; Seidenari et al. 2000). As a result, the scaled child models do not exactly reproduce the dimensions and anatomy of children. A more realistic approach suggests making a piecewise reduction of the adult model with respect to the main anatomical parameters. The adult model is divided in different parts and nonuniform downscaling is applied to each of these (morphing deformation) (Wang and Fujiwara 2003). This is known as the "Child-Like" approach. For example, Bit-Babik et al. (2005) obtained a child head by using complex scaling procedures to an adult head model to attain anatomic correctness. Nonuniform scaling of an adult head model has also been performed in studies by Christopoulou, Koulouridis, and Nikita (2009) to 
obtain a 10 -year-old child head based on statistical measurement data of head- and facial-age-related parameters.

However, inaccuracies in the size of the nonuniform downscaled child models are still present. Furthermore, aside from the physical size, the tissue electromagnetic properties vary with age. As a result, recently, whole-body child models have been developed based on MRI or CT databases of children. In research of Wiart et al. (2008), several MRI datasets of children at different ages from three different French hospitals were used to provide segmented versions of child head models. Six child head models at different ages $(5,6,8$, 9, 12, and 15 years old) were built using this approach, as illustrated in Figure 5.7. The model of a 7-year-old child based on MRI scans and consisting of approximately 20 different tissues was presented in the studies of Schoenborn, Burkhardt, and Kuster (1998). Furthermore, Dimbylow and Bolch (2007) have recently reported the use of a series of pediatric voxel models (a 9-month-old male, 4- and 8-year-old females, and 11- and 14-year-old males) with $2 \mathrm{~mm}$ resolution, based on data acquired from CT imaging.

\subsection{Females}

Several models of females have been developed in the literature, as illustrated in Table 5.1.

Pregnant female anatomically based models have also been developed recently. Although most of the calculated SAR values of the fetus or embryo models are similar or lower than that of the mother, dosimetry in pregnant female models is required for a more comprehensive risk assessment of RF exposure of fetuses and embryos (ICNIRP 2009; Kawai et al. 2010). Models of pregnant females include the model representing nine gestational ages ( Wu et al. 2006; Figure 5.8a), a truncated body model of a mother

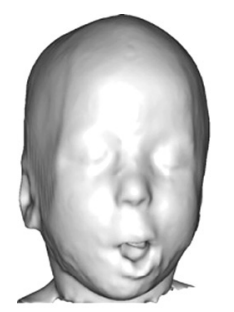

5 years old

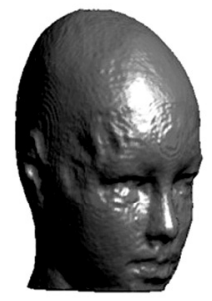

9 years old

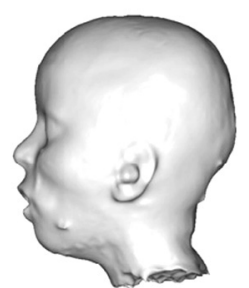

6 years old

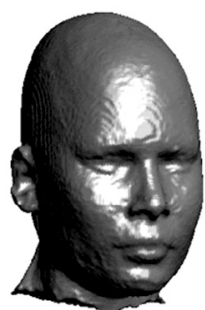

12 years old

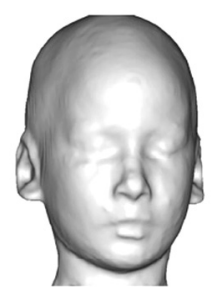

8 years old

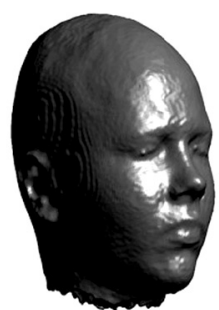

15 years old

FIGURE 5.7 Child head models based on MRI datasets of children at different ages (From Wiart, J., A. Hadjem, M. Wong, and I. Bloch. 2008. Phys Med Biol, 53, 95. With permission.) 
TABLE 5.1 Female Analytical Models Used in the Literature

\begin{tabular}{lccccc}
\hline Reference & $\begin{array}{c}\text { Age } \\
(\text { years })\end{array}$ & $\begin{array}{c}\text { Height } \\
(\mathrm{cm})\end{array}$ & $\begin{array}{c}\text { Weight } \\
(\mathrm{kg})\end{array}$ & $\begin{array}{c}\text { Voxel Size } \\
\left(\mathrm{mm}^{3}\right)\end{array}$ & $\begin{array}{c}\text { Number of } \\
\text { Tissue Types }\end{array}$ \\
\hline Mazzurana et al. (2004) & 30 & 163 & 47 & $2 \times 2 \times 2$ & $\mathrm{n} / \mathrm{a}$ \\
Liu, Collins, and Smith (2005) & $\mathrm{n} / \mathrm{a}$ & 165 & 85.5 & $5 \times 5 \times 5$ & 36 \\
Dimbylow (2005a,b) & 23 & 165 & 58 & $2 \times 2 \times 2$ & 41 \\
Nagaoka et al. (2004) & 22 & 160 & 53 & $2 \times 2 \times 2$ & 51 \\
\hline
\end{tabular}

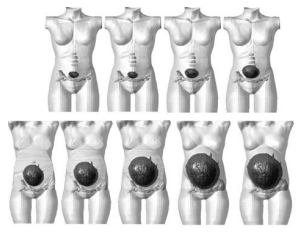

(a)

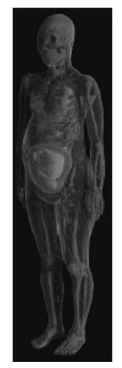

(d)

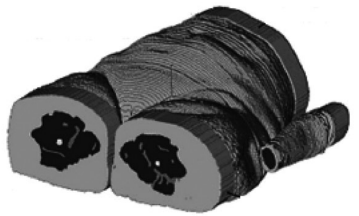

(b)
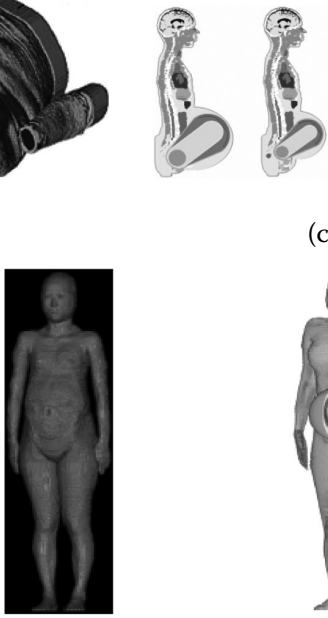

(f)

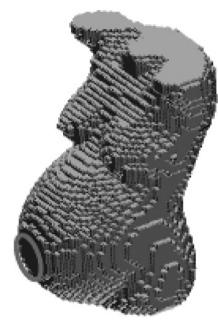

(e)

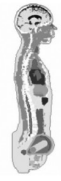

(c)
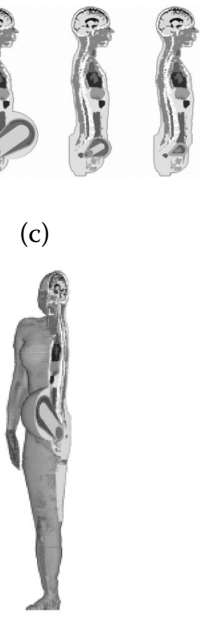

(g)

FIGURE 5.8 (See color insert.) Analytical pregnant female models developed in the research of (a) From Wu, D., S. Shami, J. Chen, and W. Kainz. 2006. IEEE Trans Microw Theory Tech, 54, 8. With permission. (b) From Hand, J. W., Y. Li, E. L. Thomas, M. A. Rutherford, and J. V. Hajnal. 2006. Magn Reson Med, 52, 802. With permission. (c) From Dimbylow, P. 2007. Phys Med Biol, 52, 802. With permission. (d) From Nagaoka, T. et al. 2007. Phys Med Biol, 52, 45. With permission. (e) From Kainz, W., D. D. Chan, J. P. Casamento, and H. I. Bassen. 2003. Phys Med Biol, 48, 60. With permission. (f) From Nagaoka, T. et al. 2006. Proc IEEE 28th Ann Conf Eng Med Biol Soc, New York. With permission. (g) From Dimbylow, P. 2006. Phys Med Biol, 51, 94. With permission.

with a 28-week-old fetus (Hand et al. 2006; Figure 5.8b), a whole-body model obtained by combining a nonpregnant female model and a mathematical fetus model (Dimbylow 2007; Figure 5.8c), a more realistic whole-body pregnant female model obtained by embedding an MRI-based fetal model inside a nonpregnant female model (Nagaoka et al. 2007; Figure 5.8d), the hybrid models described by Kainz et al. (2003) (Figure 5.8e) of a mother/fetus at 34 weeks and at 28 weeks gestation (Nagaoka et al. 2006; Figure 5.8f), as well as those representing mother and fetus at $8,13,26$, and 38 weeks gestation (Dimbylow 2006; Figure 5.8g). 


\subsubsection{Tissue Dielectric Properties}

Human tissues contain insulating materials (lipids) and electrical charges (ions, electrically polarized molecules, etc.). Therefore, they can be viewed as a weakly conducting medium (dielectric). The dielectric properties of human tissues are directly related to the amount of RF energy that is absorbed and converted into heat, due to the increase in their molecular translational and rotational kinetic energy. Exact knowledge of the dielectric properties of biological tissues has become essential in RF dosimetry in order to enable more accurate assessment of human exposure to RF EM fields emitted by mobile communication devices.

The currently most comprehensive, complete, and best-known database of RF dielectric properties of body tissues is based on the work of Gabriel et al., which provides dispersive parameter models for several important body tissues (Gabriel, Gabriel, and Corthout 1996). Before this work was published, most data on the dielectric properties of tissues used in numerical dosimetry were obtained from measurements of animals, such as pigs, sheep, or rabbits. Based on these measurements, Gabriel et al. proposed a parametric model using a Cole-Cole analysis for several body tissues in the frequency range from $10 \mathrm{~Hz}$ to $20 \mathrm{GHz}$. This model has been used extensively in numerical dosimetry for humans and animals.

Several other research efforts have been performed to assess the dielectric properties of human tissues. For example, Schmid et al. (2007). presented results for the dielectric properties of human gray matter tissue based on a sample of tissues. The sample was larger than ever reported before with respect to the number of brains, number of measurement locations, and freshness of the tissue. In the studies of Lazebnik et al. (2007b), the results of a large-scale, multi-institutional study characterizing the dielectric properties of normal breast tissue samples obtained from reduction surgeries were reported. Parameters for Debye models describing the dielectric properties of normal and malignant breast tissues were derived in the studies of Lazebnik et al. (2007a). Finally, O'Rourke et al. (2007) have recently characterized the dielectric properties of in vivo and ex vivo human liver tissues between $0.5 \mathrm{GHz}$ and $20 \mathrm{GHz}$.

However, the electrical properties of human tissues are not known with a high degree of precision and accurate knowledge of these properties is currently evolving. Almost all the work mentioned in Gabriel, Gabriel, and Corthout (1996) is based either on measurements on excised animal tissues about $2 \mathrm{~h}$ after death or, to a very small extent, on human autopsy material obtained more than $24 \mathrm{~h}$ after death. Only a few publications have reported in vivo measurements on animal tissues (Stuchly and Stuchly 1984; Surowiec et al. 1986). Furthermore, some data produced by other workers (Campbell and Land 1992) deviate significantly from the frequently used values in Gabriel, Gabriel, and Corthout (1996). Gabriel et al. have discussed the uncertainties in the measurement of dielectric properties and highlighted the major contribution of random variations from repeat measurements (Gabriel and Peyman 2006). These ambiguities can have important consequences on computed dosimetric quantities and may result in significantly different SAR distributions inside the human body (Gandhi, Lazzi, and Furse 1996; Hombach et al. 1996; Okoniewski and Stuchly 1996). For example, it appears that variations up to $50 \%$ in the convective coefficient between the head 
and the environment cause variations in the peak temperature increase of about $10 \%$ (Wang and Fujiwara 1999).

Regarding children dosimetry, the most widely accepted database of biological tissues' dielectric properties (Gabriel, Gabriel, and Corthout 1996) lacks data for children. As a result, in EM dosimetry of children, the dielectric properties of biological tissues for adults are so far being used. Nevertheless, age-dependent changes of the tissues' dielectric properties have been repeatedly reported (Conil et al. 2008; Gabriel 2005; Keshvari, Keshvari, and Lang 2006; Schmid and Uberbacher 2005). The dielectric properties, that is, permittivity and conductivity, have been demonstrated to decrease with age due to the changes of water content and organic composition of tissues (Penman, Rezazadeh, and Gabriel 2001). Higher conductivity has been found for the brain and skull of newborn rats compared to adult rats, at $900 \mathrm{MHz}(16 \%$ and $43 \%$, respectively), which suggests a possibility of SAR increase due to the higher tissue conductivity. A lower increase of permittivity has also been observed $(9.9 \%$ and $33 \%$, respectively). Furthermore, recently, a significant dependence of the dielectric properties of white matter and spinal cord on age was reported while no age-related variation has been found for the gray matter (Penman et al. 2007). In the research of Wang, Fujiwara, and Watanabe (2006), an empirical formula has been derived for the complex permittivity of various tissues as a function of the total body water (TBW), according to Lichtenecker's exponential law. With the use of the aforementioned formula, the dielectric properties in 7-year-old and 3-year-old child head models have been derived. Finally, a systematic evaluation of the age-dependent changes of the dielectric properties of a large number of different tissues has recently been published (Peyman et al. 2009). The establishment of a database for children's dielectric properties should be an essential and urgent task.

\subsubsection{Computational Methods}

Analytical and numerical methods have been developed over the last 40 years to understand coupling of electromagnetic fields to biological bodies. Analytical methods are restricted to very simple configurations, but they provide valuable insight into the physical mechanisms, yield typical parameters, and they are used for testing of numerical methods.

Studies on complex-shaped inhomogeneous bodies have been based on the use of boundary or volume techniques. In boundary techniques, the space is divided into linear, homogeneous, and isotropic domains, the boundaries of which are discretized. In volume techniques, the space is discretized directly. Boundary techniques are more efficient for geometrically simple configurations with a low surface to volume ratio, while volume techniques are preferable in modeling nonhomogeneous materials and more complex geometries. The numerical methods used to evaluate the power absorption into biological bodies mainly include the method of moments (MoM), the finite difference time domain (FDTD) method, the finite integration technique (FIT) and the finite elements method (FEM) (Lin and Bernardi 2007). Hybrid methods derived from the combination of these methods and other methods for EM propagation characterization are also used. 


\subsubsection{Analytical Methods}

Analytical techniques are so-called because, in contrast to numerical techniques, they consist of some solution to Maxwell's equation that is not based on a direct numerical solution and does not require the inversion of large matrices.

Much work to approach the problem of interaction of EM radiation with parts of the human body in an analytical way has been done in early dosimetric calculations (Cerri, De Leo, and Rosellini 1997; Durney, Massoudi, and Iskander 1986; Forgy et al. 1997; Lu et al. 1996; Meier et al. 1997; Sullivan, Borup, and Gandhi 1987; Zhou and van Oosterom 1992). The main restriction consists in adopting a simplified model of the whole body or parts of it. The choice of these highly simplified geometries is essential due to the necessity of characterizing a structure resembling the human body and/or parts of it and having at the same time a closed form of the wave equation.

Regarding the field source, most of the papers adopt a plane wave as incident field, while some others consider an elementary dipole. For example, the problem of interaction of a homogeneous sphere with a simple waveform (e.g., plane wave, short dipole) has been treated in Lin (1976) using analytical methods. In Zhou and van Oosterom (1992), the effort was focused on the evaluation of potential distribution inside spherical or spheroid volumes; these studies were related to a theoretical approach of electroencephalography (EEG) and evoked potential for the localization of brain activity. A quasistatic approximation was used because of the low frequency range of interest but the method was applied to layered anisotropic media.

Finally, most of the papers deal with the evaluation of the EM field, and therefore of the SAR, inside the simplified body model at RF or for a pulsed excitation. In the formulation of the problem, the EM field is usually described in terms of spherical wave vector eigenfunctions consisting of a combination of Legendre and spherical Bessel functions. The expansion coefficients are determined by the boundary conditions after applying the mode orthogonality and accounting for the external fields.

Although the analytical solutions do not provide detailed dosimetry information for actual human bodies, they contribute to qualitative analyses. They are particularly useful to test numerical codes, identify the structure resonant frequencies that represent conditions of maximum power deposition inside the human body, evaluate the effect of dielectric and geometric parameters spread, commonly inferred from the literature, and select the parameters of greater influence on SAR distribution.

\subsubsection{Numerical Methods}

From the end of the 1970s, numerical calculation methods for dosimetric studies have attracted great attention due to their advantage in modeling the anatomy of a human body. The constant evolution of computer systems (e.g., parallel systems) offers new possibilities for the execution of numerical codes with high computing requirements, thus facilitating more realistic and accurate modeling.

\subsection{Method of Moments}

The Method of Moments (MoM) was introduced by Harrington in 1967 in a very general formulation (Harrington 1967). It was mainly used in the 1980s for numerical 
calculations of the whole-body average SAR in relatively coarse block human models. The reference levels of incident electric field or power density in various guidelines were derived mainly from the MoM calculations of the whole-body average SAR.

Nowadays, MoM denotes methods that solve the EM problems with charges, fields, and current distributions as unknowns in integral equations. The MoM is especially popular for problems with perfectly conducting objects, such as antennas or scattering from other metal structures. Modeling of a finitely conducting ground, layered media, etc., can be introduced via Green's functions. MoM is mainly implemented using pulse functions to describe the unknown electric field in each mathematical cell of the model (the electric field has a constant value everywhere inside the mathematical cell), while linear basis functions have also been used instead of pulse functions. A variant MoM based on the tensor integral equation with volumetric discretization was introduced in the mid 1970s to study the absorption in human models (Gandhi 1980; Hagmann, Gandhi, and Durney 1979). The scatterer was subdivided into N blocks, in which a constant volume current was expanded with one unknown for each spatial dimension. The electric field integral equation (EFIE) was evaluated in the centers of the cells. This led to a system of equations with $3 \mathrm{~N}$ unknowns and a dense matrix. Furthermore, a surface-discretization MoM using electric and magnetic surface currents for each domain allows modeling of arbitrary lossy and dielectric domains.

However, the MoM is generally not appropriate for simulating the interactions between mobile communication devices and the human body. Since the source is generally a regular object, whose geometry is often described by simple equations, the radiation problem can be efficiently analyzed by MoM. This approach requires only the discretization of the domain where the current flows, without analyzing the space surrounding the source, because of the use of the suitable Green's function. On the other hand, lossy dielectric materials cannot be modeled. The EM characterization of a physically and geometrically complex object can be very difficult or impossible and time consuming, when approached with the MoM. Furthermore, as the cost of the solution of the MoM with volume discretization is roughly proportional to $\mathrm{N}^{3}$, the problem size grows so fast that the method does not allow a reasonably fine discretization of the human body. This also limits its application at frequencies higher than several hundred $\mathrm{MHz}$ because smaller size blocks are required for higher frequencies, that is, shorter wavelength.

Moment method matrix equations were originally solved by direct methods. Later iterative methods, such as the conjugate gradient method were also used. Recently there have been some breakthroughs in iterative methods coupled with good preconditioners and the faster approximation of the matrix-vector products used during the solution process. This speedup might again improve the competitiveness of the MoM approach.

The use of MoM for dosimetric studies is reported in Chuang (1994) and Karimullah, Chen, and Nyquist (1980), while MoM is used in Groot et al. (1997) and Pan, Bahrwas, and Wolff (1997) for antenna performance studies.

\subsection{Finite-Difference Time Domain}

The Finite-Difference Time-Domain (FDTD) algorithm, first proposed by Yee (1966), is the direct discretization of the time-dependent Maxwell's equations by expressing the spatial and time derivatives in a central finite-difference form implemented in a mesh 
of cubic cells (Yee cells), where the geometries under study are spatially approximated (Taflove and Hagness 2000). The cell size must be small enough to permit accurate results at the highest frequency of interest, taking into account that the materials present directly affect the wavelength. Once the cell size is selected, the maximum time step is determined by the Courant stability condition (Taflove and Hagness 2000).

FDTD has been used in several EM problems, such as RF microwave antennas and circuit design, and radar scattering from objects of various types, and it has been widely applied to EM dosimetry in models of human beings and other animals. In applying the FDTD method for numerical dosimetry calculation, the Yee cells correspond completely to the voxels in biological models. This technique has the following main advantages (Kuster 2002):

1. The ability to model complex geometries in a straightforward manner

2. Its direct derivation from Maxwell's equations, which allows the modeling of lossy and nonlinear materials without special treatment at interfaces

3. The possibility to obtain results for a broad frequency range

4. The linear increase of the computer memory requirement and the computation time with the number of voxels

5. The lack of the requirement to invert large matrices, which allows the calculation of models of the human body composed of million voxels (high spatial resolution)

6. Its suitability to evaluate the sensitivity of the results upon different parameters (e.g., dependence of the absorption upon anatomy, posture, tissue parameters)

However, a disadvantage of the method has been that the EM fields in the space surrounding the body must also be calculated. This implies that the surrounding space must also be modeled by mathematical cells. This greatly increases the number of cells for which calculations must be made, thus requiring a large amount of computer memory. Another drawback of the method is that there is no internal check for assessing the quality of the solution, that is, the quantitative uncertainty of the resulting field distribution. Coarse discretization or too small distances between the scatterer and absorbing boundaries can cause errors that are difficult to detect. However, the use of a sufficiently wide margin between the scatterers and the boundaries results in a negligible error from boundary modeling and an improved accuracy of the far-field transformation. Furthermore, major difficulties may be encountered in handling the sources. Describing small dimensions may lead to a compromise on the dimension of the space-discretization step, while significant difficulties are encountered in modeling antenna structures not conforming to the used grid, such as helical antennas (Cavagnaro and Pisa 1996; Nikita et al. 2000a). Numerical artifacts or interface effects in the representation of inhomogeneous body structures can be a significant source of error when modeling nonhomogeneous space with the discrete FDTD scheme. In studies where the field interaction is highly dependent on the shape of the boundary, this can lead to substantial error in the calculation. Improvements in the modeling process by implementing algorithms relying on boundary-fitted grids should be used in order to minimize staircasing effects.

Nevertheless, since the 1990s, the use of FDTD has become the most powerful simulation technique in the area of dosimetry and has been widely used to assess human exposure to spatially uniform or nonuniform (far-field or near-field) EM fields from 
extremely low-frequency (ELF) to microwave frequencies. It is powerful in dealing with a complex penetrable object such as the human head and has dominated over the other methods for the study of dosimetric problems related to mobile communications as well as for wireless device antenna design (Bernardi, Cavagnaro, and Pisa 1996; Cavagnaro and Pisa 1996; Christ and Kuster 2005; Gandhi and Chen 1995; Gandhi, Lazzi, and Furse 1996; Lazzi and Gandhi 1997; Lu et al. 1996; Martens et al. 1995; Martinez-Burdalo et al. 2005; Okoniewski and Stuchly 1996; Watanabe et al. 1996). This is due to the fact that enhanced computer power has become available at lower cost and intensive research work in the fields of source modeling, absorbing boundary conditions, and error checking has facilitated the simulation with more confidence in the obtained results. Several approaches for accurate absorbing boundary conditions at the boundary of the computational domain have been proposed (Berenger 1994; Bernsten and Hornsleth 1994; Mur 1981), offering excellent approximation of open space with a minimum of "white space." Negligible differences in the calculated SAR distributions or the radiation patterns are obtained regardless of the boundary conditions that are used. Furthermore, the use of a nonuniform grid has been proposed (Okoniewski and Stuchly 1996) to reduce the memory requirements, while parallelized versions of FDTD codes can be used to reduce the execution time. Other notable improvements are in the area of uncertainty assessments and graphical user interfaces (Kuster 2002).

\subsection{Finite Integration Technique}

The Finite Integration Technique (FIT), introduced by Weiland (1977), is based on the discretization of the integral form of Maxwell's equations and transforms them into a set of matrix equations, the Maxwell grid equations (MGEs), on an orthogonal dual grid pair. It is a method conceptually slightly different from the FDTD technique, which however leads to the same numerical scheme. The FIT can be thought of as equivalent to the FDTD for time-domain problems (Weiland 1990).

The use of FIT for mobile communication dosimetric calculations is reported in numerous studies (Hombach et al. 1996; Meier et al. 1997; Schoenborn, Burkhardt, and Kuster 1998).

\subsection{Finite Element Method}

The Finite Element Method (FEM) was introduced by Silvester and Ferrari (1996). Space is discretized in a finite element mesh with linear or polynomial expansion functions within each element. The unknowns are associated with the field values in the nodes. A variational method or a method of weighted residuals is used to obtain a system of equations with a sparse matrix. In wave propagation problems, the FEM is often used in the frequency domain. For a long time, its applicability for solving the Helmholtz equation was quite limited because of problems with spurious modes. Open domains were another limitation, as no efficient radiation boundary conditions were available. Therefore, FEM was often paired with a boundary element method in a hybrid scheme.

Although penalty methods can be used to avoid the corruption of results with spurious solutions, the advent of edge-element techniques that prevent the possibility of spurious modes altogether seem more promising. In the edge-element technique the unknowns are associated with the field tangential to the edges of the basic element. 
Within each element, vector expansion functions are used that are tangential to all element surfaces of which the edge is part and normal to all other surfaces. The edgeelement technique is also much better suited to nonhomogeneous problems, since no internal boundary conditions between elements with different electromagnetic properties need to be enforced. Furthermore, in contrast to the conventional, node-based finite element technique, the edge-element technique possesses a direct way of controlling discretization errors in the solution by looking at the behavior of the normal components of the field between elements.

The finite element mesh gives, in principle, a very high flexibility for discretizing almost any arbitrary geometry. However, the generation of meshes in $3 \mathrm{D}$ is still a formidable task. While there are reasonably good techniques for the discretization of technical structures, the difficulty of generating finite element models for the typically very nonhomogeneous problems in dosimetry currently prevents wider use.

Nowadays, FEM is used extensively in commercially available software to resolve submillimeter induced currents, electric and magnetic fields, and SAR at lower frequencies. The use of FEM to study the interaction of a cellular phone with the human head is reported in Rakotomalala (2007), while in Forgy et al. (1997), FEM is applied to study the performance of a special antenna for portable handsets.

\subsection{Hybrid Techniques}

Currently, none of the commercially available codes seem to be completely satisfactory for all cases of interest. A large deficiency is seen in the capability to perform reliable uncertainty assessments. Existing methods should be combined into new hybrid approaches, keeping all the advantages and eliminating the disadvantages of component methods. For example, FDTD has become the most widely acceptable numerical technique in the area of EM dosimetry. However, when exposure in an urban environment has to be modeled, the calculation burden of FDTD is prohibitive. In order to discretize the huge dimensions of the region to be studied, huge memory and CPU time are required.

On the other hand, field propagation in large environments can be efficiently studied by using Kirchhoff's integral (KI) or ray-tracing (RT) techniques. KI is a time-domain near-field to near-field transformation that can be derived starting from Green's theorem and Maxwell's equations. RT is a frequency-domain technique based on geometrical optics and the uniform theory of diffraction (UTD), and is able to study field propagation both in free space and in the presence of reflecting surfaces and diffracting edges. Both KI and RT can easily evaluate field distribution produced by sources radiating in free space but are unsuitable to predict field distribution in the presence of scatterers of arbitrary shape.

A way to overcome the problems and drawbacks specific to each method is to use hybrid techniques. The FDTD method can be used for the evaluation of the field in confined volumes containing the antenna and/or scattering objects as well as for the calculation of the SAR. The KI or RT methods can be used for the modeling of field propagation in the empty space between these volumes. Following this approach, the hybrid multiple-region/FDTD (MR/FDTD) (Bernardi et al. 2002a; Johnson and Rahmat-Samii 1997) and ray-tracing/FDTD (RT/FDTD) (Bernardi et al. 2000a, 2002a, 
2003) techniques have been developed. Another typical hybrid technique is the combination of the MoM and the FDTD method (Mangoud, Abd-Alhameed, and Excell 2000; Mochizuki et al. 2004). Such an approach is commonly used in the SAR calculation of a helical antenna next to a human head because the FDTD method has difficulties in modeling a curved wire.

\subsubsection{Uncertainty Assessment}

Error evaluation and its a posteriori prediction are important issues in all numerical simulations. Although we may have the wishful thinking that codes can be treated as a "black box," the reality is that erroneous results can be obtained due to geometrical or numerical modeling difficulties. In EM, considerations relating to numerical error have only appeared sporadically, but will continue to become of greater concern as we rely more heavily on numerical simulations for designs and other purposes. For risk assessment, it is important to investigate the uncertainty associated with dosimetry. Uncertainty is defined as the amount by which the estimated value may depart from the correct value.

Many different uncertainty components must be considered, which can be grouped into three main categories: the simulation uncertainty, the body model uncertainty, and the source uncertainty. The simulation uncertainty is related to the uncertainty in assessing a parameter of interest (e.g., the spatial peak SAR value), assuming that the source modeling and position as well as the body modeling are error free. For example, in evaluating the spatial peak SAR value, different tasks are involved, such as the evaluation of the electric field distribution, the calculation of the SAR distribution, spatial integration procedures, and search routines to localize the peak SAR value averaged over $1 \mathrm{~g}$ or $10 \mathrm{~g}$. The simulation uncertainty corresponds to errors generated from the discretization in space and time, such as numerical artifacts or errors generated in the treatment of material boundaries. Due to the lossy media encountered, some other FDTD errors (e.g., phase velocity changes in the grid or errors from boundary modeling) are expected to have almost no impact on the total uncertainty in FDTD dosimetry.

The body modeling uncertainty describes the uncertainty in the simulated parameters of interest produced by the deviation of the numerical representation of the body model with respect to the theoretical definition, as described in the test case. This uncertainty in the simulated parameters of interest related to the exact way in which the body model is built in the Yee grid represents a very critical component in the total uncertainty assessment. For example, simple models have long been considered as typical worst-case models and some reports of comparison of various realistic voxel models have been published (Kainz et al. 2005). It is however noted that simpler models may provide considerably higher doses (SAR or induced current density) or artificial phenomena such as the appearance of maximum local SAR in the deep region of the model (Lin 2002).

Since in numerical methods the device must be replaced by a numerical model, a significant error can be produced. Source uncertainty describes the uncertainty in the results obtained, caused by deviations from the defined device geometry, settings (output power, etc.), and position. The crucial parameters to model are the accurate locations, magnitudes, and distributions of the highest surface currents on the device and the antenna. Furthermore, the accurate definition of the antenna position against the 
body model in the discretized space is of major importance. Finally, the presence of reflecting objects (metallic or dielectric walls) nearby the emitting antenna has been shown to affect the source and radically change the dosimetric quantities (Bernardi, Cavagnaro, and Pisa 1996). In some papers, extensions of the treatments developed in order to describe more accurately real situations have been mentioned.

The accuracy of the numerical results produced is mainly checked with the following methods:

1. Convergence tests to check the convergence and stability of the numerical technique (Lazzi and Gandhi 1997; Massoudi et al. 1979; Meier et al. 1997; Zhou and Oosterom van 1992). Sufficient number of iterations ensures that the system has reached its steady-state.

2. Further analysis of the obtained results, by considering the specific implementation details of the numerical method. For example, FDTD even when applied for the analysis of well-defined canonical cases may lead to significant differences, depending on the specific implementation details (discretization in space and time, details of building the body model and the source in the Yee grid, details of the procedure for SAR averaging, distance between scatterers and absorbing boundaries, and simulation time). Boundary conditions that require truncating the region used for FDTD calculations is also a source of uncertainty although reported significance of this effect (PML boundaries) on the whole-body SAR has not been consistent among related studies (Findlay and Dimbylow 2006; Laakso, Ilvonen, and Uusitupa 2007; Wang et al. 2006).

3. Comparisons with measurements (Hombach et al. 1996; Karimullah, Chen, and Nyquist 1980; Meier et al. 1997; Toftgard, Hornsleth, and Andersen 1993). In order to allow easy comparison with experimental results, numerical canonical problems have been defined in complete agreement with experimental canonical problems.

4. Comparisons with analytical or semianalytical methods. For example, in the studies of Koulouridis and Nikita (2004), Cerri, De Leo, and Rosellini (1997), and Lazzi and Gandhi (1997), the accuracy of the numerical results is checked against the results obtained for spherical canonical problems by using a semianalytical method, based on Green's functions theory.

5. Comparisons with other numerical methods (Dimbylow and Mann 1994; Gandhi, Lazzi, and Furse 1996; Meier et al. 1997; Nikita et al. 2000a). It is highly recommended to validate the dosimetry by comparing with the results obtained with other methods. Canonical problems are still used as a reference to validate numerical results (Anderson 2003).

6. Interlaboratory comparisons. Some standard organizations have undertaken interlaboratory comparison for evaluating the uncertainty of SAR calculations. A recent study reported that a standard deviation of $30 \%$ was found in 12 separate SAR calculations of head models exposed to the near field of a cellular phone with the same anatomically based models and exposure conditions (Beard et al. 2006). An interlaboratory comparison of whole-body SAR calculations and the uncertainty of the calculations are given in Dimbylow, Hirata, and Nagaoka (2008). 
Obviously, the possibility to estimate the exact error bound of the results or to obtain results with a desired accuracy would be extremely desirable, thus eliminating the need for further (experimental or numerical) validation. However, the exact estimation of an error for complicated EM problems, such as the interaction of near-field sources with complex dielectric structures, is not yet possible. While international standards exist for the evaluation of uncertainty in the maximum local SAR values for compliance tests of cellular phones (IEEE 2003; IEC 2005), procedures to evaluate the uncertainty of numerical dosimetry have not been established.

\subsection{Experimental Dosimetry}

Experimental dosimetry has been used for over 25 years to obtain SAR distributions for far-field and near-field actual exposure conditions (Balzano, Garay, and Manning 1995; Balzano, Garay, and Steel 1978; Cleveland and Athey 1989; Gandhi and Chen 1995; Johnson and Guy 1972; Schmid, Egger, and Kuster 1996; Stuchly and Stuchly 1995). It enables accurate assessment of the induced field strengths under realistic conditions and thus, is widely applied for validation of the simulation results as well as for compliance testing purposes. The weaknesses of experimental dosimetry include restriction to liquid tissue-simulating media, limited spatial resolution of larger than $1 \mathrm{~mm}^{3}$, and limited applicability inside small substructures since the dimensions of the measurement instrumentation must be considerably smaller than that of the substructure under study (Cavagnaro and Pisa 1996).

\subsubsection{Human Phantoms}

It is very difficult to measure the internal electric and magnetic field strength or temperature elevation in the actual human body exposed to RF EM fields emitted by mobile communication devices by using noninvasive methods. Therefore, a phantom, a surrogate of the human body, which has electrical properties equivalent to those of the human body, is used for experimental dosimetry.

Several kinds of materials have been developed to realize the tissue electrical properties. Their references may be found in international standards on RF dosimetry (IEC 2005; IEEE 2002). Liquids and gels placed inside an enclosing shell that gives the phantom the shape of the body to be modeled (most commonly made of plexiglass) have been developed for RF dosimetry (Johnson and Guy 1972; Hartsgrove, Kraszewski, and Surowiec 1987). These materials are easy to prepare and their electrical properties are easily adjustable. Fluids rather than gels are preferred to facilitate rapid movement of the probe tip sensor placed inside the phantom. However, it is worth noting that the electrical properties of liquids and gels are not stable enough due to water evaporation. Furthermore, although dry phantoms with fine stability have also been developed, they are generally expensive and require complex and skilled procedures (Kobayashi et al. 1993; Nikawa, Chino, and Kikuchi 1996).

In the simplest phantoms, homogeneous liquids and gels are used. For example, the experimental verification of compliance of mobile communication devices with the basic limits (ICNIRP 1998; IEEE 2005) is usually performed by using plastic shell 
head models filled with homogeneous liquid. The most widely used homogeneous head phantom, SAM (specific anthropomorphic mannequin), which has been proposed by the IEEE (2003) and IEC (2005) standards for compliance testing is shown in Figure 5.9a. SAM has also been adopted by the European Committee for Electrotechnical Standardization (CENELEC; CENELEC 2001), the Association of Radio Industries and Businesses in Japan (ARIB 2002), and the Federal Communications Commission in the USA (FCC 2001). The conservativeness of the SAM phantom regarding dosimetry in children's heads has been assessed in the studies of Monebhurrun (2010) while the effect of replacing the lossy pinna of the phantom with a lossless spacer has been investigated by Kanda et al. (2002). Results questioning the conservativeness of a lossless ear spacer for the evaluation of the peak SAR in the head have been obtained in Christ and Kuster (2005). Prior to the introduction of the SAM phantom by the standardization bodies, the generic twin phantom of Figure $5.9 \mathrm{~b}$ was used and several cellular phone models were authorized based on measurements with this phantom (FCC 2003). In research by Cooper et al. (2002), the liquid filled shell human phantom of Figure 5.9c was used, which is thinner across the width of the body than the corresponding numerical visible

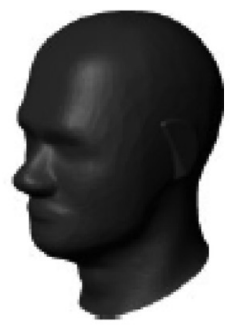

(a)

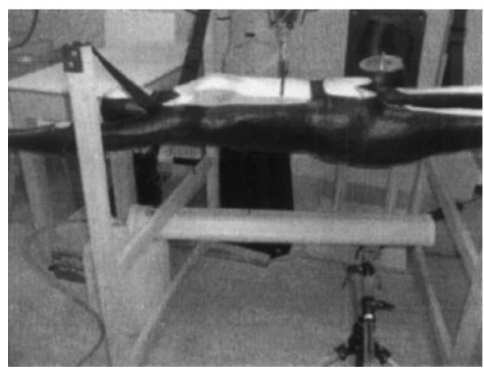

(c)

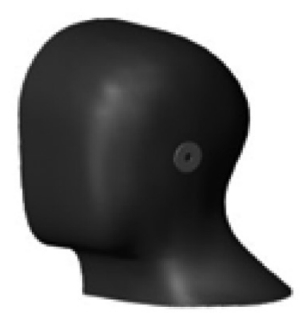

(b)

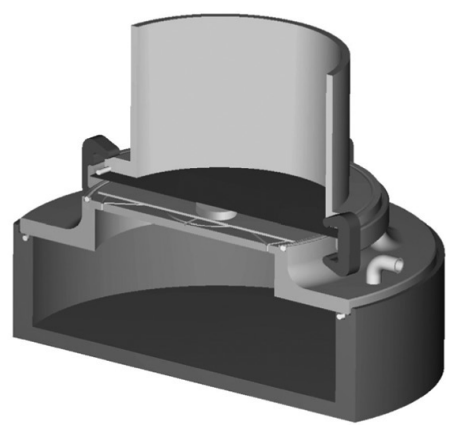

(d)

FIGURE 5.9 Homogeneous phantoms used in the literature: (a) SAM (From Christ, A. et al. 2005. Bioelectromagnetics, 26, 37. With permission.), (b) generic twin phantom (From Christ, A. et al. 2005. Bioelectromagnetics, 26, 37. With permission.), (c) phantom corresponding to the visible human model (From Cooper, J., B. Marx, J. Buhl, and V. Hombach. 2002. Bioelectromagnetics, 23, 43. With permission.), and (d) novel vacuum phantom (From Kuhn, S., E. Cabot, A. Christ, M. Capstick, and N. Kuster. 2009. Phys Med Biol, 54, 508. With permission.) 
human model, while in the research of Kuhn et al. (2009), the novel vacuum phantom of Figure 5.9d was developed. Several other homogenous phantoms can be found in Balzano, Garay, and Steel (1978), Olsen and Griner (1989), and Schmid, Egger, and Kuster (1996).

To provide realism for experimental modeling, several investigators have developed realistically shaped, heterogeneous models that are filled with several different tissuesimulant liquids/gels to represent specific parts of the human body (e.g., skin, skull, eyes). These models do not have the anatomical details of anatomically based numerical body models. However, they are a considerable improvement over homogeneous experimental phantoms in not increasing the SAR artificially. For example, in the research of Stuchly et al. (1987) the whole-body phantom of Figure 5.10a was developed, which simulates a heterogeneous structure with solid material for bone and liquid material for high-water-content tissues such as muscle. Several heterogeneous head phantoms have also been developed for SAR evaluation, as illustrated in Figure 5.10b (Cleveland and Athey 1989) and Figure 5.10c (Okano et al. 2000).

However, in a heterogeneous phantom, it is difficult to keep the materials of different compositions from mixing with each other. Therefore, procedures such as surrounding the tissue-simulant liquids/gels with thin plastic wraps or other separators are commonly used. Furthermore, since the human anatomy is quite complex, such phantoms consisting generally of two to five tissue-simulant materials (and therefore, two to five tissue types) do not necessarily match the anatomical details of the region that is to be modeled. Some authors have, therefore, focused on using homogeneous tissuesimulating fluids and homogeneous phantoms. In general, simplicity of the model and convenience in its use have often been cited as the reasons for using homogeneous models for compliance testing of mobile communication devices.

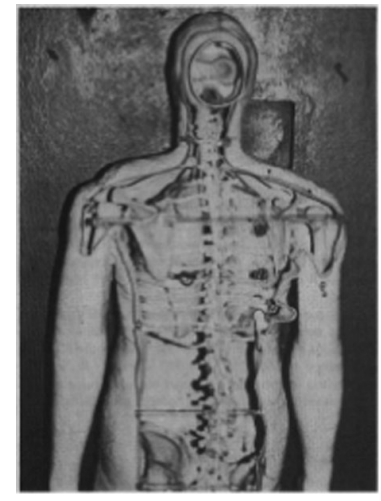

(a)

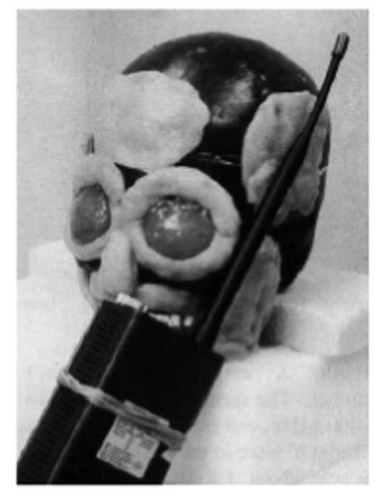

(b)

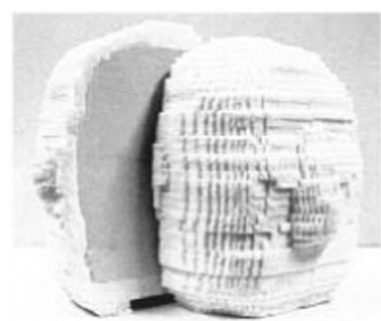

(c)

FIGURE 5.10 Heterogeneous phantoms used in the literature: (a) whole-body phantom (From Stuchly, S. S., A. Kraszewski, M. Stuchly, G. Hartsgrove, and J. Spiegel. 1987. IEEE Trans Biomed Eng, 34, 7. With permission.), (b) head phantom (From Cleveland, R. F., and T. W. Athey. 1989. Bioelectromagnetics, 10, 2. With permission.), and (c) head phantom (From Okano, Y., K. Ito, I. Ida, and M. Takahashi. 2000. IEEE Trans Microw Theory Tech, 48, 103. With permission.) 


\subsubsection{Measurement Devices and Equipment}

The criteria for performing scientifically sound measurements in experimental dosimetry have been outlined in Kuster, Balzano, and Lin (1997a). The measurement procedure should be comprehensible and evident to both experts and nonexperts and should provide reproducible results, independent of the phantom or the measurement devices and equipment used. Furthermore, the measured dosimetric quantities should not underestimate the actual maximum exposure occurring in real-life situations.

Three methods are generally used to measure external electric and magnetic fields emitted by mobile communication devices: portable RF measurement devices, spectrum analyzers, and personal exposure monitors. Portable RF measurement devices include broadband instruments with RF field sensors. These provide a relatively simple and convenient means for measuring electric and magnetic field strengths. On the contrary, spectrum analyzers are narrowband instruments. Finally, exposure estimates can be made using body-worn personal exposure monitors, which are often used in epidemiological studies.

When selecting a measurement device to assess exposure to RF fields, a number of key factors must be taken into account. For example, broadband portable RF measurement devices are relatively spectral insensitive and have a slow response time. To overcome these limitations, narrowband measurements with spectrum analyzers are sometimes preferred instead. Furthermore, the type of the personal exposure monitor used in epidemiological studies highly depends on the environment in which subjects are exposed. Workers on antenna sites could wear cheap, pocket-sized devices, whereas more sensitive instruments have been developed to capture relatively low-level exposures of the general population over a range of frequency bands used in mobile communication systems. Other factors such as the peak power limitations of the sensor, polarization aspects of the field, dynamic range, and capability to measure in near- and far-fields depending on the circumstances of the field measurement, also determine the choice of instrumentation.

Portable RF measurement devices consisting of field probes (sensors) are most commonly utilized for experimental dosimetry of the human body exposed to RF EM fields emitted by mobile communication devices. In order to achieve a satisfactory measurement precision, the probes must be optimized for each particular application with respect to sensitivity, isotropy, linearity, spatial resolution, field distortion (boundary effects, etc.), and immunity (ELF fields, secondary modes of reception, etc.). Furthermore, field probes must be calibrated at each frequency and inside the phantom materials under interest. Various calibration systems for field probes have been developed (Jokela, Hyysalo, and Puranen 1998; Meier et al. 1996).

The dimensions of the field probe are typically on the order of 4-5 mm (Balzano, Garay, and Manning 1995; Schmid, Egger, and Kuster 1996). Immersing such field probes into the tissue-simulant material of the experimental phantom displaces a significant amount of liquid/gel. This displacement might influence the field distribution that one is trying to measure, particularly at the higher frequencies. Field distortions in the vicinity of the probe can result in large errors when the probe is close to the boundaries and field distortion inside the probe can significantly impair its spherical isotropy. 
Furthermore, the size of the field probe limits the resolution of the measurements by several millimeters. Smaller field probes would therefore be needed for dosimetry at higher wireless communication frequencies. Probe-size reduction generally raises the upper frequency limit, improves spatial resolution, and reduces uncertainty due to boundary effects, but it also results in lower sensitivity and mechanical robustness.

Several measurement devices and equipment have been described in the literature. A measurement system (scanner) explicitly designed for the evaluation of human exposure to cellular phones was analyzed in Kuster, Kastle, and Schmid (1997b) and Schmid, Egger, and Kuster (1996). The key components of the scanner are the probes. Several kinds of probes that are based on diode-loaded field sensors and provide improved performance have been reported. They range from standard dosimetric probes to highly specialized probes, such as those for in vitro and in vivo assessment, for assessment of the field polarization, and others. Major advancements have been achieved in spherical isotropy, spatial resolution, sensitivity, bandwidth, linearity, immunity against secondary modes of reception, calibration accuracy, uncertainty assessments, and more.

An improved version of a scanner was described in studies by Kuster (2002) and is illustrated in Figure 5.11a. This scanner incorporates a high precision robot, isotropic field probes with diode-loaded dipole sensors, an optical proximity sensor for automated positioning of the probe with respect to the phantom surface, and sophisticated software for data processing and measurement control. In Blanch, Romeu, and Cardama (2002), an anechoic chamber was used for antenna measurements. The experimental setup is shown in Figure 5.11b. The measurement procedure is the same that is followed in standard spherical near-field measurements for antenna pattern determination. The near field is sampled over a sphere that encloses the antenna under test. The distance between the emitting antenna and the measurement field probe equals $5 \mathrm{~m}$. As a last example, in Kuhn et al. (2009), dosimetric measurements were performed with a DASY5 NEO, the fifth generation of near-field scanners, described in works of Schmid, Egger, and Kuster (1996) and illustrated in Figure 5.11c.

The near future will see new measurement devices and equipment that are more accurate and flexible and provide enhanced spatial resolution (in the submillimeter range). Furthermore, today, detector diode-loaded probes allow only the assessment of the

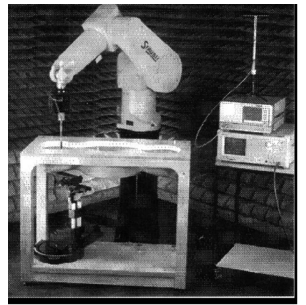

(a)

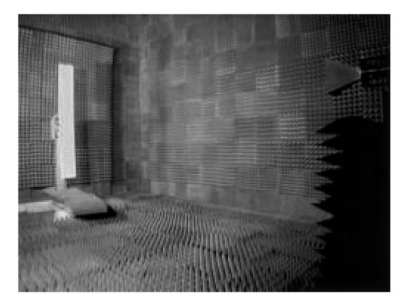

(b)

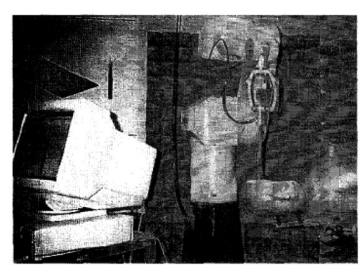

(c)

FIGURE 5.11 Measurement systems for experimental dosimetry (a) From Kuster, N. 2002. Wireless Phones Health II, 1, 19. With permission. (b) From Blanch, S., J. Romeu, and A. Cardama. 2002. IEEE Trans Antennas Propag, 50, 92. With permission. (c) From Schmid, T., O. Egger, and N. Kuster. 1996. IEEE Trans Microw Theory Tech, 44, 13. With permission. 
magnitude and cannot provide any phase information about the measured field. Future applications will require development of more advanced scanners providing not only amplitude but also phase information (Su et al. 2006).

\subsubsection{Uncertainty Assessment}

Basic information regarding uncertainty evaluation in experimental dosimetry can be found in (Iskra, McKenzie, and Cosic 2010; Vulevic and Osmokrovic 2010). In a more generic sense, the uncertainty components to be considered can be grouped into three main categories: the measurement uncertainty, the phantom uncertainty, and the source uncertainty.

The measurement uncertainty comprises the uncertainty in the calibration procedures as well as other measurement factors that will affect the overall uncertainty. These include temperature and drift effects, resolution of the display, perturbation of measurement by people or objects present in the surrounding environment, and the degree of repeatability.

The type and positioning of the measurement probes also insert some sort of uncertainty. For example, performance tests conducted on several commercially available probes equipped with orthogonally positioned sensors showed deviations from isotropy from \pm 1.5 up to $\pm 3.4 \mathrm{~dB}$ (Kuster 2002), while in the studies of Manning and Gabriel (2000) the way that the probe is articulated through the entry penetration at the top of head model was found to highly influence the SAR values.

Furthermore, the assessment of a dosimetric quantity, such as the SAR, starting from an unknown field distribution requires field measurements in several hundred points, data processing, and extrapolation/interpolation between the measured points. Such a task incorporates many different error components, all of which must be thoroughly analyzed in such a manner that is valid for all evaluations.

Phantom uncertainty includes the uncertainty with respect to the maximum exposure occurring in realistic conditions. For example, an assessment of the uncertainties in the estimation of the absolute SAR values for several allowances for the upright geometry of head phantoms has been performed in Manning and Gabriel (2000). In the case of personal monitoring systems, the perturbation of the impressed field by the phantom may result in considerable uncertainty. For instance, the field strength recorded by a body-worn instrument may differ from that recorded by the same instrument in the same position with the phantom absent by up to $10-15 \mathrm{~dB}$ close to body resonance frequencies (few 10s of $\mathrm{MHz}$ ), depending on the direction of incidence and the polarization of the radiation (ICNIRP 2009).

Finally, source uncertainty incorporates deviations of the device tested from those of mass production, manufacturing tolerances, and device position with respect to the human body.

Repeatable experimental dosimetry is essential to reduce the uncertainties. For example, in the case of exposure assessment to the radiation of cellular phones, the large standard deviations observed between different samples of phones indicate that the uncertainty can only be assessed by evaluating a sufficient number of randomly selected samples. Intercomparison of several experimental methodologies is also required. 
Great effort has recently been devoted to the intercomparison of experimental compliance procedures (Davis et al. 2006). It is also highly recommended to select relevant and accurate dosimetry techniques for each purpose and to validate the evaluated dose by comparing between numerical and experimental dosimetry. For example, in the studies of Lazzi, Gandhi, and Sullivan (2000) and Nicolas et al. (2001), the feasibility of mixed experimental/numerical procedures to compute the induced SAR beginning from measured exposure field distributions was studied. For the purposes of uncertainty assessment, a comprehensive investigation in Kainz et al. (2005) compared the SAM phantom to 14 anatomically correct head models in order to systematically evaluate whether or not SAM is conservative and assess the corresponding phantom uncertainty.

\subsection{Exposure Assessment}

\subsubsection{Cellular Phones}

Since the early 1980s when analog cellular radio systems were introduced in Europe, the cellular mobile telephone industry has undergone rapid growth. In many countries the take-up rate is approaching and sometimes exceeding $100 \%$.

Different countries have different cellular phone operating systems and slightly different frequencies are used. The digital system (global system for mobile communication, GSM) started in 1991 and has, recently, increased to be the most common phone operating system. This system uses dual band, 900 and $1800 \mathrm{MHz}$, for communication. From 2003, the third generation of cellular phones (3G), or universal mobile telecommunication system (UMTS) cellular phones, have started operating in some countries at $1900 \mathrm{MHz}$. Other countries are using slightly different systems. With adaptive power control technology, cellular phones operate at the lowest power necessary for acceptable communications in order to reduce human exposure to the radiated EM fields.

\subsubsection{Cellular Phone Modeling}

The way in which cellular phones are modeled varies from plane-wave and simple monopoles or dipoles to more complicated structures and realistic phone geometries. While real cellular phones are most commonly used in experimental dosimetry, the accurate numerical modeling of commercial cellular phones is one of the main challenges in numerical dosimetry. Experimental investigations of the numerical cellular phone models may also be performed.

Plane-wave illumination of the human body has been considered in the studies of Lin (1976), Lu et al. (1996), and Sullivan, Borup, and Gandhi (1987), while illumination by a short dipole (Zhou and van Oosterom 1992) has also been examined. Dipoles of various lengths placed in close proximity to the human head have been analyzed in research of Chen and Wang (1994), Chuang (1994), Dimbylow and Mann (1994), Hombach et al. (1996), Karimullah, Chen, and Nyquist (1980), Lazzi, Gandhi, and Sullivan (2000), Martens et al. (1995), and Meier et al. (1997). Three different numerical models of the dipole have generally been adopted. The first model is the "infinitely thin wire" approximation, obtained by setting to zero the tangential electric field component along the 
dipole's axis, with the exception of the feeding gap (Bernardi, Cavagnaro, and Pisa 1996; Martens et al. 1995). The second is the "thin" model in which a static approximation is applied and the electric and magnetic field components are assumed to vary as $1 / r$ near the wire, where $r$ is the distance from the wire center (Lazzi, Gandhi, and Sullivan 2000). The third is the "thick wire" approximation, obtained by assigning the copper conductivity value to each cell belonging to the dipole, with the exception of the antenna feed-point (Chen and Wang 1994).

A better phone model is composed by a monopole or a dipole mounted on a conducting box or a dielectric-coated conducting box (Bernardi, Cavagnaro, and Pisa 1996; Dimbylow and Mann 1994; Gandhi, Lazzi, and Furse 1996; Katsibas et al. 1998; Lazzi and Gandhi 1997; Martens et al. 1995; Nicolas et al. 2001; Okoniewski and Stuchly 1996; Toftgard, Hornsleth, and Andersen 1993). This is because the first cellular phones were equipped with antennas that behaved mostly as dipoles or monopoles. For example, in Nikita et al. (2000a), the cellular phone case was modeled as a conducting box of $120 \mathrm{~mm}$ (length) $\times 55 \mathrm{~mm}$ (width) $\times 20 \mathrm{~mm}$ (depth) with the monopole antenna centered on the upper side of the box (Figure 5.12a). The front face of the metal box was covered with a Plexiglas dielectric insulator of $0.5 \mathrm{~cm}$ thickness and the size of the feeding gap was set

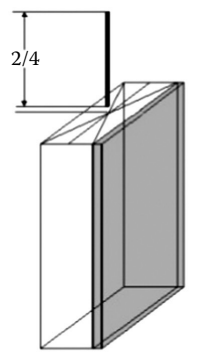

(a)

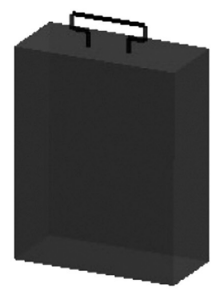

(d)

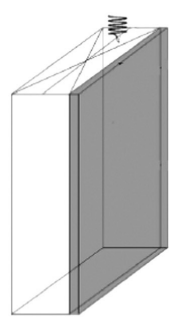

(b)

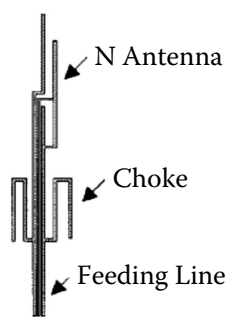

(e)

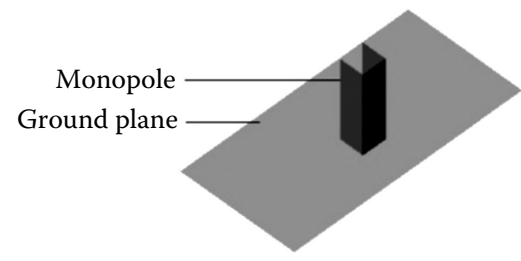

(c)

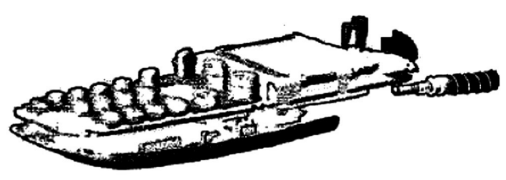

(f)

FIGURE 5.12 Cellular phone models used in the study of (a) Data from Nikita, K. S. et al. 2000a. IEEE Trans Microw Theory Tech 48:2676-85. (b) Data from Koulouridis, S., and K. S. Nikita. 2004. IEEE Trans Electromagn Compat 46:62-70. (c) Data from Yildirim, B. S., and E. A. El Sharaway. 1996. Analysis of a magnetically shielded cellular phone antenna using Finite Difference Time Domain method. In Proc IEEE MTT-S Int Microw Symp Dig, IEEE, 979-82. (d) Data from Katsibas, K. et al. 1998. IEEE Trans Antennas Propag 46:260-6. (e) From Pan, S., A. Bahrwas, and I. Wolff. 1997. IEEE Trans Antennas Propag, 45, 83. With permission. (f) Chavannes, N., R. Tay, N. Nikoloski, and N. Kuster. 2003. IEEE Antennas Propag Mag, 45, 66. With permission. 
to $0.25 \mathrm{~cm}$. Such models for the phone-radiating element are very often used nowadays also, since they represent a sort of canonical exposure source.

Recently, the need for more compact terminals and dual-band operation has given rise to new antenna types, such as helical antennas (Koulouridis and Nikita 2004, Figure 5.12b). However, regarding numerical dosimetry, helical antennas do not align to the FDTD grid. As a result, only large structures have been studied employing a pure FDTD scheme, in which the electric field components along the helix wires are set to zero (Bernardi, Cavagnaro, and Pisa 1996; Caputa et al. 2000; Cavagnaro and Pisa 1996; Christ et al. 2010; Troulis, Scanlon, and Evans 2003). For smaller structures, published reports have either employed a stack of electric dipoles and magnetic loop sources with relative weights obtained from analytical expressions for the helix far-field (Rowley, Waterhouse, and Joyner 2002) or a hybrid MoM/FDTD technique (Dimbylow, Khalid, and Mann 2003; Mangoud, Abd-Alhameed, and Excell 2000). Investigations using FDTD, properly modified through the use of a graded mesh, are presented in the studies of Bernardi et al. (2001) and Cerri et al. (1998).

Some special antenna designs, such as a magnetically shielded monopole on a ground plane (Yildirim and El-Sharawy 1996; Figure 5.12c), vertical folded loop antenna (Katsibas et al. 1998; Figure 5.12d), and N antenna (Pan, Bahrwas, and Wolff 1997; Figure 5.12e) have also been analyzed. Furthermore, flush-mounted types of antennas that blend into the handset such as side mounted, top-mounted, and back-mounted antennas have been considered. Planar-inverted-F, bent-inverted-F, printed-folded-loop antennas that can be efficiently and conveniently integrated with a handheld transceiver unit are analyzed in Bernardi et al. (2000b), Katsibas et al. (1998), Li et al. (2000), and Rowley and Waterhouse (1999).

Finally, in order to model the correct shape of cell phones, both CAD files provided by cellular phone manufacturers (Gandhi et al. 1999) and topometric sensors (Schiavoni et al. 2000) have been used. Recently, CAD files have also been used to model the internal structures (printed circuit board, battery, keypad, and buttons) of the phone. For example, the development and validation of the numerical model of the Motorola T250 is described in detail in Chavannes et al. (2003; Figure 5.12f). An alternate approach to a suitable numerical model of the cellular phone was proposed by Pisa et al. (2005). It starts with a simplified model which includes only the main phone parts (antenna, keyboard, internal box, and plastic coating) having "realistic" dimensions and electric properties. These are subsequently tuned using an optimization procedure.

\subsubsection{Phone Positioning}

In cellular phone dosimetry studies, the transceiver has to be appropriately positioned against the numerical head model utilized. For canonical modeling of the human head, positioning of the cellular phone against the ear canal of the numerical head model is straightforward. An example case is illustrated in Figure 5.13. Point E corresponds to the projection of the ear canal on the dielectric front face of the handset.

Regarding anatomical head models, guidelines exist that describe the standardization of phone positioning. The EN 50361-2001 (CENELEC 2001) and IEEE 1528-2003 (IEEE 2003) standards recommend measurement techniques for determining the peak spatial-average SAR in the human head from wireless communication devices, such as 


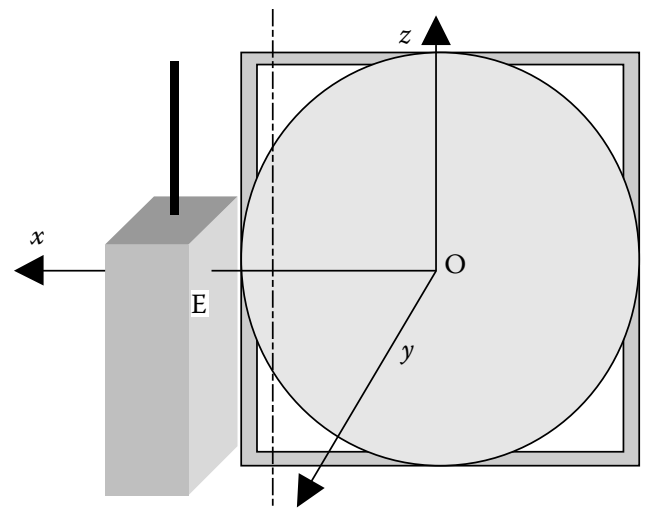

FIGURE 5.13 Appropriate positioning of the cellular phone model against the numerical or experimental phantom.
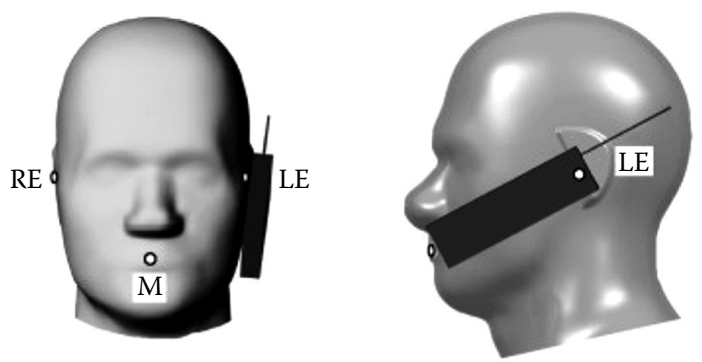

(a)

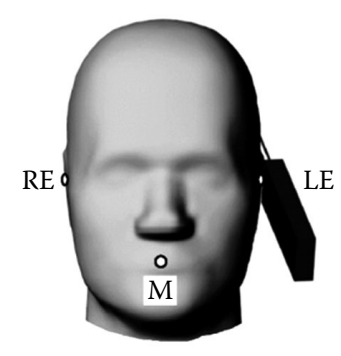

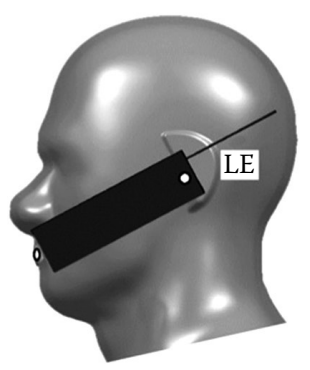

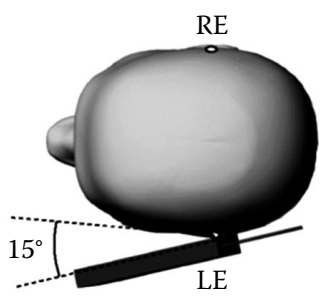

(b)

FIGURE 5.14 SAM with cell phone on (a) "cheek" (b) "tilt" position. RE = Right Ear, LE = Left Ear, $\mathrm{M}=$ Mouth. (From Beard, B. B. et al. 2006. IEEE Trans Electromagn Compat, 48, 407. With permission.)

cellular phones. These define four cellular phone positions for the standardized head model SAM: "cheek" and "tilt" for right and left ear use, as illustrated in Figure 5.14a and b, respectively (Beard et al. 2006). In the "cheek" position, the body of the cellular phone is placed against the cheek, while in the "tilt" position a projecting antenna is placed close to the head. These typical positions are expected to produce representative 
SAR results for typical exposure conditions, without being possibly able to provide the worst-case SAR.

However, the aforementioned definitions for "cheek" and "tilted" are not directly applicable to anatomical head models other than SAM. Anatomical head models have varying reference planes and points, pinna shape, and size and misalignment with the computational grid. Therefore, positioning of the cellular phone on anatomical head models other than SAM is a complex and nonstandardized procedure. In order to assure that positioning of the cell phone relative to the head model is the same among different investigators and to avoid ambiguities in the numerical dosimetry results of several research groups, as in Kainz et al. (2005), the phone positioning definitions for the SAM model were extended and a formal definition was proposed for the positioning of cellular phones next to anatomical head models. A procedure for determining reference planes and reference points based only on anatomical characteristics of the head model was described and, based on these, the "cheek" and "tilted" positions were subsequently defined. All definitions were based on anatomical characteristics of the head and tested by evaluating SAR data in 14 different head models and comparing them with the SAM model used in the CENELEC and IEEE standard procedures.

\subsubsection{Dosimetry Results}

The results of the cellular phone dosimetry studies are usually given in the form of crosssectional graphs, 2D contour plots or 3D color graphs of SAR distributions, often averaged over $1 \mathrm{~g}$ or $10 \mathrm{~g}$ of tissue for immediate comparison with the exposure guidelines (IEEE 2005; ICNIRP 1998). The derived dosimetric quantities have been found to highly depend on the numerical/experimental phantom used, the EM radiation frequency, the phone model (or, equivalently, the antenna type) and the distance between the cellular phone and the head.

The dependence of the SAR values on the head phantom has been extensively studied in the literature. In the "cellular phone standard" (CEPHOS) project, the head model was found to strongly disturb the symmetry of the antenna radiation pattern in the direction of the head. A cubic box head model drastically reduced the radiation in the head direction, while in the case of a spherical head model, there was a less dramatic but significant reduction in power radiated toward the head. Homogeneous sphere head models gave increased values for local and averaged SARs compared to layered ones. Furthermore, a large number of studies evaluating the peak spatial SAR in the SAM phantom with respect to anatomical head models concluded that the peak spatial SAR assessed with the SAM is a conservative measure for the exposure (Christ et al. 2005; Dominguez, Raizer, and Carpes 2002; Martens 2005). The handling of the phone will also have a considerable impact on the EM interaction between cellular phone and human. For example, in Table 5.2, two handset-handling scenarios are considered at $900 \mathrm{MHz}$ and the peak SAR values along with the power absorbed in the head and hand are recorded (Al-Mously and Abousetta 2008). A heterogeneous high-resolution European female head model, a mobile phone equipped with an external antenna, and an antenna input power of $600 \mathrm{~mW}$ have been assumed. In a certain usage pattern of the cellular phone with a left-side external antenna, the maximum percentage difference of the spatial peak $1 \mathrm{~g}$ avg SAR values in the head due to the right hand-hold alteration may 
TABLE 5.2 Peak SAR and Power Absorption in Tissues for the Handset at Cheek and Tilt Positions, Considering Two Handling Scenarios

\begin{tabular}{llcc}
\hline & & \multicolumn{2}{c}{ Handling Scenarios } \\
\cline { 3 - 4 } Handset Position & Dosimetric Parameters & $\begin{array}{c}\text { Grasping the Lower } \\
\text { Part of the Handset }\end{array}$ & $\begin{array}{c}\text { Grasping the Upper } \\
\text { Part of the Handset }\end{array}$ \\
\hline Cheek position & Peak 1 g avg SAR in head (W/kg) & 3.38 & 2.52 \\
& Peak 1 g avg SAR in hand (W/kg) & 1.54 & 5.16 \\
& Absorbed power in head (mW) & 250.4 & 209.1 \\
& Absorbed power in hand (mW) & 97.5 & 208.3 \\
\multirow{5}{*}{ Tilt position } & Peak 1 g avg SAR in head (W/kg) & 2.61 & 1.99 \\
& Peak 1 g avg SAR in hand (W/kg) & 1.86 & 5.11 \\
& Absorbed power in head (mW) & 184.6 & 159.2 \\
& Absorbed power in hand (mW) & 114.0 & 237.1 \\
\hline
\end{tabular}

Source: Data from Al-Mously, S. I., and M. M. Abousetta. 2008. Int J Antennas Propag 2008:1-22.

reach over $42 \%$ (whereas it may reach over $86 \%$ with the cellular phone having a bottommounted internal patch antenna).

Second, dosimetric studies have shown that the location and absolute value of the maximum value of the local SAR are dependent on the antenna frequency. In the high frequency case, the antennas are physically smaller and power is absorbed more superficially than power at lower frequencies. Accordingly, at higher frequencies, the regions of high SAR values are more concentrated and the maximum local SAR is higher than that lower frequencies (for the same radiated power), because of the difference in the penetration depth. For example, a recent interlaboratory collaboration for computational comparison of spatial peak SARs reported the SAR values depicted in Table 5.3 for the head tissue of three human head models assuming an output power of $1 \mathrm{~W}$ (Beard et al. 2006).

Third, the phone model and hence, the antenna type have been found to deposit the RF energy at different anatomical localizations in the head. As a result, the SAR distribution and maximum $1 \mathrm{~g}$ avg and $10 \mathrm{~g}$ avg SAR values differ between different phone models. For example, Figure 5.15 comparatively shows the maximum values of local SAR, and 1 and $10 \mathrm{~g}$ avg SAR in an adult and child head model exposed to two different radiating elements (linear/helical) (Koulouridis, Christopoulou, and Nikita 2005). The antenna input power is assumed to be equal to $125 \mathrm{~mW}$. In general, studies suggest that the maximum 1 or $10 \mathrm{~g}$ average spatial peak SAR is induced by a helical antenna with a metal box, and this is followed by the $\lambda / 4$ wavelength monopole antenna, the $3 / 8$ or $5 / 8(\lambda)$ monopole antenna, the $\lambda / 2$ wavelength dipole, and the back-mounted planarinverted-F antenna, while actual cellular phones have been found to cause even lower SAR values (Wiart et al. 2005). Moreover, numerical simulations comparing the SAR values obtained in a human head model from an optimized phone model with those obtained by a nonoptimized phone model showed that the nonoptimized model can lead to SAR values as much as three times the values obtained from the optimized model (Pisa et al. 2005). These findings can be explained by the current distribution along the antenna and box simulating the cellular phone model. 
TABLE 5.3 Peak SAR and Standard Deviation (STD) for the Head Tissue of Three Head Models at 835 and $1900 \mathrm{MHz}$ and Cheek-Handling Position. All Values Are Normalized to the Mean SAR Values of the SAM Model

\begin{tabular}{lllccc}
\hline & & 7-Year-Old Japanese Male (\%) & Visible Human (\%) & SAM (\%) \\
\hline $835 \mathrm{MHz}$ & \multirow{2}{*}{1 g avg SAR } & Mean & 54.9 & 71.7 & 100 \\
& & STD & 4.7 & 6.3 & 5.4 \\
& \multirow{2}{*}{$10 \mathrm{~g}$ avg SAR } & Mean & 44.2 & 70.7 & 100 \\
& & STD & 3.3 & 9.3 & 5.2 \\
$1900 \mathrm{MHz}$ & \multirow{2}{*}{ g g avg SAR } & Mean & 63.1 & 71.6 & 100 \\
& & STD & 20.3 & 18.2 & 19.1 \\
& \multirow{2}{*}{$10 \mathrm{~g}$ avg SAR } & Mean & 60.7 & 66.1 & 100 \\
& & STD & 17.7 & 18.2 & 15.2 \\
\hline
\end{tabular}

Source: Data from Beard, B. B. et al. 2006. IEEE Trans Electromagn Compat 48:397-407.

Maximum SAR $=f$ (radiating element)

$\boldsymbol{\square}$ Local SAR (adult) $\boldsymbol{\Delta}$ Local SAR (child) $\boldsymbol{\square} 1 \mathrm{~g}$ SAR (adult) $\boldsymbol{\square} 1 \mathrm{~g}$ SAR (child) $\boldsymbol{\square} 10 \mathrm{~g}$ SAR (adult) $\boldsymbol{\square} 10 \mathrm{~g}$ SAR (child)

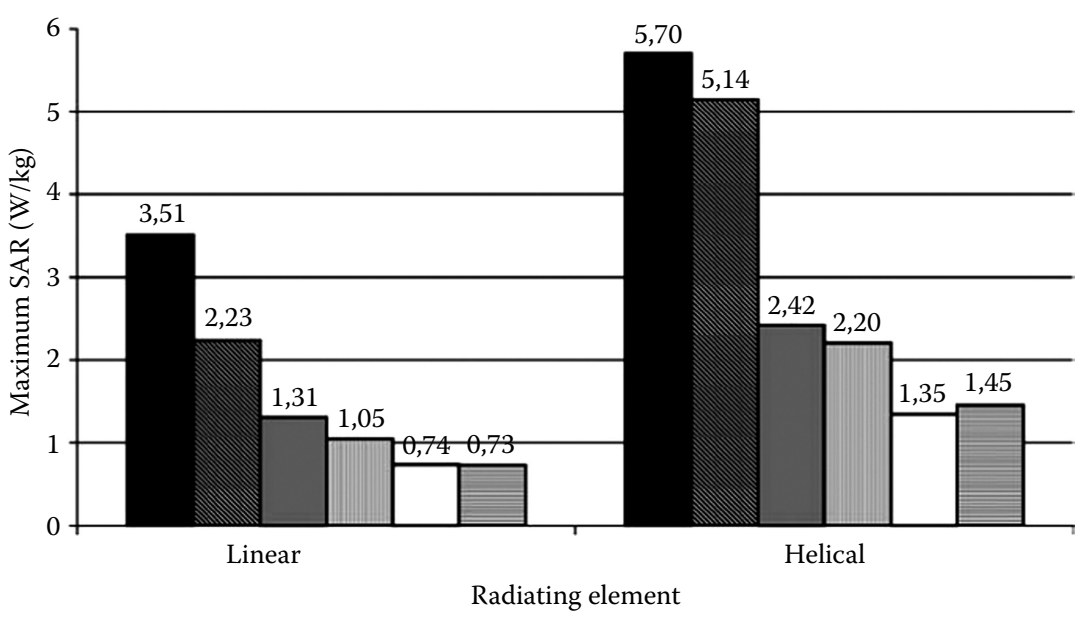

FIGURE 5.15 Peak SAR values in adult/child head models exposed to two different radiating elements (linear/helical) for an antenna input power of $125 \mathrm{~mW}$.

Lists of SAR values for different phones are published on various Web pages and values ranging from 0.1 to $1.8 \mathrm{~W} / \mathrm{kg}$ can be found. Similar results have been reported in Ali et al. (2007) for 21 different phones, with the $10 \mathrm{~g}$ avg SAR ranging from 0.3 to $1.7 \mathrm{~W} / \mathrm{kg}$. Kuster, Kastle, and Schmid (1997b) measured 16 different European digital phones and found a very wide variation in the SAR values. The phone with the lowest value, when averaged over a $10 \mathrm{~g}$ tissue, had a SAR of $0.28 \mathrm{~W} / \mathrm{kg}$ and the one with the highest value had $1.33 \mathrm{~W} / \mathrm{kg}$, all normalized to an antenna input power of $0.25 \mathrm{~W}$. If the averaging was done over $1 \mathrm{~g}$ of tissue, the SAR value went from $0.42 \mathrm{~W} / \mathrm{kg}$ to $2 \mathrm{~W} / \mathrm{kg}$. However, when 
the phone was slightly tilted toward the head of the user, the value went from a low of 0.2 to a high of $3.5 \mathrm{~W} / \mathrm{kg}$.

Finally, it is expected that as the distance between the cellular phone and the head model increases, the SAR distribution becomes weaker and more uniform. This is indeed the case in Figure 5.16, which illustrates the local SAR distribution normalized to $1 \mathrm{~W} / \mathrm{kg}$, in $\mathrm{dB}$, for a realistic adult head model in the proximity of a cellular phone (Christopoulou, Koulouridis, and Nikita 2009). The cellular phone is equipped with a small helical monopole antenna at $1710 \mathrm{MHz}$ and is assumed to be placed at a distance of (a) $0 \mathrm{~mm}$ and (b) $10 \mathrm{~mm}$ from the head model. The $x z$ and $x y$ slices where peak local SAR is calculated are depicted, while the antenna total radiated power is $125 \mathrm{~mW}$. Considering the same simulation scenario, peak SAR values and absorbed power levels are shown in Table 5.4 for varying separation distances.

The effect of metallic implants inside the head of a cellular phone user (e.g., skull plate, bone plate, fixtures), or wearable metallic elements (e.g., spectacles and earrings), on the SAR distribution has drawn scientific interest. An implant dimension close to the field half-wavelength can induce high current on the metallic object, modify the EM field distribution close to the metallic object and have a high influence on the RF energy absorbed by the human body. Moreover, it has been found that the thinner the element, the greater the increase in local SAR values. The increase in SAR values when metallic objects are implanted or attached to the human ear has been confirmed both numerically and experimentally (Fayos-Fernandez et al. 2006; Virtanen, Keshvari,
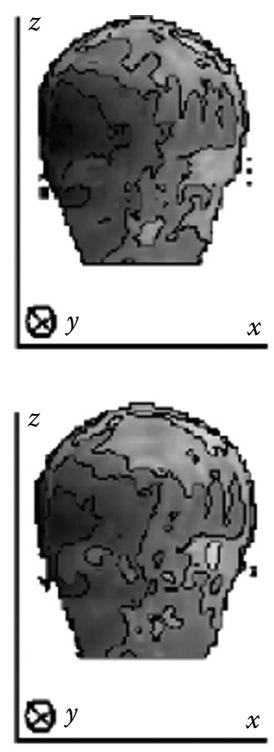

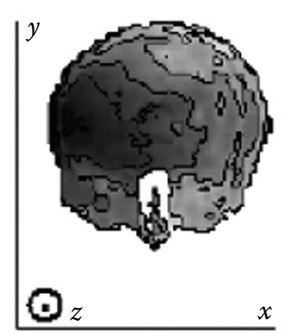

(a)

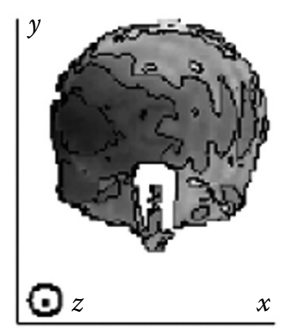
.

(b)

FIGURE 5.16 Local SAR distribution normalized to $1 \mathrm{~W} / \mathrm{kg}$ in $\mathrm{dB}$ for a realistic adult head model exposed to a cellular phone placed at a distance of (a) $0 \mathrm{~mm}$ and (b) $10 \mathrm{~mm}$ away from the head model, on the $x z$ and $x y$ slices where peak local SAR is calculated. 
TABLE 5.4 Peak SAR Values and Absorbed Power for Varying Separation Distance between the Realistic Head Model and the Cellular Phone of Figure 5.16

\begin{tabular}{lcccc}
\hline $\begin{array}{l}\text { Distance } \\
(\mathrm{mm})\end{array}$ & $\begin{array}{c}\text { Local SAR } \\
(\mathrm{W} / \mathrm{kg})\end{array}$ & $\begin{array}{c}\text { 1 g avg SAR } \\
(\mathrm{W} / \mathrm{kg})\end{array}$ & $\begin{array}{c}\text { 10 g avg SAR } \\
(\mathrm{W} / \mathrm{kg})\end{array}$ & $\begin{array}{c}\text { Absorbed } \\
\text { Power }(\mathrm{mW})\end{array}$ \\
\hline 0.0 & 5.70 & 2.42 & 1.35 & 82.81 \\
2.5 & 3.08 & 1.40 & 0.90 & 67.42 \\
5.0 & 2.21 & 0.95 & 0.68 & 55.88 \\
7.5 & 1.62 & 0.72 & 0.49 & 46.91 \\
10.0 & 1.23 & 0.56 & 0.37 & 39.86 \\
\hline
\end{tabular}

and Lappalainen 2006, 2007). A maximum increase of 3 in the averaged SAR has been obtained for some worst-case situations in a cylindrical two-layer phantom in the studies of Fayos-Fernandez et al. (2006).

Furthermore, several studies have addressed the issue of cellular phone dosimetry in children (Christ et al. 2010a, 2010b; Christopoulou, Koulouridis, and Nikita 2009; ICNIRP 2009; Kwon et al. 2010; Lin 2003; Wiart et al. 2008). Higher SAR values have generally been assessed in children (smaller) models due to two concurrent effects: differences in anatomical proportions result in a larger extent of penetration of absorbed energy for the smaller head models, while the separation distance between the cellular phone antenna and the child's head is decreased due to the smaller ear size. Figure 5.17 shows the local SAR distribution, normalized to $1 \mathrm{~W} / \mathrm{kg}$ in $\mathrm{dB}$ for realistic adult and child head models in the proximity of a cellular phone equipped with a small helical monopole antenna at $1710 \mathrm{MHz}$ on the $x z$ and $x y$ slices where peak local SAR is calculated (the cellular phone is assumed to be placed at a distance of $0 \mathrm{~mm}$ from the head model) (Christopoulou, Koulouridis, and Nikita 2009). High SAR regions in the child head are more extended as compared to those in the adult head. Considering the same simulation scenario, peak SAR values and absorbed power levels are shown in Table 5.5.

Furthermore, in the study of Gandhi, Lazzi, and Furse (1996), the computed SAR values in an adult and two linearly scaled child head models with adult dielectric properties have shown that although the peak $1 \mathrm{~g}$ avg SAR values were similar at $1900 \mathrm{MHz}$, an increase up to $50 \%$ in the $1 \mathrm{~g}$ avg SAR values was found at $835 \mathrm{MHz}$ for the smaller head sizes. In the studies of Gandhi and Kang (2002), computed results showed that the $1 \mathrm{~g}$ avg peak SAR values for both the head (pinna excluded) and the brain tissues increase monotonically with the reducing head size for all cellular phone dimensions and antenna types under test. Similarly, in the study by Beard et al. (2006), the majority of the $1 \mathrm{~g}$ avg and $10 \mathrm{~g}$ avg peak SAR values were higher in the child than the adult model, especially for the $835 \mathrm{MHz}$ phone in tilt position when normalized to antenna current. A study using child-sized (CS) and child-like (CL) head models showed that since the brain is closer to the cellular phone in the case of the CS or CL heads, the SAR in the child brain models is slightly higher than that of the adult (Hadjem et al. 2005). More recently, Wiart et al. (2008) reported that exposure of the cerebral cortex of children is higher than in adults. 

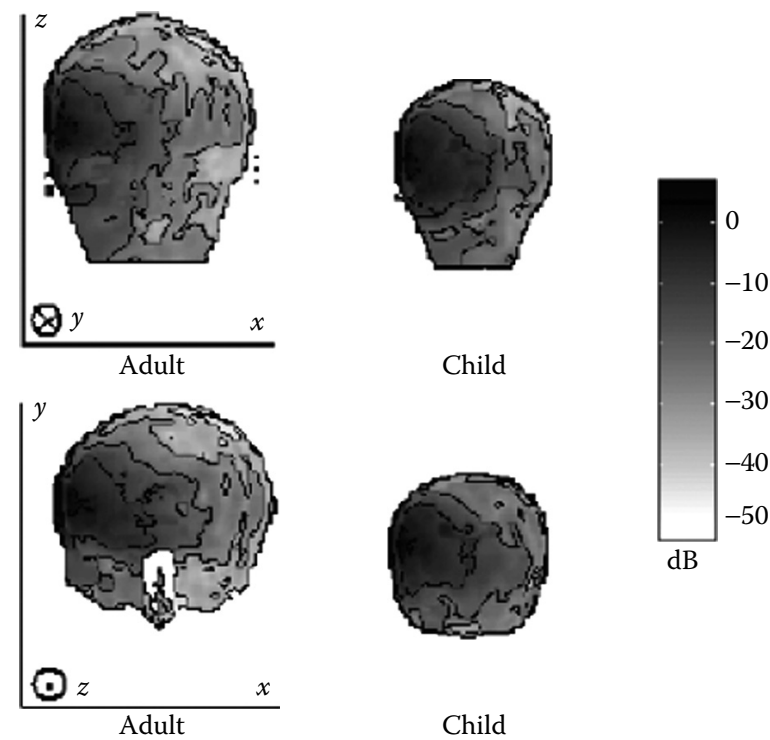

FIGURE 5.17 Local SAR distribution normalized to $1 \mathrm{~W} / \mathrm{kg}$ in $\mathrm{dB}$ for realistic adult and child head models exposed to a cellular phone placed at a distance of $0 \mathrm{~mm}$ away from the head models, on the $x z$ and $x y$ slices where peak local SAR is calculated.

TABLE 5.5 Peak SAR Values and Absorbed Power for the Head Models of Figure 5.17

\begin{tabular}{lcccc}
\hline $\begin{array}{l}\text { Head } \\
\text { Model }\end{array}$ & $\begin{array}{c}\text { Local SAR } \\
(\mathrm{W} / \mathrm{kg})\end{array}$ & $\begin{array}{c}\text { 1 g avg SAR } \\
(\mathrm{W} / \mathrm{kg})\end{array}$ & $\begin{array}{c}\text { 10 g avg SAR } \\
(\mathrm{W} / \mathrm{kg})\end{array}$ & $\begin{array}{c}\text { Absorbed } \\
\text { Power }(\mathrm{mW})\end{array}$ \\
\hline Adult & 5.70 & 2.42 & 1.35 & 82.81 \\
Child & 5.14 & 2.20 & 1.45 & 82.45 \\
\hline
\end{tabular}

In contrast, there exist some reports that have revealed no significant differences in the absolute extent of SAR penetration, $1 \mathrm{~g}$ avg and $10 \mathrm{~g}$ avg SAR, and peak SAR values between adult and child head models (Bit-Babik et al. 2005; ICNIRP 2009; Schoenborn, Burkhardt, and Kuster 1998). Some of the apparent conflicts and contrasting conclusions between the two sets of studies are attributable to uncontrolled or poorly understood variables such as different phantom models, cellular phone orientations and positioning (e.g., fixed distance vs. decreasing distance with decreasing pinna thickness), radio frequencies, and parameters used for SAR normalization (output power or antenna current), or procedures adopted for SAR averaging (Bit-Babik et al. 2005; Wang and Fujiwara 2003). The paucity of data on age-dependent tissue conductivity and permittivity is also a limitation.

Dosimetry regarding exposure of fetuses to EM radiation emitted by cellular phones has also been performed. In the works of Nagaoka et al. (2007) and Togashi et al. (2008), the induced current and average SAR of fetuses were shown to be generally similar or lower than those of the mother, while in that of Wu et al. (2006), using nine different 
pregnancy stages, it was shown that significant increase of SAR and temperature elevation takes place at late pregnancy stage.

Finally, the possible changes the absorption could have when the phone is used in a closed environment have been assessed in the literature. For example, when the cellular phone is used inside a car, metallic walls are present near the EM source that could change its radiating properties and perhaps cause a higher absorption in the human head (Dominguez, Raizer, and Carpes 2002).

\subsubsection{Parametric Studies}

Regarding cellular phone dosimetry, the dependence of the power absorbed by the human head on the human subject (age and tissue dielectric properties), the phone-head distance, and the phone positioning has also been parametrically studied in the literature.

Kuster, Kastle, and Schmid (1997b) reported a distribution of slightly compressed ear thickness ranging from 3 to 10 or more $\mathrm{mm}$, with a mean value of about $5 \mathrm{~mm}$ in order to show that the distance between the radiating source and the human head, as well as the intermediate tissue and the ear thickness, are factors that strongly influence the absorbed electromagnetic power. Furthermore, in the research of Hadjem et al. (2005), exposure of several child head models to two cellular phones equipped with patch antennas were studied and no significant difference in the power budget and the $10 \mathrm{~g}$ avg SAR was found. Both uniform (CS) and nonuniform (CL) downscaling procedures were adopted to derive the child head models of the study.

In the works of Koulouridis, Christopoulou, and Nikita (2005), a comparative dosimetric assessment for various head models (adult and child) and cellular phones operating at $1800 \mathrm{MHz}$ was carried out. Variations in the size and anatomy of the human head, the tissue dielectric properties, and the distance between the human head and the cellular phone were taken into account. Helical antennas mounted on cellular phones produced higher SAR values compared to linear ones due to the greater field concentration by the physically shorter helical antenna. Children and adults were found to absorb similar levels of EM power and morphological changes in child head models did not affect the radiation behavior of the system. On the other hand, the increase of the phonehead distance and the decrease of the dielectric properties significantly reduced the SAR values. Quantitative results are depicted in Figures 5.18 and 5.19.

Finally, a recent study presented detailed parametric results for adult and children head models exposed to small helical antennas at $1710 \mathrm{MHz}$ (Christopoulou, Koulouridis, and Nikita 2009). The peak local and $1 \mathrm{~g}$ avg SAR values induced in realistic and spherical head models were found to exponentially decrease with the increase in separation distance, while in spherical head models the $10 \mathrm{~g}$ avg SAR values were linearly dependent on the separation distance. Furthermore, the results revealed that comparable power levels (difference lower than 12\%) were absorbed by adult and child head models. The uniformly scaled down child head models led to higher $10 \mathrm{~g}$ avg SAR (4\%-37\%) and power absorption (up to 9\%) values for realistic exposure scenarios. These trends were less emphasized $(\sim 12 \%$ and up to $2 \%$, respectively) in canonical exposure scenarios. Assessment of the peak SAR value dependence upon separation distance and tissue dielectric properties concluded in a $60 \%-80 \%$ decrease for a $1 \mathrm{~cm}$ increase in distance and an up to $16 \%$ decrease for a $110 \%$ to $90 \%$ decrease in dielectric property values, 


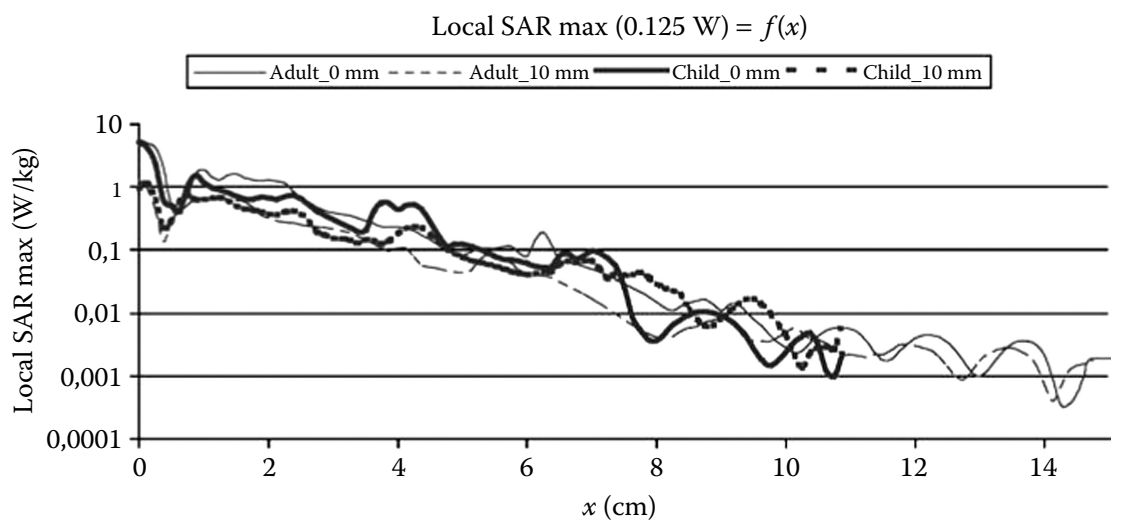

FIGURE 5.18 Local SAR variation along the $x$-axis (ear-to-ear) of anatomical adult and child head models exposed to a cellular phone with a mounted helical antenna, placed at a distance of 0 or $10 \mathrm{~mm}$ away from the head model.

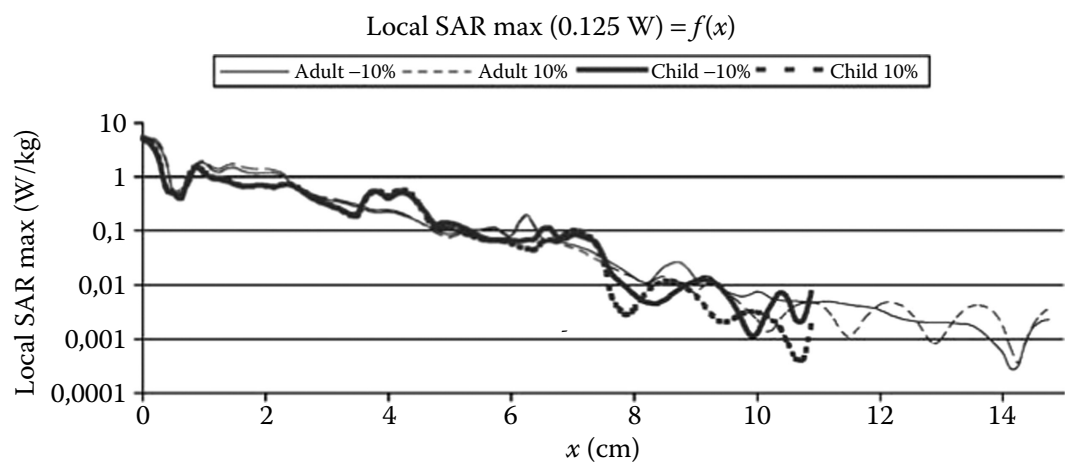

FIGURE 5.19 Local SAR variation along the $x$-axis (ear-to-ear) of anatomical adult and child head models exposed to a cellular phone with a mounted helical antenna, placed at a distance of $0 \mathrm{~mm}$ away from the head model. Dielectric properties variation $-10 \%$ to $10 \%$.

for realistic exposure scenarios. These trends were respectively less (55\%-65\%) and more (up to 24\%) emphasized in the corresponding canonical exposure scenarios.

Future parametric studies should further examine different EM sources with varied relative positioning in the proximity of the head. Multiparametric studies involving variation of dielectric properties along with separation distance and varied positioning of the cellular phone should also be carried out in order to extend the present studies' results.

\subsubsection{Wired and Wireless (Bluetooth) Hands-Free Devices}

Cellular phones are held against the head while a call is made. Typically, the distance from the antenna to the head is less than $2 \mathrm{~cm}$ and the user is in the near-field of the EM source. Wired and wireless (Bluetooth) hands-free devices enable the cellular phone to be removed from the head region. However, health concerns do remain. 
In the case of wired hands-free operation, because of the presence of an RF-energycarrying conductor connecting the cellular phone to the audio earpiece, there is a need to investigate the interaction between the cellular phone, the user, and any associated wiring, particularly if the latter passes close to the cellular phone's antenna. The SAR in the head is generally found to depend on the output power of the cellular phone, the coupling between the antenna and cable, the external attenuation, and the potential cable specific attenuation (Bit-Babik et al. 2003; Kuhn et al. 2009; Manning and Gabriel 2000; Troulis, Scanlon, and Evans 2003). No significant differences in performance between the various models of hands-free cable have become apparent, even though the microphones are in different places on different cables and some contain push buttons (Manning and Gabriel 2000).

Bit-Babik et al. (2003) and Kuhn et al. (2009) reported on measured and simulated SAR results within the user's head for a wired hands-free scenario at GSM 900/1800 and UMTS 1950, respectively. Example cases are illustrated in Figure 5.20. They concluded that the presence of the human body attenuates the EM field along the wire, thus reducing the energy that is absorbed by the user's head. The maximum spatial peak SAR in the head was found to be more than five times lower than the maximum-allowed exposure limits. Numerical results of the SAR in the heads of two anatomical models (Duke and Billie) are displayed in Table 5.6, assuming positioning of a $900 \mathrm{MHz}$ cellular phone in the pants and shirt pockets (Kuhn et al. 2009). The SAR is found to be considerably higher in the case of the shirt pocket position, resulting from less attenuation along the hands-free wire.

Similarly, in studies by Troulis, Scanlon, and Evans (2003), the coupling between a body-worn cellular phone antenna and a hands-free cable at $1800 \mathrm{MHz}$ was examined. The layout considered included an anatomically based adult male body model, with a

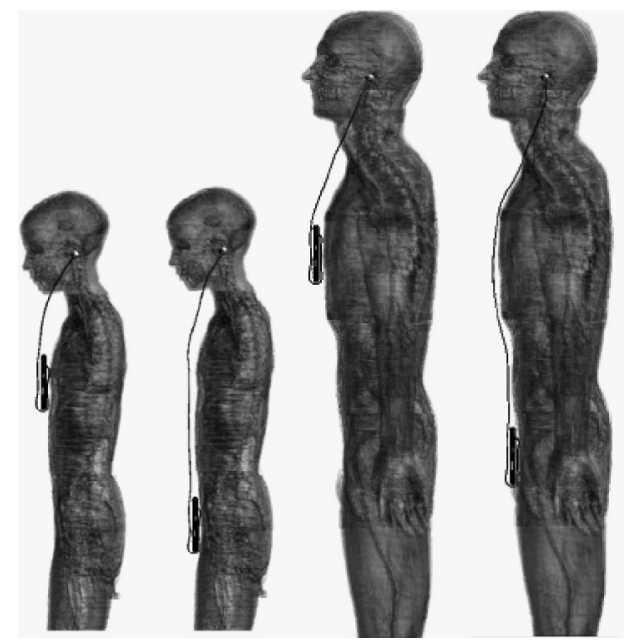

FIGURE 5.20 Example cases of human body models with cellular phones and wired hands-free devices. (From Kuhn, S., E. Cabot, A. Christ, M. Capstick, and N. Kuster. 2009. Phys Med Biol, 54, 508. With permission.) 
TABLE 5.6 $10 \mathrm{~g}$ avg SAR in the Head of Two Anatomical Models (Duke and Billie) from a Cellular Phone Connected to a Hands-Free Device at $900 \mathrm{MHz}$

\begin{tabular}{llll}
\hline \multirow{2}{*}{ Handset Position } & & \multicolumn{2}{l}{$10 \mathrm{~g}$ avg SAR $(\mathrm{W} / \mathrm{kg})$} \\
\cline { 3 - 4 } Pants pocket & Orientation & Duke & Billie \\
\cline { 3 - 4 } Shirt pocket & Back side & 0.045 & 0.036 \\
& Front side & 0.004 & 0.04 \\
& Back side & 0.366 & 0.039 \\
& Front side & 0.006 & 0.016 \\
\hline
\end{tabular}

Source: Data from Kuhn, S. et al. 2009. Phys Med Biol 54:5493-508.

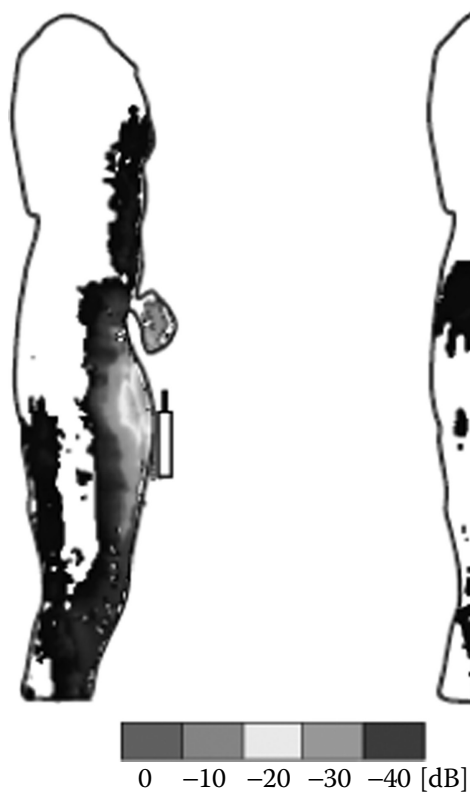

(a)

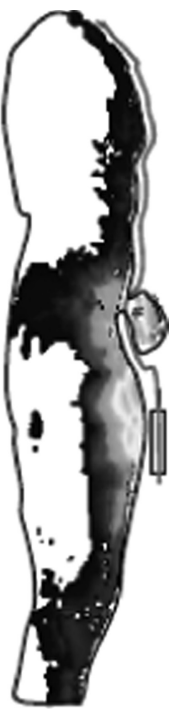

(b)

FIGURE 5.21 Qualitative comparison of the unaveraged SAR distribution in the user's body for (a) cellular phone-only and (b) cellular phone with hands-free cases $(0 \mathrm{~dB}=4.5 \mathrm{~W} / \mathrm{kg})$. (From Troulis, S. E., W. G. Scanlon, and N. E. Evans. 2003. Phys Med Biol, 48, 84. With permission.)

handset enclosure, antenna, and proximate conducting wire laid over the body surface. The wire elements used for the different modeling scenarios considered ranged over $90 \%$ of the body height. Figure 5.21 compares a plot of the unaveraged SAR values in a longitudinal slice of the user's body that incorporates the highest proportion of the handsfree lead. The cellular phone-only (Figure 5.21a) and the hands-free (Figure 5.21b) cases are considered. The hands-free lead changes the SAR distribution, extending higher values toward the user's chest according to the route taken. 
However, some specific configurations with wired hands-free devices have been found to give rise to measurable SAR levels in the head (Manning and Gabriel 2000). Such conditions are considered to be extremely unlikely and should not be encountered in normal use. This involves leading the earpiece cable close to or around the phone's antenna. The effects of such cable routing are sensitively affected by the exact configuration employed especially with external antennas. As a result of further exploratory tests, it was found that exposure from wired hands-free devices was raised when the earpiece cable is placed in contact with the cheek of the phantom as compared to when the earpiece cable hangs vertically downwards. Defining such reproducible conditions has proven to be difficult.

In the case of wireless hands-free operation (Bluetooth technology), the added effect of the exposure to the antenna of the cellular phone, which also has a Bluetooth antenna, plus the exposure to the Bluetooth antenna of the hands-free device, have to be considered. Few, yet indicative, dosimetric studies have been performed so far. In the study by Wong et al. (2007), a Bluetooth internal antenna was presented in the presence of the user's head, but no calculation of the absorption in the head was made. In the research by Martinez-Burdalo et al. (2009), several geometrical configurations were considered to simulate possible exposure situations of a person to the fields from Bluetooth antennas operating at $2400 \mathrm{MHz}$. Figure 5.22 shows a 2D plot of the $10 \mathrm{~g}$ avg SAR distribution through the body cross-section where the maximum is located, for exposure to a wireless hands-free Bluetooth antenna placed near the user's head. Results show that the exposure levels in worst-case situations studied are lower than those obtained when analyzing exposure to cellular phones. This could be expected because of the low power of the signals and the distance between the human and the antennas, with both field and SAR values being far below the limits established by the guidelines.

An important issue that must be taken into account in dosimetry studies for wired and wireless (Bluetooth) hands-free operation of cellular phones is the part of the body where the cellular phone is placed. In regular cellular phone use, the anatomical area

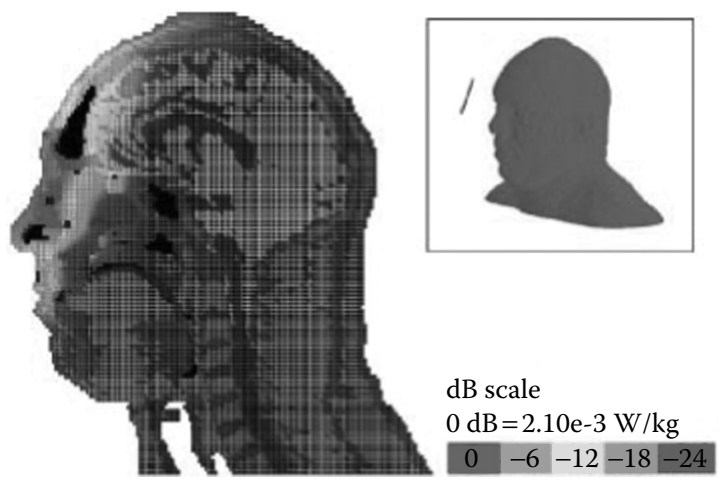

FIGURE 5.22 (See color insert.) Contour plot of the $10 \mathrm{~g}$ avg SAR distribution through the body cross-section where the maximum is located, for exposure to a hands-free Bluetooth antenna at a distance of $8 \mathrm{~cm}$ from the head. (From Martinez Burdalo, M., A. Martin, A. Sanchis, and R. Villar. 2009. Bioelectomagnetics, 30, 51. With permission.) 
with the highest exposure is the ipsilateral (same) side of the brain as used during the call. However, when the cellular phone is mounted on another part of the body (e.g., chest, waist, hip), that anatomical area receives the highest RF exposure (Mild and Wilen 2009).

For example, in the study by Kang and Gandhi (2002), the 1 and $10 \mathrm{~g}$ avg peak SAR values for four typical cellular phones operating at 835 and $1900 \mathrm{MHz}$ were calculated, assumed as though they were placed in a shirt pocket. Several clothing thicknesses were taken into account. It was found that both the 1 and $10 \mathrm{~g}$ avg peak SAR values reduced monotonically with increasing separation from the phantom. Moreover, it was found that the SAR values in the chest when the telephones are placed with the antennas closer to the body ("back" position) could be up to 2.1 times higher at $835 \mathrm{MHz}$ and up to 5.8 times higher at $1900 \mathrm{MHz}$ with respect to the SAR values found when the antennas are placed far from the body ("front" position). Similarly, in the research of Manning and Gabriel (2000) it was shown that if the cellular phone is in a pocket, there will be a lower body dose by ensuring that the keypad of the phone is facing the body.

In the study by Han et al. (2006), the SAR distribution in a human body model exposed to the EM field radiated by a $2400 \mathrm{MHz}$ planar-inverted-F antenna (PIFA) used in a Bluetooth device and located right before the user's chest was examined. As expected, the highest SAR areas were found to be at the body surface near the antenna. The $10 \mathrm{~g}$ avg peak SAR of lung, small intestine, and blood were found to be comparatively high. However, for all cases under consideration, and assuming an antenna output power below $80 \mathrm{~mW}$, the peak SAR levels were lower than the maximum-allowed exposure levels. Similarly, Martinez-Burdalo et al. (2009) modeled, at a distance of $5 \mathrm{~mm}$ away from the user's chest, two half-wavelength dipole antennas, operating at 1800 and $2400 \mathrm{MHz}$, respectively. The antennas simulated the radiating sources of a cellular phone in the two GSM bands. The maximum calculated value for the $1 \mathrm{~g}$ avg SAR was located near the chest region and equaled $1.16 \mathrm{~W} / \mathrm{kg}$, which is clearly below the basic restrictions limit. Figure 5.23 shows a contour plot of the $10 \mathrm{~g}$ avg SAR distribution through the body

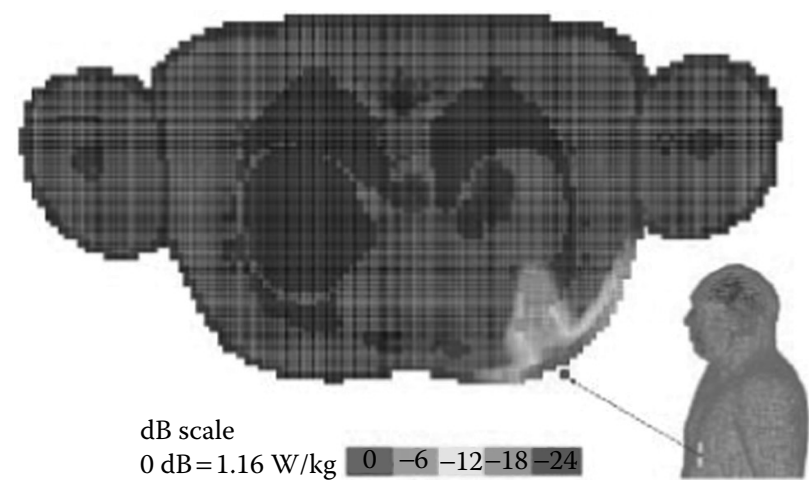

FIGURE 5.23 (See color insert.) Contour plot of the $10 \mathrm{~g}$ avg SAR distribution through the body cross-section where the maximum is located, for exposure to a GSM antenna located $5 \mathrm{~mm}$ away from the human chest. (From Martinez Burdalo, M., A. Martin, A. Sanchis, and R. Villar. 2009. Bioelectomagnetics, 30, 51. With permission.) 
cross-section where the maximum value is located. In all analyzed situations, with the maximum radiated power allowed for these technologies, the exposure levels were lower than those obtained from exposure to cellular phones and far below the limits established by the guidelines.

Placing of the cellular phone on other parts of the body has also been studied. In the study by Han et al. (2006), the coupling between a hip-mounted $1800 \mathrm{MHz}$ cellular phone fitted with a monopole antenna and a $1 \mathrm{~m}$ long wire representing a handsfree wire was investigated. For the scenarios considered, all peak SAR values were well within international guidelines on EM exposure. In the study by Troulis, Scanlon, and Evans (2003), an increase of the SAR values and deposited power was found when the phone was placed at the waist of the body with respect to the case in which the phone was placed close to the ear.

Overall, even under unlikely worst-case scenarios, wired and wireless hands-free devices offer very substantial reductions in SAR compared to the normal use of the phone against the head. In general, wired hands-free devices lead to a considerable reduction of the exposure of the head. On the other hand, wireless (Bluetooth) handsfree devices cause a low but constant level of exposure. From a precautionary point of view, the use of hands-free devices is nowadays recommended by many authorities (Mild and Wilen 2009).

\subsubsection{Base Stations}

In cellular telecommunication systems, the network is divided into cells (typically modeled as regular hexagons) and each cell is equipped with a permanently installed base station, as shown in Figure 5.24. In urban areas the cells have a radius ranging from 1 to $5 \mathrm{~km}$, while in rural regions the radius can be up to $10 \mathrm{~km}$. The steady increase in the number of subscribers of mobile telecommunication systems is pushing toward an enhancement of the systems' capacity, and an increase in the number of base stations being installed. As a result, great social concern has risen about the potential health hazard of people's exposure

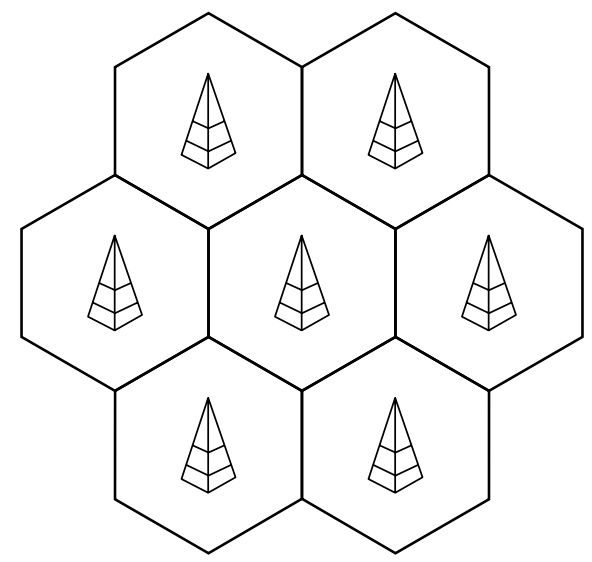

FIGURE 5.24 Division of a geographical area into regular hexagon cells. 
to the field radiated by base-station antennas and several recent studies have addressed this issue. Research on base-station dosimetry is particularly important since people can be exposed to the field radiated by base stations for a long time.

RF emissions vary depending on the design and power of the base station. Basestation antennas situated inside the buildings have low transmission powers, usually less than $1 \mathrm{~W}$. Regarding human exposure, these low power antennas can be considered to be safe at any distance. Most powerful antennas, with transmission powers usually less than $40 \mathrm{~W}$, can also be used. However, since exposure limits may be exceeded in such cases, these antennas are generally sited on high places, so that the general public cannot access the area immediately surrounding the antenna.

In Europe, standards are under development describing the requirements and procedures to verify that the general public has no access to areas where exposure to EM fields radiated by base-station antennas exceed the limits. Most base-station antennas are highly directive. In order to illustrate how the antenna beam is spatially localized, Figure 5.25 shows the electric field distribution of a UMTS base-station antenna placed at $60 \mathrm{~cm}$ above the ground (Lacroux et al. 2008). The antenna operates at $2140 \mathrm{MHz}$ and is composed of four half-wavelength dipoles and a rear metallic reflector at a distance of $3.5 \mathrm{~cm}$. The ICNIRP exposure guidelines (ICNIRP 1998) specify a 6 min averaging time. Since the radiated energy is concentrated to a main beam in front of the antenna, the time-averaged exposure is expected to be well below the limits for a person passing with normal walking speed in front of a typical base-station antenna.

In early dosimetric studies, attention was focused on the determination of simplified and efficient numerical analysis to evaluate field levels near a base-station antenna but no dosimetric analysis was performed (Altman et al. 2002; Balzano and Faraone 2001). To assess exposure to base stations, various measures were used in future studies. In 2003, Santini et al. (2003) regarded the distance to a base station as a relevant surrogate for RF exposure. They obtained information from 530 people responding to a questionnaire about nonspecific health symptoms and about distance to the base station. Respondents living at various distances (less than $300 \mathrm{~m}$ ) from base stations were compared with a reference group living more than $300 \mathrm{~m}$ from the base stations. However, it was shown

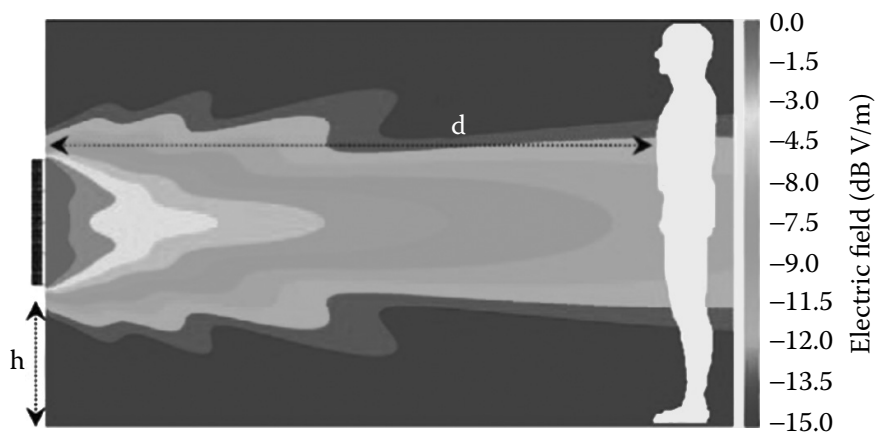

FIGURE 5.25 (See color insert.) Electric field distribution radiated by an isolated base-station antenna. (From Lacroux, F. et al. 2008. Ann Telecommun, 63, 64. With permission.) 
that distance to a base station was not an accurate measure to assess human RF exposure (Neitzke et al. 2007; Neubauer et al. 2007; Schuz and Mann 2000). As a result, future procedures for the assessment of base-station safety, with respect to human exposure, were mainly based on a comparison of the average exposure field value with reference levels suggested by exposure guidelines (EC 1999; FCC 2001; ICNIRP 1998; IEEE 2005). Since reference levels have been derived from basic restrictions under the assumption of uniform plane-wave exposure, their validity is demonstrated only under this particular condition.

However, in the vicinity of the base station, in the radiating near-field region, the antenna field is far from being uniform due to the high directivity on the vertical plane of typical base-station antennas. Similarly, in an urban environment, due to environmental reflections, such as those produced by buildings and the ground, field nonuniformities arise again. Therefore, it is not obvious that compliance of the average field level with reference levels ensures that basic limits on SAR are respected. For example, in the study by Martinez-Burdalo et al. (2005), the procedures for safety assessment in the close proximity of cellular communication base-station antennas at three different frequencies $(900,1800$, and $2170 \mathrm{MHz})$ were analyzed. For antenna-body distances in the near zone of the antenna, the fact that averaged field values were below the reference levels could, at certain frequencies, not guarantee guidelines compliance based on basic restrictions. Similarly, in the research of Lacroux et al. (2008), a numerical dosimetry study for a typical base-station antenna used in $3 \mathrm{G}$ systems was presented. The compliance of the averaged power density to the reference levels was not enough to ensure compliance to localized SAR.

As a result, a problem arises regarding the field value that should be compared with the reference one. This has resulted in different regulatory bodies issuing different standards for the calculation and measurement of EM fields related to human exposure to basestation antennas. These standards, under some aspects, do not agree with each other.

According to the IEEE exposure guidelines (IEEE 2005), in the case of nonuniform exposure conditions, the field should be averaged on a vertical surface equivalent to the projection of the human body. These guidelines are largely at the base of the Federal Communications Commission (FCC) regulations (FCC 2001). The ICNIRP guidelines (1998) state that, in order to establish whole-body averaged SAR compliance (only), the field should be averaged on a vertical surface equivalent to the projection of the human body. On the other hand, local SAR compliance should be directly assessed through a dosimetric study. The CENELEC has issued a compliance procedure in order to avoid a complete dosimetric assessment (CENELEC 2002). This procedure allows local SAR compliance evaluation through a comparison of the peak value of the exposure field with the corresponding reference level (conservative approach). Finally, there are other procedures which suggest that, if compliance is evaluated numerically rather than experimentally, the numerical analysis can be performed neglecting the presence of the environment (free-space condition), thus eliminating the problem of field nonuniformities (IEC 2002).

Due to the many contrasting approaches to base-station compliance assessment presented above, typical realistic urban environment exposure situations together with exposure in close proximity of the antenna must be studied in order to characterize the exposure to the field emitted by a base-station antenna. 
In the study by Bernardi et al. (2000a), human exposure to the EM field radiated by a rooftop-mounted base-station antenna operating around $900 \mathrm{MHz}$ in an urban environment was analyzed. The incident field and SAR distributions obtained in the three typical exposure scenarios of Figure 5.26 are shown in Figures 5.27, 5.28, and Figure 5.29, respectively. Each figure shows the distribution of the rms value of the electric field on the vertical plane containing the antenna maximum radiation direction in the absence of the subject and in the presence of the subject, as well as the SAR distribution inside the subject in the same plane. The obtained results show the strong nonuniformity of the field distribution produced by base-station antennas in urban environments and the importance of an accurate modeling of the real environment in which the exposure takes place. As expected, the lowest field levels are experienced by the subject standing in the street due to the high distance from the antenna and to the angled position with respect to the antenna pointing direction. In order to better identify the power absorbed inside the main body organs, the $1 \mathrm{~g}$ avg SAR values are reported in Table 5.7.

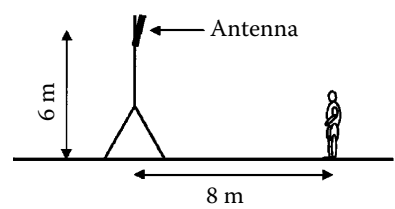

(a)

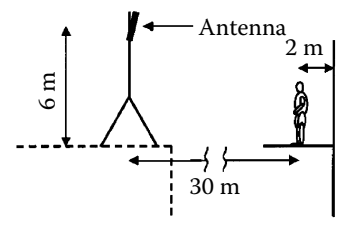

(b)

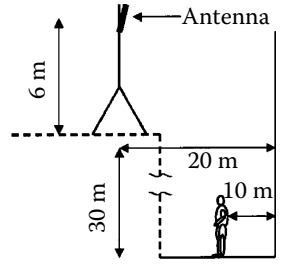

(c)

FIGURE 5.26 Exposure scenarios (a) subject on the building roof, (b) subject on the balcony, and (c) subject on the street. (From Bernardi, P., M. Cavagnaro, S. Pisa, and E. Piuzzi. 2000. IEEE Trans Microw Theory Tech, 48, 11. With permission.)

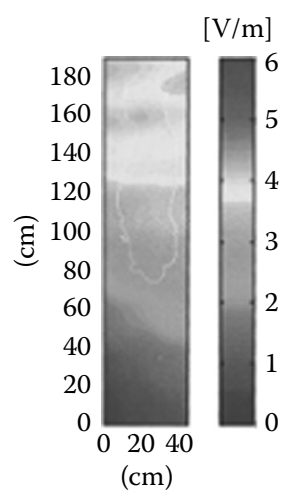

(a)

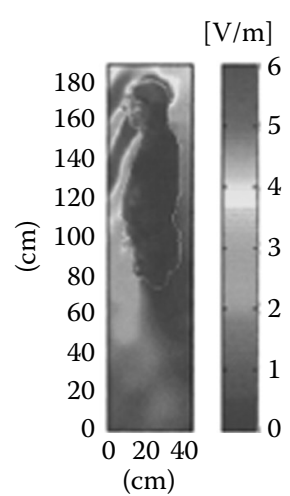

(b)

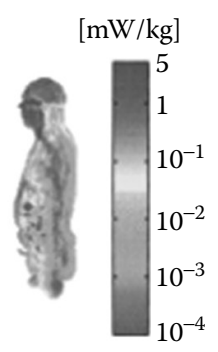

(c)

FIGURE 5.27 (See color insert.) Subject on the building roof: (a) Erms when the subject is absent (b) Erms when the subject is present (c) SAR distribution inside the subject. (From Bernardi, P., M. Cavagnaro, S. Pisa, and E. Piuzzi. 2000. IEEE Trans Microw Theory Tech, 48, 11. With permission.) 


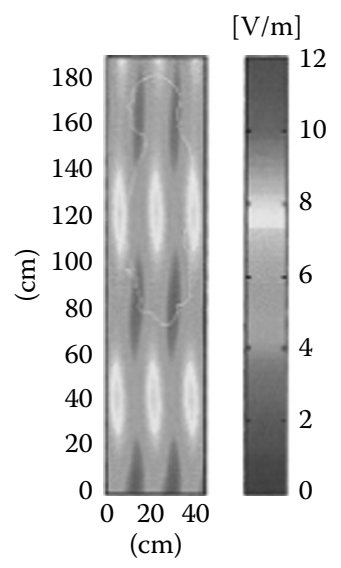

(a)

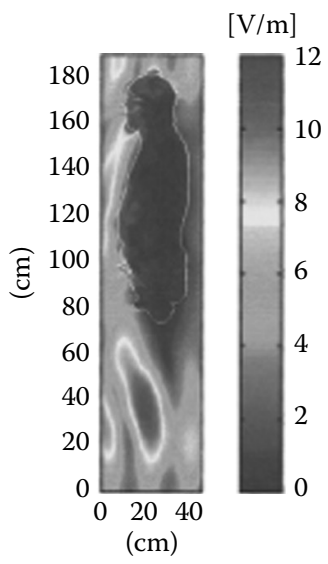

(b)

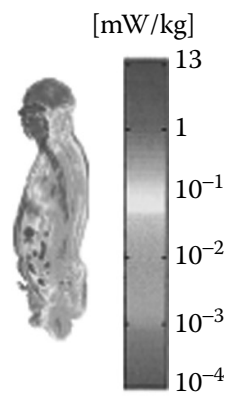

(c)

FIGURE 5.28 (See color insert.) Subject on the balcony: (a) Erms when the subject is absent (b) Erms when the subject is present (c) SAR distribution inside the subject. (From Bernardi, P., M. Cavagnaro, S. Pisa, and E. Piuzzi. 2000. IEEE Trans Microw Theory Tech, 48, 11. With permission.)

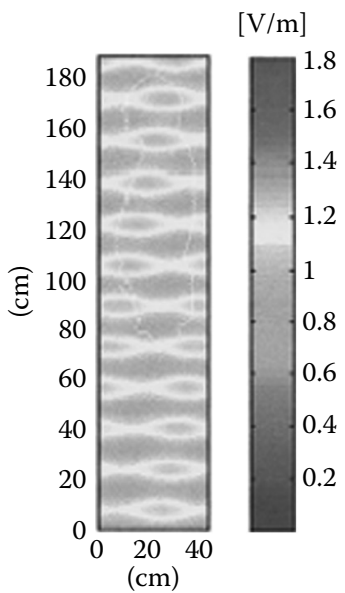

(a)

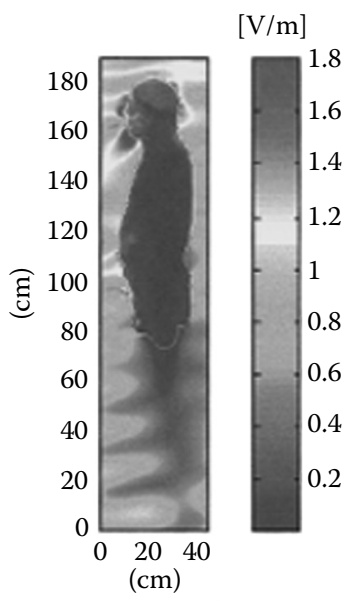

(b)

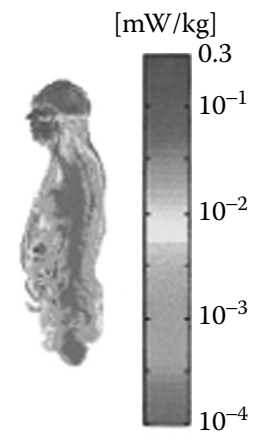

(c)

FIGURE 5.29 (See color insert.) Subject on the street: (a) Erms when the subject is absent (b) Erms when the subject is present (c) SAR distribution inside the subject. (From Bernardi, P., M. Cavagnaro, S. Pisa, and E. Piuzzi. 2000. IEEE Trans Microw Theory Tech, 48, 11. With permission.)

Similarly, in the research of Bernardi et al. (2002b), a four-element GSM base-station antenna operating in an urban environment at $947.5 \mathrm{MHz}$ has been considered. Differences between maximum and average values of the exposure field up to $50 \%$ have been obtained, evidencing the nonuniformity of the field distribution. In the study by Bernardi et al. (2003), different exposure situations for a subject standing inside a room of a building with 
TABLE 5.7 Maximum $1 \mathrm{~g}$ avg SAR Values (mW/kg) of Main Body Organs for the Exposure Scenarios of Figure 5.26

\begin{tabular}{lccc}
\hline Main Body Organs & Scenario (a) & Scenario (b) & Scenario $(\mathrm{c})$ \\
\hline Brain & 1.38 & 2.71 & 0.13 \\
Eyes & 1.67 & 3.44 & 0.07 \\
Heart & 0.39 & 1.57 & 0.02 \\
Kidney & 0.04 & 0.13 & $<0.01$ \\
Liver & 0.51 & 1.42 & 0.03 \\
Pancreas & 0.10 & 0.35 & 0.01 \\
\hline
\end{tabular}

Source: Data from Bernardi, P., M. Cavagnaro, S. Pisa, and E. Piuzzi. 2000a. IEEE Trans Microw Theory Tech 48(11):1996-2002.

a window facing a rooftop-mounted GSM 900 and a UMTS base-station antenna were analyzed. A comparison of the numerical results obtained in the realistic environments with those computed in free space has evidenced underestimations on SAR values up to a factor of two. These underestimations could be expected to be even higher for environments presenting more reflective and diffractive characteristics. As a result, compliance testing carried out in free space can yield nonconservative results. Complex exposure situations were also analyzed in the research of Bernardi et al. (2000a), where the numerical technique employed allowed representation of corner-reflector-like urban scenarios.

Exposure-field and SAR values well below reference levels and basic limits proposed in the main international protection standards have been obtained in all the situations. Analysis of the obtained results (in terms of SAR values) suggests that, when field nonuniformities (typical of realistic urban environments) are present, only the wholebody averaged SAR value is related to the average field value. This is true provided that the averaging procedure is appropriately chosen to cover all the volume occupied by the subject and not only a vertical surface. On the other hand, local SAR values show a more complex relation with the exposure field. Considering only the local-averaged field value for compliance assessment might lead to an underestimation of the real exposure level, while using the local peak of the field might lead to a remarkable overestimation.

On the basis of the results from measurements and calculations, members of the public would not be exposed in excess of the ICNIRP guidelines while standing on the ground near any of the base stations. For example, in the study by Cooper et al. (2006), based on information provided by the operators of GSM networks in the United Kingdom, the distribution of 32837 base stations was found to have a low antenna height, typically 3-6 m, and a power of a few watts. The compliance distances, in terms of the ICNIRP general public reference levels, ranged from 0.1 to $0.8 \mathrm{~m}$ in front of the antennas. Assuming isotropic radiation patterns, the minimum height at which the reference level could theoretically be exceeded near any of the base-station antennas was $2.4 \mathrm{~m}$ above ground level. Real antennas do not have isotropic radiation patterns and so, again, lesser distances would be expected with a more detailed assessment.

The most worrying exposure to base-station antennas are for people working with antenna installation and maintenance of base stations as well as people performing construction or other tasks on the roof in close proximity to the antenna (maintenance and 
operator personnel, janitors, painters, chimney sweepers, window cleaners, technical staff dealing with repair or maintenance of roof, roof-racks, and gutters or with heating, ventilation, and air conditioners). For such situations where exposure levels exceed the general public limits, there is a need for a careful investigation and clarification of operational control procedures, measurements, and instructions, elaborated and aimed to protect the health and safety of workers.

To protect themselves, workers must take into account several issues concerning their exposure to RF fields emitted by base-station antennas. First, modern base-station antennas are sector antennas that transmit only in the forward direction. Due to the high directivity on the vertical plane of the base-station antenna considered, the highest field levels are not obtained on the roof of the building where the antenna is located, but rather on the nearby building placed in the direction of the maximum antenna radiation (Bernardi et al. 2002b). Moreover, when working behind the antenna, it is likely that the RF fields are at the background level due to the very good directivity of the transmission. Second, the antenna might be placed on a small mast, chimney, or higher on the building wall. In this case, the antenna will send the transmission over the heads of people working below antennas. The same principle applies if one is working higher than the antenna.

According to the above, exposure from base-station antennas is low and cannot represent a risk for human health from the thermal point of view. Only when working very close to the antenna is there a need for precautionary measures. However, the possibility of long-term nonthermal effects of these fields is an open issue. This problem can be addressed through epidemiological studies, which, in order to be effective, require an accurate evaluation of the internally induced field (Burgi et al. 2010; Khurana et al. 2010).

\subsubsection{Wi-Fi Systems}

Wireless fidelity (Wi-Fi) is a term used to describe the way user terminal equipment (e.g., computers, PDAs, pocket PCs, cellular phones) can wirelessly connect to a network in the absence of a cable infrastructure. Instead, the connection is made using RF EM waves, along which the data are sent in pulses. This is a relatively new wireless access technology that has taken off at a rapid pace. The first wireless systems were developed in the early 1990s (e.g., WaveLAN). However, it was not until 1999 that wireless technologies were widely accepted. The most common 802.11g protocol has only been around since 2003. The network device providing the bridge between the wireless and wired networks is called the Wi-Fi access point, which is a radio transceiver itself. The user terminal equipment should be equipped with a wireless network interface card for communication with the access points.

All Wi-Fi wireless products are required to be evaluated to ensure conformance with the RF emission safety guidelines (ICNIRP 1998; IEEE 2005). These evaluations are done in accordance with the various regulations and guidelines adopted or recommended by regulatory agencies around the world. The Wi-Fi Alliance is conducting additional studies to confirm, in a variety of settings, that RF exposure to Wi-Fi products consistently falls well below the international exposure limits. In addition, the organization is sponsoring an independent review of the scientific studies that may be relevant to Wi-Fi products. At the same time, the Mobile Manufacturers Forum and the GSM Association 
are also supporting a wide variety of international research into RF safety. Many of the supported research projects also involve national and international health agencies.

While several studies present dosimetry results for animals exposed to Wi-Fi radiation (Wu et al. 2010), dosimetric studies for human beings have also been performed. In certain studies, measurements of the Wi-Fi signals have been made by considering access points only. Four scenarios were considered in the study of Martinez-Burdalo et al. (2009) to simulate possible exposure situations of a person to the fields from $\mathrm{Wi}-\mathrm{Fi}$ access point antennas operating at $2400 \mathrm{MHz}$, as illustrated in Figure 5.30. In all cases,

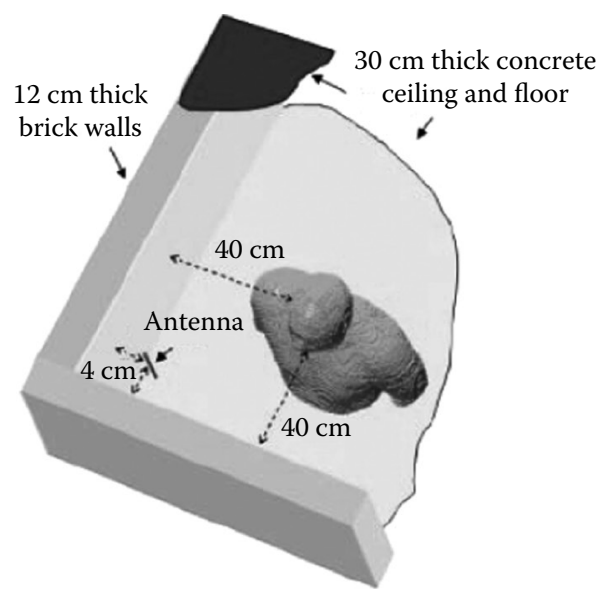

(a)

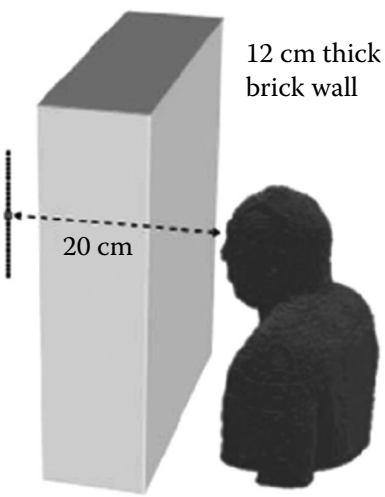

(c)

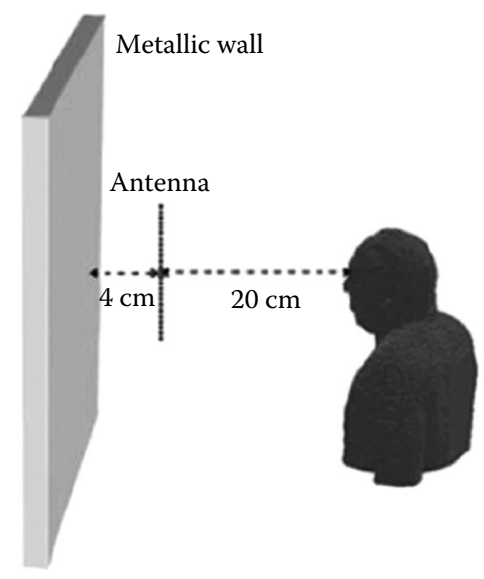

(b)

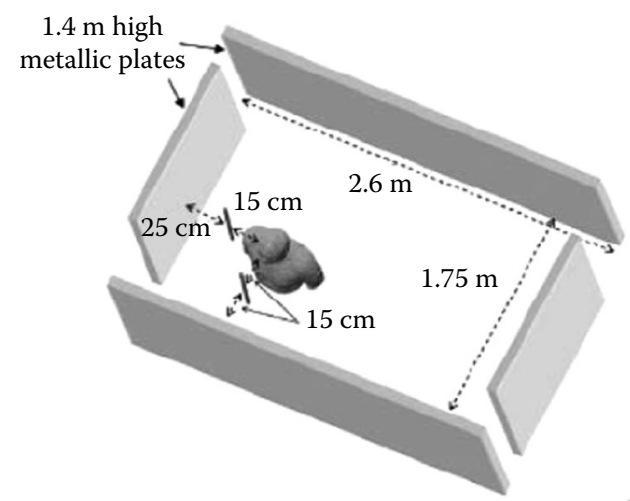

(d)

FIGURE 5.30 Scenarios of exposure to wireless access point antennas analyzed. (From Martinez Burdalo, M., A. Martin, A. Sanchis, and R. Villar. 2009. Bioelectromagnetics, 30, 51. With permission.) (a) Subject in a room with a vertical antenna near one of the corners, (b) subject with a vertical antenna in front of his eyes and a metallic reflecting plate behind the antenna, (c) subject in front of a vertical antenna that is on the opposite side of a thick brick wall, and (d) subject in the presence of two desktop antennas surrounded by flat plates, simulating metallic cabinets, behind the antennas. 


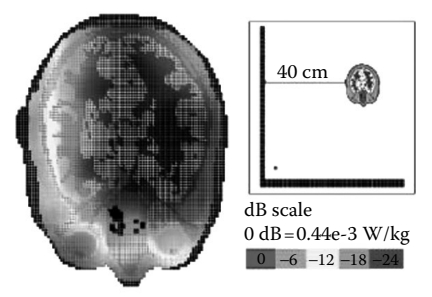

(a)

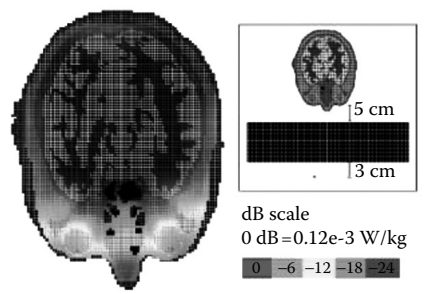

(c)

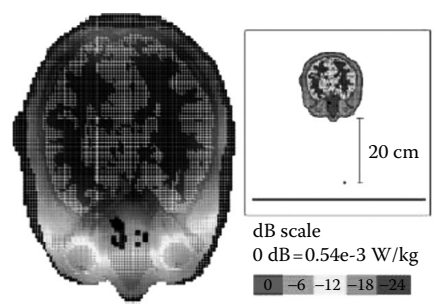

(b)

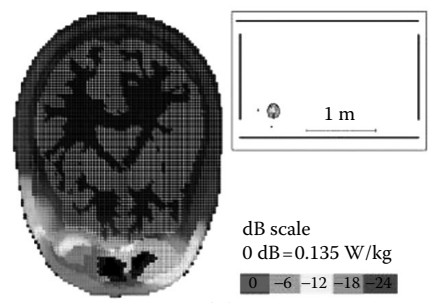

(d)

FIGURE 5.31 (See color insert.) Contour plots of the $10 \mathrm{~g}$ avg SAR distribution in the plane where the maximum is located, for the four scenarios of exposure depicted in Figure 5.30. (From Martinez Burdalo, M., A. Martin, A. Sanchis, and R. Villar. 2009. Bioelectromagnetics, 30, 51. With permission.)

the access point antennas were simulated as vertically polarized half-wavelength dipoles. Brick walls and metallic cabinets were included to consider the influence of the environment. Figure 5.31 shows contour plots of the $10 \mathrm{~g}$ avg SAR distribution inside the head of the exposed person in the plane where the maximum is located, for the four analyzed situations of exposure. In all scenarios, local field values in the position where the person can be located were below the ICNIRP reference level for $2400 \mathrm{MHz}$. However, several differences were observed between the four exposure situations. Higher levels were obtained with the geometry in Figure 5.31d, which can be explained by the presence of two antennas that cause the radiated power to be double in this case and four metallic plates that increase the field in all sides of the head due to reflections. The maximum $10 \mathrm{~g}$ avg SAR values obtained for geometries Figure 5.31a and $\mathrm{c}$ are equal. This happens because part of the antenna radiation is absorbed by the brick walls, despite the fact that the antenna-head distance is shorter in the exposure scenario (Figure 5.31c).

Other dosimetric studies have only considered exposure to Wi-Fi enabled mobile terminals. For example, Wang and Fujiwara (2005) carried out one of the few SAR studies in a sitting-adult human voxel model with a laptop and a $5200 \mathrm{MHz}$ monopole antenna. They calculated that peak localized SAR values of $20.5 \mathrm{~mW} / \mathrm{kg}$ in the hands and 0.264 $\mathrm{mW} / \mathrm{kg}$ in the torso could be achieved under certain exposure conditions. However, since the model used was homogeneous, the study concluded that the analysis should be extended to anatomically heterogeneous human models.

Finally, several dosimetric studies assessing exposure to mixed scenarios with both Wi-Fi access points and mobile terminals exist in the literature. Bornkessel and Wuschek (2006) considered exposure around access points and mobile terminals with a maximum power of $100 \mathrm{~mW}$. A strong dependence on distance was found, while all measured 
values were far below the limits of the international guidelines set in EC (1999). The highest exposure was found for immediate contact to a Wi-Fi notebook and equaled about $1.2 \%$ of the limit. At more than $98 \%$ of all measurement points the limit consumption was below $0.1 \%$ of the limit. It was also shown that public exposure is dominated by the mobile terminal and not by the access points.

Exposure to both Wi-Fi access points and mobile terminals (laptops) was also considered in a study by Foster at the University of Pennsylvania (Foster 2007). The study considered 55 locations around the world and presented a total of 356 measurements. By considering a distance of $1 \mathrm{~m}$ from laptops while uploading large files, the exposure levels were found to be of the order of up to $\mu \mathrm{W} / \mathrm{m}^{2}$. The median power density was a factor of 10 higher than the exposure level. However, in all cases under consideration the measured Wi-Fi signal levels were very far below the international exposure limits (ICNIRP 1998; IEEE 2005) and in nearly all cases far below other RF signals present in the same environment. Finally, Cortel-Carrasco et al. (2006) performed measurements of the electric field near a Wi-Fi $802.11 \mathrm{~b}$ router as well as numerical simulations of the electric field surrounding a wireless laptop computer with two hands over the keyboard. In their study, they assumed that wireless channel occupation could be theoretically considered as a means of estimating the actual power emitted by the device.

Wi-Fi dosimetry for children has also been performed. Exposure of a 10-year-old sitting child model to EM fields at the frequencies of 2500 and $5000 \mathrm{MHz}$ (commonly used by Wi-Fi devices) was studied in Findlay and Dimbylow (2010). In all situations considered, the SAR values calculated were considerably below basic restrictions. Exposure to half-wavelength dipoles showed that the highest localized SAR calculated was in the head for a $2400 \mathrm{MHz}$ dipole antenna at $3 \mathrm{~cm}$ distance and $100 \%$ duty factor. The SAR value for this scenario was $8.17 \mathrm{~W} / \mathrm{kg}$ per W. However, under more realistic exposure conditions, with the antenna operating at $100 \mathrm{~mW}$ at $31 \mathrm{~cm}$ distance and $10 \%$ duty factor, the peak localized SAR was found equal to $1.8 \mathrm{~mW} / \mathrm{kg}$. This value is considerably below the ICNIRP basic restriction of $2 \mathrm{~W} /$ $\mathrm{kg}$. Calculations were also performed for typical Wi-Fi exposure scenarios using inverted-F antennas (IFAs) mounted on laptops, as shown in Figure 5.32. The SAR intensity plots for the IFAs mounted on the bottom left (BL), top right (TR), and bottom center (BC) positions at $2400 \mathrm{MHz}$ are shown in Figure 5.33a, b, and c, respectively. Using an IFA operating at $100 \mathrm{~mW}$ with a duty factor of $10 \%$ and assuming that the human body is placed $34 \mathrm{~cm}$ away from the antenna, the maximum peak localized SAR was found to be $3.99 \mathrm{~mW} / \mathrm{kg}$ in the torso region. Increasing the duty factor to $100 \%$, while keeping the IFA operating at $100 \mathrm{~mW}$ increased the highest localized SAR value in the head to $5.7 \mathrm{~mW} / \mathrm{kg}$. This value is less than $1 \%$ of the SAR calculated in the head for a typical cellular phone exposure scenario (MartinezBurdalo et al. 2004). Furthermore, most of the energy was found to be absorbed in the hands region. Moving the antenna to the top of the screen increased the SAR absorbed in the head, as expected. Changing the laptop casing from plastic to a perfect conductor significantly increased the SAR values. The localized SAR was highly dependent on antenna position and frequency but typically was two to three times higher than that calculated for a plastic casing.

The World Health Organization's (WHO) latest fact sheet on wireless networks says that "Recent surveys have indicated that RF exposures from base stations and wireless technologies in publicly accessible areas (including schools and hospitals) are normally thousands of times below international standards" and concludes that "Considering the 


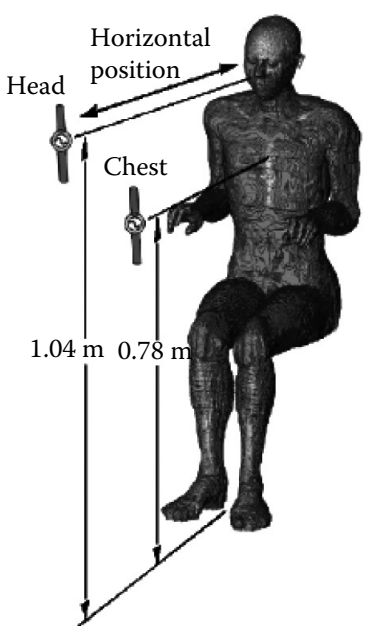

(a)

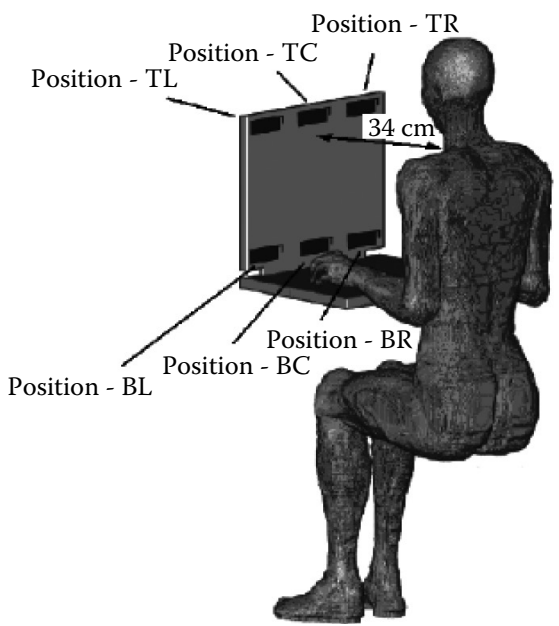

(b)

FIGURE 5.32 Exposure conditions for the subject placed in front of (a) a half-wavelength antenna and (b) an IFA mounted on a laptop. (From Findlay, R. P., and P. Dimbylow. 2010. Phys Med Biol 55:N405-N411.)

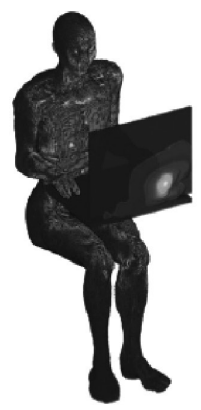

(a)

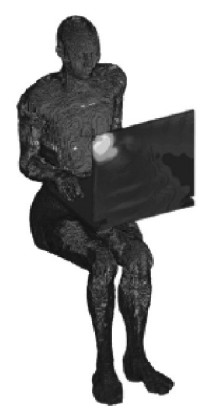

(b)

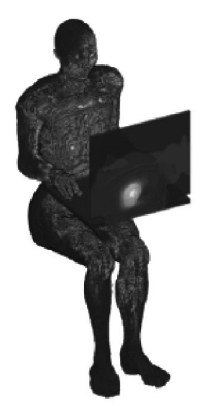

(c)

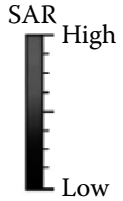

FIGURE 5.33 (See color insert.) SAR intensity plots for the IFA mounted on the (a) bottom left (BL), (b) top right (TR), and (c) bottom center (BC) positions of the laptop of Figure 5.32. (From Findlay, R. P., and P. Dimbylow. 2010. Phys Med Biol 55:N405-N411.)

very low exposure levels and research results collected to date, there is no convincing scientific evidence that the weak RF signals from base stations and wireless networks cause adverse health effects" (WHO 2006). Furthermore, the general position of the United Kingdom's Health Protection Agency (HPA) is that there is no consistent evidence to date that Wi-Fi adversely affects the health of the general population and that there is no reason why Wi-Fi should not continue to be used in schools (HPA 2007). This position slightly contrasts with that of the French Health and Security Agency, which is recommending that people reduce their exposure to wireless technologies because of health concerns (Afsset 2009). 


\subsection{Conclusions}

In this chapter, a summary was provided regarding the numerical and experimental techniques used to assess the interaction between the EM fields emitted by mobile communication devices and the human body. Canonical and anatomically detailed models used for computational exposure assessment were presented, while phantoms, measurement devices, and equipment utilized for experimental dosimetry were described. Results of recent dosimetry studies were summarized and conformance with international guidelines designed to limit human exposure were discussed.

Regarding cellular phone dosimetry, typical SAR values were found to range from 0.1 to $1.8 \mathrm{~W} / \mathrm{kg}$, while similar or slightly higher SAR values were generally assessed in children. Furthermore, parametric studies have indicated the dependence of the SAR values upon phone to head separation distance, tissue dielectric properties, and the head's anatomy. The use of wired and wireless (Bluetooth) hands-free devices to reduce exposure was then considered, and its influence on computed SAR values in the human head and the part of the human body where the cellular phone is placed was assessed. The potential health hazard of people's exposure to the field radiated by base-station antennas was also explored. The problem of which field value should be compared with the reference one was discussed and precautionary measures were found to be required only when working very close to the antenna. Finally, the results of dosimetric studies related to adult and child exposure to $\mathrm{Wi}-\mathrm{Fi}$ access points and $\mathrm{Wi}$-Fi equipped mobile terminals were presented.

In all studies, simulated and experimentally derived dosimetric parameters were found to be well below the exposure guidelines set by national and international organizations. However, the health hazards occurring from long-term exposure to these new technologies are far from settled. All organizations agree that with any new technology it is a sensible precautionary approach to keep the situation under ongoing review.

\section{Acknowledgment}

The authors would like to gratefully thank their colleague Maria Christopoulou for valuable discussions.

\section{References}

Ackerman, M. J. 1998. The Visible Human Project. Proc IEEE 86(3):504-11.

Adey, W. R. 1993. Biological effects of electromagnetic fields. J Cell Biochem 51:410-6. Afsset (French Health and Security Agency). 2009. Limit Exposure to Mobile Phones: French Experts, Agence France-Presse (AFP). http://www.informationliberation .com/?id=27697 (date accessed: 1 May 2011).

Al-Mously, S. I., and M. M. Abousetta. 2008. Anticipated impact of hand-hold position on the electromagnetic interaction of different antenna types/positions and a human in cellular communications. Int J Antennas Propag 2008:1-22.

Ali, M., M. G. Douglas, A. T. M. Sayem, A. Faraone, and C. K. Chou. 2007. Threshold power of canonical antennas for inducing SAR at compliance limits in the 300-3000 $\mathrm{MHz}$ frequency range. IEEE Trans Electromagn Compat 49:143-52. 
Allen, S. J., E. R. Adair, K. S. Mylacraine, W. Hurt, and J. Ziriax. 2003. Empirical and theoretical dosimetry in support of whole body resonant RF exposure $(100 \mathrm{MHz})$ in human volunteers. Bioelectromagnetics 24:502-9.

Allen, S. J., E. R. Adair, K. S. Mylacraine, W. Hurt, and J. Ziriax. 2005. Empirical and theoretical dosimetry in support of whole body radio frequency (RF) exposure in seated human volunteers at $220 \mathrm{MHz}$. Bioelectromagnetics 26:440-7.

Altman, Z., B. Begasse, C. Dale, A. Karwowski, J. Wiart, M. F. Wong, and L. Gattoufi. 2002. Efficient models for base station antennas for human exposure assessment. IEEE Trans Electromagn Compat 44:588-92.

Anderson, V. 2003, Comparisons of peak SAR levels in concentric sphere head models of children and adults for irradiation by a dipole at $900 \mathrm{MHz}$. Phys Med Biol 48:3263-75.

ARIB. 2002. ARIB STD-T56. Specific Absorption Rate (SAR) Estimation for Cellular Phone. Association of Radio Industries and Businesses (ARIB), Japan.

Balzano, Q., and A. Faraone. 2001. Peak and average RF safety compliance levels near radio base station antennas-Prediction formulas and numerical validation. In Proc IEEE Int Symp Electromagn Compat IEEE, 780-5. Montreal, Canada.

Balzano, Q., O. Garay, and T. Manning. 1995. Electromagnetic energy exposure of simulated users of portable cellular telephones. IEEE Trans Veh Technol 44:390-403.

Balzano, Q., O. Garay, and F. R. Steel. 1978. Energy deposition in simulated human operators of $800 \mathrm{MHz}$ portable transmitters. IEEE Trans Veh Technol 27(4):174-81.

Beard, B. B., W. Kainz, T. Onishi, T. Iyama, S. Watanabe, O. Fujiwara, J. Wang et al. 2006. Comparisons of computed mobile phone induced SAR in the SAM phantom to that in anatomically correct models of the human head. IEEE Trans Electromagn Compat 48:397-407.

Berenger, J. P. 1994. A perfectly matched layer for the absorption of electromagnetic waves. J Comput Phys 4:185-200.

Bernardi, P., M. Cavagnaro, R. Cicchetti, S. Pisa, E. Piuzzi, and O. Testa. 2003. A UTD/ FDTD investigation on procedures to assess compliance of cellular base station antennas with human exposure limits in a realistic urban environment. IEEE Trans Microw Theory Tech 51(12):2409-17.

Bernardi, P., M. Cavagnaro, P. D’Atanasio, E. Palma, S. Pisa, and E. Piuzzi. 2002a. FDTD, multiple-region/FDTD, raytracing/FDTD: A comparison on their applicability and accuracy for human exposure evaluation. Int J Numer Model 15:579-93.

Bernardi, P., M. Cavagnaro, and S. Pisa. 1996. Evaluation of the SAR distribution in the human head for cellular phones used in a partially closed environment. IEEE Trans Electromagn Compat 38(3):357-66.

Bernardi, P., M. Cavagnaro, S. Pisa, and E. Piuzzi. 2000a. Human exposure to radio base station antennas in urban environment. IEEE Trans Microw Theory Tech 48(11):1996-2002.

Bernardi, P., M. Cavagnaro, S. Pisa, and E. Piuzzi. 2000b. Specific absorption rate and temperature increases in the head of a cellular phone user. IEEE Trans Microw Theory Tech 48(7):1118-26.

Bernardi, P., M. Cavagnaro, S. Pisa, and E. Piuzzi. 2001. A graded-mesh FDTD code for the study of human exposure to cellular phones equipped with helical antennas. Appl Comput Electromagn Soc J 16:90-6. 
Bernardi, P., M. Cavagnaro, S. Pisa, and E. Piuzzi. 2002b. Numerical evaluation of human exposure to radio base station antennas. IEEE Trans Microw Theory Tech 48:1996-2002.

Bernsten, S., and S. N. Hornsleth. 1994. Retarded lime absorbing boundary conditions. IEEE Trans Antennas Propag 42:1059-64.

Bit-Babik, G., C. K. Chou, A. Faraone, A. Gessner, M. Kanda, and Q. Balzano. 2003. Estimation of the SAR in the human head and body due to radiofrequency radiation exposure from handheld mobile phones with hands-free accessories. Radiat Res 159:550-7.

Bit-Babik, G., A. W. Guy, C. K. Chou, A. Faraone, M. Kanda, A. Gessner, J. Wang et al. 2005. Simulation of exposure and SAR estimation for adult and child heads exposed to radiofrequency energy from portable communication devices. Radiat Res 163:580-90.

Blanch, S., J. Romeu, and A. Cardama. 2002. Near field in the vicinity of wireless basestation antennas: An exposure compliance approach. IEEE Trans Antennas Propag 50:685-92.

Bornkessel, C., and W. Wuschek. 2006. Exposure measurements in different WLANscenarios. In Proc Int Conf and COST 281 Workshop on Emerging EMF Technol. Graz, Austria: Potential Sensitive Groups and Health.

Burgi, A., P. Frei, G. Theis, E. Mohler, C. Braun-Fahrländer, J. Fröhlich, G. Neubauer et al. 2010. A model for radiofrequency electromagnetic field predictions at outdoor and indoor locations in the context of epidemiological research. Bioelectromagnetics 31:226-36.

Burkhardt, M., and N. Kuster. 2000. Appropriate modeling of the ear for compliance testing of handheld MTE with SAR safety limits at 900/1800 MHz. IEEE Trans Microw Theory Tech 48:1927-34.

Campbell, A. M., and D. V. Land. 1992. Dielectric properties of female human breast tissue measured in vitro at 3.2 GHz. Phys Med Biol 37:193-210.

Caon, M. 2004. Voxel-based computational models of real human anatomy: A review. Radiat Environ Biophys 42:229-35.

Caputa, K., M. A. Stuchly, M. Skopec, H. I. Bassen, P. Ruggera, and M. Kanda. 2000. Evaluation of electromagnetic interference from a cellular telephone with hearing aid. IEEE Trans Microw Theory Tech 48:2148-54.

Cavagnaro, M., and S. Pisa. 1996. Simulation of cellular phone antennas by using inductive lumped elements in the 3D-FDTD algorithm. Microw Opt Technol Lett 13(6):324-6.

CENELEC. 2001. CENELEC EN 50361. Basic Standard for the Measurement of Specific Absorption Rate Related to Human Exposure to Electromagnetic Fields from Mobile Phones (300 MHz-3 GHz), European Committee for Electrotechnical Standardization (CENELEC), Belgium.

CENELEC. 2002. CENELECEN 50383. Basic Standard for the Calculation and Measurement of Electromagnetic Field Strength and SAR Related to Human Exposure from Radio Base Stations and Fixed Terminal Stations for Wireless Telecommunication Systems (110 MHz-40 GHz), European Committee for Electrotechnical Standardization (CENELEC), Belgium.

Cerri, G., R. De Leo, and G. Rosellini. 1997. Evaluation of electromagnetic power deposition in a spherical multilayer head in the near field of a linear antenna. Wireless Networks 3(6):499-510. 
Cerri, G., P. Russo, A. Schiavoni, G. Tribellini, and P. Bielli. 1998. A new MoM-FDTD hybrid technique for the analysis of scattering problems. Electron Lett 34(5):438-40.

Chavannes, N., P. Futter, R. Tay, K. Pokovic, and N. Kuster. 2006. Reliable prediction of mobile phone performance for different daily usage patterns using the FDTD method. Proc IEEE Int Workshop Antenna Technol (IWAT '06), IEEE, 345-8. White Plains, NY.

Chavannes, N., R. Tay, N. Nikoloski, and N. Kuster. 2003. Suitability of FDTD-based TCAD tools for RF design of mobile phones. IEEE Antennas Propag Mag 45:52-66.

Chen, K., A. Gelb, and R. Renaut. 2003. Improving tissue segmentation of human brain MRI through preprocessing by the Gegenbauer reconstruction method. Neuroimage 20:489-502.

Chen, H. Y., and H. H. Wang. 1994. Current and SAR induced in a human head model by electromagnetic fields irradiated from a cellular phone. IEEE Trans Microw Theory Tech 42(12):2249-54.

Christ, A., N. Chavannes, N. Nikoloski, H. U. Gerber, K. Pokovic, and N. Kuster. 2005. A numerical and experimental comparison of human head phantoms for compliance testing of mobile telephone equipment. Bioelectromagnetics 26:125-37.

Christ, A., M. C. Gosselin, M. Christopoulou, S. Kuhn, and N. Kuster. 2010a. Agedependent tissue-specific exposure of cell phone users. Phys Med Biol 55:1767-83.

Christ, A., M. C. Gosselin, S. Kuhn, and N. Kuster. 2010b. Impact of pinna compression on the RF absorption in the heads of adult and juvenile cell phone users. Bioelectromagnetics 31:406-12.

Christ, A., and N. Kuster. 2005. Differences in RF energy absorption in the heads of adults and children. Bioelectromagnetics 26:S31-44.

Christ, A., T. Samaras, A. Klingenbock, and N. Kuster. 2006. Characterization of the electromagnetic near-field absorption in layered biological tissue in the frequency range from $30 \mathrm{MHz}$ to $6000 \mathrm{MHz}$. Phys Med Biol 51:4951-65.

Christensen, H. C., J. Schuz, M. Kosteljanet, H. S. Poulsen, J. D. Boice, J. K. McLaughlin, and C. Johansen. 2005. Cellular telephones and risk for brain tumours: A population-based, incident case-control study. Neurology 64:1189-95.

Christensen, H. C., J. Schuz, M. Kosteljanet, H. S. Poulsen, J. Thomsen, and C. Johansen. 2004. Cellular telephone use and risk of acoustic neuroma. Am J Epidemiol 159:277-83.

Christopoulou, M., S. Koulouridis, and K. S. Nikita. 2009. Parametric study of power absorption patterns induced in adult and child head models by small helical antennas. Prog Electromagn Res 94:49-67.

Chuang, H. R. 1994. Human operator coupling effects on radiation characteristics of a portable communication dipole antenna. IEEE Trans Antennas Propag 42(4):556-60.

Cleveland, R. F., and T. W. Athey. 1989. Specific sbsorption rate (SAR) in models of the human head exposed to handheld UHF portable radios. Bioelectromagnetics 10(2):173-186.

Conil, E., A. Hadjem, F. Lacroux, M. F. Wong, and J. Wiart. 2008. Variability analysis of SAR from $20 \mathrm{MHz}$ to $2.4 \mathrm{GHz}$ for different adult and child models using finitedifference time-domain. Phys Med Biol 53:1511-25.

Cooper, T. G., S. M. Mann, M. Khalid, and R. P. Blackwell. 2006. Public exposure to radio waves near GSM microcell and picocell base stations. J Radiol Prot 26(2):199. 
Cooper, J., B. Marx, J. Buhl, and V. Hombach. 2002. Determination of safety distance limits for a human near a cellular base station antenna, adopting the IEEE standard or ICNIRP guidelines. Bioelectromagnetics 23:429-43.

Cortel-Carrasco, A., A. Gati, M. Wong, and J. Wiart. 2006. WiFi exposure assessment. Abstracts of the XXVIII Annual Meeting of the Bioelectromagnetics Society BEMS' 03, Cancun, Mexico.

Curto, S., and M. J. Ammann. 2006. Electromagnetic interaction between resonant loop antenna and simulated biological tissue. Microw Opt Technol Lett 48(12):2418-21.

Curto, S., and M. J. Ammann. 2007. Electromagnetic coupling mechanism in a layered human tissue model as reference for $434 \mathrm{MHz}$ RF medical therapy applicators. Proc IEEE Antennas and Propag Soc Int Symp, IEEE, 3185-8. Honolulu, HI.

Davis, C. C., B. B. Beard, A. Tillman, J. Rzasa, E. Merideth, and Q. Balzano. 2006. International intercomparison of specific absorption rates in a flat absorbing phantom in the near-field of dipole antennas. IEEE Trans Electromagn Compat 48(3):579-88.

Dimbylow, P. 1998. FDTD calculation of the whole-body averaged SAR in an anatomically realistic voxel model of the human body from $1 \mathrm{MHz}$ to $1 \mathrm{GHz}$. Phys Med Biol 42:479-90.

Dimbylow, P. 2002. Fine resolution calculations of SAR in the human body for frequencies up to 3GHz. Phys Med Biol 47:2835-46.

Dimbylow, P. 2005a. Development of the female voxel phantom, NAOMI, and its application to calculations of induced current densities and electric fields from applied low frequency magnetic and electric fields. Phys Med Biol 50:1047-70.

Dimbylow, P. 2005b. Resonance behavior of whole-body averaged specific energy absorption rate (SAR) in the female voxel model, NAOMI. Phys Med Biol 50:4053-63.

Dimbylow, P. 2006. Development of pregnant female, hybrid voxel-mathematical models and their application to the dosimetry of applied magnetic and electric fields at $50 \mathrm{~Hz}$. Phys Med Biol 51:2383-94.

Dimbylow, P. 2007. SAR in the mother and foetus for RF plane wave irradiation. Phys Med Biol 52:3791-802.

Dimbylow, P., and W. Bolch. 2007. Whole-body-averaged SAR from $50 \mathrm{MHZ}$ to $4 \mathrm{GHz}$ in the University of Florida child voxel phantoms. Phys Med Biol 52:6639-49.

Dimbylow, P., and O. P. Gandhi. 1991. Finite-difference time-domain calculations of SAR in a realistic heterogeneous model of the head for plane-wave exposure from 600 MHz to $3 \mathrm{GHz}$. Phys Med Biol 36:1075-89.

Dimbylow, P., A. Hirata, and T. Nagaoka. 2008. Intercomparison of whole-body averaged SAR in European and Japanese voxel phantoms. Phys Med Biol 53:5883-97.

Dimbylow, P., M. Khalid, and S. Mann. 2003. Assessment of specific energy absorption rate (SAR) in the head from a TETRA handset. Phys Med Biol 48:3911-26.

Dimbylow, P., and S. M. Mann. 1994. SAR calculations in an anatomically realistic model of the head for mobile communication tranceivers at $900 \mathrm{MHz}$ and $1.8 \mathrm{GHz}$. Phys Med Biol 39:1537-53.

Dominguez, H., A. Raizer, and W. P. Carpes. 2002. Electromagnetic fields radiated by a cellular phone in close proximity to metallic walls. IEEE Trans Magn 38:793-6. 
Durney, C. H., H. Massoudi, and M. F. Iskander. 1986. Radiofrequency Radiation Dosimetry Handbook. Brooks Air Force Base, San Antonio, TX: USAF School of Aerospace Medicine.

EC (European Commission). 1999. Council recommendation (1999/519/EC) of 12 July 1999 on the limitation of exposure of the general public to electromagnetic fields (0 Hz to $300 \mathrm{GHz}$ ). Off J Eur Communities L199:59-70.

Fayos-Fernandez, J., C. Arranz-Faz, A. M. Martinez-Gonzalez, and D. SanchezHernandez. 2006. Effect of pierced metallic objects on SAR distributions at $900 \mathrm{MHz}$. Bioelectromagnetics 27:337-53.

FCC (Federal Communication Commission). 2001. Evaluating compliance with FCC guidelines for human exposure to radio frequency electromagnetic fields. Supplement C to OET Bulletin 65 (Edition 97-01), FCC, Washington, DC. 20554.

FCC (Federal Communications Commission). 2003. Equipment authorization system generic search, 445 12th Street. SW Washington, DC. 20554. http://gullfoss2.fcc .gov/prod/oet/cf/eas/reports/GenericSearch.cfm (date accessed: 1 May 2011).

Findlay, R. P., and P. Dimbylow. 2005. Effects of posture on FDTD calculations of specific absorption rate in a voxel model of the human body. Phys Med Biol 50:3825-35.

Findlay, R. P., and P. Dimbylow. 2006. FDTD calculations of specific energy absorption rate in a seated voxel model of the human body from $10 \mathrm{MHz}$ to $3 \mathrm{GHz}$. Phys Med Biol 51:2339-52.

Findlay, R. P., and P. Dimbylow. 2010. SAR in a child voxel phantom from exposure to wireless computer networks (Wi-Fi). Phys Med Biol 55:N405-11.

Forgy, E. A., J. Chen, W. C. Chew, and J. M. Jin. 1997. A comparison of the BCG-FFT and FD-TD methods for the 3-D human head absorption problem. In Proc IEEE Antennas Propag Soc Int Symp, IEEE, 1202-5. Montreal, Canada.

Foster, K. R. 2007. Radiofrequency exposure from wireless LANs utilizing WiFi technology. Health Phys 92:280-9.

Gabriel, C. 2005. Dielectric properties of biological tissue: Variation with age. Biolectromagnetics 26:S12-8.

Gabriel, C., S. Gabriel, and E. Corthout. 1996. The dielectric properties of biological tissues. Med Phys 41:2231-93.

Gabriel, C., and A. Peyman. 2006. Dielectric measurement: Error analysis and assessment of uncertainty. Phys Med Biol 51:6033-46.

Gandhi, O. P. 1980. State of the knowledge for electromagnetic absorbed dose in man and animals. Proc IEEE 68:24-32.

Gandhi, O. P., and J. Y. Chen. 1995. Electromagnetic absorption in the human head from experimental $6 \mathrm{GHz}$ handheld tranceivers. IEEE Trans Electromagn Compat 37:547-58.

Gandhi, O. P., Y. G. Gu, J. Y. Chen, and H. I. Bassen. 1992. SAR and induced current distributions in a high-resolution anatomically based model of a human for plane-wave exposures 100-915 MHz. Health Phys 63:281-90.

Gandhi, O. P., and G. Kang. 2002. Some present problems and a proposed experimental phantom for SAR compliance testing of cellular telephones at 835 and $1900 \mathrm{MHz}$. Phys Med Biol 47:1501-18. 
Gandhi, O. P., G. Lazzi, and C. M. Furse. 1996. Electromagnetic absorption in the human head and neck for mobile telephones at 835 and $1900 \mathrm{MHz}$. IEEE Trans Microw Theory Tech 44:1884-97.

Gandhi, O. P., G. Lazzi, A. Tinniswood, and Q. S. Yu. 1999. Comparison of numerical and experimental methods for determination of SAR and radiation patterns of handheld wireless telephones. Bioelectromagnetics 20:93-101.

Gjonaj, E., M. Bartsch, M. Clemens, S. Schupp, and T. Weiland. 2002. High-resolution human anatomy models for advanced electromagnetic field computations. IEEE Trans Magn 38:357-60.

Groot, R. J., A. A. Efanov, E. Krenz, and J. P. Phillips. 1997. Simulation of portable UHF antennas in the presence of certain dielectric structures using the numerical electromagnetics code. In Proc 13th Annual Review of Progress in Applied Computational Electromagn, Applied Computational Electromagnetics Society (ACES), 839-43. Monterey, CA.

Hadjem, A., D. Lautru, C. Dale, M. F. Wong, V. F. Hanna, and J. Wiart. 2005. Study of specific absorption rate (SAR) induced in two child head models and in adult heads using mobile phones. IEEE Trans Microw Theory Tech 53:4-11.

Hagmann, M. J., O. P. Gandhi, and C. H. Durney. 1979. Numerical calculation of electromagnetic energy deposition for a realistic model of man. IEEE Trans Microw Theory Tech 27:804-9.

Han, Y. N., Y. H. Lu, J. L. Zhang, and X. L. Zhu. 2006. The interaction between PIFA for Bluetooth applications and a high-fidelity human body model. In Proc 4th AsiaPacific Conf Environm Electromagn, IEEE, 331-5. Dalian, China.

Hand, J. W., Y. Li, E. L. Thomas, M. A. Rutherford, and J. V. Hajnal. 2006. Prediction of specific absorption rate in mother and fetus associated with MRI examinations during pregnancy. Magn Reson Med 55:883-93.

Harrington, R. F. 1967. Matrix methods for field problems. Proc IEEE 55:136-49.

Hartsgrove, G., A. Kraszewski, and A. Surowiec. 1987. Simulated biological materials for electromagnetic radiation absorption studies. Bioelectromagnetics 8:29-36.

Hepworth, S. J., M. J. Schoemaker, K. R. Muir, A. J. Swerdlow, M. J. van Tongeren, and P. A. McKinney. 2006. Mobile phone use and risk of glioma in adults: Case-control study. Br Med J 332:883-7.

Hirata, A., S. Matsuyama, and T. Shiozawa. 2000. Temperature rises in the human eye exposed to EM waves in the frequency range 0.6-6 GHz. IEEE Trans Microw Theory Tech 42:386-93.

Hombach, V., K. Meier, M. Burkhardt, E. Kuhn, and N. Kuster. 1996. The dependence of EM energy absorption upon human head modeling at $900 \mathrm{MHz}$. IEEE Trans Microw Theory Tech 44:1865-73.

HPA (Health Protection Agency). 2007. Press Releases. Archive for 2007. http://www.hpa .org.uk/web/HPAwebFile/HPAweb_C/1254510322363 (date accessed: 1 May 2011).

ICNIRP (International Commission on Non-Ionizing Radiation Protection). 1998. Guidelines for limiting exposure to time-varying electric, magnetic, and electromagnetic fields up to $300 \mathrm{GHz}$. Health Phys 74:494-522. 
ICNIRP (International Commission on Non-Ionizing Radiation Protection). 2009. Exposure to High Frequency Electromagnetic Fields, Biological Effects and Health Consequences (100 kHz-300 GHz). ICNIRP.

ICRP (International Commission on Radiation Protection). 1994. ICRP publication 66: Human respiratory tract model for radiological protection. ICRP 24:1-3.

IEC (International Electrotechnical Commission). 2005. IEC Standard 62209-1. Human Exposure to Radio Frequency Fields from Hand-Held and Body-Mounted Wireless Communication Devices-Human Models, Instrumentations, and Procedures. IEC.

IEC (Italian Electrotechnical Committee). 2002. IEC Standard 211-10. Guidance for Carrying Out a Base Radio Station Complying with Limits for Human Exposure to High Frequency Electromagnetic Fields. IEC.

IEEE. 2002. IEEE Standard C.95.3-2002. IEEE Recommended Practice for Measurements and Computations of Radio Frequency Electromagnetic Fields with Respect to Human Exposure to such Fields, $100 \mathrm{kHz}-300 \mathrm{GHz}$. IEEE.

IEEE. 2003. IEEE Standard 1528 SCC34. IEEE Recommended Practice for Determining the Peak Spatial-Average Specific Absorption Rate (SAR) in the Human Head from Wireless Communications Devices: Measurement Techniques. IEEE.

IEEE. 2005. IEEE Standard C.95.1-2005. IEEE Standard for Safety Levels with Respect to Human Exposure to Radio Frequency Electromagnetic Fields, $3 \mathrm{kHz}$ to $300 \mathrm{GHz}$. IEEE.

Iskra, S., R. McKenzie, and I. Cosic. 2010. Factors influencing uncertainty in measurement of electric fields close to the body in personal RF dosimetry. Radiat Prot Dosimetry 140:25-33.

Johnson, C. C., and A. W. Guy. 1972. Nonionizing electromagnetic wave effects in biological materials and systems. Proc IEEE 60:692-718.

Johnson, J. M., and Y. Rahmat-Samii. 1997. MR/FDTD: A multiple-region finitedifference-time domain method and its application to microwave analysis and modeling. Microw Opt Technol Lett 14:101-5.

Jokela, K., P. Hyysalo, and L. Puranen. 1998. Calibration of specific absorption rate (SAR) probes in waveguide at $900 \mathrm{MHz}$. IEEE Trans Instrum Meas 47:432-8.

Kainz, W., A. Christ, K. Honegger, E. Hahn, J. Shen, W. Rascher, R. Janka et al. 2007. The "Virtual Family"-Novel CAD based anatomical models of two adults and two children for dosimetry and implant evaluations. In Proc Annual Meeting of the Bioelectromagnetics Society, BEMS, 38-40. Kanazawa, Japan.

Kainz, W., D. D. Chan, J. P. Casamento, and H. I. Bassen. 2003. Calculation of induced current densities and specific absorption rates (SAR) for pregnant women exposed to hand-held metal detectors. Phys Med Biol 48:2551-60.

Kainz, W., A. Christ, T. Kellom, S. Seidman, N. Nikoloski, B. Beard, and N. Kuster. 2005. Dosimetric comparison of the specific anthropomorphic mannequin (SAM) to 14 anatomical head models using a novel definition for the mobile phone positioning. Phys Med Biol 50:3423-45.

Kan, P., S. E. Simonsen, J. L. Lyon, and J. R. W. Kestle. 2007. Cellular phone use and brain tumor: A meta-analysis. J Neurooncol 86:71-8. 
Kanda, M., Q. Balzano, P. Russo, A. Faraone, and G. Bit-Babik. 2002. Effects of earconnection modeling on the electromagnetic-energy absorption in a human-head phantom exposed to a dipole antenna field at $835 \mathrm{MHz}$. IEEE Trans Electromagn Compat 44:4-10.

Kang, G., and O. P. Gandhi. 2002. SARs for pocket-mounted mobile telephones at 835 and 1900 MHz. Phys Med Biol 47:4301-13.

Karimullah, K., K. Chen, and D. P. Nyquist. 1980. Electromagnetic coupling between a thin-wire antenna and a neighboring biological body: Theory and experiment. IEEE Trans Microw Theory Tech 28:1218-25.

Katsibas, K. D., C. A. Balanis, P. A. Tirkas, and C. R. Birtcher. 1998. Folded loop antenna for mobile hand-held units. IEEE Trans Antennas Propag 46:260-6.

Kawai, H., T. Nagaoka, S. Watanabe, K. Saito, M. Takahashi, and K. Ito. 2010. Computational dosimetry in embryos exposed to electromagnetic plane waves over the frequency range of $10 \mathrm{MHz}-1.5 \mathrm{GHz}$. Phys Med Biol 55:N1.

Keshvari, J., R. Keshvari, and S. Lang. 2006. The effect of increase in dielectric values on specific absorption rate (SAR) on eye and head tissues following 900, 1800 and 2450 $\mathrm{MHz}$ radio frequency (RF) exposure. Phys Med Biol 51:1463-77.

Khurana, V. G., L. Hardell, J. Everaert, A. Bortkiewicz, M. Carlberg, and M. Ahonen. 2010. Epidemiological evidence for a health risk from mobile phone base stations. Int $J$ Occup Environ Health 16:263-7.

Klaeboe, L., K. G. Blaasaas, and T. Tynes. 2007. Use of mobile phones in Norway and risk of intracranial tumours. Eur J Cancer Prev 16:158-64.

Kobayashi, T., T. Nojima, K. Yamada, and S. Uebayashi. 1993. Dry phantom composed of ceramics and its application to SAR estimation. IEEE Trans Microw Theory Tech 41:136-40.

Koenig, W. J., J. M. Donovan, and J. M. Pensler. 1995. Cranial bone grafting in children. Plast Reconstr Surg 95:1-4.

Koulouridis, S., M. Christopoulou, and K. S. Nikita. 2005. Comparative assessment of power absorption in heads of adults and children exposed to the radiation of cellular phones at $1800 \mathrm{MHz}$. Environmentalist 25:223-32.

Koulouridis, S., and K. S. Nikita. 2004. Study of the coupling between human heads and cellular phone helical antennas. IEEE Trans Electromagn Compat 46:62-70.

Kriticos, H. N., and H. P. Schwan. 1979. Potential temperature rise induced by electromagnetic field in brain tissues. IEEE Trans Biomed Eng 26:29-34.

Kuhn, S., E. Cabot, A. Christ, M. Capstick, and N. Kuster. 2009. Assessment of the radiofrequency electromagnetic fields induced in the human body from mobile phones used with hands-free kits. Phys Med Biol 54:5493-508.

Kuster, N. 2002. Latest progress in dosimetry and near-field evaluations for mobile communications devices. Wireless Phones Health II 1:7-19.

Kuster, N., Q. Balzano, and J. C. Lin. 1997a. Mobile Communications Safety. Chapman \& Hall, London, UK.

Kuster, N., R. Kastle, and T. Schmid. 1997b. Dosimetric evaluation of handheld mobile communications equipment with known precision. IEEE Trans Commun 80:645-52. 
Kwon, M. S., M. Huotilainen, A. Shestakova, T. Kujala, R. Näätänen, and H. Hämäläinen. 2010. No effects of mobile phone use on cortical auditory change-detection in children: An ERP study. Bioelectromagnetics 31:191-9.

Laakso, I., S. Ilvonen, and T. Uusitupa. 2007. Performance of convolutional PML absorbing boundary conditions in finite-difference time-domain SAR calculations. Phys Med Biol 52:7183-92.

Lacroux, F., E. Conil, A. C. Carrasco, A. Gati, M. F. Wong, and J. Wiart. 2008. Specific absorption rate assessment near a base-station antenna $(2,140 \mathrm{MHz})$ : Some key points. Ann Telecommun 63:55-64.

Lahkola, A., A. Auvinen, J. Raitanen, M. J. Schoemaker, H. C. Christensen, M. Feychting, C. Johansen et al. 2007. Mobile phone use and risk of glioma in 5 north European countries. Int J Cancer 120:1769-75.

Lazebnik, M., M. Okoniewski, J. H. Booske, and S. C. Hagness. 2007a. Highly accurate Debye models for normal and malignant breast tissue dielectric properties at microwave frequencies. IEEE Microw Wirel Compon Lett 17:822-4.

Lazebnik, M., D. Popovic, L. McCartney, C. B. Watkins, M. J. Lindstrom, J. Harter, S. Sewall et al. 2007b. A large-scale study of the ultrawideband microwave dielectric properties of normal breast tissue obtained from reduction surgeries. Phys Med Biol 52:2637-56.

Lazzi, G., and O. P. Gandhi. 1997. Realistically tilted and truncated anatomically based models of the human head for dosimetry of mobile telephones. IEEE Trans Electromagn Compat 39:55-61.

Lazzi, G., O. P. Gandhi, and D. Sullivan. 2000. Fast and efficient FDTD simulations of personal wireless devices for SAR compliance testing and antenna design. In Proc IEEE Antennas Propag Soc Int Symp, IEEE, 1088-91. Salt Lake City, UT.

Li, L. W., M. S. Leong, P. S. Kooi, and T. S. Yeo. 2000. Specific absorption rates in human head due to handset antennas: A comparative study using FDTD method. J Electromag Waves Appl 14:987-1000.

Lin, J. C. 1976. Electromagnetic pulse interaction with mammalian cranial structures. IEEE Trans Biomed Eng 23:61-5.

Lin, J. C. 2000. The development of human exposure standards for RF fields. J Radiats Biol Ecol Russ Acad Sci 40:425-8.

Lin, J. C. 2002. Cellular mobile telephones and children. IEEE Antennas Propag Mag 44:142-5.

Lin, J. C. 2003. Risk to children from cellular telephone radiation. IEEE Microw Mag 4:20-6.

Lin, J. C. 2007. Dosimetric comparison between different possible quantities for limiting exposure in the RF band: Rationale for the basic one and implications for guidelines. Health Phys 92:547-53.

Lin, J. C. 2009. Carcinogenic Effect of Wireless Communication Radiation in Rodents. In: Advances in Electromagnetic Fields in Living Systems, Vol. 5, 35-82. New York: Springer.

Lin, J. C., and P. Bernardi. 2007. Computational methods for predicting field intensity and temperature change. In Bioengineering and Biophysical Aspects of Electromagnetic Fields, ed. F. Barnes, and B. Greenebaum, Chap. 10, 293-380. Boca Raton, FL: CRC Press. 
Lin, J. C., and Z. W. Wang. 2005. SAR and temperature distributions in canonical head models exposed to near- and far-field electromagnetic radiation at different frequencies. Electromagn Biol Med 24:405-21.

Liu, W., C. M. Collins, and M. B. Smith. 2005. Calculations of B1 distribution, specific absorption rate, and intrinsic signal-to-noise ratio for a body-size birdcage coil loaded with different human subjects at 64 and $128 \mathrm{MHz}$. Appl Magn Reson 29:5-18.

Lonn, S., A. Ahlbom, H. C. Christensen, C. Johansen, J. Schuz, S. Edstrom, G. Henriksson et al. 2006. Mobile phone use and risk of parotid gland tumor. Am J Epidemiol 164:637-43.

Lu, Y., J. Ying, T. K. Tan, and K. Arichandran. 1996. Electromagnetic and thermal simulations of 3-D human head model under RF radiation by using the FDTD and FD approaches. IEEE Trans Magn 32:1653-6.

Mangoud, M. A., R. A. Abd-Alhameed, and P. S. Excell. 2000. Simulation of human interaction with mobile telephones using hybrid techniques over coupled domains. IEEE Trans Microw Theory Tech 48:2014-21.

Manning, M. I., and C. H. B. Gabriel. 2000. SAR tests on mobile phones used with and without personal hands-free kits, SAR Test Report for DTI 0083.

Martens, L. 2005. Electromagnetic safety of children using wireless phones: A literature review. Bioelectromagnetics 7:S133-7.

Martens, L., J. Moerloose, D. Zutter, J. Poorter, and C. Wagter. 1995. Calculation of the electromagnetic fields induced in the head of an operator of a cordless telephone. Radio Sci 30:283-90.

Martinez-Burdalo, M., A. Martin, M. Anguiano, and R. Villar. 2004. Comparison of FDTD-calculated specific absorption rate in adults and children when using a mobile phone at 900 and $1800 \mathrm{MHz}$. Phys Med Biol 49:345-54.

Martinez-Burdalo, M., A. Martin, M. Anguiano, and R. Villar. 2005. On the safety assessment of human exposure in the proximity of cellular communications base station antennas at 900, 1800 and $2170 \mathrm{MHz}$. Phys Med Biol 50:4125.

Martinez-Burdalo, M., A. Martin, A. Sanchis, and R. Villar. 2009. FDTD assessment of human exposure to electromagnetic fields from WiFi and Bluetooth devices in some operating situations. Bioelectromagnetics 30:142-51.

Mason, P. A., W. D. Hurt, T. J. Walters, J. A. D’Andrea, P. Gajsek, K. L. Ryan, D. A. Nelson, K. I. Smith, and J. M. Ziriax. 2000. Effects of frequency, permittivity, and voxel size on predicted specific absorption rate values in biological tissue during electromagnetic field exposure. IEEE Trans Microw Theory Tech 48:2050-8.

Massoudi, H., C. H. Durney, P. W. Barber, and M. F. Iskander. 1979. Electromagnetic absorption in multilayered cylindrical models of man. IEEE Trans Microw Theory Tech 27:825-9.

Mazzurana, M., L. Sandrini, A. Vaccari, C. Malacarne, L. Cristoforetti, and R. Pontalti. 2004. Development of numerical phantoms by MRI for RF electromagnetic dosimetry: A female model. Radiat Prot Dosimetry 111:445-51.

Meier, K., M. Burkhardt, T. Schmid, and N. Kuster. 1996. Broadband calibration of E-field probes in lossy media. IEEE Trans Microw Theory Tech 44:1954-62. 
Meier, K., V. Hombach, R. Kaestle, R. Yew-Siow, and N. Kuster. 1997. The dependence of electromagnetic energy absorption upon human-head modeling at $1800 \mathrm{MHz}$. IEEE Trans Microw Theory Tech 45:2058-62.

Mild, K. H., and J. Wilen. 2009. Occupational exposure in wireless communication. In Advances in Electromagnetic Fields in Living Systems, Health Effects of Cell Phone Radiation, ed. J. C. Lin, Vol. 5, 199-219. New York: Springer.

Mochizuki, S., S. Watanabe, M. Taki, Y. Yamanaka, and H. Shirai. 2004. A new iterative MoM/FDTD formulation for simulating human exposure to electromagnetic waves. IEICE Trans Electron E87-C:1540-7.

Monebhurrun, V. 2010. Conservativeness of the SAM phantom for the SAR evaluation in the child's head. IEEE Trans Magn 46:3477-80.

Mur, G. 1981. Absorbing boundary conditions for the finite-difference approximation of the time-domain electromagnetic field equations. IEEE Trans Electromagn Compat 23:377-82.

Nagaoka, T., T. Togashi, K. Saito, M. Takahashi, K. Ito, T. Ueda, H. Osada, H. Ito, and S. Watanabe. 2006. An anatomically realistic voxel model of the pregnant woman and numerical dosimetry for a whole-body exposure to RF electromagnetic fields. In Proc IEEE 28th Ann Conf Eng Med Biol Soc, IEEE, 5463-7. New York.

Nagaoka, T., T. Togashi, K. Saito, M. Takahashi, K. Ito, and S. Watanabe. 2007. An anatomically realistic whole-body pregnant-woman model and specific absorption rates for pregnant-woman exposure to electromagnetic plane waves from $10 \mathrm{MHz}$ to $2 \mathrm{GHz}$. Phys Med Biol 52:6731-45.

Nagaoka, T., S. Watanabe, K. Sakurai, E. Kunieda, M. Taki, and Y. Yamanaka. 2004. Development of realistic high-resolution whole-body voxel models of Japanese adult males and females of average height and weight, and application of models to radio-frequency electromagnetic field dosimetry. Phys Med Biol 49:1-15.

Neitzke, H. P., J. Osterhoff, K. Peklo, and H. Voigt. 2007. Determination of exposure due to mobile phone base stations in an epidemiological study. Radiat Protein Dosim 124:35-9.

Neubauer, G., M. Feychting, Y. Hamnerius, L. Kheifets, N. Kuster, I. Ruiz, J. Schuz, R. Uberbacher, J. Wiart, and M. Roosli. 2007. Feasibility of future epidemiological studies on possible health effects of mobile phone base stations. Bioelectromagnetics 28:224-30.

Nicolas, E., D. Lautru, F. Jacquin, M. F. Wong, and J. Wiart. 2001. Specific absorption rate assessments based on a selective isotropic measuring system for electromagnetic fields. IEEE Trans Instrum Meas 50:397-401.

Nikawa, Y., M. Chino, and K. Kikuchi. 1996. Soft and dry phantom modeling material using silicone rubber with carbon fiber. IEEE Trans Microw Theory Tech 44:1949-53.

Nikita, K. S., M. Cavagnaro, P. Bernardi, N. K. Uzunoglu, S. Pisa, E. Piuzzi, J. N. Sahalos et al. 2000a. A study of uncertainties in modeling antenna performance and power absorption in the head of a cellular phone user. IEEE Trans Microw Theory Tech 48:2676-85.

Nikita, K. S., G. S. Stamatakos, N. K. Uzunoglu, and A. Karafotias. 2000b. Analysis of the interaction between a layered spherical human head model and a finite length dipole. IEEE Trans Microw Theory Tech 48:2003-13. 
O'Rourke, A. P. O., M. Lazebnik, J. M. Bertram, M. C. Converse, S. C. Hagness, J. G. Webster, and D. M. Mahvi. 2007. Dielectric properties of human normal, malignant and cirrhotic liver tissue: In vivo and ex vivo measurements from 0.5 to $20 \mathrm{GHz}$ using a precision open-ended coaxial probe. Phys Med Biol 52:4707-19.

Okano, Y., K. Ito, I. Ida, and M. Takahashi. 2000. The SAR evaluation method by a combination of thermographic experiments and biological tissue-equivalent phantoms. IEEE Trans Microw Theory Tech 48:2094-103.

Okoniewski, M., and M. A. Stuchly. 1996. A study of the handset antenna and human body interaction. IEEE Trans Microw Theory Tech 44:1855-64.

Olsen, R. G., and T. A. Griner. 1989. Outdoor measurements of SAR in a full-size human model exposed to $29.2 \mathrm{MHz}$ near-field irradiation. Bioelectromagnetics 10:162-71.

Pan, S., A. Bahrwas, and I. Wolff. 1997. N antennas and their applications in portable handsets. IEEE Trans Antennas Propag 45:1475-83.

Peyman, A., C. Gabriel, E. H. Grant, G. Vermeeren, and L. Martens. 2009. Variation of the dielectric properties of tissues with age: The effect on the values of SAR in children when exposed to walkie-talkie devices. Phys Med Biol 54:227-41.

Penman, A., S. J. Holden, S. Watts, R. Perrott, and C. Gabriel. 2007. Dielectric properties of porcine cerebrospinal tissues at microwave frequencies: In vivo, in vitro and systematic variation with age. Phys Med Biol 52:2229-45.

Penman, A., A. A. Rezazadeh, and C. Gabriel. 2001. Changes in the dielectric properties of rat tissue as a function of age at microwave frequencies. Phys Med Biol 46:1617-29.

Pisa, S., M. Cavagnaro, V. Lopresto, E. Piuzzi, G. A. Lovisolo, and P. Bernardi. 2005. A procedure to develop realistic numerical models of cellular phones for an accurate evaluation of SAR distribution in the human head. IEEE Trans Microw Theory Tech 53:1256-65.

Rakotomalala, A. 2007. A FEM model of human head to show the risk of brain tumors when using cell phone over a long period of time. In Proc 8th Int Confon Mathematics and Computers in Biology and Chemistry, World Scientific and Engineering Academy and Society (WSEAS) 66-8. Vancouver, Canada.

Rowley, J. T., and R. B. Waterhouse. 1999. Performance of shorted microstrip patch antennas for mobile communications handsets at $1800 \mathrm{MHz}$. IEEE Trans Antennas Propag 47:815-22.

Rowley, J. T., R. B. Waterhouse, and K. H. Joyner. 2002. Modeling of normal-mode helical antennas at $900 \mathrm{MHz}$ and $1.8 \mathrm{GHz}$ for mobile communication handsets using the FDTD technique. IEEE Trans Antennas Propag 50:812-20.

Santini, R., P. Santini, P. Le Ruz, J. M. Danze, and M. Seigne. 2003. Survey of people living in the vicinity of cellular phone base stations. Electromagn Biol Med 22:41-9.

Schiavoni, A., P. Bertotto, G. Richiardi, and P. Bielli. 2000. SAR generated by commercial cellular phones-phone modeling, head modeling, and measurements. IEEE Trans Microw Theory Tech 48:2064-71.

Schmid, T., O. Egger, and N. Kuster. 1996. Automated E-field scanning systems for dosimetric assessments. IEEE Trans Microw Theory Tech 44:105-13. 
Schmid, G., and R. Uberbacher. 2005. Age dependence of dielectric properties of bovine brain and ocular tissues in the frequency range of $400 \mathrm{MHz}$ to $18 \mathrm{GHz}$. Phys Med Biol 50:4711-20.

Schoemaker, M. J., A. J. Swerdlow, A. Ahlbom, A. Auvinen, K. G. Blaasaas, and E. Cardis. 2005. Mobile phone use and risk of acoustic neuroma: Results of the interphone case-control study in five north European countries. Br J Cancer 93:842-8.

Schoenborn, F., M. Burkhardt, and N. Kuster. 1998. Differences in energy absorption between heads of adults and children in the near field of sources. Health Phys 74:160-8.

Schuz, J., E. Bohler, G. Berg, B. Schlehofer, I. Hettinger, K. Schlaefer, J. Wahrendorf, K. Kunna-Grass, and M. Blettner. 2006. Cellular phones, cordless phones, and the risks of glioma and meningioma (Interphone Study Group, Germany). Am J Epidemiol 163:512-20.

Schuz, J., and S. Mann. 2000. A discussion of potential exposure metrics for use in epidemiological studies on human exposure to radiowaves from mobile phone base stations. J Expo Anal Environ Epidemiol 10:600-5.

Seidenari, S., G. Giusti, L. Bertoni, C. Magnoni, and G. Pellacani. 2000. Thickness and echogenicity of the skin in children as assessed by $20 \mathrm{MHz}$ ultrasound. Dermatology 201:218-22.

Silvester, P., and R. Ferrari. 1996. Finite Elements for Electrical Engineers. New York: Cambridge University Press.

Stuchly, M. A., and S. S. Stuchly. 1984. Permittivity of mammalian tissues in vivo and in vitro. Advances in experimental techniques and recent results. Int J Electron 56:443-56.

Stuchly, M. A., and S. S. Stuchly. 1995. Experimental radio and microwave dosimetry. In Handbook of Biological Effects of Electromagnetic Fields, ed. C. Polk and E. Postow, 295-336. Boca Raton, FL: CRC Press.

Stuchly, S. S., A. Kraszewski, M. Stuchly, G. Hartsgrove, and R. J. Spiegel. 1987. RF energy deposition in a heterogeneous model of man. IEEE Trans Biomed Eng 34:951-7.

Su, C. M., C. H. Wu, K. L. Wong, S. H. Yeh, and C. L. Tang. 2006. User's hand effects on EMC internal GSM/DCS mobile phone antenna. In Proc IEEE Antennas Propag Soc Int Symp, IEEE, 2097-100. Albuquerque, NM.

Sullivan, D. 1990. Three-dimensional computer simulation in deep regional hyperthermia using the finite-difference time-domain method. IEEE Trans Microw Theory Tech 38:204-11.

Sullivan, D. M., D. T. Borup, and O. P. Gandhi. 1987. Use of the finite-difference timedomain in calculating EM absorption in human tissues. IEEE Trans Biomed Eng 34:148-57.

Surowiec, A. J., S. S. Stuchly, M. Keaney, and A. Swarup. 1986. In vivo and in vitro dielectric properties of feline tissues at low radiofrequencies. Phys Med Biol 31:901-9.

Taflove, A., and S. C. Hagness. 2000. Computational Electrodynamics: The Finite-Difference Time-Domain Method. Boston, MA: Artech House.

Takebayashi, T., S. Akiba, Y. Kikuchi, M. Taki, K. Wake, S. Watanabe, and N. Yamaguchi. 2006. Mobile phone use and acoustic neuroma risk in Japan. Occup Environ Med 63:802-7. 
Toftgard, J., S. N. Hornsleth, and J. B. Andersen. 1993. Effects on portable antennas of the presence of a person. IEEE Trans Antennas Propag 41:739-46.

Togashi, T., T. Nagaoka, S. Kikuchi, K. Saito, S. Watanabe, M. Takahashi, and K. Ito. 2008. FDTD calculations of specific absorption rate in fetus caused by electromagnetic waves from mobile radio terminal using pregnant woman model. IEEE Trans Microw Theory Tech 56:554-9.

Troulis, S. E., W. G. Scanlon, and N. E. Evans. 2003. Effect of a hands-free wire on specific absorption rate for a waist-mounted $1.8 \mathrm{GHz}$ cellular telephone handset. Phys Med Biol 48:1675-84.

Uusitupa, T., I. Laakso, S. Ilvonen, and K. Nikoskinen. 2010. SAR variation study from 300 to $5000 \mathrm{MHz}$ for 15 voxel models including different postures. Phys Med Biol 55:1157.

Virtanen, H., J. Keshvari, and R. Lappalainen. 2006. Review interaction of radio frequency electromagnetic fields and passive metallic implants: A brief review. Bioelectromagnetics 27:431-9.

Virtanen, H., J. Keshvari, and R. Lappalainen. 2007. The effect of authentic metallic implants on the SAR distribution of the head exposed to 900,1800 and $2450 \mathrm{MHz}$ dipole near field. Phys Med Biol 52:1221-36.

Vulevic, B., and P. Osmokrovic. 2010. Evaluation of uncertainty in the measurement of environmental electromagnetic fields. Radiat Protein Dosim 141:173-7.

Wang, J., and O. Fujiwara. 1999. FDTD computation of temperature rise in the human head for portable telephones. IEEE Trans Microw Theory Tech 47:1528-34.

Wang, J., and O. Fujiwara. 2003. Comparison and evaluation of electromagnetic absorption characteristics in realistic children for $900 \mathrm{MHz}$ mobile telephones. IEEE Trans Microw Theory Tech 51:966-71.

Wang, J., and O. Fujiwara. 2005. EM interaction between a $5 \mathrm{GHz}$ band antenna mounted PC and a realistic human body model. IEICE Trans Commun E88-B:2604-8.

Wang, J., O. Fujiwara, S. Kodera, and S. Watanabe. 2006. FDTD calculation of whole-body average SAR in adult and child models for frequencies from $30 \mathrm{MHz}$ to $3 \mathrm{GHz}$. Phys Med Biol 51:4119-27.

Wang, J., O. Fujiwara, and S. Watanabe. 2006. Approximation of aging effect on dielectric tissue properties for SAR assessment of mobile telephones. IEEE Trans Electromagn Compat 48:408-13.

Wang, J., O. Fujiwara, S. Watanabe, and Y. Yamanaka. 2004. Computation with a parallel FDTD system of human-body effect on electromagnetic absorption for portable telephones. IEEE Trans Microw Theory Tech 52:53-8.

Watanabe, S., M. Taki, T. Nojima, and O. Fujiwara. 1996. Characteristics of the SAR distributions in a head exposed to electromagnetic fields radiated by a hand-held portable radio. IEEE Trans Microw Theory Tech 44:1874-83.

Weiland, T. 1977. A discretization method for the solution of Maxwell's equations for six-component fields. Electron Commun 31:116-20.

Weiland, T. 1990. Maxwell's grid equations. Frequenz 44:9-16.

Wells, W. M., W. E. L. Grimson, R. Kikinis, and F. A. Jolesz. 1996. Adaptive segmentation of MRI data. IEEE Trans Med Imaging 15:429-42. 
WHO (World Health Organization). 2006. Electromagnetic Fields and Public Health. WHO fact sheet 304. http://www.who.int/mediacentre/factsheets/fs304/en/index. html (date accessed: 1 May 2011).

Wiart, J., A. Hadjem, N. Gadi, I. Bloch, M. F. Wong, A. Pradier, D. Lautru, V. F. Hanna, and C. Dale. 2005. Modeling of RF head exposure in children. Bioelectromagnetics 26:S19-30.

Wiart, J., A. Hadjem, M. Wong, and I. Bloch. 2008. Analysis of RF exposure in the head tissues of children and adults. Phys Med Biol 53:3681-95.

Wong, K. L., M. R. Hsu, W. Y. Li, S. W. Su, and A. Chen. 2007. Study of the Bluetooth headset antenna with the user's head. Microw Opt Technol Lett 49:19-23.

Wu, T., A. Hadjem, M. F. Wong, A. Gati, O. Picon, and J. Wiart. 2010. Whole-body newborn and young rats' exposure assessment in a reverberating chamber operating at 2.4 GHz. Phys Med Biol 55:1619.

Wu, D., S. Shamsi, J. Chen, and W. Kainz. 2006. Evaluations of specific absorption rate and temperature increase within pregnant female models in magnetic resonance imaging birdcage coils. IEEE Trans Microw Theory Tech 54:4472-8.

Yee, K. S. 1966. Numerical solution of initial boundary value problems involving Maxwell's equations in isotropic media. IEEE Trans Antennas Propag 14:585-9.

Yildirim, B. S., and E. A. El-Sharawy. 1996. Analysis of a magnetically-shielded cellular phone antenna using finite difference time domain method. In Proc IEEE MTT-S Int Microw Symp Dig, IEEE, 979-82. San Francisco, CA.

Zankl, M., and A. Wittman. 2001. The adult male voxel model "Golem" segmented from whole-body CT patient data. Radiat Environ Biophys 40:153-62.

Zhou, H., and A. van Oosterom. 1992. Computation of the potential distribution in a four-layer anisotropic concentric spherical volume conductor. IEEE Trans Biomed Eng 39:154-8. 



\section{6}

\section{Medical Devices and Systems Exposure and Dosimetry}

6.1 Introduction 331

6.2 Medical Therapy

Thermotherapy $•$ Magnetic

Stimulation - Magnetic

Therapy - Magnetic Targeting - Magnetic

Navigation - Electronic Implants

6.3 Medical Diagnosis

Magnetic Resonance Imaging • Transcranial

Magnetic Stimulation - Magnetic

Tracking - Magnetic Navigation - Telemedicine

6.4 Electromagnetic Compatibility

Sources • Risk Management

6.5 Summary.

References.

\subsection{Introduction}

Electromagnetic fields (EMFs) have gained increasing importance in the field of health care. The major applications are based on direct interaction of EMF either to deliver energy for treatment such as stimulating nerve and muscle cells by induced electric current densities or by converting field energy into heat, or to acquire diagnostic information such as on distribution and binding status of nuclei by magnetic resonance imaging (MRI) or triggering functional responses such as by transcranial magnetic stimulation (TMS).

However, in the meanwhile, emerging new applications for monitoring, targeting, tracking, and navigating are widening the exposure scenarios by introducing EMF sources into the body such as electronic implants and capsular endoscopes with incorporated data transmitting capability or injecting magnetic sources such as magnetic nanoparticles into tissues. Such intracorporal EMF sources expose tissues directly with no benefit of separation distance and consequent field amplitude decrease. 
Magnetic fields and EMFs are widely used in medicine (Figure 6.1), whereas the applications of electric fields play a minor role. They are mainly restricted to capacitive applicators such as in diathermy or in very strong electric fields causing spark discharges to the body surface generated by fulgurators or radio-frequency (RF) surgery devices to destroy or cut tissues and/or treat cancer.

Other applications with electrodes fed and directly inserted into the target to generate high-amplitude intracorporal electric field strengths such as for electroporation of cell membranes to enable transferring drugs or nuclear acids into cells are not discussed here. Similarly, electric stimulation of nerve and muscle cells by surface electrodes for diagnostic and/or therapeutic application or functional stimulation is excluded. RF surgical devices are only discussed with regard to their stray fields but not to their intended use for cutting, coagulating, or ablating tissue.

According to the legal definition, a medical device is any instrument, apparatus, appliance, software, material and other article, active or nonactive, intended by its manufacturer to be used specifically for human medical purposes such as diagnosis, therapy or rehabilitation, and birth control. Medical systems are combinations of devices, medical with other medical or nonmedical devices that are required to achieve the intended medical purpose (EC 1993, 2007).

The definition of a medical device includes also material such as magnet strips for magnet therapy that expose the patient to static magnetic fields or magnetic (nano) particles intended for medical use.

It is evident that electromedical devices may expose patients to EMFs intentionally such as diathermy or MRI. However, electromedical devices may also expose patients

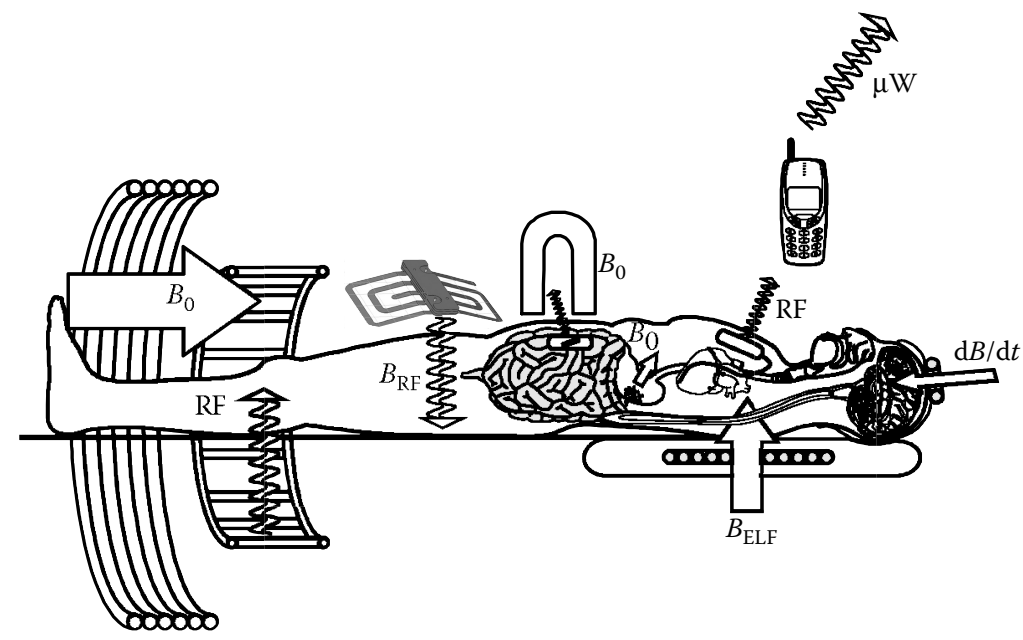

FIGURE 6.1 Electromagnetic field (EMF) application in medicine with static magnetic fields $B_{0}$, extremely low-frequency (ELF) magnetic fields $B_{\mathrm{ELF}}$, magnetic transients $\mathrm{d} B / \mathrm{d} t$, radio-frequency electromagnetic fields (RF), and microwaves $(\mu \mathrm{W})$ for magnetic resonance imaging, diathermy, magnetic navigation and device tracking, capsular endoscope, magnetic therapy, transcranial magnetic stimulation, tracking nanoparticles, and remote data transmission. 
and/or staff unintentionally to RF stray fields from surgical devices, to RF EMF from telemetric data transmission from electromedical implants, or to magnetic fields from incorporated devices, active or nonactive, because of their generation by electric current flow or by the physical nature of ferromagnetic or superparamagnetic material (e.g., magnetic nanoparticles).

Electromagnetic coupling comprises a wide range of frequencies, from static magnetic fields (e.g., MRI, permanent magnets for navigation and tracking) to extremely low-frequency (ELF) electric and magnetic fields (e.g., magnet therapy), and to RF EMFs (e.g., diathermy, hyperthermia, and MRI) and microwave radiation (e.g., diathermy and telemedicine). In addition, it includes fast magnetic field transients (e.g., TMS and MRI).

For clarity, it should be kept in mind that from a physical point of view the umbrella term electromagnetic could be slightly misleading. In the wide frequency range extending from static fields until microwaves, the physical nature of these fields as well as the governing mechanisms of interaction and propagation changes. In the static case and in the ELF range, electric and magnetic fields are decoupled and need to be considered separately. Only at higher frequencies in the RF range, electric and magnetic fields are tied together (coupled) and justify the term electromagnetic. Only in close vicinity to the antenna (in the reactive near field), depending on the kind of antenna (rod or loop antenna), either electric or magnetic fields may dominate and the counterpart neglected. For small antenna dimensions, this applies up to a distance of about half the wavelength of the EMF. Up to frequencies of $500 \mathrm{MHz}$, this distance is larger than the body diameter of $30 \mathrm{~cm}$.

\subsection{Medical Therapy}

In medical therapy, electric fields, magnetic fields, and EMFs are applied to stimulate nerve and/or muscle cells, induce electric fields to stimulate healing, or deliver heat as a primary goal or as a vehicle for inducing a secondary effect such as drug delivery or activation.

\subsubsection{Thermotherapy}

Excess heating of the target region can be subdivided into the following (Habash et al. 2006):

- Long-term low-temperature diathermia (e.g., elevating temperatures to $40^{\circ} \mathrm{C}-41^{\circ} \mathrm{C}$ for 6-72 hours)

- Moderate-temperature hyperthermia (e.g., elevating temperatures to $42^{\circ} \mathrm{C}-45^{\circ} \mathrm{C}$ for 15-60 minutes)

- High-temperature hyperthermia or thermal ablation (e.g., elevating temperatures up to $50^{\circ} \mathrm{C}$ for $4-6$ minutes)

Therefore, these applications differ with regard to amplitude and duration of EMF exposure. The biological interactions may occur at the molecular, cellular, organ, and/or system level. EMF delivers heat by converting EMF energy into kinetic energy of moving particles. This is achieved by accelerating intracorporal particles by electric or magnetic 
forces acting at charged particles or magnetic moments, respectively, causing translational, oscillating, and/or rotational movements. In a macroscopic point of view, heat is defined by the frequency of collisions of particles and hence their statistical movement (Brownian motion).

\subsubsection{Hyperthermia}

Hyperthermia aims at increasing the temperature of target tissue sufficiently long above a critical temperature to damage cancer cells. To protect healthy tissue, sophisticated treatment planning is required, usually based on numerical modeling. Energy delivery by EMG can be performed either by direct absorption by the tissue or by targeted absorption by introducing ferromagnetic or superparamagnetic parts and particles into the target tissue.

\subsection{Direct Electromagnetic Field Heating}

As the result of their specific biological properties (e.g., reduced heat dissipating ability, lower interstitial $\mathrm{pH}$, and increased proliferation rate), tumor cells are more susceptible to heat than normal cells. However, individual thermal susceptibility of different tumor cell types can lead to different responses, from complete regression to growth delay. However, individual reactions might also include heat-induced growth stimulation. Repeated thermal exposure with sublethal doses may result in a transient increase of cellular resistance to heat (Seegenschmiedt, Fessenden, and Vernon 1996). The response to thermal dose is fairly linear. Therefore, the thermal dose $D$ can be described by temperature increase $\Delta T$ multiplied by exposure time $t$ (Sapareto and Dewley 1984):

$$
D=\Delta T t
$$

The heat effect depends on temperature increase $\Delta T$ multiplied with its duration $t$, for example, heating to $44^{\circ} \mathrm{C}$ for 180 minutes is equivalent to $45^{\circ} \mathrm{C}$ for 90 minutes (Dewey et al. 1982; Sapareto and Dewey 1984). The tissue temperature increase $\mathrm{d} T / \mathrm{d} t$ is dependent on energy delivery and dissipation by conductive and convective heat transport. With the mass density $\rho$, the specific thermal capacity of tissue $C_{\mathrm{wT}}$ and blood $C_{\mathrm{wB}}$, the thermal conductivity $k_{\mathrm{T}}$ of tissue, the volumetric perfusion rate $w_{\mathrm{b}}$, the metabolic rate $Q_{\text {met }}$, and heating power $P$, this can be described by the following equation (Pennes 1948; Seegenschmiedt, Fessenden, and Vernon 1996):

$$
\frac{\mathrm{d} T}{\mathrm{~d} t}=\frac{1}{\rho C_{\mathrm{wT}}}\left[\Delta\left(k_{\mathrm{T}}-\Delta T\right)-w_{\mathrm{b}} C_{\mathrm{wB}}\left(T-T_{\mathrm{c}}\right)+P+Q_{\mathrm{met}}\right]
$$

The localized heating to critical temperatures is one important modality of the synergistic trimodal cancer therapy (heat, radiation, and chemicals). The initial phase of hyperthermia is characterized by direct heat-induced cellular injury. In the second phase, indirect tissue damage is characterized by a progression of tissue injury even after cessation of initial heating. This progressive injury may involve several factors, including apoptosis, microvascular damage, ischemia-reperfusion injury, Kupffer cell activation, altered cytokine expression, and alterations in the immune response (Nikfarjam, 
Muralidharan, and Christophi 2005). Heat delivery can be supported by modulating blood flow, administrating thermo-sensitizing agents, and introducing ferromagnetic particles or elements into the target tissue.

Heating is performed with hyperthermia devices with different antenna using frequencies dedicated for industrial, scientific, and medical use (ISM frequencies), in particular 13.5 MHZ, 21.7 MHZ, 433 MHz, and 2.45 GHz and output power up to $500 \mathrm{~W}$ for short-wave devices (IEC 60601-2-3) and $250 \mathrm{~W}$ for microwave devices (IEC 60601-2-6). Different applicators are used for superficial heating (e.g., magnetic loop, capacitor, linear or two-dimensional antenna arrays, and helical antenna) and deep heating (e.g., inductive, capacitive applicators, biconal dipole antenna, transverse electromagnetic (TEM) applicator, and annular ring or linear phased array).

Focusing EMF to deep target regions is achieved by real-time shaping and by selecting power deposition patterns of configurations with multiple antennas based on the result of treatment planning systems and numerical simulation with individual patient data. To avoid excess superficial heating and/or to modulate the intracorporal treatment pattern, a fluid-filled bolus is inserted between the body surface and the applicator.

Quantitative exposure data are sparse. However, new methods for noninvasive in vivo temperature measurement (e.g., based on MRI) are expected to fill the gap. Specific absorption rate (SAR) values within the target region can be estimated with the thermal capacity $c_{\mathrm{W}}$ and the differential temperature rise $\delta T / \delta t$ from the following:

$$
\mathrm{SAR}=c_{\mathrm{W}} \delta T / \delta t
$$

A survey of SAR values derived from temperature-time curves of in vivo measurements resulted in $10 \mathrm{~W} / \mathrm{kg}$ at $100 \mathrm{~W}$ output power (Wust et al. 1995; Seegenschmiedt, Fessenden, and Vernon 1996). Exposure lasts for several minutes. With the specific electric conductivity $\sigma$ and the mass density $\rho$, the intracorporal electric field strength $E_{\mathrm{i}}$ can be derived with the simplified assumption of plane waves to $E_{\mathrm{i}}=135 \mathrm{~V} / \mathrm{m}$ (with the wave propagation impedance $Z=377 \Omega$, which equals $B=0.45 \mu \mathrm{T}$ ) from the following:

$$
\operatorname{SAR}=\left(\sigma E_{\mathrm{i}}^{2}\right) / \rho=\left(\sigma B^{2} Z^{2}\right) /\left(\mu^{2} \rho\right)
$$

\subsection{Targeted Hyperthermia}

Efficiency and targeting of EMF heating can be increased by introducing ferromagnetic parts or superparamagnetic particles into the target region. Nanoparticles of ferromagnetic or ferrimagnetic material may exhibit a single magnetic domain and act like a large magnetic moment, which is composed of all individual magnetic moments and exhibits superparamagnetism. This happens if their dimension is small enough, about $3-50 \mathrm{~nm}$ depending on the material. Usually targeted hyperthermia is applied for several minutes with $100-500 \mathrm{kHz}$ and fields of $10-100 \mathrm{mT}$.

Because of their magnetic anisotropy, nanoparticles usually are oriented in only two stable positions, namely parallel or antiparallel to each other. The most widely used magnetic nanoparticles for hyperthermia consist of iron oxide. Such superparamagnetic iron oxide nanoparticles are termed SPIONs. Other metallic nanoparticles of Co, Fe, 
or Fe-Co exhibit higher magnetization compared to iron oxide and allow even better heating. However, with respect to metallic nanoparticles, the main issue concerns their biocompatibility and hence protection against oxidation and their potential toxicity. To increase their biocompatibility, particles may be coated by inorganic material (silica and gold). As an example, to generate a biocompatible coating, $\mathrm{CoFe}_{2} \mathrm{O}_{4}$ nanoparticles may be layered with polygalacturonic acid.

When an external magnetic field is applied to an assembly of superparamagnetic nanoparticles, their magnetic moments tend to align along and oscillate with the applied field. Therefore, in addition to heating by induced eddy currents, the magnetic particles are also heated due to the alternating change of magnetism that follows a hysteresis curve. The area $A$ of this hysteresis loop (expressed in $\mathrm{J} / \mathrm{g}$ ) is dissipated in the environment under the form of thermal energy. For Fe-Co nanoparticles, $A$ amounts to $1.5 \mathrm{~mJ} / \mathrm{g}$; for Co nanoparticles, it increases to $3.25 \mathrm{~mJ} / \mathrm{g}$. It reaches $5.6 \mathrm{~mJ} / \mathrm{g}$ for $\mathrm{Fe}$ nanoparticles.

In a simplified approach, the SAR for this magnetic heating mechanism can be estimated by multiplying the hysteresis loop area $A$ (in Ws/kg) by the frequency $f$ of the alternating magnetic field:

$$
\mathrm{SAR}=f \cdot A
$$

This formula is simplified. It neglects the influence of the magnetocrystalline anisotropy; the concentration of nanoparticles; and their size, the temperature, and the magnetic field strength $H$. The SAR of nanoparticles depends on the square of the magnetic field strength, $H^{2}$. As the size of particles increases (e.g., to micrometer), they exhibit multiple magnetic domains, and heat production becomes proportional to $H^{3}$ (Ramachandran and Mazuruk 2004).

Targeted EMF heating was first applied in treating glioblastoma. This type of tumor is characterized by the fact that recurring metastases are not growing anywhere but only at the boundary of the initial surgical cavity inside the brain. Therefore, the growth of metastases can be inhibited by recurrent EMF heating with a head coil. To restrict heating to this area only, after tumor resection, the surgical cavity was coated with metallic particles (Ni particles) in the millimeter range (Heppner 1982).

For applications not restricted to a special kind of tumor, ferromagnetic needles consisting of sintered $\mathrm{MgFeO}_{4}$ may be introduced into the tumor (e.g., breast and kidney) and heated by EMF (e.g., $540 \mathrm{kHz}, 5 \mathrm{mT}$ ) with $2 \mathrm{~kW}$ generator output power. After 30-minute heating, local tissue temperatures reached $61^{\circ} \mathrm{C}$. Nuclei of the tumor cells became hyperchromatin immediately after heating. Subsequently, the injured area increased progressively until 3 days after heating and tumors disappeared without complications (Watanabe et al. 2009; Yukumi et al. 2009).

Another approach is injecting ferromagnetic or superparamagnetic nanoparticles into the tumor and subsequent targeted heating by RF EMF. Due to their smaller size, considerably higher fields are required for heating nanoparticles (e.g., $153 \mathrm{kHz}$, $129 \mathrm{mT}$ ) (Ramachandran and Mazuruk 2004; Johannsen et al. 2010). The advantage is improved localization and increased safety because of inherently limited heating due to the low Curie temperature of $62^{\circ} \mathrm{C}$ (Kaman et al. 2009). The Curie temperature is 
the critical temperature beyond which the previously ferromagnetic material becomes paramagnetic.

\subsubsection{Diathermy}

In diathermy tissue, temperature is moderately elevated to $40^{\circ} \mathrm{C}-41^{\circ} \mathrm{C}$ to protect tissue from unintended damage. Using EMF is the first choice for delivering heat to tissues and joints to treat inflammations, improve blood circulation, and relieve pain. Tissues are heated by absorbing RF EMF at frequencies $27.12 \mathrm{MHz}, 433 \mathrm{MHz}$, and $2.45 \mathrm{GHz}$. However, short-wave diathermy (SWD) and hence the frequency $27.12 \mathrm{MHz}$ is most widely used. According to the safety standard of medical SWD devices IEC 60601-2-3 (IEC 1999), the output power of these devices is limited to $500 \mathrm{~W}$.

In contrast to hyperthermia, the application of diathermy is not based on treatment planning. Treatment is performed just by placing capacitive or inductive applicators over the target region and selecting the output power by a thumb rule. Based on the patient's feedback, the output setting may be adjusted to avoid discomfort and pain. Therefore, intracorporal regions outside the patient's target zone might be considerably overexposed (Figure 6.2).

Investigations of intracorporal SAR distributions induced by SWD showed that therapeutic heating efficiency and target heat load vary considerably. Capacitive and inductive applicators exhibit quite different heating patterns and efficiency.

\subsection{Unintended Side Effects}

6.2.1.2.1.1 Patient Within the patient, at maximum power setting, basic restrictions for the general population can be exceeded below applicators up to a depth of about $10 \mathrm{~cm}$. Although heating is intended in the treatment zone, it might lead to unintentional side effects if the vulnerable regions outside the treatment area are affected. Unintentional exposures of vulnerable regions well outside the target area may affect eyes, lenses, central nervous system (CNS), and gonads. Investigations showed that

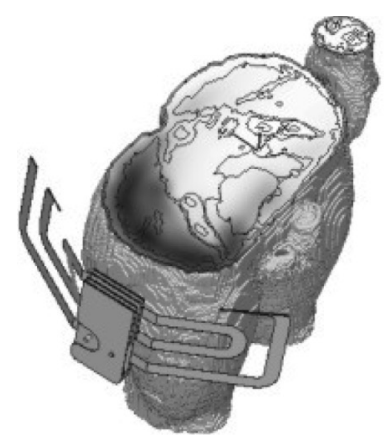

(a)

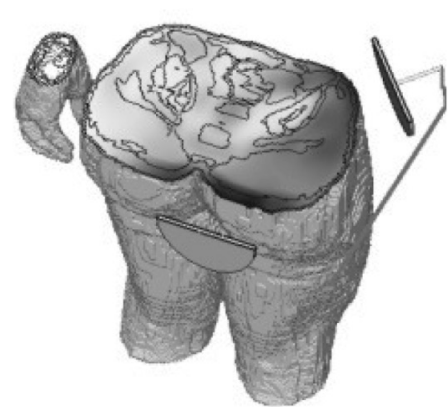

(b)

FIGURE 6.2 Diathermic heating of the hip with (a) inductive and (b) capacitive applicator. (From Leitgeb, N., A. Omerspahic, and F. Niedermayr. 2010. Exposure of non-target tissue in medical diathermy. Bioelectromagnetics 31:12-9. With permission.) 
exposure could reach up to $26 \mathrm{~W} / \mathrm{kg}$ at gonads and up to $7 \mathrm{~W} / \mathrm{kg}$ at the fetus. Such high values might lead to unacceptable side effects, in particular because this could be avoided by proper choice of the type of applicator or proper adjustment of output power (Leitgeb, Omerspahic, and Niedermayr 2010).

These results reinforce warnings that sensitive regions may be subjected to unintended overexposures such as the eye lens (because of the impaired heat exchange and hence the risk of cataracts) and reproductive organs. If such overexposures were encountered, they were associated with inductive applicators rather than capacitive ones.

Numerical investigations with a pregnant woman model concentrated on treatment locations close to the amniotic sac (hip and spine). It could be demonstrated that fetal overexposure can occur, however, not at any treatment. During hip treatment with inductive applicators, fetal exposure exceeded the exposure limit up to 3.6-fold (Leitgeb, Omerspahic, and Niedermayr 2010).

6.2.1.2.1.2 Staff It is well known that emitted RF stray fields may interfere with other electromedical devices and electronic implants (Irnich, de Bakker, and Bisping 1978; Pinski and Trohman 2002).

However, there are also established risks of overexposing medical staff. Measurement programs in the working environment (Li and Feng 1999; Martin, McCallum, and Heaton 1990; Moseley and Davison 1981; Shah and Farrow 2007, Shields, O'Hare, and Gormley 2004; Stuchly et al. 1982; Tuschl et al. 1999) found stray RF EMF considerably exceeding International Commission on Non-Ionizing Radiation Protection (ICNIRP)'s exposure limits (ICNIRP 1998). Occupational limits for medical staff were exceeded up to a distance of $2 \mathrm{~m}$ from devices and for accompanying persons up to $2.5 \mathrm{~m}$.

Except for epidemiologic studies performed on pregnant women subjected to diathermy (Lerman, Jacubovich, and Green 2001; Quellet-Hellstrom and Stewart 1993), so far, little scientific attention has been given to excess patient's exposure. Guidelines for use (NHMRC 1985) restrict or even ban the treatment of pregnant women but only generally recommend reducing the exposure of sensitive regions such as eye lenses and gonads without giving specific advice, and other guidelines aim at protecting the operator only (HC 2008). An investigation among 116 senior physiotherapists in 41 Irish hospital-based physiotherapy departments discovered inadequate safety practices and the urgent need for guidelines for safety of operators, patients, and the public during diathermy (Shields, Gormley, and O'Hare 2006).

\subsubsection{Heat Therapy}

In heat therapy, also called thermotherapy, heat is applied to the body via different sources, among them electrical medical devices such as electric heating blanks, pads, and mattresses or low-temperature infrared cabins with intended medical use.

\subsection{Heating Blankets}

Because the patient is in close vicinity to the wiring of electric heating blankets, pads, and mattresses, local exposure to magnetic fields can be relevant. However, it decreases rapidly with increasing distance and varies considerably depending on the placement of heating wires. Lowest magnetic fields are produced by bifilar mounted filaments 


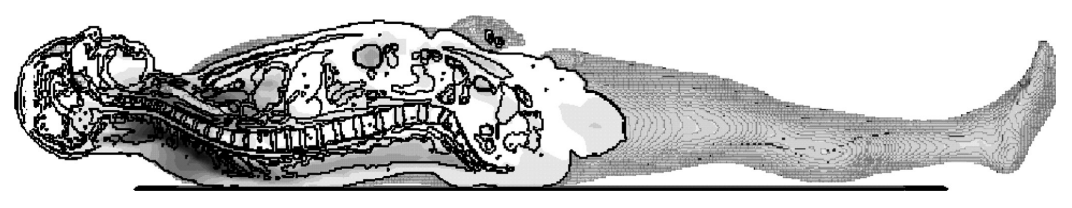

FIGURE 6.3 Intracorporal current density distribution induced by a patient heating blanket. (Data from Leitgeb, N. 2010. Safety of Electromedical Devices. Law-Risks-Opportunities. Vienna, Austria: Springer. With permission.)

compared to conventional meander-like wiring. However, if measured according to the existing regulations, magnetic induction values become low due to averaging over the $100 \mathrm{~cm}^{2}$ sensing area. In the case of electronic power control of the devices, a complex frequency spectrum is generated, which apart from mains frequency exhibits a series of harmonics. The frequency-weighted sum resulted in an equivalent for $50 \mathrm{~Hz}$ of $0.6 \mu \mathrm{T}$ (Leitgeb 2008). Consequently, current densities induced in the CNS remain low and amount to about $8 \%$ of the ICNIRP's basic restriction (Figure 6.3; Leitgeb 2007).

\subsection{Infrared Cabins}

Therapeutic heat may also be generated by infrared radiation emitted in cabins, which are situated in medical premises as well as at homes. Infrared sources may either be hightemperature spot sources such as quartz bulbs emitting radiation in the near infrared range $(0.38-3 \mu \mathrm{m})$ or low-temperature large-area electric heating elements emitting infrared radiation in the mid-infrared range (3-50 $\mu \mathrm{m})$ as defined by ISO $20473(2007)$. High-temperature cabins require a larger distance to the ELF magnetic source. In contrast, in low-temperature infrared cabins, patients stay close to the heating elements. Therefore, large areas of their bodies are exposed to ELF magnetic fields. However, the induction remains well below the exposure limits and amounts to about some microtesla.

\subsubsection{Magnetic Stimulation}

Noninvasive transcutaneous magnetic stimulation has become a valuable tool in medical diagnosis and therapy such as transcranial stimulation of the brain, transcutaneous magnetic functional stimulation, or magnetic defibrillation of the heart.

Magnetic stimulators contain a high-voltage $(400 \mathrm{~V}$ to several $\mathrm{kV})$ discharge system generating discharge pulses with high peak currents (4 kA to several $10 \mathrm{kA}$ ), which produce very intense brief magnetic field pulses. They last up to a few milliseconds with peak values of up to $10 \mathrm{~T}$. Due to high currents, resistive loss within the coil may cause considerable heating and consequently requires adequate coil design and heat management.

\subsubsection{Transcranial Magnetic Stimulation}

The stimulation of localized cortical regions results in alteration of movement, mood, and behavior. Investigations are still on the way to study TMS's applicability in a number of psychiatric and neuropsychiatric disorders. Today, in medical therapy, TMS may be used for repetitive TMS (rTMS) of the cortex with repetition frequencies of $1-60 \mathrm{~Hz}$ 
to treat chronic neuropathic pain and various neuropathic disorders including schizophrenia (Demirtas-Tatlidede et al. 2010) although double-blind investigations could not find an effect (Mogg et al. 2007) or missed statistical evidence for an effect (Dlabac-de Lange, Knegtering, and Aleman 2010). Other studies suggested some therapeutic effect in treating depression by rTMS over the prefrontal cortex. However, a meta-analysis concluded that there was only limited efficacy (Couturier 2005).

The rheobase for brain-cell stimulation (the minimum value required for even long stimulating time) has been calculated to be $20 \mathrm{~V} / \mathrm{m}$ (Reilly 1998). For durations of magnetic pulses longer than several milliseconds, it could be shown that cellular excitability can be influenced by induced fields above a few volts per meter (Jefferys et al. 2003). For reliable brain-cell stimulation, the induced electric field needs to exceed $100 \mathrm{~V} / \mathrm{m}$. Amplitudes of applied magnetic pulses amount to 2-3 T, pulse durations are 50-600 $\mu \mathrm{s}$, and gradients extend up to $10,000 \mathrm{~T} / \mathrm{s}$ during rise times up to $100 \mu \mathrm{s}$.

The depth until which tissue can be stimulated depends on the coil design and can extend, for example, up to 8 - $\mathrm{cm}$ deep. Although standard coils such as figure-8 coils or butterfly coils are limited to superficial brain regions, deeper brain stimulation can be achieved by sophisticated coil configurations (Roth et al. 2010).

\subsubsection{Magnetic Defibrillation}

Magnetic cardiac stimulation could overcome problems of conventional electric cardiac defibrillators associated with electrode contacting and placement. Compared to nerve stimulation, magnetic cardiac stimulation requires considerably higher energies (Pastore et al. 2010). However, compared to electric cardiac defibrillation, it offers the advantage to be quickly applicable without any preparation of the patient (undressing and preparing electrodes with contact gel) and avoids risks from high contact voltage of up to $5 \mathrm{kV}$ (IEC 60601-2-4).

Cardiac stimulation requires current densities above the fibrillation threshold, which has been reported to be about $40-50 \mathrm{~A} / \mathrm{m}^{2}$ with pulse durations of about $1 \mathrm{~ms}$ (Irnich 1994). For magnetic cardiac defibrillation, coil design must allow in-depth stimulation and provide sufficient high-pulse parameters to achieve reliable coverage of cardiac muscle (Pastore et al. 2010).

\subsubsection{Magnetic Therapy}

Magnetic fields, in spite of being suspected to cause childhood leukemia if emitted from power lines or transformer stations (IARC 2002), are frequently considered a last resort of people with various health complaints. Aggressive marketing and fanciful therapeutic promises have made them a big business. The dispute whether magnet therapy is beneficial or hogwash is ongoing (Flamm 2007; Pieber, Herceg, and Paternostro-Sluga 2010; Kröling et al. 2009). Magnetic therapy device manufacturers provide long lists of indications and disorders from angina, depression, headache, and toothache to rheumatism. Authorities are reluctant in generally accepting the clinical efficiency of magnetic therapy devices. The U.S. Food and Drug Administration (FDA) has restricted acceptance of magnetic therapy only to some indications of proven clinical evidence (FDA 2006); the German Federal Board of Physicians and Health Insurance has classified 
magnetic therapy not eligible for reimbursement because of unproven efficiency (HMR 2004); and in an ordinance to the medical devices law, the Austrian government has banned magnetic therapy devices from the lay market and restricted their distribution. Magnetic therapy devices are allowed to be distributed only based on medical prescriptions for individual patients (MFTGV 2003).

\subsubsection{Sinusoidal Fields}

Devices are equipped with Helmholtz coils generating fairly homogenous magnetic fields, or with pads, pillows, and mattresses with integrated flat spiral coils, which emit very inhomogeneous alternating current $(\mathrm{AC})$ magnetic fields. The used frequency range is $1 \mathrm{~Hz}-1 \mathrm{kHz}$, and amplitudes are usually below limits of exposure guidelines (ICNIRP 1998) and range from some nT to about $100 \mu \mathrm{T}$. The exceptions are devices for treating wounds and bone fractures where magnetic inductions can reach several millitesla. Because pads, pillows, and mattresses with integrated flat spiral coils generate magnetic fields that rapidly decrease with distance, the exposure parameter can hardly be defined. In fact, different parts of the body are quite differently exposed. Therefore, if there were a dose-dependent healing effect, it is very uncertain whether the required exposure conditions could be achieved in the relevant target area, and if so, whether they could be maintained over a sufficiently long period in view of patient movements during the intended treatment time of about $30 \mathrm{~m}$ per session (Figure 6.4).

\subsubsection{Pulsed Fields}

Scientific studies have proven the validity of treatment by electrical stimulation. To generate intracorporal electric signals, three types of technologies are clinically available: (1) contact currents, (2) capacitive coupling, and (3) inductive coupling (Gan and Glazer 2006). Inductive coupling by pulsed magnetic therapy (PMT), also called pulsed EMF therapy (PEMF), is most commonly used in orthopedics for the treatment of nonunion fractures or failed fusions (pseudarthrosis).

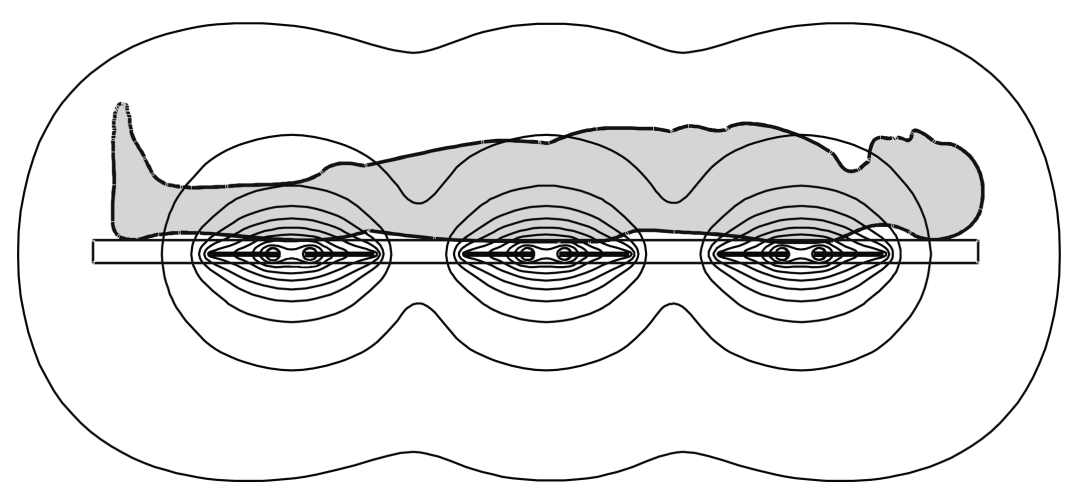

FIGURE 6.4 Inhomogeneous alternating current (AC) magnetic field distribution generated by three flat coils integrated within a magnetic mattress. 
A series of magnetic pulses induces electrical fields, which stimulate bone repair (e.g., $72 \mathrm{mT}, 50 \mathrm{~Hz}$ ). There are several PEMF stimulation devices approved by the FDA that are widely available to patients for use. These devices are considered to provide additive support to bone growth and repair (Grana, Marcos, and Kokubu 2008). The effectiveness of PEMF in the management of osteoarthritis has been investigated with different outcomes. In a meta-analysis, it has been concluded that pulsed EMFs improve clinical scores and function in patients with osteoarthritis of the knee and should be considered adjuvant therapies in the management of osteoarthritis (Vavken et al. 2009). In a study including 57 cases of tibial pseudarthrosis, surgical treatment was combined with PEMF in 22 cases. Of the PEMF treated group (22), 20 (91\%) fractures healed, and two (9\%) did not. From the not PEMF treated group (35), 29 (83\%) healed, and six (17\%) did not. PMT was considered clinically relevant both with regard to healing and time to union (Cebrián et al. 2010).

Studies have also investigated the effectiveness of PEMF in healing soft-tissue wounds, suppressing inflammatory responses at the cell membrane level to alleviate pain, and increasing range of motion. Pieber, Herceg, and Paternostro-Sluga (2010) reported on two small and three large studies describing analgesic benefits of PEMF treatment, whereas in one large study, no beneficial effect could be found. Kroeling et al. (2009) concluded that the current evidence for PEMF shows that these modalities might be more effective in treating neck pain than placebo but not compared to other interventions.

\subsubsection{Static Magnetic Fields}

Magnetic devices that are offered for medical treatment include magnetic bracelets, insoles, magnetic straps for wrist and knee, back and neck braces, and pads, pillows, and mattresses.

Two main types of magnets exist:

1. Static or permanent magnets in which the magnetic field is generated by the spin of electrons within the ferromagnetic material itself

2. Magnet coils either cylindrical with concentric windings or flat with spiral windings in which the magnetic field is generated by electric currents

Permanent magnets can be of compact material and are marketed in various strengths, typically with inductions at their surface between 5 and $500 \mathrm{mT}$. In addition, magnetic foils (plastic foils coated with magnetic particles) are offered with surface fields of less than $1 \mathrm{mT}$. However, it needs to be noted that the fields are very inhomogeneous and amplitudes decrease very rapidly with distance.

Costantino et al. (2007) treated wrist and hand fractures with permanent magnets inserted into a plaster cast of hand and wrist fractures and reported on reduced union time of $35 \%$, however, with no control group. Permanent magnets (necklace) were not more effective for pain relief than placebo (Pieber, Herceg, and Paternostro-Sluga 2010; Pittler, Brown, and Ernst 2007; Finegold 2006; Flamm 2006). In a book, Colbert et al. (2009) analyzed 56 studies, 42 in patients and 14 in healthy volunteers. The most common indication was osteoarthritis of the knee, and other applications concerned 
peripheral neuropathy, peripheral vessel disease, wound and ulcer healing, obstructive sleep apnea, tinnitus, and a variety of chronic pain syndromes. A multivariate analysis did not reveal significant results $(p>0.2)$. In general, studies suffered from poor description. The parameters most often insufficiently described were related to magnetic exposure (magnet dimension and strength and distance to target tissue). Overall, the current evidence is considered to be lacking or of low quality that a static magnetic field could reduce pain or disability (Kroeling et al. 2009; Colbert et al. 2009).

\subsection{Interference}

Neodymium-iron-boron $(\mathrm{NdFeB})$ magnets are small in size but produce strong magnetic fields, which may interfere with the function of cardiac pacemakers and implantable cardioverter-defibrillators (ICDs). The maximum distance resulting in device interference was $3 \mathrm{~cm}$. No significant differences were found with respect to device manufacturer and device types (Wolber et al. 2007).

\subsubsection{Magnetic Targeting}

Ferromagnetic or superdiamagnetic (nano)particles are introduced into the patient for targeting tumors or for local delivery of drugs or heat. SPIONs have diverse diagnostic and potential therapeutic applications in the CNS. Their application may improve disease detection, therapeutic monitoring, and treatment efficacy especially in the context of antiangiogenic chemotherapy and anti-inflammatory medications (Weinstein et al. 2010).

Because they are incorporated into the body and are in close contact with tissues, at their surface, magnetic particles generate local static magnetic fields, which expose the target region and their pathway out of the body.

Magnetic vehicles, namely magnetic capsules and nanoparticles, are attractive for delivery of therapeutic agents. They can be targeted to specific locations in the body through the application of a magnetic field gradient and locally deliver therapeutic agents. The magnetic localization of a therapeutic agent results in the concentration of the therapy at the target site, consequently reducing or eliminating the systemic drug side effects (Polyak and Friedman 2009).

To support drug delivery, SPIONs are coated by organic substances (e.g., phospholipids, fatty acids, polysaccharides, and polymers). The largest class of particles (0.3$3.5 \mu \mathrm{m})$ is applied orally, the most common sizes $(60-150 \mathrm{~nm})$ and ultra-small SPIONs (USPIO) of about $10-40 \mathrm{~nm}$ are injected.

However, their therapeutic use in treating CNS pathologies in vivo is limited by their inability to traverse biological barriers that may cause insufficient local accumulation and retention. This could be overcome by the combined use of focused ultrasound and magnetic targeting, which synergistically delivers therapeutic doses of magnetic nanoparticles across the blood-brain barrier (BBB) (Liu et al. 2010).

Hollow capsules are loaded with active substances (e.g., a dye) and coated with magnetite $\left(\mathrm{Fe}_{3} \mathrm{O}_{4}\right)$ nanoparticles. Magnetic fields $(295 \mathrm{kHz}, 3.9 \mathrm{mT})$ may be successfully utilized also for triggering drug delivery by using magnetic nanoparticles for drug transport and releasing substances on demand. This is enabled by selective electromagnetic 
heating of magnetite particles and heat-induced phase transition in the lipid membrane (Katagiri, Nakamura, and Koumoto 2010). Selective heating of magnetic particles allows maintaining low bulk temperatures outside the target region (McGill et al. 2009). As an example, cytotoxicity of magnetic paclitaxel nanoparticles (MPNPs) against glioma was studied in glioma-bearing rats. After intravenous injection and magnetic targeting with a $500 \mathrm{mT}$ magnet, drug content in glioma and its antitumor activity was increased, and hence, the advantage of magnetic targeting for local chemotherapy of malignant glioma was demonstrated (Zhao et al. 2010).

Superparamagnetic nanoparticles encapsulated in aerosols and guided by external magnetic fields were also applied to treat lung carcinomas (Hallmark et al. 2010). With these, drug-carrying magnetic aerosols were delivered through pulmonary pathways directly to the tumor cells. This allowed drug deposition only in target cells, while healthy lung regions remained unaltered and consequently protected from side effects (Dahmani et al. 2009).

\subsubsection{Magnetic Navigation}

Magnetic navigation systems consist of two external electromagnets placed on opposite sides of the patient, which guide, for example, an ablation catheter. A small magnet at its tip allows navigating it to the target area, for example, in the heart. The accuracy of this procedure is higher than that with manual navigation (Chen et al. 2010).

The development of magnetically controlled medical devices (e.g., catheters) and anchored intracorporal surgical instruments, retractors, and cameras have helped advance surgical practice. Magnetic anchoring guidance systems (MAGS) are composed of an internal surgical instrument controlled by an external handheld magnet. Because they do not require a dedicated surgical port, invasiveness is reduced. The application of a swallowable pill camera used also in capsule endoscopy results in fewer instrument collisions, improves surgical working space, and provides an image comparable to that in standard laparoscopy. Therefore, the use of MAGS has further enhanced the use of minimally invasive techniques.

As an example, it allows meeting or exceeding the benefits of current-day fixed-trocar laparoscopy (Cadeddu et al. 2009; Scott and Cadeddu 2009). It allows reducing the number of transabdominal trocars to a single site and thus minimizing surgery invasiveness of laparoscopic surgery to a single port.

\subsubsection{Electronic Implants}

Due to the position of electronic implants inside the body and hence because of their immediate contact to tissue, active electronic implants may lead to different kinds of tissue exposure to EMF. This exposure may be generated for the following reasons:

- Direct exposure to ELF and/or direct current (DC) magnetic fields generated during operation by internal electric currents

- Exposure to RF EMF continuous transmission of energy via transmitting coils placed on the body surface and exposing the interjacent tissue 
- Exposure to pulsed fields from a body-worn device to operate the medical implant (e.g., to trigger pulse delivery of implanted functional stimulators and stimulate nerves (e.g., for breathing) or control skeletal muscles)

- Continuous wireless transmission of signals to achieve the intended use (e.g., acoustic signals received by the belt-worn master device to the in-the-ear hearing aid)

- Remote wireless data transmission to read out stored data or to monitor devices' operation, for which active implants are equipped with functions, for example, for saving events, parameter sequences, and electrocardiograms

- Occasional EMF transmission to (re)program implanted devices (e.g., cardiac pacemakers or cardioverter defibrillators)

\subsection{Medical Diagnosis}

\subsubsection{Magnetic Resonance Imaging}

\subsubsection{Principle}

MRI has become a major medical imaging modality, which allows noninvasive anatomic, functional, and molecular imaging with still growing areas of application.

The operation principle of MRI makes use of intrinsic nuclear magnetic moments, in particular of hydrogen. These are usually in random positions with magnetic fields compensating each other. If a strong external static magnetic field is applied (e.g., along the body axis), these magnetic moments align either parallel or antiparallel to it. Because there is a surplus of parallel alignment, the atomic contributions add to a macroscopic magnetization, which can be measured. The efficiency of alignment (and magnetization amplitude) increases with increasing external static magnetic induction.

If an adequate (resonant) external RF EMF is acting on the aligned nuclei, they are able to absorb energy and start spinning around the field lines of the external static field. This leads to precession of the macroscopic magnetization. However, due to basic atomic laws, nuclear absorption of RF EMF quantum energy is only possible in case of (magnetic) resonance. It occurs if the frequency of the RF EMF equals the nucleus-specific Larmor frequency $\omega_{\mathrm{L}}$. For hydrogen, $\omega_{\mathrm{L}}$ amounts to $42.58 \mathrm{MHz} / \mathrm{T}$. This resonance frequency strictly depends on the atomic gyromagnetic ratio $\gamma$ and the magnetic field $B$ at the site of the nucleus according to the following:

$$
\omega_{\mathrm{L}}=\gamma \cdot B
$$

The spinning nuclei generate a macroscopic magnetization, which is inclined relative to the direction of the external field and hence exhibits a transverse magnetic component. The angle of inclination (flip angle) increases proportional to the duration of the $\mathrm{RF}$ pulse. In a receiving coil, the transversal RF EMF component induces a damped sinusoidal signal, which is used as primary information for MRI.

One of the advantages of MRI is that more than just one tissue-characterizing parameter can be derived from the amplitude and time course of the received signal. 
- Its amplitude is dependent on the number of involved hydrogen nuclei and hence on the water content of tissue.

- Its time course is mainly influenced by two different mechanisms. The first is the release of the absorbed RF EMF energy quantum and return of nuclei to their basic state. Consequently the longitudinal magnetization is recovering at the expense of the transversal vector component. The time constant of this mechanism is dependent on the tissue structure and is called spin-lattice time constant $T_{1}$.

- The second mechanism responsible for the signal decay is dephasing of the spinning nuclei due to local $B$-field variations. These are caused by the influence of neighboring nuclear magnetic moments (spins). Fluctuating local $B$-fields cause fluctuating Larmor resonance frequencies and consequently varying phase angles of nuclear spins. The vectorial summation of increasingly dephased magnetic components results in the ongoing reduction of the macroscopic transverse magnetization. Because nuclei continue spinning, they still keep their absorbed energy and do not return to longitudinal alignment. Therefore, the longitudinal magnetic vector component remains unaffected. The time constant of this mechanism is dependent on tissue density and rigidity and is called spin-spin time constant $T_{2}$.

Both time constants are valuable for tissue discrimination and hence are used for imaging. Therefore, in addition to the initial RF pulse to tip the macroscopic magnetization to a flip angle, measurement of both time constants requires specific sequences of RF pulses differing in their duration and time interval (e.g., inversion recovery sequence to measure $T_{1}$ or spin echo sequence for $T_{2}$ ). To acquire sufficient independent information for image generation, this complex structure of RF pulse sequences is rapidly repeated.

Unfortunately, the receiving coil is measuring a signal originating from an intracorporal volume that is not capable of localizing the exact signal origin. To allow this, two additional measures are needed: (1) the application of a longitudinal static magnetic field gradient $B(z)$. This assures that only nuclei within a small cross-sectional slice are able to absorb the RF energy, which fulfill the condition that their Larmor frequency $\omega_{\mathrm{L}}=\gamma \cdot B(z)$ equals the RF frequency $\omega_{\mathrm{RF}}$. (2) Spatial coding is performed by applying transverse static magnetic field gradients $B(x)$ and $B(y)$, which code the site of spinning nuclei by different Larmor frequencies and hence widen the original monofrequent measured signal into a broadband signal. Consequently, frequency analysis of the measured broadband signal allows identifying the integral signal components originating from all nuclei along a line in the cross section, which exhibits the same Larmor frequency. From a series of such integral projections of different orientations, a numerical reconstruction algorithm is able to generate a high-resolution cross-sectional anatomic image of the body. With selectable weighting of the three basic parameters (hydrogen density and time constants $T_{1}$ and $T_{2}$ ), MRI offers many possibilities to display and differentiate tissues with selectable contrast.

However, the gyromagnetic ratio $\gamma$ does not only depend on the atomic nucleus as such but also on its type of chemical binding to other nuclei. This results in a chemical (frequency) shift $\Delta \omega_{\mathrm{L}}$, which increases proportional to $B$, and allows molecular imaging. To provide this information to medical diagnosis and improve the signal-to-noise ratio, high static magnetic fields are required. 


\subsubsection{Exposure}

The functional principle of MRI involves different kinds of magnetic fields and EMFs:

1. Static magnetic field (exhibiting a longitudinal gradient). The amplitude of this field must be increased to get higher signal amplitudes, improved signal-to-noise ratio, or a resolvable chemical frequency shift. Consequently, MRI development goes toward higher static magnetic fields. The conventional devices today apply $1.5 \mathrm{~T}$ or $3 \mathrm{~T}$, whereas experimental devices with fields up to $11 \mathrm{~T}$ are already available. Because of excessively increasing resistive heating loss, such high fields must be generated by superconducting magnetic coils cooled down to temperatures of liquid Helium $\left(-269^{\circ} \mathrm{C}\right)$.

On the other hand, costs and weight of devices increase with increasing magnetic induction. Therefore, for commercial reasons, attempts are made to get sufficient image quality with lower induction. Although currently low-field MRI already use only some $100 \mathrm{mT}$, future ultralow-field low-cost devices could operate with static magnetic fields well below $100 \mathrm{mT}$ down to $1 \mathrm{mT}$, for example, by overcoming the requirement for a constant static magnetic field (Trahms and Burghoff 2010; Matlashov et al. 2009; Bindonisti et al. 2004; Durand et al. 2002).

2. Switched gradient fields are applied in the three spatial directions (longitudinal, transverse lateral, and transverse anterior-posterior). The shape of these gradients varies with the applied RF pulse sequence. The gradient rise time is typically $0.2-1 \mathrm{~ms}$ with rates of change between 20 and $150 \mathrm{Tm}^{-1} \mathrm{~s}^{-1}$ (HPA 2008; ICNIRP 2004).

3. RF EMFs depending on the assessed tissue parameter exhibit a complex time signature. Due to the proportionality of the magnetic resonance frequency and the static magnetic field, the frequency of the applied RF EMF increases with the amplitude of the static magnetic field. Conventional devices apply $63.9 \mathrm{MHz}(1.5 \mathrm{~T})$ and $127.8 \mathrm{MHz}$ (3 T). However, frequencies reached $462 \mathrm{MHz}(11 \mathrm{~T})$ in already available experimental devices. On the other hand, there has been development of low-cost MRI with low fields that need only $12.8 \mathrm{MHz}(300 \mathrm{mT})$; in the future with further field reductions, frequencies may be even much lower, such as $42.58 \mathrm{kHz}$ for $1 \mathrm{mT}$ MRI.

RF fields are usually generated by birdcage coils driven in quadrature, which produce circular polarized fields. Apart from its linear dependence on field amplitude, inductive heating increases with the square of frequency. Therefore, prevention from excess heating SARs is limited for different reference masses: whole body, partial body, or local. In fact, heating is one of the limiting factors in MRI development (Pediaditis, Leitgeb, and Cech 2008; Hand et al. 2006).

\subsubsection{Unintended Side Effects}

Because the intended purpose of MRI does not include therapeutic effects, any associated adverse health-relevant effect needs to be considered an unintended side effect. Such effects can arise from fields used for imaging such as static magnetic fields, switched gradients, and RF EMF pulses but include also acoustic noise, electromagnetic interference (EMI) with other devices, and attractive forces on ferromagnetic objects. 


\subsection{Static Magnetic Fields}

One of the basic interaction mechanisms of static magnetic fields is generation of forces, in particular,

- Translational forces within gradient fields on diamagnetic, paramagnetic, or ferromagnetic molecules (e.g., hemoglobin, collagen, and fibrin), particles (e.g., magnetite), and parts (such as metallic implants)

- Rotational or twisting forces on magnetic dipoles until alignment with the magnetic field vector

- Electrodynamic forces on moving electrical charged particles leading to potential differences perpendicular to movement (Hall voltage) or increased blood flow resistance

In addition, on a microscopic level, static magnetic fields can alter electron spin states and hence influence the speed and balance of chemical reactions and/or the transit time of free radicals, which modifies their destructive potential.

Electric flow potentials induced by static magnetic fields can change the $\mathrm{T}$ wave of electrocardiograms (associated with ventricular contraction). This can be observed above $100 \mathrm{mT}$ (Tenforde et al. 1983). In higher static magnetic fields due to the contributions of electric voltages across the aorta and other blood vessels, recorded electrographs (ECGs) could become even uninterpretable. Induced electric field strengths may also cause current densities, for example, $100 \mathrm{~mA} / \mathrm{m}^{2}$ at the atrial sinus at $5 \mathrm{~T}$ (Kinuichi et al. 1996). They may increase up to $220 \mathrm{~mA} / \mathrm{m}^{2}$ at $11 \mathrm{~T}$. However, this still remains well below the cardiac fibrillation threshold of 40-50 A/m² (Irnich 1994).

Electric potentials may also be induced in objects moving through static magnetic fields. Because high static magnetic fields are generated by electric currents flowing in supraconducting coils, they cannot be simply switched on and off on demand but persist after imaging. Therefore, people close to the patient tunnel such as medical staff, service engineers, and room cleaners may move through static fields. Also patients are moving through the field when moved into or out of the magnet.

The impact of movement has been studied by numerical simulation. It was demonstrated that induced electric current densities increase with velocity of the movement and the amplitude of the magnetic gradient. The ICNIRP's basic restriction for workers $\left(40 \mathrm{~mA} / \mathrm{m}^{2}\right)$ can be exceeded in the vicinity of devices with $1.5 \mathrm{~T}$ or more even if motion is slow such as $1 \mathrm{~m} / \mathrm{s}$. Due to different external field profiles, no strict scaling law exists. However, restrictions are expected to be met at a compliance distance of $1 \mathrm{~m}$ to the magnet (Crozier and Chadwick 2007; Crozier and Liu 2005).

Experimental investigations revealed that transient sensory effects such as vertigo, nausea, metallic taste, and even magnetophosphenes (flickering light sensations) may be experienced during movement through gradient static fields, although with considerable interpersonally varying sensitivity. Threshold for motion-induced magnetophosphenes in sensitive people was determined to be $1 \mathrm{~T} / \mathrm{s}$ for movements longer than 1 second or in field gradients of $1 \mathrm{~T}^{2} / \mathrm{m}$ for postural sways (ICNIRP 2009b).

In addition, MRI scanners present a unique set of safety risks for a health care facility. In the space surrounding an MRI scanner due to attractive forces of magnetic gradient fields, ferromagnetic objects may be accelerated and become dangerous projectiles 
with consequential risks. In addition, inside the patient, ferromagnetic implants such as aneurysm clips could be twisted, rip the blood vessel, and cause even fatal internal bleeding (Sommer et al. 2004). Such risks can occur already in static fields of several millitesla. Therefore, gates are installed preventing metallic objects from approaching the magnet. However, experience shows that a residual risk might still remain, for example, if in an emergency case a ferromagnetic fire extinguisher is brought into the vicinity of the magnet (ICNIRP 2009a, IEC 2010).

\subsection{Switched Magnetic Gradient Fields}

Time-varying magnetic fields induce electric currents in conducting objects. Induced intracorporal current densities may become large enough to stimulate nerve and muscle cells and hence cause involuntary movements and/or painful sensations. This limits enhancing switched magnetic gradients. In addition, they contribute to potential excess heating. Their relative contribution to heating increases with decreasing static field strength (and decreasing RF frequency); however, their contribution can be neglected in comparison to RF EMF heating. Therefore, the safety standard of MRI devices limits gradient fields, in particular, the time derivative $\mathrm{d} B / \mathrm{d} t$, which depends on the duration and mode of device operation (IEC 60601-2-33) because of their stimulatory effects.

\subsection{Pulsed Radio-Frequency Electromagnetic Fields}

In MRI, the frequency of RF EMFs extends from $42.6 \mathrm{kHz}$ until $426 \mathrm{MHz}$. Their energy is absorbed by tissues and converted to heat. The exposure is usually characterized by the average power deposited per unit mass, which is called specific absorption rate and is given in watt per kilogram. Depending on the kind of exposure, the reference mass for SAR assessment may be the whole body $\left(\mathrm{SAR}_{\mathrm{WB}}\right)$, partial body such as head, trunk, and extremities, or local such as any $1 \mathrm{~g}\left(\mathrm{SAR}_{\mathrm{lg}}\right)$ or $10 \mathrm{~g}\left(\mathrm{SAR}_{10 \mathrm{~g}}\right)$ tissue mass. In pregnant women, the fetus merits specific attention. Therefore, prevention from excess heating as described by SAR values becomes one of the limiting factors in MRI development (Pediaditis, Leitgeb, and Cech 2008; Hand et al. 2006).

Numerical investigations into an anatomical model of a pregnant woman (SILVY) showed that for the same magnetic induction in the center of the birdcage coils, the SARs exhibit a quadratic increase with frequency (Figure 6.5). The largest local $S_{10} R_{10 g}$ values were found in the extremities. They exceeded the whole-body SAR by about 24 -fold. The whole-body $\mathrm{SAR}_{\mathrm{WB}}$ of the mother and the fetus were almost similar; however, high partial-body SAR values were identified in the trunk of the mother. Local SAR $\mathrm{R}_{10 \mathrm{~g}}$ values of the fetus were about fourfold smaller (Pediaditis, Leitgeb, and Cech 2008).

\subsection{Contrast Agents}

To improve contrast in magnetic resonance images, the most commonly used contrast agents are based on chelates of gadolinium $(\mathrm{Gd})$, which are delivered intravenously. However, due to their unique magnetic properties and the ability to function at the cellular and molecular level of biological interactions, magnetic nanoparticles have become an attractive alternative both as contrast agents for MRI and as carriers for drug delivery. 


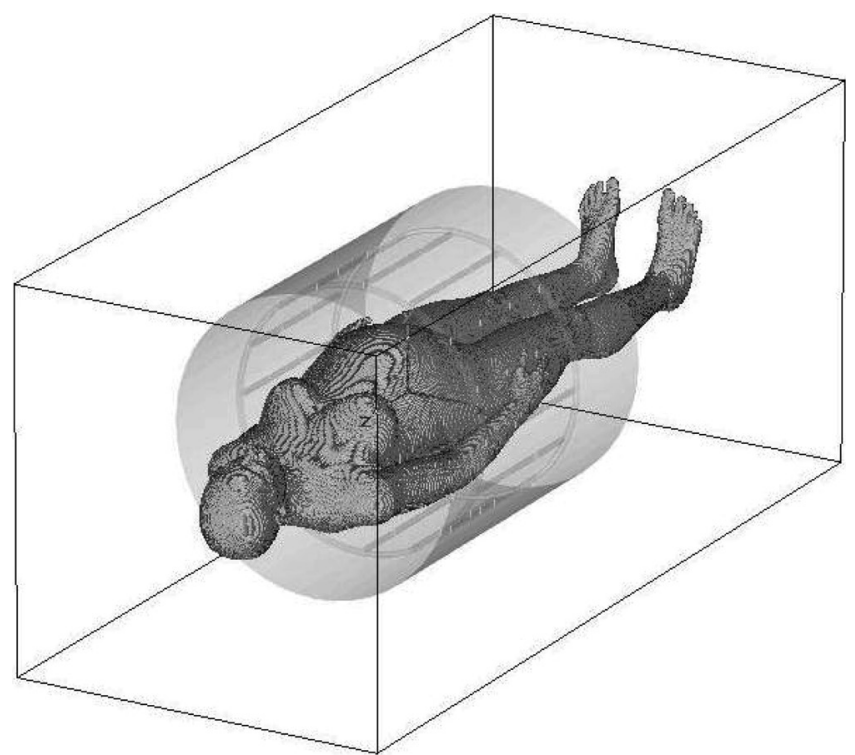

FIGURE 6.5 Numerical model of a pregnant woman SILVY inside a magnetic resonance imaging (MRI) birdcage coil. (Data from Pediaditis, M., N. Leitgeb, and R. Cech. 2008. Phys Med Biol 53:7187-95.)

In the latter case, highly specific targeting agents and other functional ligands such as fluorophores and permeation enhancers may act at the cellular level.

Such nanoparticles are useful for MRI-based detection, diagnosis, and treatment of malignant tumors, and cardiovascular and neurological diseases. Magnetic nanoparticle contrast agents are applied to examine BBB dysfunction and investigate tumors and other neuroinflammatory pathologies, the cerebrovasculature system using perfusionweighted MRI sequences, and in vivo cellular tracking in CNS disease or injury. Novel syntheses of nanoparticles are expected to rapidly expand the range of applications in patients with brain tumor, cerebral ischemia or stroke, carotid atherosclerosis, multiple sclerosis, and traumatic brain injury or epilepsy.

\subsection{Magnetization}

In the vicinity of MRI scanners, their static magnetic fields, in particular of very high field devices, could magnetize ferromagnetic objects and hence permanently change magnetic background fields. However, investigations demonstrated that residual magnetism in an MRI suite after controlled ramp down of an 8-T superconducting magnet was not significantly elevated compared with magnetic fields in the environment (Sammet et al. 2010).

\subsection{Acoustic Noise}

Magnetic field gradients cause rapid changes of currents within gradient coils which in turn induce strong Lorentz forces. These act between wires and make them vibrate and thus generate acoustic noise. In particular in ultra-high speed imaging, noise levels can become as high as $140 \mathrm{~dB}$. 
In addition, inside high-field MRI, RF pulses may induce thermoelastic pressure waves inside the head, which also may contribute to auditory sensations. For a given SAR, the peak acoustic pressures generated in the anatomic head model were essentially the same at 64, 300, and $400 \mathrm{MHz}(1.5,7.0$, and 9.4 T). Exposing the cochlea to $4 \mathrm{~W} / \mathrm{kg}$ generates pressures in the anatomic head that are comparable to the auditory threshold pressure of $20 \mathrm{mPa}$ of human sound perception. Moreover, results indicate that the peak acoustic pressure in the brain is two to three times the auditory threshold of the FDA guideline $(8 \mathrm{~W} / \mathrm{kg})$. However, even at a high SAR of $20 \mathrm{~W} / \mathrm{kg}$, causing an acoustic pressure in the brain of more than seven times the auditory threshold, at the cochlea, the sound pressure levels would not be more than $17 \mathrm{~dB}$ above the perception threshold (Lin and Wang 2010).

\subsection{Contraindications}

In general, patients with metallic implants are contraindicated for MRI due to the risk of excess heating by induced electric currents and the consequential tissue damage. In the case of ferromagnetic implants, risks due to magneto-mechanical effects are added. Electromagnetic compatibility problems may arise with electronic implants due to induction of interference voltages and consequently cause malfunction or damage of electronic components. In the case of cardiac pacemakers, the risk associated with static magnetic field induced switching to another operating mode and/or alterations of programmed parameters is added. For functional testing, switching from the programmed synchronous mode (triggered or demand) to the fixed-frequency asynchronous mode is foreseen by placing a permanent magnet onto the chest wall. In static magnetic fields, above $1 \mathrm{mT}$ internal reed relays can be activated unintentionally. Therefore, pacemaker patients should keep a safe distance large enough to assure that the static magnetic fringe field has decreased below $0.5 \mathrm{mT}$ (IEC 60601-2-33). The first MRI compatible pacemakers are becoming available.

It could be shown that meeting exposure limits of the mother does not protect the fetus from overexposure (Figure 6.6). Exposure of the mother is limited by the partialbody local $\mathrm{SAR}_{10 \mathrm{~g}}$ value. If the mother is exposed up to the limit for normal operation conditions, the fetal SAR exceeds limits such as 1.8-fold the local SAR $\mathrm{R}_{10 \mathrm{~g}}$ and 7.4-fold the fetal whole-body $\mathrm{SAR}_{\mathrm{WB}}$. Therefore, the fetal exposure turns out to be the limiting parameter for MRI investigations of the mother (Pediaditis, Leitgeb, and Cech 2008).

\subsubsection{Limiting Exposure}

For the general population and workers, there are already guidelines, recommendations, standards, or legal regulations limiting exposures to nonionizing EMFs in the entire range from static fields until the border to optical infrared radiation (ICNIRP 1998; IEEE 2002a,b, 2005; EC 1999, 2004). However, patients are exempted from such regulations. Medical devices may expose patients even to much higher levels provided their benefit outweighs potential risks (IEC 60601-1, IEC 60601-2-33). Manufacturers have to systematically analyze and assess risks according to existing rules (Leitgeb 2010). In medical practice, it is the physician who is responsible to balance risks and benefits to an individual patient. With regard to MRI examinations, a three-tier approach is defined to 

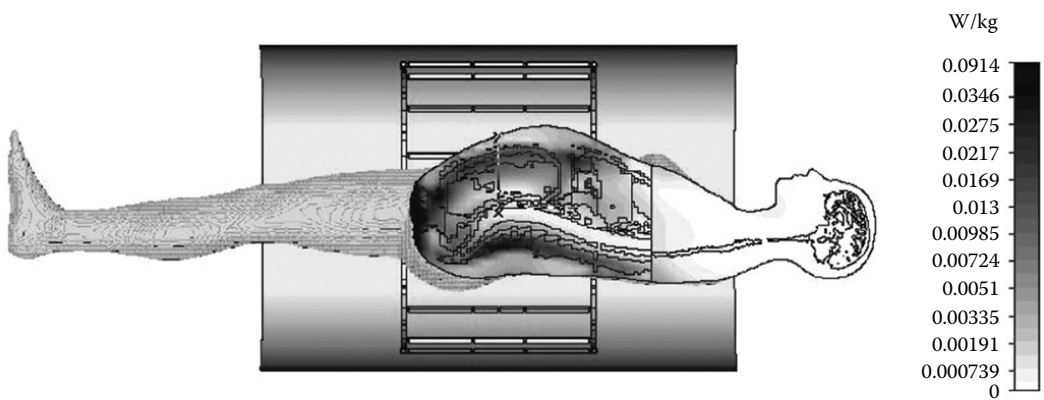

FIGURE 6.6 SAR distribution within the sagittal plane of a pregnant woman SILVY and the fetus during 4-T magnetic resonance imaging (MRI) with $170 \mathrm{MHz}$ radio-frequency electromagnetic field (from PF EMF). (Data from Pediaditis, M., N. Leitgeb, and R. Cech. 2008. Phys Med Biol 53:7187-95.)

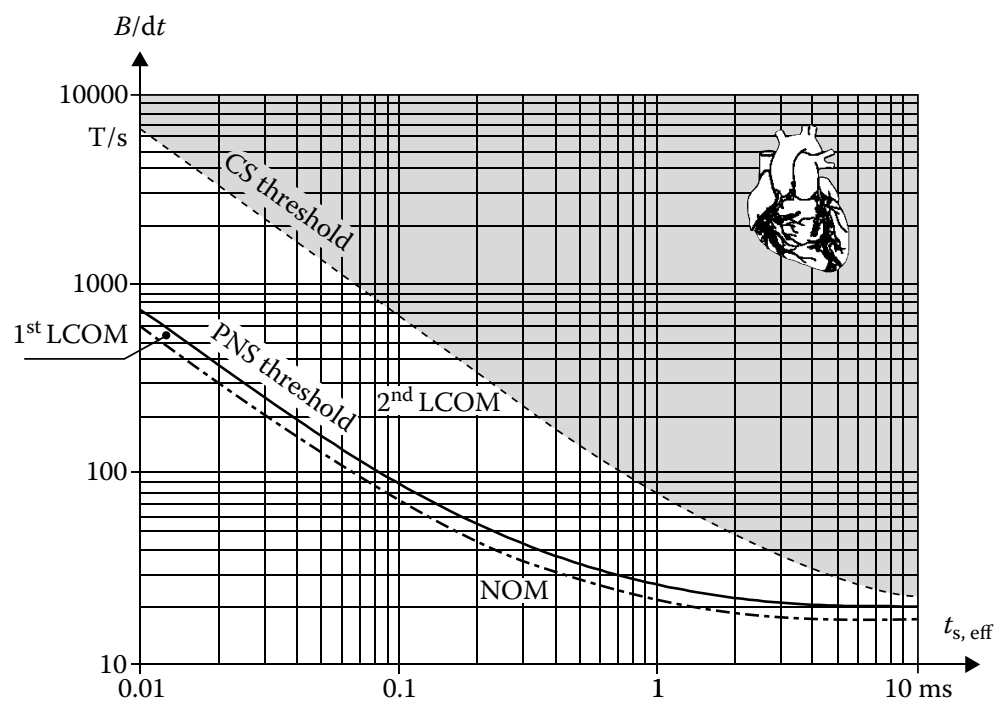

FIGURE 6.7 Operating modes of magnetic resonance imaging (MRI) with regard to switched magnetic gradients. $\mathrm{NOM}=$ normal operation mode; 1 st LCOM $=$ first-level controlled operating mode; 2nd LCOM = second-level controlled operating mode; PNS = peripheral nerve stimulation; and CS = cardiac stimulation. (From IEC 60601-2-33. 2010. Medical Electrical Equipment. Part 2-33: Particular Requirements for Basic Safety and Essential Performance Of Magnetic Resonance Equipment for Medical Diagnosis. Geneva, Switzerland: Safety Standard International Electrotechnology Communications. With permission.)

protect patients as good as possible without hindrance to medical routine and still allowing flexibility for research and further development. Three operating modes are defined for routine, specific examinations, and experimental procedures with graduated regulations and organizational requirements (ICNIRP 2009a,b; IEC 60601-2-33). They account for potential adverse effects of stimulation (Figure 6.7) and heating and are as follows: 


\subsection{Normal Operating Mode}

This mode includes routine examinations with MRI outputs that are low enough to avoid any physiologic stress to the patient. Switched magnetic gradients should not induce electric field strengths exceeding $80 \%$ of the threshold of peripheral nerve stimulation. If not differently determined individually or justified by shorter durations, the induced electric field strength is limited to $E_{\mathrm{i}} \leq 2.2 \mathrm{~V} / \mathrm{m}$. In the case of whole-body exposure, the gradient is limited to $\mathrm{d} B / \mathrm{d} t \leq 20 \mathrm{~T} / \mathrm{s}$. To avoid undue heat stress, SAR values should meet the following limits: $\mathrm{SAR}_{\mathrm{WB}} \leq 2 \mathrm{~W} / \mathrm{kg}, \mathrm{SAR}_{\mathrm{PP}} \leq 2 \mathrm{M}_{\mathrm{EX}} / \mathrm{M}_{\mathrm{WB}} \mathrm{W} / \mathrm{kg}, \mathrm{SAR}_{\mathrm{HEAD}} \leq 3.2 \mathrm{~W} / \mathrm{kg}$.

\subsection{First-Level Controlled Operating Mode}

This mode is dedicated for special MRI examinations. It requires controlling patients by medical supervision because one or more electromagnetic parameters may be large enough to cause physiological stress to patients. Switched magnetic gradients should not induce electric field strengths exceeding $100 \%$ of the threshold of peripheral nerve stimulation. Although the exposure limit of the head equals those of normal operation mode, the $S A R_{W B}$ values for the whole-body and partial-body exposure are increased by a factor of 2. Therefore, the following limits should be met: $\mathrm{SAR}_{\mathrm{WB}} \leq 4 \mathrm{~W} / \mathrm{kg}, \mathrm{SAR}_{\mathrm{PP}} \leq$ $4 \mathrm{M}_{\mathrm{EX}} / \mathrm{M}_{\mathrm{WB}} \mathrm{W} / \mathrm{kg}, \mathrm{SAR}_{\mathrm{HEAD}} \leq 3.2 \mathrm{~W} / \mathrm{kg}$.

\subsection{Second-Level Controlled Operating Mode}

In this mode, apart from controlling patients by medical supervision, an ethical approval is required because one or more electromagnetic parameters exceed first-level controlled operating mode limits and may be high enough to produce significant risks for patients (Table 6.1).

TABLE 6.1 Reference Levels for Static Magnetic Fields $B_{0}$ and Basic SAR Restrictions for the Public and Magnetic Resonance Imaging (MRI) Patients

\begin{tabular}{|c|c|c|c|c|c|c|c|}
\hline & \multirow[b]{2}{*}{$\begin{array}{c}\mathrm{B}_{0, \mathrm{WB}} \\
\mathrm{T}\end{array}$} & \multirow[b]{2}{*}{$\begin{array}{l}\mathrm{SAR}_{\mathrm{WB}} \\
\mathrm{W} / \mathrm{kg}\end{array}$} & \multirow[b]{2}{*}{$\begin{array}{l}\mathrm{SAR}_{\mathrm{PB}} \\
\mathrm{W} / \mathrm{kg}\end{array}$} & \multirow[b]{2}{*}{$\begin{array}{l}\text { SAR }_{\text {HEAD }} \\
\mathrm{W} / \mathrm{kg}\end{array}$} & \multicolumn{3}{|c|}{ Local RF EMF Transmit Coils } \\
\hline & & & & & $\begin{array}{l}\text { SAR }_{\text {HEAD }} \\
\mathrm{W} / \mathrm{kg}\end{array}$ & $\begin{array}{l}\mathrm{SAR}_{\text {TRUNK }} \\
\mathrm{W} / \mathrm{kg}\end{array}$ & $\begin{array}{l}\text { SAR }_{\text {LIMBS }} \\
\mathrm{W} / \mathrm{kg}\end{array}$ \\
\hline General public & $0.4^{\mathrm{a}}$ & $0.08^{\mathrm{a}}$ & - & - & - & $0.4^{\mathrm{a}}$ & $0.4^{\mathrm{a}}$ \\
\hline $\begin{array}{l}\text { Patients with medical } \\
\text { implants }\end{array}$ & $0.0005^{\mathrm{b}}$ & - & - & - & - & - & - \\
\hline Workers & $2-8^{c, d}$ & $0.4^{\mathrm{a}}$ & - & $2-8^{\mathrm{c}, \mathrm{d}}$ & $2-8^{\mathrm{c}, \mathrm{d}}$ & $2-8^{c, d}$ & $8^{\mathrm{a}}$ \\
\hline Normal operating mode & $4^{\mathrm{c}}$ & $2^{\mathrm{b}}$ & $2-10^{\mathrm{b}, \mathrm{e}}$ & $3.2^{\mathrm{b}}$ & $10^{\mathrm{b}}$ & $10^{\mathrm{b}}$ & $20^{\mathrm{b}}$ \\
\hline $\begin{array}{l}\text { First-level controlled } \\
\text { operating mode }\end{array}$ & $8^{c}$ & $4^{\mathrm{b}}$ & $4-10^{\mathrm{b}, \mathrm{e}}$ & - & $20^{\mathrm{b}}$ & $20^{\mathrm{b}}$ & $40^{\mathrm{b}}$ \\
\hline $\begin{array}{l}\text { Second-level controlled } \\
\text { operating mode }\end{array}$ & $>8^{c}$ & $>4^{\mathrm{b}}$ & $>4-10^{\mathrm{b}, \mathrm{e}}$ & $>3.2^{\mathrm{b}}$ & $>20^{\mathrm{b}}$ & $>20^{\mathrm{b}}$ & $>40^{\mathrm{b}}$ \\
\hline $\begin{array}{l}\text { a ICNIRP (2009a). } \\
\text { b IEC } 60601-2-33 \text {. } \\
\text { c ICNIRP (2009b). } \\
{ }^{\mathrm{d}} \text { Head and trunk, for } \mathrm{s} \\
\text { e } 10 \times \mathrm{M}_{\text {exposed }} / \mathrm{M}_{\mathrm{WB}} \text {. } \\
\text { WB = whole body; PB }\end{array}$ & $\begin{array}{l}\text { ific task } \\
\text { artial bo }\end{array}$ & $\begin{array}{l}\text { in contr } \\
\text { y. }\end{array}$ & lled envirc & ment. & & & \\
\hline
\end{tabular}




\subsubsection{Transcranial Magnetic Stimulation}

In medical diagnosis, TMS is a noninvasive method to study brain functions and functional connectivity, for example, to investigate motor pathways or to evaluate diagnostic parameters such as central motor conduction time (CMCT), motor threshold (MT), motor evoked potential (MEP) amplitude, central silent period threshold (cSPT), and duration.

The CMCT is a sensitive parameter to detect myelopathic abnormalities even in the case of negative radiological findings. It may also be useful to detect upper motor neuron involvement in amyotrophic lateral sclerosis. The diagnostic sensitivity may be increased by using the triple stimulation technique (TST), by combining several parameters such as CMCT, MT, and silent period, or by studying multiple muscles. In peripheral facial nerve palsies, TMS may be used to localize the site of nerve dysfunction and clarify the etiology. TMS parameters may also help in detecting lesions in multiple sclerosis. Abnormalities in CMCT or TST may correlate with motor impairment and disability.

Cerebellar stimulation may detect lesions in the cerebellum or the cerebellar output pathway (Chen et al. 2008). TMS may also be useful in diagnosis of neurophysiological disorders such as Parkinson and Alzheimer diseases. The ipsilateral silent period that measures transcallosal inhibition is a potential method to distinguish between different Parkinson syndromes. Prolonged CMCT might help identifying the genetic Parkinson type (Perretti et al. 2010). Short-latency afferent inhibition (SAI), which is related to central cholinergic transmission, is reduced in Alzheimer's disease.

TMS may also be used to create a map of the somatotopic organization of the motor cortex. Advantageous over other noninvasive brain imaging techniques is the functional response of the stimulated cortical area. TMS has been shown to be effective in mapping cortical motor areas and in the functional assessment of motor tracts, for example, in the follow-up of recovery from stroke (Ruohonen and Karhu 2010).

The amplitudes of magnetic fields required for diagnostic TMS are lower than for TMS therapy. The amplitude of the magnetic gradient depends on pulse time and depth of the stimulation target zone but needs to exceed $20 \mathrm{~T} / \mathrm{s}$.

\subsubsection{Magnetic Tracking}

Magnetic tracking systems determine the location of objects (e.g., a catheter) that contain a magnetic sensor or marker. This requires a magnetic field of known geometry generated by orthogonal field coils. In spite of the use of magnetic fields, due to widespread unfortunate habit, this technique is frequently called electromagnetic tracking (EMT). In contrast to an optical tracking system, this approach does not need line of sight and hence allows tracking of intracorporal objects. When an object is placed inside the magnetic field, voltages are induced in the sensor, which are indicating the position and orientation of the object. Sensors might be coils in which signals are induced either by $8-12 \mathrm{kHz}$ AC fields or by switched DC fields. Another solution is tracking the position of a permanent magnet or transponder incorporated in the medical device. Inaccuracies might occur if the tracking field is disturbed by the presence of metallic objects or EMI (Zhang et al. 2006; Wagner et al. 2002).

Magnetic tracking is applied for a variety of applications such as minimally invasive surgery, catheterization, and assisting radiation therapy by tracking movable tumors (Keall et al. 2010). 


\subsubsection{Magnetic Navigation}

Endoscopy by miniaturized swallowable capsules with integrated imaging and transmission systems has become the gold standard for the endoscopic examination of the gastrointestinal tract. Video capsule endoscopes contain one or two cameras, a light source, batteries, electromagnetic transmitter and receiver, and magnetic elements for navigation. Due to their miniaturization, capsules are small enough (e.g., $26 \times 11 \mathrm{~mm}$ ) to be swallowed with water, usually after a 12-hour fast.

The capsule is propelled via peristalsis through the gastrointestinal tract and can be magnetically navigated. For this purpose, capsules contain two diagonally mounted rotatable permanent magnets. To steer the capsule, a static magnetic field is produced by an external permanent magnet or an electromagnetic coil. In addition, capsules may incorporate an electromagnetic actuator, which is controlled by wireless communication.

The combined use of external static magnetic fields and internal actuation to move small permanent intracapsular magnets allow camera steering and orientation with high accuracy (Valdastri et al. 2010). However, the exponential decrease of magnetic field strength with distance has major implications for precision of the remote control.

Currently, there are two capsule endoscopes available: PillCam ESO and PillCam SB for the evaluation of the esophagus and the small bowel, respectively. The PillCam ESO has two cameras on each end and captures images at a rate of 7 frames per second by each camera, whereas the PillCam SB has one camera and captures images at a rate of 2 frames per second. Both capsule endoscopes transmit the image data to a recording device worn on the patient's waist using a RF signal in the wireless local area network (WLAN) or Bluetooth bandwidth (Li, Leighton, and Sharma 2007). After incorporation and some delay to account for the transit time to reach the target region, images are continually acquired with an image storing speed of up to 18 frames per second (Eliakim 2010; Waterman and Gralnek 2009; Delvaux and Gay 2008).

The mean gastric transit time is about 63 minutes (10-319 minutes) and the mean intestinal transit time about 194 minutes (70-322 minutes). The capsule reaches the right colon in about 5-8 hours and is finally excreted naturally.

Capsule endoscopy is applied for the examination of the small bowel and other organs in the gastrointestinal tract, including the esophagus and colon in several clinical situations such as obscure gastrointestinal bleeding, suspected Crohn's disease, and surveillance of polyposis syndromes. In addition, they help screening for colorectal cancer, esophageal varices, and Barrett's esophagus.

Patients are exposed by the incorporated field source to static magnetic fields from the intracorporal permanent magnetic navigation elements and RF EMF emitted by the transmitting antenna.

\subsubsection{Telemedicine}

Wireless medical telemetry from medical device to medical device in a patient's body, from the body-worn device to an external monitoring device, or from a medical device to a network (e.g., bedside data acquisition and monitoring) can directly cause exposure 
to RF EMF exposure or impose a risk due to EMI from other intentional and unintentional transmitters, resulting in the disruption of medical device function or network service.

Communication with medical electronic implants is possible via an interface placed on the body surface. Currently, special mobile phones or telephone modems are available for remote transmission of data that are stored in the implants. Depending on the system, remote transmission can be carried out automatically without requiring patient action or with manual help from the patient responding to an individual call from the health service. Remote patient monitoring is based on existing mobile phone networks. The use of WLAN technology enables even bidirectional data transmission that allows for interrogating and programming including transmission of parameters and electrocardiograms in real time without the use of the traditional programming head.

Communication via electromagnetic information transfer includes the following:

- Continuous transmission of signals (e.g., acoustic signals received by the beltworn master device to the in-the-ear hearing aid)

- Recurrent transmission to read out stored data (e.g., episodes of cardiac dysfunction)

Communication is usually restricted to specific frequencies allocated for industrial, scientific, and/or medical use (ISM frequencies) such as $6.8 \mathrm{MHz}, 13.6 \mathrm{MHz}, 27 \mathrm{MHz}$, $41 \mathrm{MHz}, 433 \mathrm{MHz}, 2.45 \mathrm{GHz}$, and $5.8 \mathrm{GHz}$. For application in telemedicine and in mobile telecommunication, short-range communication bluetooth or WLAN could be used or general telecommunication services such as global system of mobile communication (GSM), universal system of mobile communication (UMTS), or long-term evolution (LTE).

Usually output power for such short-range transmission is low, and the exposure is several orders of magnitudes below the basic restrictions (Figure 6.8).

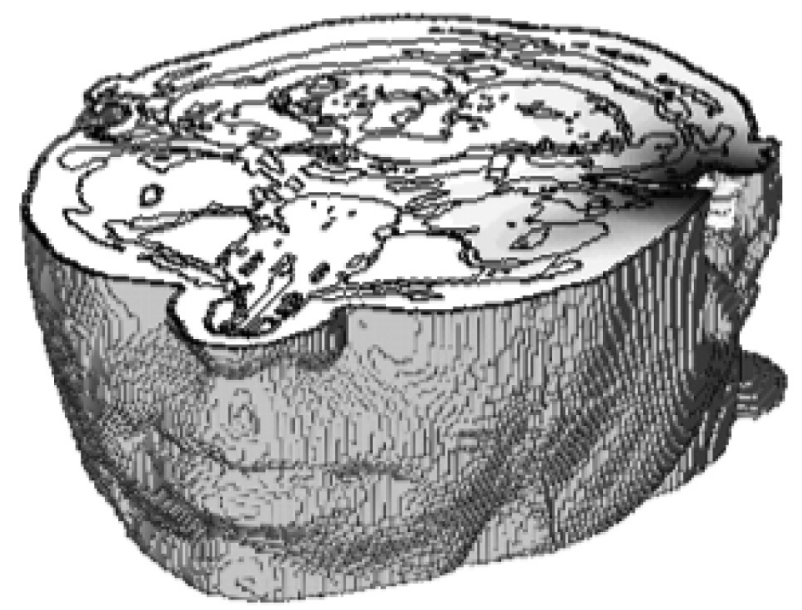

FIGURE 6.8 Intracorporal SAR distribution in a cross-section through an in-the-ear hearing aid, caused by the telecommunication link to a belt-worn transmitter. Voxel SAR values are in the nanowatt per kilogram range. 


\subsection{Electromagnetic Compatibility}

\subsubsection{Sources}

Medical devices may emit EMFs in an unintended manner either as fringe fields or stray fields of intended EMF emission (e.g., magnetic therapy devices, diathermy devices, and MRI) or as an unintended side effect of their intended function (e.g., RF surgery devices) or just as a side effect of electric energy consumption or transformation of voltages (e.g., transformer).

Another source of interference is from the integration of wireless technology into the infrastructure of hospitals (smart building concepts) for (bedside) monitoring, recordkeeping, and intercommunication (of equipment and personnel purposes), as well as RF-based identification (RFID), control, and tracking.

These emissions may have the following consequences:

- Unintended exposure of sensitive regions of the patient outside the treatment zone (e.g., diathermy and hyperthermia)

- Exposure of tissues along the communication pathway during data transmission from or to implanted devices

- Unintended exposure of internal organs by incorporated EMF sources (e.g., magnets of capsule endoscopes and ferromagnetic nanoparticles)

- Exposure of medical staff (e.g., surgeons during RF surgery, medical assistants during diathermy, or when moving the patient in or out of MRI devices)

- Exposure of accompanying persons, for example, during open MRI examinations of children

- Exposure of the general public, for example, in waiting areas or aisles due to outreaching fields from field sources like MRI

- Exposure of workers, service personnel, or room cleaning service (e.g., around MRI scanners or around beam guiding magnets of ultra-high energy ion therapy devices)

- EMI with implanted electronic devices of patients (which may, for example, inhibit cardiac pacemaker pulse delivery, change the dose rate of implanted drug delivery devices, and induce noise in electronic hearing aids) (Gustrau et al. 2002)

- EMI with other medical electronic devices. This may have severe consequences in the case of patient monitors (e.g., ECG monitor) or life-supporting devices (e.g., infusion pumps). EMI can cause critical care medical devices to malfunction with severe consequences such as inappropriate therapy, patient injury, and mortality (COMAR 1998).

- Degradation of biosignals (e.g., electrocardiogram and electroencephalogram) during patient monitoring

\subsubsection{RF Surgery}

During RF surgery, in the worst case, a surgeon's hands are exposed to a continuous and pulsed RF wave whose magnetic field strength is $0.75 \mathrm{~A} / \mathrm{m}$ (electric field strength $400 \mathrm{~V} / \mathrm{m}$ ). Occasionally, stray radiation may exceed ICNIRP's occupational exposure guidelines, especially close to the patient return plate. Of particular concern is 
electromagnetic interference (EMI) with life-support devices (ventilators and electrocardiographic devices), which may fail to operate correctly. Most clinically relevant interference occurred when an RF surgery device was used within $0.3 \mathrm{~m}$ of other medical equipment (De Marco and Maggi 2006). In the vicinity of electrosurgical devices, medical staff could be critically exposed up to a distance of $70 \mathrm{~cm}$ from the active electrode and its supplying cable. Considerable overexposure was encountered when the electrode cable was placed directly on the surgeon's body. The current flowing through the arm of the surgeon holding the electrode can exceed the permissible value of $40 \mathrm{~mA}$, and electric field strength may exceed $1000 \mathrm{~V} / \mathrm{m}$ (Gryz and Karpowicz 2006).

\subsubsection{Diathermy}

In SWD, 10 units in six departments were examined with regard to their stray fields. It was found that stray fields from capacitive SWD fell below operator limits only at $2 \mathrm{~m}$ (electric field strength 4.8-39.8 V/m; magnetic field strength $0.015-0.072 \mathrm{~A} / \mathrm{m}$ ) and at $1 \mathrm{~m}$ for inductive SWD $(0-36 \mathrm{~V} / \mathrm{m} ; 0.01-0.065 \mathrm{~A} / \mathrm{m})$. Capacitive pulsed SWD fields fell below the limits at $1.5 \mathrm{~m}(1.2-19.9 \mathrm{~V} / \mathrm{m} ; 0.002-0.045 \mathrm{~A} / \mathrm{m})$ and at $1 \mathrm{~m}$ for inductive pulsed SWD $(0.7-4.0 \mathrm{~V} / \mathrm{m} ; 0.009-0.03 \mathrm{~A} / \mathrm{m})$. An extra $0.5 \mathrm{~m}$ was required before fields fell below recommended limits for other personnel. These results demonstrate that under a worst-case scenario emissions from SWD exceed the guidelines for operators at distances currently recommended as safe (Shields, O'Hare, and Gormley 2004).

In eight physiotherapy departments, 11 microwaves, four SWD, and 15 magneto therapy devices were analyzed, and cases of exposures exceeding occupational limits were identified (Maccá et al. 2008).

\subsubsection{Risk Management}

To manage risks from such interference several measures may be taken:

- Developing guidelines for use (Gombotz et al. 2009; Joshi 2009) and performing treatment planning (Habash et al. 2007) to minimize unintended exposure during treatment (e.g., diathermy and hyperthermia)

- Identifying zones of restricted access

- Warning against EMF around powerful EMF sources (e.g., MRI)

- Preventing excessive exposure of workers by workplace assessment, training, exposure minimization, and/or shielding as required by worker protection legislation (e.g., EC 2004)

- Improving immunity of medical devices against EMI as required by the electromagnetic compatibility (EMC) standard for medical devices (IEC 60601-1-2) or for medical implants (IEC 45502-1). As an example, in the frequency range $150 \mathrm{kHz}-$ $80 \mathrm{MHz}$, the required immunity level is $E_{\mathrm{i}}=3 \mathrm{~V} / \mathrm{m}$ for general medical devices and $10 \mathrm{~V} / \mathrm{m}$ for life-supporting medical devices (IEC 60601-1-2). Implanted cardiac pacemakers should not work outside the manufacturer's tolerances up to a signal input interference voltage of $2 \mathrm{mV}_{\mathrm{pp}}$ (IEC 45502-1) 
- Maintaining a safe distance from RF EMF emitting devices. In the frequency range $800 \mathrm{MHz}-2.5 \mathrm{GHz}$, depending on the RF EMF output power $P$ and the immunity level $E_{\mathrm{i}}$, the medical devices should maintain a safe distance $d$ :

$$
d=\frac{k \times \sqrt{P}}{E_{\mathrm{i}}}
$$

with $k=23$ for life-supporting and $k=7$ for all other medical devices. As an example, this results in a safe distance of medical devices to GSM handsets of $1.2 \mathrm{~m}$ for $900 \mathrm{MHz}$ (output power, $0.25 \mathrm{~W}$ ) or $0.8 \mathrm{~m}$ for $1.8 \mathrm{GHz}$. Walkie talkies used by rescue services should even be kept at a distance of $5.1 \mathrm{~m}$ (IEC 60601-1-2)

- Immunity of a device cannot be recognized from its appearance. Therefore, maintaining a safe distance is an important preventive measure. Because walls are no major shield, this needs to be observed also in rooms and aisles adjacent to critical locations (e.g., intensive care units)

- Reducing the ambient field level in locations with critical assessment of biosignals. Standards limit rms power frequency magnetic levels for biosignal recording to $141 \mathrm{nT}$ for electrocardiograms (ECG), $71 \mathrm{nT}$ for electroencephalograms (EEG), and $35 \mathrm{nT}$ for electromyograms (EMG) (ÖVE E 8007). This requires already timely measures such as appropriate site selection, design, construction, and layout of health care facilities (Leitgeb 2010; Cesar, Ramos, and Tahan 2009).

\subsection{Summary}

Medical applications make use of EMFs over a wide range of frequencies and amplitudes (Figure 6.9). Medical device development is characterized by the fact that exposure of the patient may not only be caused by external devices operated at some distance or in contact to the body such as transmitting coils for delivering energy and commands to implanted stimulators. Medical devices may also be incorporated, and they become EMF sources in close contact to tissue and organs such as transmitters of electronic implants for data exchange, permanent magnets for navigation, or magnetic nanoparticles for tracking, targeting, heating, and MRI contrast enhancement.

Magnetic therapy devices apply static and alternating magnetic fields in the frequency range of a few $\mathrm{Hz}$ until several $\mathrm{kHz}$. With regard to amplitudes, two ranges can be differentiated: Weak-field applications below existing limits with lacking evidence of clinical efficiency, and emissions above limits usually for wound healing and bone fracture treatment. Diathermy and hyperthermia applications are restricted to ISM frequencies and generate intended exposures of the patient well above limits but may also cause relevant exposures of medical staff and unintended exposure of sensitive regions within the patient. The use of ferromagnetic parts and superparamagnetic (nano)particles offered new possibilities of targeted EMF heating for cancer treatment or drug delivery and moved tumor tracking. Required RF EMF amplitudes increase with decreasing size of particles. A major EMF source is MRI imaging with high static magnetic fields and intense RF EMF as well as high magnetic gradients with development toward higher static fields as well as toward low-cost low-field devices. 


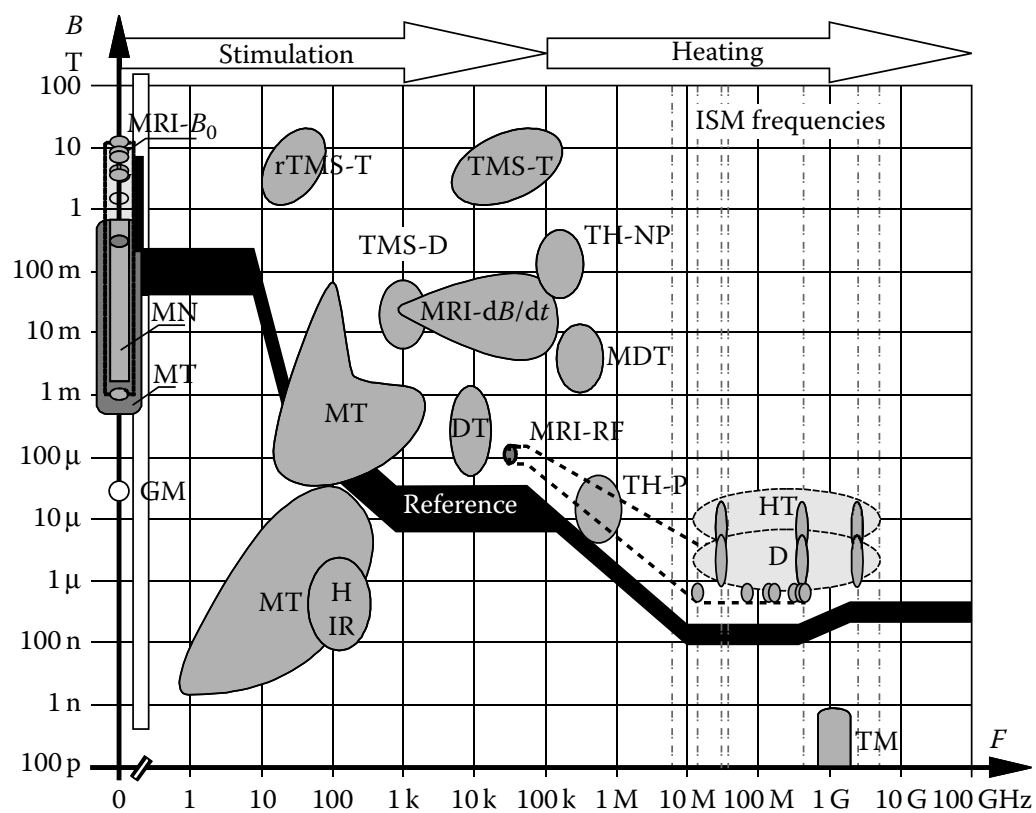

FIGURE 6.9 Magnetic emissions from medical devices. Reference $=$ reference values limiting exposure of the general population (lower boundary) and workers (upper boundary); $\mathrm{GM}=$ geomagnetic field; $\mathrm{MT}=$ magnet therapy; $\mathrm{MN}=$ magnetic navigation; $\mathrm{MRI}=$ magnetic resonance imaging; TMS-T $=$ transcranial therapeutic magnetic stimulation; $\mathrm{rTMS}-\mathrm{T}=$ repetitive transcranial therapeutic magnetic stimulation; TMS-D = transcranial diagnostic magnetic stimulation; TH-NP = teleheating of nanoparticles; TH-P = teleheating of ferromagnetic parts; $\mathrm{MDT}=$ magnetic drug targeting; DT = magnetic device tracking; D = diathermy; HT = hyperthermia; and $\mathrm{TM}=$ telemedicine.

Although even high exposures of patients are justified if risks are outweighed by benefits in several applications, there is a need to prevent staff, accompanying persons, or the fetus from unintended excess exposure. It is an ongoing challenge to identify and quantify critical exposure scenarios and implement adequate risk management strategies to minimize unnecessary exposure and protect patients and workers from potential health risks.

\section{References}

Bindonisti, C. P., J. Choukiefe, G. Tastevin, A. Vignaud, and P. J. Nacher. 2004. MRI of the lung using hyperpolarized ${ }^{3} \mathrm{He}$ at very low magnetic field (3mT). Magn Res Mater Phys 16:255-8.

Cadeddu, J., R. Fernandez, M. Desai, R. Bergs, C. Tracy, S. J. Tang, P. Rao, M. Desai, and D. Scott. 2009. Novel magnetically guided intra-abdominal camera to facilitate laparoendoscopic single-site surgery: Initial human experience. Surg Endosc 23(8):1894-9. 
Cebrián, J. L., P. Gallego, A. Francés, P. Sánchez, E. Manrique, F. Marco, and L. LópezDurán. 2010. Comparative study of the use of electromagnetic fields in patients with pseudoarthrosis of tibia treated by intramedullary nailing. Int Orthop 34(3):437-40.

Cesar, M., G. Ramos, and C. M. V. Tahan. 2009. An assessment of the electric power quality and electrical installation impacts on medical electrical equipment operations at health care facilities. Am J Appl Sci 6(4):638-45.

Chen, R., D. Cros, A. Curra, V. Di Lazzaro, J. P. Lefaucheur, M. R. Magistris, K. Mills et al. 2008. The clinical diagnostic utility of transcranial magnetic stimulation: Report of an IFCN committee. Clin Neurophysiol 119(3):504-32.

Chen, J., P. I. Hoff, E. Solheim, P. Schuster, M. K. Off, and O. J. Ohm. 2010. Magnetic navigation for ablation of cardiac arrhythmias. Tidsskr Nor Laegeforen 130(15):1467-70.

Colbert, A. P., H. Wahbeh, N. Harlingg, E. Connelly, H. C. Schiffke, C. Forsten, W. L. Gregory et al. 2009. Static magnetic field therapy: A critical review of treatment parameters. Evid Based Complement Alternat Med 6(2):133-9.

COMAR. 1998. Radiofrequency interference with medical devices. IEEE Med Biol Mag 17(2):111-4.

Costantino, C., F. Pogliacomi, F. Passera, and G. Concari. 2007. Treatment of wrist and hand fractures with natural magnets: Preliminary report. Acta Biomed 78(3):198-203.

Couturier J. L. 2005. Efficacy if rapid-rate repetitive transcranial magnetic stimulation in the treatment of depression: A systematic review and meta-analysis. J Psych Neurosci 30(2):83-90.

Crozier, S., and P. Chadwick. 2007. Assessment of electromagnetic fields around magnetic resonance imaging (MRI) equipment. Health and Safety Executive Report RR570, Norwich UK.

Crozier, S., and F. Liu. 2005. Numerical evaluation of the fields induced by body motion in or near high-field MRI scanners. Prog Biophys Mol Biol 87:267-78.

Dahmani, Ch., S. Götz, T. Weyh, R. Renner, M. Rosenecker, and C. Rudolph. 2009. Respiration triggered magnetic drug targeting in the lungs. Conf Proc IEEE Eng Med Biol Soc 5440-3. Minneapolis, USA.

De Marco, M., and S. Maggi. 2006. Evaluation of stray radiofrequency radiation emitted by electrosurgical devices. Phys Med Biol 51(14):3347-58.

Delvaux, M., and G. Gay. 2008. Capsule endoscopy: Technique and indications. Best Pract Res Clin Gastroenterol 22(5):813-37.

Demirtas-Tatlidede, A., C. Freitas, J. R. Cromer, L. Safar, D. Ongur, W. S. Stone, L. J. Seidman, J. D. Schmahmann, and A. Pascual-Leone. 2010. Safety and proof of principle study of cerebellar vermal theta burst stimulation in refractory schizophrenia. Schizophr Res 124(1-3):91-100.

Dewey W. C., L. E. Hopwood, S. A. Sapareto, and L. D. Gerweck. 1982. Cellular responses to combinations of hyperthermia and radiation. Radiology 123:463-74.

Dlabac-de Lange, J. J., R. Knegtering, and A. Aleman. 2010. Repetitive transcranial magnetic stimulation for negative symptoms of schizophrenia: Review and meta-analysis. J Clin Psychiatry 71(4):411-8.

Durand, E., G. Guillot, L. Darasse, G. Tastevin, P. J. Nacher, A. Viggnaud, D. Vatttalo, and J. Bitton. 2002. CPMG measurements and ultrafast imaging in human lungs with hyperpolarized Helium-3 at low field (0.1T). Magn Reson Med 47:75-87. 
EC. 1993. EU Directive 93/42/EEC: Medical Devices. Amended by MDD 2007/47/EC European Commission, OJEU L 169/9. Brussels, Belgium.

EC. 1999. Limiting the public exposure to electromagnetic fields $(0 \mathrm{~Hz}-300 \mathrm{GHz})$. Council recommendation 1999/519/EC. Brussels, Belgium.

EC. 2004. Minimum health and safety requirements regarding the exposure of workers to the risk arising from physical agents (electromagnetic fields). EU directive 2004/40/ EC. Brussels, Belgium.

EC. 2007. EU Directive 2007/47/EC: Medical Devices. Amendment to MDD 93/42/EEC. European Commission, OJEU L 247/31. Brussels, Belgium.

Eliakim, R. 2010. Video capsule endoscopy of the small bowel. Curr Opin Gastroenterol 26(2):129-33.

FDA. 2006. Reclassigfication petition for the non-invasive bone growth stimulator. Docket \# 2005P-0121.

Finegold, L. 2006. Magnet therapy. Extraordinary claims, but no proved benefits. BMJ 332(7532):4.

Flamm, B. L. 2006. Magnet therapy: A billion-dollar boondoggle. Sceptical Inquire 30:26-8.

Flamm, B. L. 2007. Magnetic therapy: Healing or hogwash? Anesth Analg 104(2): 290-4.

Gan, J. C., and P. A. Glazer. 2006. Electrical stimulation therapies for spinal fusions: Current concepts. Eur Spine J 15(9):1301-11.

Gombotz, H., M. Anelli Monti, N. Leitgeb, M. Nürnberger, and B. Strohmer. 2009. Perioperative management of patients with implanted pacemakers or cardioverterdefibrillators. Anaesthesist 58:465-98.

Grana, D. R., H. J. Marcos, and G. A. Kokubu. 2008. Pulsed electromagnetic fields as adjuvant therapy in bone healing and peri-implant bone formation: an experimental study in rats. Acta Odontol Latinoam 21(1):77-83.

Gryz, K., and J. Karpowicz. 2006. Electromagnetic hazards from electrosurgeryassessment of occupational exposure to electromagnetic field and currents induced in the body. Rocz Panstw Zakl Hig 57(2):165-75.

Gustrau, F., A. Bahr, S. Goltz, and S. Eggert. 2002. Active medical implants and occupational safety-measurement and numerical calculation of interference voltage. Biomed Technol 247(Suppl 1 Pt 2):656-9.

Habash, R. W. Y., R. Bansal, D. Krewski, and H. T. Alhafid. 2006. Thermal therapy, Part 1: An introduction to thermal therapy. Crit Rev Biomed Eng 344(6):459-89.

Habash, R. W. Y., R. Bansal, D. Krewski, and H. T. Alhafid. 2007. Thermal therapy, Part 4: Electromagnetic and thermal dosimetry. Crit Rev Biomed Eng 344(6):123-82.

Hallmark, B., N. J. Darton, T. James, P. Agrawal, and N. K. H. Slater. 2010. On the magnetic field strength required to capture superparamagnetic nanoparticles in flow systems. J Nanopart Res doi:12:2951-65.

Hand, J. W., Y. Li, E. L. Thomas, M. A. Rutherford, and J. V. Hajanal. 2006. Prediction of specific absorption rate in mother and fetus associated with MRI examinations during pregnancy. Magn Reson Med 55:883-93.

HC. 2008. Short wave diathermy. Guidelines for limiting radiofrequency exposure. Health Canada, Safety Code 25. www.hc-sc.gc.ca (Last accessed September 28, 2010). 
Heppner, F. 1982. New technologies to combat malignant tumours of the brain. Anticancer Res 2(1-2):101-9.

HMR. 2004. Guidelines on remedies. German Fed Board Phys Health Insur BAnz 108a, June 9, Germany.

HPA. 2008. Protection of Patients and Volunteers Undergoing MRI Procedures. Chilton: RCE-7, Health Protection Agency.

IARC. 2002. Monographs on the Evaluation of Cancer Risks to Human, Volume 80: NonIonizing Radiation, Part 1: Static and Extremely Low-Frequency (ELF) Electric and Magnetic Fields. Geneva. Switzerland: WHO.

ICNIRP. 1998. Guidelines for limiting exposure to time-varying electric, magnetic and electromagnetic fields (up to $300 \mathrm{GHz}$ ). Health Phys 74(4):494-522.

ICNIRP. 2004. Statement on medical magnetic resonance (MR) procedures: Protection of patients. Health Phys 87(2):197-216.

ICNIRP. 2009a. Guidelines on limits of exposure to static magnetic fields. Health Phys 96:504-14.

ICNIRP. 2009b. Amendment to the ICNIRP "Statement on medical magnetic resonance (MR) procedures: Protection of patients." Health Phys 97(3):259-61.

IEC 60601-1. 2005. Medical Electrical Equipment. Part 1: General Requirements for Basic Safety and Essential Performance. Geneva, Switzerland: Safety Standard International Electrotechnology Communications.

IEC 60601-1-2. 2007. Medical Electrical Equipment. Part 1-2: General Requirements for Basic Safety and Essential Performance-Collateral Standard: Electromagnetic Compatibility-Requirements and Tests. Geneva, Switzerland: Safety Standard International Electrotechnology Communications.

IEC 60601-2-3 + A1. 1999. Medical Electrical Equipment. Part 2: Particular Requirements for the Safety of Short-Wave Therapy Equipment. Geneva, Switzerland: Safety Standard International Electrotechnology Communications.

IEC 60601-2-4. 2002. Medical Electrical Equipment. Part 2: Particular Requirements for the Safety of Cardiac Defibrillators. Geneva, Switzerland: Safety Standard International Electrotechnology Communications.

IEC 60601-2-6. 1984. Medical Electrical Equipment. Part 2: Particular Requirements for the Safety of Microwave Therapy Equipment. Geneva, Switzerland: Safety Standard International Electrotechnology Communications.

IEC 60601-2-33. 2010. Medical Electrical Equipment. Part 2-33: Particular Requirements for Basic Safety and Essential Performance of Magnetic Resonance Equipment for Medical Diagnosis. Geneva, Switzerland: Safety Standard International Electrotechnology Communications.

IEEE. 2002a. Recommended practice for measurements and computations of radio frequency electromagnetic fields with respect to human exposure to such fields, 100 kHz-300 GHz. IEEE standard C.95.3-2002. New York.

IEEE. 2002b. Standard for safety levels with respect to human exposure to electromagnetic fields 0-3 kHz. IEEE standard C.95.6-2002. New York.

IEEE. 2005. Standard for safety levels with respect to human exposure to radio frequency electromagnetic fields $3 \mathrm{kHz}$ to $300 \mathrm{GHz}$. IEEE standard C.95.1-2005. New York. 
Irnich, W. 1994. Electrostimulation by time-varying magnetic fields. Magnet Reson Mater Phys Biol Med 2(1):43-9.

Irnich, W., J. M. de Bakker, and H. J. Bisping. 1978. Electromagnetic interference in implantable pacemakers. Pacing Clin Electrophysiol J 1(1):52-61.

ISO 20473. 2007. Optics and Photonics. Spectral Bands. Geneva, Switzerland: International Organization for Standardization.

Jefferys, J. G. R., J. Deans, M. Bikson, and J. Fox. 2003. Effects of weak electric fields on the activity of neurons and neuronal networks. Radiat Protein Dosim 106:321-3.

Johannsen, M., B. Thiesen, P. Wust, and A. Jordan. 2010. Magnetic nanoparticle hyperthermia for prostate cancer. Int J Hyperthermia 26(8):790-5.

Joshi, G. P. 2009. Perioperative management of outpatients with implantable cardioverter defibrillators. Curr Opin Anaesthesiol 22(6):701-4.

Kaman O., E. Pollert, P. Veverka, M. Veverka, E. Hadová, K. Knizek, M. Marysko, P. Kaspar, M. Klementová, V. Grünwaldová, S. Vasseur, R. Epherre, S. Mornet, G. Goglio, and E. Duguet. 2009. Silica encapsulated manganese perovskite nanoparticles for magnetically induced hyperthermia without the risk of overheating. Nanotechnology 20:275610(1)-275610(7).

Katagiri, K., M. Nakamura, and K. Koumoto. 2010. Magnetoresponsive smart capsules formed with polyelectrolytes, lipid bilayers and magnetic nanoparticles. ACS Appl Mater Interfaces 2(3):768-73.

Keall, P. J., A. Sawant, B. Cho, D. Ruan, J. Wu, P. Poulsen, J. Petersen, L. J. Newell, H. Cattell, and S. Korreman. 2011. Electromagnetic-guided dynamic multileaf collimator tracking enables motion management for intensity-modulated arc therapy. Int J Radiat Oncol Biol Phys 79(1):312-20.

Kinuishi, Y., H. Yamagushi, and T. S. Tenforde. 1996. Theoretical analysis of magnetic field interactions with aortic blood flow. Bioelectromagnetics 17:21-3.

Kroeling, P., A. Gross, C. H. Goldsmith, S. J. Burnie, T. Haines, N. Graham, and A. Brant. 2009. Electrotherapy for neck pain. Cochrane Database Syst Rev 7(4):CD004251.

Leitgeb, N. 2007. Metrological assessment of general population's exposure to ELF and RF electromagnetic fields generated from the use of electrical appliances. BfS Research Report StSch 4446. Munich, Gernamy.

Leitgeb, N. 2008. Assessment of multiple frequency ELF electric and magnetic field exposure. Phys Med Biol 53:431-43.

Leitgeb, N. 2010. Safety of Electromedical Devices. Law-Risks-Opportunities. Vienna, Austria: Springer.

Leitgeb, N., A. Omerspahic, and F. Niedermayr. 2010. Exposure of non-target tissue in medical diathermy. Bioelectromagnetics 31:12-9.

Lerman, Y., R. Jacubovich, and M. S. Green. 2001. Pregnancy outcome following exposure to shortwaves among female physiotherapists in Israel. Am J Ind Med 39(5):499-506.

Li, C. Y., and C. K. Feng. 1999. An evaluation of radio frequency exposure from therapeutic diathermy equipment. Ind Health 37(4):465-8.

Li, F., J. A. Leighton, and V. K. Sharma. 2007. Capsule endoscopy: A comprehensive review. Minerva Gastroenterol Dietol 53(3):257-72. 
Lin, J. C., and Z. Wang. 2010. Acoustic pressure waves induced in human heads by RF pulses from high-field MRI scanners. Health Phys 98(4):603-13.

Liu, H. L., M. Y. Hua, H. W. Yang, C. Y. Huang, P. C. Chu, J. S. Wu, I. C. Tseng et al. 2010. Magnetic resonance monitoring of focused ultrasound/magnetic nanoparticle targeting delivery of therapeutic agents to the brain. Proc Natl Acad Sci USA 107(34):15205-10.

Maccà, I., M. L. Scapellato, M. Carrieri, A. Pasqua di Bisceglie, B. Saia, and G. B. Bartolucci. 2008. Occupational exposure to electromagnetic fields in physiotherapy departments. Radiat Prot Dosimetry 128(2):180-90.

Martin, C. J., H. M. McCallum, and B. Heaton. 1990. An evaluation of radiofrequency exposure from therapeutic diathermy equipment in the light of current recommendations. Clin Phys Physiol Meas 11(1):53-63.

Matlashov, A. N., V. S. Zotev, R. H. Kraus Jr., H. Sandin, A. V. Urbaitis, P. I. Volegov, and M. A. Espy. 2009. SQUIDs for magnetic resonance imaging at ultra-low magnetic field. PIERS Online 5(5):466-70.

McGill, S. L., C. L. Cuylear, N. L. Adolphi, M. Osinski, and H. D. Smyth. 2009. Magnetically responsive nanoparticles for drug delivery applications using low magnetic field strengths. IEEE Trans Nanobiosci 8(1):33-42.

MFTGV. 2003. Ordinance of magnetic field therapy devices. Austrian BGBl II. 343/2003.

Mogg, A., R. Purvis, S. Eranti, F. Contell, J. P. Taylor, T. Nicholson, R. G. Brown, and D. M. McLoughlin. 2007. Repetitive transcranial magnetic stimulation for negative symptoms of schizophrenia: A randomized controlled pilot study. Schizophr Res 93(1-3):221-8.

Moseley, H., and M. Davison. 1981. Exposure of physiotherapists to microwave radiation during microwave diathermy treatment. Clin Phys Physiol Meas 2(3):217-21.

NHMRC. 1985. Code of practice for the safe use of shortwave diathermy units. Australian National Health and Medical Research Council, www.nhmrc.gov.au

Nikfarjam, M., V. Muralidharan, and C. Christophi. 2005. Mechanisms of focal heat destruction of liver tumors. J Surg Res 127(2):208-23.

ÖVE/ÖNORM E 8007. 2007. Electrical Installations in Hospitals and Locations for Medical Use Outside Hospitals. Vienna, Austria: Austrian safety standard, ASI.

Pastore, A. P., A. De Leo, R. De Leo, G. Della Chiara, V. Mariani Primiani, F. Moglie, and G. Cerri. 2011. A realistic model for the analysis of heart magnetic stimulation. IEEE Trans Biomed Eng 58(2):291-300.

Pediaditis, M., N. Leitgeb, and R. Cech. 2008. RF-EMF exposure of fetus and mother during magnetic resonance imaging. Phys Med Biol 53:7187-95.

Pennes, H. H. 1948. Analysis of tissue and arterial blood temperatures in the resting human forearm. Appl Physiol 1(2):93-121.

Perretti, A., A. De Rosa, L. Marcantonio, V. Iodice, A. Estraneo, F. Manganelli, B. Garavaglia, A. Filla, L. Santoro, and G. De Michele. 2011. Neurophysiological evaluation of motor corticospinal pathways by TMS in idiopathic early-onset Parkinson's disease. Clin Neurophysiol 122(3):546-9.

Pieber, K., M. Herceg, and T. Paternostro-Sluga. 2010. Electrotherapy for the treatment of painful diabetic peripheral neuropathy: A review. J Rehabil Med 42(4):289-95. 
Pinski, S. L., and R. G. Trohman. 2002. Interference in implanted cardiac devices, part II. Pacing Clin Electrophysiol 25(10):1496-509.

Pittler, M. H., E. M. Brown, and E. Ernst. 2007. Static magnets for reducing pain: Systematic review and meta-analysis of randomized trials. CMAJ 177(7):736-42.

Polyak, B., and G. Friedman. 2009. Magnetic targeting for site-specific drug delivery: Applications and clinical potential. Expert Opin Drug Deliv 6(1):53-70.

Quellet-Hellstrom, R., and W. F. Stewart. 1993. Miscarriages among female physical therapists who report using radio- and microwave-frequency electromagnetic radiation. Am J Epidemiol 138(10):775-86.

Ramachandran, N., and K. Mazuruk. 2004. Magnetic microspheres and tissue model studies for therapeutic applications. Ann N Y Acad Sci 1027:99-109.

Raman, J. D., D. J. Scott, and J. A. Cadeddu. 2009. Role of magnetic anchors during laparoendoscopic single site surgery and NOTES. J Endourol 23(5):781-6.

Reilly, J. P. 1998. Applied Bioelectricity: From Electric Stimulation to Electropathology, 194411. New York: Springer.

Roth, Y., A. Amir, Y. Leykovitz, and A. Zangen. 2010. Three-dimensional distribution of the electric field induced in the brain by transcranial magnetic stimulation using figure-8 and deep H coils. J Clin Neurophys 24(1):2-9.

Ruohonen, J., and J. Karhu. 2010. Navigated transcranial magnetic stimulation. Neurophysiol Clin 40(1):7-17.

Sammet, S., R. M. Koch, F. Aguila, and M. V. Knopp. 2010. Residual magnetism in an MRI suite after field-rampdown: What are the issues and experiences? J Magn Reson Image 31(5):1272-6.

Sapareto, S. A., and W. C. Dewey. 1984. Thermal dose determination in cancer therapy. Int J Radiat Oncol Biol Phys 10:787-800.

Seegenschmiedt, M. H., P. Fessenden, and C. C. Vernon, eds. 1996. Thermo-Radiotherapy and Thermo-Chemotherapy. Vol. 1. Berlin, Germany: Springer.

Shah, S. G., and A. Farrow. 2007. Investigation of practices and procedures in the use of therapeutic diathermy: A study from the physiotherapists' health and safety perspective. Physiother Res Int 12(4):228-41.

Shields, N., J. Gormley, and N. O’Hare. 2006. Short-wave diathermy: Current clinical and safety practices. Physiother Res Int 7:191-202.

Shields, N., N. O’Hare, and J. Gormley. 2004. An evaluation of safety guidelines to restrict exposure to stray radiofrequency radiation from short-wave diathermy units. Phys Med Biol 49(13):2999-3015.

Sommer, T., D. Maintz, A. Schmiedel, M. Hackenbroch, U. Hofer, H. Urbach, C. Pavlidis, F. Träber, H. Schild, and M. Höher. 2004. High field MR imaging: Magnetic field interactions of aneurysm clips, coronary artery stents and iliac artery stents with a 3.0 Tesla MR system. Rofo 176(5):731-8.

Stuchly, M. A., M. H. Repacholi, D. W. Lecuyer, and R. D. Mann. 1982. Exposure to the operator and patient during short wave diathermy treatments. Health Phys 42(3):341-66.

Tenforde, T. S., C. T. Gaffey, B. R. Moyer, and T. F. Budinger. 1983. Cardiovascular alterations in Macaca monkeys exposed to stationary magnetic fields. Automedia 144:271-93. 
Trahms, L., and M. Burghoff. 2010. NMR at very low fields. Magn Reson Image 28(8):1244-50.

Tuschl, H., G. Neubauer, H. Garn, K. Duftschmid, N. Winker, and H. Brusl. 1999. Occupational exposure to high frequency electromagnetic fields and its effect on human immune parameters. Int J Occup Med Environ Health 12(3):239-51.

Valdastri, P., C. Quaglia, E. Buselli, A. Arezzo, N. Di Lorenzo, M. Morino, A. Menciassi, and P. Dario. 2010. A magnetic internal mechanism for precise orientation of the camera in wireless endoluminal applications. Endoscopy 42(6):481-6.

Vavken, P., F. Arrich, O. Schuhfried, and R. Dorotka. 2009. Effectiveness of pulsed electromagnetic field therapy in the management of osteoarthritis of the knee: A metaanalysis of randomized controlled trials. J Rehab Med 41(6):406-11.

Wagner, A., K. Schicho, W. Birkfellner, M. Figl, R. Seemann, F. König, and R. Ewers. 2002. Quantitative analysis of factors affecting intraoperative precision and stability of optoelectronic and electromagnetic tracking systems. Med Phys 29(5):905-12.

Watanabe, Y., K. Sato, S. Yukumi, M. Yoshida, Y. Yamamoto, T. Doi, H. Sugishita et al. 2009. Development of a second-generation radiofrequency ablation using sintered $\mathrm{MgFe}(2) \mathrm{O}(4)$ needles and alternating magnetic field for human cancer therapy. Biomed Mater Eng 19(2-3):101-10.

Waterman, M., and I. M. Gralnek. 2009. Capsule endoscopy of the esophagus. J Clin Gastroenterol 43(7):605-12.

Weinstein, J. S., C. G. Varallyay, E. Dosa, S. Gahramanov, B. Hamilton, W. D. Rooney, L. L. Muldoon, and E. A. Neuwelt. 2010. Superparamagnetic iron oxide nanoparticles: diagnostic magnetic resonance imaging and potential therapeutic applications in neurooncology and central nervous system inflammatory pathologies, a review. J Cereb Blood Flow Metab 30(1):15-35.

Wolber, T., S. Ryf, C. Binggeli, J. Holzmeister, C. Brunckhorst, R. Luechinger, and F. Duru. 2007. Potential interference of small neodymium magnets with cardiac pacemakers and implantable cardioverter-defibrillators. Heart Rhythm 4(1):1-4.

Wust, P., H. Stahl, J. Löffel, M. Seebass, H. Fähling, K. Petermann, and R. Felix. 1995. Noninvasive prediction of SAR distributions with electro-optical E-field sensor. Int J Hyperthermia 11:295-310.

Yukumi, S., Y. Watanabe, A. Horiuchi, T. Doi, K. Sato, M. Yoshida, Y. Yamamoto et al. 2009. Repeated inductive heating using a sintered $\mathrm{MgFe} 2 \mathrm{O} 4$ needle for minimally invasive local control in breast cancer therapy. Int J Hyperthermia 25(6):416-21.

Zhang, H., F. Banovac, R. Lin, N. Glossop, B. J. Wood, D. Lindisch, E. Levy, and K. Cleary. 2006. Electromagnetic tracking for abdominal interventions in computer aided surgery. Comput Aided Surg 11(3):127-36.

Zhao, M., C. Liang, A. Li, J. Chang, H. Wang, R. Yan, J. Zhang, and J. Tai. 2010. Magnetic paclitaxel nanoparticles inhibit glioma growth and improve the survival of rats bearing glioma xenografts. Anticancer Res 30(6):2217-23. 


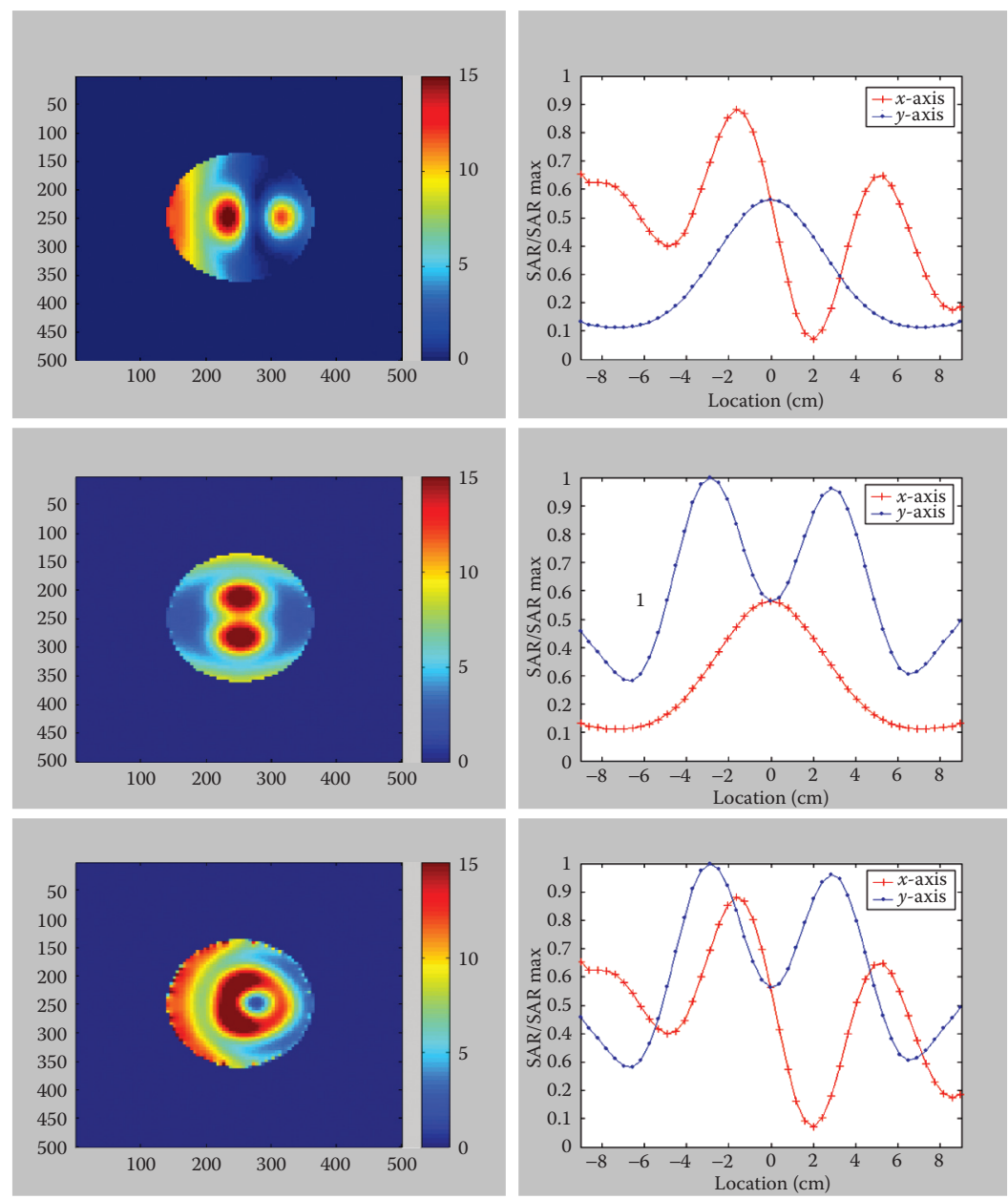

FIGURE 1.11 Specific absorption rate distribution inside a brain sphere under plane-wave exposure: The three columns represent values for the $x y, y z$, and $z x$ planes, respectively. The corresponding line distributions along (red) the direction and transverse to the direction (blue) of propagation are shown at the bottom (diameter $=18 \mathrm{~cm}$ and frequency $=400 \mathrm{MHz}$ ). The direction of wave propagation is along the $x$ axis. 


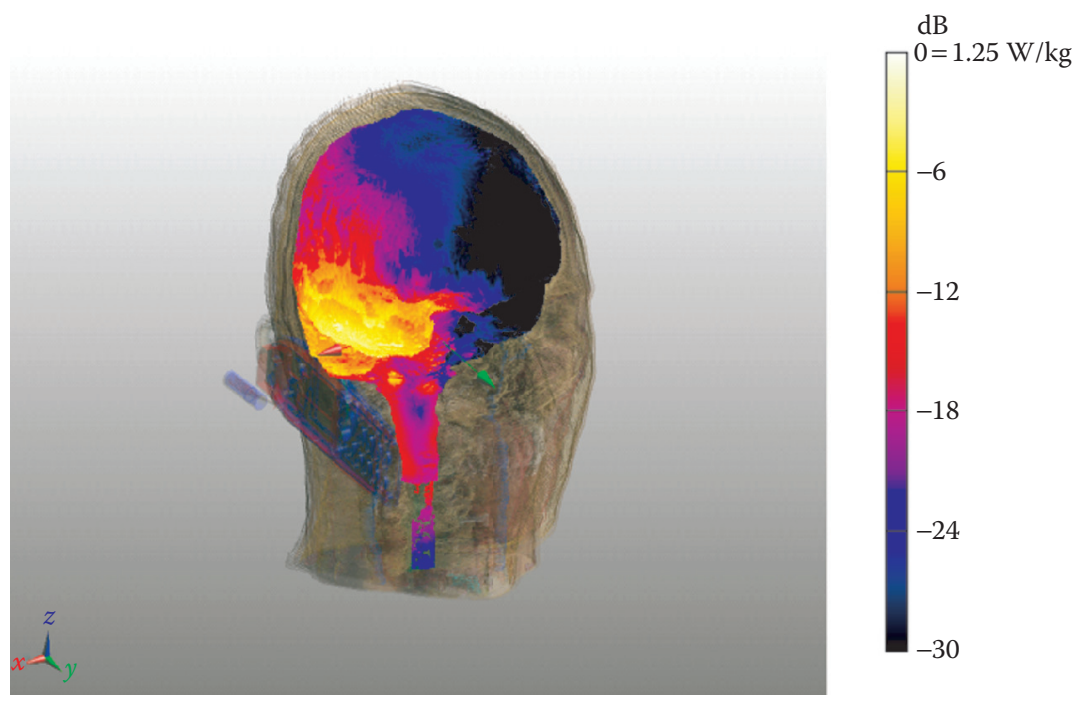

FIGURE 1.12 Distribution of specific absorption rate inside the head of a young European male irradiated by a $902 \mathrm{MHz}$ cellular mobile telephone: Radiofrequency radiation is absorbed mostly in the temporal cortical region of the brain; $0 \mathrm{~dB}$ in the scale equals $1.25 \mathrm{~W} / \mathrm{kg}$. (Courtesy of Myoung Soo Kwon, University of Turku, Finland.)
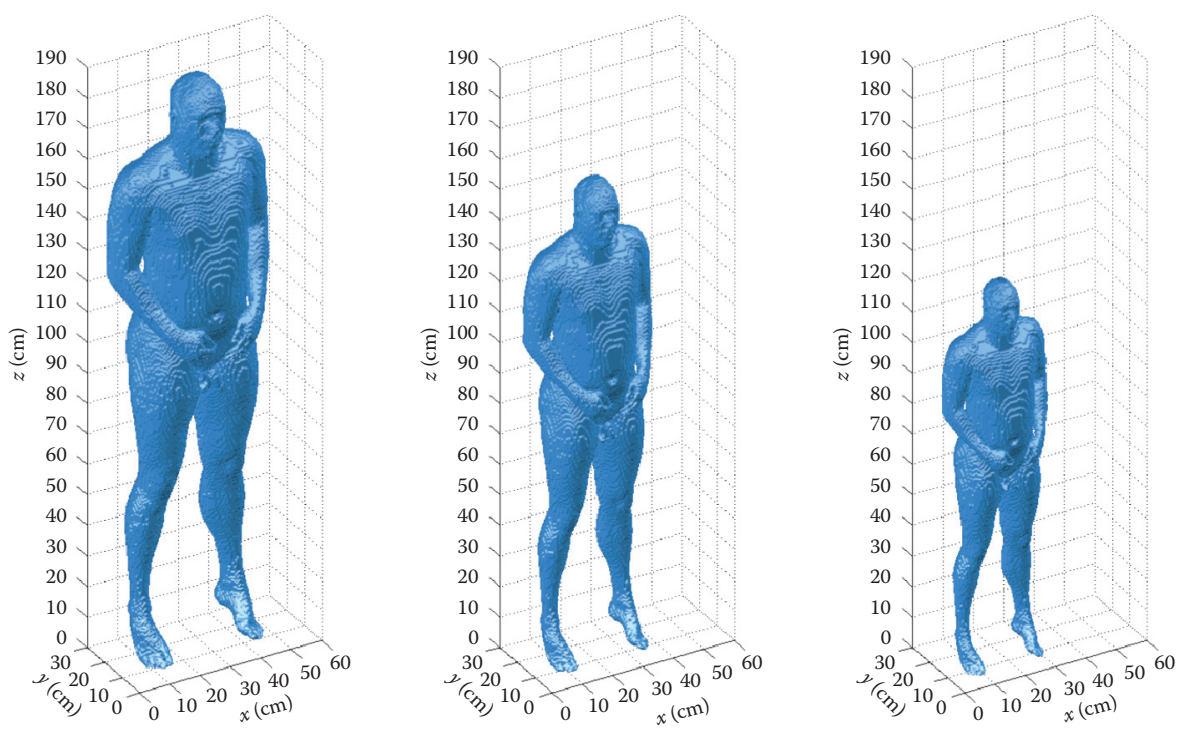

FIGURE 1.13 Adult (left), 13-year-old child-scaled (middle), and 7-year-old child-scaled (right) phantom body models. (From Piuzzi, E., P. Bernardi, M. Cavagnaro, S. Pisa, and J. C. Lin. 2011. Analysis of adult and child exposure to uniform plane waves at mobile communication systems frequencies (900 MHz-3 GHz). IEEE Trans Electromagn Compat 53:38-47. With permission.) 

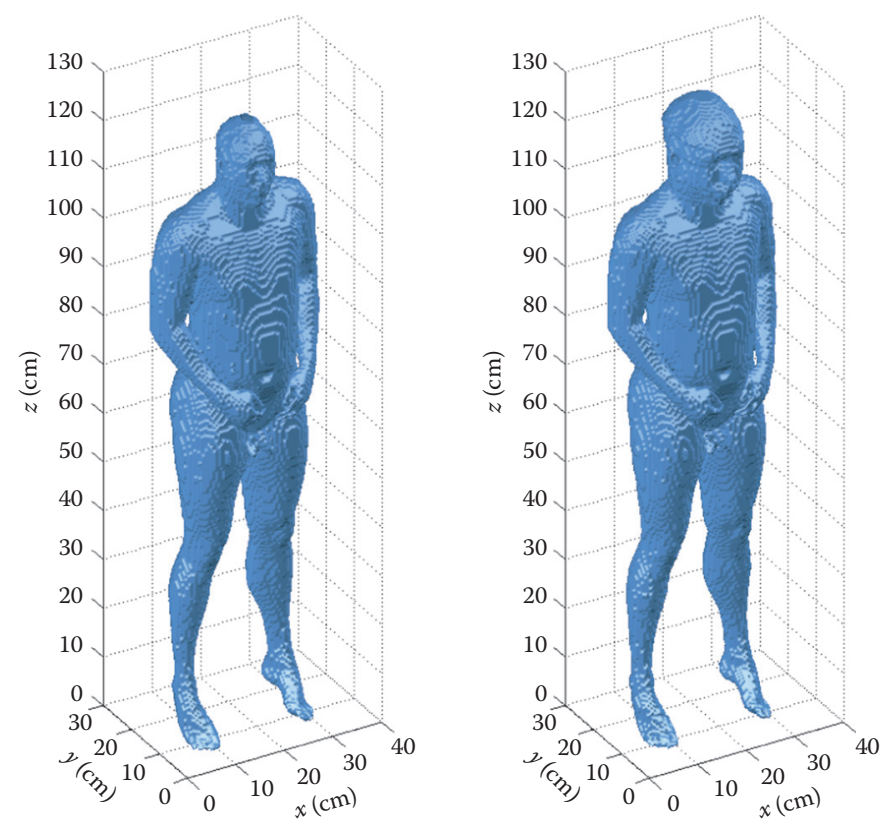

FIGURE 1.14 Comparison of 7-year-old child-scaled (left) and childlike (right) body models. (From Piuzzi, E., P. Bernardi, M. Cavagnaro, S. Pisa, and J. C. Lin. 2011. Analysis of adult and child exposure to uniform plane waves at mobile communication systems frequencies (900 MHz-3 GHz). IEEE Trans Electromagn Compat 53:38-47. With permission.)

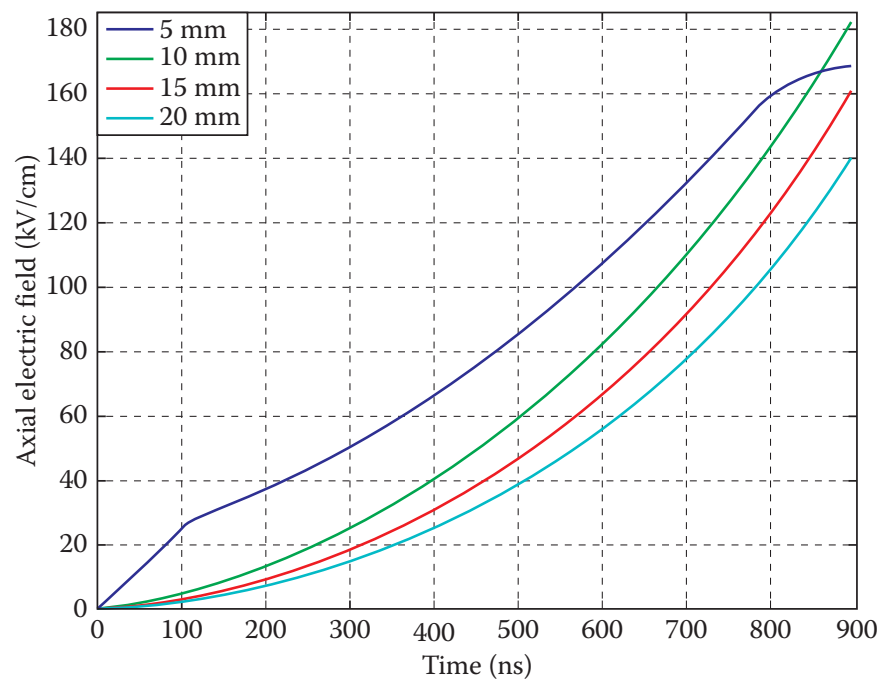

FIGURE 2.11 Calculated axial electric fields at the center of the $2.5-\mu \mathrm{m}$ radius neuron for the trapezoidal external pulse at locations of $5,10,15$, and $20 \mathrm{~mm}$ downstream. The excitation source was taken to be $1 \mathrm{~mm}$ from the nerve axis. 

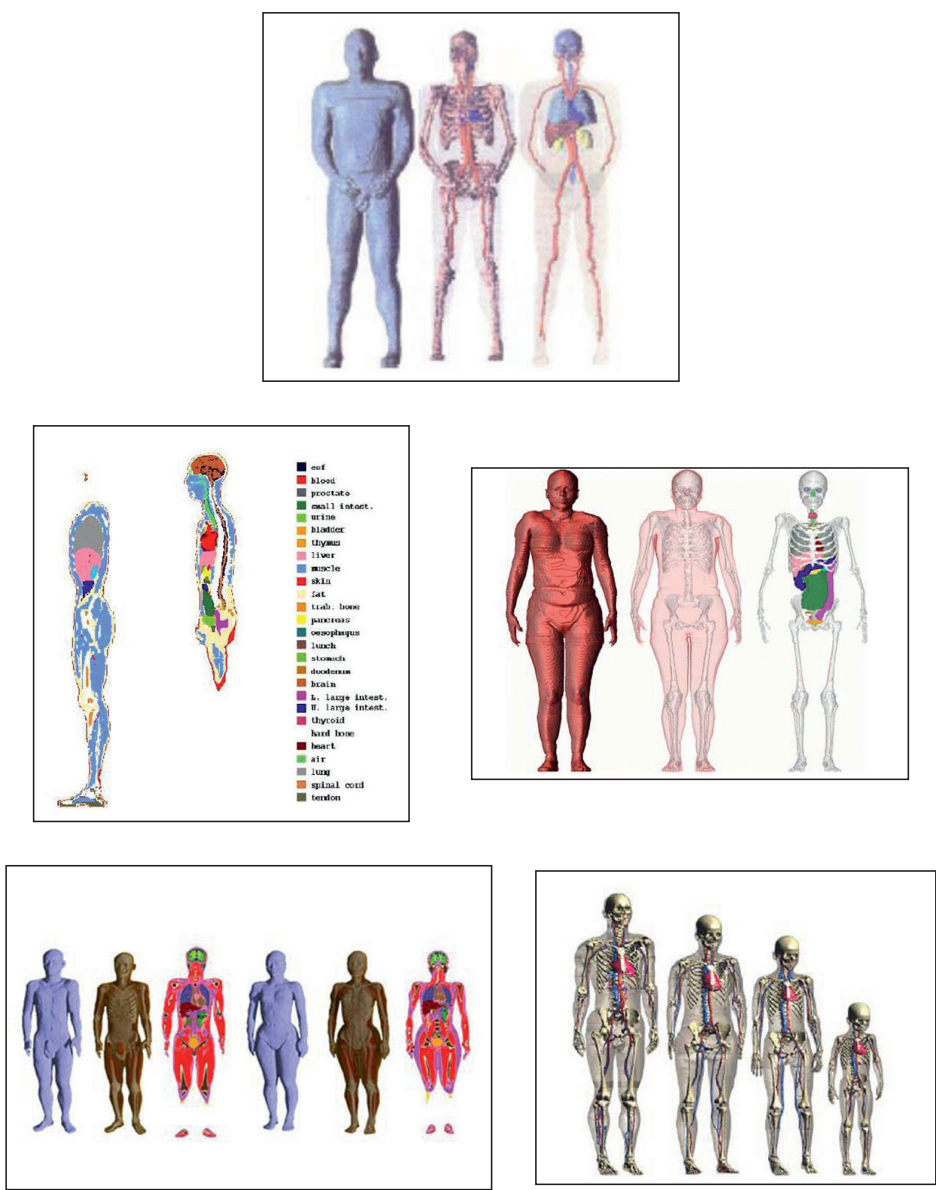

FIGURE 4.5 The visualized realistic phantom human models. The top figure: the external view, skeleton, major vessels, and organs of the three-dimensional anatomical body phantom model developed by the University of Victoria. (From Stuchly, M. A., and T. W. Dawson. 2000. Interaction of low-frequency electric and magnetic fields with the human body. Proc IEEE 88:641-64. With permission.) The middle two figures: high-resolution three-dimensional image of a male body model called NORMAN (left). The body is divided into over 8 million 2-mm voxels. The tissue type of each voxel is defined (http://www.hpa.org.uk/Topics/Radiation/UnderstandingRadiation/ InformationSheets/info_EmfModelling/); volume-rendered images of NAOMI (right). (From Dimbylow, P. J. 2005. Development of the female voxel phantom, NAOMI, and its application to calculations of induced current densities and electric fields from applied low frequency magnetic and electric fields. Phys Med Biol 50:1047-70. With permission.) The bottom two figures: the orthogonal section display and bone enhancement image for both Japanese male and female models (left) (courtesy of T. Nagaoka, NICT) and the high-resolution whole-body human models of the Virtual Family developed by the Foundation for Research on Information Technologies in Society (right). (From Christ, A. et al. 2010. The Virtual Family-development of anatomical CAD models of two adults and two children for dosimetric stimulations. Phys Med Biol 55:N23-38. With permission.) 


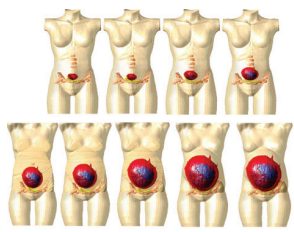

(a)

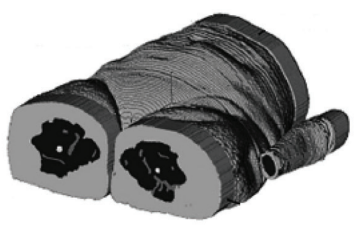

(b)
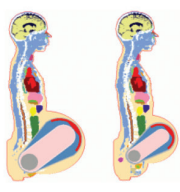

(d)

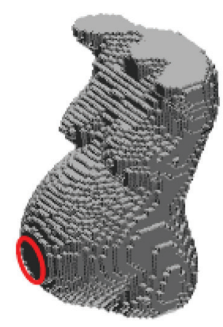

(e)

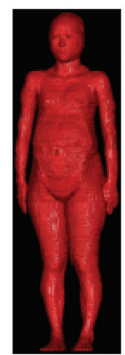

(f) (c)

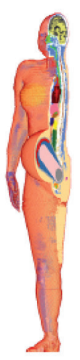

(g)

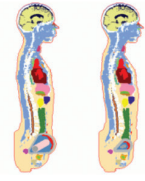

FIGURE 5.8 Analytical pregnant female models developed in the research of (a) From Wu, D., S. Shami, J. Chen, and W. Kainz. 2006. IEEE Trans Microw Theory Tech, 54, 8. With permission. (b) From Hand, J. W., Y. Li, E. L. Thomas, M. A. Rutherford, and J. V. Hajnal. 2006. Magn Reson Med, 52, 802. With permission. (c) From Dimbylow, P. 2007. Phys Med Biol, 52, 802. With permission. (d) From Nagaoka, T. et al. 2007. Phys Med Biol, 52, 45. With permission. (e) From Kainz, W., D. D. Chan, J. P. Casamento, and H. I. Bassen. 2003. Phys Med Biol, 48, 60. With permission. (f) From Nagaoka, T. et al. 2006. Proc IEEE 28th Ann Conf Eng Med Biol Soc, New York. With permission. (g) From Dimbylow, P. 2006. Phys Med Biol, 51, 94. With permission.

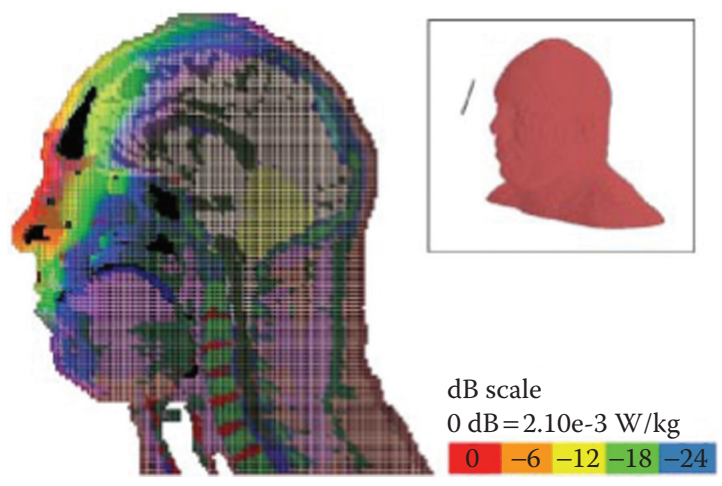

FIGURE 5.22 Contour plot of the $10 \mathrm{~g}$ avg SAR distribution through the body cross-section where the maximum is located, for exposure to a hands-free Bluetooth antenna at a distance of $8 \mathrm{~cm}$ from the head. (From Martinez Burdalo, M., A. Martin, A. Sanchis, and R. Villar. 2009. Bioelectomagnetics, 30, 51. With permission.) 


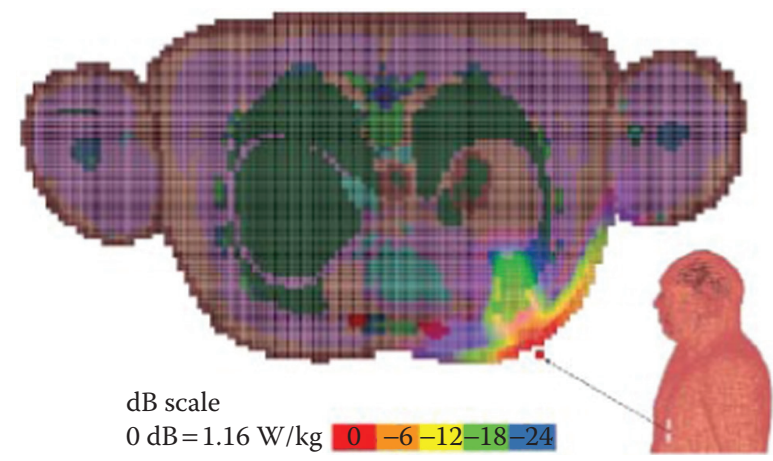

FIGURE 5.23 Contour plot of the $10 \mathrm{~g}$ avg SAR distribution through the body cross-section where the maximum is located, for exposure to a GSM antenna located $5 \mathrm{~mm}$ away from the human chest. (From Martinez Burdalo, M., A. Martin, A. Sanchis, and R. Villar. 2009. Bioelectomagnetics, 30, 51. With permission.)

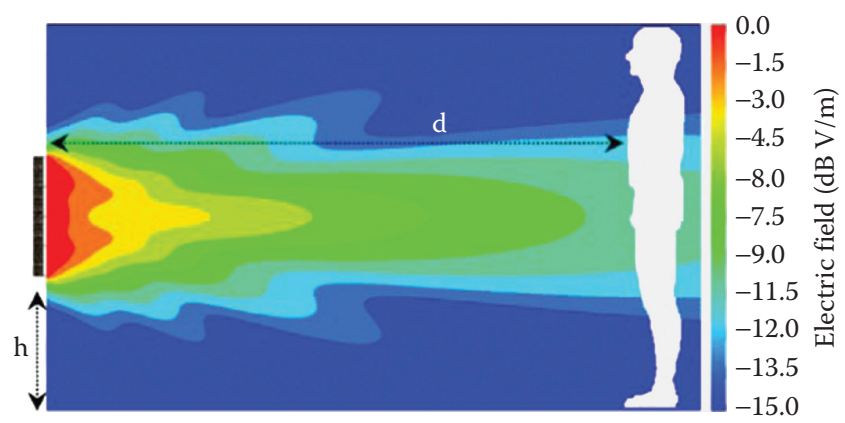

FIGURE 5.25 Electric field distribution radiated by an isolated base-station antenna. (From Lacroux, F. et al. 2008. Ann Telecommun, 63, 64. With permission.)

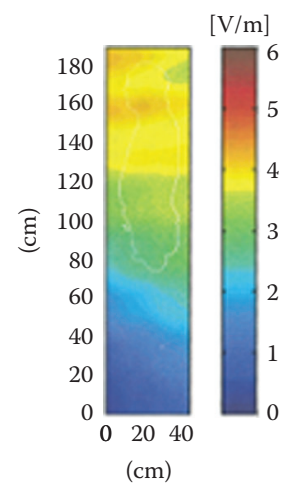

(a)

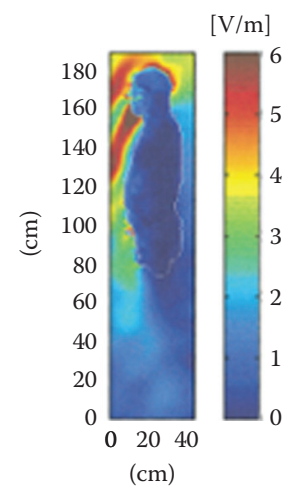

(b)

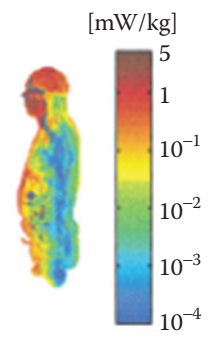

(c)

FIGURE 5.27 Subject on the building roof: (a) Erms when the subject is absent (b) Erms when the subject is present (c) SAR distribution inside the subject. (From Bernardi, P., M. Cavagnaro, S. Pisa, and E. Piuzzi. 2000. IEEE Trans Microw Theory Tech, 48, 11. With permission.) 


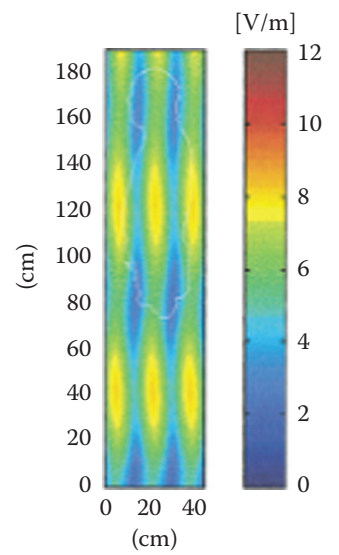

(a)

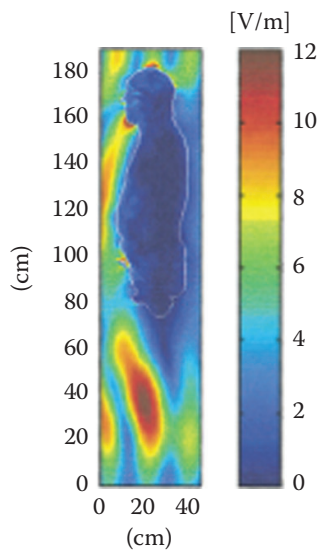

(b)

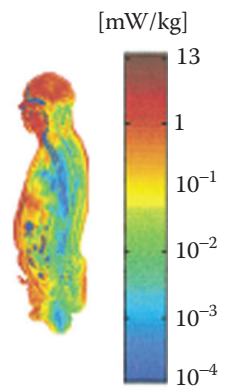

(c)

FIGURE 5.28 Subject on the balcony: (a) Erms when the subject is absent (b) Erms when the subject is present (c) SAR distribution inside the subject. (From Bernardi, P., M. Cavagnaro, S. Pisa, and E. Piuzzi. 2000. IEEE Trans Microw Theory Tech, 48, 11. With permission.)

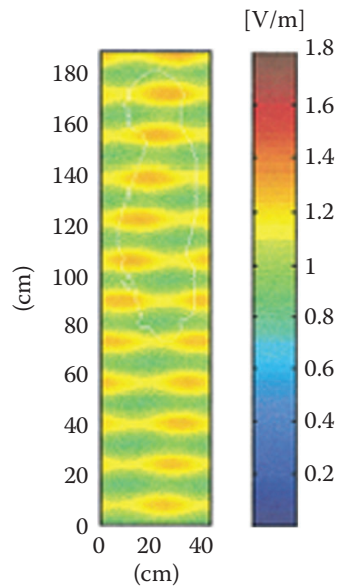

(a)

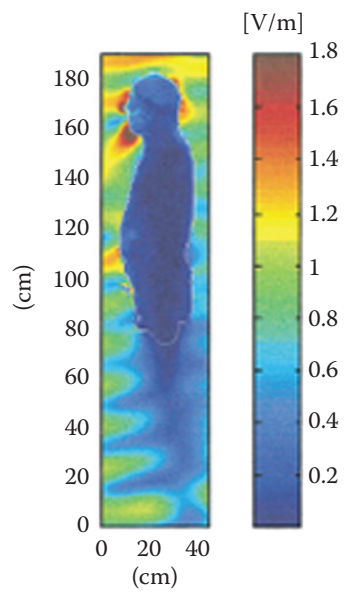

(b)

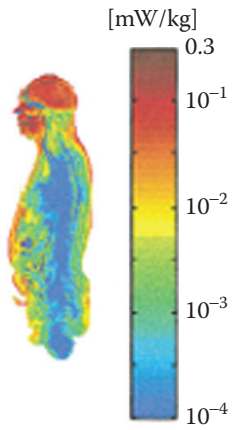

(c)

FIGURE 5.29 Subject on the street: (a) Erms when the subject is absent (b) Erms when the subject is present (c) SAR distribution inside the subject. (From Bernardi, P., M. Cavagnaro, S. Pisa, and E. Piuzzi. 2000. IEEE Trans Microw Theory Tech, 48, 11. With permission.) 


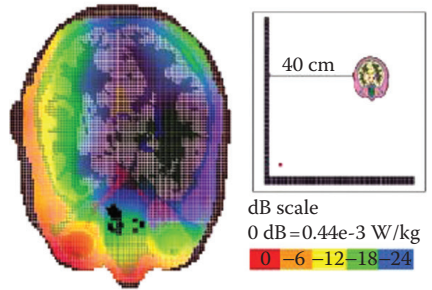

(a)

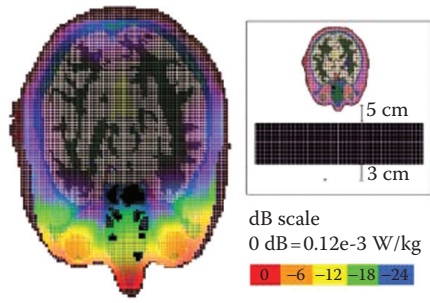

(c)

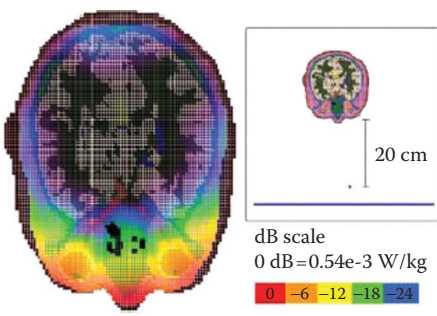

(b)

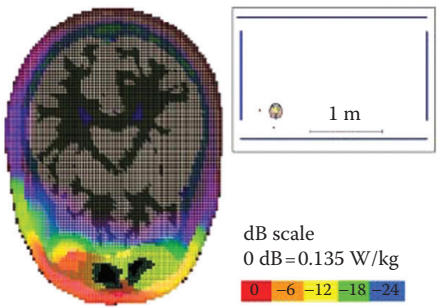

(d)

FIGURE 5.31 Contour plots of the $10 \mathrm{~g}$ avg SAR distribution in the plane where the maximum is located, for the four scenarios of exposure depicted in Figure 5.30. (From Martinez Burdalo, M., A. Martin, A. Sanchis, and R. Villar. 2009. Bioelectromagnetics, 30, 51. With permission.)

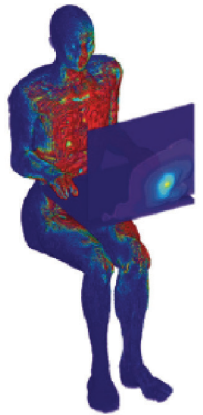

(a)

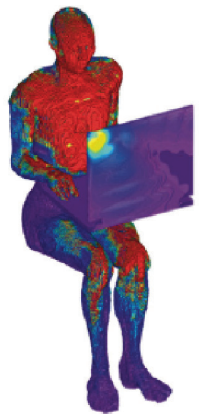

(b)

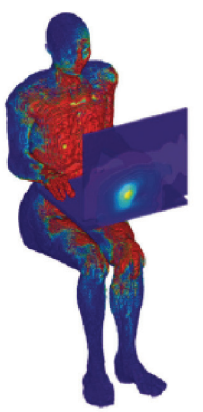

(c)

FIGURE 5.33 SAR intensity plots for the IFA mounted on the (a) bottom left (BL) (b) top right (TR) (c) bottom center (BC) positions of the laptop of Figure 5.32. (From Findlay, R. P., and P. Dimbylow. 2010. Phys Med Biol 55:N405-N411.) 


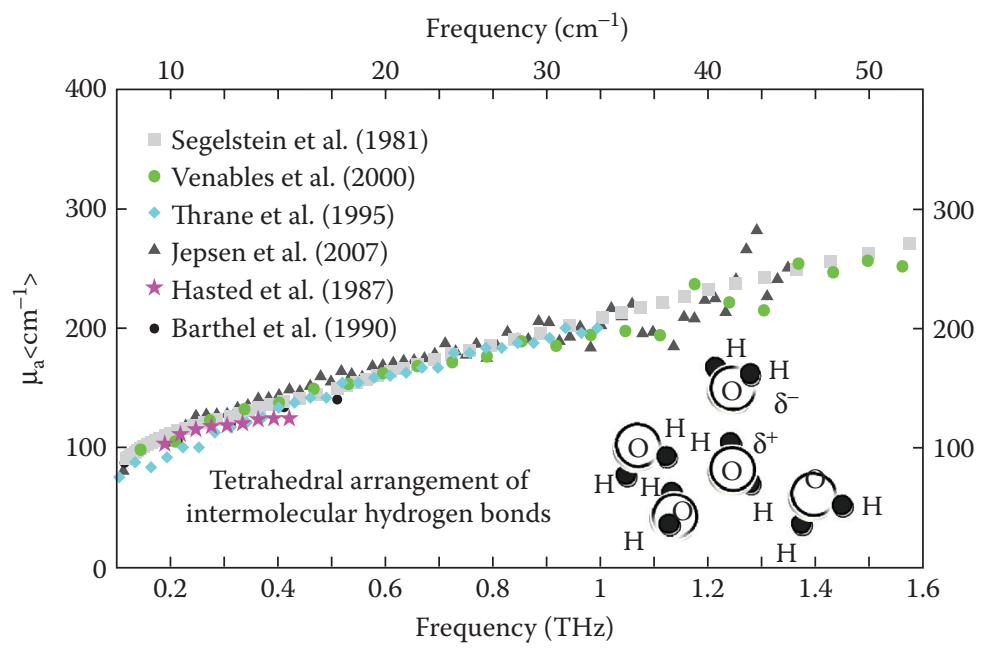

(a)

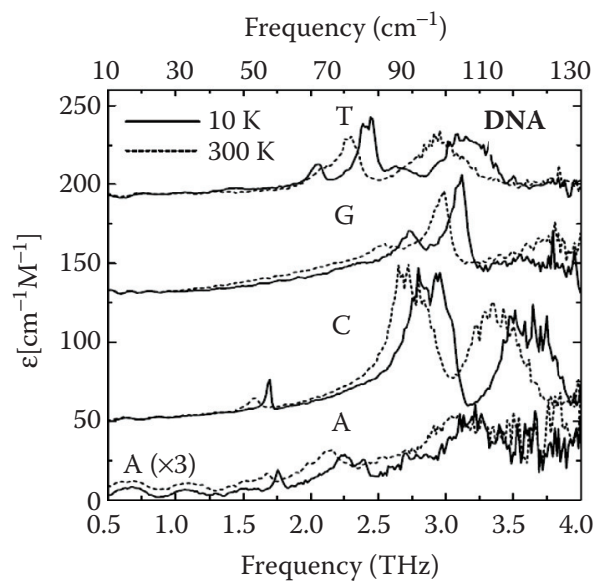

(b)

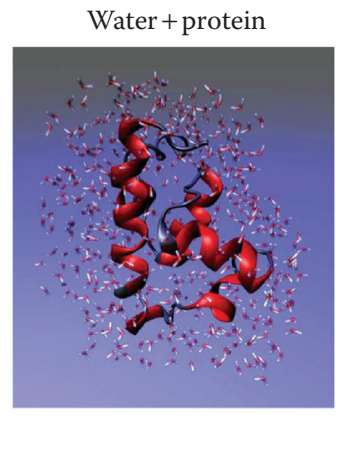

(c)

FIGURE 7.2 (a) Frequency-dependent absorption coefficient of water and image of hydrogen bonds in water. (Data from Segelstein, D. 1981. The Complex Refractive Index of Water. Kansas City, MO: University of Missouri; Hasted, J. B., S. K. Husain et al. 1987. Infrared Phys 27(1):11-5; Barthel, J., and H. Hetzenauer. 1990. Dielectric Specta of Some Common Solvents in the Microwave Region, Water and Lower Alcohols. Amsterdam: Elsevier; Thrane, L., R. H. Jacobsen et al. 1995. Chem Phys Lett 240(4):330-3; Venables, D. S., A. Chiu et al. 2000. J Chem Phys 113(8):3243-8; Jepsen, P. U., U. Moller et al. 2007. Opt Express 15(22):14717-37.) Hydrogen-bond rearrangements, intermolecular bends, occur on the picosecond timescale, corresponding to $40-60 \mathrm{~cm}^{-1}$. (b) Frequency dependent absorption coefficient of DNA nucleobases A, C, T, and G. Each nucleobase has unique spectral resonances from 0.5 to $4.0 \mathrm{THz}$. (Reprinted with permission from Fischer, B. M., M. Walther et al. 2002. Phys Med Biol 47(21):3807-14.) (c) Cartoon of water molecules dancing around a protein molecule. (Reprinted with permission from Leitner, D. M., M. Gruebele et al. 2008. HFSP J 2(6):314-23.) 


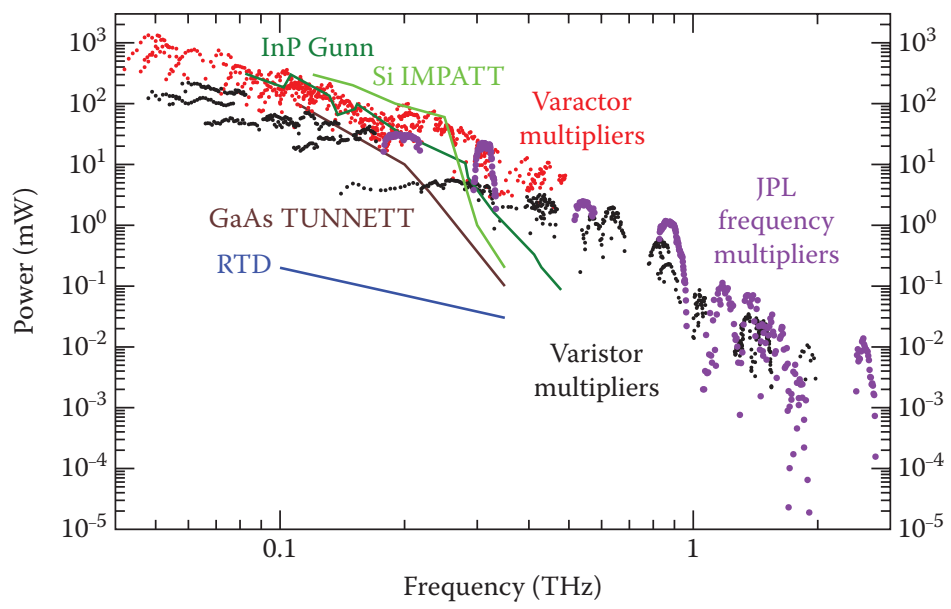

FIGURE 7.8 Peak performance of solid-state electronic THz sources using frequency multiplication of microwave technology. InP Gunn, Si impact ionization avalanche transit-time device (IMPATT), GaAs tunneling transit-time diode (TUNNETT), and resonant tunneling diode (RTD). (Data courtesy of Dr. H. Eisele, University of Leeds, UK.) Varactor multiplier and varistor multiplier data. (Courtesy of Dr. Hessler, Virginia Diodes, Inc., Charlottesville, VA. Jet Propulsion Laboratory (JPL) frequency multiplier unit. (Data courtesy of Peter Siegel, JPL SWAT Team.)

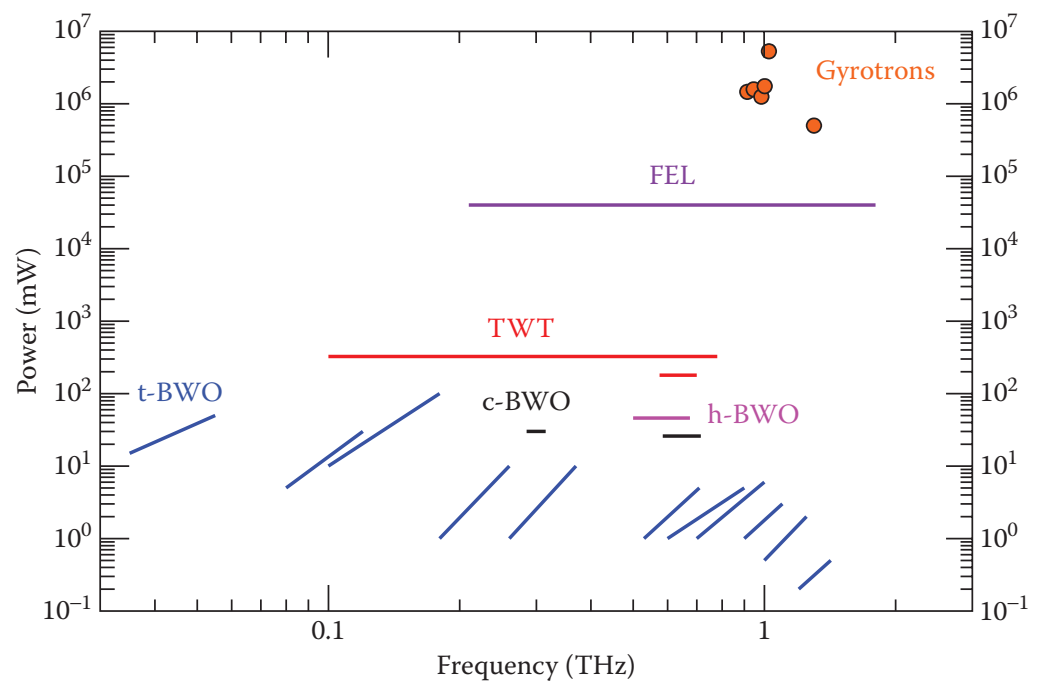

FIGURE 7.10 Peak performance of $\mathrm{THz}$ sources that use electron accelerators or electron vacuum tubes. t-backward wave oscillator (BWO). (Data courtesy of Dr. A. Gershteyn (Insight Product Company LLC.) c-BWO, h-BWO, travelling wave tube (TWT) data courtesy of Dr. Gerald Mearini (Teraphysics LLC.) Gyrotron. (Data courtesy of Dr. Glyavin (Nizhny Novgorod, Russia; Glyavin, M. Y., A. G. Luchinin et al. 2008. Phys Rev Lett 100(1):015101-3.) Free-electron laser (FEL.) (Data courtesy of Dr. Michael Klopf (Jefferson Laboratory, Newport News, VA). Note: FEL and gyrotrons are pulsed sources (pulse power is plotted), whereas other sources are CW sources. 
1895: The first X-ray image

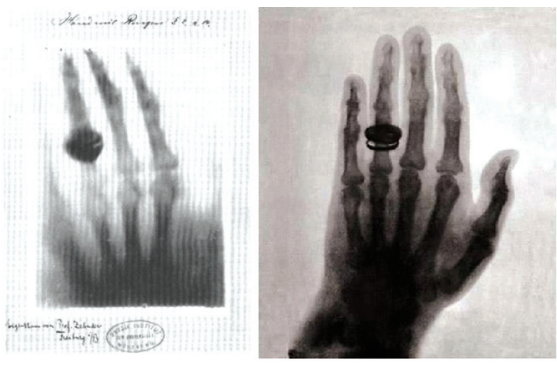

(a)
1995: The first T-ray image

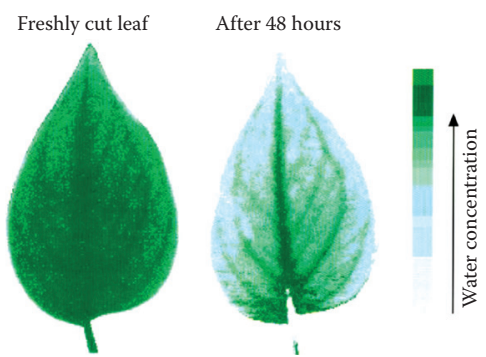

(b)

FIGURE 7.12 X-ray versus T-ray. (a) 1895: The year of the first X-ray image. Photograph of the hand of Frau Rantgen. (Reprinted from Mould, R. F. 1995. Phys Med Biol 40(11):1741. With permission.) (b) 1995: The year of the first T-ray image. THz image of a leaf. (Reprinted from Hu, B. B., and M. C. Nuss. 1995. Opt Lett 20(16):1716-8. With permission. (1995, Optical Society of America.)

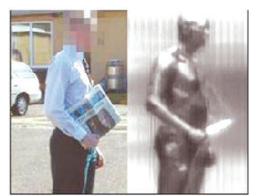

(a)

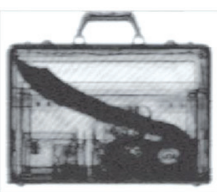

(b)

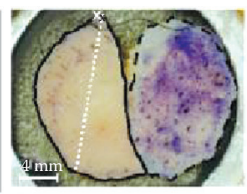

(c)

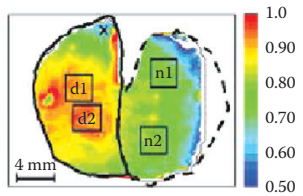

(d)

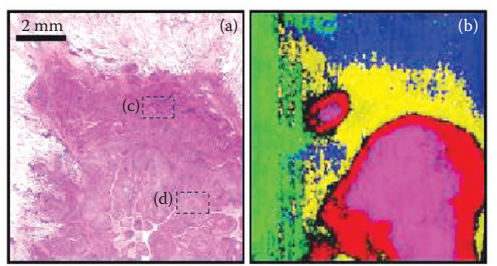

(e)

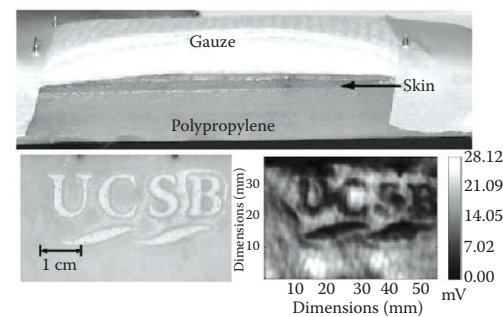

(f)

FIGURE 7.13 THz imaging applications. (a) THz image of concealed knife behind a newspaper. (Reprinted from Clery, D. 2002. Science 297:761-2. With permission. @2002, Science.) (b) $0.2 \mathrm{THz}$ CW image of a briefcase holding a large knife. (Reprinted from Karpowicz, N., H. Zhong et al. 2005. Appl Phys Lett 86(5):054105. With permission. (C2005, America Institute of Physics.) (c) Visible image of cancerous skin (left) and healthy skin (right). (d) THz image of cancerous skin (left) and healthy skin (right). (Reprinted from Woodward, R. M., V. P. Wallace et al. 2003. J Invest Dermatol 120(1):72-8. With permission. (2003, Nature.) (e) Visible histological image (left) and THz image (right) of squamous cell carcinoma in lung tissue. (Reprinted from Brun, M. A., F. Formanek et al. 2010. Phys Med Biol 55(16):4615. With permission. (O2010, Institute of Physics.) (f) Reflective THz imaging of porcine skin burns: experimental setup (top), photograph of burn (left), and THz image of burn through 10 layers of medical gauze (right). (Reprinted from Taylor, Z. D., R. S. Singh et al. 2008. Opt Lett 33(11):1258-60. With permission. @2008, Optical Society of America.) 


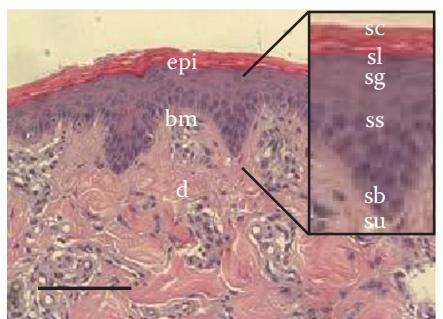

(a)

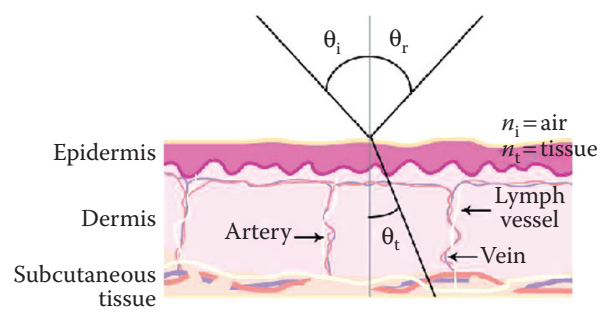

(b)

FIGURE 7.14 (a) Histological cross section of porcine skin tissue (hematoxylin and eosin stain at $40 \times$ magnification). epi $=$ epidermis, $\mathrm{bm}=$ basement membrane, $\mathrm{d}=$ dermis, $\mathrm{sc}=$ stratum corneum, $\mathrm{sl}=$ stratum lucidum, $\mathrm{sg}=$ stratum granulosum, $\mathrm{ss}=$ stratum spinosum, and $\mathrm{sb}=$ stratum basale. (b) Cartoon image of THz light incident on skin at an angle $\theta_{\mathrm{i}}$. A portion of the THz beam is specularly reflected with an angle $\theta_{\mathrm{r}}$ and the remainder is transmitted at $\theta_{\mathrm{t}}$.

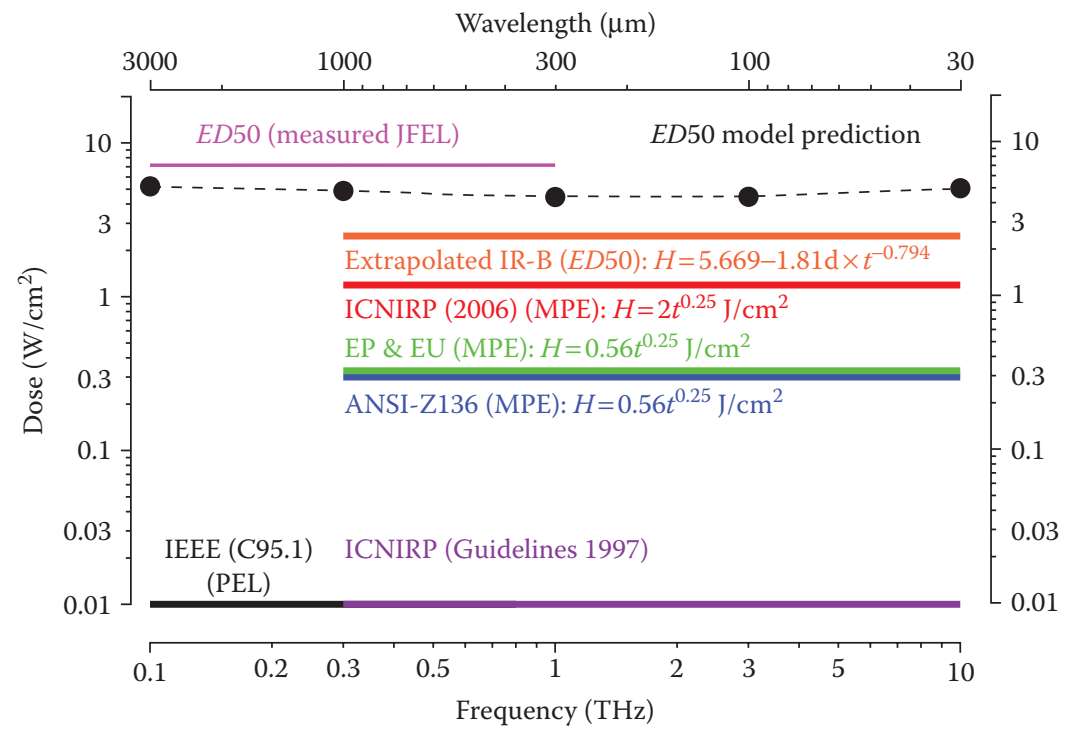

FIGURE 7.19 Summary plot of empirical data, computational modeling data, and current terahertz radiation exposure standards. The defined exposure limits $\left(\mathrm{W} / \mathrm{cm}^{2}\right)$ are plotted versus frequency $(\mathrm{THz})$ and wavelength. The plot includes the following: experimentally determined ED50 using a free-electron laser (FEL; pink), computational modeling ED50 predictions (dashed black), empirical equation designed for use at IR wavelengths (orange). (Data from Chen et al. 2005.) ICNIRP IR-C statement (red). (Data from Protection 2006.) EP \& EU (green), ANSI-Z136.1 MPE values (blue). (Data from Erdreich and Klauenberg 2001; Union 2006), ICNIRP guidelines for MPE (purple). (Data from ICNIRP 1996), and IEEE C95.1 suggested PEL (solid-black line). (Data from Erdreich and Klauenberg 2001.) 


\section{Terahertz Radiation:} Sources, Applications, and Biological Effects

7.1 Introduction 370

The Terahertz Region: Fundamental Units with

Biological Relevance - Nonionizing Radiation:

"X-Rays" versus "T-Rays" • Spectroscopic

Fingerprint Region for Biological Materials

7.2 Terahertz Sources: Conventional and

State-of-the-Art.

Direct Generation Laser Sources • Electronic

Solid-State Terahertz Devices: Frequency

Up-Conversion - Accelerating Electron-Based

Terahertz Sources - Nonlinear Optical Terahertz

Sources: Frequency Down-Conversion

7.3 Terahertz Applications.

Spectroscopy - Imaging

7.4 Terahertz Skin Interactions.

Skin Anatomy - Fundamental Principles of

Terahertz-Tissue Optics • Optical Properties

of Skin - Photothermal Mechanisms and

Classic Heat Transfer - Thermal History:

Temperature Damage Zones and Associated

Biological Effects - Tissue Damage at Terahertz

Frequencies - Terahertz Safety Standards

7.5 Biological Effects at a Cellular and

Biomolecular Level.

Background on Cellular Stress Response and

Cellular Death - Terahertz Bridge-The First

International Project on Terahertz Bioeffects

(2001-2004) - Recent Studies on Terahertz

Gerald J. Wilmink Bioeffects (2004-2010) • Cellular Effects

Jessica E. Grundt

of Terahertz Radiation 


\author{
Direct Effects of Terahertz Radiation on Cellular \\ Organelles and Biomolecules - Genotoxic \\ Effects of Terahertz Radiation - Does Terahertz \\ Radiation Trigger Signature Gene Expression \\ Profiles in Human Cells? \\ 7.6 Conclusion and Future Prospects

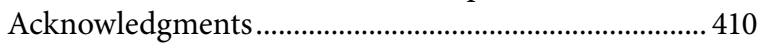 \\ References.................................................................... 411
}

\title{
7.1 Introduction
}

Every second the Sun emits a countless number of photons. These photons whirl around the universe and inundate our everyday lives with electromagnetic (EM) energy. Many of these photons are visible to us as they scatter throughout the atmosphere, but the vast majority of them are completely unnoticed. In fact, roughly $98 \%$ of the photons released since the Big Bang have been emitted from a region of the EM spectrum that is not visible to us-the Terahertz (THz) region (Trentham 1999).

Given their natural abundance, one may assume that much is known about the effects that $\mathrm{THz}$ photons elicit when they interact with biological structures. But this is not the case. In fact, to date, fewer biological studies have been conducted at $\mathrm{THz}$ frequencies than for any other type of EM radiation. Data are scarce at $\mathrm{THz}$ frequencies because high-power sources have historically been unavailable for this region. However, this has changed over the past decade, where a surge of recent research activity has resulted in the development of many new types of sources. These sources have bridged the proverbial "THz Gap," and they have also proven to be incredibly valuable research tools. In addition, they are increasingly being integrated into a host of practical medical, military, and security applications. For example, THz imaging and sensing techniques are being tested at airports for security screening (Brijot 2010; Thruvision 2010), at major hospitals for cancer and burn diagnosis (Woodward et al. 2002; Woodward et al. 2003; Wallace et al. 2004; Wallace et al. 2004; Wolbarst and Hendee 2006; Bourne et al. 2008; Oh et al. 2008; Singh et al. 2008; Oh et al. 2009; Suen et al. 2009), and at border patrol checkpoints for identification of concealed explosives, drugs, and weapons (Federici et al. 2005; Dobroiu et al. 2006; Bogue 2009).

Widespread use of new $\mathrm{THz}$ applications has prompted concerns regarding the health effects associated with this type of radiation. Such concerns have made it imperative that bioeffects data be available for proper health hazard evaluation, the development of empirically-based safety standards, and safe exploitation of $\mathrm{THz}$ technologies. Fortunately, researchers have recently responded to these concerns by performing timely investigations to examine the biological effects associated with $\mathrm{THz}$ radiation. (Smye et al. 2001; Clothier and Bourne 2003; Fedorov et al. 2003; Scarfi et al. 2003; Walker 2003; Ramundo-Orlando et al. 2007; Zeni et al. 2007; Korenstein-Ilan et al. 2008; Olshevskaya et al. 2008; Cherkasova et al. 2009; Homenko et al. 2009.) These scientific reports, however, have been performed by researchers from various disciplines, thus, they are scattered in journals with research foci ranging from engineering to physics to biology. Unfortunately, a comprehensive chapter has not yet appeared in the literature, which both collects the information gleaned from these recent works, and also organizes 
it into a logical structure that provides readers with a framework to better understand $\mathrm{THz}$ bioeffects. The need to fill this void prompted us to write this book chapter.

The purpose of this chapter is twofold. First, to provide readers with a common reference that contains the necessary background concepts in biology, physics, and $\mathrm{THz}$ technology, which are required to both conduct and evaluate $\mathrm{THz}$ biological research. Second, to provide a review of the $\mathrm{THz}$ bioeffects reports that have been published in the literature.

The contents of this chapter are divided into six sections. The first section provides a general introduction to the $\mathrm{THz}$ spectral region. The second and third sections then provide an overview of $\mathrm{THz}$ sources and applications, respectively. Following the description of $\mathrm{THz}$ sources and applications, the fourth section then provides an overview of the fundamental concepts that are required to understand the interaction of $\mathrm{THz}$ radiation with biological systems. This section begins with details regarding the composition and function of skin, the primary biological target for $\mathrm{THz}$ radiation. This discussion is followed where emphasis is placed on the fundamental biophysical mechanisms that govern THz-skin interactions, including a discussion on energy deposition, temperature transients, and conventional thermal effects. The concepts described in this section are particularly valuable for the proper analysis of the pleotropic effects observed in $\mathrm{THz}$ bioeffects studies. The fifth section then provides a comprehensive review of $\mathrm{THz}$ bioeffects studies performed to date. We end this chapter with a conclusion section where we address challenges and future opportunities in this field.

\subsubsection{The Terahertz Region: Fundamental Units with Biological Relevance}

The $\mathrm{THz}$ spectral band is a region of the EM spectrum that neighbors the infrared (IR) region on the high-frequency side and the microwave (MW) region on the lowfrequency side (Figure 7.1). This region is defined as frequencies ranging from 0.1 to $10 \mathrm{THz}\left(1 \mathrm{THz}=10^{12} \mathrm{~Hz}\right)$, or the wavelengths ranging from 30 to $3000 \mu \mathrm{m}$. The size of a $\mathrm{THz}$ wave has biological relevance in regards to the following general concepts: spatial resolution of imaging applications, scattering properties in biological tissues, and direct biomolecular excitation.

First, modalities that employ $\mathrm{THz}$ wavelengths can provide images with higher spatial resolution than counterpart systems, which use longer wavelengths, such as millimeterwave imaging systems (i.e., Rayleigh criterion). The practical value of this property was first demonstrated in 1995 at Bell Laboratories (Hu and Nuss 1995). In this study, Hu and Nuss illustrated that $\mathrm{THz}$ waves could be collimated and focused down to the diffraction limit of a few hundred microns at the surface of a biological sample. This finding was particularly compelling because it demonstrated that $\mathrm{THz}$-imaging approaches permit the accurate measurement of biochemical compositions.

In addition to resolution, the size of a $\mathrm{THz}$ wave is also relevant to the scattering processes associated with imaging applications. This feature stems from the fact that $\mathrm{THz}$ waves are larger in size than many biological structures: mammalian cells (10-25 $\mu \mathrm{m})$, arterioles $(30 \mu \mathrm{m})$, collagen fibrils $(20-50 \mu \mathrm{m})$, human hair $(50-70 \mu \mathrm{m})$, and the thickness of epidermis $(\sim 500-1500 \mu \mathrm{m})$. Because photons are known to be scattered most 
Electromagnetic spectrum

\begin{tabular}{|c|c|c|c|c|c|c|c|}
\hline \multirow{2}{*}{$\begin{array}{l}\text { Frequency }(\mathrm{Hz}) \\
\text { Spectral bands }\end{array}$} & \multicolumn{7}{|r|}{$0^{20} 10^{21}$} \\
\hline & Radio waves & $\mu$-wave & $\mathrm{THz}$ & IR & UV & $\mathrm{X}$-rays & $\gamma$-rays \\
\hline Frequency (THz): & $\mathrm{v}$ & 0.1 & & $\begin{array}{r}1.0 \\
+.1\end{array}$ & & 10.0 & \\
\hline Wavelength $(\mu \mathrm{m})$ : & $\lambda=c / v$ & $\begin{array}{r}3000 \\
\end{array}$ & & $\begin{array}{r}300 \\
\quad 1 .\end{array}$ & & 30 & \\
\hline Wave number $\left(\mathrm{cm}^{-1}\right)$ : & $k=1 / \lambda$ & 3.3 & & $\begin{array}{r}3.34 \\
. . .\end{array}$ & & 334.0 & \\
\hline Period (picoseconds): & $\tau=1 / v$ & $\begin{array}{r}10.0 \\
\text { ب. }\end{array}$ & & $\begin{array}{r}1.0 \\
1 .\end{array}$ & & 0.1 & \\
\hline Photon energy (meV): & $E=h v$ & 0.4 & & $\begin{array}{r}4.1 \\
. .1 \\
\end{array}$ & & $\begin{array}{l}41.0 \\
. .\end{array}$ & \\
\hline Temperature (K): & $T=h v / k_{\mathrm{b}}$ & 4.8 & & $\begin{array}{r}47.8 \\
\end{array}$ & & بـ & \\
\hline
\end{tabular}

FIGURE 7.1 The Terahertz (THz) band of the electromagnetic spectrum. $\mu$-wave $=$ microwave; IR = infrared; VIS = visible; and UV = ultraviolet. Note: $c$ is the speed of light $\left(2.998 \times 10^{10} \mathrm{~m} / \mathrm{s}\right), h$ is Plank's constant $\left(4.136 \times 10^{-12} \mathrm{eV} \cdot \mathrm{s}\right)$, and $K_{\mathrm{b}}$ is Boltzmann's constant $\left(8.617 \times 10^{-5} \mathrm{eV} \cdot \mathrm{K}^{-1}\right)$.

strongly by structures whose size matches their wavelength (Wang and Wu 2007), THz waves suffer minimal Rayleigh scattering and primarily undergo Mie scattering processes as they pass through biological tissues. This feature is currently being exploited in THz near-field microscopy.

Finally, biological macromolecules have metastable states with very high dipole moments, and as a result, they are strongly excited at frequencies near $0.1 \mathrm{THz}$ (Frohlich 1975). This feature gives $\mathrm{THz}$ waves the unique ability to directly excite and induce resonance effects in cells and biomolecules. Because such interactions presumably would have both positive and negative implications, research groups are actively conducting investigations to better elucidate these coupling and excitation mechanisms.

\subsubsection{Nonionizing Radiation: “X-Rays" versus “T-Rays"}

$\mathrm{THz}$ photons range in quantum energy between 0.4 and $4.1 \mathrm{meV}$ (Figure 7.1). These energy levels are several orders of magnitude below that required for molecular ionization ( several eVs). Therefore, "T-rays" are classified as nonionizing EM radiation. It is interesting to note that the quantum energy of a $\mathrm{THz}$ photon is one million times lower than that of an ionizing X-ray photon.

\subsubsection{Spectroscopic Fingerprint Region for Biological Materials}

$\mathrm{THz}$ radiation interacts strongly with the rotation of many molecular gases and the collective vibrational motions of biological liquids and solids. Interestingly, such interactions are stronger in the $\mathrm{THz}$ region than in neighboring spectral regions. In fact, the interaction strength in the $\mathrm{THz}$ region is exponentially larger than in the IR region and 
roughly $10^{3}$ to $10^{6}$ times stronger than in the millimeter-wave region (Albert et al. 2001). This property makes $\mathrm{THz}$ spectroscopy a very valuable research tool, and many experts contend that this property may be the single most important feature of the THz spectral region (Mittleman 2003).

Numerous gases, liquids, and biological materials undergo intermolecular vibrations, intramolecular vibrations, collective motions, and molecular rotations at $\mathrm{THz}$ wave numbers (3-334 $\left.\mathrm{cm}^{-1}\right)$ or periods (0.1-10 ps) (see Figure 7.1). As a consequence, this region is intrinsically rich with unique spectroscopic signatures that are intrinsically unavailable in other spectral bands. Here, we briefly discuss the properties of water and several biological macromolecules in this spectral range. For a more detailed discussion, we refer the reader to several excellent books and articles (Albert et al. 2001; Beard et al. 2002; Globus 2003; Mittleman 2003; Svanberg 2004; Fischer 2005; Pivonka et al. 2007; Dexheimer 2008).

Covering $\sim 70 \%$ of the Earth's surface and accounting for $\sim 60 \%-70 \%$ of the total weight of an average human being, water is the most ubiquitous chemical substance on planet Earth. Water exhibits a plethora of unique chemical and physical properties that make it essential to human life: (1) Water is "the universal solvent" in which all biomolecules (i.e., carbohydrates, proteins, and nucleic acids) are dissolved in; (2) Water is required for the proper folding and function of all biomolecules (Pal et al. 2002a,b; Pal et al. 2003a,b; Pal and Zewail 2004); (3) Water consists of oxygen atoms that have a higher electronegativity (i.e., $\delta^{-}$) than hydrogen atoms; (4) Water is a polar molecule with a large dipole moment; and (5) Water molecules form a tetrahedral arrangement of intermolecular hydrogen bonds to its neighboring molecules (Figure 7.2a).

$\mathrm{THz}$ radiation interacts strongly with water due to the slow relaxation time of water molecules. This property is closely associated with rotational dynamics and intermolecular hydrogen-bond rearrangements (i.e., intermolecular bends), which occur on the picosecond timescale (Ladanyi and Skaf 1993; Russo et al. 2003). Therefore, in order to probe and capture these dynamics, experimental methods must also function on the picosecond timescale (Figure 7.2a). Millimeter-wave and IR spectroscopic methods permit measurements on the $\sim 100$ ps and subpicosecond timescale, respectively. However, THz spectroscopic techniques can capture dynamics on the 0.1-10.0 ps timescale. This fundamental property makes $\mathrm{THz}$ tools ideal for probing water dynamics in biological structures.

In addition to water, many biological macromolecules (e.g., carbohydrates, lipids, proteins, and DNA) undergo vibrational motion on the picosecond timescale. These collective modes include intramolecular vibrations (i.e., stretch, bond, and torsion), intermolecular vibrations (i.e., van der Waals forces and hydrogen bonding), and molecular rotations (i.e., slow relaxation of water). For example, carbohydrates, which are essential molecules for energy storage and structural maintenance, exhibit several distinct resonances in the $\mathrm{THz}$ region (typically, below $200 \mathrm{~cm}^{-1}$ ) (Walther et al. 2000; Globus et al. 2002; Xie 2002; Globus 2003). In addition, monosaccharide isomers (i.e., glucose, galactose, mannose, and fructose) exhibit vastly different spectra at $\mathrm{THz}$ wave numbers $\left(70-100 \mathrm{~cm}^{-1}\right)$, despite the fact that they have very similar molecular structures (Fischer 2005). Studies have also shown that the photoisomerization of retinal, an integral step in the vision process, exhibits a vibrational mode at $\mathrm{THz}$ wave numbers $\left(\sim 60 \mathrm{~cm}^{-1}\right.$; Wang et al. 1994). 


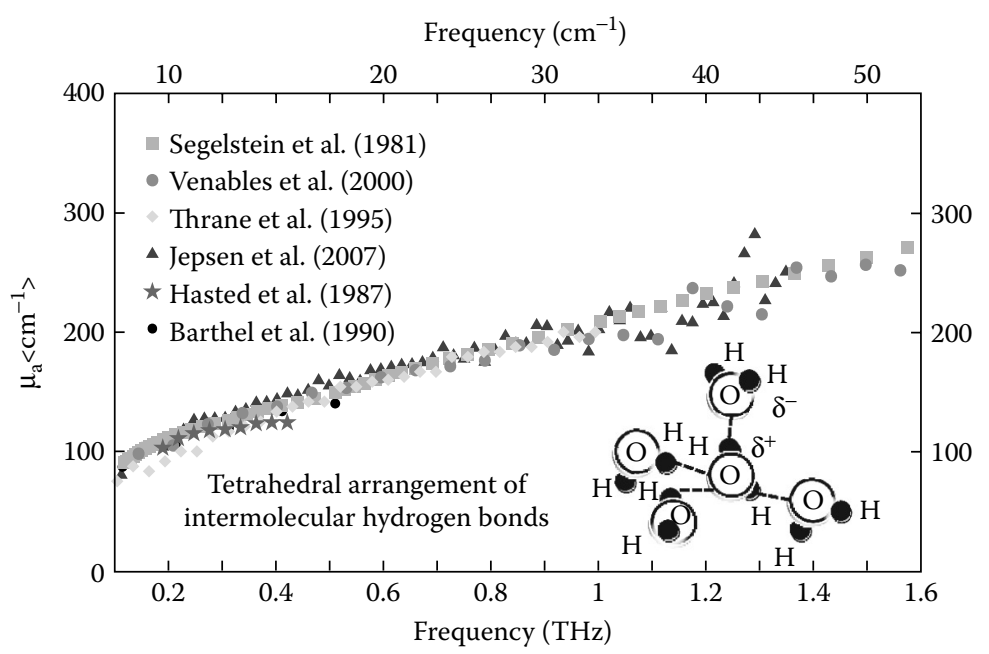

(a)

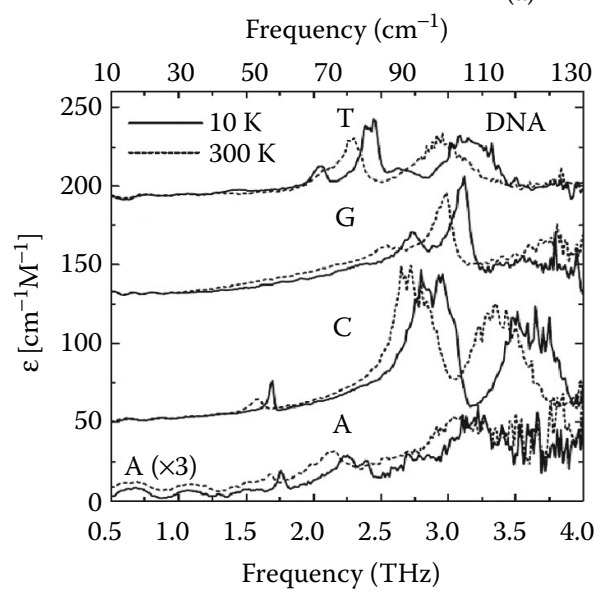

(b)

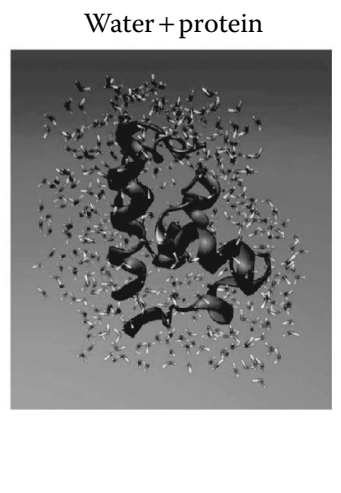

(c)

FIGURE 7.2 (See color insert.) (a) Frequency-dependent absorption coefficient of water and image of hydrogen bonds in water. (Data from Segelstein, D. 1981. The Complex Refractive Index of Water. Kansas City, MO: University of Missouri; Hasted, J. B., S. K. Husain et al. 1987. Infrared Phys 27(1):11-5; Barthel, J., and H. Hetzenauer. 1990. Dielectric Specta of Some Common Solvents in the Microwave Region, Water and Lower Alcohols. Amsterdam: Elsevier; Thrane, L., R. H. Jacobsen et al. 1995. Chem Phys Lett 240(4):330-3; Venables, D., and C. Schmuttenmaer. 2000. J Chem Phys 113(24):11222-36; Jepsen, P. U., U. Moller et al. 2007. Opt Express 15(22):14717-37.) Hydrogen-bond rearrangements, intermolecular bends, occur on the picosecond timescale, corresponding to $40-60 \mathrm{~cm}^{-1}$. (b) Frequency dependent absorption coefficient of DNA nucleobases A, $\mathrm{C}, \mathrm{T}$, and G. Each nucleobase has unique spectral resonances from 0.5 to $4.0 \mathrm{THz}$. (Reprinted with permission from Fischer, B. M., M. Walther et al. 2002. Phys Med Biol 47(21):3807-14.) (c) Cartoon of water molecules dancing around a protein molecule. (Reprinted with permission from Leitner, D. M., M. Gruebele et al. 2008. HFSP J 2(6):314-23.) 
Several studies have also shown that DNA nucleobases (i.e., A, T, C, and G) exhibit resonances between $33.4 \mathrm{~cm}^{-1}$ and $117 \mathrm{~cm}^{-1}$ (Fischer et al. 2002; Xie 2002; Fischer 2005), whereas DNA films exhibit modes from 300 to $500 \mathrm{~cm}^{-1}$ (Globus et al. 2002; Figure 7.2b). A growing number of research groups are using $\mathrm{THz}$ spectroscopic approaches to investigate the important role that water plays at the surface of biomolecules. Most notably, Havenith's group recently demonstrated that water molecules actually "dance" with proteins during solvation (Figure 7.2c). Finally, transmembrane proteins have also been shown to exhibit collective modes in the THz region ( at $\left.115 \mathrm{~cm}^{-1}\right)$ (Xie 2002). In summary, water molecules, biomolecules, and water-protein solvation processes all exhibit dynamics that occur on the picosecond timescale. Therefore, $\mathrm{THz}$ spectroscopic tools are uniquely suited to probe these dynamics.

\subsection{Terahertz Sources: Conventional and State-of-the-Art}

Historically, fewer sources have been available for the $\mathrm{THz}$ region than for neighboring millimeter-wave and IR spectral bands. For many years, the lack of suitable sources enticed scientists from both the millimeter wave and IR research communities to develop sources for this uncharted territory. Developing bright $\mathrm{THz}$ sources has been a challenging task for two fundamental reasons. First, conventional photonic approaches are limited because few materials exist that have a small enough band gap to directly generate $\mathrm{THz}$ radiation. A band gap is the difference in energy between the valence and conduction band of a solid, and band-gap spacing is directly correlated with the output wavelength of laser sources. The second principle is that the intrinsic transit time and resistance-capacitance effects of conventional electronic devices causes their output power to roll off with increases in frequency (i.e., power $\sim 1 / f^{2}-1 / f^{3}$ ) (Siegel 2002). As a result, the output power of conventional electronic sources drops off at $\mathrm{THz}$ frequencies.

Although an extensive review on $\mathrm{THz}$ sources is beyond the scope of this chapter, a general understanding of modern sources is necessary for subsequent discussions on THz-induced bioeffects. For further details about THz sources, we refer the reader to several excellent reviews and books (Ferguson and Zhang 2002; Siegel 2002; Wolbarst and Hendee 2006; Hosako et al. 2007; Lee and Wanke 2007; Liu et al. 2007; Tonouchi 2007; Williams 2007; Lee 2009; Sirtori 2009). THz sources are typically categorized by their principle operational scheme. The most commonly used schemes are the following: (1) direct generation laser sources; (2) solid-state electronic devices (frequency up-conversion); (3) accelerating electron-based sources; and (4) nonlinear optical effect sources (frequency down-conversion).

\subsubsection{Direct Generation Laser Sources}

Far-IR gas lasers (FIR), electrically pumped solid-state lasers, and quantum cascade lasers (QCLs) are the three most common types of direct generation $\mathrm{THz}$ laser sources. The FIR laser is the oldest $\mathrm{THz}$ source and has initial uses dating back as early as the 1960s (Crocker et al. 1964; Dodel 1999; Figure 7.3a). FIR systems, also referred to as optically pumped molecular gas lasers, consist of a tunable $\mathrm{CO}_{2}$ optical pump laser 


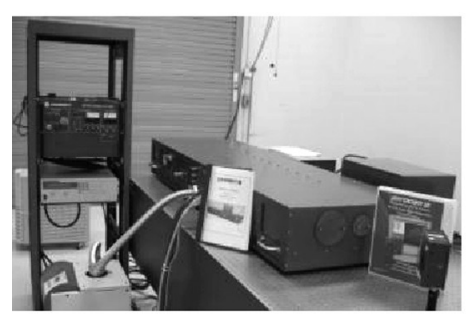

(a)

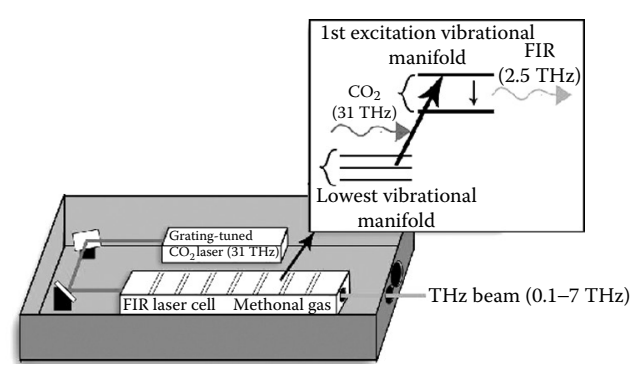

(b)

FIGURE 7.3 (a) Photograph of far-infrared (FIR) molecular gas THz laser source (CoherentDEOS, Bloomfield, CT). (b) Schematic of primary elements, lasing scheme, and operation principle used in a FIR THz laser.

$(\lambda=9-11 \mu \mathrm{m}, \sim 50 \mathrm{~W})$, a vacuum envelope for molecular gases, a pump frequency reference lock, and intracavity waveguides. Lasing action is achieved using the $\mathrm{CO}_{2}$ laser to excite the vibrational levels of gas molecules, which have transition frequencies in the $\mathrm{THz}$ spectrum (i.e., methanol $\left(\mathrm{CH}_{3} \mathrm{OH}\right)$ Figure $\left.7.3 \mathrm{~b}\right)$.

FIR lasers exhibit several performance characteristics that make them an excellent source for THz bioeffects studies. First, they provide high levels of average output power, typically on the order of $\sim 100 \mathrm{~mW}$ at many frequency lines (Dalzell et al. 2010; Wilmink et al. 2010; Wilmink et al. 2010; Wilmink et al. 2011; Wilmink and Roach 2010). Second, FIRs are widely tunable to hundreds of discrete frequency lines across the $\mathrm{THz}$ spectral band. Fortunately for the FIR user, tuning or "hopping" to each discrete frequency line is straightforward and is achieved by simply adjusting the pump laser wavelength and the gas type and pressure. Third, FIRs generate coherent, monochromatic, narrow linewidth (typically, on the order of $50 \mathrm{kHz}$ ), continuous wave (cw) $\mathrm{THz}$ radiation. The final, and perhaps the most attractive feature of FIRs, is that they are easy to operate and maintain. Probably the main limitations of FIR lasers is their large footprint, appreciable weight, and expense. Systems typically cost in excess of several hundred thousand dollars.

The p-type germanium laser ( $\mathrm{p}-\mathrm{Ge}$ ) is the most common type of electrically pumped direct generation $\mathrm{THz}$ laser source (Figure 7.4a). Invented in the early 1980s, the p-Ge establishes lasing action using the streaming motion and the population inversion of two Landau levels (Komiyama 1982; Andronov et al. 1984; Komiyama et al. 1985). Population inversion is established by hot carriers in Ge crystals that are positioned in crossed electric and magnetic fields (Reichertz et al. 1997; Lee 2009). Figure 7.4b is an energy diagram of population inversion and stimulated $\mathrm{THz}$ emission in a $\mathrm{p}-\mathrm{Ge}$ source. In brief, high electric fields cause a heavy hole $(\mathrm{HH})$ to accelerate up to an excited state. Subsequently, it emits an optical phonon and thereby returns to a lower stable Landau state in a light hole (LH) band. Stable LH Landau levels then return to a lower LH band and in the process emit $\mathrm{THz}$ photons.

$\mathrm{p}$-Ge lasers are an attractive $\mathrm{THz}$ source because they are magnetically tunable from 1 to $4 \mathrm{THz}$, provide narrow line widths of $\sim 20 \mathrm{MHz}$, repetition rates of up to $45 \mathrm{kHz}$, laser 


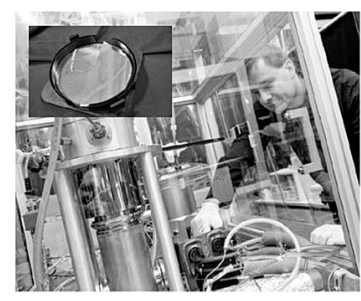

(a)

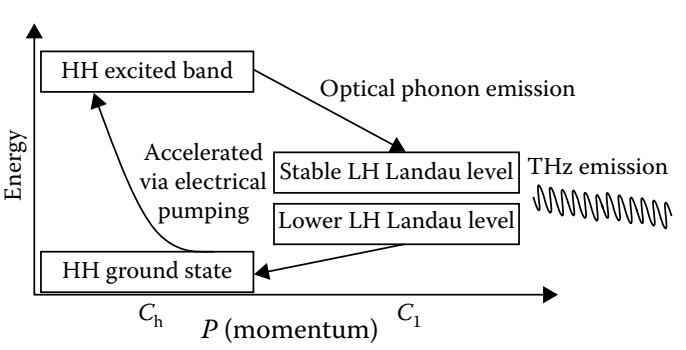

(b)

FIGURE 7.4 (a) Photograph of p-type Germanium (p-Ge) THz laser. (Image courtesy of Dr. Brundermann, Germany.) (b) Energy diagram of population inversion and stimulated $\mathrm{THz}$ emission in a p-type Ge laser with crossed electric and magnetic fields.

pulse lengths up to $32 \mu$ s, and peak powers greater than $10 \mathrm{~W}$ (Brundermann et al. 1995; Bergner et al. 2005). The main limitation of $\mathrm{p}$-Ge sources is that they require magnetic field in excess of 1 Tesla, and cryogenic operating conditions (Brundermann et al. 1995; Bründermann and Röser 1997; Lee 2009).

Recent advances in nanotechnology have resulted in the development of the newest type of THz laser source: the THz-QCL (Figure 7.5 [a through d]; Faistet al. 1994; Hosako et al. 2007; Williams 2007; Sirtori 2009; Wade et al. 2009). QCLs are semiconductor lasers that achieve emission through the use of multiple quantum well structures and intersubband transitions. QCLs require two fundamental processes for emission: intersubband transitions and cascading. An intersubband transition involves electrons that undergo transitions in one period of the superlattice and are injected or "tunneled" into a subsequent period. These electrons then undergo another intersubband transition, and this "cascading" process is repeated until the electron reaches the end of the superlattice, ultimately resulting in the emission of a $\mathrm{THz}$ photon (Belkin et al. 2007). Figure 7.5d is a schematic of intersubband structures and the cascading process. Perhaps the most critical distinguishing feature of QCLs is that they use a single electron to create the emission of multiple photons; thus, their quantum efficiencies are greater than unity. This feature permits QCLs to provide higher output powers than conventional semiconductor laser diodes, which use interband transitions to generate a single photon.

The first operational QCL was reported in 1994 by scientists from Bell Laboratory (Faist et al. 1994). This source provided optical lasing action at $4.2 \mu \mathrm{m}(75 \mathrm{THz})$ and generated peak powers exceeding $8 \mathrm{~mW}$. Then, almost a decade later, Kohler et al. unveiled the first THz-QCL. This source operated at $50 \mathrm{~K}$ and provided $2 \mathrm{~mW}$ of power at $4.4 \mathrm{THz}$ (Kohler et al. 2002). More recent THz-QCLs have been shown to provide lasing action at frequencies ranging from 1.5 to $4.5 \mathrm{THz}$, and typically emit a few mWs of power at $100 \mathrm{~K}$ and $200 \mathrm{~mW}$ at cryogenic temperatures (Belkin et al. 2007; Williams 2007; Sirtori 2009; Wade et al. 2009; Figure 7.6).

New design methods are currently being explored to develop more efficient THz QCLs, which operate at room temperature, emit radiation at lower frequencies $(0.1-1.5 \mathrm{THz})$, 


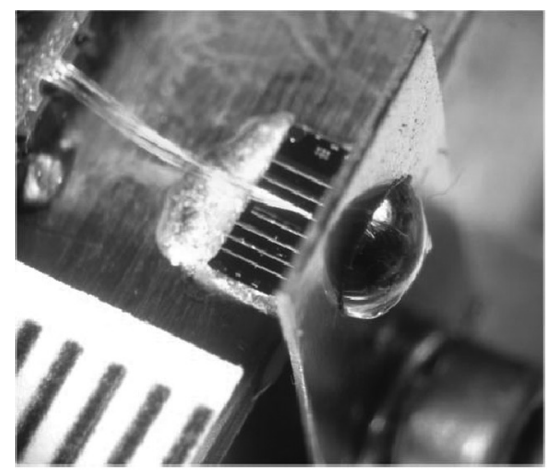

(a)

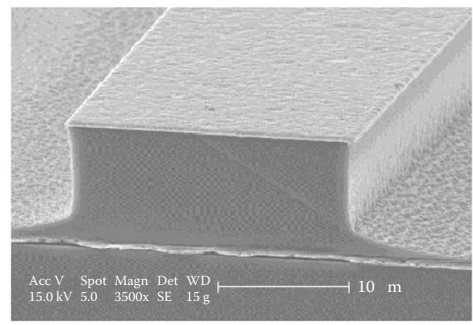

(c)

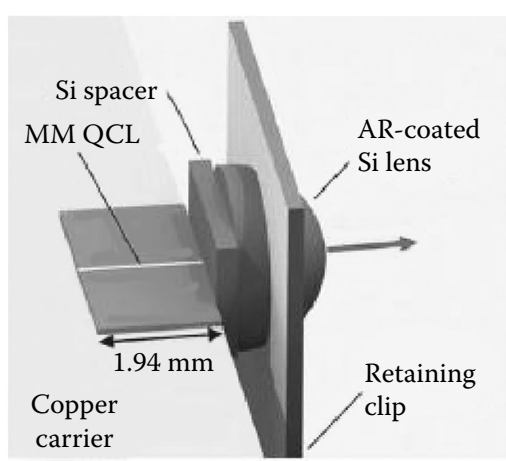

(b)

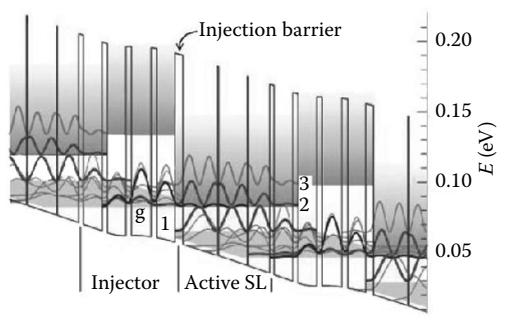

(d)

FIGURE 7.5 (a) Photograph of a THz quantum cascade laser (QCL). (Courtesy of Dr. Allen Lee, Longwave Photonic, MA.) (b) Schematic of principle elements: QCL, silicon spacer, and lens. (Reprinted from Wei Min Lee, A., Q. Qin et al. 2007. Opt Lett 32(19):2840-2. With permission. (2007, Optical Society of America.) (c) SEM image of mesa facet of THz QCL. (d) Schematic of conduction band structure. (Reprinted from Kohler, R. 2002. Nature 417:156-9. With permission. (C2002, Macmillan Publishers Ltd.)

and that better collimate the ever diverging $\mathrm{THz}$ beam. For example, in a recent study, Yu et al. demonstrated that spoof surface plasmon structures can be used to dramatically reduce beam divergence and increase their power collection efficiency by a factor of 6 (Yu et al. 2010). If scientists continue to address these challenges, the compact nature and high power of THz-QCLs make them a very promising source for future bioeffects studies. For additional details on THz QCLs, we refer the reader to an excellent review by Williams (2007).

\subsubsection{Electronic Solid-State Terahertz Devices: Frequency Up-Conversion}

In recent years, many electronic solid-state devices have been developed to generate modest power levels of $\mathrm{THz}$ radiation. However, these systems are limited because the technologies and techniques that they use provide limited output power at higher $\mathrm{THz}$ frequencies (Siegel 2002). Figure 7.7a contains the images of several state-of-the-art 


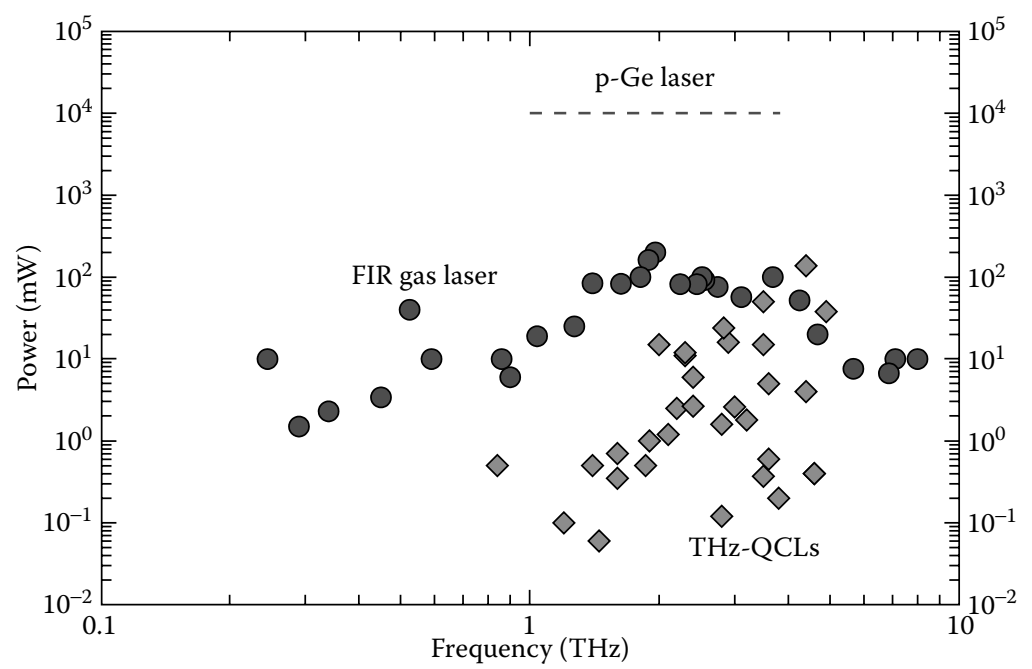

FIGURE 7.6 Peak performance of direct generation THz laser sources. Peak output power $(\mathrm{mW})$ plotted versus frequency (THz). Far-infrared (FIR) data, p-type Germanium (p-Ge), and quantum cascade laser (QCL). (Data provided courtesy of Dr. Gerald J. Wilmink, Dr. Eric Bundermann, and Dr. Benjamin S. Williams, respectively.)

(a) InP Gunn $(0.48 \mathrm{THz})$

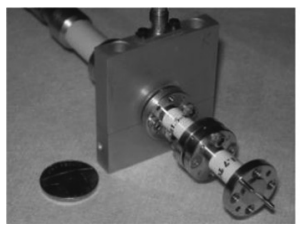

Frequency multiplier $(1.9 \mathrm{THz})$

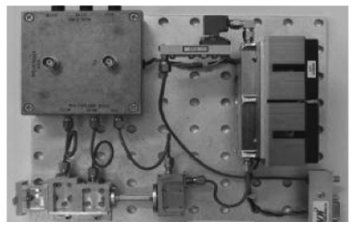

Frequency multiplier $(2.7 \mathrm{THz})$

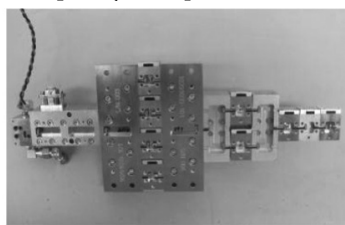

(b) Frequency multiplication of microwaves (up-conversion)

Microwave oscillator: Gunn diode

Frequency multiplier element

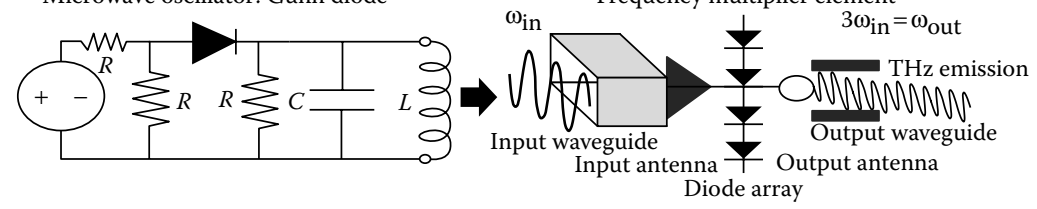

FIGURE 7.7 (a) Photographs of state-of-the-art solid-state electronic THz sources that use frequency multiplication of microwave technology. Image of InP Gunn device operating at 0.48 THz. (Courtesy of Dr. H. Eisele, University of Leeds, UK.) Frequency multiplier unit operating from 1.4 to 1.9 THz and 2.7 THz. (Courtesy of Dr. Hessler, Virginia Diodes, Inc., Charlottesville, VA and Dr. Peter Siegel, founder JPL Submillimeter Wave Advanced Technology Team [JPL SWAT]). (b) Schematic representation of primary components of a typical frequency multiplier $\mathrm{THz}$ source. Circuit diagram for microwave oscillator and frequency multiplier unit, which collects, transmits, and up-converts signal to output $\mathrm{THz}$ frequencies. 
electronic devices. Electronic devices typically consist of a microwave synthesizer or oscillator, and a frequency multiplier element, which consists of an array of schottky barrier diodes (SBDs). In brief, the oscillator functions to generate "seed" microwave radiation, and the SBD array functions to multiply the frequency of the incoming microwave radiation to $\mathrm{THz}$ frequencies (i.e., frequency up-conversion).

Several features contribute to the utility of electronic sources. First, they provide high average output power (typically, $\sim 100 \mathrm{~mW}$ at lower $\mathrm{THz}$ frequencies). Second, they generate narrow line-width $\left(10^{-6}\right), \mathrm{cw}$ THz radiation. Finally, electronic sources are rugged, compact, and operate at room temperature. Due to these properties, solid state electronic $\mathrm{THz}$ sources are commonly used in both basic and applied research. However, despite their incredible efficiency at lower $\mathrm{THz}$ frequencies, such approaches are limited and are only capable of generating a few $\mathrm{mWs}$ of power at higher frequencies. In fact, the output power of electronic sources has been reported to drop off between $1 / \mathrm{f}^{2}$ and $1 / \mathrm{f}^{3}$ with increases in frequency. Thus, significant advances must be made in order to create electronic sources that operate more efficiently at these higher frequencies.

In recent years, several groups have addressed these fundamental challenges. A few notable examples of recent progress include: InP and GaAs Gunn diodes (Eisele and Kamoua 2006; Eisele 2010), frequency multiplier units based on SBDs, impact ionization avalanche transit-time devices (IMPATTs) (Mukherjee et al. 2007; Mukherjee and Roy 2010), tunneling transit-time diodes (TUNNETT) (Buniatyan et al. 2004; Nishizawa et al. 2008; Ryzhii 2009), and resonant tunneling diodes (RTDs) (Asada 2008). Figure 7.7a contains an image of the InP Gunn diode created by Dr. Eisele, a professor from the University of Leeds in the United Kingdom (Eisele and Kamoua 2006; Eisele 2010). This particular system uses a $160 \mathrm{GHz}$ oscillator seed and up-converts it to produce a third harmonic at $0.48 \mathrm{THz}$. This source generates greater than $100 \mathrm{~mW}$ of power at $0.1 \mathrm{THz}$ and $0.1 \mathrm{~mW}$ at $0.48 \mathrm{THz}$ (Figure 7.8). Varactors, varistors, and other advanced frequency-multiplier systems are also being developed at NASA Jet Propulsion Laboratory (JPL) and Virginia Diodes, Inc. (Figure 7.7a). These systems provide nearly a milliwatt of power at frequencies greater than $1 \mathrm{THz}$. Overall, electronic solid-state devices continue to be reliable sources for generation of low-frequency $\mathrm{THz}$ radiation. With future advances in fabrication techniques, such sources may find increased use in THz bioeffects studies.

\subsubsection{Accelerating Electron-Based Terahertz Sources}

Several THz sources use electron accelerators and periodic beam undulation techniques to generate bright $\mathrm{THz}$ radiation. Common examples include backward wave oscillators (BWOs), travelling wave tubes (TWTs), klystrons, gyrotrons, and free-electron lasers (FELs). Interestingly, despite their striking differences in appearance and size, all of these devices function using the same general operation principle. Basically, an electron beam is accelerated, collimated, and modulated using magnets and an external structure (i.e., comb grating in a BWO and a wiggler in a FEL). The external structure functions to create a periodic acceleration of the electrons in the beam, which directly results in the generation of $\mathrm{THz}$ radiation. Further details regarding the specific elements and operation principles of BWOs and FELs are provided below. 


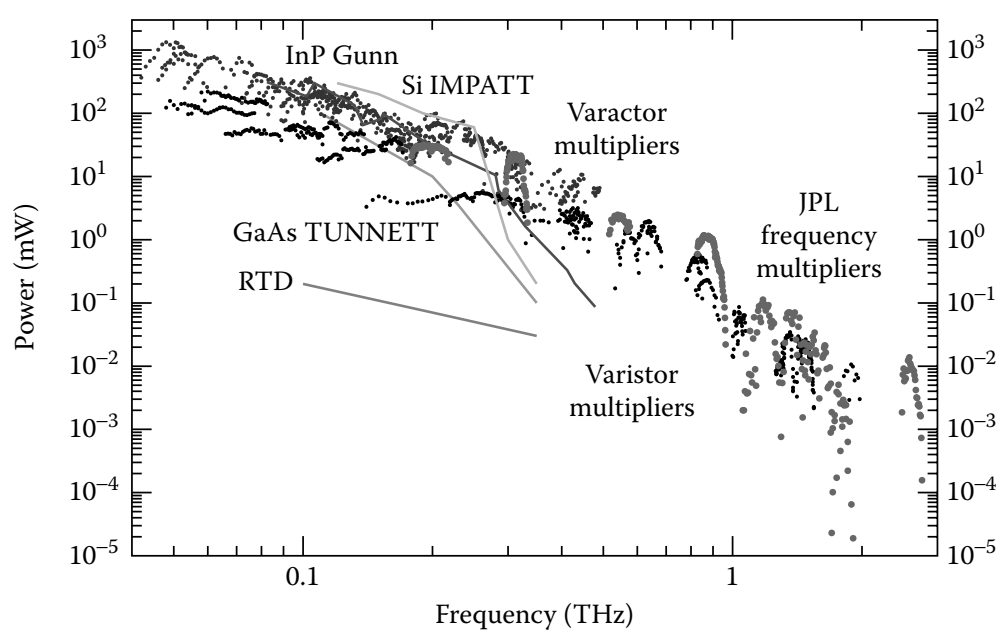

FIGURE 7.8 (See color insert.) Peak performance of solid-state electronic THz sources using frequency multiplication of microwave technology. InP Gunn, Si impact ionization avalanche transit-time device (IMPATT), GaAs tunneling transit-time diode (TUNNETT), and resonant tunneling diode (RTD). (Data courtesy of Dr. H. Eisele, University of Leeds, UK.) Varactor multiplier and varistor multiplier data. (Courtesy of Dr. Hessler, Virginia Diodes, Inc., Charlottesville, VA. Jet Propulsion Laboratory (JPL) frequency multiplier unit. (Data courtesy of Peter Siegel, JPL SWAT Team.)

\subsubsection{Backward Wave Oscillators}

BWOs, also called carcinotrons or backward wave tubes, are tabletop devices that use electron vacuum tubes to generate $\mathrm{THz}$ radiation. The name $\mathrm{BWO}$ was given to these sources because they use an electron beam that travels backward, or in the opposite direction of a travelling EM wave. For years after their first demonstration in 1951 (Kompfner and Williams 1953; Daniel and Bernard 1959; Rudolf 1961), BWOs were primarily developed and used in Russia. In more recent years, however, many U.S. and European companies have begun commercializing BWOs (Figure 7.9a).

BWOs typically consist of the following elements: a magnetic housing system, a magnet (typically, 1 Tesla or greater), high-voltage power supplies $(2-6.5 \mathrm{kV})$, comb grating, a cooling system, a waveguide, and an electron gun (cathode and anode). Figure 7.9b contains a schematic of the primary elements and principle operation of a BWO. The initial step in BWO operation involves heating the cathode to temperatures of $\sim 1200^{\circ} \mathrm{C}$. Heating causes electrons to be emitted and travel toward the anode. This process is accelerated using an electric field, which is directed between the cathode and anode. Once a sufficient number of electrons are created, this results in the generation of an electron beam. The magnetic system is then used to collimate the beam. The comb grating is then used to drive the electrons into "bunches," which, in turn, create surface waves. Once the velocity of the surface wave equals that of the electron beam, energy is delivered to the EM wave. The power of the generated EM wave has its group velocity directed opposite to the direction of motion of the electrons, and the output $\mathrm{THz}$ wave is coupled out through a waveguide. In a BWO, the frequency of the generated wave is 
BWO frequency synthesizer

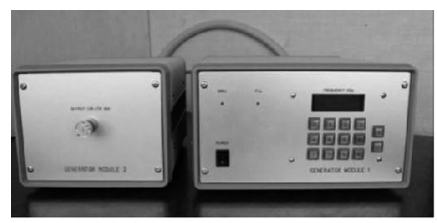

Compact BWO

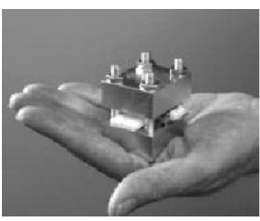

(a)

\section{JLabs THz FEL}

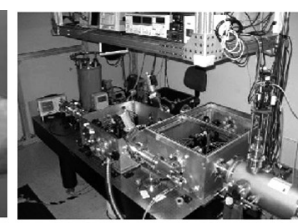

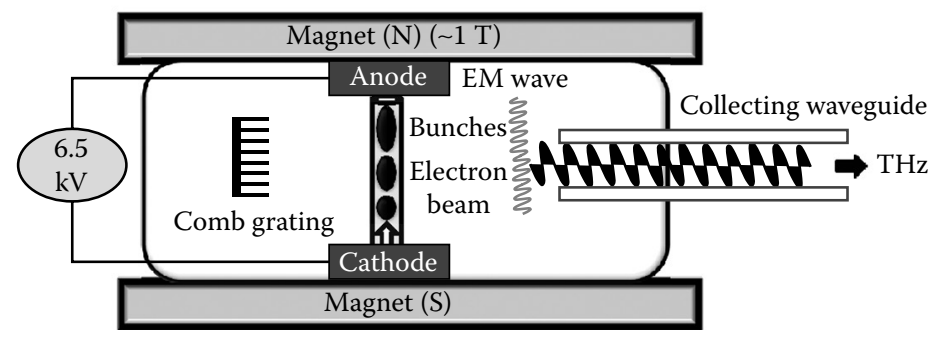

(b)

FIGURE 7.9 (a) Photographs of backward wave oscillators (BWOs) and free-electron laser (FEL) $\mathrm{THz}$ sources. Images of BWO frequency synthesizer and recently developed compact BWO. (Courtesy of Dr. A. Gershteyn (Insight Product Company LLC) and Dr. Gerald Mearini (Teraphysics LLC, Cleveland, $\mathrm{OH}$ ), respectively. Image of THz FEL at Jefferson Laboratory courtesy of Dr. Michael Klopf.) (b) Schematic of primary elements and operation of electron accelerators used in BWOs.

controlled by the velocity of the electron beam; as a result, the output $\mathrm{THz}$ frequency can be directly adjusted by altering the bias voltage. Typical BWO sources are tunable over a wide range of frequencies $(0.035-1.42 \mathrm{THz})$, provide modest power levels $(0.2-100 \mathrm{~mW})$, and offer narrow line widths (1-10 MHz; Figure 7.10).

BWOs were employed in many of the initial THz bioeffects studies (Il'ina et al. 1979). However, several issues have limited more recent use of these devices. First, BWOs are very expensive due to the sophisticated engineering and development approaches that are required to create them. Second, they have limited portability due to their large and a cumbersome magnetic housing system (i.e., 27 Liters, $100 \mathrm{lbs}, 1 \mathrm{~T}$ ). Third, they have short working lifetimes ( $\sim 500$ hours). This is primarily because the electron vacuum tubes used in BWOs quickly wear down due to continuous exposure to high temperatures $\left(1200^{\circ} \mathrm{C}\right)$, voltages $(6.5 \mathrm{kV})$, and pressures $\left(10^{-8}\right.$ Torr $)$.

In recent years, several companies have developed compact $\mathrm{THz}$ BWOs to transition vacuum electronic based systems from $\mathrm{MW}$ to $\mathrm{THz}$ frequencies (Figure 7.9a). These companies are using CVD diamond technology and sophisticated microfabrication techniques to reduce the size of their devices by a factor of 10 . These miniaturized vacuum electronic devices are compact, light, continuously tunable, and provide $\sim 325 \mathrm{~mW}$ of power at frequencies ranging from 0.1 to $0.78 \mathrm{THz}$ (Mearini 2010; Figure 7.10). Miniaturized BWOs show promise for use not only in basic science research, but also in a host of practical applications. 


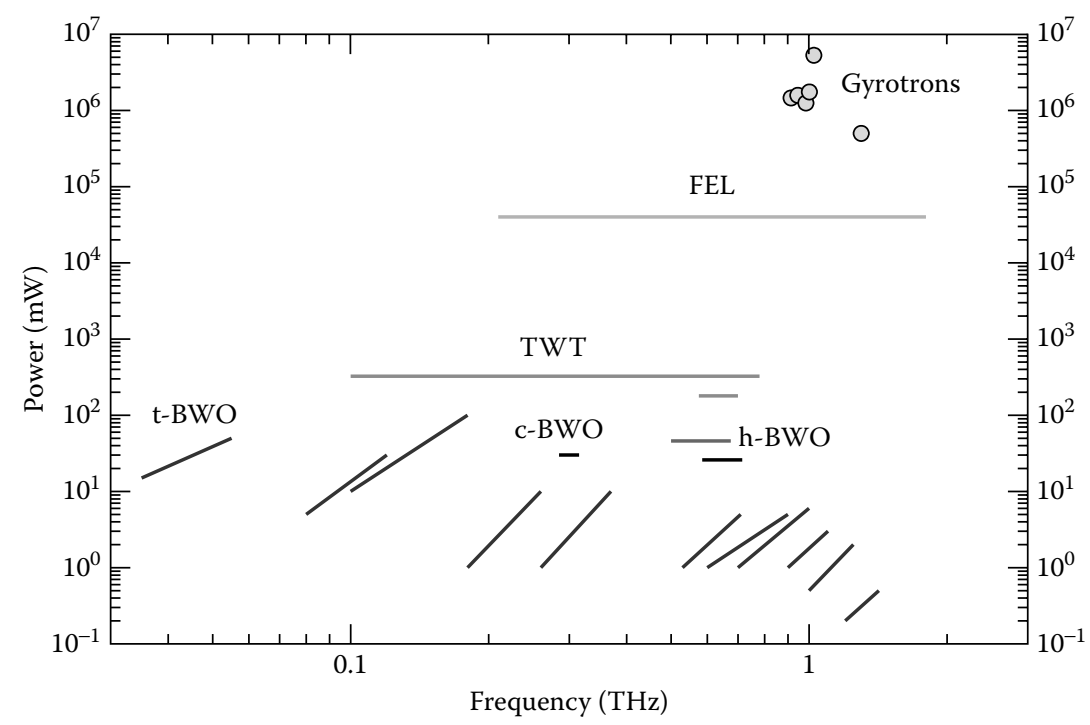

FIGURE 7.10 (See color insert.) Peak performance of THz sources that use electron accelerators or electron vacuum tubes. t-backward wave oscillator (BWO). (Data courtesy of Dr. A. Gershteyn (Insight Product Company LLC.) c-BWO, h-BWO, travelling wave tube (TWT) data courtesy of Dr. Gerald Mearini (Teraphysics LLC.) Gyrotron. (Data courtesy of Dr. Glyavin (Nizhny Novgorod, Russia; Glyavin, M. Y., A. G. Luchinin et al. 2008. Phys Rev Lett 100(1):015101-3.) Free-electron laser (FEL.) (Data courtesy of Dr. Michael Klopf (Jefferson Laboratory, Newport News, VA). Note: FEL and gyrotrons are pulsed sources (pulse power is plotted), whereas other sources are CW sources.

\subsubsection{Terahertz Free-Electron Lasers}

Over the past few decades, numerous FELs have been developed to create high power $\mathrm{THz}$ radiation. Four THz FELs are located in the United States (Jefferson Laboratory, UCSB, University of Hawaii, and Stanford), and several are in operation in Japan, Korea, Netherlands, Germany, Australia, France, Russia, and Italy.

$\mathrm{THz}$ FELs consist of two primary components: a large electron accelerator (typically, linac or electrostatic) and a wiggler magnetic array. The electron accelerator serves to provide a relativistic electron beam, while the magnetic field of the wiggler functions to undulate the electron beam. The modulation of the electron beam causes the oscillation of electrons, resulting in emission of bright $\mathrm{THz}$ radiation.

$\mathrm{THz}$ FELs are ideal sources for bioeffects studies because they are widely tunable both in terms of frequency and in mode of operation (cw and pulsed). For instance, the THz FEL at Jefferson Laboratory emits very high levels of average power on the order or $40 \mathrm{~W}$, high pulse energy of roughly $3 \mathrm{~nJ} /$ pulse, narrow pulsewidth (350 fs), and a $75 \mathrm{MHz}$ repetition rate (Figure 7.10) (Carr et al. 2002). The primary disadvantages of FELs, which are basically small scale particle accelerators, are they have a large footprint, require teams of researchers to run, and are expensive to engineer, maintain, and operate. Despite these limitations, FELs provide the highest output power of any modern $\mathrm{THz}$ source, and will probably continue to be the most commonly used 
source for THz bioeffects studies. (Zenil et al. 2007; Scarfi et al. 2003; Doria et al. 2004; Grosse 2002; Berns et al. 1990; Berns et al. 1994; Berns and Bewley 1987.)

\subsubsection{Nonlinear Optical Terahertz Sources: Frequency Down-Conversion}

Today's most ubiquitous pulsed $\mathrm{THz}$ sources use laser-driven emitters (i.e., diodepumped fiber lasers) and nonlinear optical effects to down-convert frequencies from the optical region. These compact systems are attractive sources because they provide monochromatic $\mathrm{THz}$ radiation with a narrow line width, efficiently operate at room temperature, and are broadly tunable. Pulsed $\mathrm{THz}$ sources typically exploit one of the following second-order $\left(\chi^{2}\right)$ nonlinear optical processes to generate $\mathrm{THz}$ radiation: (1) difference frequency generation (DFG), (2) optical rectification (OR), and (3) optical parametric oscillation (OPO). In this section, we provide a brief overview of each of these systems. For a more comprehensive review, we refer the reader to the following review articles (Suizo and Kawase 2008; Vodopyanov 2008).

\subsubsection{Difference Frequency Generation}

DFG sources were first demonstrated in the 1960s using a quartz crystal placed inside the cavity of a ruby laser (Zernike and Berman 1966). These studies demonstrated that $\mathrm{THz}$ radiation could be generated through the interaction of two optical laser beams with nonlinear crystals. The optical sources typically used in DFG systems are narrowband IR $(\lambda=0.8-2.1 \mu \mathrm{m})$ laser beams with slightly different frequencies $\left(\omega_{1}\right.$ and $\left.\omega_{2}\right)$. When these sources interact with nonlinear crystals, most commonly gallium selenide or arsenide (GaSe or GaAs), they generate emission at a beat frequency equal to the difference between the two input frequencies $\left(\omega_{\mathrm{THz}}=\omega_{1}-\omega_{2}\right)$. DFG systems are attractive $\mathrm{THz}$ sources for spectroscopy applications because they are easily tunable, provide $\mathrm{THz}$ radiation with frequencies ranging from 0.2 to $6 \mathrm{THz}$, and provide high-peak powers ranging from $10^{2}$ to $10^{5} \mathrm{~mW}$ (Shi et al. 2002). However, due to their low average power, DFG sources are not commonly used for $\mathrm{THz}$ bioeffects studies. The primary challenge with DFG techniques is that the nonlinear optical materials have very high absorption coefficients, which make $\mathrm{THz}$ generation inefficient. In recent years, several methods have been developed to overcome this limitation. Among these, quasi-phase-matching (QPM) techniques show tremendous promise (Suizo and Kawase 2008).

\subsubsection{Optical Rectification}

In OR sources, $\mathrm{THz}$ radiation is generated using nonlinear media and difference frequency mixing approaches between the Fourier components of an optical pulse. OR systems typically consist of an optical pump source, most often a femtosecond (fs) optical laser source, and thin electro-optic nonlinear crystals. OR sources have been shown to generate broadband $\mathrm{THz}$ radiation with pulse energies of $10 \mu \mathrm{J}$ and a peak power of $100 \mu \mathrm{W}$. Such systems are commonly used in THz spectroscopy systems.

\subsubsection{Optical Parametric Oscillation}

Optical parametric generation is a second-order $\left(\chi^{2}\right)$ nonlinear optical process where the photon of a pump pulse is converted into two photons with lower quantum energies. 
TABLE 7.1 Monochromatic-Tunable Terahertz (THz)-Wave Sources Based on Nonlinear Frequency Conversion

\begin{tabular}{lccc}
\hline & \multicolumn{3}{c}{ THz Source } \\
\cline { 2 - 4 } & $\begin{array}{c}\text { THz-wave } \\
\text { Parametric Generation } \\
\text { (TPG) }\end{array}$ & $\begin{array}{c}\text { THz-wave } \\
\text { Parametric Oscillator } \\
\text { (TPO) }\end{array}$ & $\begin{array}{c}\text { Injection-seeded } \\
\text { THz-wave Parametric } \\
\text { Generation (Is-TPG) }\end{array}$ \\
\hline Tuning by & THz grating & Cavity rotation & Seeding \\
Frequency range & $0.9-3.8 \mathrm{THz}$ & $0.9-2.7 \mathrm{THz}$ & $0.6-3.0 \mathrm{THz}$ \\
Line width & $>1 \mathrm{THz}$ & $\sim 30 \mathrm{GHz}$ & $\sim 100 \mathrm{MHz}$ \\
Output power & $10 \mathrm{~nJ} /$ pulse & $20 \mathrm{~nJ} /$ pulse & $100 \mathrm{~nJ} /$ pulse \\
\hline
\end{tabular}

Source: Data courtesy of Dr. Kodo Kawase.

Typically, the pump pulse $\left(\omega_{\mathrm{p}}\right)$ and the idler pulses $\left(\omega_{\mathrm{i}}\right)$ are at near-IR optical frequencies, whereas the signal photon is at THz frequencies $\left(\omega_{\mathrm{TH} z}\right)$. The sum of $\omega_{\mathrm{THz}}$ and $\omega_{\mathrm{I}}$ are equal in quantum energy to the $\omega_{\mathrm{p}}$ (i.e., $\omega_{\mathrm{p}}=\omega_{\mathrm{THz}}+\omega_{\mathrm{I}}$ ). These processes are most commonly exploited using lithium niobate crystals $\left(\mathrm{LiNbO}_{3}\right) . \mathrm{LiNbO}_{3}$ is an ideal material for $\mathrm{THz}$ wave generation because it is transparent over a wide wavelength range, and it has a large nonlinear absorption coefficient at wavelengths where pump lasers have the most power. OPO sources often use a Q-switched mode-locked Nd:YAG laser as the pump source $(\lambda=1.064 \mu \mathrm{m}, 7 \mathrm{ps}$ pulse duration, $50 \mathrm{MHz}$ repetition rate, and $10 \mathrm{~W}$ average power). These sources are continuously tunable from 1 to $3 \mathrm{THz}$, and their output power is directly proportional to the idler intensity.

In recent years, several novel approaches have been used to increase the idler intensity, and in turn, the $\mathrm{THz}$ output power. Notable approaches include the following: THz-wave parametric oscillator (TPO), THz-wave parametric generation (TPG), and injection-seeded TPG (is-TPG). Kawase et al. recently reported on the development of a widely tunable is-TPG source with the following specifications: frequency $(0.7-2.4 \mathrm{THz})$, high-peak power $(>200 \mathrm{~mW})$, spectral resolution $\left(<100 \mathrm{MHz}\right.$ and $\left.0.003 \mathrm{~cm}^{-1}\right)$, and room temperature operation (Suizo and Kawase 2008). See Table 7.1 for a comparison of these systems.

\subsection{Terahertz Applications}

$\mathrm{THz}$ spectroscopy and imaging technologies are finding widespread use in biomedical, medical, military, and defense applications. In this section, we provide an overview of the history and practical uses for these technologies.

\subsubsection{Terahertz Spectroscopy}

Over the past decade, numerous groups have developed THz time-domain spectroscopy (THz-TDS) techniques to characterize the frequency-dependent optical properties of 
biological liquids (Kindt and Schmuttenmaer 1996; Venables and Schmuttenmaer 1998; Venables and Schmuttenmaer 2000; Venables et al. 2000). The vast majority of these studies have used a photoconductive (PC) switch technique that was pioneered at Bell Laboratories in the 1980s (Auston 1975; Auston et al. 1984; Fattinger and Grischkowsky 1989; Grischkowsky et al. 1990; Taylor et al. 2008). In brief, the PC switch technique uses a pulse from an fs laser-often times a Ti-sapphire laser-to excite a biased PC antenna consisting of metallic striplines deposited on semiconductor materials. Upon interaction with the semiconductor material, the optical pulse generates photocarriers. These photocarriers are then accelerated using a direct current (DC) bias, and in turn create photocurrent within the PC antenna. These time-varying photocurrents, occurring in the subpicosecond range, thereby emit broadband EM radiation at $\mathrm{THz}$ frequencies.

$\mathrm{THz}$-TDS devices that use Ti-sapphire lasers and operate in transmission mode can provide accurate laboratory-based measurements; however, they are not suitable for in vivo clinical applications for several reasons. First, Ti-sapphire lasers are impractical tools, as they are incredibly large, heavy, and expensive. Second, biological tissues consist primarily of water, which is known to strongly absorb $\mathrm{THz}$ radiation $\left(\mu_{\mathrm{a}}=300 \mathrm{~cm}^{-1}\right.$ at $1.5 \mathrm{THz}$; Kindt and Schmuttenmaer 1996). Therefore, tissues must be sectioned into very thin slices $(\leq 200 \mu \mathrm{m})$ in order to make spectroscopic measurements in transmission mode. Valuable spectroscopic information can be gained using sectioned tissues; however, the sectioning process can dehydrate and alter tissue morphology. Because these alterations can drastically affect the tissue's optical properties, ideally THz-TDS measurements should be performed on intact human tissues using reflection based measurements.

In more recent years, several $\mathrm{THz}$ spectrometers have been developed to measure the optical properties of intact human tissues using a reflection based geometry. Figure 7.11 contains photographs of several "state-of-the-art" $\mathrm{THz}$ spectrometers. Rather than making transmission-based measurements, these systems measure spectra in reflection mode (Pickwell et al. 2004; Pickwell et al. 2005; Ashworth et al. 2009; Huang et al. 2009; Huang et al. 2009; Wilmink et al. 2011; Pickwell-MacPherson and Wallace 2009).

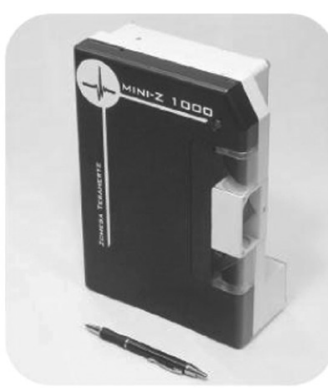

(a)

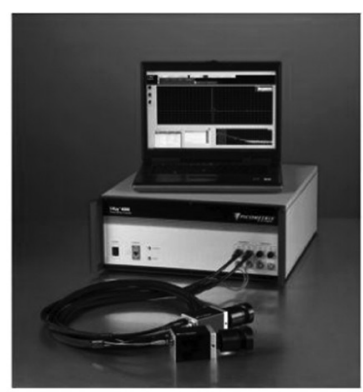

(b)

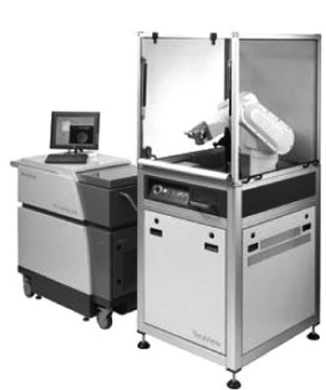

(c)

FIGURE 7.11 Photographs of several "state-of-the-art" THz spectrometers and imaging systems. (a) Mini-Z 1000. (Courtesy of Zomega THz Corp, Troy, NY, USA.) (b) T-Ray ${ }^{\mathrm{TM}} 4000 \mathrm{TD}-\mathrm{THz}$ system (Courtesy of Picometrix Inc., Ann Arbor, MI, USA.) (c) TPI ${ }^{\mathrm{TM}}$ Imaga 2000. (Courtesy of TeraView Ltd, Cambridge, UK). 
These systems are presently being used to detect and diagnose cancer, burns, and other disorders (Chan et al. 2007; Oh et al. 2008; Ashworth et al. 2009; Oh et al. 2009). The contrast and sensitivity of these systems are due to the fact that the optical properties of healthy, burned, and diseased tissues vary widely at $\mathrm{THz}$ frequencies. In addition, these properties have been shown to vary with several other factors: organism (e.g., human, rat, and porcine) (Pickwell et al. 2004; Pickwell et al. 2005; Huang et al. 2009; PickwellMacPherson and Wallace 2009; Sun et al. 2009), tissue type (e.g., skin, muscle, adipose, liver, and heart) (Fitzgerald et al. 2003; Pickwell et al. 2004; He 2006), preparation technique (e.g., freshly excised vs. frozen-thawed), and fixation duration (Sun et al. 2009). Overall, $\mathrm{THz}$ spectrometers that permit reflection-based measurements have proven to be useful for a host of medical and biomedical applications.

\subsubsection{Terahertz Imaging}

From a historical perspective, it is interesting to note that the first X-ray image was collected in 1895, while the first T-ray image was not captured until 1995, exactly 100 years later. Figure 7.12 contains the photographs of the first X-ray and T-ray images. The first $\mathrm{X}$-ray images provided definitive evidence that $\mathrm{X}$ ray machines would be very useful in examination of bone structures. Similarly, the first T-ray images provided visual proof that $\mathrm{THz}$ imaging systems would be useful in accurately resolving hydration levels in leaves and other biological structures. Since their initial demonstration 15 years ago, significant progress has been made in the refinement of these approaches. Many factors have contributed to these advancements, including the increased availability of suitable sources and the elevated interest in the $\mathrm{THz}$ research field.

One of the most unique properties of $\mathrm{THz}$ radiation is that it penetrates many common nonpolar dielectric materials (e.g., leather, plastics, and paper), yet is reflected strongly off most metals (e.g., silver is $\sim 0.996$ and gold is $\sim 0.994$ ) (Oh et al. 2009). This property has been the cornerstone to many security applications. Figure 7.13 ( $\mathrm{a}$ and b) contains

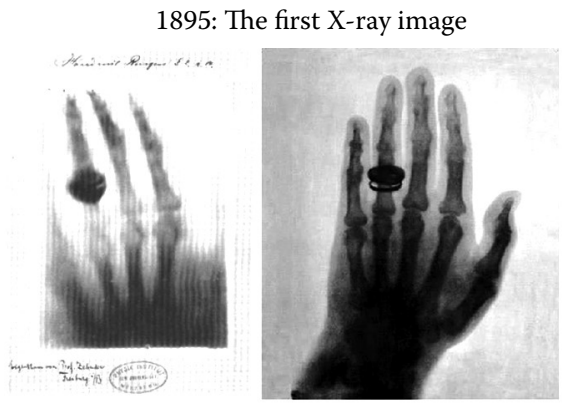

(a)
1995: The first T-ray image

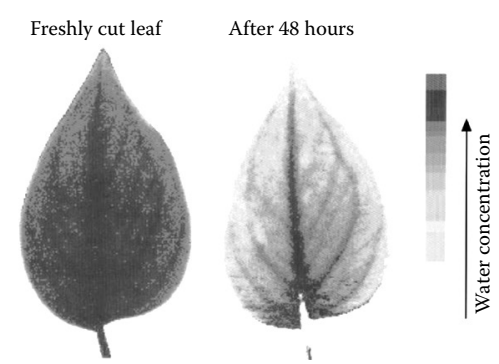

(b)

FIGURE 7.12 (See color insert.) X-ray versus T-ray. (a) 1895: The year of the first X-ray image. Photograph of the hand of Frau Rantgen. (Reprinted from Mould, R. F. 1995. Phys Med Biol 40(11):1741. With permission.) (b) 1995: The year of the first T-ray image. THz image of a leaf. (Reprinted from Hu, B. B., and M. C. Nuss. 1995. Opt Lett 20(16):1716-8. With permission. ๑1995, Optical Society of America.) 
a few of the earliest images that demonstrate the ability of $\mathrm{THz}$ applications to resolve concealed weapons through both paper and leather briefcases (Clery 2002; Karpowicz et al. 2005). Over the past decade, such approaches have been refined, and groups have demonstrated that high-resolution $\mathrm{THz}$ imaging operating at $0.6 \mathrm{THz}$ is capable of detecting weapons concealed by clothing at standoff ranges up to $25 \mathrm{~m}$ (Cooper et al. 2008). Such approaches are also being used to identify concealed explosives and drugs (Liu et al. 2007). In addition to active imaging, several companies are also beginning to commercialize passive $\mathrm{THz}$ imaging systems for covert screening in airports.

$\mathrm{THz}$ imaging techniques are also actively being developed for biomedical purposes. $\mathrm{THz}$ medical imaging techniques were first demonstrated in 2002 (Woodward et al. 2002; Woodward et al. 2003). The results of this study underscored the ability of $\mathrm{THz}$ imaging tools to aid in the diagnosis of basal cell carcinoma (BCC) - the most common cancer worldwide. Figure 7.13 (c and d) contains the visible and $\mathrm{THz}$ images collected in this study. More recent reports have showed that $\mathrm{THz}$ imaging modalities are not only useful for distinguishing spectral differences between normal and cancerous tissue, but also are suitable for resolving differences within tumors (Figure 7.13e). In addition to

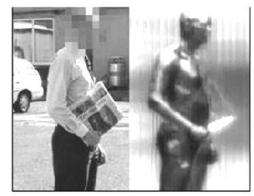

(a)

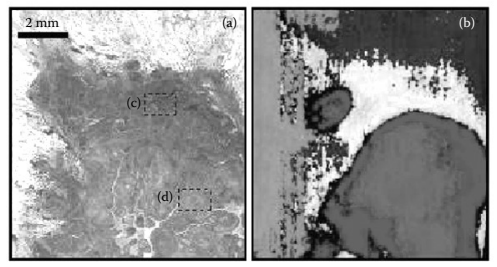

(e)

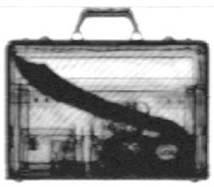

(b)

(c)

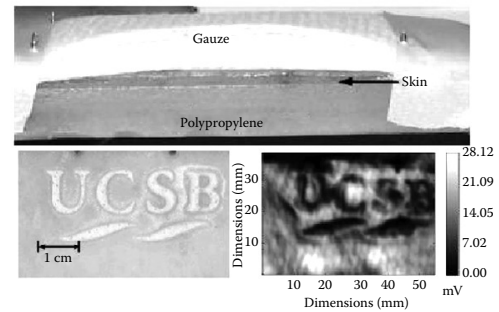

(f)

FIGURE 7.13 (See color insert.) THz imaging applications. (a) THz image of concealed knife behind a newspaper. (Reprinted from Clery, D. 2002. Science 297:761-2. With permission. ๑2002, Science.) (b) $0.2 \mathrm{THz} \mathrm{CW}$ image of a briefcase holding a large knife. (Reprinted from Karpowicz, N., H. Zhong et al. 2005. Appl Phys Lett 86(5):054105. With permission. (2005, America Institute of Physics.) (c) Visible image of cancerous skin (left) and healthy skin (right). (d) THz image of cancerous skin (left) and healthy skin (right). (Reprinted from Woodward, R. M., V. P. Wallace et al. 2003. J Invest Dermatol 120(1):72-8. With permission. @2003, Nature.) (e) Visible histological image (left) and $\mathrm{THz}$ image (right) of squamous cell carcinoma in lung tissue. (Reprinted from Brun, M. A., F. Formanek et al. 2010. Phys Med Biol 55(16):4615. With permission. (2010, Institute of Physics.) (f) Reflective THz imaging of porcine skin burns: experimental setup (top), photograph of burn (left), and $\mathrm{THz}$ image of burn through 10 layers of medical gauze (right). (Reprinted from Taylor, Z. D., R. S. Singh et al. 2008. Opt Lett 33(11):1258-60. With permission. @2008, Optical Society of America.) 
cancer, THz imaging approaches are also being developed to better identify skin-surface abnormalities, such as burns, scars, and wounds (Bogue 2009; Oh et al. 2009; PickwellMacPherson and Wallace 2009; Suen et al. 2009). In 2008, a study demonstrated that a reflective THz-pulsed imaging system could be used to obtain high-resolution images of skin burns through 10 layers of dry medical gauze. Figure 7.13f contains the photographs of the experimental setup, visible burn images, and $\mathrm{THz}$ images for burned skin through gauze. In summary, $\mathrm{THz}$ spectroscopy and imaging approaches are being used in many biomedical, medical, and security applications.

\subsection{Terahertz Skin Interactions}

In this section, we provide details regarding the composition of skin, the primary biologic target for $\mathrm{THz}$ radiation. Then, we outline the fundamental principles of tissue optics that govern $\mathrm{THz}$ skin interactions, including a discussion of the optical properties of skin at $\mathrm{THz}$ frequencies. We then discuss photothermal mechanisms and the thermal effects associated with each temperature damage zone. Finally, we discuss computational and empirical approaches to measure THz-induced tissue damage. The importance of tissue damage measurements is then discussed in the context of determining safe exposure standards at $\mathrm{THz}$ frequencies.

\subsubsection{Skin Anatomy}

Skin covers the entire human body and is therefore our largest organ. At the most basic level, skin provides several vital functions: (1) provide protective barrier from the outside world, (2) receive sensory information, (3) control evaporation and prevent fluid loss, (4) regulate heat loss, and (5) provide a water-resistant barrier to minimize loss of essential nutrients. The skin barrier can be breached by a host of common chemical, mechanical, and thermal stressors. In addition, unique stressors, such as ultraviolet (UV), IR, and $\mathrm{THz}$ radiation can also cause damage to the skin barrier. When skin is exposed to external stressors it responds by eliciting a dynamic repair response to combat the insult. This response takes place at a systemic, tissue, cellular, and molecular level.

Skin tissue consists of an outer epidermis, an underlying dermis, and a hypodermis (Figure 7.14). The epidermis consists primarily of water, keratin proteins, keratinocytes, Langerhans, and melanocytes. In order to provide various levels of protection, the thickness of the epidermis varies across the human body between 50 and $1000 \mu \mathrm{m}$. Epidermal thickness is controlled by the keratinocytes, which are genetically programmed to undergo kertinization or stratification processes. Figure 7.14a contains a histological cross section of the five epithelial layers. From the outside moving inward, the layers include the following: stratum corneum (sc), stratum lucidum (sl), stratum granulosum (sg), stratum spinosum (ss), and stratum basale (sb).

The inner sb layer consists of a single layer of cells, which are highly concentrated with melanin pigment granules. Melanin granules are produced by melanocytes and are transferred to keratinocytes by cytocrine secretion. The color of human skin (i.e., Fitzpatrick type) varies with the concentration and type of melanin granules. Although 


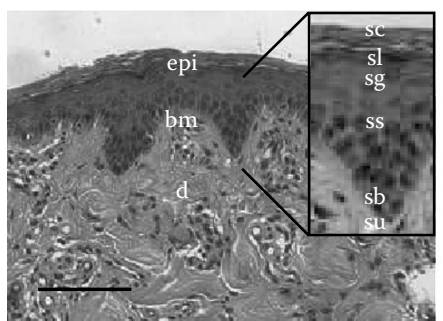

(a)

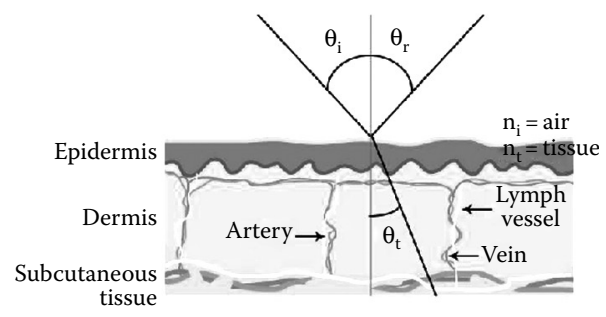

(b)

FIGURE 7.14 (See color insert.) (a) Histological cross section of porcine skin tissue (hematoxylin and eosin stain at $40 \times$ magnification). epi $=$ epidermis, $b m=$ basement membrane, $d=$ dermis, $\mathrm{sc}=$ stratum corneum, $\mathrm{sl}=$ stratum lucidum, $\mathrm{sg}=$ stratum granulosum, $\mathrm{ss}=$ stratum spinosum, and $\mathrm{sb}=$ stratum basale. (b) Cartoon image of $\mathrm{THz}$ light incident on skin at an angle $\theta_{\mathrm{i}}$. A portion of the THz beam is specularly reflected with an angle $\theta_{\mathrm{r}}$ and the remainder is transmitted at $\theta_{\mathrm{t}}$.

spectroscopic studies have not been performed for melanin at $\mathrm{THz}$ frequencies; however, studies at optical frequencies have shown that melanin broadly absorbs from $\lambda=200$ to $1200 \mathrm{~nm}$, and that its absorption decreases with increases in wavelength $[4,5]$. Assuming this trend continues into the $\mathrm{THz}$ region, melanin most likely does not strongly absorb $\mathrm{THz}$ radiation. Aside from providing color to skin, the sb layer also has structural significance, owing to its participation in the formation of the basement membrane (bm). The bm consists of type IV collagen, laminin, entactin/nidogen, and sulfated proteoglycans. It provides structural support for cells, anchorage for neighboring cells, and stimuli for cell growth and migration. It is interesting to note that few studies have been conducted to characterize the optical properties of collagen at $\mathrm{THz}$ frequencies (Tewari et al. 2009).

Immediately below the epidermis is the dermis. The dermis ranges in thickness between 300 and $4000 \mu \mathrm{m}$, and its primary functions are to provide skin with strength, shape, and structural integrity. The dermis consists of fibroblasts attached to an extracellular matrix (ECM). The ECM consists of fibrillar collagen $(-20-50 \mu \mathrm{m}$ thickness) that is embedded within a ground substance. The ground substance consists of water, collagen, elastin, glycosaminoglycans (GAGs), and proteoglycans. GAGs are very hydrophilic and typically sequester a volume of water $\sim 1000$ times their own volume (Vogel 2003). This feature causes most of the water present in the skin to reside in the ground substance of the dermis. In recent years, numerous studies have been conducted to investigate water solvation dynamics and to determine the optical properties for different types of water molecules (i.e., free, bound, bulk)(Pal et al. 2002a,b; Pal et al. 2003a,b; Pal and Zewail 2004; Heugen et al. 2006).

Both type I and III fibrillar collagens consist of three a chains. These chains aggregate into a triple helices forming tropocollagen (TC) molecules. TC aggregates to form microfibrils, fibrils, and sheets of lamellae. Normal fibrillar collagen exhibits a characteristic periodicity of $\sim 67 \mathrm{~nm}$; thus, loss of this banding pattern is commonly used as an indication of tissue thermal denaturation. Several microscopic techniques permit the 
visualization of this banding pattern, such as polarization and transmission electron microscopy (TEM), are commonly used for tissue damage evaluation (See Section 7.4.7 for further details regarding the practical significance of these measurements.)

\subsubsection{Fundamental Principles of Terahertz-Tissue Optics}

When $\mathrm{THz}$ photons interact with skin, a fraction of the photons are reflected at the material boundary, and the remaining photons are transmitted into the skin. Figure $7.14 \mathrm{~b}$ is a graphical representation of an incident $\mathrm{THz}$ wave being reflected and transmitted into human skin. Assuming an unit incident irradiance $E_{0}\left(\mathrm{Wm}^{-2}\right)$, the light transmitted into skin can be defined as: $T=1-R$, where $T$ and $R$ represent the ratio of transmitted and specular reflected photons, respectively. Surface losses are primarily due to the differences in the index of refraction ( $n$ ) between air and tissue, whereas the transmission losses are governed by the tissue's optical properties (i.e., absorption and scattering coefficients, $\mu_{\mathrm{a}}$ and $\mu_{\mathrm{s}}$, respectively).

The first step in THz-tissue optics is to accurately determine the magnitude of specular reflected light at the surface of the skin. Neglecting polarization, the magnitude of specular loses can be computed using Fresnel's equation:

$$
R=\left(\frac{n_{\mathrm{i}}-n_{\mathrm{t}}}{n_{\mathrm{i}}+n_{\mathrm{t}}}\right)^{2}
$$

where $n_{\mathrm{i}}$ is the refraction of air $(n=1)$ and $n_{\mathrm{t}}$ is the index of refraction for skin at different $\mathrm{THz}$ frequencies. Figure $7.14 \mathrm{~b}$ is a graphical illustration of Fresnel reflection losses. In this figure, $\mathrm{THz}$ light is incident on skin at an angle $\theta_{i}$, a portion of the $\mathrm{THz}$ beam is specularly reflected with an angle $\theta_{r}$, and the remainder is transmitted at $\theta_{t}$. Figure 7.15 contains data for the $n$ values of porcine skin, and their corresponding specular reflection values (Wilmink and Roach 2010; Wilmink et al. 2011) The data show that the $n$ values for skin range from 2.2 at $0.1 \mathrm{THz}$ to 1.8 at $1.6 \mathrm{THz}$. These values correspond to specular reflection values ranging between $15 \%$ and $9 \%$. Given these first principle calculations, it is clear that the air-tissue interface leads to appreciable surface loses, which are greatest at lower $\mathrm{THz}$ frequencies.

After calculating the percentage of incident $\mathrm{THz}$ radiation that is reflected off the surface of skin, the next step of tissue optics is to account for the transmission of the remaining light. The remaining $85 \%-91 \%$ of the $\mathrm{THz}$ photons that are transmitted into the tissue can be determined using Beer's law:

$$
E(z)=E_{0} \mathrm{e}^{\left(-\mu_{\mathrm{a}} z\right)}
$$

where $E_{0}$ is the irradiance or power density at the skin surface $\left(\mathrm{W} / \mathrm{m}^{2}\right), E(z)$ the fluence at a depth $z\left(\mathrm{~W} / \mathrm{m}^{2}\right), z$ the tissue depth, and $\mu_{\mathrm{a}}$ the absorption coefficient $(1 / \mathrm{m})$ for the tissue at a given wavelength. The penetration depth $(\delta)$, which is defined as the inverse of the absorption coefficient, represents the depth to which light will penetrate to an intensity of $1 / \mathrm{e}(37 \%)$ of $E_{0}$. 

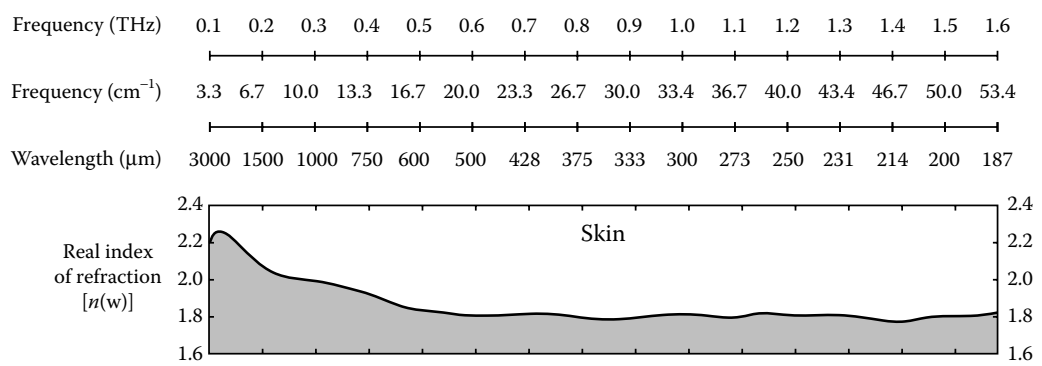

(a)

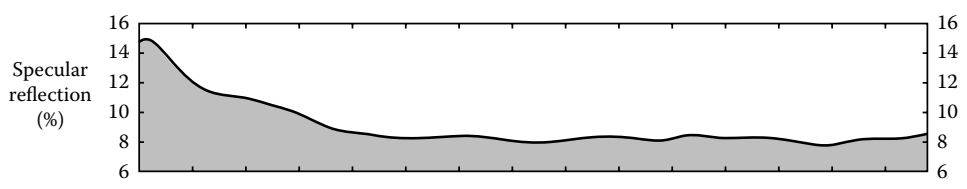

(b)

FIGURE 7.15 (a) Index of refraction (n) for porcine skin as a function of frequency (THz). Data collected using THz-TDS. (Data from Wilmink et al. 2011.) (b) Specular reflection (percentage) for porcine skin as a function of frequency (THz). Values were calculated using Fresnel equation with $\theta_{i}=0$ (incident $\mathrm{THz}$ laser beam normal to skin surface.).

\subsubsection{Optical Properties of Skin}

In order to implement Beer's law and calculate the distribution of $\mathrm{THz}$ energy in skin, the optical properties of skin must be characterized at $\mathrm{THz}$ frequencies. As described above in Section 7.3.1, several research groups have measured these properties. Figure 7.16 (a through c) contains data from Pickwell et al. (2005) and Wilmink et al. 2011. These studies show that $n$ values for fresh tissue ranged between 2.2 and 1.8 , whereas $n$ values for the frozen-thawed samples was greater and ranged between 2.6 and 2.0 .

The data also show that the magnitude of $\mu_{\mathrm{a}}$ increased with frequency. At lower THz frequencies $(0.1-0.8 \mathrm{THz})$ fresh and frozen-thawed skin exhibited comparable values for $\mu_{\mathrm{a}}\left(\sim 50-180 \mathrm{~cm}^{-1}\right)$. In contrast, for frequencies greater than $0.8 \mathrm{THz}$, fresh tissue exhibited $\mu_{\mathrm{a}}$ values that were $\sim 10 \%-100 \%$ greater than those measured for frozenthawed tissues. Using the $\mu_{\mathrm{a}}$ values, the $\delta$ for the skin samples (Figure 7.16c) is maximal $(\delta \approx 200 \mu \mathrm{m})$ at $0.1 \mathrm{THz}$ and minimal at $1.6 \mathrm{THz}(\delta \approx 50 \mu \mathrm{m})$.

\subsubsection{Photothermal Mechanisms and Classic Heat Transfer}

When $\mathrm{THz}$ radiation is transmitted into skin, the optical energy is absorbed by target chromophores (i.e., bulk, free, and bound water). Once absorbed, this energy is converted into heat, causing photothermal effects to occur. Using the incident $\mathrm{THz}$ 


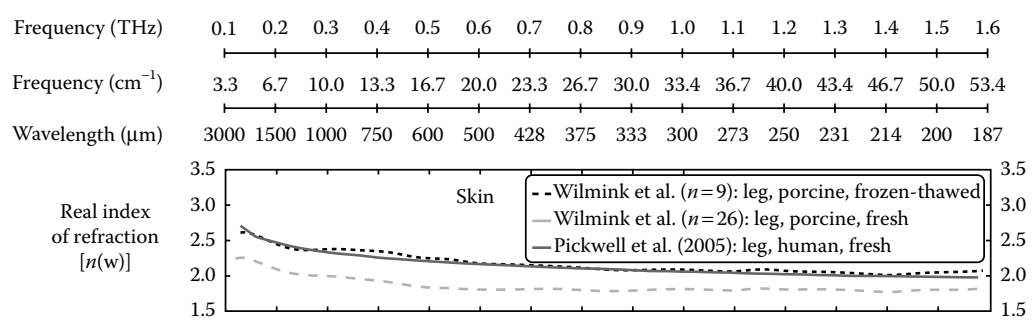

(a)

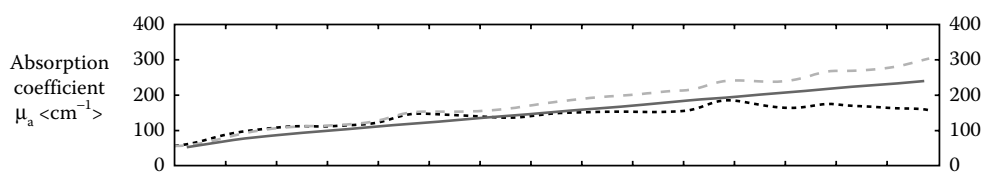

(b)

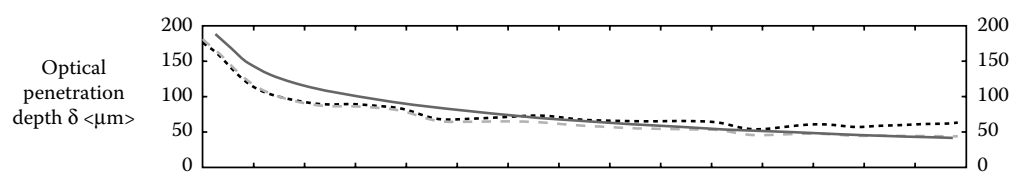

(c)

FIGURE 7.16 Optical properties of porcine skin plotted versus frequency (THz). (a) Real index of refraction $(n)$. (b) Absorption coefficient $\left(\mu_{\mathrm{a}}\right)$. (c) Optical penetration depth $(\delta)$. (Data from Wilmink et al. 2009; Wilmink et al. 2011; and Pickwell et al. 2005.) Data are expressed as means \pm SD, with $n=9$ or 26 .

irradiance and $\mu_{\mathrm{a}}$ for skin, the rate and amount of energy that is deposited into the tissue can be calculated. This rate is typically defined as the rate of heat generation $S\left(\mathrm{~W} / \mathrm{m}^{3}\right)$ :

$$
S(r, z)=\mu_{\mathrm{a}}(r, z) E_{0}(r, z)
$$

where $E_{0}(r, z)$ is the irradiance at some point $(r, z)$ in the tissue $\left(\mathrm{W} / \mathrm{m}^{2}\right)$. Using $S$, the temperature rise in the tissue can be calculated using the following relationship:

$$
\Delta T=\frac{S \Delta t}{\rho c}
$$

where $\Delta T$ is the temperature rise $\left({ }^{\circ} \mathrm{K}\right), \Delta t$ is the duration of the heat exposure (seconds), $\rho$ is the density of skin that is heated $\left(\mathrm{g} / \mathrm{m}^{3}\right)$, and $c$ is the specific heat of the tissue $(\mathrm{J} / \mathrm{g} \mathrm{K})$.

Depending on the duration of the $\mathrm{THz}$ exposure, heat transfer occurs via the classic paths of heat transfer: conduction, convection, radiation, and evaporation. Conduction is the primary mode of heat transport in tissue, and it is driven by a temperature gradient, which is typically described using Fourier's law:

$$
q_{\text {cond }}^{\prime \prime}=-k A \frac{\Delta T}{\Delta X}
$$


where $q_{\text {cond }}$ is the heat flux (W), $k$ is the thermal conductivity $(\mathrm{W} / \mathrm{m} \cdot \mathrm{K}), A$ is the area $\left(\mathrm{m}^{2}\right)$, and $\Delta T / \Delta X$ is the temperature gradient in the direction of heat flow. Combining Equations 7.4 and 7.5 results in the following general heat transfer equation:

$$
k \nabla^{2} T+S=\rho c \frac{\partial T}{\partial t}
$$

where the first term is the temperature gradient in all directions, $S$ is the heat generation, and the right-hand side of the equation is the change in temperature over time. This equation basically describes the "build up" and distribution of heat in the irradiated tissue.

Convection plays a role in heat transfer when a fluid present can circulate the heat away from an area. Consequently, convection should be considered at tissue surfaces where heat can be lost to the surrounding air. Convection is described by the following:

$$
q_{\text {conv }}^{\prime \prime}=h\left(T_{\mathrm{S}}-T_{\mathrm{a}}\right)
$$

where $q_{\text {conv }}^{\prime \prime}$ is the heat flux $\left(\mathrm{W} / \mathrm{m}^{2}\right), h$ the convection coefficient $\left(\mathrm{W} / \mathrm{m}^{2} \cdot \mathrm{K}\right), T_{\mathrm{s}}$ the temperature of the tissue, and $T_{\mathrm{a}}$ the surrounding temperature.

Radiative heat transport is defined as the spontaneous emission of EM waves by matter. This type of radiation is attributed to the changes in the electron configuration of the atoms within matter. Radiation mechanisms are not prominent for the transfer of heat to surrounding tissue, and such mechanisms are not expected to play a major role in the heat transfer of $\mathrm{THz}$ skin interactions. However, for completeness, the StefanBoltzmann law that describes these processes is provided:

$$
q_{\mathrm{rad}}=\sigma \varepsilon\left(T_{\mathrm{S}}^{4}-T_{\mathrm{a}}^{4}\right)
$$

where $q_{\mathrm{rad}}$ is the heat flux $(\mathrm{W}), \sigma$ is the Stefan-Boltzmann constant $\left(5.67 \times 10^{-8} \mathrm{Wm}^{-2} \mathrm{~K}^{-4}\right)$, $\varepsilon$ is emissivity (i.e., a measure of how well a surface radiates energy), $T_{\mathrm{s}}$ is the tissue temperature, and $T_{\mathrm{a}}$ is the surrounding temperature.

\subsubsection{Thermal History: Temperature Damage Zones and Associated Biological Effects}

When $\mathrm{THz}$ radiation is passed into skin, chromophores absorb the photons and heating occurs. Heating triggers a temperature rise that increases the kinetic energy of the molecules. Once the kinetic energy is greater than the intramolecular bonds that hold the molecules together, the molecules begin to denature. In an attempt to better characterize these effects, the term thermal history was established. Thermal history is defined as the temperature profile and time at which the tissue is subjected to elevated temperatures. Thermal-induced biological effects are commonly grouped into three temperature damage zones: low temperature $\left(43^{\circ} \mathrm{C}-100^{\circ} \mathrm{C}\right)$, middle temperature $\left(100^{\circ} \mathrm{C}-300^{\circ} \mathrm{C}\right)$, and high temperature $\left(300^{\circ} \mathrm{C}-1000^{\circ} \mathrm{C}\right)$. Figure 7.17 contains data for the effects commonly 


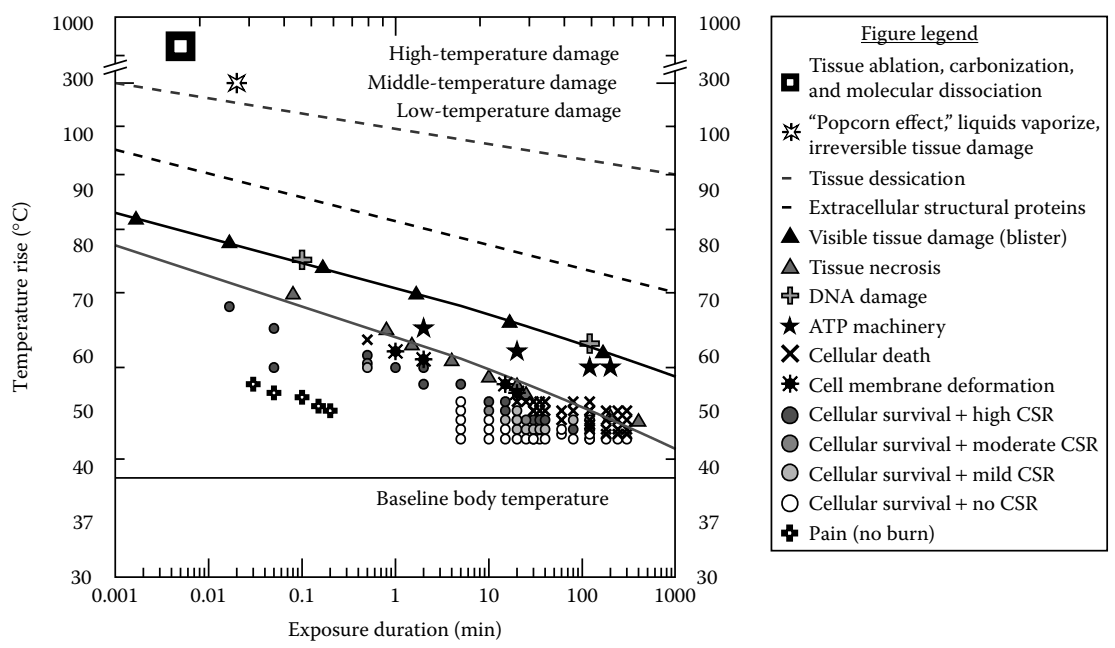

FIGURE 7.17 Thermal effects correlated with each thermal damage zone. (Data from Welch and Gemert 1995; Beckham et al. 2004; Wilmink et al. 2006; Beckham et al. 2008; Wilmink et al. 2008; Wilmink et al. 2009; and Wilmink et al. 2010.)

observed in each temperature damage zone. Knowledge of thermal history and conventional thermal effects are important factors to analyze the specific effects caused by $\mathrm{THz}$ radiation. Specifically, knowledge of thermal effects can help the reader determine whether the effects reported in $\mathrm{THz}$ bioeffects studies are fully attributable to the $\mathrm{THz}$ induced temperature rise or whether other "microthermal" mechanisms may be contributing to the observed effects.

\subsubsection{Low-Temperature Zone}

Besides THz FELs, most modern $\mathrm{THz}$ source do not have sufficient output power to generate tissue temperature rises greater than $100^{\circ} \mathrm{C}$. Therefore, the low-temperature damage zone is presently the most relevant for analysis of $\mathrm{THz}$ bioeffects studies. The most common biological effects associated with the low-temperature zone are the following: (1) intracellular biomolecular level: deactivation of enzymes, protein unfolding or denaturation, and acceleration of cellular metabolism; (2) organelle level: cell shrinkage and nanoporation of lipid membrane; (3) cellular level: cell death (e.g., apoptosis and necrosis); and (4) tissue level: birefringence loss, membrane rupture, and collagen hyalinization.

Numerous intracellular biomolecules can become damaged when tissue is heated to $40^{\circ} \mathrm{C}-50^{\circ} \mathrm{C}$ for several minutes or longer. These effects are primary because mild thermal doses can directly disrupt the hydrogen and disulfide bonds that maintain the tertiary structure of proteins. Damage to biomolecules is generally defined as being reversible or irreversible, where reversible damage with lower thermal doses and irreversible damage with higher thermal doses. When intracellular proteins are reversibly damaged, mammalian cells typically activate a cellular stress response (CSR) to repair the damage (see Figure 7.17). In contrast, when proteins are irreversibly damaged, cells 
activate proteolysis mechanisms to degrade and remove the proteins (See Section 7.5.1 for more details on the CSR.).

In addition to directly damaging biomolecules, thermal effects to cellular organalles are also observed for the low-temperature damage zone. A few examples include: cell shrinkage, nanoporation of lipid membrane, disruption of mitochondria function, and increased membrane permeability. Interestingly, if the magnitude of the thermal stress is severe, such effects typically result in necrosis, a traumatic-type of cell death. In contrast, if the magnitude of the thermal stress is severe, yet the cell has adequate resources available (i.e., Adenosine triphosphate (ATP), oxygen, etc.), then it preferentially activates apoptotic pathways, a means of controlled "cellular death."

Several thermal effects associated with the low-temperature zone also manifest themselves at a tissue level. A few notable effects include structural protein denaturation, birefringence loss, and collagen hyalinization (Henriques and Moritz 1947; Moritz 1947; Moritz and Henriques 1947). Structural proteins, such as fibrillar collagen, have higher energy bonds, are less labile than intracellular proteins, and are typically damaged when tissue temperatures reach $60^{\circ} \mathrm{C}$ for $\sim 1$ minute or longer (Vogel and Venugopalan 2003) (See Figure 7.17). In contrast, structural membrane proteins can withstand slightly higher temperatures and become compromised as the temperature rises to $70^{\circ} \mathrm{C}-80^{\circ} \mathrm{C}$.

\subsubsection{Medium- and High-Temperature Zones}

$\mathrm{THz}$ sources that have the output power to cause biological effects associated with the middle and higher temperature zones are currently not available. However, such sources are expected to be developed in the near future. Therefore, knowledge of these effects will become more relevant, especially as higher power $\mathrm{THz}$ sources are developed for use in new therapeutic applications. Envisaged applications include therapeutic heating, biostimulation, laser skin resurfacing, thermal preconditioning, laser surgical ablation and/or cutting, or even tumor ablation. In brief, the medium-temperature damage zone region is characterized by water-dominated effects (i.e., vaporization, irreversible fibrillar collagen damage, and the "popcorn effect"), whereas the high-temperature region is characterized by tissue ablation, carbonization, and molecular dissociation. At these elevated temperatures, structural proteins such as fibrillar collagen are irreversibly damaged leading to further coagulation and visible tissue whitening. Coagulation signifies a lethal end point for the tissue. Another irreversible change is the birefringence loss that occurs when the regular arrangement of collagen molecules is disrupted. Birefringence is a rotation in the angle of polarization of the tissue. Alterations in collagen microstructure can be detected by transmission polarizing microscopy (TPM), TEM, and highresolution multiphoton microscopy (MPM) using second-harmonic generation.

\subsubsection{Tissue Damage at Terahertz Frequencies}

\subsubsection{Arrhenius Integral and Characterizing Terahertz-Induced Tissue Damage}

The Arrhenius integral is a rate process that is commonly used to characterize tissue damage, coagulation, or birefringence loss as a function of temperature and time. 
Thermal damage has been shown to be exponentially dependent on temperature and linearly dependent on time of exposure (Beckham et al. 2004), and is commonly defined using the following equation:

$$
\Omega=\ln \left(\frac{C_{0}}{C(t)}\right)=\int_{0}^{t_{\mathrm{p}}} A \exp \left(-E_{\mathrm{a}} / R T\right) \mathrm{d} t
$$

where $\Omega$ is the tissue damage, $A$ the frequency factor (i.e., damage rate [1/second]), $E_{\mathrm{a}}$ the activation energy $(\mathrm{J} / \mathrm{mole}), T$ the temperature of exposure $\left({ }^{\circ} \mathrm{K}\right), R$ the gas constant $(8.32 \mathrm{~J} / \mathrm{mol} \mathrm{K})$, and the integral is over the time of the heat exposure. The Arrhenius integral takes into account temperature-time history of the sample to predict tissue damage based on the damage threshold. The threshold for tissue damage is usually determined by pathological analysis and is a ratio of the concentration of native (undamaged) tissue before irradiation exposure $\left(C_{0}\right)$ to the concentration of native tissue at the end of the exposure time $(\mathrm{Ct})$. The threshold for determination of damaged to undamaged tissue is arbitrarily selected. Importance of tissue damage calculations will become more clear in discussions regarding $\mathrm{THz}$ safety standards (Section 7.4.7).

\subsubsection{Computational Modeling and Empirical Measures of Terahertz-Induced Skin Damage}

Conventional computational modeling techniques have been used to simulate the propagation of $\mathrm{THz}$ radiation in biological tissues. Such efforts have also been extended to predict tissue-damage thresholds for skin exposed to $\mathrm{THz}$ radiation (Ibey et al. 2008). A recent study examined these processes using a two-dimensional, cylindrically symmetrical tissue construct (Ibey et al. 2008). In this work, an approximate solution to the bioheat equation was computed using source terms generated from either an optical or a radio-frequency model. The optical model source term was estimated from a simple linear absorption model (Eq. 7.2), whereas the radio-frequency model source term was formulated using finite-difference time-domain (FDTD) computational electrodynamic techniques (Ibey et al. 2008). The FDTD techniques were used to predict the specific absorption rate (SAR) generated within single and multislab skin models. Each source term was entered into the bioheat equation to predict the thermal history (temperature and time) for THz-exposed skin. After the bioheat equation was used to determine the tissue's thermal history, damage thresholds were computed using the Arrhenius equation (see Eq. 7.9). Figure 7.18 contains the plots of the predicted temperature rise and tissue-damage thresholds at $\mathrm{THz}$ frequencies. Knowledge of tissue-damage thresholds is vital for the safe use of $\mathrm{THz}$ radiation in real-world applications. Such information is also critical in the design of effective medical procedures.

Our research group has also conducted several experiments to explore $\mathrm{THz}$-induced tissue damage (McQuade et al. 2007; Dalzell et al. 2010). The results of these studies have been used to provide empirical data to support current $\mathrm{THz}$ standards. In these studies, we used the FEL at Jefferson Laboratory $\left(v=0.1-1.0 \mathrm{THz}, \mathrm{E}=2.0-14.0 \mathrm{~mW} / \mathrm{cm}^{2}\right.$, 2 seconds) and wet chamois cloths. Thresholds were determined using conventional damage score determination and probit analysis techniques, and tissue temperatures were measured using IR thermographic techniques. Using the experimental data, 


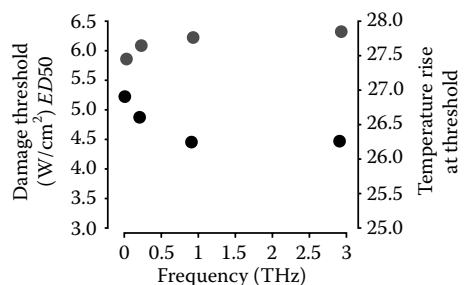

(a)

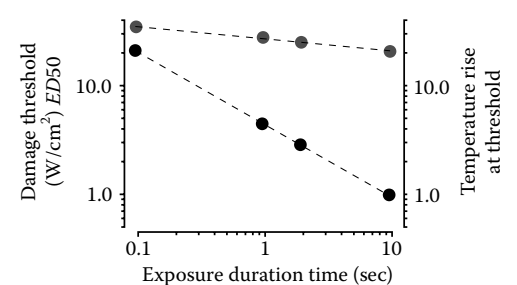

(b)

FIGURE 7.18 Computational modeling predictions of damage thresholds and temperature rises for tissues exposed to $\mathrm{THz}$ radiation. (a) Plot of the predicted damage thresholds and temperature rises for several THz frequencies (1-second exposures). (b) Plot of the predicted damage thresholds and temperature rises for tissues irradiated at a frequency of $1 \mathrm{THz}$ for exposure durations of 0.1, 1.0, 2.0, and 10.0 seconds. (Reprinted with permission from Dalzell, D. R., J. McQuade et al. 2010. Damage Thresholds for Terahertz Radiation. Optical Interactions with Tissues and Cells XXI: SPIE.)

we determined that the tissue-damage threshold (ED50) was $7.16 \mathrm{~W} / \mathrm{cm}^{2}$, a value which was consistent with that predicted with our computational models.

\subsubsection{Terahertz Safety Standards}

Safety standards are established to ensure that workers and the general population are protected against adverse health effects associated with EM radiation. Several international organizations define safety standards: European Parliament \& Union (EP \& EU), International Commission on Non-Ionizing Radiation Committee (ICNIRP), American National Standards Institute (ANSI), Institute of Electrical and Electronics Engineers (IEEE), European Committee for Electrotechnical Standardization (CENELEC), Australia/New Zealand (AUS/NZ), and the National Radiological Protection Board (NRPB). These organizations typically use different guidelines to independently define their own safety standard; as a result, there is great disparity between the current $\mathrm{THz}$ standards. However, although each organization uses different guidelines, they all rely heavily on published experimental data to determine the most suitable standard. Therefore, the investigations that provide additional empirical data are valuable and contribute towards the global harmonization of current standards (Erdreich and Klauenberg 2001).

Safety standards do exist for $\mathrm{THz}$ frequencies; however, these current values may be limited because they have been determined using extrapolated estimates from neighboring spectral regions. In addition, few studies have collected experimental data to support these standards. Overall, experimental studies are needed to ensure the safe use of THz radiation devices and to avoid unnecessarily strict restrictions on output power, which may preclude the timely usage of these new devices and/or applications.

Defining appropriate safety standards for the $\mathrm{THz}$ region is complicated for several reasons. First, because the $\mathrm{THz}$ frequency band spans from 0.1 to $10 \mathrm{THz}$, it falls under adherence to both laser safety standards $(0.3-10 \mathrm{THz})$ and radio-frequency radiation safety standards $(0.1-0.3 \mathrm{THz})$. This feature provides an intrinsic challenge because the 


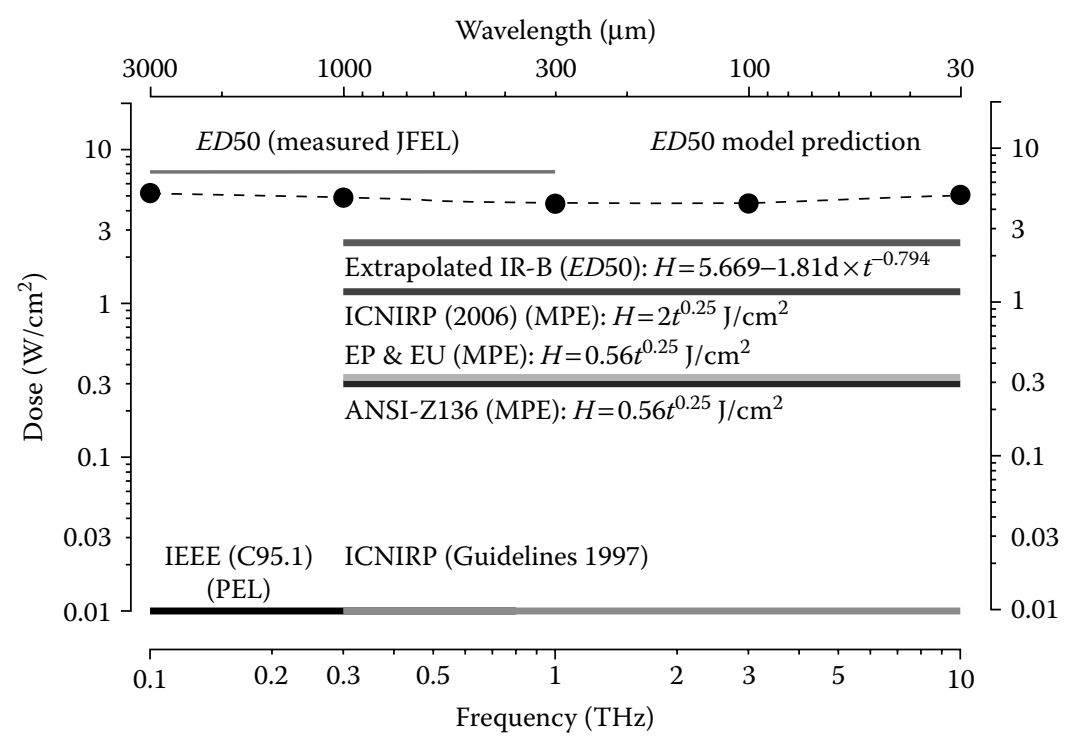

FIGURE 7.19 (See color insert.) Summary plot of empirical data, computational modeling data, and current terahertz radiation exposure standards. The defined exposure limits $\left(\mathrm{W} / \mathrm{cm}^{2}\right)$ are plotted versus frequency $(\mathrm{THz})$ and wavelength. The plot includes the following: experimentally determined ED50 using a free-electron laser (FEL; pink), computational modeling ED50 predictions (dashed black), empirical equation designed for use at IR wavelengths (orange). (Data from Chen et al. 2005.) ICNIRP IR-C statement (red). (Data from Protection 2006.) EP \& EU (green), ANSI-Z136.1 MPE values (blue). (Data from Erdreich and Klauenberg 2001; Union 2006), ICNIRP guidelines for MPE (purple). (Data from ICNIRP 1996), and IEEE C95.1 suggested PEL (solid-black line). (Data from Erdreich and Klauenberg 2001.)

laser and radio-frequency standards are defined using different end point determinants; the laser standard (ANSI Z136.1) uses tissue-damage thresholds (ED50), whereas the radio-frequency standard (IEEE C95.1) is defined using a psychophysical perception of pain and/or heating, typically associated with work stoppage." Due to these differences, current safety standards vary widely across the $\mathrm{THz}$ band.

Figure 7.19 is a plot that compares our computational modeling and empirical data to current international standards (Dalzelle et al. 2010). In the plot, irradiance or power density $\left(\mathrm{W} / \mathrm{cm}^{2}\right)$ is plotted versus $\mathrm{THz}$ frequency. Interestingly, the empirically determined $E D 50$ of $7.16 \mathrm{~W} / \mathrm{cm}^{2}$ is roughly $40 \%$ greater than the value predicted with our computational models $\left(\sim 5 \mathrm{~W} / \mathrm{cm}^{2}\right)$ (Dalzell et al. 2010). Using equations designed to predict thresholds at short IR wavelengths, other research groups predicted the threshold to be roughly 2.5 $\mathrm{W} / \mathrm{cm}^{2}$ at $\mathrm{THz}$ frequencies (Chen et al. 2005). With regard to current $\mathrm{THz}$ safety standards, the ICNIRP statement recommends Maximal permissible exposure (MPE) of $1.19 \mathrm{~W} / \mathrm{cm}^{2}$, whereas both the EP \& EU and ANSI-Z136 suggest MPEs around $0.3 \mathrm{~W} / \mathrm{cm}^{2}$. In addition, the initial ICNIRP report suggests a MPE of $0.01 \mathrm{~W} / \mathrm{cm}^{2}$, a value which is in well agreement with the permissible exposure limit (PEL) set by IEEE C95.1. Overall, future studies must be conducted in order to establish standards that are supported by experimental data. 


\subsection{Biological Effects at a Cellular and Biomolecular Level}

$\mathrm{THz}$ irradiation of human skin can cause thermal gradients that result in appreciable increases in tissue temperature. These spatio-temporal thermal gradients are the driving force for THz-induced skin damage (as discussed in Section 7.4.). In addition to damaging the tissue, these thermal gradients can also cause damage to resident skin cells (i.e., keratinocytes, fibroblasts, etc). In this section, we provide an overview of the cellular processes that are typically activated in cells exposed to environmental stressors, such as heat or $\mathrm{THz}$ radiation. We begin with a discussion of the core proteins and intracellular signaling pathways that are associated with the cellular stress response (CSR). We then discuss the biological effects that $\mathrm{THz}$ radiation causes at a cellular, organelle, and biomolecular level. Finally, we conclude with recent data, which suggests that human cells express a signature gene expression profile when exposed to $\mathrm{THz}$ radiation.

\subsubsection{Background on Cellular Stress Response and Cellular Death}

Mammalian cells frequently encounter conditions that can lead to stress. A few wellcharacterized external stressors include hyperthermia, hypothermia, hypoxia, ionizing radiation, and EM energy. Internal stressors include ATP depletion, oxidative stress, and pathogenic stimuli. At the molecular level, these stressors can exert strain on intracellular biomolecules (e.g., lipids, proteins, and DNA). If the applied strain is appreciable and exceeds the cells homeostatic regulatory mechanisms, these biomolecules can undergo structural modifications that preclude them from functioning properly. In addition, if the magnitude of the strain exceeds the cell's capacity for repair (i.e., irreversible damage), then such exposures can lead to cell death via apoptotic or necrotic mechanisms.

In order to survive and adapt to stressful conditions, all mammalian cells have evolved a molecular defense reaction called the cellular stress response. The CSR is rapidly activated in response to stress and primarily involves the following intracellular signaling pathways: redox, DNA sensing and repair, molecular chaperones, proteolysis, energy metabolism, and apoptosis (Kultz 2003). Many proteins are associated with these pathways; however, a group of 44 evolutionary conserved proteins have recently been identified as core mediators (Kultz 2005). These proteins, collectively referred to as minimal stress proteins, are regulated by cells at both the transcriptional and posttranscriptional levels. At the transcription level, the mRNAs for these stress proteins are transcribed for rapid de novo protein synthesis; whereas at the posttranscriptional level, regulation is executed on the pool of existing mRNAs, where some are selected for translation and others are suppressed.

When skin is exposed to $\mathrm{THz}$ radiation, resident skin cells may undergo reversible and irreversible changes. A few common cellular and molecular effects include the following: activation of the CSR, cellular membrane deformation, cell death via necrotic mechanisms, damage to ATP machinery, and DNA damage (Figure 7.20). When cells are exposed to severe hyperthermic stress, they typically exhibit a signature stress 


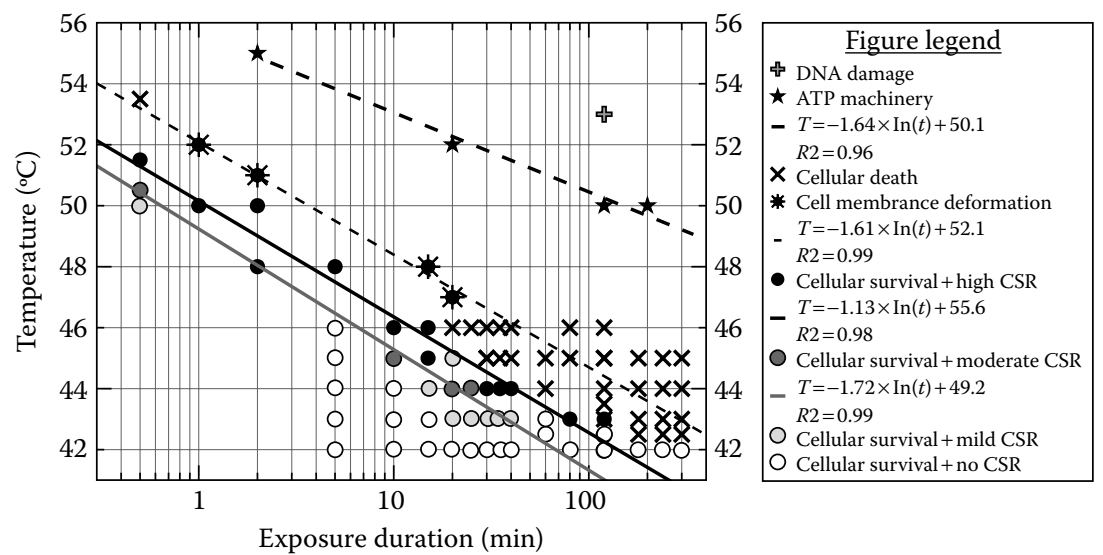

FIGURE 7.20 Cellular effects corresponding to different temperatures. (Data from Welch and Gemert 1995; Beckham et al. 2004; Wilmink et al. 2006; Beckham et al. 2008; Wilmink et al. 2008; Wilmink et al. 2009; and Wilmink et al. 2010.)

response profile, which consists primarily of many types of heat shock proteins (HSPs). Figure 7.21 ( $\mathrm{a}$ and b) contains mRNA microarray and qPCR data for human dermal fibroblasts exposed to hyperthermic stress $\left(44^{\circ} \mathrm{C}\right.$ for 40 minutes) (Wilmink et al. 2010). This data shows that the hyperthermic treatment group expressed transcripts for 32 of the 44 minimal stress proteins, and of these, 23 encoded for molecular chaperone proteins-primarily heat shock proteins (HSPs). The most well-characterized HSPs are HSPA1A (Hsp70), HSPA6 (Hsp70), HSPA4L (Hsp70L), DNAJA4 (Hsp40), DNAJB1 (Hsp40), and HSPH1 (Hsp105). In summary, the pathways and proteins associated with the CSR are well characterized for many common stressors; however, few studies have carefully examined the cellular response to exotic stressors, such as $\mathrm{THz}$ radiation. Because mammalian cells have predominantly evolved survival mechanisms to cope with common stressors (i.e., heat, $\mathrm{pH}$ changes, etc.), studies that investigate the response that $\mathrm{THz}$ radiation induces in cells will improve our current understanding of the CSR.

\subsubsection{Terahertz Bridge-The First International Project on Terahertz Bioeffects (2001-2004)}

Prior to the twenty-first century, only a few $\mathrm{THz}$ bioeffects studies were reported in the literature. Then, in early 2000 , a surge of $\mathrm{THz}$ research activity led to an increase in the number of THz-based applications. These innovative applications not only garnered significant scientific interest, but they also prompted concerns regarding the hazards and biological effects associated with $\mathrm{THz}$ radiation. With a scarcity of $\mathrm{THz}$ bioeffects data available at the time, Dr. Gallarano led efforts to organize an international research effort entitled the "THz-Bridge" project (Gallerano 2004). During the 2001-2004 timeframe, studies under the purview of the $\mathrm{THz}$-Bridge project sought to answer two fundamental questions: (1) What mechanism governs the interaction of $\mathrm{THz}$ radiation with cells and biomolecules? and (2) Does $\mathrm{THz}$ radiation causes genotoxic effects in human cells? 

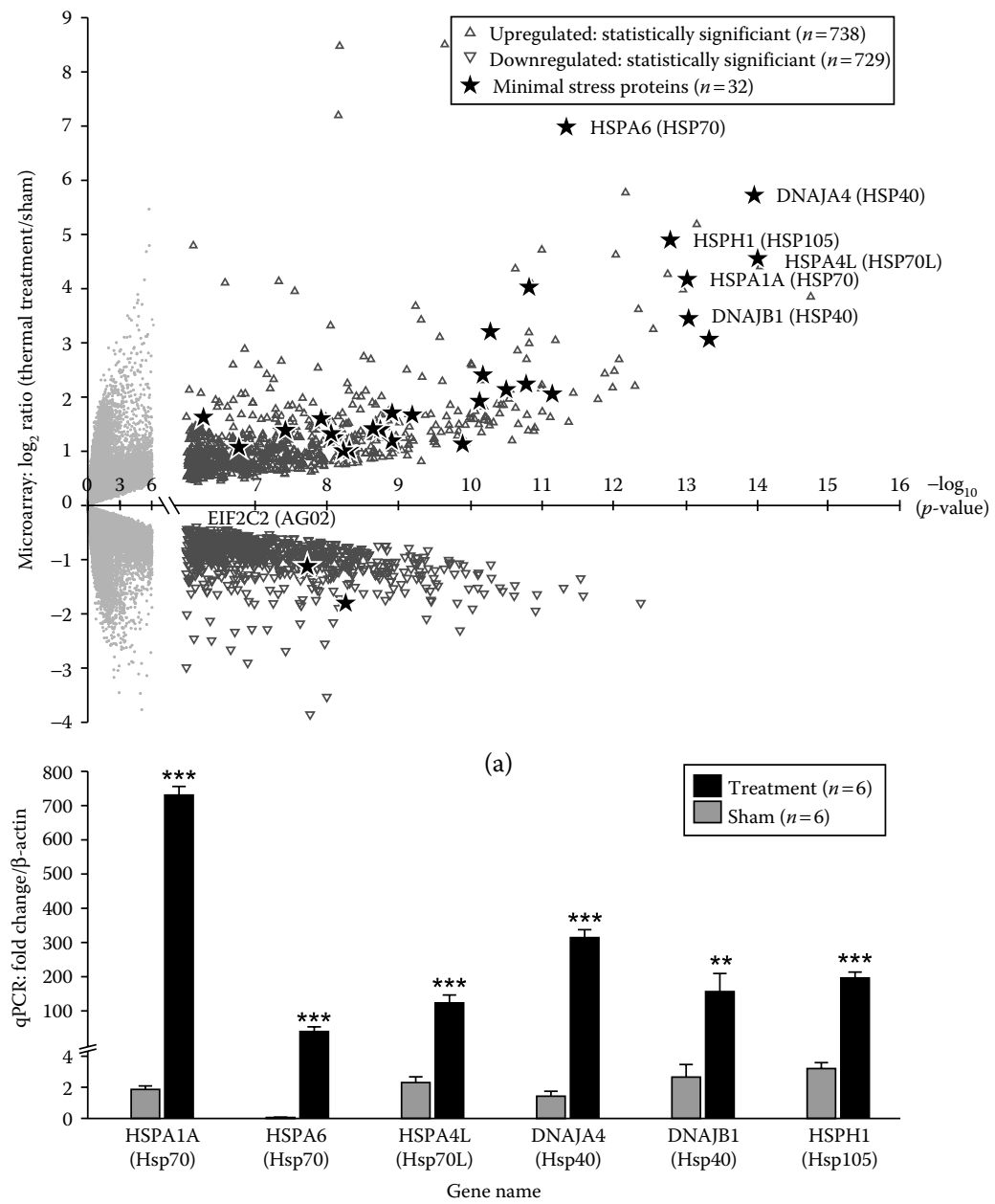

(b)

FIGURE 7.21 Human dermal fibroblasts exposed to thermal stress express an appreciable cellular stress response, as evidenced by the marked up-regulation of minimal stress proteins. Dermal fibroblasts were exposed to a hyperthermia protocol $\left(44^{\circ} \mathrm{C}\right.$ for 40 minutes), and RNA was extracted 4 hours after exposure. Microarrays and qPCR analyses were conducted for control and treatment groups with $n=6$ for each group. (a) Volcano plot of mRNA microarray data, in which expression is plotted versus the level of statistically insignificant targets, filled circles; significant targets, triangles; minimal stress proteins, filled stars. Data are expressed as means. (b) Gene expression for minimal stress proteins (HSPA1A, HSPA6, HSPA4L, DNAJA4, DNAJB1, and HSPH1) using qRT-PCR. The mRNA expression fold values $\left(2^{-\Delta \Delta C t}\right)$ were measured for sham and treatment groups with $n=6$ for each group. Values were calculated in relation to $\beta$-actin and normalized to a separate RNA calibrator (sham, gray bar; treatment, black bar). Data are expressed as means $\pm \mathrm{SD}$; ${ }^{* *} p<10^{-5},{ }^{*} p<10^{-3}$; between indicated groups. With kind permission from Springer Science and Business Media. (From Wilmink, G. J., C. L. Roth et al. 2010. Cell Stress Chaperone 15(6):1027-38. With permission. (2010). 
In regard to the first question, most studies associated with the THz-Bridge project found that THz-induced bioeffects are mediated primarily via photothermal mechanisms (i.e., thermal effects). This finding is not surprising given the fact that $\mathrm{THz}$ frequencies are known to be strongly absorbed by water, the main constituent and primary tissue chromophore at $\mathrm{THz}$ frequencies (Wilmink et al. 2011).

In contrast, mixed results were reported for the second question regarding the genotoxic nature of THz radiation (Berns and Bewley 1987; Scarfi et al. 2003; Zeni et al. 2007; Korenstein-Ilan et al. 2008). For example, Korenstein-Ilan et al. (Korenstein-Ilan et al. 2008) observed both genotoxic and epigenetic effects in lymphocytes exposed to $0.1 \mathrm{THz}$ radiation, whereas neither of these effects were observed in other comparable studies (Scarfi et al. 2003; Zeni et al. 2007). In addition to affecting DNA, several studies report that $\mathrm{THz}$ radiation can also impair intracellular protein and enzymatic processes (Cherkasova et al. 2009; Homenko et al. 2009). These findings are particularly compelling because the energy of THz photons is not high enough to directly disrupt chemical bonds. All in all, the THzBridge project made several landmark discoveries in the area of research on THz bioeffects. In addition, the project served to raise several poignant questions regarding the mechanism by which $\mathrm{THz}$ radiation interacts, couples, and subsequently damages biomolecules.

\subsubsection{Recent Studies on Terahertz Bioeffects (2004-2010)}

Since 2004, theoretical models have been developed that postulate that specific $\mathrm{THz}$ frequencies may be able to couple directly to biomolecules via coherent excitations (Frohlich 1975) and/or nonlinear resonance mechanisms (Chitanvis 2006; Alexandrov et al. 2009). This coupling mechanism is believed to be due to that fact that $\mathrm{THz}$ radiation (i.e., $\mathrm{THz}=10^{12} \mathrm{~Hz}$ ) oscillates on the same timescale as the natural phonon frequencies of double-stranded DNA (dsDNA) (Chitanvis 2006; Alexandrov et al. 2009). Furthermore, such coupling has been suggested to lead to the creation of localized openings (bubbles) between the DNA strands (Chitanvis 2006; Alexandrov et al. 2009). In turn, these openings then drive the dsDNA to "unzip," thereby interfering with normal transcription processes. Since these processes could cause biological effects, which may not be fully attributable to conventional thermal effects, these studies have motivated researchers to reconsider the concept of "microthermal" or "nonthermal" effects. In addition, these studies have contributed to the emergence of several fundamental questions: (1) Do mammalian cells exposed to specific $\mathrm{THz}$ exposure conditions (frequency, exposure duration, irradiance, pulsed vs. CW, etc.) exhibit specific cellular effects (i.e., morphological changes, viability, growth kinetics)? (2) What direct impact does $\mathrm{THz}$ radiation have on cellular plasma membranes and intracellular biomolecules (i.e., lipids, proteins, and DNA)? (3) Is THz radiation genotoxic to mammalian cells, and if so, at what exposure conditions are such effects observed? and (4) Do mammalian cells exposed to $\mathrm{THz}$ radiation exhibit a signature gene expression profile that is not observed in hyperthermic-treated cells?

In an effort to guide a discussion which addresses the above questions, we have created a table that summarizes the findings from most THz bioeffects studies that have been conducted to date. (Table 7.2; Clothier and Bourne 2003; Fedorov et al. 2003; Scarfi et al. 2003; Ramundo-Orlando et al. 2007; Zeni et al. 2007; Korenstein-Ilan et al. 2008; Olshevskaya et al. 2008; Cherkasova et al. 2009; Homenko et al., 2009.) In this table, we provide the 


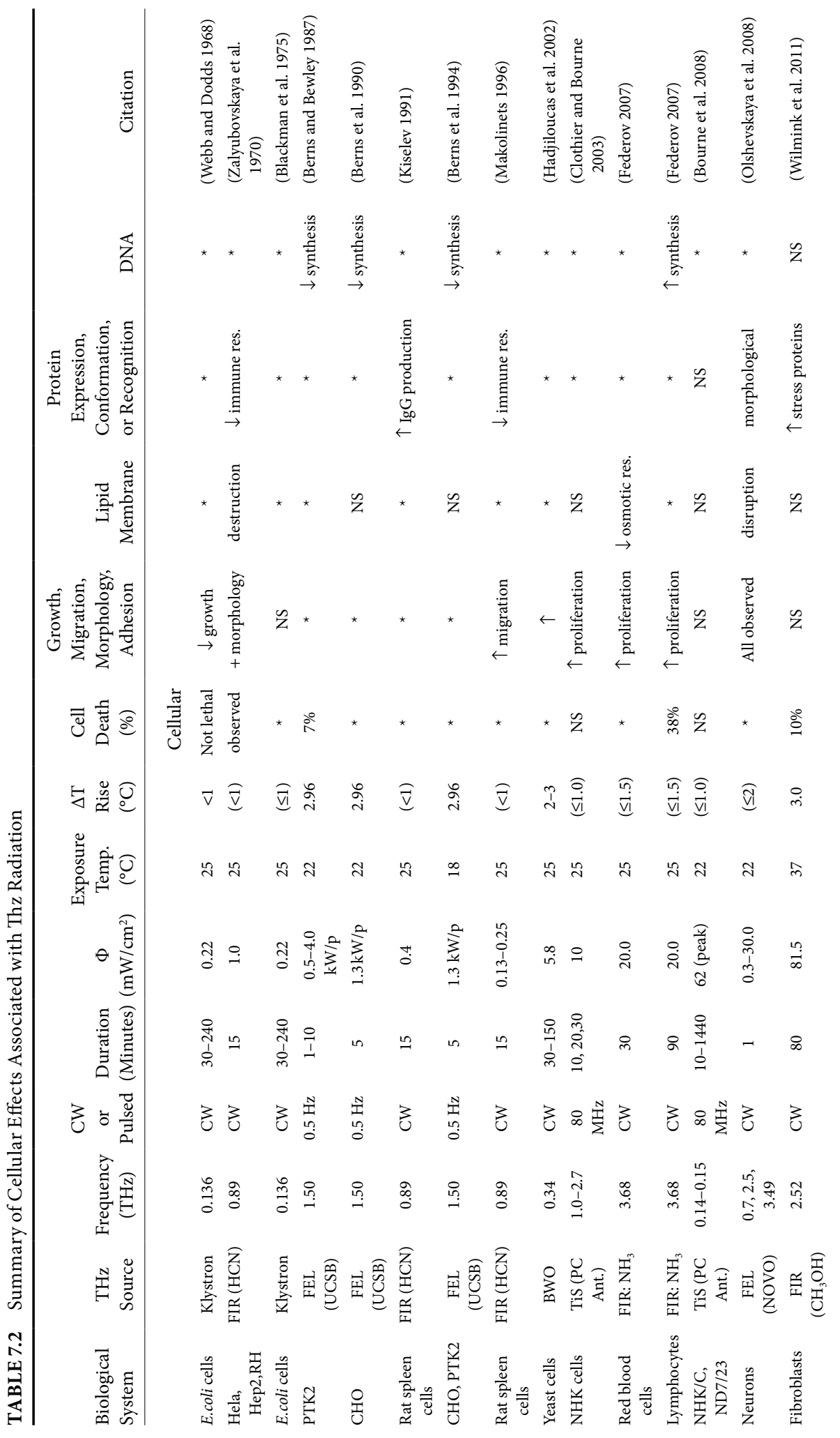




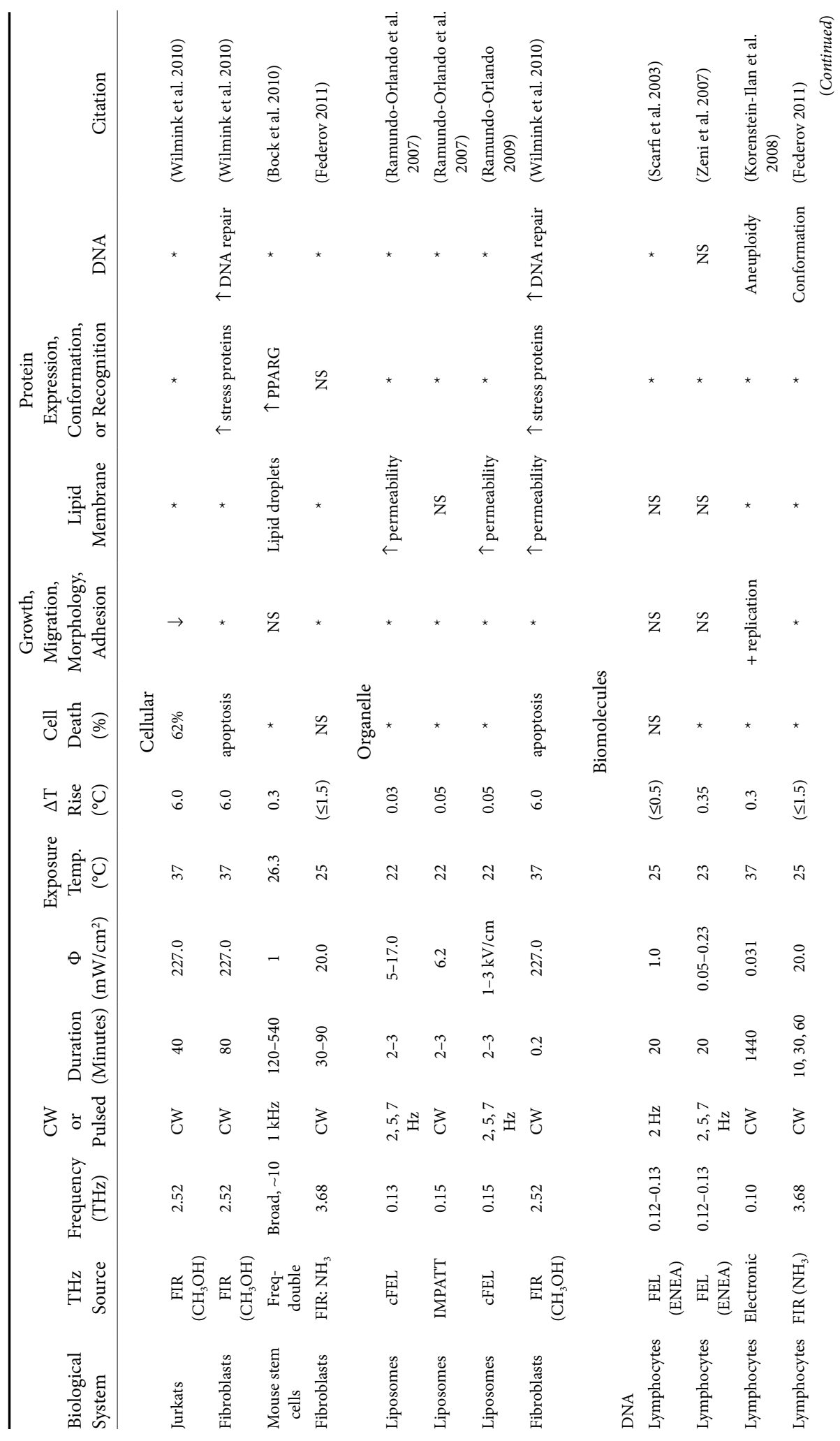




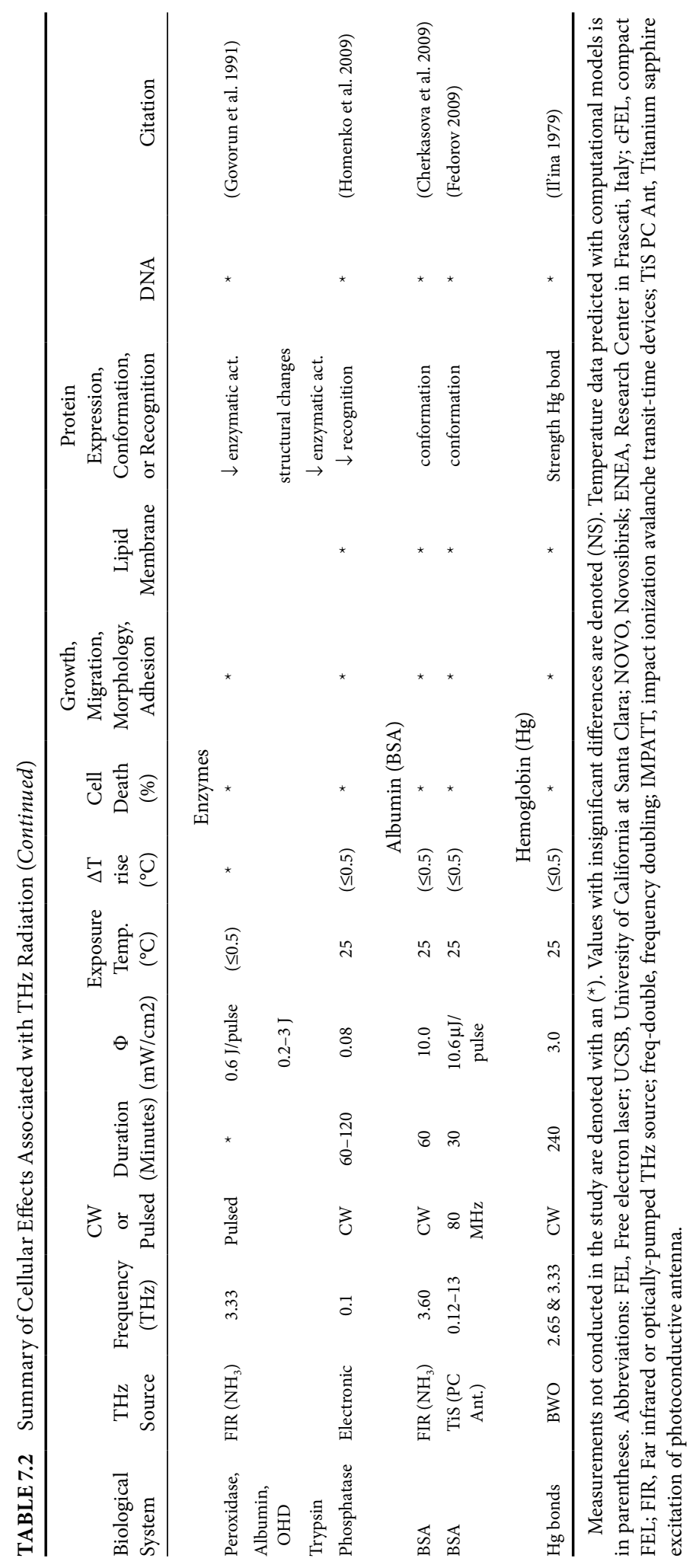


exposure parameters used in each study: frequency ( $\mathrm{THz}$ ), delivery mode (CW vs. pulsed), exposure duration, and incubation temperature during exposure. In addition, we provide a detailed list of the bioeffects that were observed: temperatures generated during exposure, viability, changes in cell growth, and structural/functional changes to lipid membranes, proteins, and DNA. After taking a cursory look at Table 7.2, it is clear that the exposure parameters, cell type, and end point evaluation techniques used in each study vary significantly. Therefore, although it may be difficult to draw overarching conclusions regarding the effects of $\mathrm{THz}$ radiation, several effects were consistently observed in many reports. We shall focus our subsequent discussion on these effects.

\subsubsection{Cellular Effects Caused by Terahertz Radiation}

In most previous studies, $\mathrm{THz}$ radiation did not cause changes at a cellular level (e.g., morphology, viability, and growth kinetics) (Scarfi et al. 2003; Zeni et al. 2007; Homenko et al. 2009; Clothier and Bourne 2003); however, a few studies observed cellular effects. Specifically, Olshevskaya et al. observed that neurons exhibited morphological changes when exposed to $2.5 \mathrm{THz}$ radiation (Olshevskaya et al. 2008), and Korenstein-Ilan et al. observed changes in growth kinetics for cells exposed to $\mathrm{THz}$ radiation for long exposure durations (e.g., 1440 minutes) (Korenstein-Ilan et al. 2008). Our group did not observe any morphological changes in THz-exposed cells, but we did observe that the exposed fibroblasts did exhibit enhanced proliferation rates (Wilmink et al. 2010). A likely explanation for our results is that the exposure conditions we used generated modest $\left(-3^{\circ} \mathrm{C}-6^{\circ} \mathrm{C}\right)$ temperature increases, conditions that are known to increase cellular proliferation rates (Wilmink et al. 2006; Wilmink et al. 2009; Figure 7.22). In addition, for the longer exposures, we observed that $\mathrm{THz}$ radiation can affect the viability and growth of dermal fibroblasts; however, these effects are most likely due to the fact

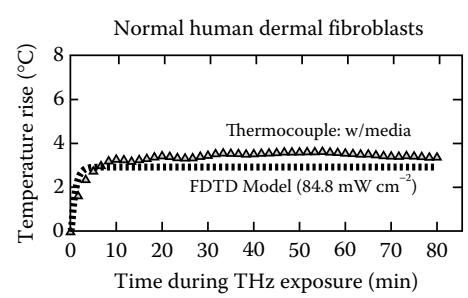

(a)

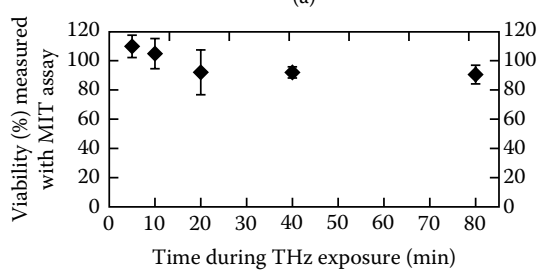

(c)

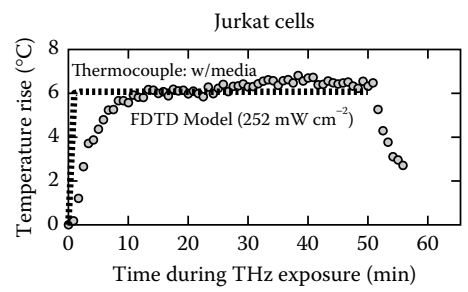

(b)

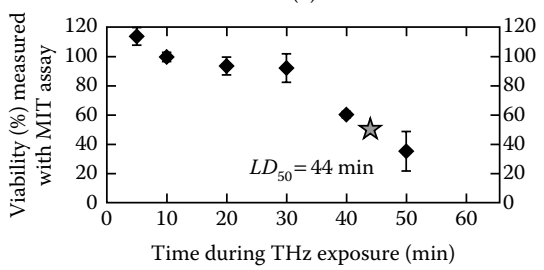

(d)

FIGURE 7.22 ( $a$ and b) Thermal history (temperature-time curves) and ( $c$ and d) viability curves for dermal fibroblasts $\left(2.52 \mathrm{THz}, 84.8 \mathrm{~mW} \mathrm{~cm}^{-2}, 5-80\right.$ minutes $)$ and Jurkat cells $(2.52 \mathrm{THz}$, $636 \mathrm{~mW} \mathrm{~cm}^{-2}, 5-80$ minutes) exposed to $\mathrm{THz}$ radiation. 
that we used the highest irradiance that has been reported to date (e.g., $\sim 2500$ times greater than most previous studies). Moreover, because our hyperthermic-exposed cells also showed signs of death, these findings further confirm that the THz-induced cell death that we observed is mediated primarily via photothermal mechanisms.

\subsubsection{Direct Effects of Terahertz Radiation on Cellular Organelles and Biomolecules}

In addition to the conflicting results reported at a cellular level, the data in Table 7.2 also suggest that mixed conclusions have been drawn regarding the impact of $\mathrm{THz}$ on cellular organelles and intracellular biomolecules (e.g., lipids, proteins, and DNA). For instance, Ramundo-Orlando et al. showed that pulsed $\mathrm{THz}$ radiation can directly affect the permeability of liposomes (Ramundo-Orlando et al. 2007). This observation is quite intriguing, and it will be interesting to see if future investigations observe similar effects in mammalian cells. In addition to lipids, Homenko et al. have also shown that $\mathrm{THz}$ radiation can affect protein activity in an antigen-antibody model (Homenko et al. 2009). Consistent with this finding, our research group recently observed that THz-exposed cells dramatically increase their intracellular HSP expression. Since HSP upregulation is a hallmark condition for intracellular protein damage, our observations suggest that $\mathrm{THz}$ radiation causes significant intracellular protein damage. However, we have not yet conducted definitive experiments to determine whether HSP upregulation is a result of direct protein damage (primary effect) or is caused by the temperature rise that generated during $\mathrm{THz}$ irradiation (secondary effect).

\subsubsection{Genotoxic Effects of Terahertz Radiation}

Mixed conclusions have also been drawn on the effect that $\mathrm{THz}$ radiation exerts on DNA. For instance, both Scarfi et al. (Scarfi et al. 2003) and Zeni et al. (Zeni et al. 2007) showed that $\mathrm{THz}$ radiation does not induce genotoxicity, while Korenstein-Ilan et al. (KorensteinIlan et al. 2008) observed signs of aneuploidy (i.e., genomic instability). Although all of these studies used roughly the same frequency $(\sim 0.1 \mathrm{THz})$ and the same cell line (lymphocytes), a few fundamental differences exist between them that may account for these conflicting results. First, Korenstein-Ilan et al. exposed cells for considerably longer exposure durations than used in Scarfi et al. 2003 and Zeni et al. 2007-1440 minutes rather than 20 minutes. Second, the groups used very different sources in their experiments: Korenstein-Ilan et al. used a frequency multiplier unit that generates incoherent CW THz radiation, whereas Scarfi and Zeni et al. both used a FEL that generates coherent, pulsed, broadband THz radiation (Scarfi et al. 2003; Zeni et al. 2007). To date, it is still not clear which of these properties may be responsible for these conflicting results.

\subsubsection{Does Terahertz Radiation Trigger Signature Gene Expression Profiles in Human Cells?}

In this final section, we provide data that our group recently collected. Our main goal for these experiments was to determine whether human cells activate a signature gene 
expression profile when exposed to $\mathrm{THz}$ radiation. In order to examine this concept in a comprehensive manner, we conducted an in vitro experiment where Jurkat cells were exposed to either $\mathrm{THz}$ radiation or a bulk heating (i.e., hyperthermic stress) protocol for 40 minutes. IR thermographic techniques were employed to ensure that both protocols had identical thermal profiles $\left(\Delta T=6^{\circ} \mathrm{C}\right)$. In contrast to our previous studies where we selected a few hundred genes to examine postexposure, in these experiments we conducted a full genetic analysis for the exposed cells. To do this, we conducted gene expression profiling using the most recently released DNA microarray gene chips. DNA microarrays are powerful technologies because they can provide a high throughput and detailed view of the entire genome and transcriptome. In these experiments, we used GeneChip Human Genome U133 Plus 2.0 Arrays, the latest gene-chip array that provides the most comprehensive coverage of all genes in the human genome. This particular genome array permits analysis on over 47,000 transcripts and variants, including more than 38,000 well-characterized human genes. Figure 7.23 contains a comparison of gene expression profiles for 24 genes expressed
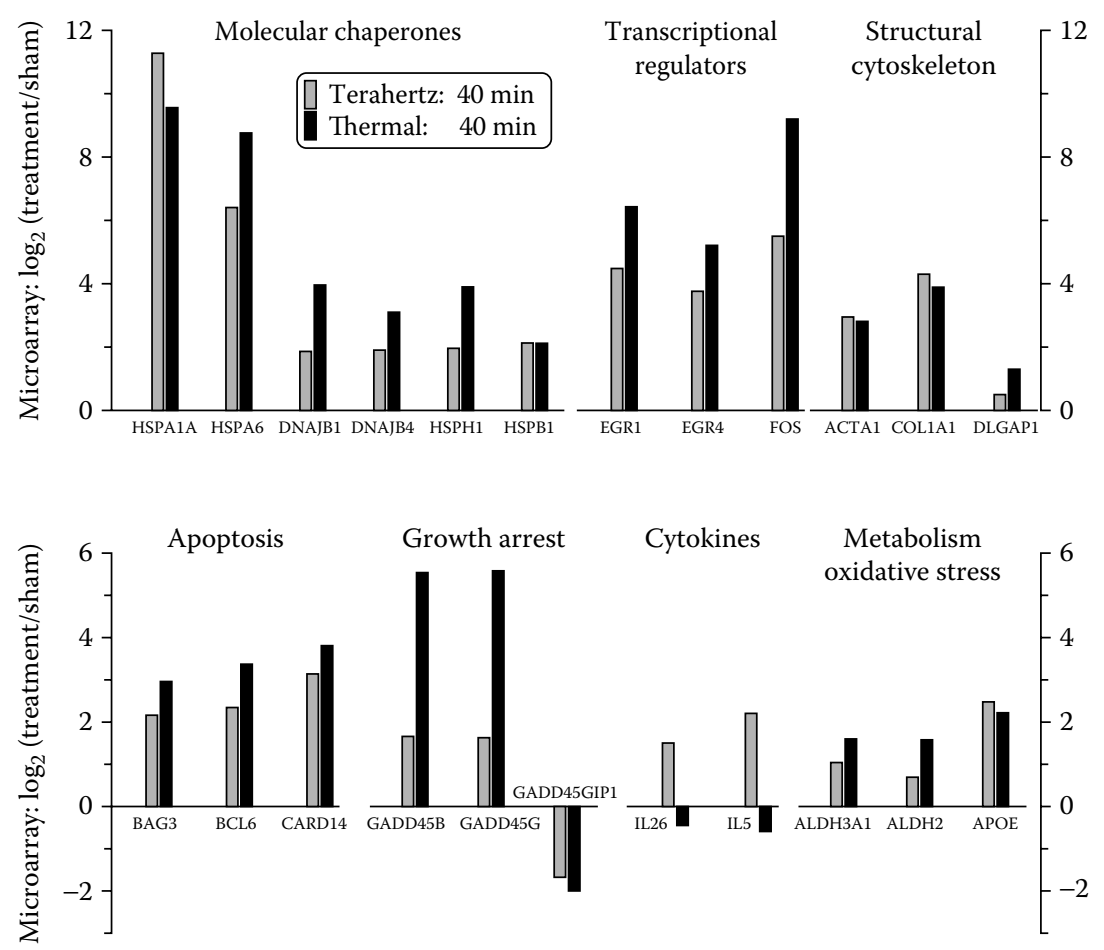

FIGURE 7.23 Comparison of microarray gene expression profiles for Jurkat cells exposed to $\mathrm{THz}$ radiation $\left(2.52 \mathrm{THz}, 636 \mathrm{~mW} \mathrm{~cm}^{-2}, 40\right.$ minutes) and thermal stress $\left(44^{\circ} \mathrm{C}\right.$ for 40 minutes). Both exposure groups exhibited identical temperature-time dosimetric profiles (temperature increases of $\left.6.0^{\circ} \mathrm{C}\right)$. 
by Jurkat cells exposed to $\mathrm{THz}$ radiation or hyperthermic stress. From this data, we drew several conclusions. First, many genes were markedly upregulated in both the $\mathrm{THz}$ and heat exposed groups. This suggests that $\mathrm{THz}$ radiation and heating both trigger dramatic cellular responses. Second, many genes were expressed in the $\mathrm{THz}$ group, which were not expressed in bulk heating group. This suggests that $\mathrm{THz}$ radiation may cause specific effects to human cells that are not observed in bulk heated cells. Over the next few months, our research group plans to conduct qPCR experiments to verify these preliminary results.

\subsection{Conclusion and Future Prospects}

In this chapter, we have provided an introduction to the $\mathrm{THz}$ spectral band, an overview of the most commonly used $\mathrm{THz}$ sources, and highlights of modern $\mathrm{THz}$ imaging and spectroscopy applications. After laying down the foundation, we then provided a comprehensive review of THz-tissue optics, thermal effects, and relevance to international safety standards. We concluded with a discussion of the biological effects of THz radiation at a cellular, organelle, and biomolecular level.

In the future, we anticipate that $\mathrm{THz}$ sources will be developed that are capable of generating greater than a few Watts of power. The types of sources that are most likely to lead the way include THz-QCLs, miniaturized BWOs, and compact FEL sources. These technological advances will likely foster the creation of many new applications that use $\mathrm{THz}$ radiation, such as in high speed wireless communications. In addition, $\mathrm{THz}$ imaging and spectroscopy technologies are expected to find widespread use in medical, military, and defense applications. As is the case with most emerging and/ or new applications, the ever increasing use of $\mathrm{THz}$ radiation is expected to continue to increase the public's concerns regarding the dangers associated with them. We believe that most of these concerns will be properly addressed in studies performed over the next 5 to 10 years. We also hope that the studies conducted during this time frame will help lay the groundwork that paves the way for the next generation of $\mathrm{THz}$ researchers.

\section{Acknowledgments}

We wish to thank the National Academy of Sciences NRC Research Associateship program and the Air Force Research Laboratory for providing us with the opportunities to conduct THz research. In particular, we would like to thank Dr. Morley Stone and Dr. Walter Kozumbo for their consistent support. This work was supported by grants provided by AFRL, Air Force Office of Scientific Research (AFOSR), HQAF SGRS Clinical Investigation Program: "Determination of Cellular Bioeffect Thresholds for Terahertz Frequencies." This work was also supported by Partnership for Education and Research in Materials (PREM) provided by the National Science Foundation (NSF), Division of Materials Research Award (\#0934218). This chapter is dedicated to my grandfather Dominic Cameratta, a courageous and loving man who passed away on December 26, 2010. 


\section{References}

Albert, S., D. T. Petkie et al. 2001. Fast scan submillimeter spectroscopy technique (FASSST): A new analytical tool for the gas phase. CHIMIA Int J Chem 55:29-34.

Alexandrov, B. S., V. Gelev, et al. 2010. DNA breathing dynamics in the presence of a terahertz field. Physics Letters A 374(10):1214-1217.

Andronov, A. A., I. V. Zverev, V. A. Kozlov, Yu. N. Nozdrin, S. A. Pavlov, and V. Shastin. 1984. Stimulated emission in the long-wavelength IR region from hot holes in Ge in crossed electric and magnetic fields. J Exp Theor Phys Lett 40:804.

Asada, M. 2008. Resonant tunneling diodes for sub-terahertz and terahertz oscillators. Jpn J Appl Phys 47:4375-84.

Ashworth, P. C., E. Pickwell-MacPherson et al. 2009. Terahertz pulsed spectroscopy of freshly excised human breast cancer. Opt Express 17(15):12444-54.

Auston, D. H. 1975. Picosecond optoelectronic switching and gating in silicon. Appl Phys Lett 26(3):101-3.

Auston, D. H., K. P. Cheung et al. 1984. Picosecond photoconducting Hertzian dipoles. Appl Phys Lett 45(3):284-6.

Barthel, J., and H. Hetzenauer. 1990. Dielectric Specta of Some Common Solvents in the Microwave Region, Water and Lower Alcohols. Amsterdam: Elsevier.

Beard, M. C., G. M. Turner et al. 2002. Terahertz spectroscopy. J Phys Chem B 106(29):7146-59.

Beckham, J. T., M. A. Mackanos et al. 2004. Assessment of cellular response to thermal laser injury through bioluminescence imaging of heat shock protein 70. Photochem Photobiol 79(1):76-85.

Beckham, J. T., G. J. Wilmink et al. 2008. Role of HSP70 in cellular thermotolerance. Lasers Surg Med 40(10):704-15.

Belkin, M. A., F. Capasso et al. 2007. Terahertz quantum-cascade-laser source based on intracavity difference-frequency generation. Nat Photonics 1(5):288-92.

Bergner, A., U. Heugen et al. 2005. New p-Ge Thz laser spectrometer for the study of solutions: Thzabsorption spectroscopy of water. Rev Sci Instrum 76(6): 063110-063110-063115.

Berns, M., W. Bewley et al. 1990. Free electron laser irradiation at 200 microns affects DNA synthesis in living cells. Proc Natl Acad Sci U S A 87(7):2810-2.

Berns, M., W. Bewley et al. 1994. Free electron laser irradiation at 200 microns inhibits DNA synthesis in living cells. J Laser Appl 6(7):165-9.

Berns, M. W., and W. Bewley. 1987. Inhibition of nucleic acid synthesis in cells exposed to 200 micrometer radiation from the Free electron laser. Photochem Photobiol 46(2):165-7.

Blackman, C. F., S. G. Benane et al. 1975. Effects of nonionizing electromagnetic radiation on single cell biologic systems. Annals N Y Acad Sci 247(1):352-66.

Bock, J., Y. Fukuyo et al. 2010. Mammalian stem cells reporgramming in response to Terahertz Radiation. PLoS Biol 5(12):e15806

Bogue, R. 2009. Terahertz imaging: A report on progress. Sens Rev 29(1):6-12.

Bourne, N., R. H. Clothier et al. 2008. The effects of terahertz radiation on human keratinocyte primary cultures and neural cell cultures. Altern Lab Anim 36(6):667-84. 
Brijot. 2010. http://www.brijot.com/contact-us/ (accessed November 15, 2010).

Brun, M. A., F. Formanek et al. 2010. Terahertz imaging applied to cancer diagnosis. Phys Med Biol 55(16):4615.

Bründermann, E., and H. Röser. 1997. First operation of a far-infrared p-germanium laser in a standard close-cycle machine at $15 \mathrm{~K}$. Infrared Phys Technol 38(4):201-3.

Brundermann, E., H. P. Roser et al. 1995. High repetition rate far-infrared p-type germanium hot hole lasers. Appl Phys Lett 67(24):3543-5.

Buniatyan, V. V., V. M. Aroutiounian et al. 2004. Silicon carbide TUNNETT diodes. Solid State Electron 48(9):1569-77.

Chan, W. L., J. Deibel et al. 2007. Imaging with terahertz radiation. Rep Prog Phys 70(8):1325-79.

Chen, B., D. C. O’Dell et al. 2005. Porcine skin ED50 damage thresholds for 2,000 nm laser irradiation. Lasers Surg Med 37(5):373-81.

Cherkasova, O., V. Fedorov et al. 2009. Influence of terahertz laser radiation on the spectral characteristics and functional properties of albumin. Opt Spectrosc 107(4):534-7.

Chitanvis, S. M. 2006. Can low-power electromagnetic radiation disrupt hydrogen bonds in dsDNA? J Polym Sci B Polym Phys 44(18):2740-7.

Clery, D. 2002. Brainstorming their way to a revolution. Science 297:761-2.

Clothier, R. H., and N. Bourne. 2003. Effects of THz exposure on human primary keratinocyte differentiation and viability. J Biol Phys 29(2):179-85.

Cooper, K. B., R. J. Dengler et al. 2008. Penetrating 3-D imaging at 4- and 25-m range using a submillimeter-wave radar. Microwave Theory Tech IEEE Trans 56(12):2771-8.

Crocker, A., H. A. Gebbie et al. 1964. Stimulated emission in the far infra-red. Nature 201(4916):250-1.

Dalzell, D. R., J. McQuade et al. 2010. Damage Thresholds for Terahertz Radiation. Optical Interactions with Tissues and Cells XXI: SPIE.

Daniel, C., and E. Bernard. 1959. Backward Travelling Wave Oscillators. United States: Csf.

Dexheimer, S. L. 2008. Terahertz Spectroscopy: Principles and Applications. Boca Raton, FL: CRC Press.

Dobroiu, A., C. Otani et al. 2006. Terahertz-wave sources and imaging applications. Meas Sci Technol 17(11):R161-74.

Dodel, G. 1999. On the history of far-infrared (FIR) gas lasers: Thirty-five years of research and application. Infrared Phys Technol 40(3):127-39.

Doria, A., G. P. Gallerano, et al. 2004. THz radiation studies on biological systems at the ENEA FEL facility. Infrared Physics \& Technology 45(5-6):339-347.

Eisele, H. 2010. $480 \mathrm{GHz}$ oscillator with an InP Gunn device. Electron Lett 46(6):422-3.

Eisele, H., and R. Kamoua. 2006. High-performance oscillators and power combiners with InP Gunn devices at 260-330 GHz. Microwave Wireless Compon Lett IEEE 16(5):284-6.

Erdreich, L. S., and B. J. Klauenberg. 2001. Radio frequency radiation exposure standards: Considerations for harmonization. Health Phys 80(5):430-9.

Faist, J., F. Capasso et al. 1994. Quantum cascade laser. Science 264(5158):553-6.

Fattinger, C., and D. Grischkowsky. 1989. Terahertz beams. Appl Phys Lett 54:490-2.

Federici, J. F., B. Schulkin et al. 2005. THz imaging and sensing for security applications: Explosives, weapons and drugs. Semicond Sci Technol 20(7):S266-80. 
Federov, V. 2001. Comparative study of the effects of infrared, submillimeter, and millimeter EM radiation. Biophysics 46(2):293-297.

Fedorov, V., S. S. Popova et al. 2003. Dynamic effects of submillimeter wave radiation on biological objects of various levels of organization. Int J Infrared Millimeter Waves 24(8):1235-54.

Federov, V. 2007. Investigation of possibility of submillimeter laser using as instrument for diagnostics in medicine (Proceedings Paper). SPIE 6734(04):1-7.

Federov, V. 2011. Study of biological effects of electromagnetic radiation of submillimeter part of terahertz range. Biomeditsiknskaya Radioelektronika.

Fedorov, V. I., A. S. Pogodin, V. G. Bespalov, S. E. Putilin, O. A. Smolyanskaya, V. Grachev Ya, S. A. Kozlov. 2009. Influence of pulsed overwide-range terahertz radiation on albumin conformation. Millimetrovye Volny v Biologii I Meditsine 2:50-58.

Ferguson, B., and X.-C. Zhang. 2002. Materials for terahertz science and technology. Nat Mater 1(1):26-33.

Fischer, B. M. 2005. Broadband THz time-domain spectroscopy of biomolecules. Math Phys Freiburg im Bresigau, Albert-Ludwigs-Universitat:246.

Fischer, B., M. Hoffmann, et al. 2005. Terahertz time-domain spectroscopy and imaging of artificial RNA. Opt Express 13(14):5205-5215.

Fischer, B. M., M. Walther et al. 2002. Far-infrared vibrational modes of DNA components studied by terahertz time-domain spectroscopy. Phys Med Biol 47(21):3807-14.

Fitzgerald, A. J., E. Berry et al. 2003. Catalogue of human tissue optical properties at terahertz frequencies. J Biol Phys 29(2):123-8.

Frohlich, H. 1975. The extraordinary dielectric properties of biological materials and the action of enzymes. Proc Natl Acad Sci U S A 72(11):4211-5.

Gallerano, G. P. 2004. THz-Bridge: Terahertz radiation in Biological Research, Investigation on Diagnostics and study of potential Genotoxic Effects. 2009, http://www.frascati. enea.it/THz-BRIDGE/ (retrieved November 15, 2010).

Globus, T. 2003. THz-spectroscopy of biological molecules. J Biol Phys 29(2):89-100.

Globus, T. R., D. L. Woolard et al. 2002. Submillimeter-wave Fourier transform spectroscopy of biological macromolecules. J Appl Phys 91(9):6105-13.

Glyavin, M. Y., A. G. Luchinin et al. 2008. Generation of 1.5-kW, $1 \mathrm{THz}$ coherent radiation from a gyrotron with a pulsed magnetic field. Phys Rev Lett 100(1):015101-3.

Govorun, V. M., V. E. Tretiakov et al. 1991. Far-infrared radiation effect on the structure and properties of proteins. Int J Infrared and Millimeter Waves 12(12):1469-74.

Grischkowsky, D., S. Keiding et al. 1990. Far-infrared time-domain spectroscopy with terahertz beams of dielectrics and semiconductors. J Opt Soc Am B 7(10):2006-15.

Hadjiloucas, S., M. Chahal et al. 2002. Preliminary results on the non-thermal effects of 200-350 GHz radiation on the growth rate of S. cerevisiae cells in microcolonies. Phys Med Biol 47(21):3831.

Hasted, J. B., S. K. Husain et al. 1987. The temperature variation of the near millimeter wavelength optical constants of water. Infrared Phys 27(1):11-5.

He, M. 2006. Far-infrared signature of animal tissues characterized by terahertz timedomain spectroscopy. Opt Commun 259(1):389-92. 
Henriques, F. C., and A. R. Moritz. 1947. Studies of thermal injury: I. The conduction of heat to and through skin and the temperatures attained therein. A theoretical and an experimental investigation. Am J Pathol 23(4):530-49.

Heugen, U., G. Schwaab et al. 2006. Solute-induced retardation of water dynamics probed directly by terahertz spectroscopy. Proc Natl Acad Sci U S A 103(33):12301-6.

Homenko, A., B. Kapilevich et al. 2009. Effects of $100 \mathrm{GHz}$ radiation on alkaline phosphatase activity and antigen-antibody interaction. Bioelectromagnetics 30(3):167-75.

Hosako, I., N. Sekine et al. 2007. At the dawn of a new era in terahertz technology. Proc IEEE 95(8):1611-23.

Hu, B. B., and M. C. Nuss. 1995. Imaging with terahertz waves. Opt Lett 20(16):1716-8.

Huang, S., P. C. Ashworth et al. 2009. Improved sample characterization in terahertz reflection imaging and spectroscopy. Opt Express 17(5):3848-54.

Huang, S. Y., Y. X. Wang et al. 2009. Tissue characterization using terahertz pulsed imaging in reflection geometry. Phys Med Biol 54(1):149-60.

Ibey, B. L., J. A. Payne et al. 2008. Comparing Finite Difference Time Domain and Monte Carlo Modeling of Human Skin Interaction with Terahertz Radiation. Optical Interactions with Tissue and Cells XIX. San Jose, CA: SPIE.

ICNIRP. 1996. Guidelines on limits of exposure to laser radiation of wavelengths between $180 \mathrm{~nm}$ and $1000 \mathrm{um}$. Health Phys 71:804-19.

Il'ina, S., G. F. Bakaushina, V. I. Gaĭduk, A. M. Khrapko, and N. B. Zinov'eva. 1979. Possible role of water in transmitting the effect of millimeter range radiation to biological objects. Biofizik 24(3):513.

Jepsen, P. U., U. Moller et al. 2007. Investigation of aqueous alcohol and sugar solutions with reflection terahertz time-domain spectroscopy. Opt Express 15(22):14717-37.

Karpowicz, N., H. Zhong et al. 2005. Compact continuous-wave subterahertz system for inspection applications. Appl Phys Lett 86(5):054105.

Kindt, J. T., and C. A. Schmuttenmaer. 1996. Far-infrared dielectric properties of polar liquids probed by femtosecond terahertz pulse spectroscopy. J Phys Chem 100(24):10373-9.

Kiselev, V. K., E. M. Kuleshov, Yu. E. Kamenev, Yu. P. Delevsky, N. A. Zarzhetskaya, Makolinets V.I. 1991. Use of submillimeter laser technology in immunological studies. Radiotekhnicheskie Systemy Millimetrovogo i Submillimetrovogo Diapazonov: 176-81.

Kohler, R. 2002. Terahertz semiconductor-heterostructure laser. Nature 417:156-9.

Kohler, R., A. Tredicucci et al. 2002. Terahertz semiconductor-heterostructure laser. Nature 417(6885):156-9.

Komiyama, S. 1982. Far-infrared emission from population-inverted hot-carrier system in p-Ge. Phys Rev Lett 48(4):271-4.

Komiyama, S., N. Iizuka et al. 1985. Evidence for induced far-infrared emission from p-Ge in crossed electric and magnetic fields. Appl Phys Lett 47(9):958-60.

Kompfner, R., and N. T. Williams. 1953. Backward-wave tubes. Proc IRE 41(11):1602-11. Korenstein-Ilan, A., A. Barbul et al. 2008. Terahertz radiation increases genomic instability in human lymphocytes. Radiation Research 170(2):224-34.

Kultz, D. 2003. Evolution of the cellular stress proteome: From monophyletic origin to ubiquitous function. J Exp Biol 206(Pt 18):3119-24. 
Kultz, D. 2005. Molecular and evolutionary basis of the cellular stress response. Annu Rev Physiol 67:225-57.

Ladanyi, B. M., and M. S. Skaf. 1993. Computer simulation of hydrogen-bonding liquids. Annu Rev Phys Chem 44(1):335-68.

Lee, Y.-S. 2009. Principles of Terahertz Science and Technology. Berlin: Springer.

Lee, M., and M. C. Wanke. 2007. Applied physics. Searching for a solid-state terahertz technology. Science 316(5821):64-5.

Leitner, D. M., M. Gruebele et al. 2008. Solvation dynamics of biomolecules: Modeling and terahertz experiments. HFSP J 2(6):314-23.

Liu, H.-B., H. Zhong et al. 2007. Terahertz spectroscopy and imaging for defense and security applications. Proc IEEE 95(8):1514-27.

Makolinets, V. I. 1996. Influence of submillimeter wave radiation on some manifestation of immunoresponsives Medithiskaya Reabilitologia, Kurortologiya i Fizioterapiya 1:33-5.

McQuade, J., N. Jindra et al. 2007. Theoretical and Experimental Bioeffects Research for High-Power Terahertz Electromagnetic Energy. San Jose, CA: SPIE.

Mearini, G. 2010. THz Source Specifications. G. J. Wilmink. Cleveland, OH: San Antonio Teraphysics Corporation.

Mittleman, D. 2003. Sensing with Terahertz Radiation. Berlin:Springer.

Moritz, A. R. 1947. Studies of thermal injury: III. The pathology and pathogenesis of cutaneous burns. An experimental study. Am J Pathol 23(6):915-41.

Moritz, A. R., and F. C. Henriques. 1947. Studies of thermal injury: II. The relative importance of time and surface temperature in the causation of cutaneous burns. Am J Pathol 23(5):695-720.

Mould, R. F. 1995. The early history of X-ray diagnosis with emphasis on the contributions of physics 1895-1915. Phys Med Biol 40(11):1741.

Mukherjee, M., N. Mazumder et al. 2007. GaN IMPATT diode: A photo-sensitive high power terahertz source. Semicond Sci Technol 22(12):1258-67.

Mukherjee, M., and S. K. Roy. 2010. Wide-Bandgap III-V nitride based avalanche transittime diode in Terahertz regime: Studies on the effects of punch through on high frequency characteristics and series resistance of the device. Curr Appl Phys 10(2):646-51.

Nishizawa, J., T. Kurabayashi et al. 2008. $706 \mathrm{GHz}$ GaAs CW fundamental-mode TUNNETT diodes fabricated with molecular layer epitaxy. Phys Status Solidi (c) 5(9):2802-4.

Oh, S. J., J. Kang et al. 2009. Nanoparticle-enabled terahertz imaging for cancer diagnosis. Opt Express 17(5):3469-75.

Oh, S. J., I. Maeng et al. 2008. Nanoparticle Contrast Agents for Terahertz Medical Imaging. Infrared, Millimeter and Terahertz Waves. IRMMW-THz 2008. 33rd International Conference.

Olshevskaya, J. S., A. S. Ratushnyak et al. 2008. Effect of terahertz electromagnetic waves on neurons systems. Computational Technologies in Electrical and Electronics Engineering, 2008. SIBIRCON 2008. IEEE Region 8 International Conference on: 210-211.

Pal, S. K., and A. H. Zewail. 2004. Dynamics of water in biological recognition. Chem Rev 104(4):2099-123. 
Pal, S. K., L. Zhao et al. 2003a. Site- and sequence-selective ultrafast hydration of DNA. Proc Natl Acad Sci U S A 100(24):13746-51.

Pal, S. K., L. Zhao et al. 2003b. Water at DNA surfaces: Ultrafast dynamics in minor groove recognition. Proc Natl Acad Sci U S A 100(14):8113-8.

Pal, S. K., J. Peon et al. 2002a. Biological water at the protein surface: Dynamical solvation probed directly with femtosecond resolution. Proc Natl Acad Sci U S A 99(4):1763-8.

Pal, S. K., J. Peon et al. 2002b. Ultrafast surface hydration dynamics and expression of protein functionality: Alpha -Chymotrypsin. Proc Natl Acad Sci US A 99(24):15297-302.

Pickwell, E., B. E. Cole et al. 2004. In vivo study of human skin using pulsed terahertz radiation. Phys Med Biol 49(9):1595-607.

Pickwell, E., A. J. Fitzgerald et al. 2005. Simulating the response of terahertz radiation to basal cell carcinoma using ex vivo spectroscopy measurements. J Biomed Opt 10(6):064021.

Pickwell-MacPherson, E., and V. P. Wallace. 2009. Terahertz pulsed imaging-a potential medical imaging modality? Photodiagn Photodyn Ther 6(2):128-34.

Pivonka, D. E., J. M. Chalmers et al. 2007. Applications of Vibrational Spectroscopy in Pharmaceutical Research and Development. Chichester, England; Hoboken, NJ: J. Wiley.

Protection, I. C. N. I. R. 2006. ICNIRP statement on far infrared radiation exposure. Health Phys 91(6):630-45.

Ramundo-Orlando, A. 2009. Terahertz Radiation Effects and Biological Applications. J Infrared, Millimeter and Terahertz Waves 30(12):1308-18.

Ramundo-Orlando, A., G. P. Gallerano et al. 2007. Permeability changes induced by $130 \mathrm{GHz}$ pulsed radiation on cationic liposomes loaded with carbonic anhydrase. Bioelectromagnetics 28(8):587-98.

Reichertz, L. A., O. D. Dubon et al. 1997. Stimulated far-infrared emission from combined cyclotron resonances in germanium. Phys Rev B 56(19):12069.

Rudolf, K. 1961. Backward Wave Tube. United States: English Electric Valve Co Ltd.

Russo, D., G. Hura et al. 2003. Hydration dynamics near a model protein surface. Biophys J 86(3):1852-62.

Ryzhii, V. 2009. Graphene tunneling transit-time terahertz oscillator based on electrically induced $\mathrm{p}-\mathrm{i}-\mathrm{n}$ junction. Appl Phys Express 2(3):034503.

Scarfi, M. R., R. Di Pietro et al. 2003. THz exposure of whole blood for the study of biological effects on human lymphocytes. J Biol Phys 29(2):171-6.

Segelstein, D. 1981. The Complex Refractive Index of Water. Kansas City, MO: University of Missouri.

Shi, W., Y. J. Ding et al. 2002. Efficient, tunable, and coherent 0.18-5.27 THz source based on GaSe crystal. Opt Lett 27(16):1454-6.

Siegel, P. H. 2002. Terahertz technology. Microwave Theory Tech IEEE Trans 50(3):910-28.

Singh, R. S., Z. D. Taylor et al. 2008. Towards THz medical imaging; reflective imaging of animal tissues. Stud Health Technol Inform 132:472-4.

Sirtori, C. 2009. Quantum cascade lasers: Breaking energy bands. Nature Photonics $3(1): 13-5$.

Smye, S. W., J. M. Chamberlain et al. 2001. The interaction between terahertz radiation and biological tissue. Phys Med Biol 46(9):R101-12. 
Suen, J. Y., P. Tewari et al. 2009. Towards medical terahertz sensing of skin hydration. Stud Health Technol Inform 142:364-8.

Suizo, K., and K. Kawase. 2008. Monochromatic-tunable terahertz-wave sources based on nonlinear frequency conversion using lithium niobate crystal. IEEE J Sel Top Quantum Electron 14(2):295-306.

Sun, Y., B. M. Fischer et al. 2009. Effects of formalin fixing on the terahertz properties of biological tissues. J Biomed Opt 14(6):064017.

Svanberg, S. 2004. Atomic and molecular spectroscopy: Basic aspects and practical applications. Verlag, Berlin, Heidelberg (Germany): Springer.

Taylor, Z. D., R. S. Singh et al. 2008. Reflective terahertz imaging of porcine skin burns. Opt Lett 33(11):1258-60.

Tewari, P., M. O. Culjat et al. 2009. Role of collagen in terahertz absorption in skin. San Jose, CA SPIE.

Thrane, L., R. H. Jacobsen et al. 1995. THz reflection spectroscopy of liquid water. Chem Phys Lett 240(4):330-3.

Thruvision. 2010. http://www.thruvision.com/index.html (accessed November 15, 2010).

Tonouchi, M. 2007. Cutting-edge terahertz technology. Nat Photonics 1(2):97-105.

Trentham, N. 1999. Hubble space telescope ultraviolet spectral energy distributions for three ultraluminous infrared galaxies. Astron J 117(5):2152.

The European Parliament and the Council of the European Union. 2006. Directive 2006/25/ EC of the European parliament and of the council. Off J Eur Union 114(1):38-59.

Venables, D. S., A. Chiu et al. 2000. Structure and dynamics of nonaqueous mixtures of dipolar liquids. I. Infrared and far-infrared spectroscopy. J Chem Phys 113(8):3243-8.

Venables, D. S., and C. A. Schmuttenmaer. 1998. Far-infrared spectra and associated dynamics in acetonitrile-water mixtures measured with femtosecond $\mathrm{THz}$ pulse spectroscopy. J Chem Phys 108(12):4935-44.

Venables, D., and C. Schmuttenmaer. 2000. Spectroscopy and dynamics of mixtures of water with acetone, acetonitrile, and methanol. J Chem Phys 113(24):11222-36.

Vodopyanov, K. 2008. Optical THz-wave generation with periodically-inverted GaAs. Laser Photonics Rev 2(1-2):11-25.

Vogel, A., and V. Venugopalan. 2003. Mechanisms of pulsed laser ablation of biological tissues. Chem Rev 103(2):577-644.

Wade, G., D. Smirnov et al. 2009. Magnetic-field-assisted terahertz quantum cascade laser operating up to $225 \mathrm{~K}$. Nature Photonics 3(1):41-5.

Walker, G. C. 2003. Two methods for modelling the propagation of terahertz radiation in a layered structure. J Biol Phys 29:141-8.

Wallace, V. P., A. J. Fitzgerald et al. 2004. Terahertz pulsed imaging of basal cell carcinoma ex vivo and in vivo. Br J Dermatol 151(2):424-32.

Wallace, V. P., P. F. Taday et al. 2004. Terahertz pulsed imaging and spectroscopy for biomedical and pharmaceutical applications. Faraday Discuss 126:255-63; discussion 303-11.

Walther, M., B. Fischer et al. 2000. Far-infrared vibrational spectra of all-trans, 9-cis and 13-cis retinal measured by $\mathrm{THz}$ time-domain spectroscopy. Chem Phys Lett 332(3-4):389-95. 
Wang, Q., R. W. Schoenlein et al. 1994. Vibrationally coherent photochemistry in the femtosecond primary event of vision. Science 266(5184):422-4.

Wang, L. V., and H.-i. Wu. 2007. Biomedical Optics: Principles and Imaging. Hoboken, NJ: Wiley-Interscience.

Ward, J. S., G. Chattopadhyay et al. 2008. Tunable Broadband Frequency-Multiplied Terahertz Sources. Infrared, Millimeter and Terahertz Waves, 2008. IRMMW-THz 2008. 33rd International Conference.

Webb, S. J., and D. D. Dodds. 1968. Inhibition of bacterial cell growth by 136 gc microwaves. Nature 218:374-5.

Wei Min Lee, A., Q. Qin et al. 2007. High-power and high-temperature THz quantumcascade lasers based on lens-coupled metal-metal waveguides. Opt Lett 32(19):2840-2.

Welch, A. J., and M. J. C. V. Gemert. 1995. Optical-Thermal Response of Laser-Irradiated Tissue. New York: Plenum Press.

Williams, B. S. 2007. Terahertz quantum-cascade lasers. Nature Photonics 1(9):517-25.

Wilmink, G. J., B. L. Ibey et al. 2011. Development of a compact terahertz time-domain spectrometer for the measurement of the optical properties of biological tissues. J Biomed Opt 16(4):047006-047010.

Wilmink, G. J., B. L. Ibey, et al. 2010. Determination of death thresholds and identification of terahertz $(\mathrm{THz})$-specific gene expression signatures. Optical interactions with tissue and cells XX. San Jose, CA; San Francisco, CA: SPIE.

Wilmink, G. J., S. R. Opalenik et al. 2006. Assessing laser-tissue damage with bioluminescent imaging. J Biomed Opt 11(4):041114.

Wilmink, G. J., S. R. Opalenik et al. 2008. In vivo optical imaging of hsp70 expression to assess collateral tissue damage associated with infrared laser ablation of skin. J Biomed Opt 13(5):054066.

Wilmink, G. J., S. R. Opalenik et al. 2009. Molecular imaging-assisted optimization of hsp70 expression during laser-induced thermal preconditioning for wound repair enhancement. J Invest Dermatol 129(1):205-16.

Wilmink, G. J., B. D. Rivest et al. 2009. The Optical Properties of Biological Tissues in the Terahertz Wavelength Range. Optical interactions with tissue and cells XX. San Jose, CA: SPIE.

Wilmink, G. J., B. D. Rivest et al. 2011. In vitro investigation of the biological effects associated with human dermal fibroblasts exposed to $2.52 \mathrm{THz}$ radiation. Lasers Surg Med 43(2):152-63.

Wilmink, G. J., B. D. Rivest et al. 2010b. Quantitative Investigation of the Bioeffects Associated with Terahertz Radiation. Optical interactions with tissues and cells XXI: SPIE.

Wilmink, G. J., and W. P. Roach. 2010. Measurement of the optical properties of skin using terahertz time-domain spectroscopic techniques. BIOS Proc SPIE 7562.

Wilmink, G. J., C. L. Roth et al. 2010. Identification of microRNAs associated with hyperthermia-induced cellular stress response. Cell Stress Chaperone 15(6):1027-38.

Wolbarst, A. B., and W. R. Hendee. 2006. Evolving and experimental technologies in medical imaging. Radiology 238(1):16-39.

Woodward, R. M., B. E. Cole et al. 2002. Terahertz pulse imaging in reflection geometry of human skin cancer and skin tissue. Phys Med Biol 47(21):3853-63. 
Woodward, R. M., V. P. Wallace et al. 2003. Terahertz pulse imaging of ex vivo basal cell carcinoma. J Invest Dermatol 120(1):72-8.

Xie, A. 2002. Excited-state lifetimes of far-infrared collective modes in proteins. J Biol Phys 28:147-54.

Yu, N., Q. J. Wang et al. 2010. Designer spoof surface plasmon structures collimate terahertz laser beams. Nat Mater 9(9):730-5.

Zalyubovskaya, N. P., R. I. Kiselev et al. 1970. To biological activity of radiation in millimeter and submillimeter ranges. Eksperimental'noy i Klinicheskoy Radiologii 6:202-5.

Zeni, O., G. P. Gallerano et al. 2007. Cytogenetic observations in human peripheral blood leukocytes following in vitro exposure to $\mathrm{THz}$ radiation: A pilot study. Health Phys 92(4):349-57.

Zernike, F., and P. R. Berman. 1966. Generation of far infrared as a difference frequency. Phys Rev Lett 16(3):117. 



\section{Index}

A

Absorbing boundary conditions (ABCs), 29, 33 Accelerating electron-based terahertz sources, 380-381

Access point, Wi-Fi, 309

Acoustic noise, 350-351

Adenosine triphosphate (ATP), 396

$\gamma$-Aminobutyric acid (GABA), 130, 135

Ampere-Maxwell law, 4

Analgesia, occurrence of, 129-130

Anatomically based models, 266-267

adults, $267-269$

children, 269-270

computed SAR in, 32

energy absorption in, 25-26

females, 270-271

Anatomical realism, 36

Anatomic head model, 351

Animal-size head model, transmitted pulse in, 49

Arrhenius integral, 396-397

Azimuthal angle, 86-87

\section{B}

Backward wave oscillators, 381-383

Band gap, THz radiation, 375

Basal cell carcinoma (BCC), 388

Base stations, exposure assessment, 303-309

$\mathrm{BBB}$, see Blood-brain barrier

Behavioral changes, in organisms, 127

BEM, see Boundary element method

Bessel functions, 91, 274

Biological dielectric properties, 97-102

Biological effects

of electromagnetic fields, 3

of magnetic fields, 116 intensities and frequencies, 123

mechanisms of, 117

on radical pair reactions, 119

Biological free radicals, 118

Biological systems, magnetic sensing mechanism, 116

Biological tissues

dielectric properties of, 200

electrical properties of, 199-200

Biomedical imaging, 40

Blood-brain barrier (BBB), 343

dysfunction, 350

Blood oxygenation level-dependent (BOLD), 161,168

Bluetooth hands-free devices, see Wired/ wireless hands-free devices

Body modeling uncertainty, 279

Body-worn devices, 35

Bone marrow-derived stromal cell (BMSC), 143,170

Bone marrow mesenchymal stem cells

(BMMSCs), 144

Bone mineral density (BMD) values, 139

Bone tissue engineering, 170

Boundary element method (BEM), 77, 215, $219,226,231,238$

Boundary techniques, 273

Brain-cell stimulation, rheobase for, 340

C

CAD, see Computer-aided design

Caenorhabditis elegans, 132

Cancer treatments, 171-173

Canonical models, 263-265

Carbamoyl cyanide m-chlorophenylhydrazone (CICCP), 152 
Cardiac pacemakers, static magnetic field in, 351

Cardiac stimulation, 340

Cardiosphere-derived cells (CDCs), 147

Cardiospheres (CSs), 147

Cell geometries, 85-90

Cell growth, 142-144

Cell membrane, 144-149

Cell-phone base stations, whole-body exposure, 35-38

Cell proliferation, 142-144

Cell suspensions, dielectric parameters, 102-105

Cellular death, 400-401

Cellular effects, 401-406 of magnetic fields, 134

by terahertz radiation, $407-408$

Cellular phone coupling of RF energy, 26

SAR, 27-28, 31-33

exposure assessment

dosimetry results, 291-297

parametric studies, $297-298$

positioning, 289-291

modeling of, 29-30, 287-289

Cellular phone standard (CEPHOS) project, 291

Cellular stress response (CSR), 400-401

Cellular telecommunication systems, 303

Central motor conduction time (CMCT), 354

Cerebellar inhibition (CBI), 160

Cerebellar stimulation, 354

Child-like approach, 269

Childlike head models, 36 absorption rate in, 34-35

Child-scaled models, 36

Circulatory system effects, 134-135

CMCT, see Central motor conduction time

Cole-cole analysis, 272

Computational methods, 273 analytical, 274

numerical, 274-279

Computer-aided design (CAD)

human models, 268

phone models, 30, 31

Constant conductivity spherical model, 50-52

Continuum approaches, for cell geometries, 85-90

Continuum mean-field approaches, spherical cells, 77,85

Laplace equation, 78

pore distribution function, 84

Smoluchowski equation, 79, 80, 83

Continuum Smoluchowski theory, 81
Contour recognition algorithms, 266

Contralesional dorsal premotor cortex

(cPMd), 160

Contrast agents for MRI, 349-350

Cortical gray matter (GM), 161

Corynebacterium glutamicum, 73

Courant condition, 28

Cryptochrome (CRY), 120, 125

Curie temperature, 336-337

Current densities, in human models, 235-237

Cylindrical geometries, Poisson's equation in, 91

\section{D}

Debye equation, 60, 61

Debye models, parameters for, 272

Diakoptic theory, 214

Diathermy, 337-338, 358

Dielectric constants, 98, 200

Dielectric parameters extracting from cell suspensions, 102-105 model for cell, 100

Dielectric properties biological, 97-102 tissues, 200, 272-273

Dielectric spheroidal model, 212-213

Difference frequency generation (DFG), 384

Differentiation in cell, 142-144

Digital system, 287

Dipole antenna, 12 electromagnetic energy from, 15 far field of, 15-16 quasistatic/near fields of, 13-15

Direct electromagnetic field heating, 334-335

Direct generation laser sources, 375-378

Discrete Fourier transform (DFT), 100

Dosimetry, 33, 199

experimental, 281 measurement devices and equipment, 284-286

uncertainty assessment, 286-287

macroscopic, 233

microscopic, 72, 233

numerical

in bioelectromagnetics, 242

canonical models, 263-265

ELF EMF, 202-205

uncertainty assessment, 279-281

$\mathrm{Wi}-\mathrm{Fi}, 312$

Double-shelled prolate spheroidal cell, $85-86,89,90$ 
Drosophila transgenesis, 125

Dugesia dorotocephala, 133

Dugesia tigrina, 132

Dysfunction, BBB, 350

E

Earnshaw's theorem, 118

Eddy currents, 202

Edge-element techniques, 277, 278

Effective isotropic radiated power (EIRP), 41

EFIE, see Electric field integral equation

EIRP, see Effective isotropic radiated power

Electrical breakdown, 73

Electric field distribution, 8

Electric field integral equation (EFIE), 275

Electric fields

in cells, modeling, 76-77

interactions

biological cells, 72

with living cells, 73-76

in tissues and nerves, 90-94

Electric flow potentials, 348

Electric pulsing, temperature changes, 94-97

Electrochemotherapy, 94

Electrodynamic forces, 348

Electrodynamic interactions, moving electrolytes, 122

Electroencephalography (EEG), 137

Electromagnetic compatibility, 351 sources, $357-358$

Electromagnetic coupling, 333

Electromagnetic (EM) radiation, 370

Electromagnetic energy, propagation of, 12-16

Electromagnetic fields (EMFs), 73, 118

Electromagnetic interference (EMI), 358

Electromagnetic pulses (EMPs)

in biological bodies, 41-43

effects of, 40

Gaussian, see Gaussian electromagnetic pulse propagation, $41,50-52$

constant conductivity spherical model for, $50-52$

transmitted, 50

waveform, 42

in spherical head model, 58

Electromagnetic tracking (EMT), 354

Electromedical devices, 332

Electronic implants, 344-345

Electronic solid-state terahertz devices, 378-380
Electrophosphene, 232

Electroporation, 75

ELF EMF, see Extremely low-frequency electromagnetic field

Embryonic cells, 133

EMFs, see Electromagnetic fields

EMPs, see Electromagnetic pulses

Enzyme-catalyzed reaction, SMF effect on, 119

Epidermal growth factor (EGF) receptors, 148

Erithacus rubecula, 125

Erythrocytes, 74

Escherichia coli, 125

European Committee for Electrotechnical Standardization, 282

European robins, 125

Experimental dosimetry, 281

measurement devices and equipment, 284-286

uncertainty assessment, 286-287

Extended SPFD (Ex-SPFD) method, 229

Extracellular matrix (ECM), 390

Extra high voltage (EHV) transmission line, 234

Extremely low-frequency electromagnetic field (ELF-EMF), 127, 148, 234

analgesic effect of, 130

biological effects of, 197-199

and biological systems, coupling between, 201-202

current densities in human models, 235-237

and development of human body model, 205-210

genotoxic effect of, 140

human models for

MRI based male and female, 207-208

phantom models, 207, 209, 210

whole-body model, 207, 210

ICNIRP guidelines, 239-242

numerical dosimetry approaches of, 202-205

research recommendations of, 244

validation of numerical dosimetry, 242-243

Extremely low-frequency (ELF) electric fields, 16, 17

coupling and dosimetry

homogeneous and heterogeneous human models, 216-217

other models, 219-220

realistically shaped models, 218-219, 220

simple and analytical models, 211-215 
current density in human model, 201, 216-217, 220

distribution, 18

human models for, 205-207

coupling of, 202

interaction, 10

spectrum, 3

Extremely low-frequency magnetic fields

coupling and dosimetry of other models, 233-234

realistically shaped models, 226-232

realistic human models, 223-225

simple and analytical models, 221-226, 226

current density in human model in, 201, 222-225

human models for, 207

coupling of, 202

phantom models, 207, 210

Extrinsic apoptotic pathway, 74

F

Faraday's law, 4, 203

Far field, of dipole antenna, 15-16

Far-IR gas lasers (FIR), 375

Fast-multipole surface-charge simulation method, 226

FCC, see Federal Communications Commission

FDTD, see Finite-difference time-domain

Federal Communications Commission (FCC), 27, 41

regulations, 305

FEM, see Finite-element method

Fenton reaction, 121-122

Ferromagnetic nanoparticles, 343

and heating, 335-336

FES, see Finite-element scheme

Field probe, dimensions of, 284

Finite-difference time-domain (FDTD), 397

algorithm, 28, 36, 275-279

method, 210, 218, 228, 230, 234, 241, 243

Finite-element method (FEM), 161, 215, 219, 222, 234, 277

numerical microdosimetry techniques, 89

Finite-element scheme (FES), 77

Finite-integration technique (FIT), 219, 229, 232,277

First-level controlled operating mode, MRI, 353
FIT, see Finite-integration technique

Free-electron lasers (FELs), 380

Free radical recombination reactions, weak SMF effects on, 119

Frequencies of magnetic fields, 123

Frequency down-conversion, 384-385

Frequency up-conversion, 378-379

Functional magnetic stimulation (FMS), 138

Functional vessel densities (FVDs), 172

\section{G}

Gadolinium (Gd), as contrast agents, 349

Gauss' electric law, 5

Gaussian electromagnetic pulse in planar biological medium, 44-48, 57 in spherical head models, $48-50,57-58$

Genotoxicity

in-vitro, 149-153

of terahertz radiation, 408

in vivo, $140-142$

Geomagnetic field, 125, 132

German Federal Board of Physicians and Health Insurance, 340

Glioblastoma treatment, 336

Glutathione (GSH) concentrations, 141

Green's function, 275, 280

Ground-penetrating radars (GPRs), 40

Gyromagnetic ratio, 346

\section{$\mathbf{H}$}

Hamster-derived insulin-secreting cells (HIT-T15), 146

Handheld transceivers, 40

Health Protection Agency (HPA), 313

Heating, magnetic nanoparticles, 335, 336

Heating blankets, 338-339

Heat therapy, 333-334, 338-339

Helianthus annuus, 154

Heterogeneous high-resolution European female head model, 291

Heterogeneous male model, 267

Heterogeneous phantom, 283

High-temperature zone, 396

Homogeneous 3D model, 268

Homogeneous head phantom, 282

Homogeneous spherical models, 234 of biological tissue, 17

Horseradish peroxidase (HRP), 119

Hugo's body model, 267 
Human body models

with cellular phones, 299

three-dimensional (3-D), 29

Human mesenchymal stem cells (hMSCs), 144

Human phantoms, 281-283

Human-size head model, transmitted pulse in, 49

Human umbilical vein endothelial cells

(HUVECs), 144

Hybrid techniques, 278-279

6-Hydroxydopamine (6-OHDA), 136

Hyperthermia

direct electromagnetic field heating, 334-335

targeted, 335-337

Hysteresis loop, 336

I

ICNIRP, see International Commission on Non-Ionizing Radiation Protection

Idealized box models, 30

IFAs, see Inverted-F antennas

IM, see Impedance method

Impact ionization avalanche transit-time devices (IMPATTs), 380

Impedance method (IM), 203, 222, 226, 229

Induced electric fields

and currents, 122-123

strengths, 348

Induced intracorporal current densities, 349

Inductive field, 14

Infinitely thin wire approximation, 287

Infrared cabins, 339

InP Gunn device, 379

Intensities of magnetic fields, 123

Intermediate frequency (IF) magnetic field, 198

International Commission on Non-Ionizing

Radiation Protection (ICNIRP),

219, 231, 239-242, 338, 348

International Commission on Radiation Protection (ICRP), 267

Intracorporal current density distribution, 339

Intracorporal electric signals, 341

Inverted-F antennas (IFAs), 312, 313

In vivo genotoxicity, 140-142

Iron ions, 121

Isotropic radiation patterns, 308

J

Japanese anatomical head model, 267-268

Joule effect, 147
K

Kirchhoff's integral (KI), 278

Kirchhoff's law, 222

$\mathbf{L}$

Laplace equation, 78, 85, 100

for prolate spheroids, 86

Larmor frequency, 345, 346

Lichtenecker's exponential law, 273

Linear curve-fitting procedure, 38

Lithiumniobatecrystals (LiNbO3), 385

Long-term plasticity (LTP), 135

Lorentz forces, 350

Low-frequency coupling, electric and magnetic fields, 17, 19-20

Low-temperature zone, 395-396

M

Macroscopic dosimetry, 233

Magnet coils, 342

Magnetically affected reaction yield (MARY) spectrum, 118

Magnetic anchoring guidance systems (MAGS), 344

Magnetic anisotropy, 335

Magnetic capsules, 343

Magnetic defibrillation, 340

Magnetic emissions, 360

Magnetic field distribution, AC current, 341

Magnetic field effects

in-vitro studies on, 142

cell membrane and metabolic activity, 144-149

genotoxicity, 149-153

on plant growth response, 153-155

in vivo studies on behavior and recognition, 127-129

biological sensing and magnetite, 125-127

circulatory system effects, 134-135

genotoxicity, 140-142

musculoskeletal system, 137-138

occurrence of analgesia, 129-130

reproduction and development, 131-134

Magnetic field gradients, 350

Magnetic fields, 7-9

antibacterial effects by, $156-157$

cellular effects of, 134 
low-frequency and, 10-11

medical applications of

magnetic resonance imaging, 165-169

magnetoencephalography, 164-165

TMS, 157-163

treatments of diseases, 171-173

Magnetic flux density, 117

Magnetic induction, 122

Magnetic interference, 343

Magnetic localization, 343

Magnetic moments, nanoparticles, 336

Magnetic nanoparticles, 343

applications, 350

selective heating of, 344

Magnetic navigation, 344, 355

Magnetic orientation, for tissue engineering, 169-171

Magnetic paclitaxel nanoparticles (MPNPs), cytotoxicity, 344

Magnetic resonance electrical impedance tomography (MREIT), 168

Magnetic resonance imaging (MRI), 165-169, 207, 226, 242, 244, 266

human models for ELF EMF, 207-208

limiting exposure, 351-353

operating modes of, 352

principle, 345-346

magnetic fields and EMFs, 347

unintended side effects, 347-351

Magnetic spin effects, in radical enzymatic reactions, 119

Magnetic stimulation magnetic defibrillation, 340

transcranial, 339-340, 354

Magnetic targeting, 343-344

Magnetic therapy, 340-341, see also specific therapies

pulsed fields, 341-342

static magnetic fields, 342-343

Magnetic tracking, 354

Magnetite, biological sensing and, 125-127

Magnetite-based magnetoreception, 126

Magnetization, 350

Magnetoencephalography, 164-165

Magnetofluid dynamics (MFD), 134

Magnetohydrodynamics (MHD), 122

Magnetophosphenes, 232

Magnetosomes, 155

Magnetotaxis, 155-156

Magnetotropism, 153-155

Mag-seeding technique, 170
Malignant glioma, 344

Matching boundary conditions, 87,88

Maxwell's equations, 3-4, 204, 242, 275, 277

Maxwell-Wagner relationship, 103

MC3T3-E1 cells, 147

Medical device, definition, 332

Medical therapy

diathermy, 337-338

heat therapy, 338-339

hyperthermia, 334-337

thermotherapy, 333-334

Medium temperature zone, 396

Messenger ribonucleic acid (mRNA), 138

Metabolic activity, 144-149

Metal ions, 121

Metallic implants, effect of, 294

Method of moment (MoM), 213, 274-275

MG63 cells, 145

Microarray gene expression, 409

Microdosimetry, 72

Microorganisms, response to magnetic fields, 153-157

Microscopic dosimetry, 233

Microtubule-associated protein 2 (MAP2), 146

Microwave cardiac ablation (MCA), 162

Millimeter waves

coupling of, 59

transmitted and reflected, 59-63

Mobile phones, see Cellular phone

MoM, see Method of moment

Motor-evoked potential (MEP), 136, 138, 162

MRI, see Magnetic resonance imaging

Musculoskeletal system, 137-138

\section{$\mathbf{N}$}

Nanoparticles, 335-336

magnetic, 343, 344

applications, 350

selective heating of, 344

magnetic moments, 336

superparamagnetism, 335

Nanosecond-pulsed electric fields, 2

Nano-second wide pulses, 40

National Institute of Information and Communication Technology

(NICT), 268

National Radiation Protection Board (NRPB), 267

Near field, of dipole antenna, 13-15

Neodymium-iron-boron (NdFeB) magnets, 343 
Nerve

electric field interactions in, 90-94

stimulation, 340

Neurodegenerative disorders, 121

Neuroendocrine system, 135-137

Neurophysiological system, 135-137

Nitric oxide (NO), 121

Node-based finite element technique, 278

Nonionizing radiation, 372

Nonlinear optical terahertz sources frequency down-conversion, 384 optical parametric oscillation, 384-385

Norepinephrine (NE), 135

Normal operating mode, MRI, 353

Norman model, 267

Numerical dosimetry in bioelectromagnetics, 242

canonical models, 263-265

ELF EMF, 202-205

uncertainty assessment, 279-281

Numerical method, 243

Numerical microdosimetry techniques, 89

Numerical model, 279

of dipole, 287-288

in SAR determination, 28

\section{O}

Optical parametric oscillation, 384-385

Optical rectification, 384

Osteoblastic cells, 149

\section{$\mathbf{P}$}

Pain, treatments of, 171-173

Parametric model, 272

Parkinson's disease (PD), 136

Parkinson syndromes, 354

PEMF therapy, see Pulsed EMF therapy

Perfectly matched layer (PML), 29

Permanent magnets, 342

Permittivity, 199, 200, 207

parameters, 61

Personal exposure monitors, 284

Photoconductive (PC) switch technique, 386

Photolyase, 125

Photothermal mechanisms, 392-394

Physical laws, electromagnetic phenomena, 3-5

PillCam ESO, 355

PillCam SB, 355

Planar-inverted-F antenna (PIFA), 302
Planar three-layer body model, 263-264

Planar tissue models, 50

Plane wave

in human body, 287

polarizations, 24

Plant growth response, magnetic effects on, 153-155

Plastic shell head models, 281-282

Pore distribution function, 84

Portable RF measurement devices, 284

Power density, for millimeter wave, 62

Poynting vector, 15

Pregnant woman SILVY model, 349, 350

Probe-size reduction, 285

Prolate spheroids, Laplace equation for, 86

p-type germanium laser (p-Ge), 376, 377

Pulsed electromagnetic fields (PEMFs), 129

Pulsed EMF (PEMF) therapy, 341-342

Pulsed fields, 341-342

Pulsed radio-frequency electromagnetic fields, 349

Pulse durations, 75

Purkinje cells, 160

\section{Q}

Quantum cascade lasers (QCLs), 375

Quasistatic electric field coupling, 16-18, 19-20

of dipole antenna, 13

low-frequency and, 10-11

Quasistatic magnetic field

coupling, $18-20$

of dipole antenna, 13

$\mathbf{R}$

Radiated energy, 304

Radiation zone, 14, 16

Radical pair theory, 119

Radiofrequency dosimetry, 25-26

Radio frequency electromagnetic field (RF EMF), 347

absorption of, 345

frequency of, 349

radiation, biological effects of, 261-262

Radiofrequency energy, orientation and polarization, 24

Radiofrequency (RF)

band, 2

emissions, 304 
energy, coupling from handheld mobile phones, 26

plane waves, characteristics, 22

radiation, 3

reflection/transmission, 21-22

surgery, 357-358

Radiofrequency (RF) field

coupling

to bodies with curvature, $22-24$

in far field/near field, 24-25, 39-40

and energy deposition, 20-21

Ray-tracing (RT) techniques, 278

Reactive field, 14

Reactive nitrogen species (RNS), 118

Reactive oxygen species (ROS), 118

Reactive region zone, 14

Regulated on activation, normal $\mathrm{T}$ cell expressed and secreted (RANTES), 148

Relative permittivity, of cell membrane, 100, 101

Remote patient monitoring, 356

Remote wireless data transmission, 345

Resonant tunneling diodes (RTDs), 380

Reverse Transcription-Polymerase Chain Reaction (RT-PCR), 132

$\mathrm{RF}$, see Radiofrequency

RF EMF, see Radio frequency electromagnetic field

Rheobase, for brain-cell stimulation, 340

Risk management, 358-359

Rotational/twisting forces, 348

RT techniques, see Ray-tracing techniques

\section{S}

Saccharomyces cerelisiae, 73

Safety risks, 348-349

SAM, see Specific anthropomorphic mannequin

SAR, see Specific absorption rate

Scalar potential finite difference (SPFD) method, 204-205, 218-219, 227-229, 241

Scanner, 285

Schottky barrier diodes (SBDs), 380

Second-level controlled operating mode, MRI, 353

Semianalytical solution method, 233-234

Sensor networks, 40

Short-term plasticity (STP), 135
Short-wave diathermy (SWD), 337, 358

Signature gene expression profile,

$\mathrm{THz}$ radiation, $408-410$

Simulation uncertainty, 279

Singlet-triplet (S-T) transitions, 125

Sinusoidal fields, 341

Skeletal muscle anisotropy, 228

Skeletal system, 138-139

Skin

anatomy, 389-391

optical properties of, 392

SMFs, see Static magnetic fields

Smoluchowski equation, 79, 80, 83

Smoluchowski theory, 85

Smooth endoplasmic reticulum (SER), 142

Spatial coding, 346

Specific absorption rate (SAR), 24, 207, 214, $262,298,335,397$

anatomical features on, 35

assessment, 349

distribution, 33, 294, 296, 300, 301, 356

fetal exposure, 351

for magnetic heating mechanism, 336

measurement of, 27,28

on millimeter-wave, 62-63

mobile phones

coupling of RF energy, 27-28, 31-33

quantity of, 26

restrictions, 353

Specific anthropomorphic mannequin

(SAM), 28

phantom, conservativeness of, 282

Spectroscopic fingerprint region, 372-375

Spectrum analyzers, 284

SPFD method, see Scalar potential finite difference method

Spherical coordinate system, 12

Spherical dielectric models, 234

Spherical head model

electromagnetic pulse waveform in, 58

Gaussian electromagnetic pulse, 48-50

Spheroidal cell model, 85-86

Spin-lattice time constant, 346

SPIONs, see Superparamagnetic iron oxide nanoparticles

Standing-wave phenomenon, 22

Static electric fields, 7-9

Static magnetic fields (SMFs), 73, 342-343, 347-349

in cardiac pacemakers, 351 
effect on enzyme-catalyzed reaction, 119

mechanisms of, 116-117

millitesla-level, 121

morphofunctional influence of, 142

Static magnets, 342

Steady state transfer function, 41

Stratum corneum (SC), 90

Streptozotocin (STZ), 130

Successive overrelaxation (SOR) method, 203

Superconducting quantum interference device (SQUID), 124

Superdiamagnetic nanoparticles, 343, 344

and heating, 335, 336

Superficial heating, in hyperthermia, 335

Superior parietal lobule (SPL), 136

Superparamagnetic iron oxide nanoparticles (SPIONs), 335

applications, 343

Superparamagnetism, in nanoparticles, 335

Supraporation, 84

Surface-charge integral equation (SCIE), 213

Surface polarization, 17

Switched magnetic gradient fields, 347 , 349,353

Sympathetic nervous system (SNS), 121

$\mathbf{T}$

Tail-flick latencies (TFLs), 130

Targeted hyperthermia, 335-337

Telemedicine, 355-356

Temperature changes, electric pulsing, 94-97

Temperature damage zones

and biological effects, 394-395

low-temperature zone, 395-396

medium- and high-temperature zones, 396

Tenebrio molitor, 133

Terahertz applications

skin interactions, 389

terahertz imaging, 387-389

terahertz spectroscopy, 385-387

Terahertz Bridge project, on bioeffects, 401-403

Terahertz free-electron lasers, 383-384

Terahertz frequencies, tissue damage, 396-398

Terahertz-induced tissue damage

characterizing, 396-397

modeling and empirical measures, 397-398
Terahertz radiation

bioeffects, 401-407

biological relevance with, 371-372

cellular effects by, $407-408$

direct effects of

on cellular organelles and

biomolecules, 408

genotoxic terahertz radiation, 406

sources

accelerating electron-based terahertz sources, 380-384

direct generation laser sources, 375-378

electronic solid-state terahertz devices, 378-380

nonlinear optical sources, 384-385

Terahertz safety standards, 398-399

Terahertz time-domain spectroscopy

(THz-TDS), 383-384

Terahertz-tissue optics, principles of, 391-392

Terahertz waves

coupling of, 59

transmitted and reflected, 59-63

Thermal history, 394-396

Thermal pain threshold (TPT), 129-130

Thermal susceptibility, tumor cell, 334

Thermographic method, 214

Thermotherapy, see Heat therapy

Thick wire approximation, 288

Thin model approximation, 288

THP-1 cells, 143

Through-wall sensing, 40

THz-wave parametric oscillator (TPO), 385

Time-domain analysis, 78

Time domain dielectric spectroscopy (TDDS), 102-103

Time-varying electromagnetic fields, 9

Time-varying magnetic fields, 122, 349

Tissue engineering, magnetic orientation for, 169-171

Tissue exposure, $344-345$

Tissue interfaces, electromagnetic phenomena, 6-7

Tissues

dielectric properties, $272-273$

electric field interactions in, 90-94

electromagnetic properties of, 5-6

TMP, see Transmembrane potential

TMS, see Transcranial magnetic stimulation

Topometric sensors, 31 
Total body water (TBW), 273

Transcranial magnetic stimulation (TMS), 124, 339-340, 354

and induced current, 157-163

Transient sensory effects, 348

Translational forces, 348

Transmembrane potential (TMP), 77, 85, 102, 219-220

magnitude of, 100, 101

values for pulse durations, 101, 102

Transmembrane voltage induction, 77, 78

classical theory of, 75

Travelling wave tubes (TWTs), 380

T-rays, 372

Triple stimulation technique (TST), 354

Tumor cell, thermal susceptibility, 334

Tunneling transit-time diodes

(TUNNETT), 380

$\mathbf{U}$

U937 cells, 142, 143

Ultra high voltage (UHV) transmission line, 234

Ultra-wideband (UWB) pulses

coupling of, 40-41, 56-57

field/current, induced, 52-56

induced current inside body, 58-59

spectrum of, 53

Uniform-downscaling approach, 269

Uniform theory of diffraction (UTD), 278

Unintended side effects, 337-338, 347-351

Universal mobile telecommunication system

(UMTS) cellular phones, 287

U.S. Food and Drug Administration

(FDA), 340

UTD, see Uniform theory of diffraction

UWB pulses, see Ultra-wideband pulses
V

Vascular endothelial growth factor (VEGF), 144

Vehicular radar, 40

Very low-frequency (VLF), human body model, 237

Video capsule endoscopes, 355

Visible human (VH) project, 29, 36, 267

Visual system, 135-137

Vitamin C, protective effects of, 133

Vitamin E, protective effects of, 133

Voltage-gated potassium channel (VGPC), 144

Voxel-based models, 268

W

Wada test, 168, 169

Wave propagation problems, 277

Wi-Fi systems, see Wireless fidelity systems

Wired/wireless hands-free devices, exposure assessment, 298-303

Wireless fidelity systems (Wi-Fi), exposure assessment, 309-313

Wireless medical telemetry, 355

Wireless personal area networks (WPAN), 40

Wireless transmission, 345

World Health Organization's (WHO), 312

$\mathbf{X}$

Xenopus embryos, 133

X-rays, 372

$\mathbf{Z}$

Zubal adult male model, 267 